\title{
PREIIMINARY REPORT
}

\author{
EASTROPAC CRUISE \\ ABOARD \\ R/V THOMAS WASHINGTON \\ 3 August to 25 september 1967
}

Prepared by

T. James Lewis

Eastern Area Cruise \#18 
PRELIMINARY REPORT

Eastern Area Cruise \#18

3 Aug to 25 Sept 1967

ITINERARY

3 August 1967 - Depart San Diego

21 August " - Reach $10^{\circ} \mathrm{S}, 119^{\circ} \mathrm{W}$

23 August " - Reach $10^{\circ} \mathrm{S}, 112^{\circ} \mathrm{W}$

26-27 August - Depart cruise track for rendezvous with $\mathrm{R} / \mathrm{V}$ UNDAUNTEED

29 August to - Special current study along $112^{\circ} \mathrm{W}$. at equator

2 September

11 September - Arrive Manzanilio, Mexico

13 September - Depart Manzanil1o, Mexico

19-23 September-Special study at south end of Guadalupe Island

25 September - Arrive San Diego

This report summarizes observations made by $T$. James Lewis on EASTROPAC cruise August 3 to September 25 aboard the Scripps Institution of Oceanography Research Vessel THOMAS WASHINGTON. The ship departed San Diego August 3 (see FIGURE I), proceeded to $20^{\circ} \mathrm{N}$, $119^{\circ} \mathrm{W}$, then south to $10^{\circ} \mathrm{S}$, then east to $112^{\circ} \mathrm{W}$, then north to the equator, with a short diversion eastward near $3^{\circ} \mathrm{S}$ to rendezvous with the other EASTROPAC vessel, R/V UNDAUNTED. After a 5-day current study near the equator, the ship proceeded north again along $112^{\circ} \mathrm{W}$ to $12^{\circ} \mathrm{N}$, and then northeastward to Manzanillo, Mexico. After two days in Manzanillo, the cruise continued westward and northwestward to Guadalupe Island for a 5-day study, and then directly to San Diego, arriving early in the morning of September 25. Observations of birds, mammals, and turtles, made on 45 days of the trip, are presented in TABLES $1-12$ and FIGURES $1-9$ at the end of this report. A total of 8,681 birds of 45 species were observed between sunrise and sunset. An additional 205 birds of 9 species were seen at night. Juan Fernandez Petrels, Sooty Terns and Wedge-tailed Shearwaters were the dominant birds in two rather small areas between $5^{\circ} \mathrm{N}$ and $7^{\circ} \mathrm{N}$ (FIGURE 3). Diurnal observations were conducted for 298.6 hours during which the ship traveled 3,040 miles. Nocturnal observations were conducted for 34.5 hours while the ship was underway.

Excellent cooperation was received from the captain and crew as well as from many of the scientific party. Use of the skiff on several occasions would not have been possible without their help. 


\section{Methods}

Observations were made from the bridge. During a few days when the wind was very calm or coming from our stern, observations were from the flying bridge. From the bridge only about three quarters of the horizon could be observed at any one time and many birds were surely missed because of this. Watch was maintained during nearly all of the diurnal hours that the ship was underway, and occasional checks were made when the ship was stopped on station.

Nocturnal observations were made while under way using a light from the Port Boat Deck. No observations were made during the last portion of trip because of the full moon. Time on station was not recorded as observation time even though occasional sightings were made during these periods.

\section{Discussion}

For convenience of analyses the trip has been divided into 8 areas: A - Aug 3-6, B - 7-16 Aug., C - 17-21 Aug., D - 23-28 Aug., E - 29 Aug.-2 Sept., F - 3-8 Sept., G - 9-10, 13-15 Sept., H - 16-18 and 24 Sept.

Area A - from San Diego to $20 \mathrm{~N}$ was dominated by Leach Storm Petrels and Northern Phalaropes. In the southern part Wedge-tailed Shearwaters, New Zealand Shearwaters, and Cook Petrels also became very important. These areas included many of the coastal birdfauna as well as some migrant ducks and landbirds.

Areas $B$ and $F$ from $20^{\circ} \mathrm{N}$ to the equator are characterized by a concentration of Pterodroma externa near $5^{\circ} \mathrm{N}$. This concentration occurs at the boundary between the North Equatorial counter current and the California coast.

Areas $C$ and $D$ from the equator to $10^{\circ} \mathrm{S}$ are very similar with a few scattered flocks of Sooty Terns in their southern parts.

Area $\mathrm{E}$, between $2^{\circ} 30^{\circ} \mathrm{N}$ and $\mathrm{S}$, along $112^{\circ} \mathrm{W}$, is the area of $\mathrm{Dr}$. B. A. Taft's current studies. August 29 th was spent at the Equator. On the 30 th to 31 st we went to $2^{\circ} 30^{\prime} \mathrm{N}$ and back to the Equator, and on September 1 and 2 , we went to $2^{\circ} 30^{\prime} \mathrm{S}$ and back. The only birds of note in this area were the Galapagos Storm Petrels following the ship, which made up nearly one-half of the observations.

Area $G$ is the coastal area off Manzanillo Mexico. In this area the predominantly land based sea birds such as boobies, terns, and gulls make up most of the observations.

Area $H$ is the final leg northbound from $20^{\circ} \mathrm{N}$ along the Baja California coast to Guadalupe, and between Guadalupe and San Diego. 
New Zealand Shearwater

This bird occurred only on August 5 about 250 miles southwest of Guadalupe Isl and.

\section{Manx Shearwater}

The listing of observations under this name means that all birds observed were not identified to race. These birds occurred south of Guadalupe and north of the Equator.

\section{Tahiti, Phoenix, and Tahiti/Phoenix Petrel}

Several of these very similar petrels were specifically identified only when they were observed at very close range.

\section{Herald Petrel} $119^{\circ} \mathrm{W}$.

Only one bird identified as Herald Petrel was seen near $7^{\circ} \mathrm{N}$ at

\section{Dark-rumped Petrel}

This Galapagos Isl and bird was in the area of high Shearwater/ Petrel populations north of the Equator at both $119^{\circ} \mathrm{W}$ and $112^{\circ} \mathrm{W}$.

\section{$\underline{P}$. externa}

Both races, Juan Fernandez Petrel and White-necked Petrel, were observed on this trip. The White-necked Petrels were separated by the heavier black border on the leading edge of the wing. This species was the predominant one for the survey but occurring almost exclusively in high concentrations at $5-7^{\circ} \mathrm{N}$ along both $119^{\circ} \mathrm{W}$ and $112^{\circ} \mathrm{W}$.

\section{Cook Petrel}

These birds were observed well off the Baja California coast.

\section{Bonin Petrel}

Mive of these Hawailan breeding birds were very distinctive when compared with the other small pterodroma. Their very dark collars and prominent slash in the wing made them unmistakable.

\section{Storm Petrel}

Birds not positively identified with white rumps were called White-rumped Storm Petrels. Birds with white rumps and almost all of the other characteristics of Leach Storm Petrel but not positively identified were called Leach-type Storm Petrels. Those called Storm Petrel sp. Were distant enough that no rump color or other characters could be observed. 
This bird was the dominant one following the ship throughout most of the trip.

\section{White-faced Storm Petrel}

Most of these birds were south of the large concentration at $5^{\circ} \mathrm{N}$ and occurred only on the eastern-most leg of the trip.

\section{Fork -tailed Petrel (?)}

This is a questionable sighting because of location but Ed Coughran and I both described the bird separately and felt that we were fairly sure of our observation.

\section{$\underline{\text { Sooty Tern }}$}

Records for this trip indicate that the Sooty Terns were using the areas of $5^{\circ}-10^{\circ} \mathrm{N}$ and $5^{\circ}-10^{\circ} \mathrm{S}$, and were virtually absent from the remaining areas. Most flocks that were closely observed contained immatures indicating they were from spring or sumer breeding populations.

\section{Mammals and Turtles}

Seven hundred five mammals of about eight species, and 12 turtles of two species were recorded (TABIE 12).

\section{Other Data Collecting Activities}

Routine collections of data proceeded as on previous EASTROPAC trips. Only one of the continuous recording environmental data buoys that had been anchored by the January EASTROPAC trip was found and serviced. A computer aboard ship made some of the oceanographic data available, but special programs had to be written depending on the type of analysis desired. These data can be obtained from BCF or Scripps. On this trip two special projects were carried out. One was a current study by Dr. B. A. Taft on the 29th August to 2 September near the Equator. The other was a vertical distribution study of a pelagic snail off the southern end of Guadalupe Island, carried out by Mr. Charles B. Miller. 


\title{
Scientific Personnel
}

\author{
\#Taft, Bruce Alan* SIO \\ Assistant Research Oceanographer \\ \#Abbott, John Lynn SIO \\ Associate Division Engineer \\ Behringer, David W. BCF \\ Student at SIO \\ Cornelius, John F. IBM \\ Customer Engineer \\ Coughran, Ed H. IBM \\ Representative \\ Daniels, Dick \\ SIO \\ Marine Technician \\ Edmond, John M. \\ SIO \\ Student \\ \#Wallace, Kenneth N.S.F. \\ High School Student \\ Judkins, David C. SIO \\ Student \\ Klapow, Laurence SIO \\ Student \\ Lewis, Thomas J. SI \\ Pacific Project \\ Michel, Fred A. SIO \\ Marine Technician \\ **Miller, Chas. B. SIO \\ Student \\ \#Powers, Robert E. SIO \\ Electrical Technician \\ Wagner, Robert W. IATMC \\ Laboratory Technician \\ \#Wong, Chi Shing SIO \\ Student \\ \#Wirth, David \\ SIO \\ Marine Technician \\ Wormuth, John H. SIO \\ Student \\ \#-Depart ship at Manzanillo \\ *-Scientist in charge \\ **-Join ship at Manzanillo \\ SIO-Scripps Institution of Oceanography \\ IATTC-Inter-Am Tropical Tuna Commission \\ NSF-National Science Foundation \\ BCF-Bureau of Commercial Fisheries \\ IBM-International Business Machines \\ SI-Smithsonian Institution
}


Area

Species or Group

Black-footed Albatross

Pink-footed Shearwater

Pale-footed Shearwater

Wedge-tailed Shearwater

New Zealand Shearwater

Sooty Shearwater

Sooty/Slenderbilled Shearwater

Christmas Shearwater

Manx Shearwater

Tahiti Petrel

Phoenix Petrel

Tahiti/Phoenix Petrel

Kermadec Petrel

Herald Petrel

Dark-rumped, Petrel

Juan Fernandez Petrel

White-necked Petrel

Pterodroma externa ssp.

Large Pterodroma sp.

Cook Petrel

Bonin Petrel

Black-winged Petrel

White-winged Petrel

Collared Petrel

Pterodroma leucoptera ssp

Smal1 Pteroaroma sp

Murphy Petre1

Pterodroma sp.

Bulver Petrel

Shearwater/Petrel sp.

Wilson (?) Storm Petrel

White-throated Storm Petrel

White-bellied Storm Petrel

Galapagos Storm Petrel

Harcourt (?) Storm Petrel

Leach Storm Petrel

(Socorro) Leach Storm Petrel Leach-type Storm Petrel sp

White-rumped storm Petrel sp

Black Storm Petrel

White-faced Storm Petrel

Fork-tailed Storm Petrel

Least Storm Petrel

Storm Petrel sp

Red-bilied Tropicbird

Red-talled Iropicbird

Tropicbira sp

Brown Pelican

Blue-footed Booby

Blue-faced Booby

Brown Booby

E E

G

H

Totals

4
1
28
16
11
12
3

\section{4}

1

8

$89 \quad 6 \quad 2$

1

16

11

12

3

3

332

1

12

311

1

16

106

71

1478

22

17

6

5

9

15

1

18

1

1

17

$17 \quad 8$

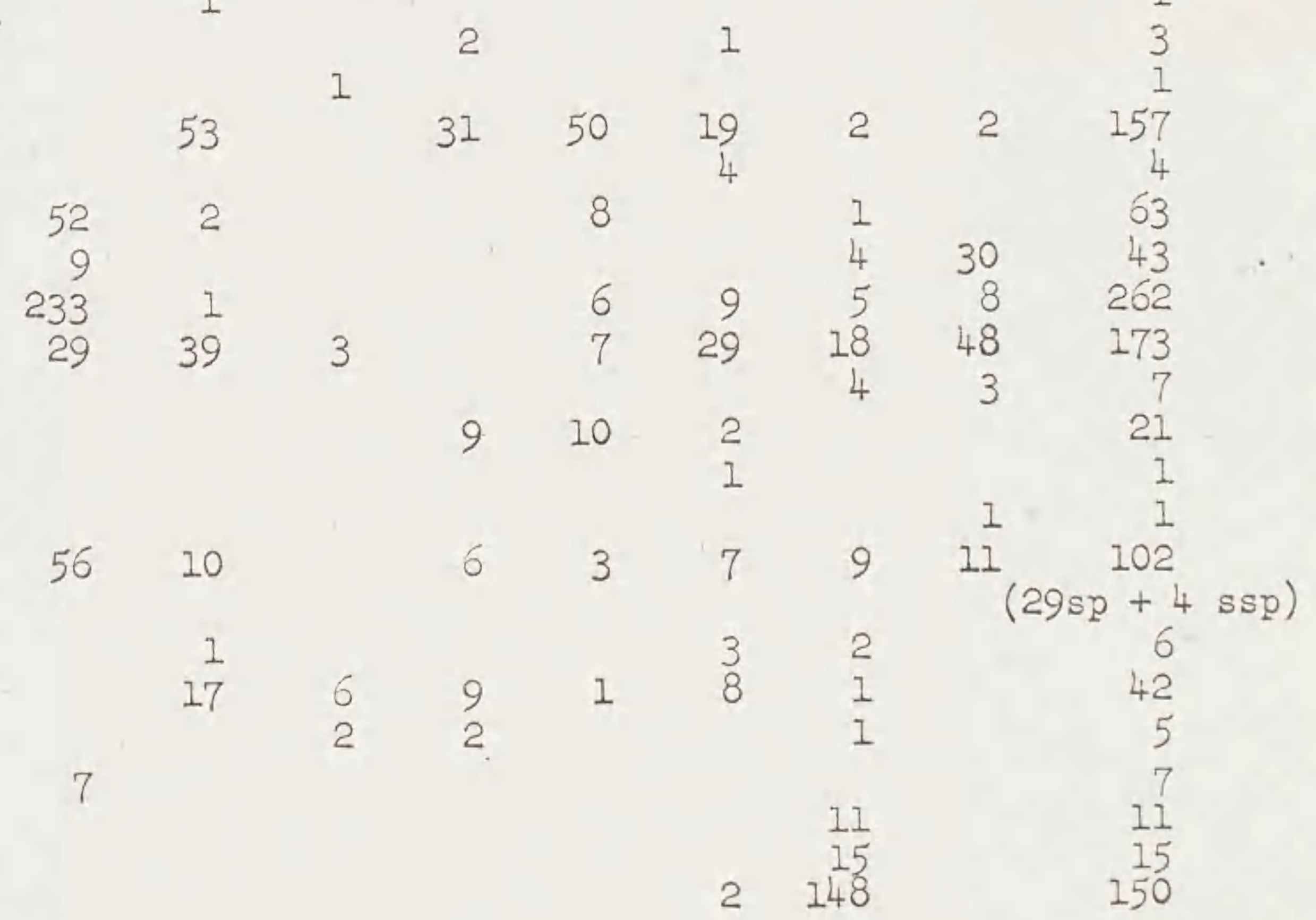


TABLE I (cont'd) p.2

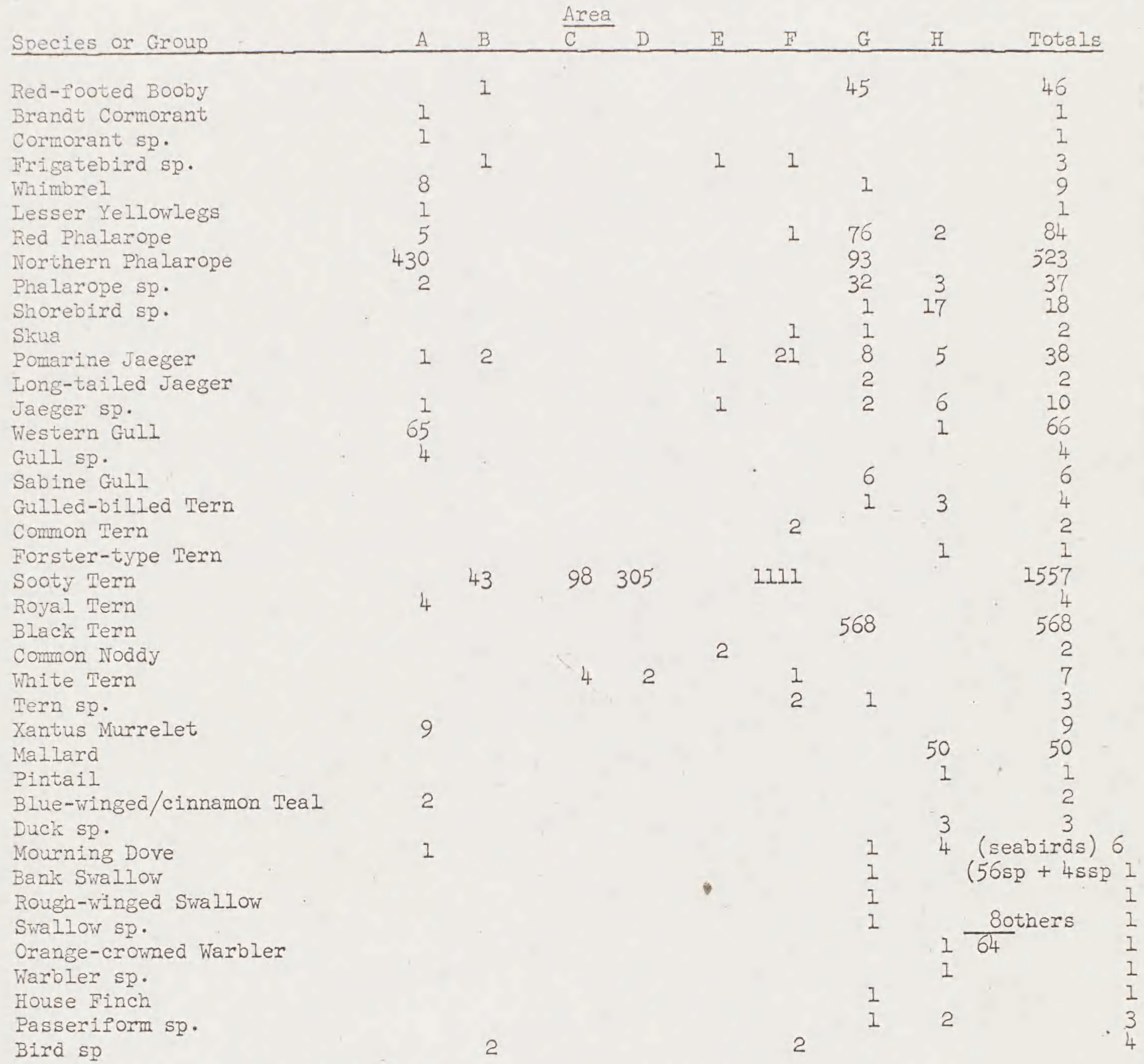

Totals Birds

No. of Species

No. Of Hours

No. of Miles

Birds per linear mile

\section{$1034 \quad 1019 \quad 199 \quad 467$}

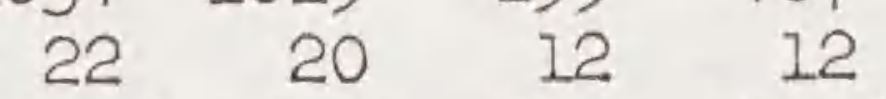

$\begin{array}{llll}27.5 & 53.0 & 34.1 & 43.6\end{array}$

$\begin{array}{llll}261 & 517 & 366 & 450\end{array}$

$\begin{array}{llll}3.97 & 1.97 & 0.54 & 1.04\end{array}$

\section{6}

11

3723

1914

209

$27 \quad 15$

$\begin{array}{lllll}27.0 & 40.0 & 35.4 & 38.0\end{array}$

$\begin{array}{llll}265 & 435 & 359 & 387\end{array}$

$\begin{array}{llll}0.44 & 8.56 & 5.33 & 0.54\end{array}$
8681

64

298.6

3040 
TABIE 2. Summary of Area A: San Diego to $20^{\circ} \mathrm{N}$, along $119^{\circ} \mathrm{W}$.

$3 \quad 4 \frac{\text { August }}{5} \quad 6 \quad$ Total

Black-footed Albatross

Pink-footed Shearwater

4

1

Wedge-tailed Shearwater

New Zealand Shearwater

Sooty Shearwater

Sooty/Slenderbilied Shearwater

Manx Shearwater

Pterodroma externa ssp

Cook Petrel

Murphy Petrel

Pterodroma sp

Shearwater/Petrel sp

Leach Storm Petrel

(Socorro) Leach Storm Petrel

Leach-type Storm Petrel

White-rumped Storm Petrel sp.

Storm Petrel sp.

\section{Brown Pelican}

Brandt Cormorant

Cormorant $\mathrm{sp}$

Whimbrel

Lesser Yellowlegs

Red Phalarope

Northern Phalarope

Phalarope sp.

Pomarine Jaeger

Jaeger sp.

Western Gull

Gull sp.

Royal Tern

Xantus Murrelet

Blue-winged/Cinnamon Teal

Mourning Dove

Total Birds

No. of Species

No. of Hours

No. of Miles

Birds/linear mile

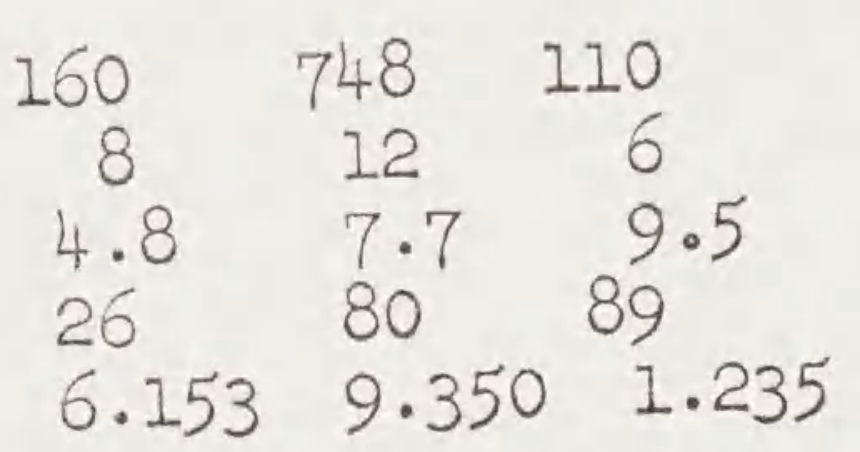

1
7
1

11

11213

12

37

$\begin{array}{lrl}1 & 1 & 1\end{array}$

1

$\begin{array}{rr}37 & 8 \\ 6 & 3\end{array}$

216

15

2

4

28

16

11

13
3

3

17

1

1

17

52

9

$15 \quad 14$

233

$15 \quad 28 \quad 13$

29

7 7

$\begin{array}{rr}1 & \\ 1 & \\ & 8 \\ & 1 \\ & 5 \\ 20 & 410 \\ 1 & \\ & 1 \\ & 1\end{array}$

1

1

8

1

430

I

65

2

1

65

4
4

9
2
1

2 
TABLE 3. SUMMARY OF AREA B: $20^{\circ} \mathrm{N}$ to Equator, along $119^{\circ} \mathrm{W}$.

\begin{tabular}{|c|c|c|c|c|c|c|c|c|c|c|c|}
\hline & 7 & 8 & 9 & 10 & $\frac{\text { August }}{11}$ & 12 & 13 & 14 & .15 & 16 & Total \\
\hline $\begin{array}{l}\text { Wedge-tailed } \\
\text { Shearwater }\end{array}$ & & & 1 & & 3 & 5 & 4 & 70 & & 6 & 89 \\
\hline $\begin{array}{l}\text { Tahiti Petrel } \\
\text { Phoenix Petrel }\end{array}$ & & & & & 1 & 1 & 1 & & I & & $\begin{array}{l}3 \\
1\end{array}$ \\
\hline $\begin{array}{l}\text { Tahiti/Phoenix } \\
\text { Petrel }\end{array}$ & & & & & 4 & & 1 & & & 1 & 6 \\
\hline $\begin{array}{l}\text { Kermadec Petrel } \\
\text { Herald Petrel }\end{array}$ & & & & & 1 & 1 & 1 & $I$ & & & \\
\hline Dark-rumped Petrel & 6 & & 3 & & 2 & 1 & 1 & 1 & & 2 & 16 \\
\hline $\begin{array}{l}\text { Juan Fernandez } \\
\text { Petrel }\end{array}$ & & & & & & 20 & 5 & $7 \overline{5}$ & 1 & 5 & 106 \\
\hline White-necked Petrel & & & & & 1 & 25 & 2 & 43 & & & 71 \\
\hline$\frac{\text { Pterodroma }}{\text { Cook Petrel }}$ externa ss & & & & & 1 & $\begin{array}{r}16 \\
2\end{array}$ & $\begin{array}{r}27 \\
4\end{array}$ & 425 & 2 & 7 & \\
\hline $\begin{array}{l}\text { Bonin Petrel } \\
\text { Black-winged Petrel }\end{array}$ & & . & & & & & 2 & $\begin{array}{l}3 \\
9\end{array}$ & & & \\
\hline $\begin{array}{l}\text { White-winged Petrel } \\
\text { Collared Petrel }\end{array}$ & & & & & & 2 & $\begin{array}{l}6 \\
1\end{array}$ & 3 & & 9 & $\begin{array}{r}0 \\
15\end{array}$ \\
\hline$\frac{\text { Pterodroma leucoptera }}{\mathrm{ssp}}$ & & & & & 1 & & & & & & \\
\hline $\begin{array}{l}\text { Smali Pterodroma } \mathrm{sp} \\
\text { Pterodroma } \mathrm{sp}\end{array}$ & & 1 & 1 & & 2 & 1 & 7 & 7 & & & 8 \\
\hline Bulwer Petrel & & & & & & & 3 & & & & \\
\hline $\begin{array}{l}\text { Shearwater/Petrel sp } \\
\text { Wilson(?) Storm } \\
\text { Petrel }\end{array}$ & 1 & 1 & & & $\begin{array}{l}2 \\
1\end{array}$ & 1 & 2 & 1 & & & 8 \\
\hline Galapagos Storm Petre & el & & & & 50 & & 1 & 2 & & & \\
\hline Leach Storm PetreI & 1 & 1 & & & & & & & & & 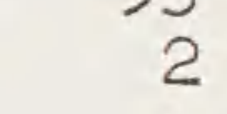 \\
\hline $\begin{array}{l}\text { Leach-type Storm } \\
\text { Petrel }\end{array}$ & & & & & & & & & & & \\
\hline $\begin{array}{l}\text { White-rumped Storm } \\
\text { Petrel sp }\end{array}$ & & 1 & & 3 & 28 & & & 4 & 2 & 1 & \\
\hline Storm Petrel sp & & & 1 & & 8 & & & 1 & & & 0 \\
\hline $\begin{array}{l}\text { Red-billed Tropic- } \\
\text { bird }\end{array}$ & & & & 1 & & & & & & & \\
\hline $\begin{array}{l}\text { Red-tailed Tropic- } \\
\text { bird }\end{array}$ & 1 & 6 & 2 & 4 & & 1 & 1 & & 2 & & \\
\hline Red-footed Booby & 1 & & & & & & & & & & \\
\hline Frigatebird sp & & & & & & & & & & 1 & \\
\hline $\begin{array}{l}\text { Pomarine Jaeger } \\
\text { Sooty Tern }\end{array}$ & & & & 1 & & & & 1 & & & \\
\hline $\begin{array}{l}\text { Sooty Tern } \\
\text { Bird sp }\end{array}$ & 1 & 1 & & & & & & 3 & & 40 & \\
\hline
\end{tabular}

Total Birds

No. of Species

12

$\begin{array}{rrrr}8 & 9 & 105 & 76 \\ 4 & 4 & 8 & 8\end{array}$

$69 \quad 649$

$\begin{array}{rr}8 & 72 \\ 5 & 7\end{array}$

1019

No. of Hours

$\begin{array}{ll}12 & 11 \\ 5 & 3\end{array}$

$5.4 \quad 4.4 \quad 4.4$

$4.4 \quad 4.0$ $\begin{array}{lllll}46 & 42 & 53 & 50 & 47 \\ 0.260 & 0.261 & 0.150 & 0.180 & 2.23\end{array}$

11

9

$\begin{array}{lll}6.8 & 6.8 & 6.5\end{array}$

No. of Miles

Birds/linear mile

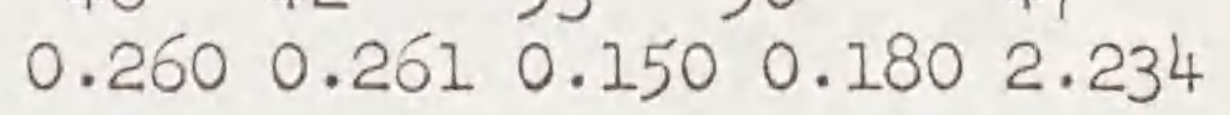
$\begin{array}{lll}58 & 52 \quad 67\end{array}$

$3 \cdot 1$

7.2

20

$1.310 \quad 1.326 \quad 9.686$

38

64

53.0

517 
TABLE 4. SUMMARY OF AREA C: Equator to $10^{\circ} \mathrm{S}$, along $119^{\circ} \mathrm{W}$

August

17

Wedge-tailed Shearwater

Christmas Shearwater

Tahiti Petrel

Phoenix Petrel

Tahiti/Phoenix Petrel

Kermadec Petrel

Juan Fernandez Petrel

White-necked Petrel

Pterodroma externa ssp

Collared Petrel

Sma 11 Pterodroma

Pterodroma sp.

Shearwater/Petrel sp.

White-bellied Storm Petrel

White-rumped Storm Petrel sp.

Rea-tailed Tropicbird

Tropicbira sp

Sooty Tern

White Tern

Total Biras

No. of Species

No. of Hours

No. of Miles

Biras per linear mile
18

19

20

21

Total

$\begin{array}{lr} & \\ 1 & 2 \\ & \\ & 1 \\ 1 & 3 \\ & \end{array}$

2

1

5
1
3

6

3

1

2

1

1

1

28

3

9

2

4

2

1

4

1

2

2
98
4

$\begin{array}{cc}5 & 20 \\ 2 & 4 \\ 5.8 & 5.7 \\ 82 & 58 \\ 0.060 & .344\end{array}$

22
5
7.4
77
0.285

14

7

8.6

79

0.177
199

12

34.1 366

0.544 
TABLE 5. SUMMARY OF AREA D: $10^{\circ} \mathrm{S}$ to Equator a long $112^{\circ} \mathrm{W}$

\begin{tabular}{|c|c|c|c|c|c|c|c|}
\hline & 23 & 24 & 25 & 26 & 27 & 28 & Total \\
\hline Wedge-tailed Shearwater & 2 & & & & & & 2 \\
\hline Tahiti Petrel & 2 & & & & & & 2 \\
\hline Phoenix Petrel & 2 & & & & & & 2 \\
\hline Tahiti/Phoenix Petrel & 2 & 2 & & & & & 4 \\
\hline Kermadec Petrel & & 1 & & & & & 1 \\
\hline Juan Fernandez Petrel & 1 & 1 & & & & & 2 \\
\hline Pterodroma externa ssp & & & I & & I & & 2 \\
\hline Large Pterodroma sp & & & & & & 1 & 1 \\
\hline Collared Petrel & 5 & II & 14 & 2 & 13 & & 45 \\
\hline Shearwater/Petrel sp. & 13 & 10 & 12 & 1 & 4 & & 40 \\
\hline White-throated Storm Petrel & & & & & 2 & & 2 \\
\hline Galapagos Storm Petrel & & & & 9 & 10 & 12 & $3 I$ \\
\hline White-faced Storm Petrel & & 4 & 2 & & 1 & 2 & 9 \\
\hline Storm Petrel sp & & 2 & 1 & 3 & & & 6 \\
\hline Rea-tailed Tropicbịa & 9 & & & & & & 9 \\
\hline Tropicioird sp & 2 & & & & & & 2 \\
\hline $\begin{array}{l}\text { Sooty Tern } \\
\text { White Tern }\end{array}$ & 103 & 194 & 7 & 1 & & & 305 \\
\hline White Tern & & 1 & 1 & & & & 2 \\
\hline Total Birds & 141 & 226 & 38 & 16 & 31 & 15 & 467 \\
\hline No. of Species & 7 & 7 & 5 & 3 & 5 & 3 & 12 \\
\hline No. of Hours & $7 \cdot 5$ & 6.8 & 6.4 & 10.0 & 8.8 & 4.1 & 43.6 \\
\hline $\begin{array}{l}\text { No. of Miles } \\
\text { Birds per linear mile }\end{array}$ & $\begin{array}{l}74 \\
1.905\end{array}$ & $\begin{array}{l}73 \\
3.095\end{array}$ & ${ }^{58} .655$ & $\begin{array}{l}92 \\
0.173\end{array}$ & $\begin{array}{l}102 \\
0.303\end{array}$ & $\begin{array}{l}51 \\
0.294\end{array}$ & $\begin{array}{l}450 \\
1.038\end{array}$ \\
\hline & & & & 0.173 & & & \\
\hline
\end{tabular}


TABLE 6. SUMMARY OF AREA E: between $2^{\circ} 30^{\prime} \mathrm{N}$ and $\mathrm{S}$, along $112^{\circ} \mathrm{W}$.

\begin{tabular}{|c|c|c|c|c|c|c|}
\hline & \multirow{2}{*}{\multicolumn{2}{|c|}{$\frac{\text { August }}{30}$}} & \multicolumn{3}{|c|}{ September } & \multirow[b]{2}{*}{ Total } \\
\hline & & & 31 & 1 & 2 & \\
\hline $\begin{array}{l}\text { Pale-footed Shearwater } \\
\text { Tahiti/Phoenix Petrel } \\
\text { Collared Petrel } \\
\text { Small Pterodroma sp } \\
\text { Pterodroma sp } \\
\text { Bulwer Petrel } \\
\text { Shearwater/Petrel } \\
\text { Galapagos Storm Petrel } \\
\text { Leach Storm Petrel } \\
\text { Leach-type Storm Petrel } \\
\text { White-rumped Storm Petrel sp } \\
\text { White-faced Storm Petrel } \\
\text { Storm Petrel sp } \\
\text { Red-tailed Tropicbird } \\
\text { Frigatebird sp } \\
\text { Pomarine Jaeger } \\
\text { Jaeger sp } \\
\text { Common Noddy }\end{array}$ & 1 & $\begin{array}{l}2 \\
5 \\
1 \\
\end{array}$ & $\begin{array}{r}1 \\
19 \\
2 \\
3 \\
5 \\
1 \\
1\end{array}$ & $\begin{array}{l}8 \\
2 \\
2 \\
1 \\
1\end{array}$ & $\begin{array}{r}6 \\
1 \\
2 \\
21 \\
4 \\
1 \\
3 \\
1 \\
\end{array}$ & $\begin{array}{r}1 \\
1 \\
16 \\
2 \\
1 \\
1 \\
4 \\
50 \\
8 \\
6 \\
7 \\
10 \\
3 \\
1 \\
1 \\
1 \\
1 \\
2\end{array}$ \\
\hline $\begin{array}{l}\text { Total Birds } \\
\text { No. of Species } \\
\text { No. of Hours } \\
\text { No. of Miles } \\
\text { Biras per linear mile }\end{array}$ & $\begin{array}{l}4 \\
3 \\
--\end{array}$ & $\begin{array}{l}18 \\
5 \\
8.6 \\
74 \\
0.243\end{array}$ & $\begin{array}{l}33 \\
4 \\
7.1 \\
79 \\
0.417\end{array}$ & $\begin{array}{l}21 \\
6 \\
9.2 \\
87 \\
0.241\end{array}$ & $\begin{array}{l}40 \\
6 \\
2.1 \\
25 \\
1.600\end{array}$ & $\begin{array}{l}116 \\
11 \\
27.0 \\
265 \\
0.438\end{array}$ \\
\hline
\end{tabular}


TABLE 7. SUMMARY OF AREA $\mathrm{F}$ : Equator to $15^{\circ} \mathrm{N}$, mostly along $112^{\circ} \mathrm{W}$.

September

$3 \quad 4 \quad 5 \quad 6$

Pink-footed Shearwater

Wedge-tailed Shearwater

Christmas Shearwater

Manx Shearwater

Tahiti Petrel

Phoenix Petrel

Tahiti/Phoenix Petrel

Kermadec Petrel

Dark-rumped Petrel

Juan Fernandez Petrel

White-necked Petrel

Pterodroma externa ssp

Black-winged Petrel

Collared Petrel.

Small Pterodroma $\mathrm{sp}$

Murphy PetreI

Pterodroma sp

Bulwer Petrel

Shearwater/Petrel sp

White-throated Storm Petrel

Galapagos Storm Petrel

Harcourt(?) Storm Petrel

Leach-type Storm Petrel

White-rumped Storm Petrel sp

White-faced Storm Petrel

Fork-tailed Storm Petrel

Storm Petrel sp

Red-billed Tropicbird

Red-tailed Tropicbird

Brown Booby

Frigatebird $s p$

Red Phalarope

Skua

Pomarine Jaeger

Common Tern

Sooty Tern

White Tern

Tern sp

Bird sp

Total Birds

No. of Species

No. of Hours

No. of Miles

Birds per linear mile

$\begin{array}{rr}1 & 2 \\ 46 & 462 \\ & 1 \\ & 2\end{array}$

2
62

2

1
1 $\quad 4$

81

81
4

76

5

76

1

9

$1 \quad 4629$

1

1

14

$\frac{1}{6}$

6

1

6

1

4

1

126

$427 \quad 681$

1

$9 \quad 823 \quad 2156$

$\begin{array}{lll}9 & 15 & 15\end{array}$

$\begin{array}{lll}6.1 & 6.8 & 7.2\end{array}$

$\begin{array}{lll}76 & 88 & 76\end{array}$

$\begin{array}{lll}0.118 & 9.352 \quad 28.368\end{array}$

4

202

2

1

1

I
3
1

8

Total

7

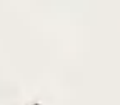
3
594
2
7

1

12

61

3

1

11

1

3

3

19

215

30

5

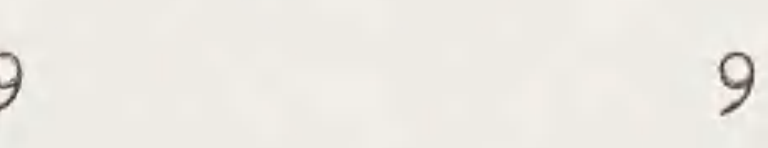

9

4

23

2

81

$$
3
$$

1

15
4

1345

14

261

31

108

3

9

1

$8 \frac{1}{88}$

1

19

4

9

29

1

2

1
1
2

1

2

1

3
2

3

1

$$
\begin{array}{r} 
\\
2 \\
1 \\
159 \\
8 \\
6
\end{array}
$$
255
11
5.8
45

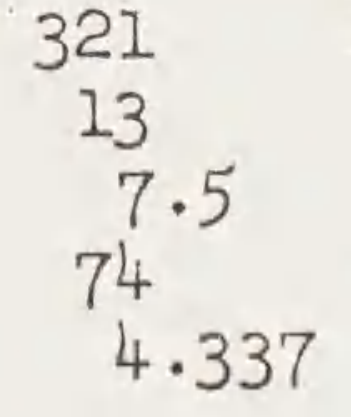

59
8
6.6
76

5.666

2.092

3723

28

40.0

435

8.56 
TABIE 8. SUMMARY OF AREA G: within 400 miles of Manzanillo, Mexico

September

9 10 (skip) 13

14

15

Total

Pink-footed Shearwater

Pale-footed Shearwater

Wedge-tailed Shearwater

Manx Shearwater

Juan Fernandez Petrel

Pterodroma externa ssp

Shearwater $/$ Petrel sp

Galapagos Storm Petrel

Leach Storm Petrel

(Socorro) Leach Storm Petrel

Leach-type Storm Petrel

White-rumped Storm Petrel sp

Black Storm Petrel

Storm Petrel sp

Red-billed Tropicbird

Red-tailed Tropicbird

Tropicbird sp

Blue-footed Booby

Blue-faced Booby

Brown Booby

Red-footed Booby

Thimbrel

Red Phalarope

Northern Phalarope

Phalarope sp

Shorebird sp

Skua

Pomarine Jaeger

Long-tailed Jaeger

10 (skip) 13

14

4

$\begin{array}{rr}2 & \\ 86 & 3 \\ 41 & \\ 301 & 19 \\ 4 & \\ 218 & 18 \\ 1 & 1 \\ 1 & \end{array}$

4

4
8

$8 \quad 137$

$1 \quad 134$

19

6
88

1
34
4

1

321

$18 \quad 5$

1

4

1

5

242

I

41

12

Jaeger $\mathrm{sp}$

Sabine Gull

Gull-billed Tern

Black Tern

Tern sp

Mourning Dove

Bank Swallow

Rough-winged Swallow

Swallow sp

House Finch

Passeriform sp

Total Birds

No. of Species

No. Of Hours

No. of Miles

Birds per linear mile

$\begin{array}{ll}1 & 3 \\ 1 & 1 \\ 1 & \end{array}$

2

1

4
2

3

11

4

1

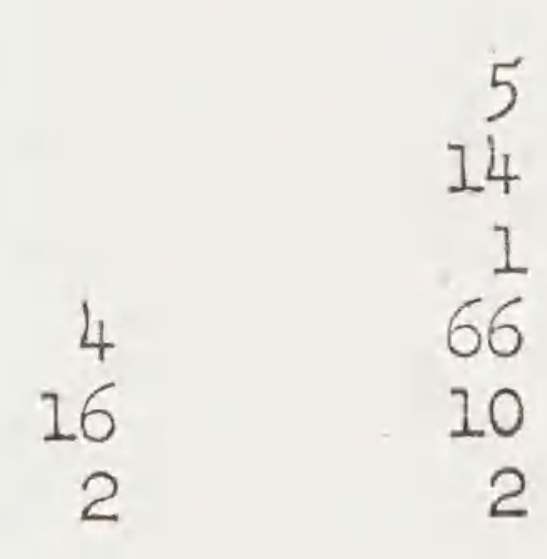

3
2
7
21

136

14

66

10

1

2

4

41

2

2

23

25

1

3

3
2
1

6

1

$568+$

1

1

1

1

I

1

691

15

9.1

87

7.942

156

12

$7 \cdot 7$

83

1.879

857
9
3.0
30
28.566

149

18

$7 \cdot 5$

72

2.069

$\begin{array}{rr}61 & 1914 \\ 7 & 27\end{array}$

$8.1 \quad 35.4$

87

359

0.701

5.33 
TABLE 9. SUMMARY OF AREA H $-20^{\circ} \mathrm{N}$ to San Diego, via Guadalupe Island

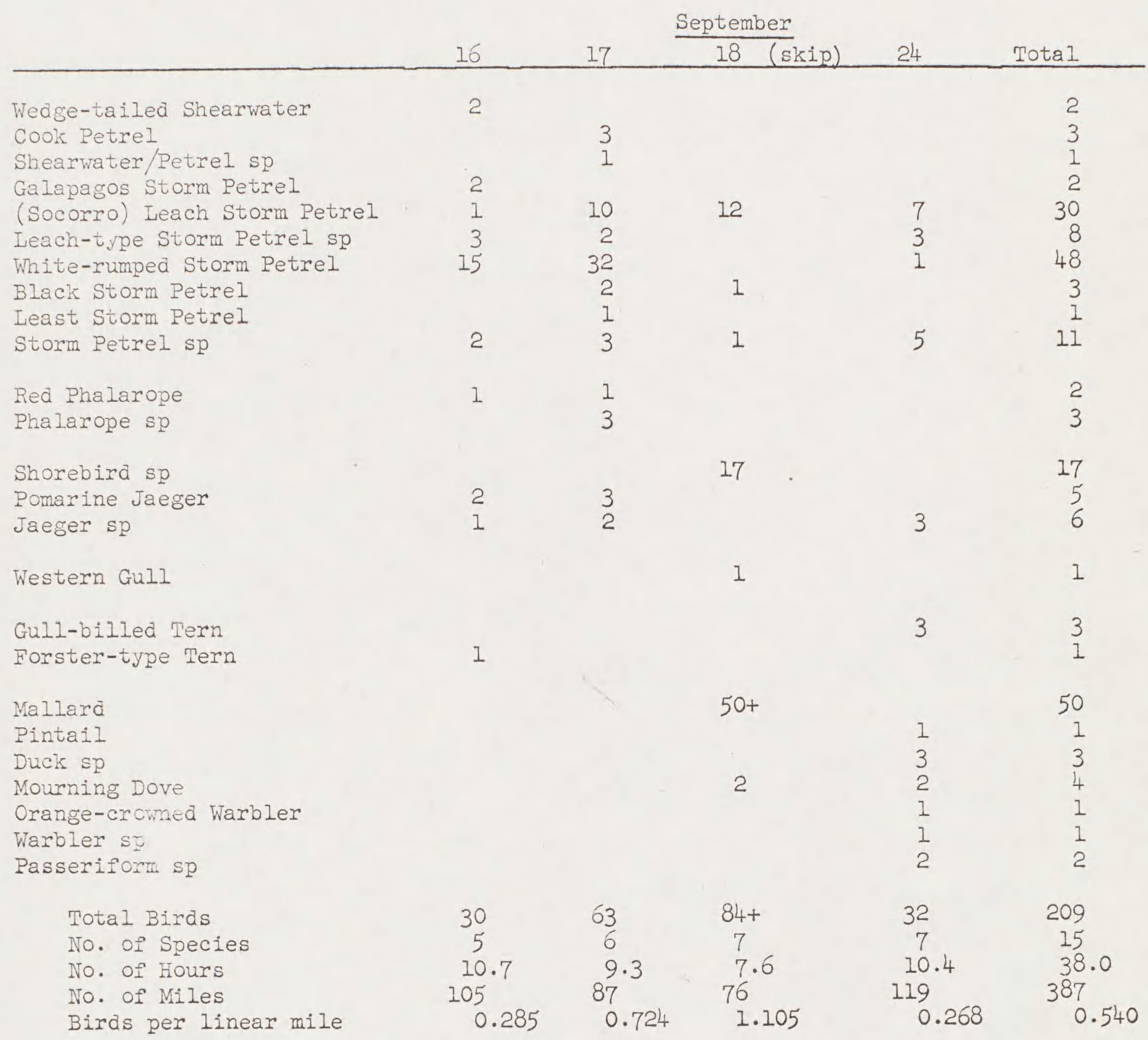




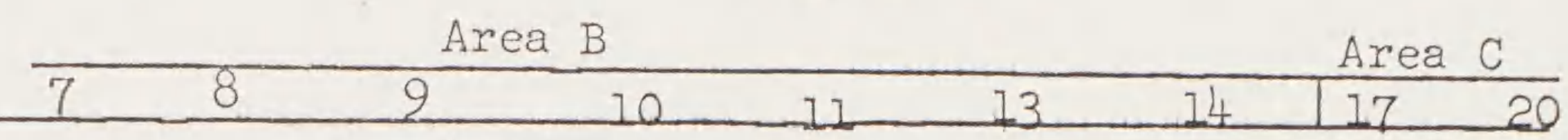

Wedge-tailed Shearwater

Pterodroma externa

Small Pterodroma

Shearwater/Petrel

White-rumped Storm Petrel sp

Storm Petrel sp

Sooty Tern

Bird $\mathrm{sp}$

Total Birds

No. of hours

\section{Petrel sp}

1

$\begin{array}{ll}1 & 1 \\ 4 & 1 \\ 2 & 1\end{array}$

1
5

1

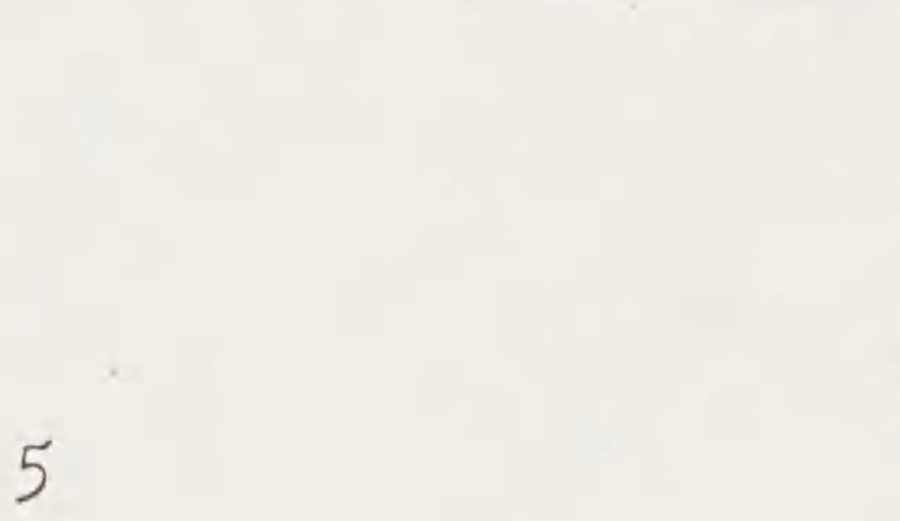

Tahiti/Phoenix Petrel Juan Fernandez Petrel

Pterodroma externa

Collared Petrel

Small Pterodroma

Shearwater/Petrel

White-rumped Storm

Petrel sp

White-faced Storm

Petrel

Storm Petrel sp

Red-tailed Tropicbird

Phalarope sp

Sooty Tern

Bird $\mathrm{sp}$

Total biras

No. of hours

$\begin{array}{lllllllll}5 & 1 & 0 & 1 & 0 & 8 & 2 & 0 & 0 \\ 2.0 & 1.0 & 1.3 & 2.0 & 0.8 & 2.0 & 2.0 & 1.0 & 2.0\end{array}$

August/September

\begin{tabular}{lllll|llll|lllll|l}
\hline \multicolumn{4}{c}{ Area $D$} & Area E F & \\
23 & 24 & 25 & 26 & 27 & 30 & 31 & 1 & 2 & 3 & 4 & 5 & 7 & Total \\
\end{tabular}

TABLE 11. SPECIMENS COLLECTED (AII skins)

Galapagos Storm Petrel 7

Leach Storm Petrel 11

White-faced Storm Petrel 2

Red-tailed Tropicbird I

Red-footed Booby 2

Red Phalarope I

Northern Phalarope 1

Parasitic Jaeger I

Western Gull 3

Orange-crowned Warbler 1

Yellow Warbler 1

Total $\overline{31}$ 
California Sea lion

13 August 1967

Seal sp

13 August 1967

Common Dolphin

553 August 1967

Right Whale Dolphin

1501 September 1967

Dolphin sp?

503 August 1967

6028 August 1966

15531 August 1967

1502 september 1967

504 September 1967

Pilot Whale

311 August 1967

False Killer Whale

319 August 1967

Killer Whale

$1 \quad 14$ August 1967

Sperm Whale

$1 \quad 17$ August 1967

Sei Whale

I $\quad 11$ August 1967

24 September 1967

Whaie $\mathrm{sp}$

$1 \quad 11$ August 1967

$4 \quad 17$ August 1966

218 August 1967

$2 \quad 31$ August 1967

I 10 September 1967

$2 \quad 17$ September 1967

Total Mammals recorded: 705 
Loggerhead (?) Turtle

$1 \quad 13$ August 1967

$1 \quad 27$ August 1967

$1 \quad 14$ September 1967
Green Turtle

$5 \quad 10$ September 1967

414 September 1967

Total turtles recorded: 12 


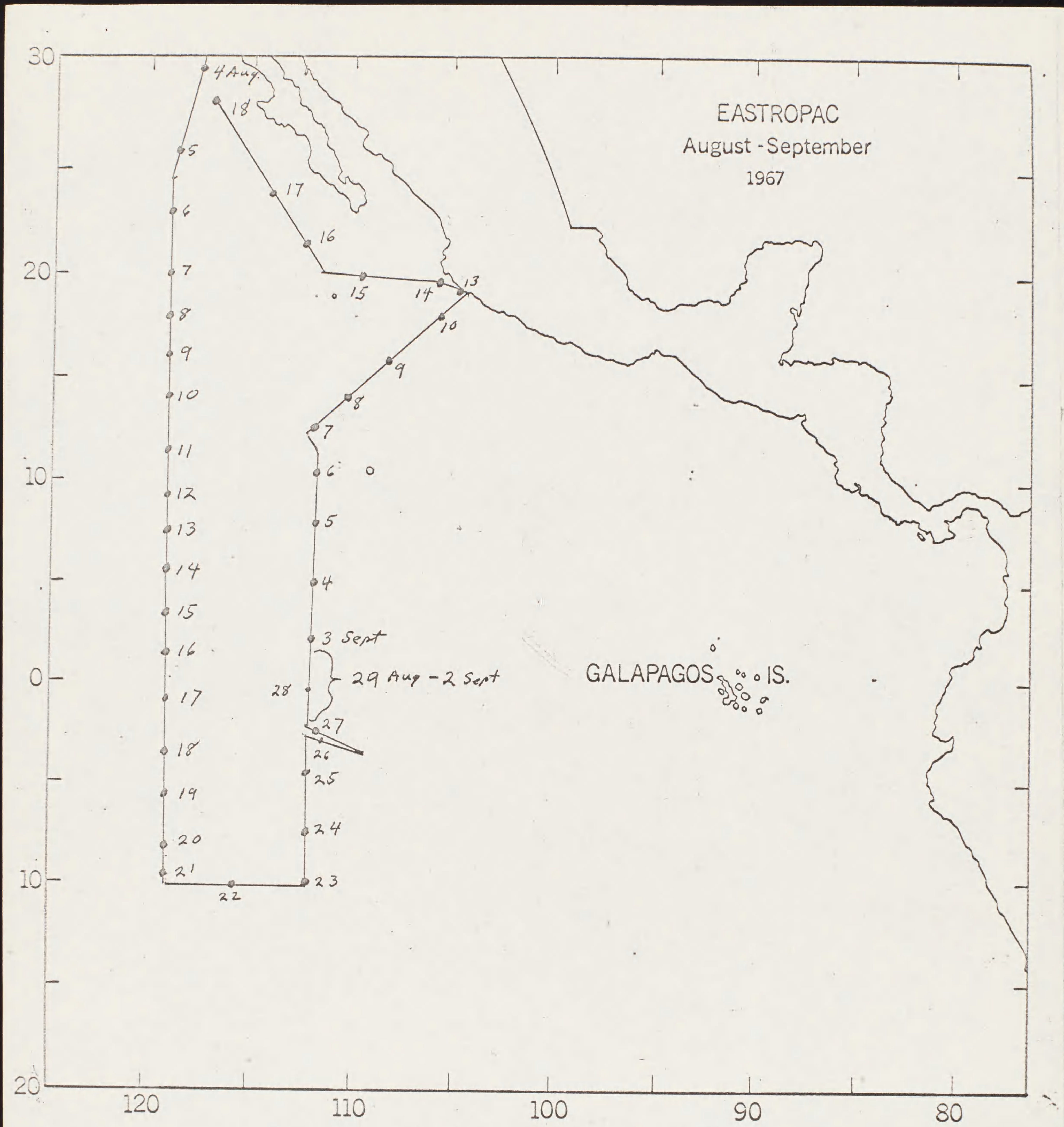

FIGURE 1. Daily Noon POsitions, R/V THOMAS WASHINGTON (EAC 18). 


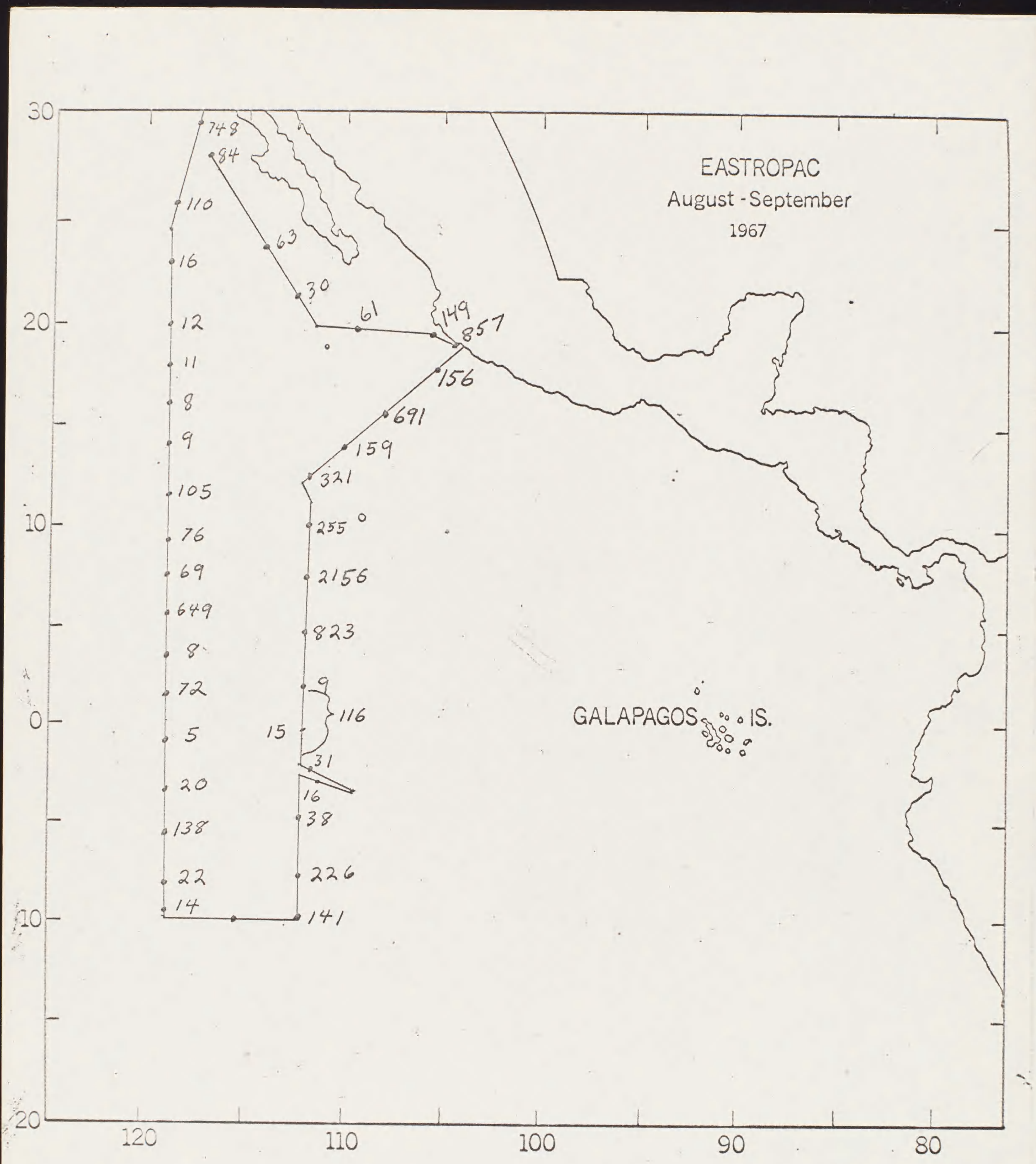

FIGURE 2. Daily Abundance of Total Birds, EAC 18. 


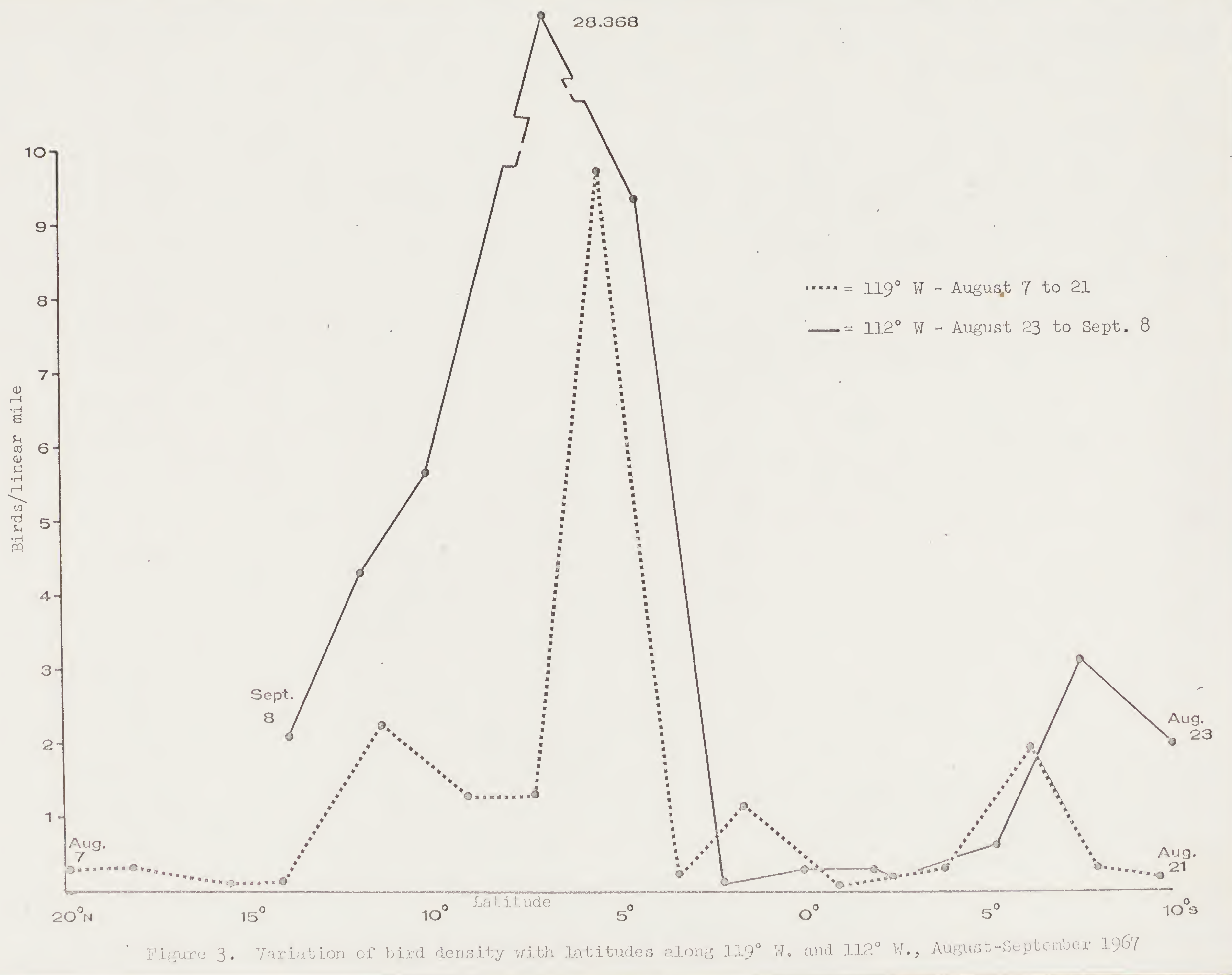




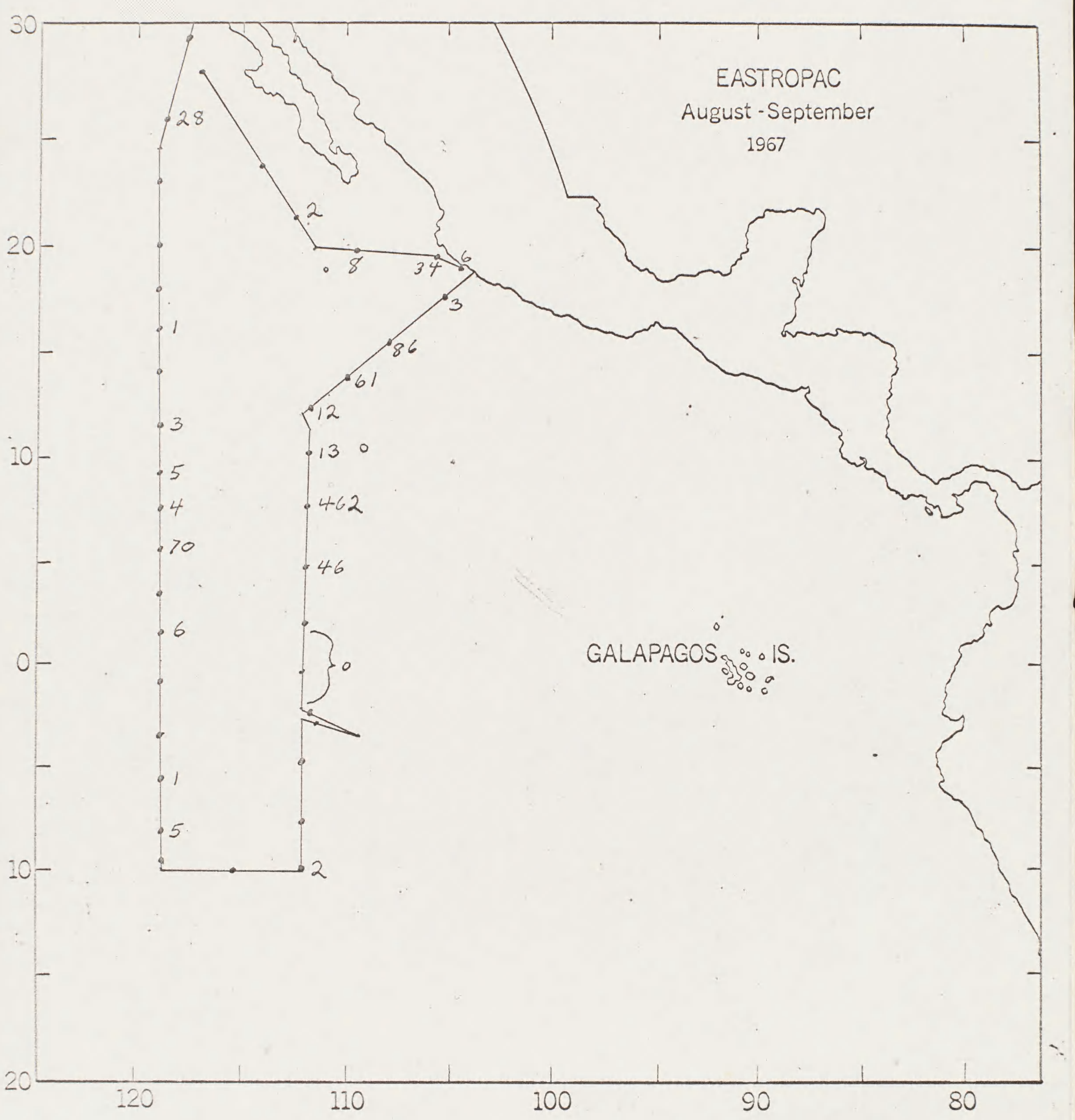

FIGURE 4. Daily Abundance of Wedge-tailed Shearwaters. 


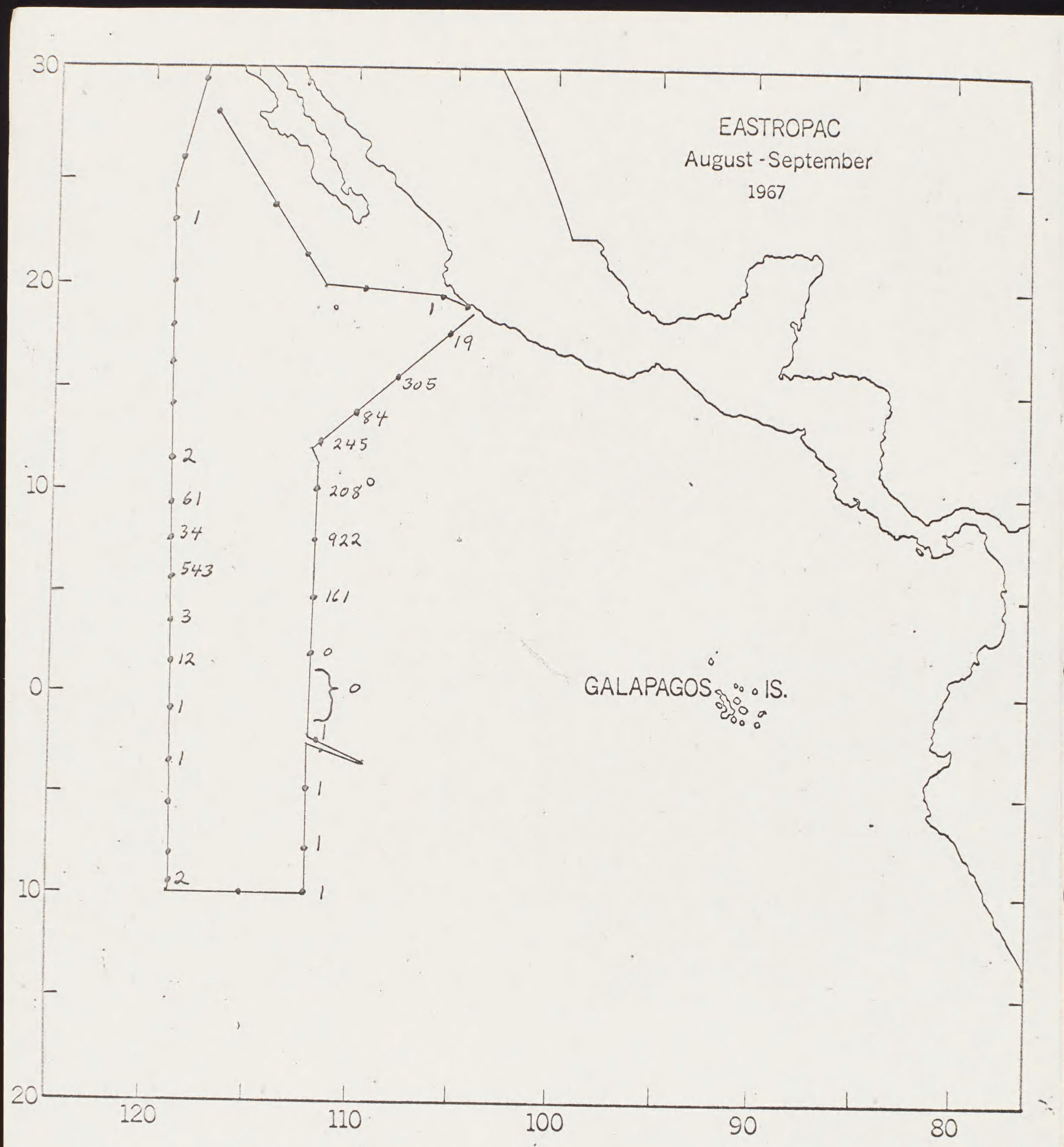

FIGURE 5. Daily Abundance of Pterodroma externa. 


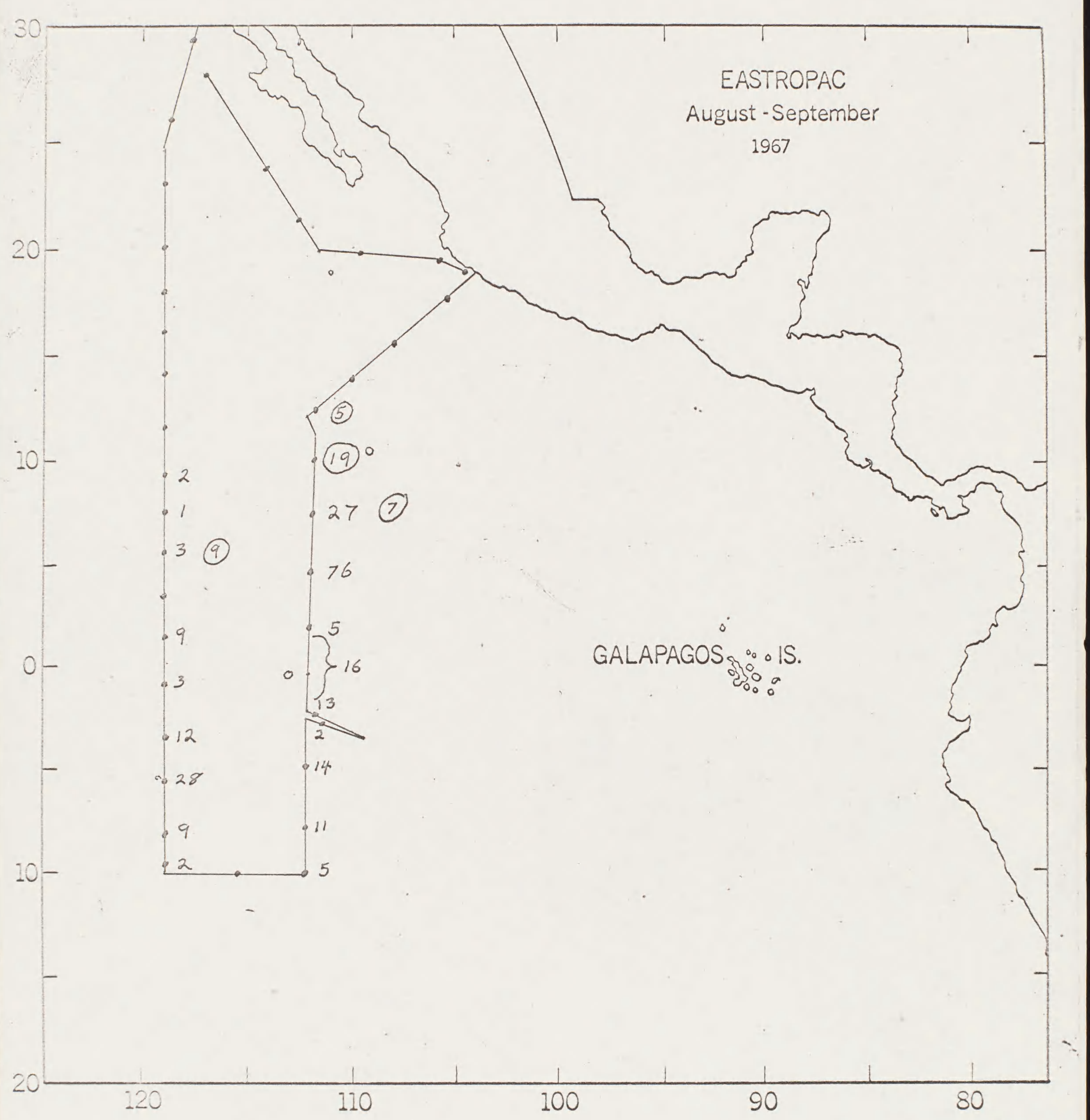

FIGURE 6. Daily Abundance of Collared Petrels (plain) and Black-winged Petrels (encircled). 


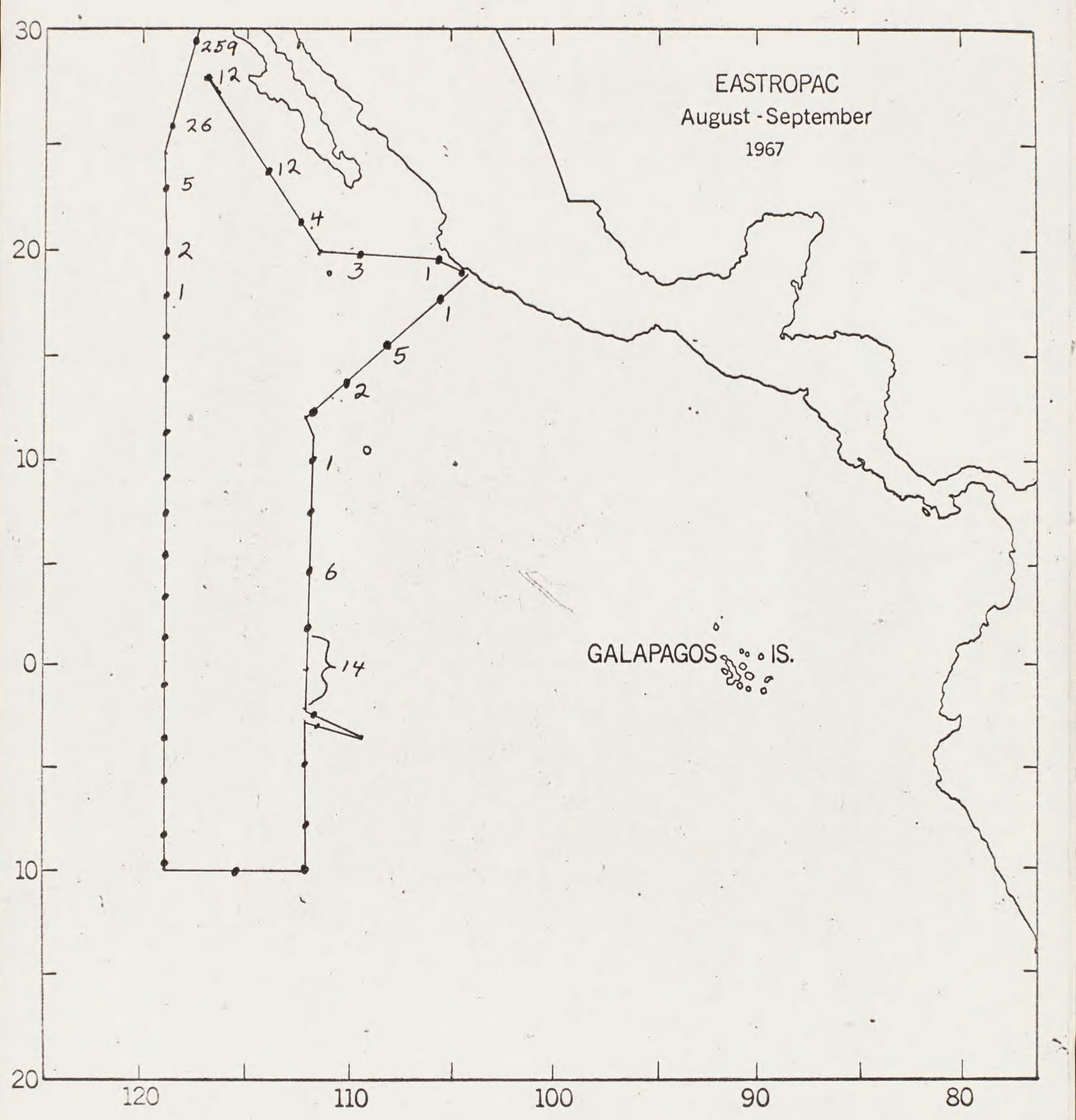

FIGURE 7. Daily Abundance of Leach and Leach-type Storm Petrels. 


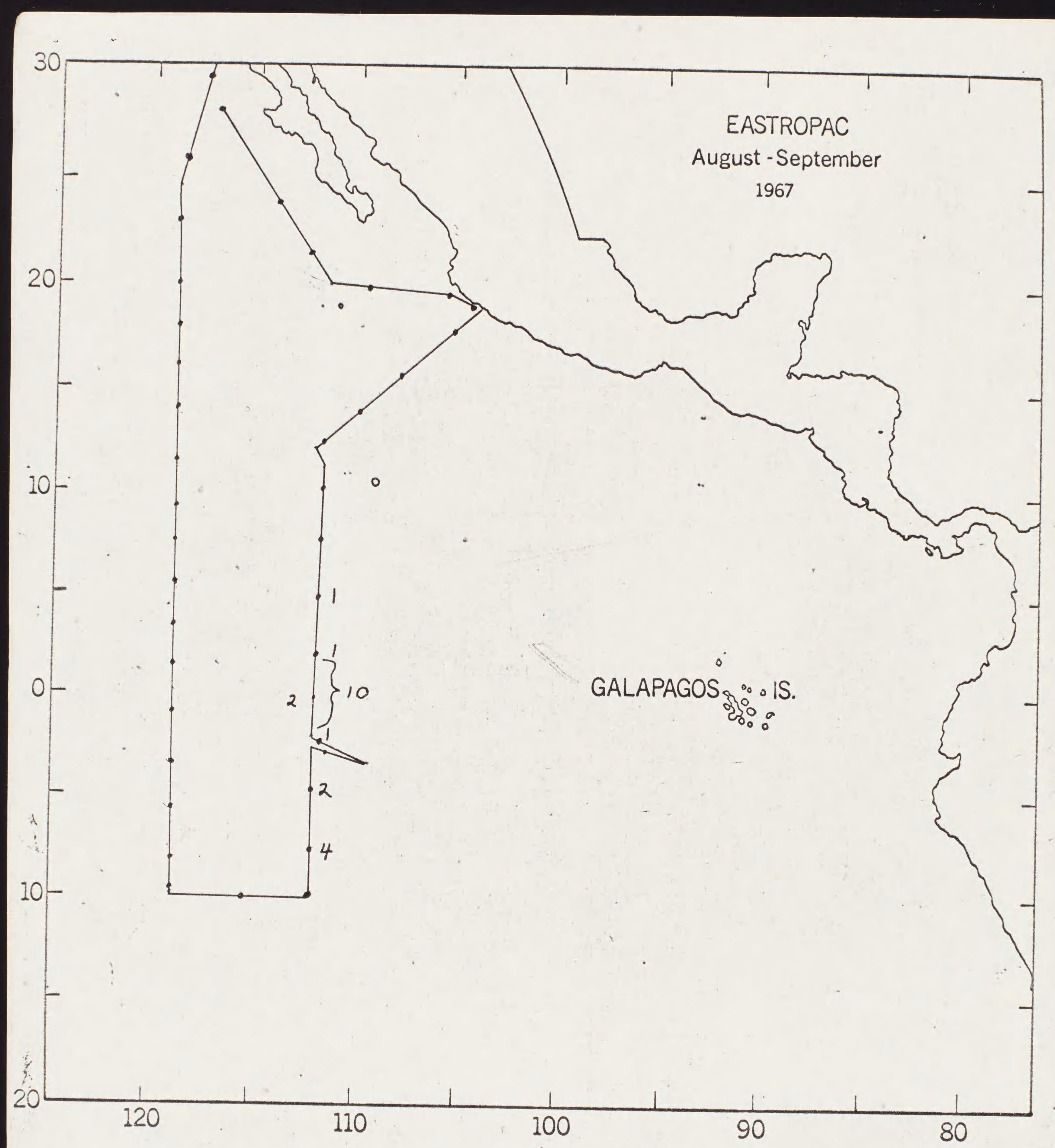

FIGURE 8. Da1ly Abundance of White-faced Storm Petrels. 


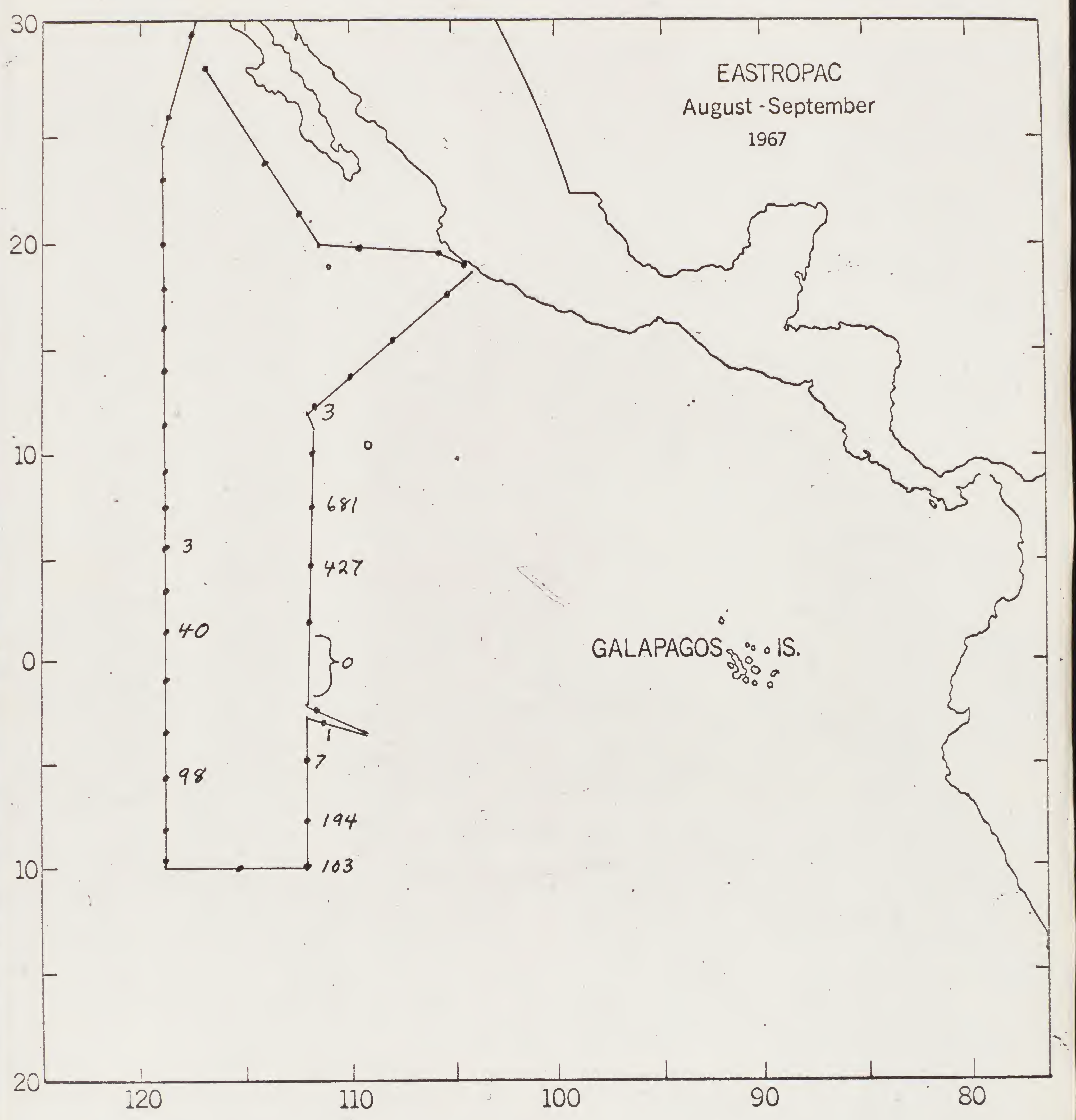

FIGURE 9. Daily Abundance of Sooty Terns. 


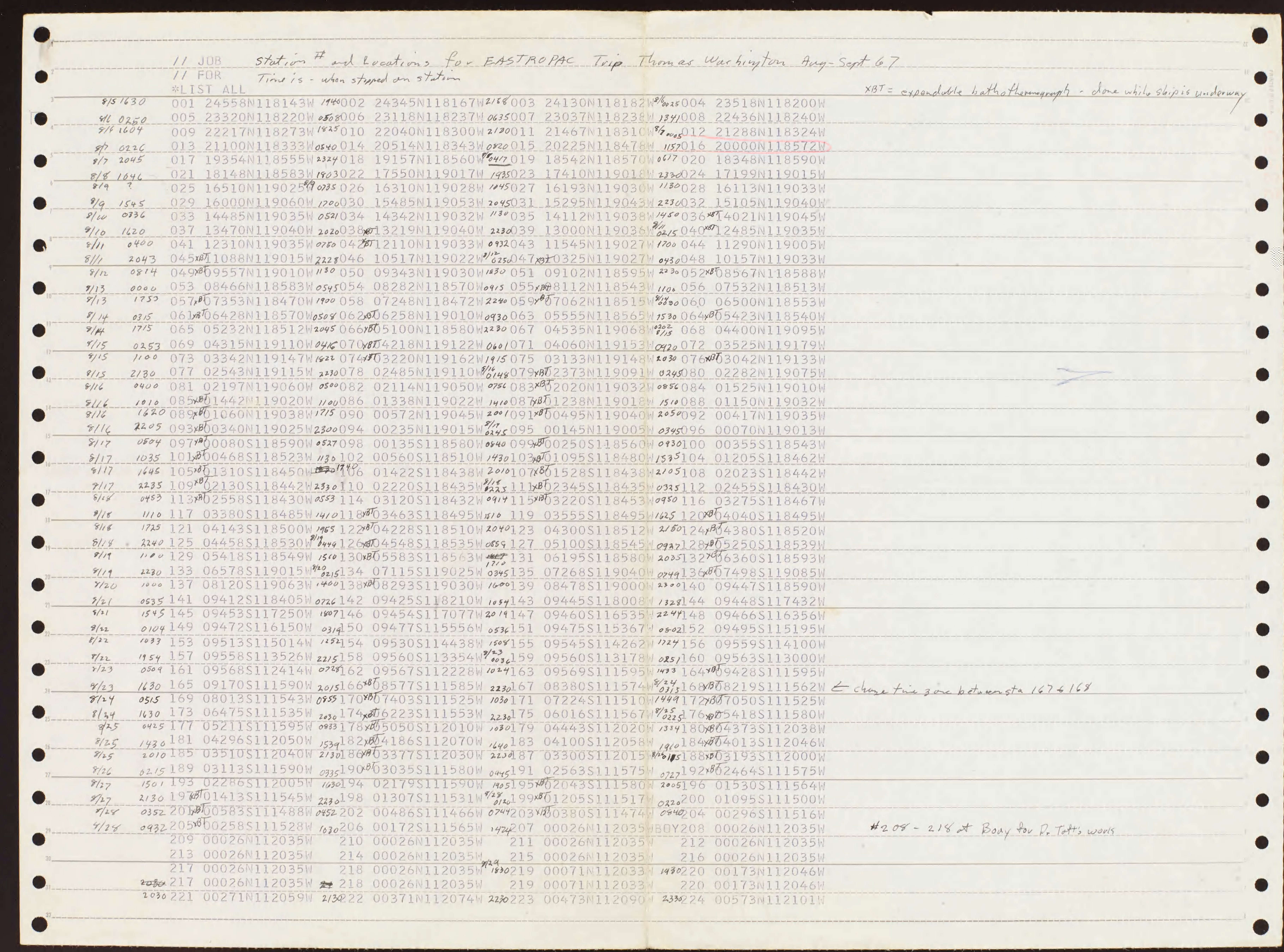




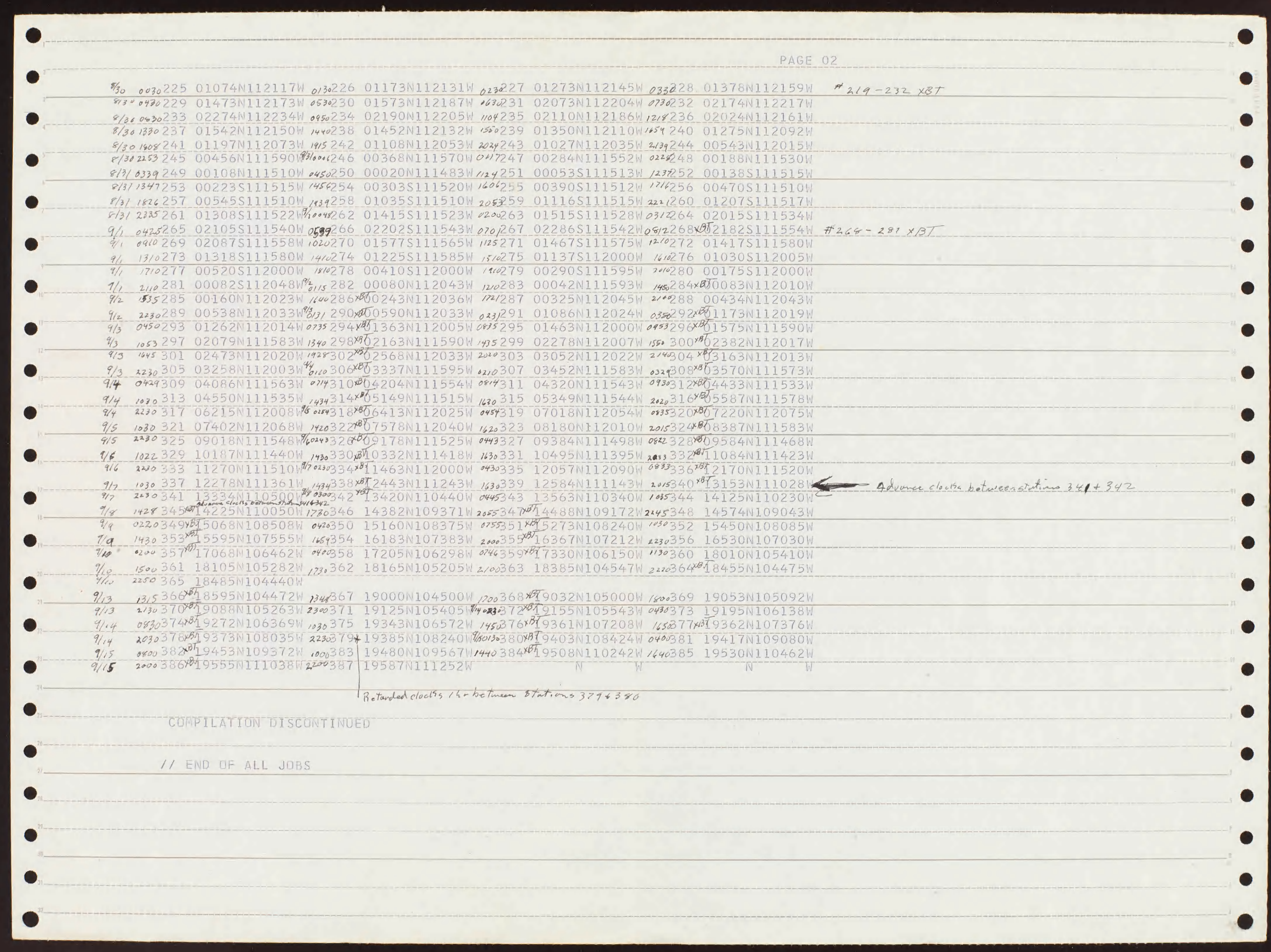




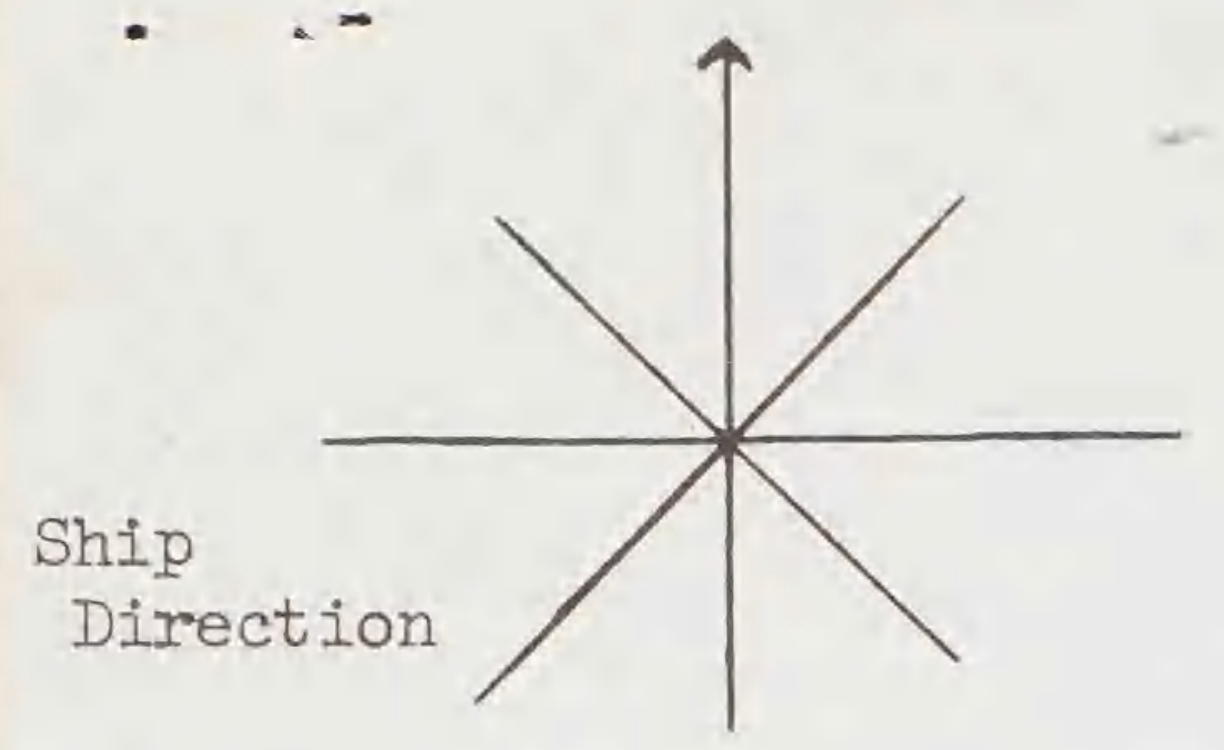

SMITHSONLAN INSTITUTION

DIVISION OF BIRDS

AT SEA DAILY LOG - E

OBSERVERS:

SPEC IMEN

or
Date 3 Aug $<7$

$\mathrm{Pg}$.\#

TIME SPECIES \# DIR. BAND NO. REMARKS

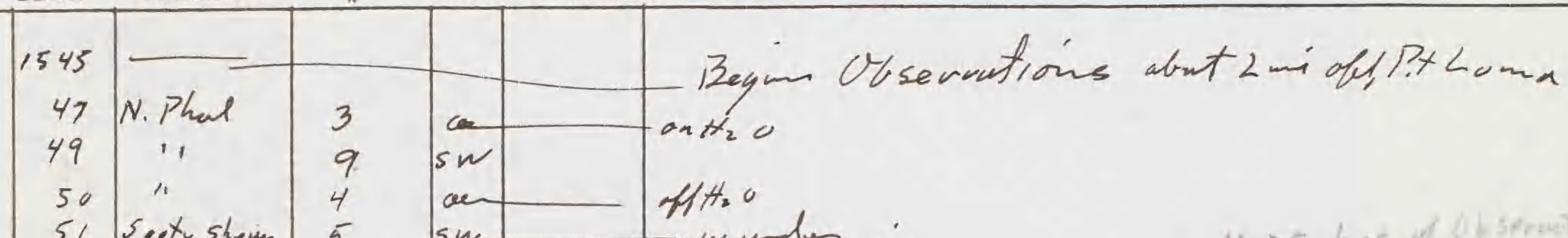

53 soxy shein

57 P.f Shear

1601

$s / 5<$

s/sit

03
04

$\begin{array}{ll}04 & W R S P \\ 13 & S / S L\end{array}$

sw

$\mathrm{H}_{\mathrm{H}} \mathrm{H} \mathrm{O}$

sw

follumis 2 Ad I Fin

cee

18

WRSP

21

23

WRSP

34

Brind

ca

46 WRSP

5) St.Pet

weed Feedin occasiandly

s

Followis centhislip oun $51, i c / 5$ calsealioin

1730

w

3 Fum Retph

ae

Nw

we

clone tir ch

Beyin

1743 Brown Pellip

1804

S/SL

- At stution

15

N. Phal

N. Phat

39
45

Brom Pel

co

40 Purposis Buird's Datphin

50

st Pet

1900 Phal sp

03 st Pet.

of st pet.

oq Keuch's Ș?

10

S/SL

E

$\$ 5$

3
6

3

ree.

Departadstatur ccto185

E

a

F

SI-MNH-958-e

Rev. 5-66 


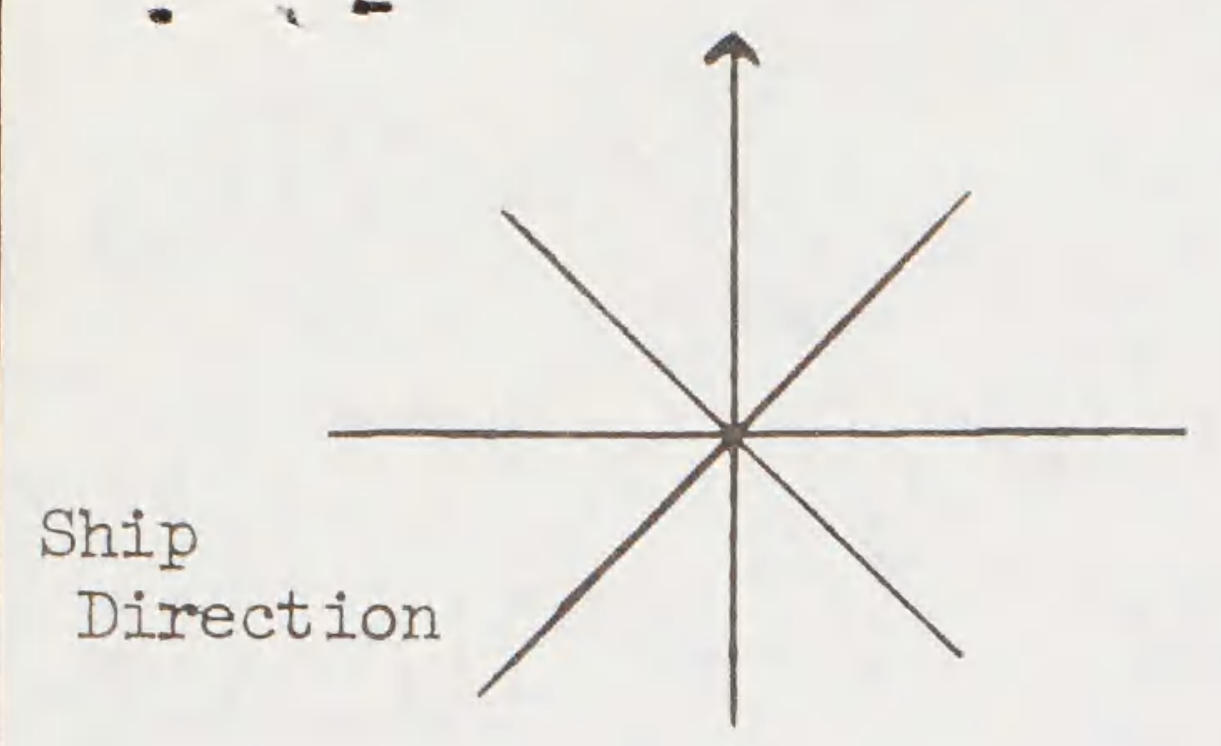

OBSERVERS :

SMITHSONIAN INSTITUTION

DIVISION OF BIRDS

AT SEA DAILY LOG - E

SPECIMEN

or
Date IA wy CI

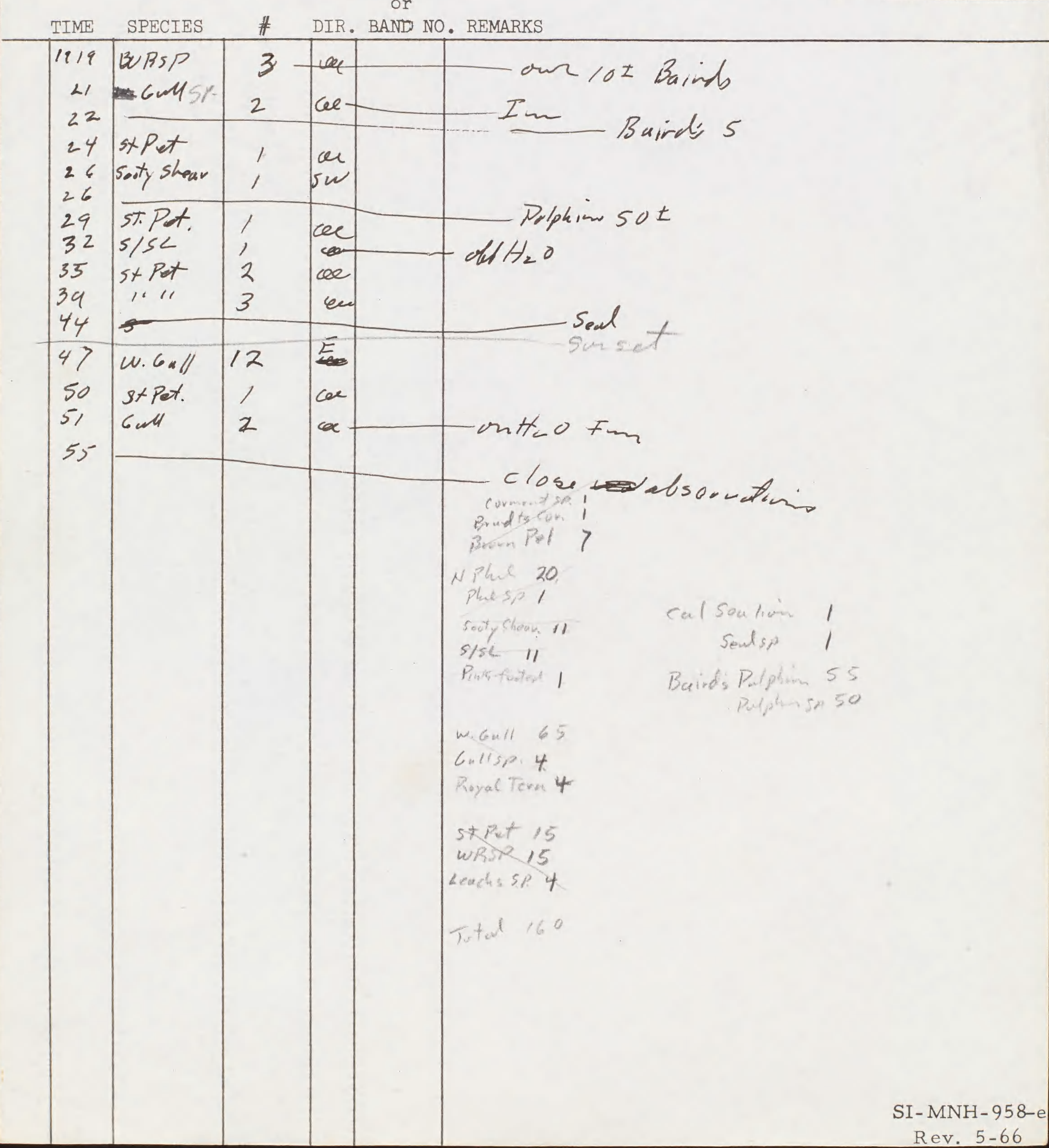




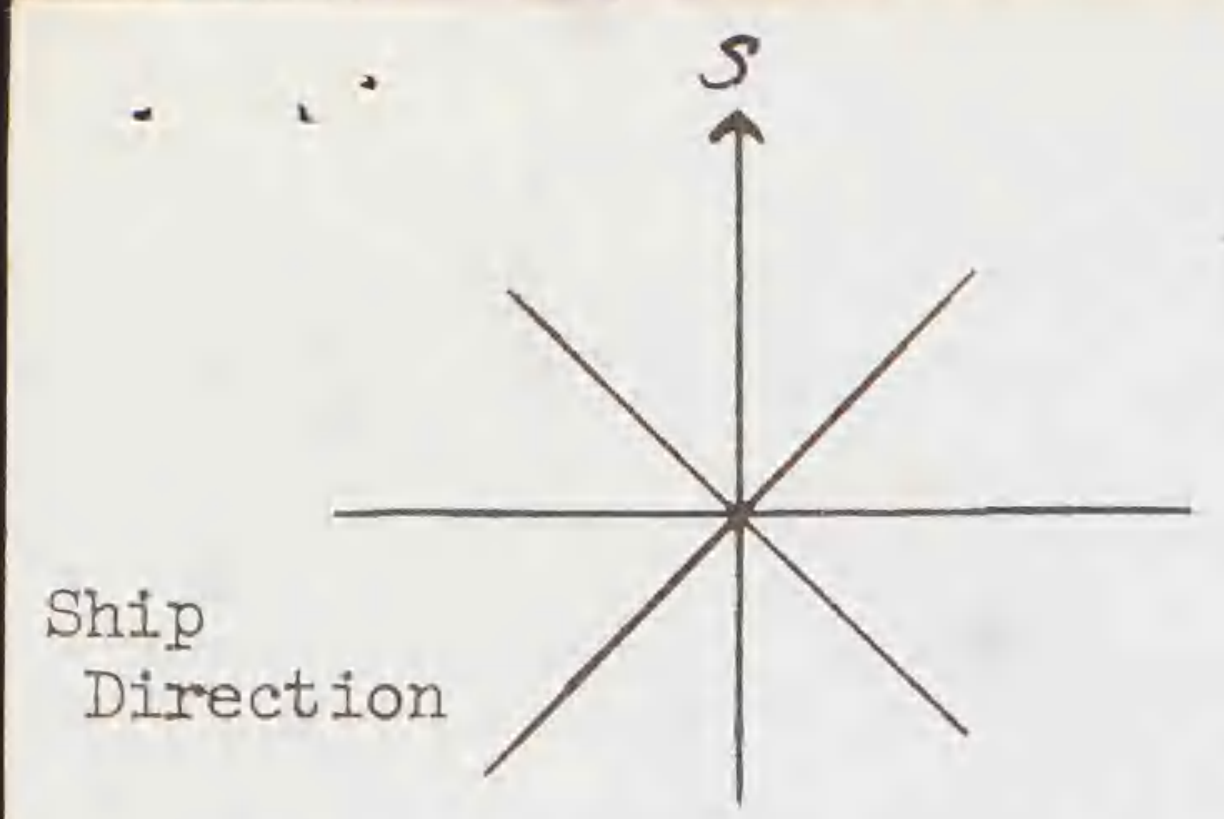

-59 miles

OBSERVERS :

SMITHSONIAN INSTITUTION

DIVISION OF BIRDS

AT SEA DAILY LOG - E

SPEC IMEN

or
7.7

chmpe tMidnight.
Date

$\mathrm{Pg} \cdot \# \mathbb{4}$ सuy 6 ?

+8 Tme Zan

-

Begmin misemation

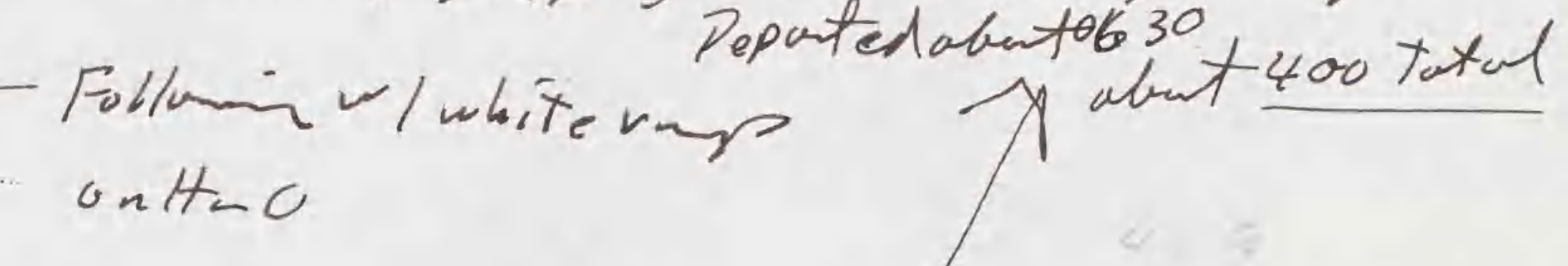

r

$N W$
$i$

43 Red Pind 1

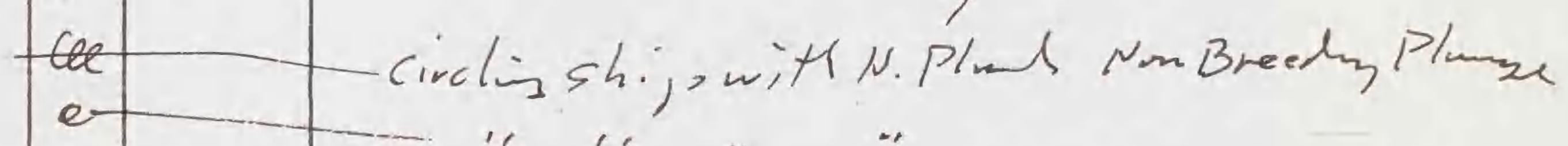

\begin{tabular}{c|c|c|}
54 & N.Phal & 6 \\
6603 & Curlew & 7
\end{tabular}

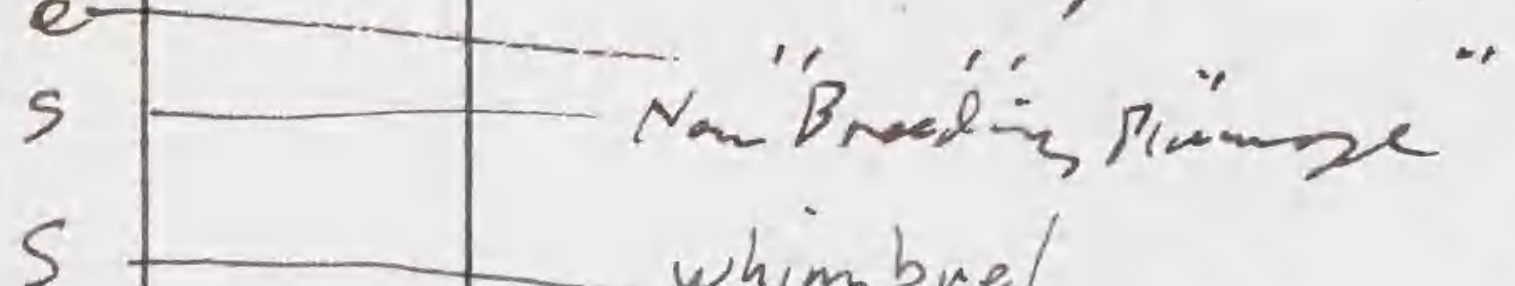
whimbuel

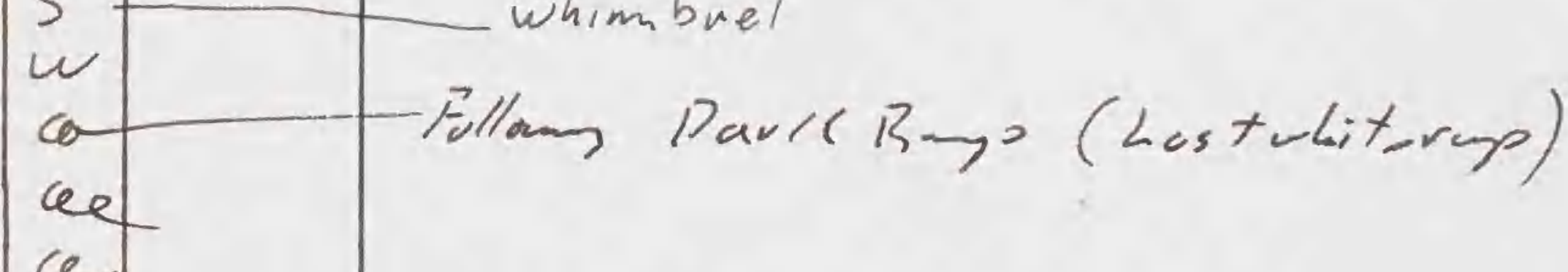

\begin{tabular}{l|l|l}
15 & heach's type & 1 \\
22 & wRsp & 2 \\
26 & $n$ & 1 \\
28 & "1 & 3 \\
29 & Leach's type & 1 \\
33 & St.Pet. & 1 \\
37 & Leachitipe & 1
\end{tabular}

40 R. Phal.

\begin{tabular}{c|l|l}
41 & Leads type & 1 \\
43 & "1. & 1 \\
47 & St.Pt & 1 \\
47 & NiPlal & 4 \\
51 & Learl's SP & 1 \\
0654 & Cooks Pot & 1 \\
& Leadistype & 3
\end{tabular}

\begin{tabular}{l|l}
$w$ \\
$w$ \\
cee \\
an
\end{tabular} \mid afy

cee

al NomBieedin Plonze

0702

cee

s

0755

58 Leach Type 1 ,

osroo

stormpot

E

03 heach', type

\begin{tabular}{l|ll|l|l}
07 & 11 & 1. & 2 & ue \\
09 & 1 & 1. & 1 & ue
\end{tabular}

\begin{tabular}{c|cc|c|c}
10 & 1 & 1 & 6 & aee \\
14 & 11 & 11 & 3 & wec \\
17 & 11 & 1 & 1 & ce
\end{tabular}

Brealsfast $\mathrm{Cl}=\mathrm{e}$

oper

cee

on $H_{2}$ O Piom? ocallury

hee
ar
ne
un
nee
wh

SI- MNH- 958-e

Rev, 5-66 


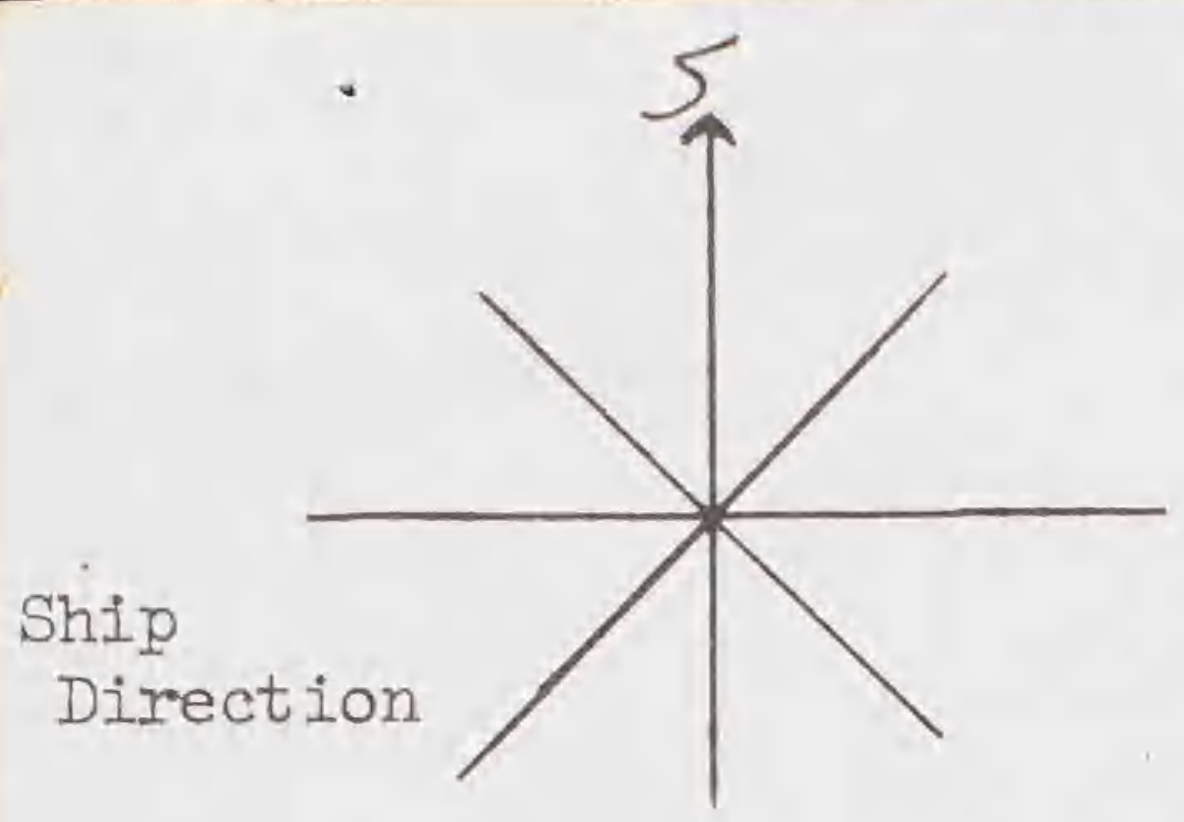

OBSERVERS :

SMITHSONIAN INSTITUTION DIVISION OF BIRDS AT SEA DAILY LOG - E

SPEC MEN

or

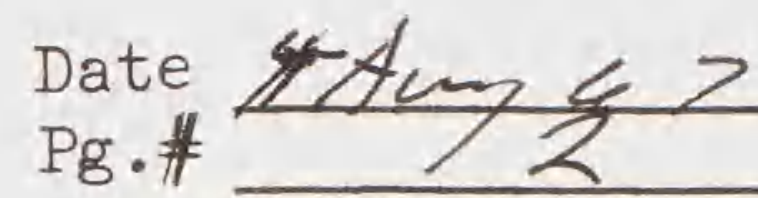

TIME SPECIES \# DIR. BAND NO. REMARKS

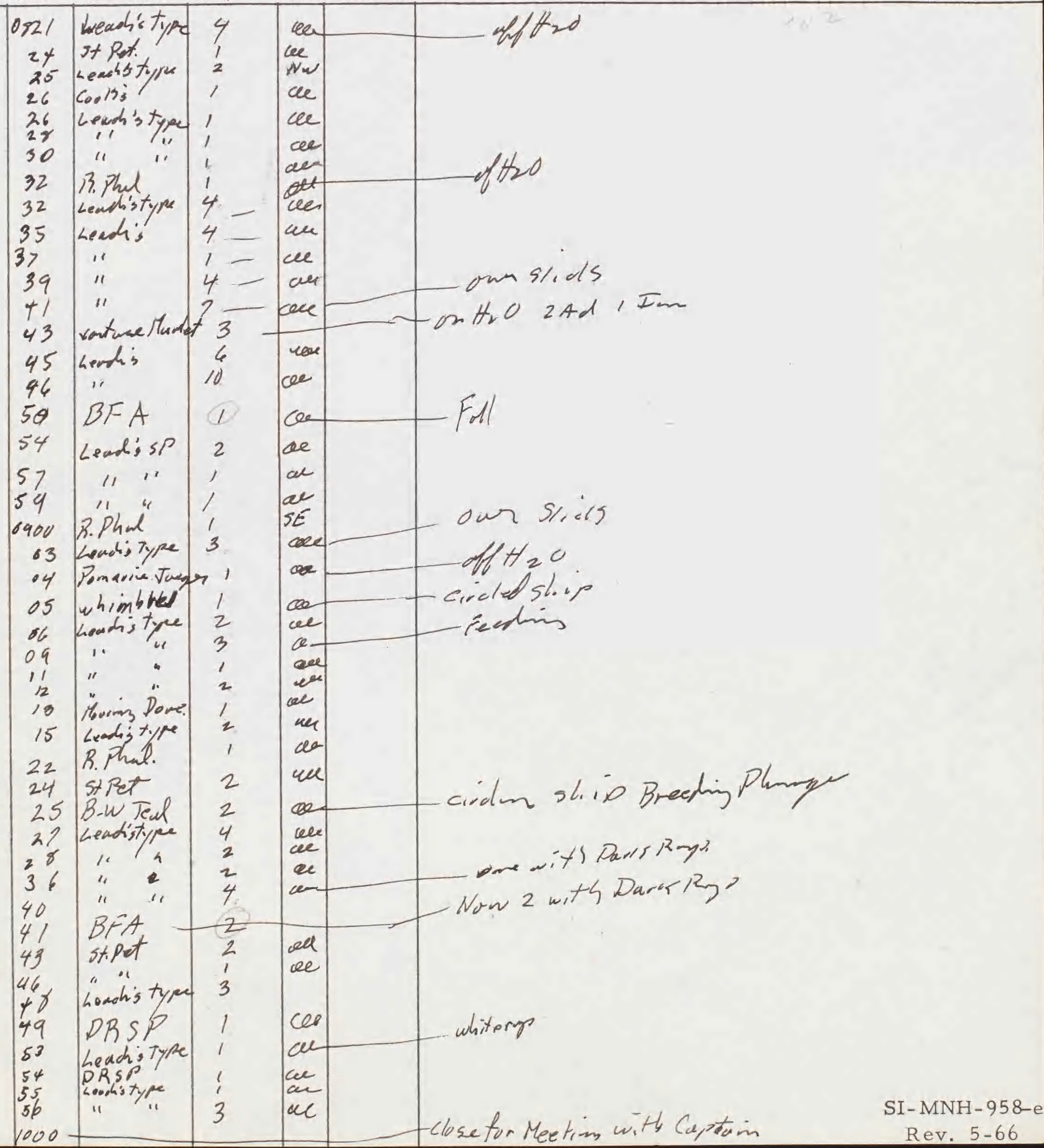


Ship

Direction

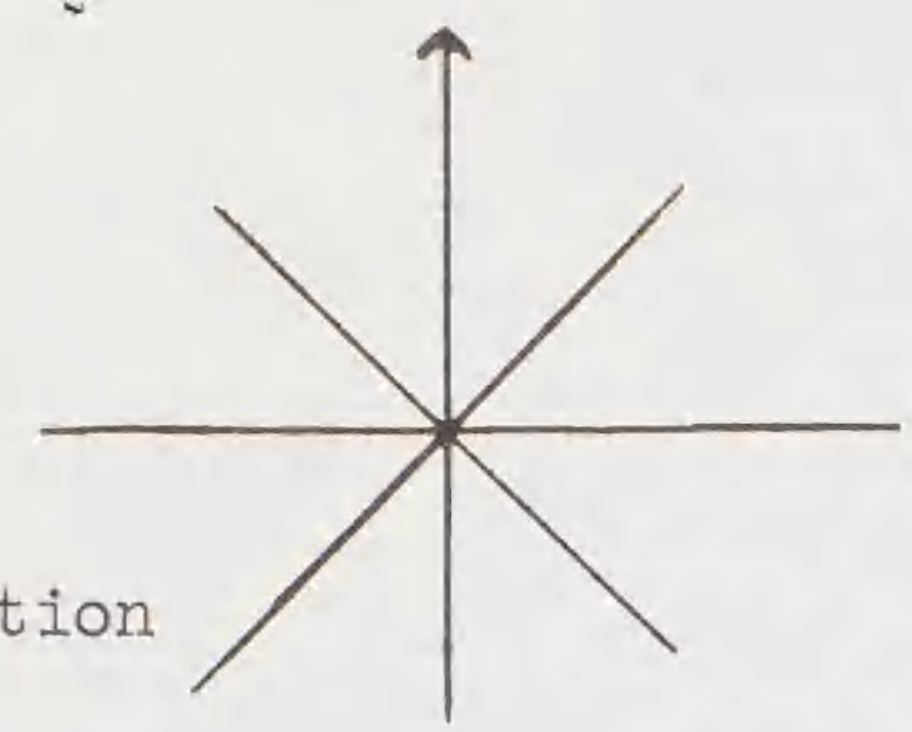

SMITHSONIAN INSTITUTION DIVISION OF BIRDS

AT SEA DAILY LOG - E

SPEC IMEN

or
Date $\frac{4 \text { Ang }}{3}$

TIME SPECIES \# DIR. BAND NO. REMARKS

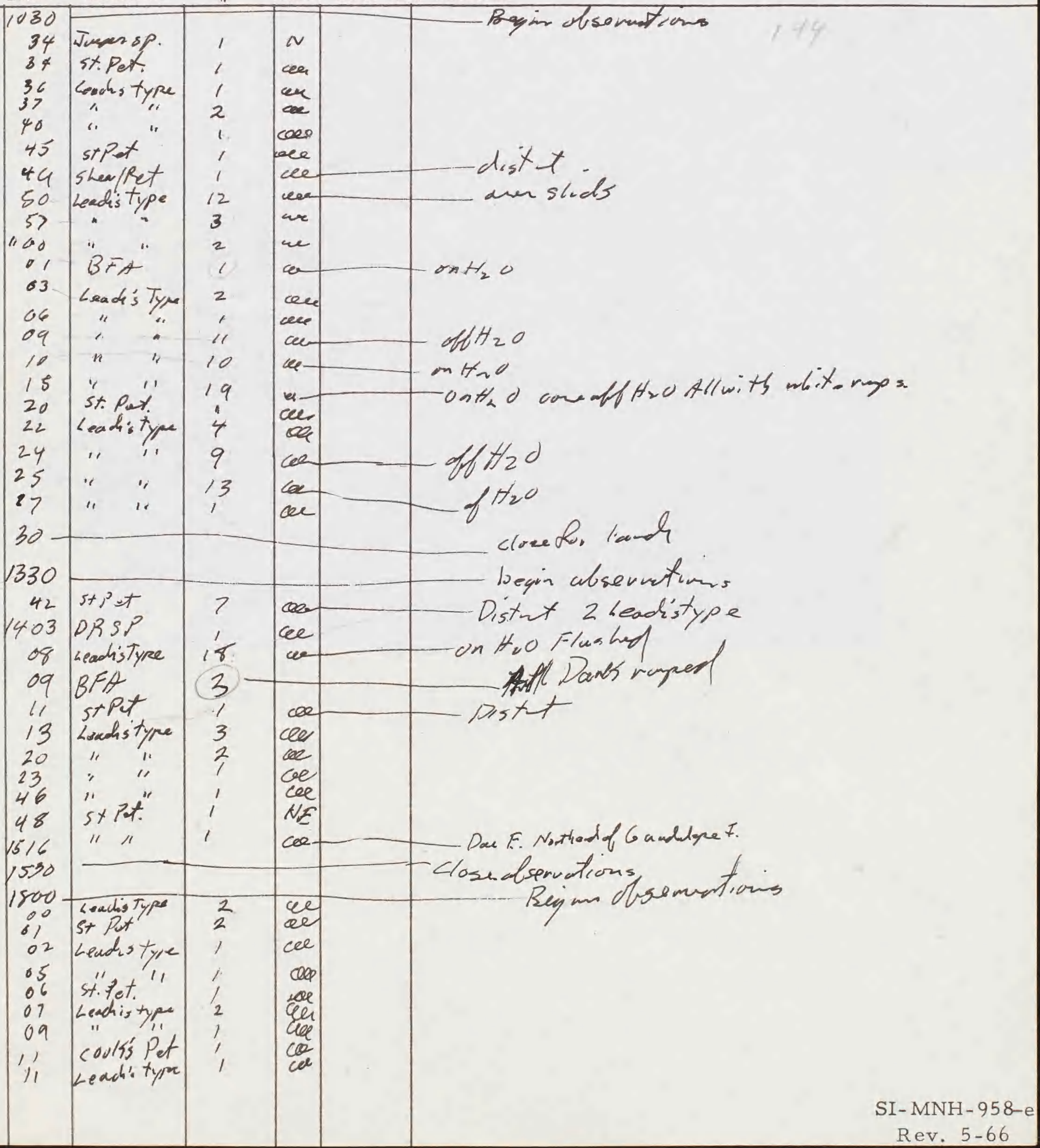




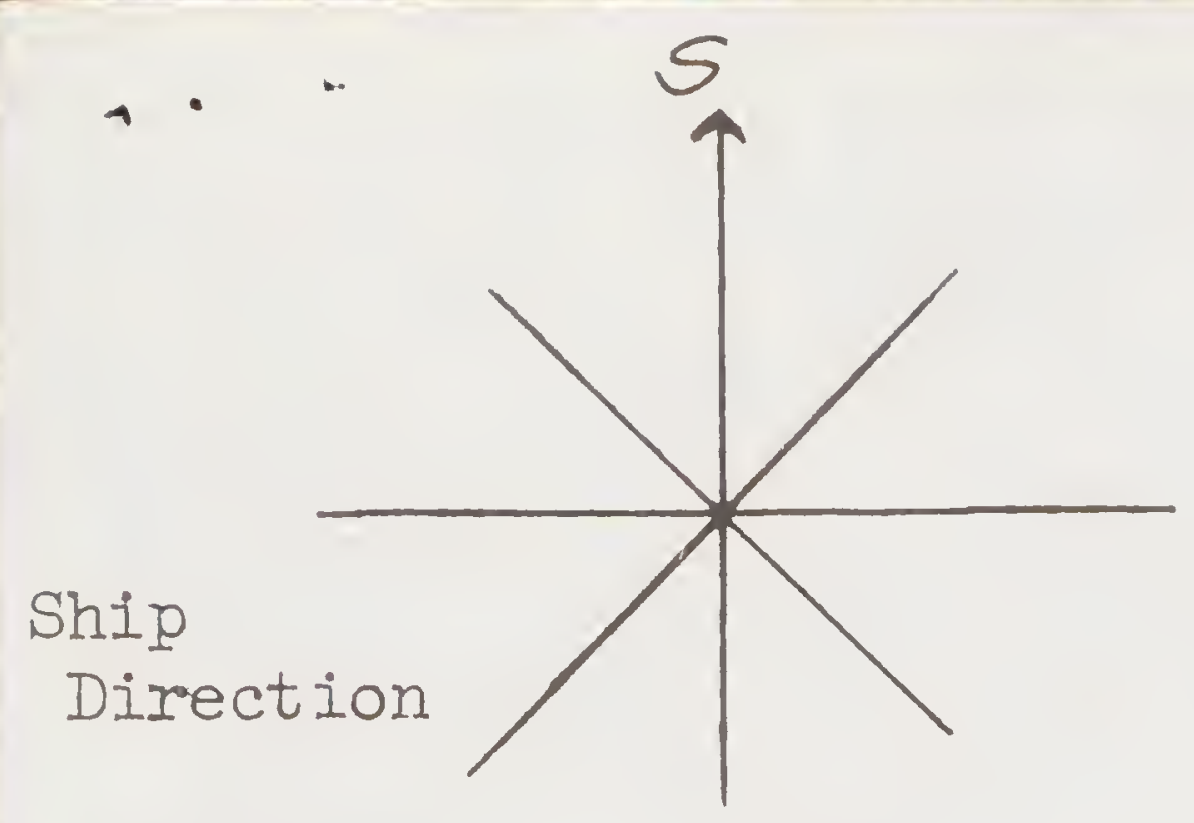

OBSERVERS :

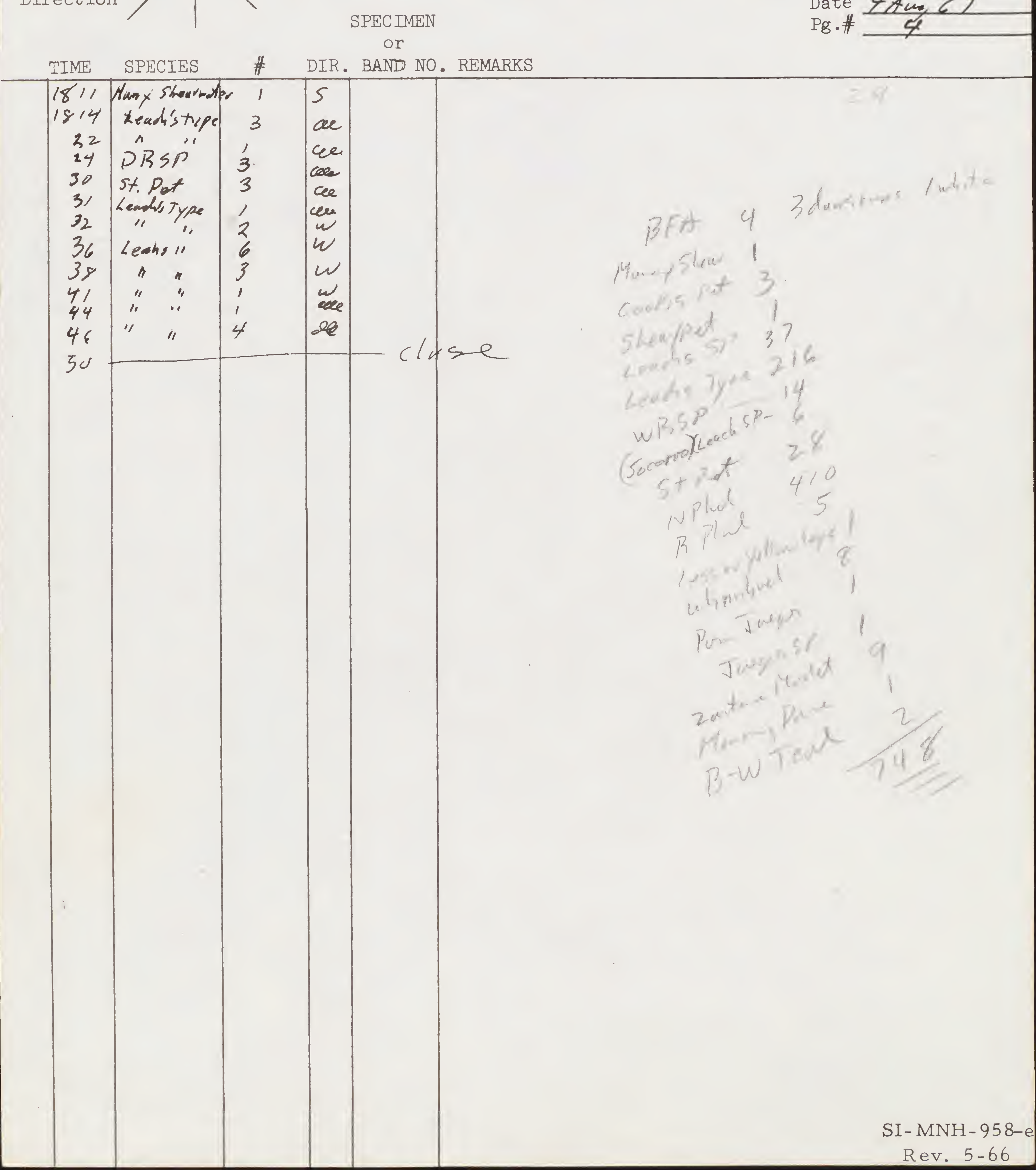




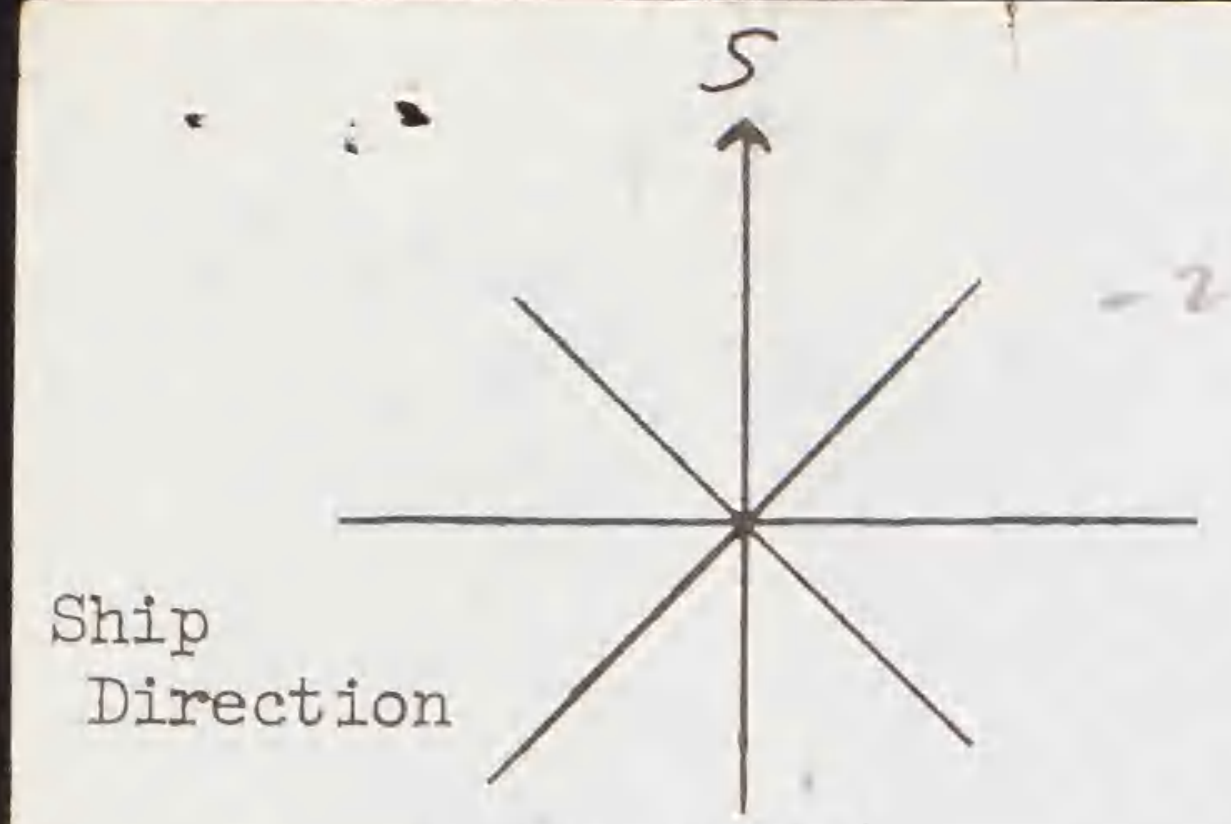

SMITHSONIAN INSTITUTION

DIVISION OF BIRDS

AT SEA DAILY LOG - E

OBSERVERS:

temp Grandest.

SPECIMEN

or

9.5 hus pobsenvition Pg.\# Stroy \&7

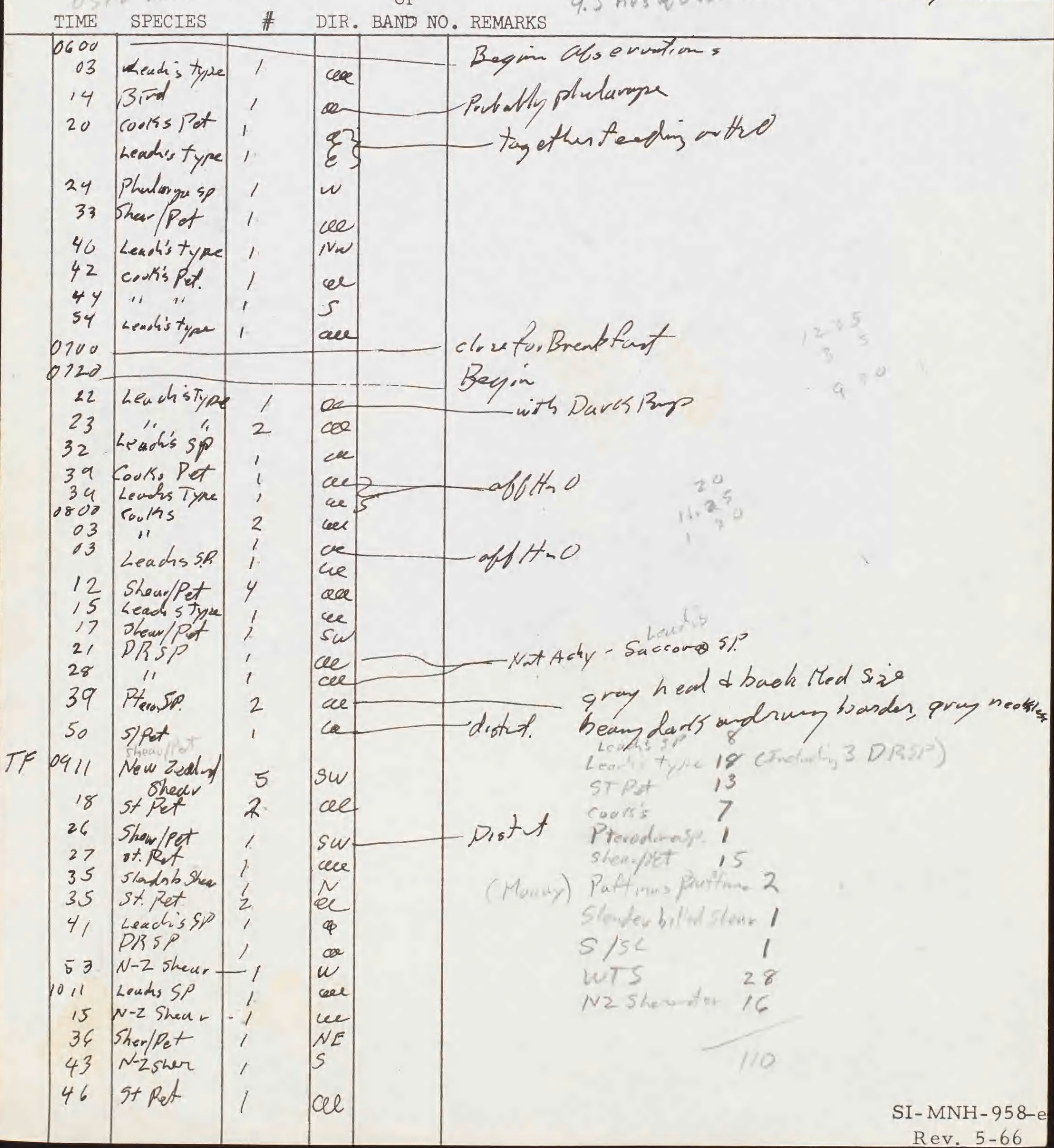




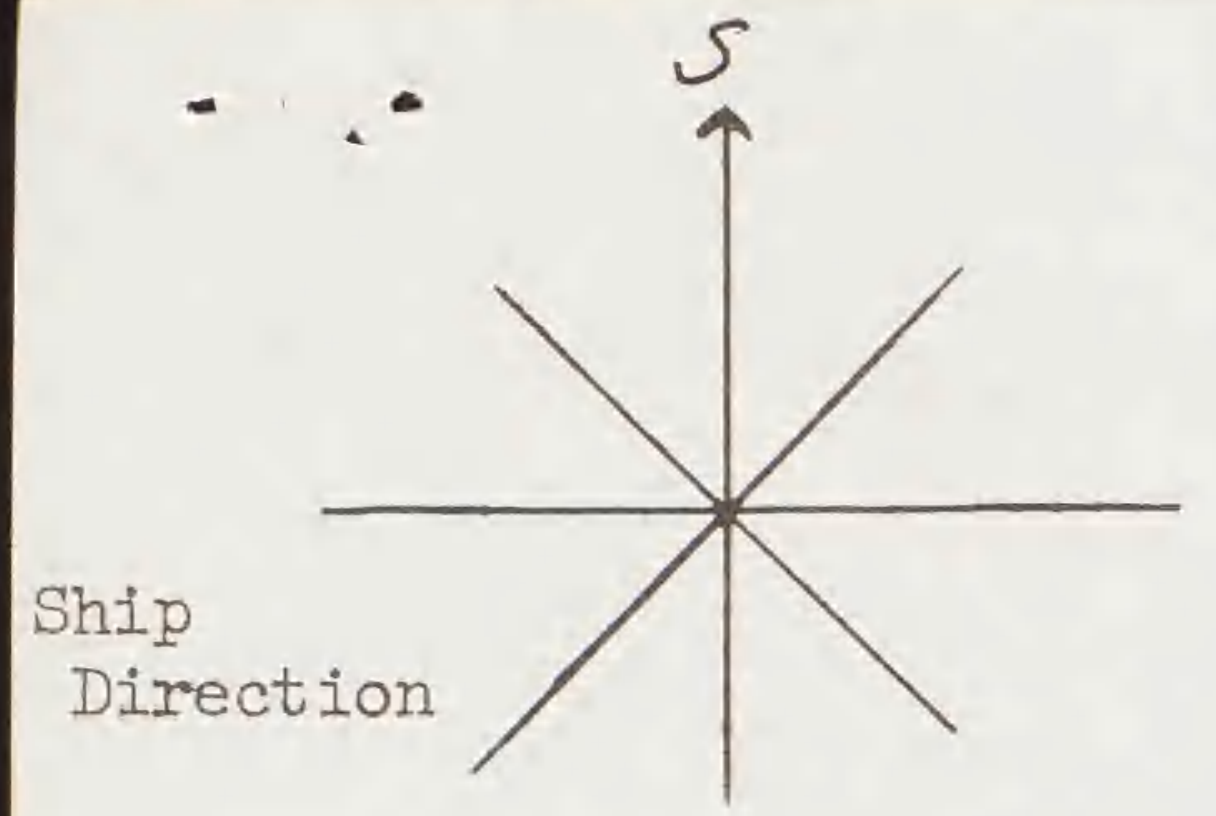

SMITHSONIAN INSTITUTION

DIVISION OF BIRDS

AT SEA DAILY LOG - E

SPEC IMEN

or

OBSERVERS :

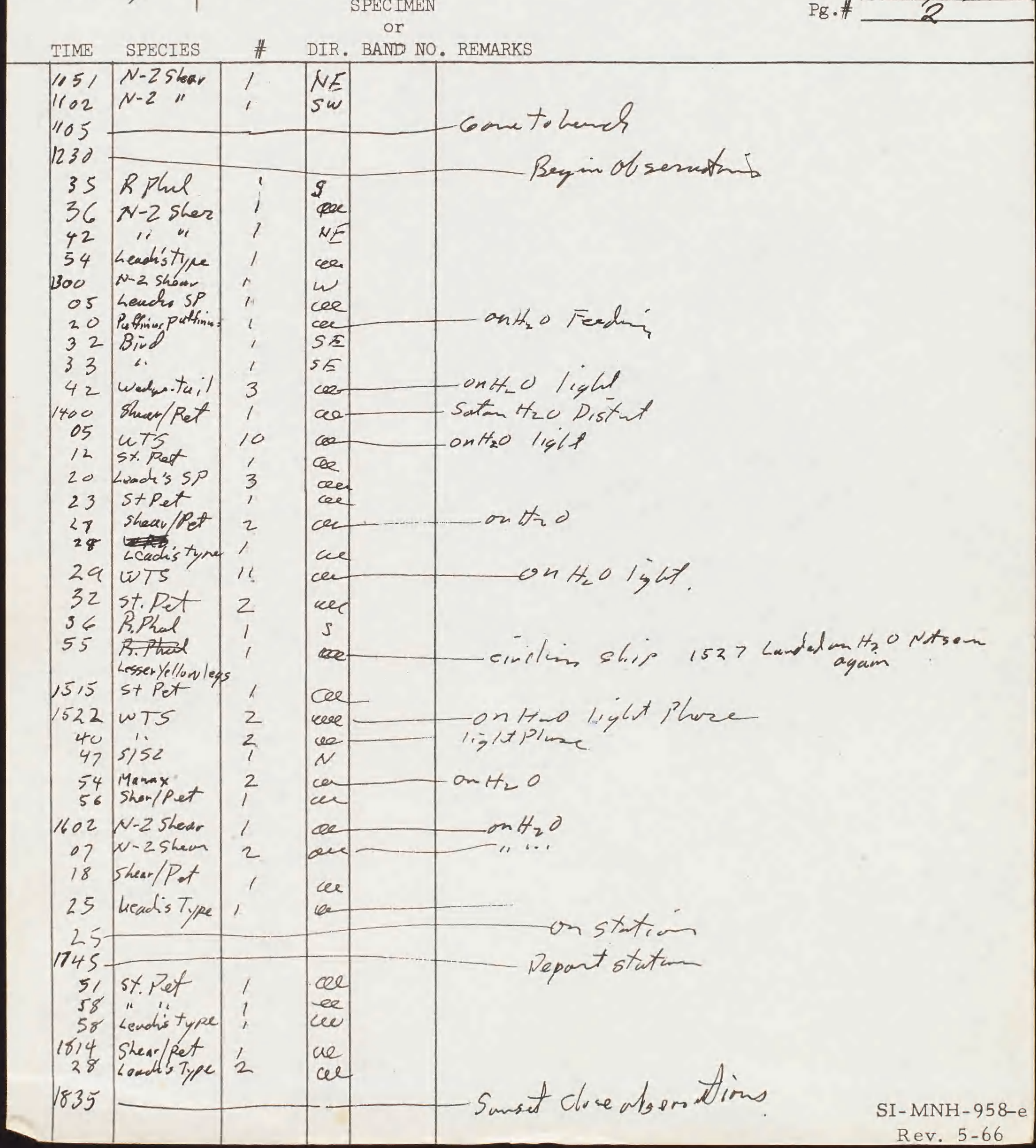




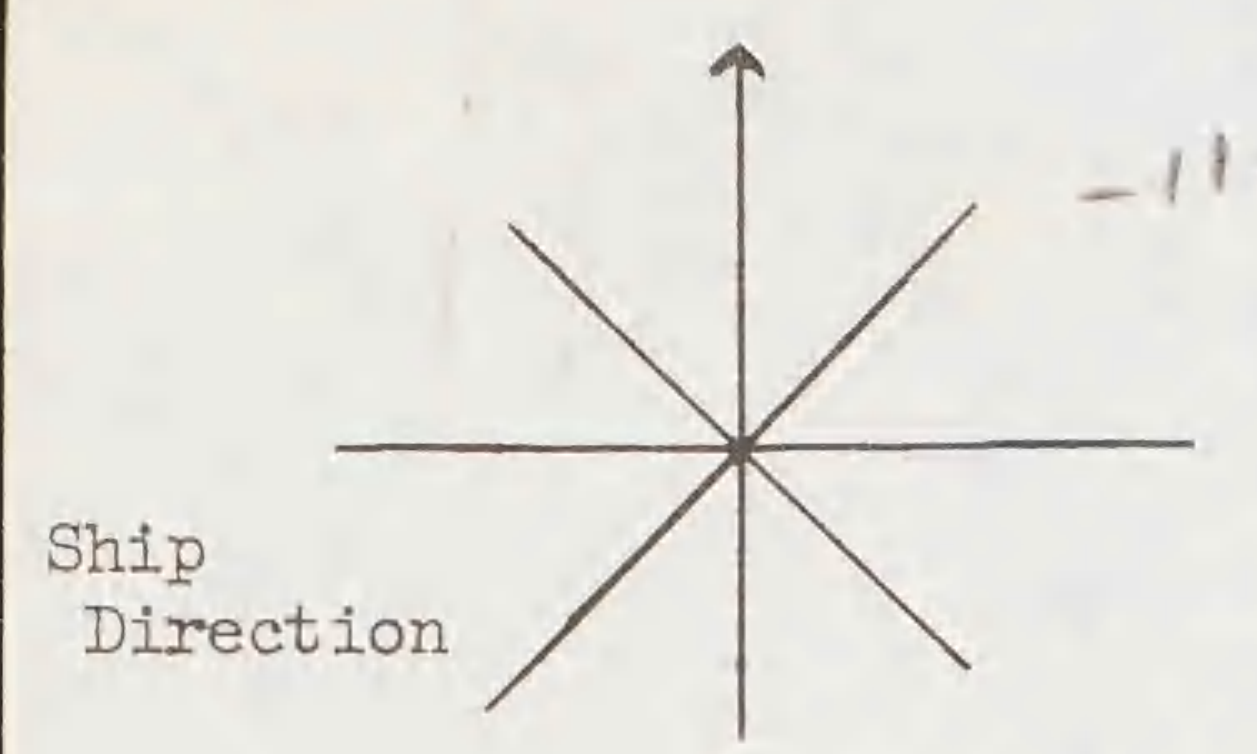

SMITHSONIAN INSTITUTION

DIVISION OF BIRDS

AT SEA DAILY LOG - E

SPECIMEN

Date 4 An g 107

or

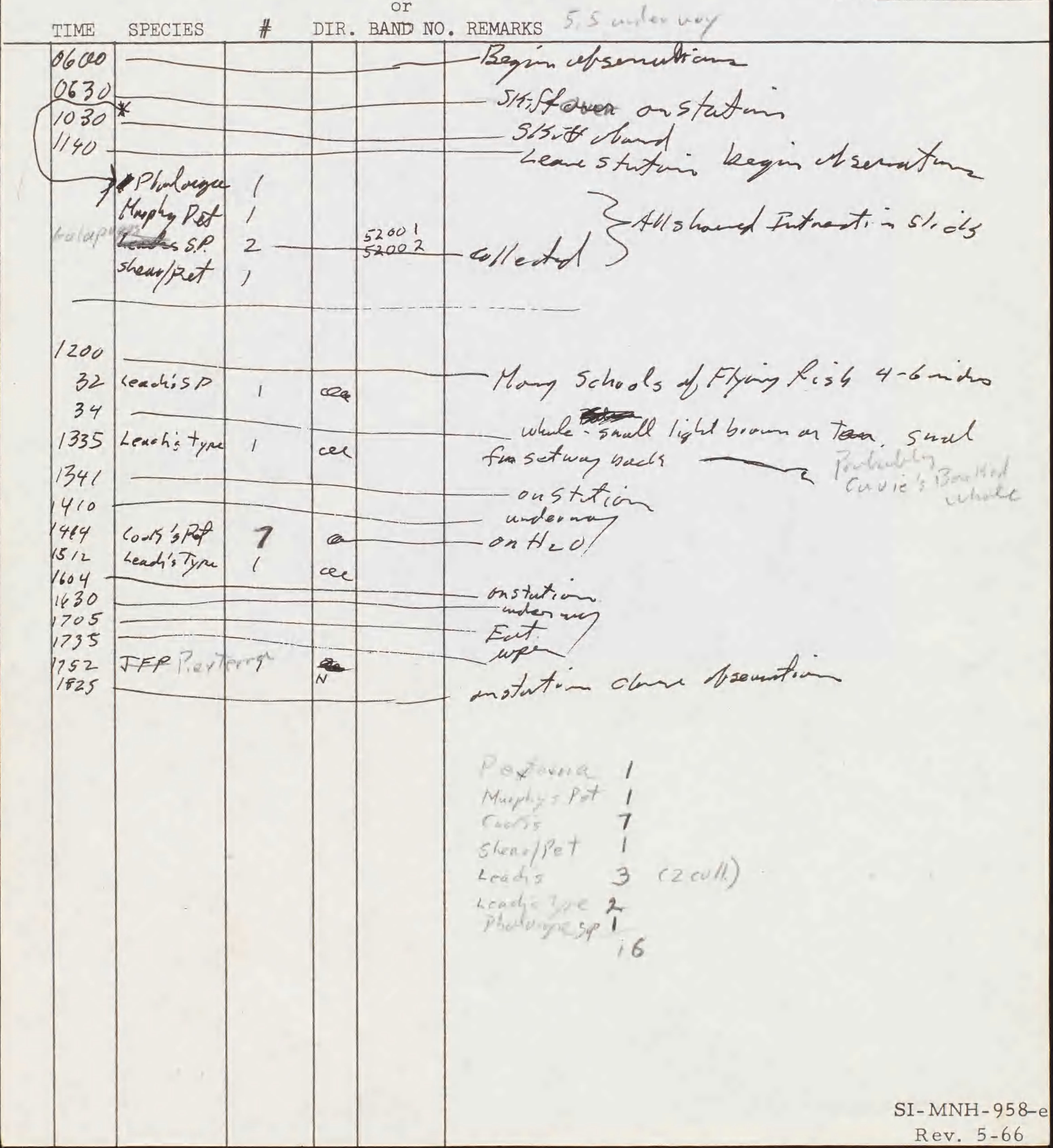




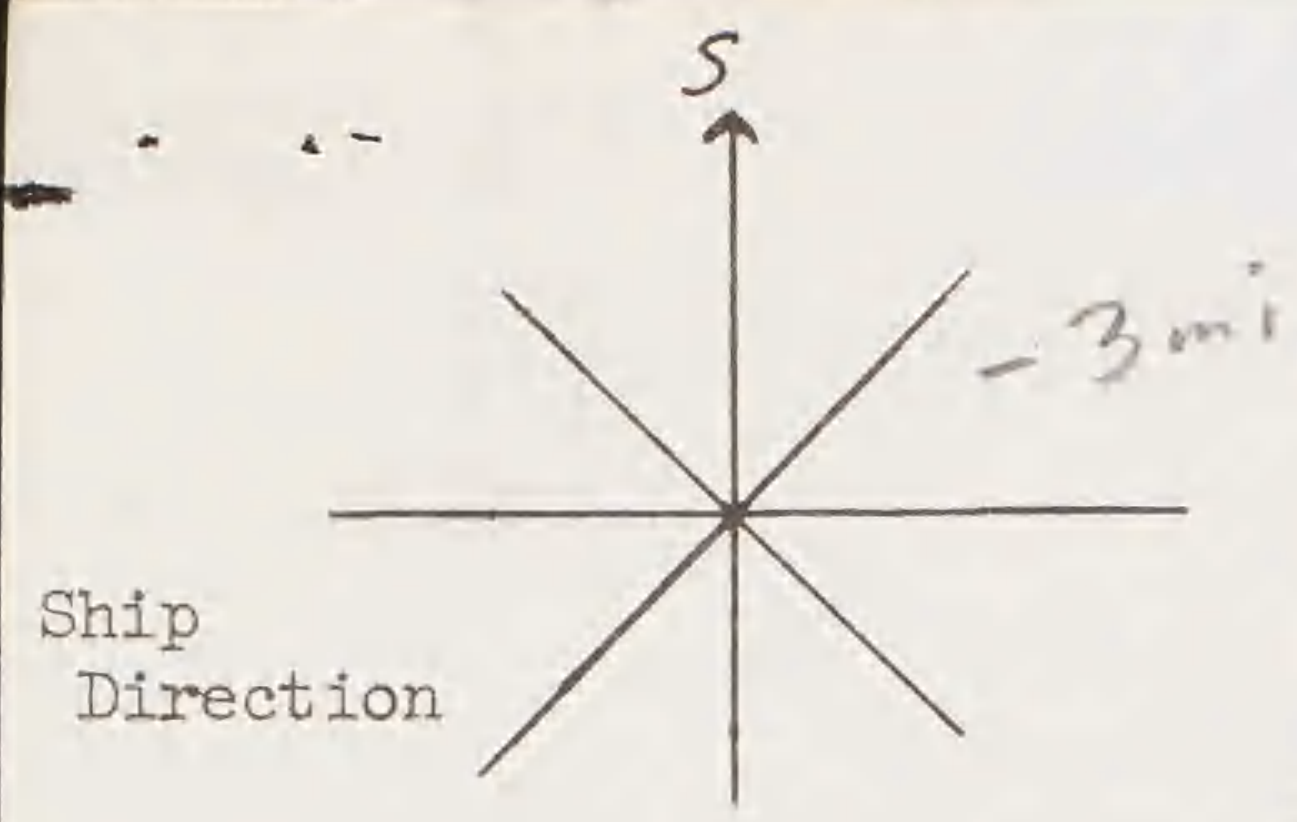

OBSERVERS :

Oueraust Harmer

SMITHSONIAN INSTITUTION

DIVISION OF BIRDS

AT SEA DAILY LOG - E

SPECIMEN

clear to lutenterame duran $x$ emery

or

Date DAmpens TL)

$\mathrm{Pg} \cdot$ \#

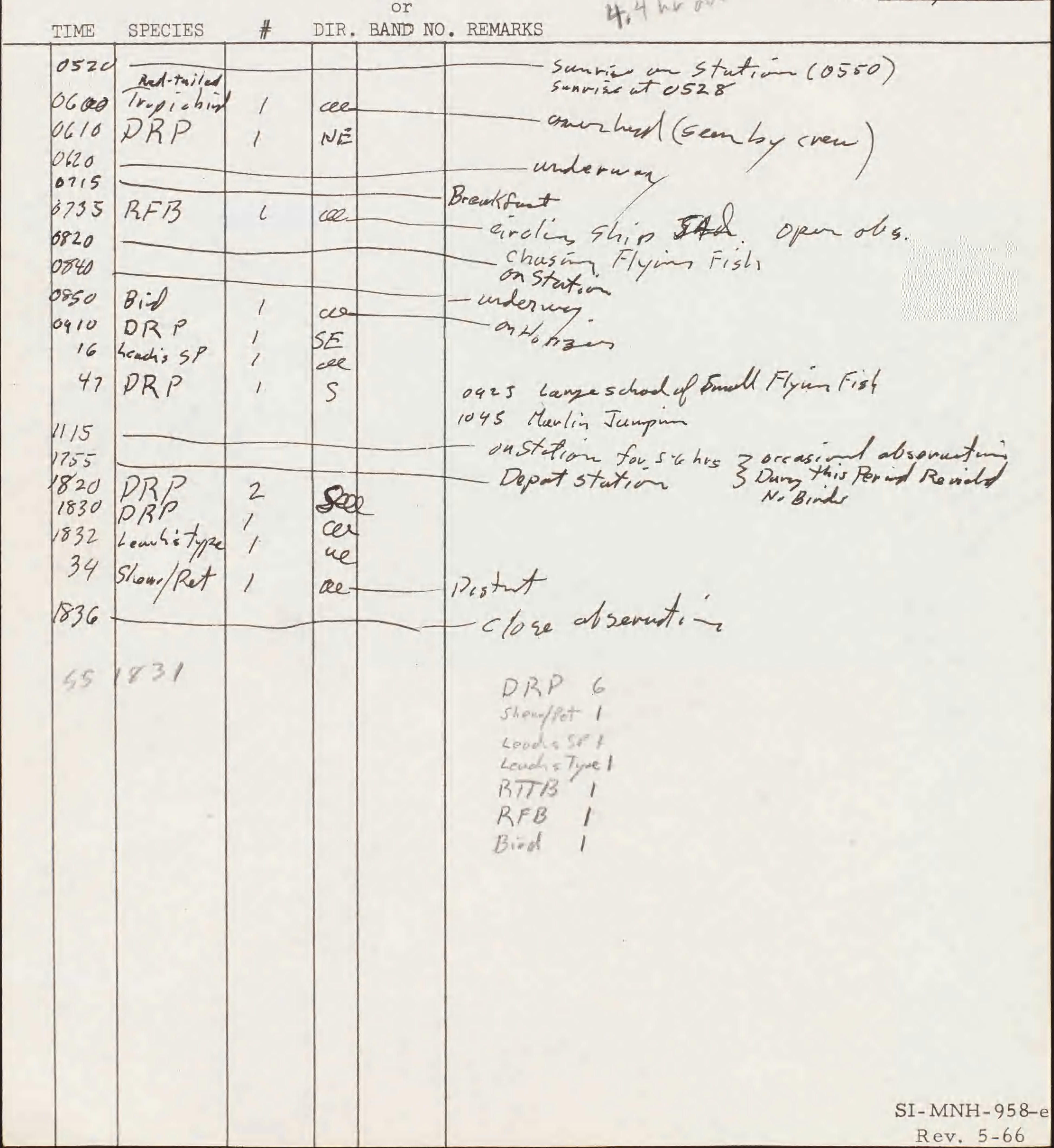




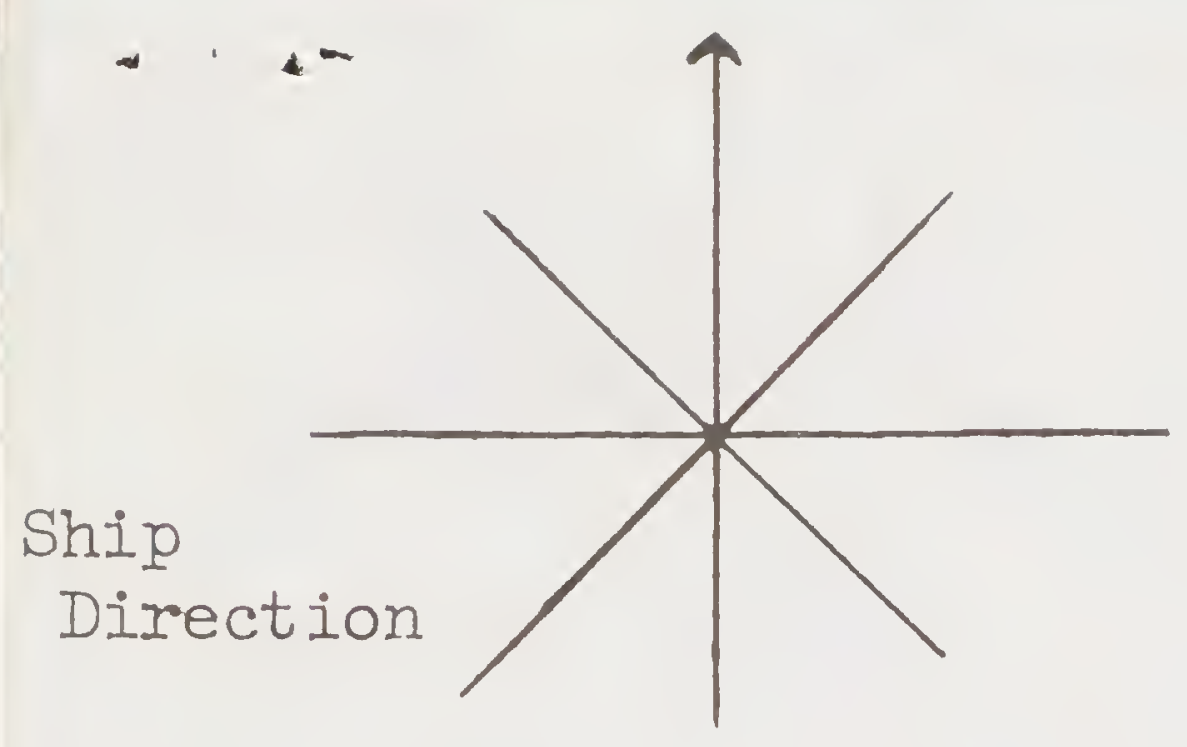

SMITHSONIAN INSTITUTION DIVISION OF BIRDS AT SEA DAILY LOG - E

OBSERVERS :

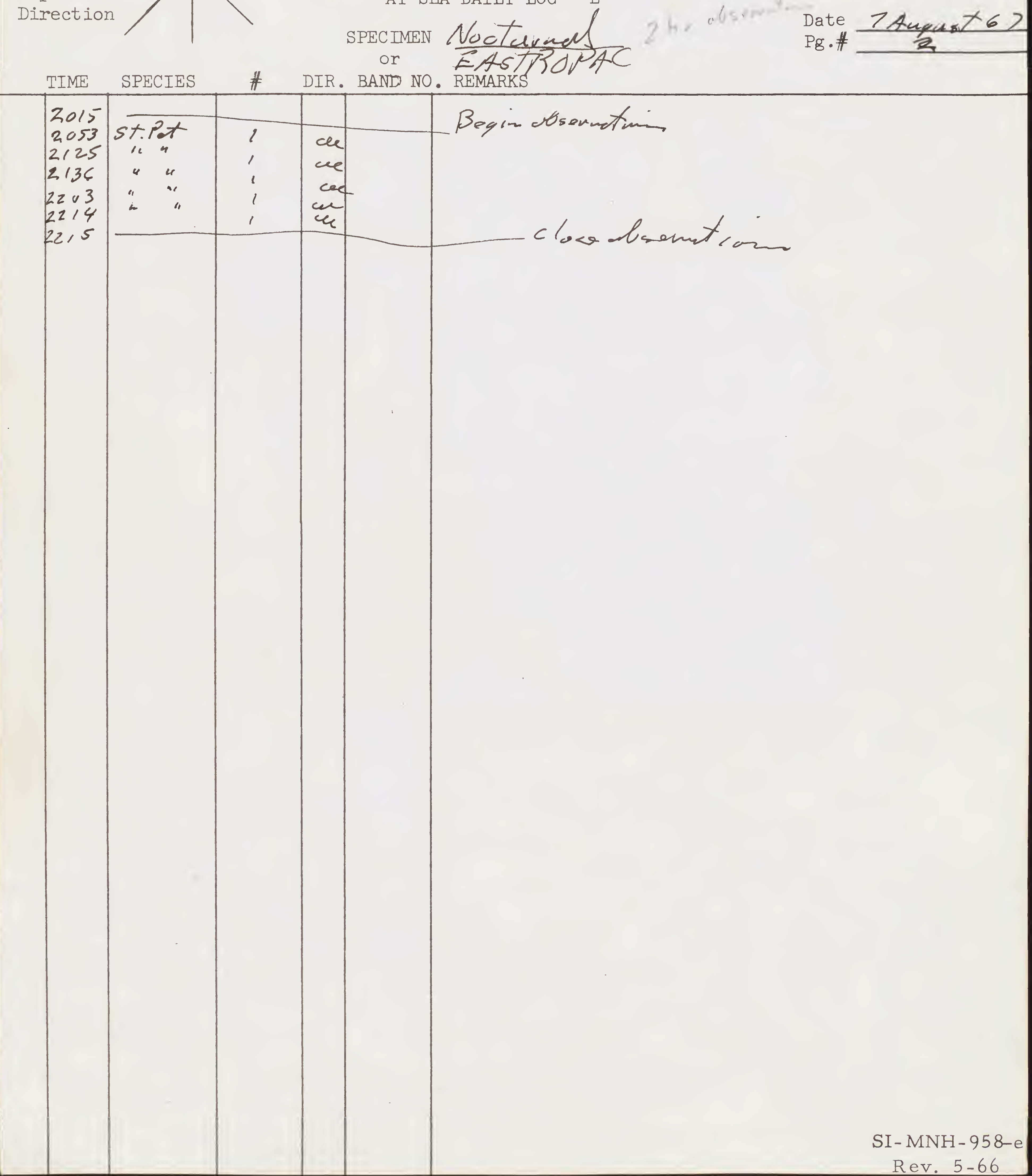




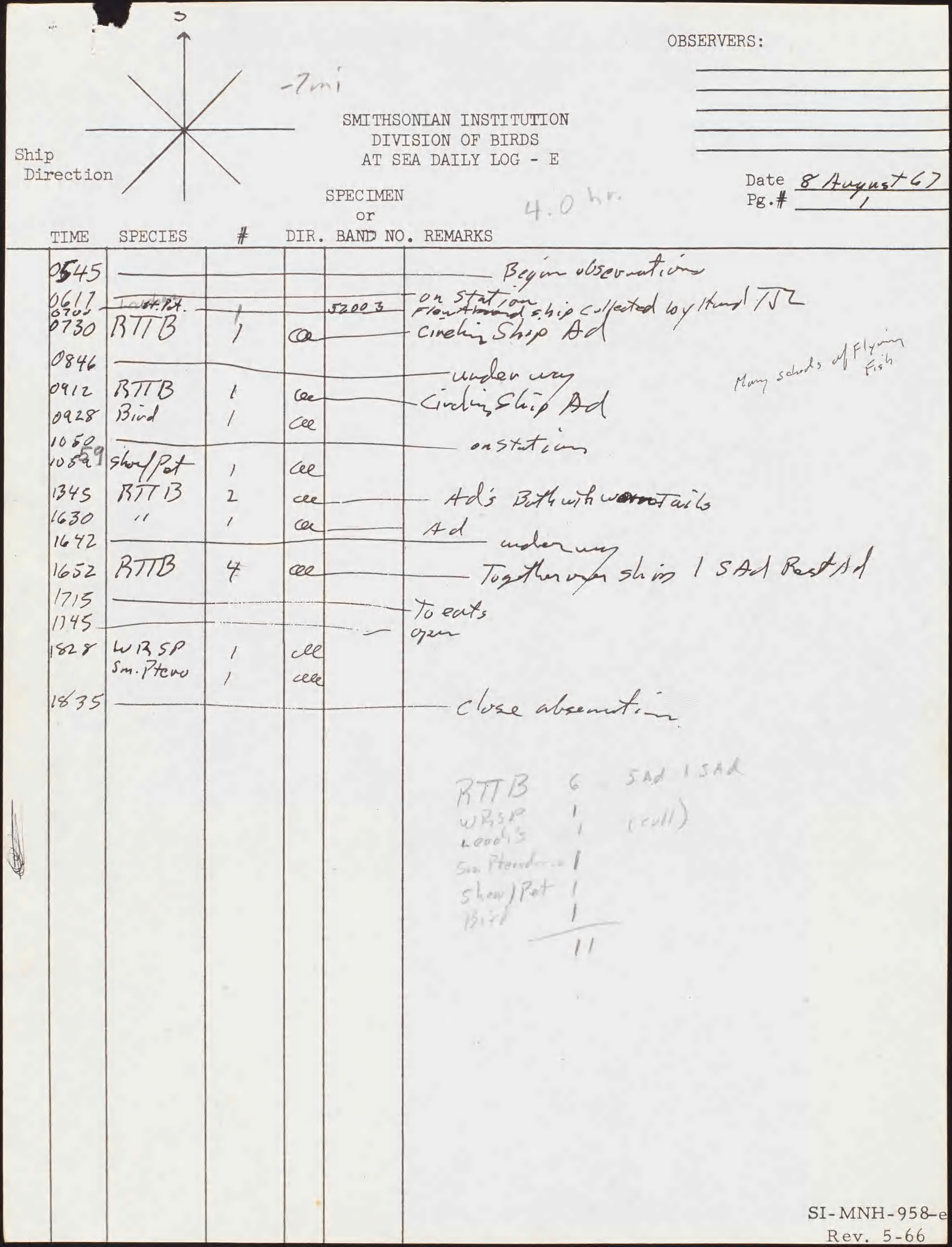




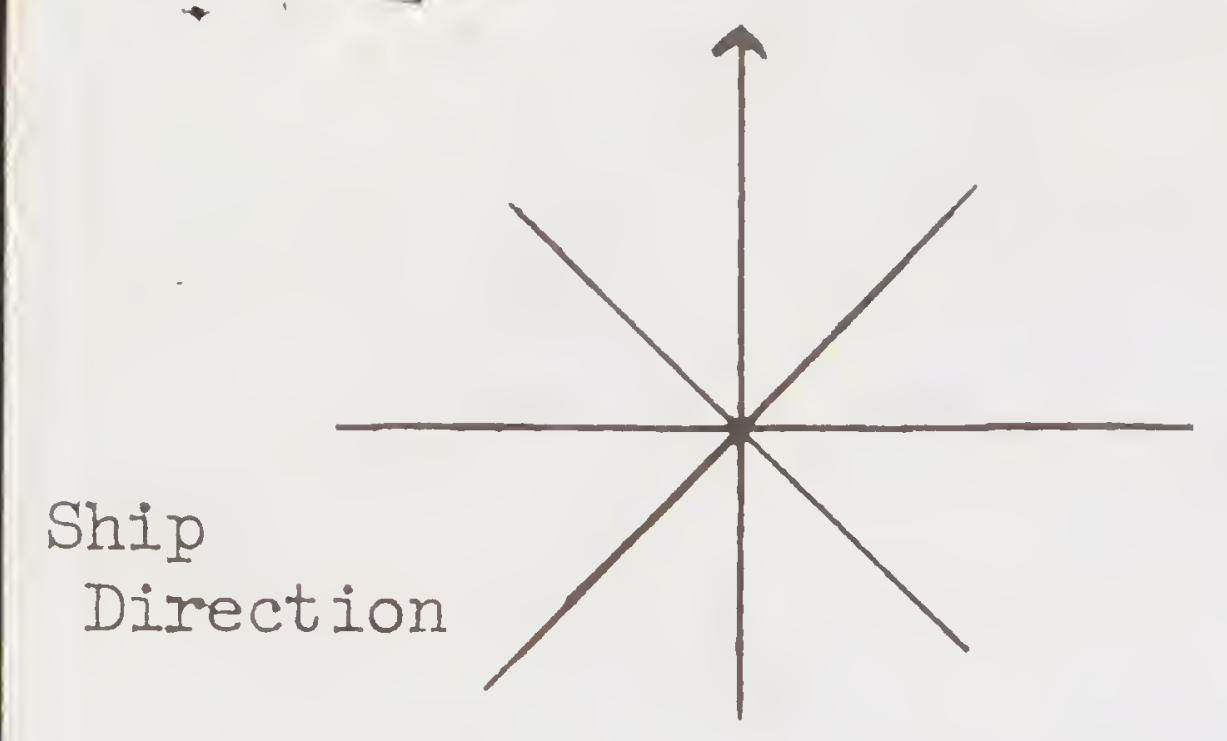

OBSERVERS :

SMITHSONIAN INSTITUTION

DIVISION OF BIRDS

AT SEA DAILY LOG - E

or

Nocturnal

DIR. BAND NO. REMARKS

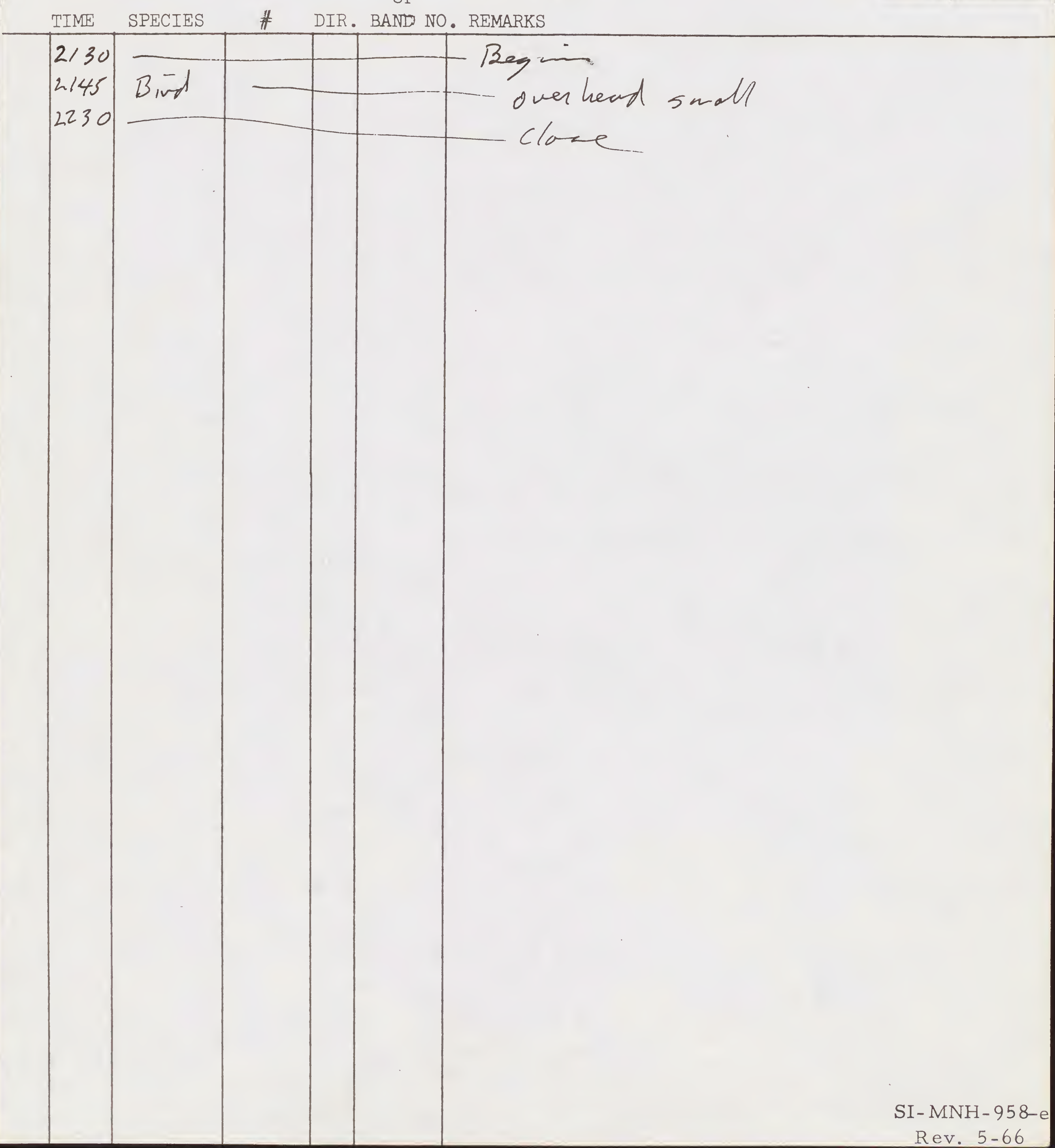




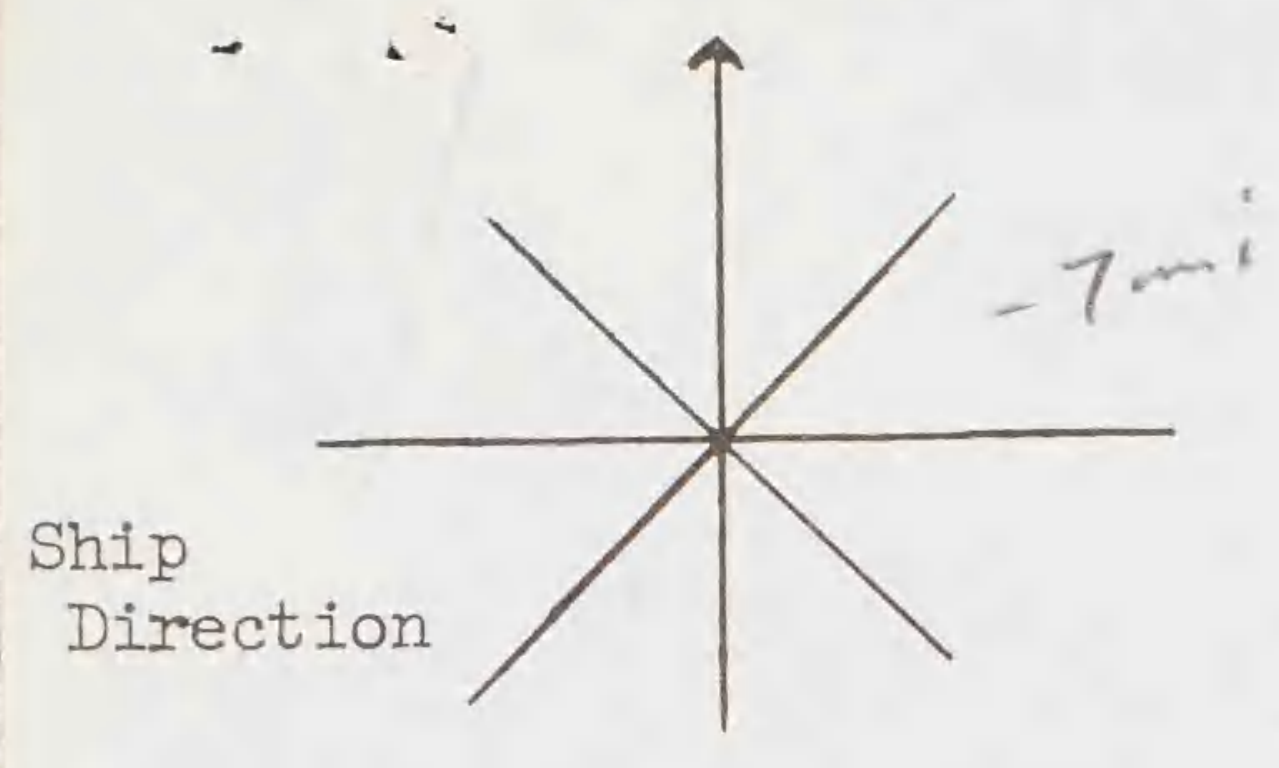

SMITHSONIAN INSTITUTION DIVISION OF BIRDS

AT SEA DAILY LOG - E

OBSERVERS :

$5.4^{h} \quad \begin{aligned} & \text { Date } 9 \text { August } 62 \\ & \text { Pg.\# }\end{aligned}$

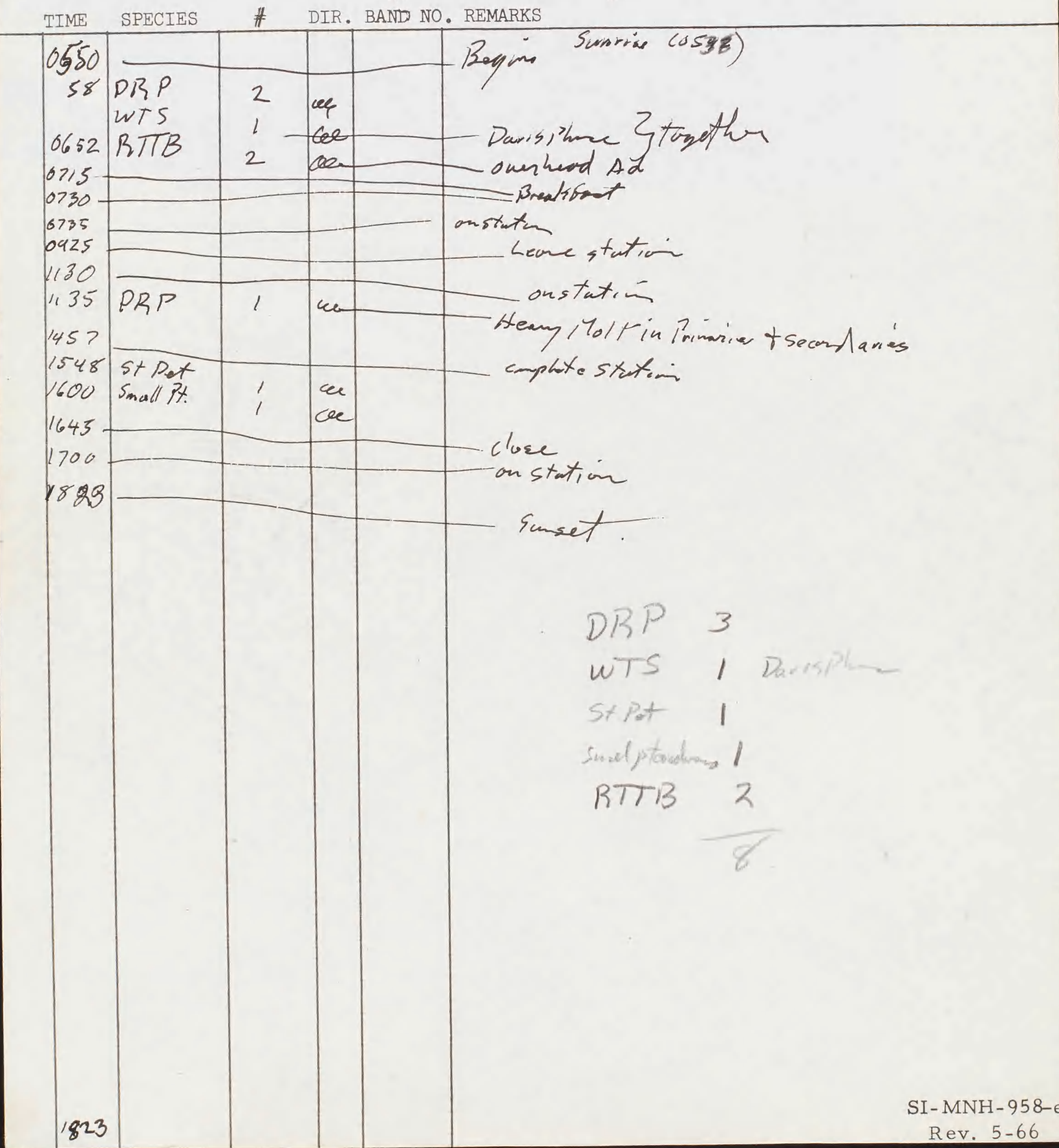




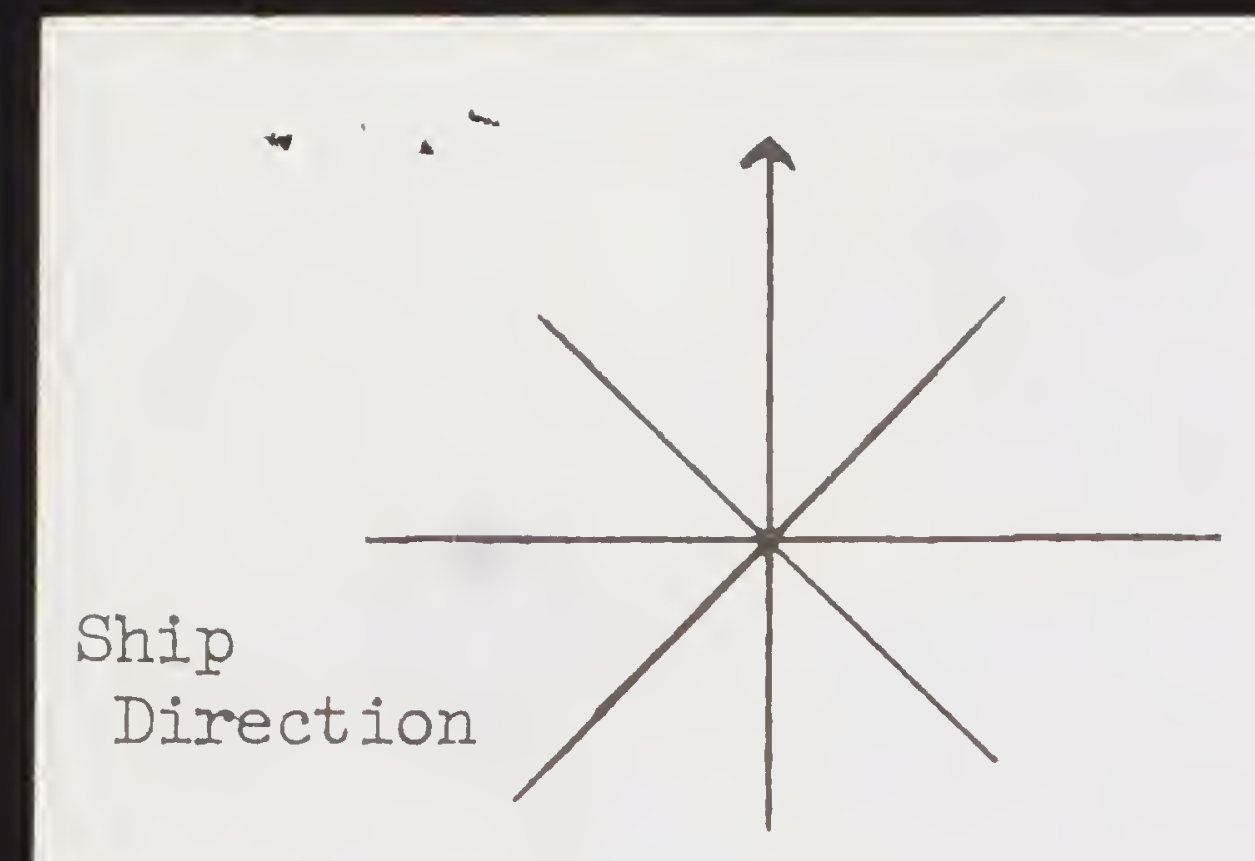

\section{OBSERVERS :}

SMITHSONIAN INSTITUTION

DIVISION OF BIRDS

AT SEA DAILY LOG - E

SPECIMEN Noct re onal

or

Date Qturnat

\# DIR. BAND NO. REMARKS

\begin{tabular}{|l|l|l|l|l|l}
2010 & - & & & & oper \\
260 & & & & & obe \\
\hline
\end{tabular}

SI- MNH - 958-e Rev. 5-66 


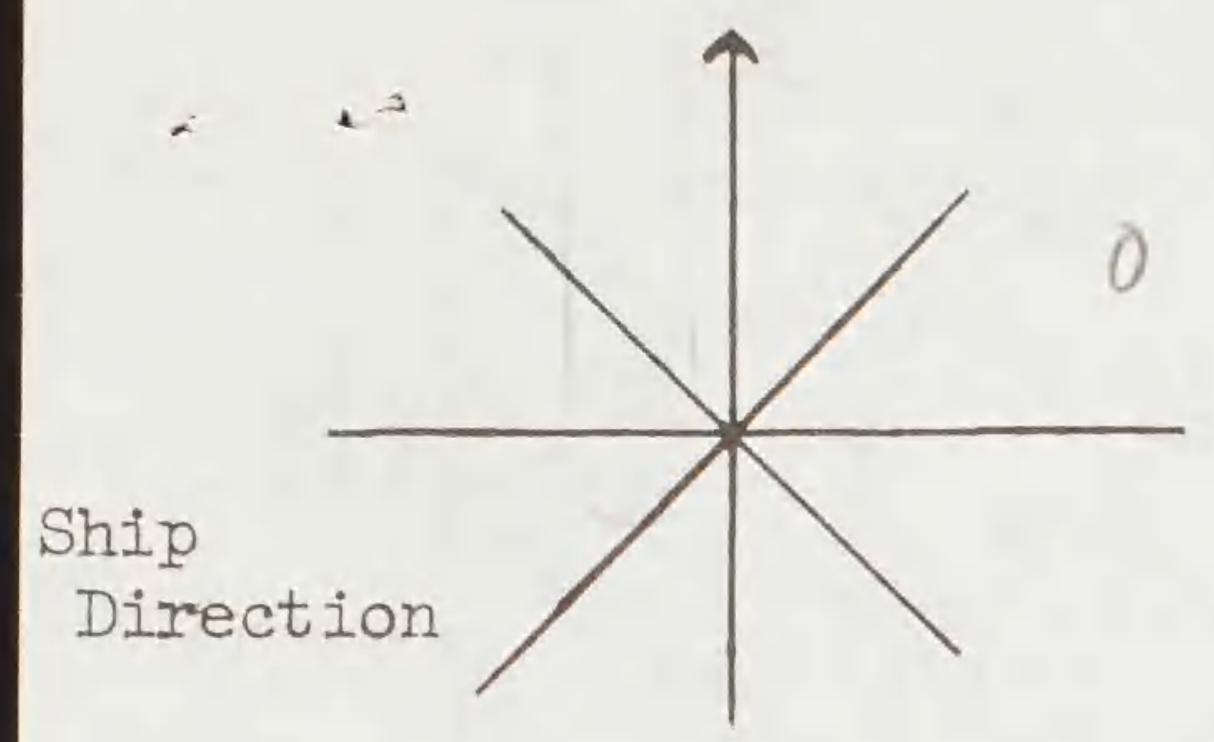

OBSERVERS :

SMITHSONIAN INSTITUTION DIVISION OF BIRDS AT SEA DAILY LOG - E

SPECIMEN

or
Date 10 Hug ns 167 $\mathrm{Pg} \cdot \#$

DIR. BAND NO. REMARKS

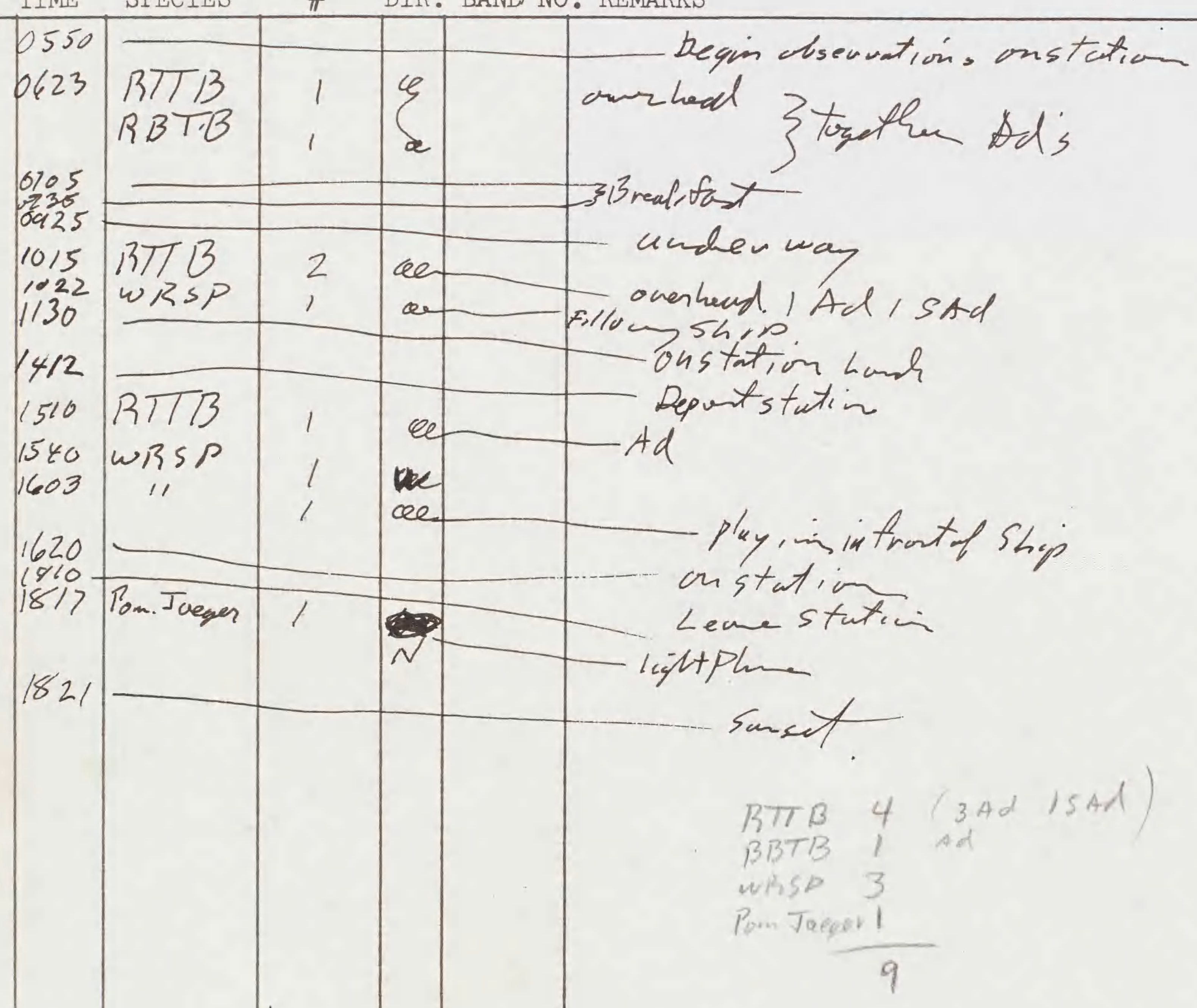

SI- MNH-958-e

1821

Rev. 5-66 


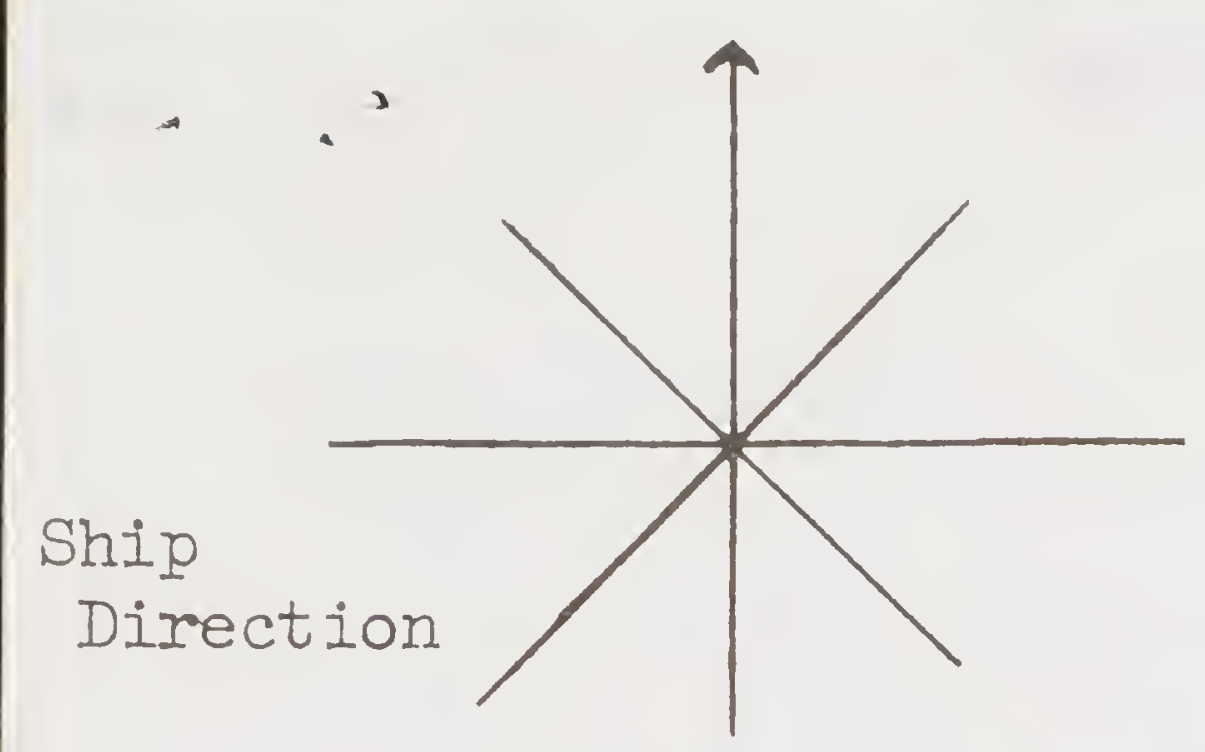

SMITHSONIAN INSTITUTION DIVISION OF BIRDS

AT SEA DAILY LOG - E

SPECIMEN Nocturnal

or

OBSERVERS :

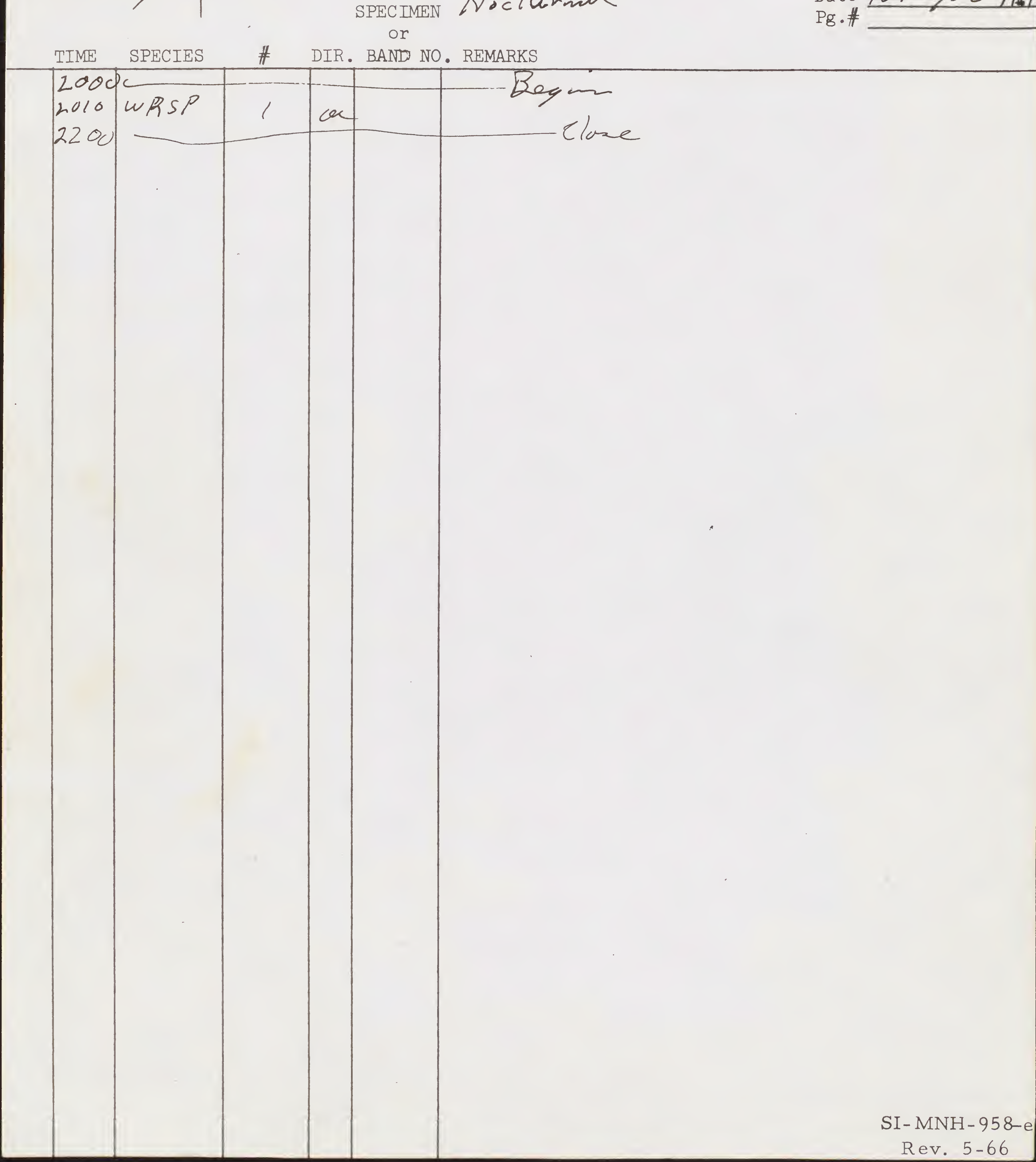




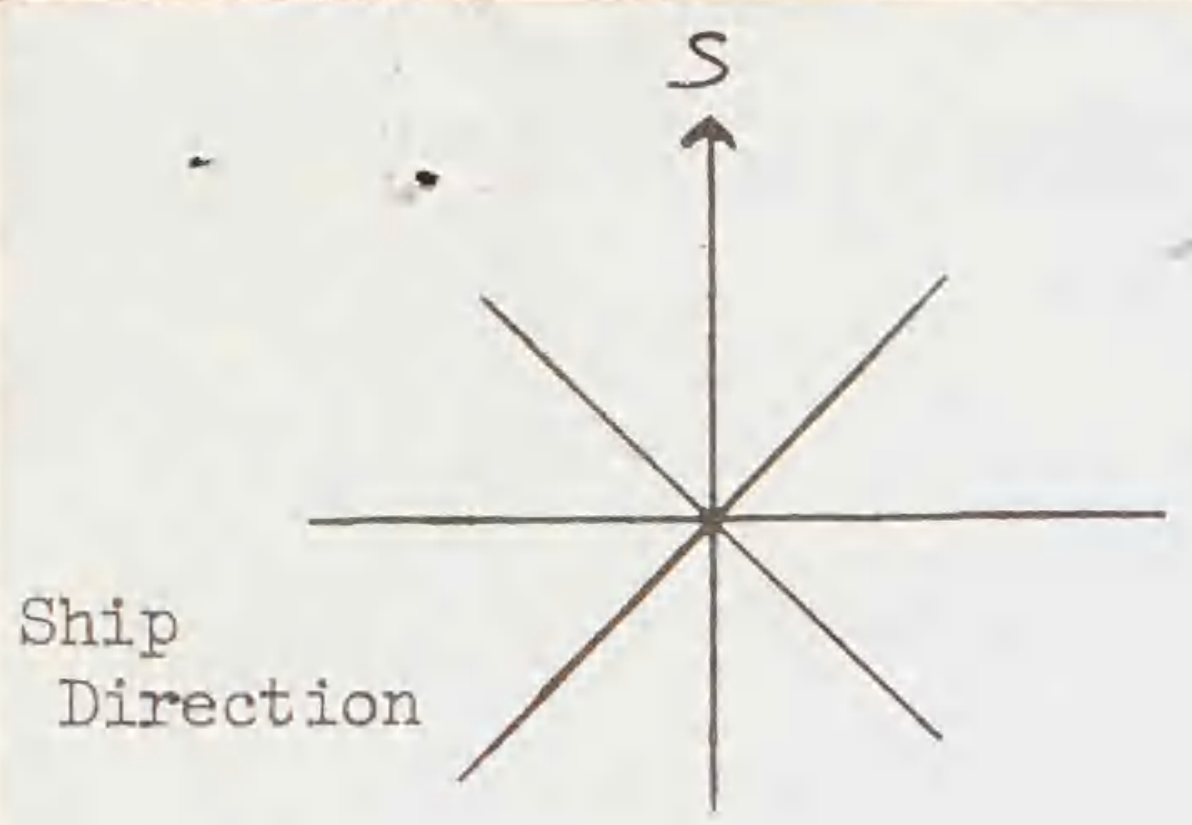

SMITHSONIAN INSTITUTION

DIVISION OF BIRDS

AT SEA DAILY LOG - E

SPECIMEN

or

OBSERVERS :

Glasysen - test Calm

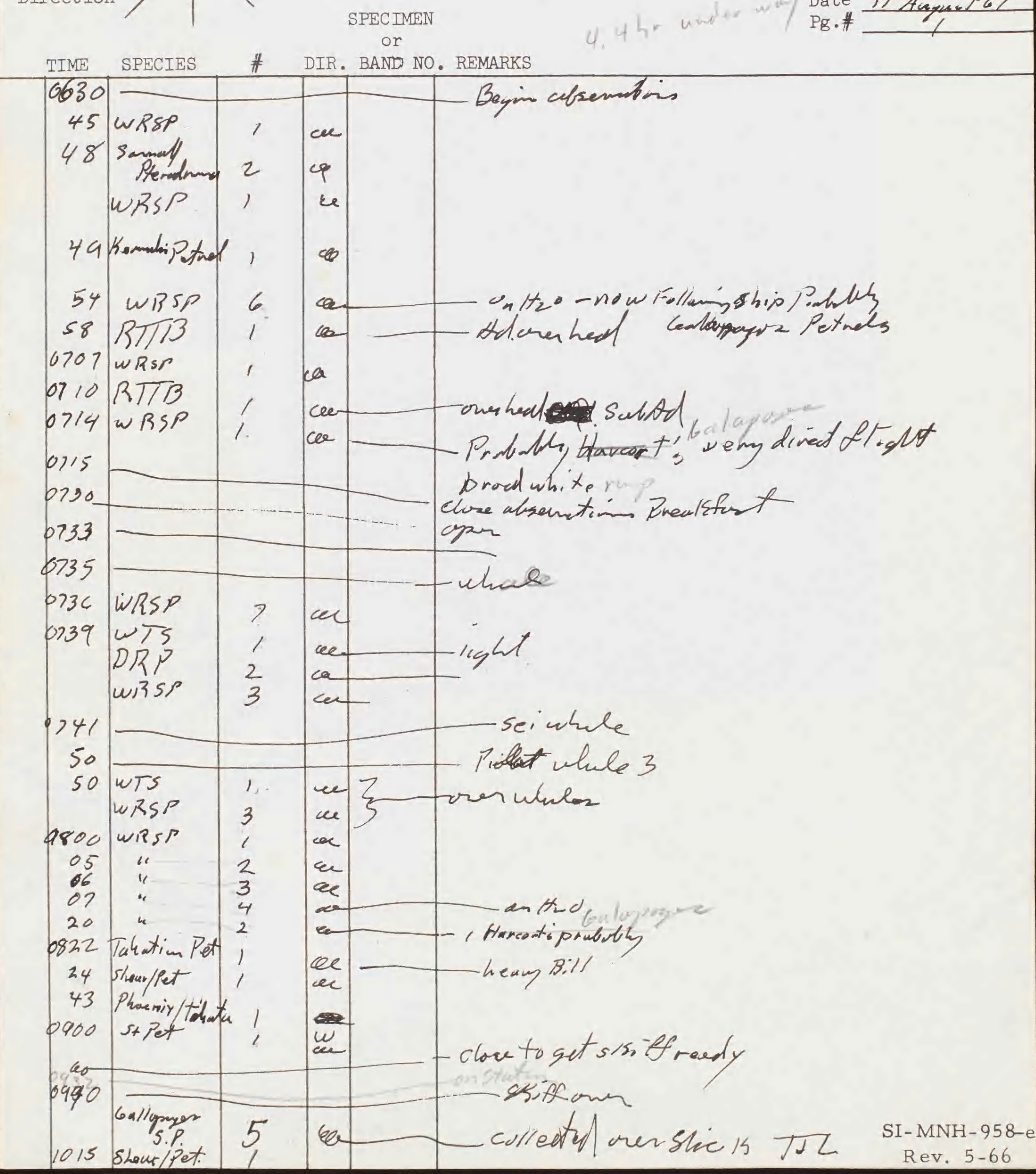




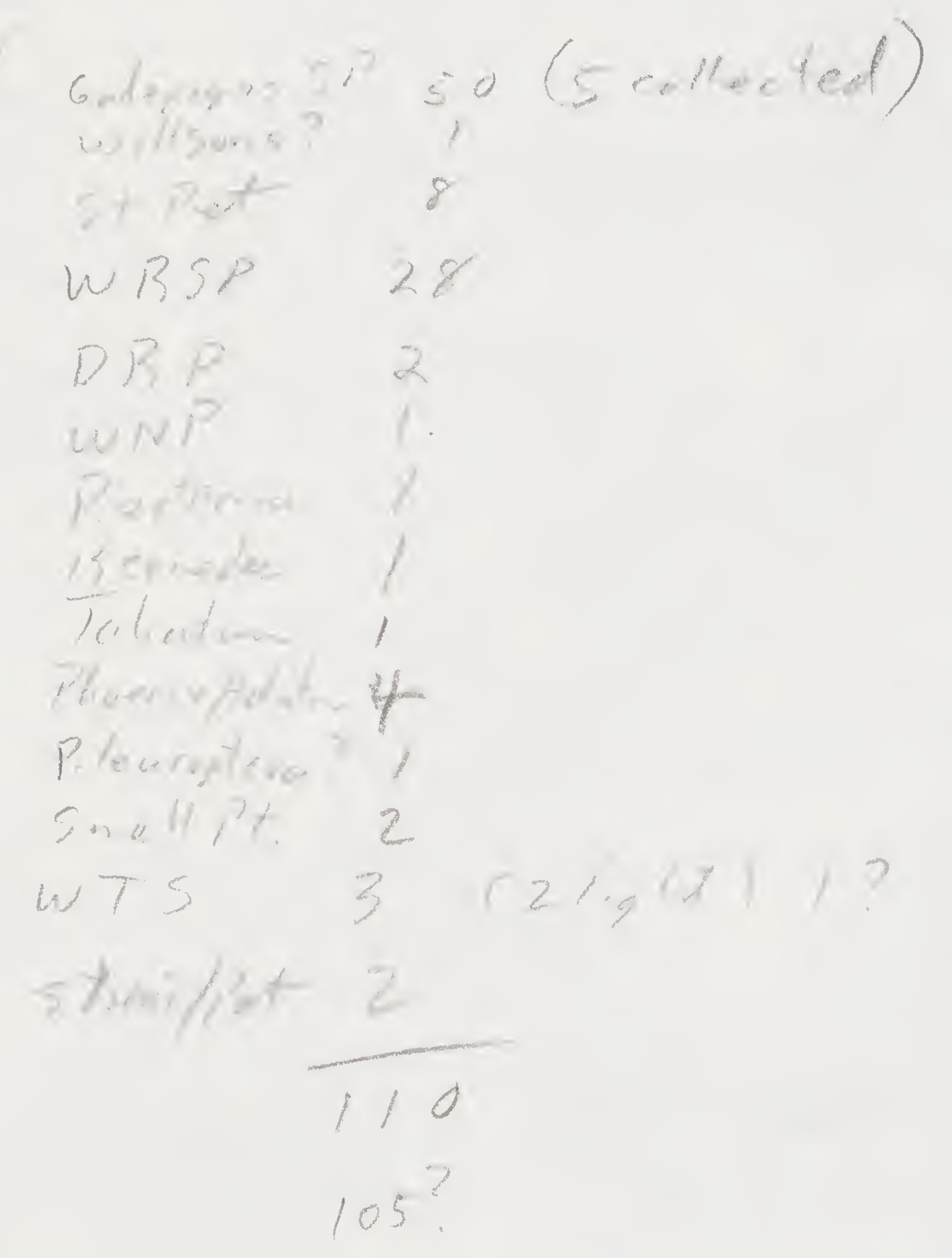




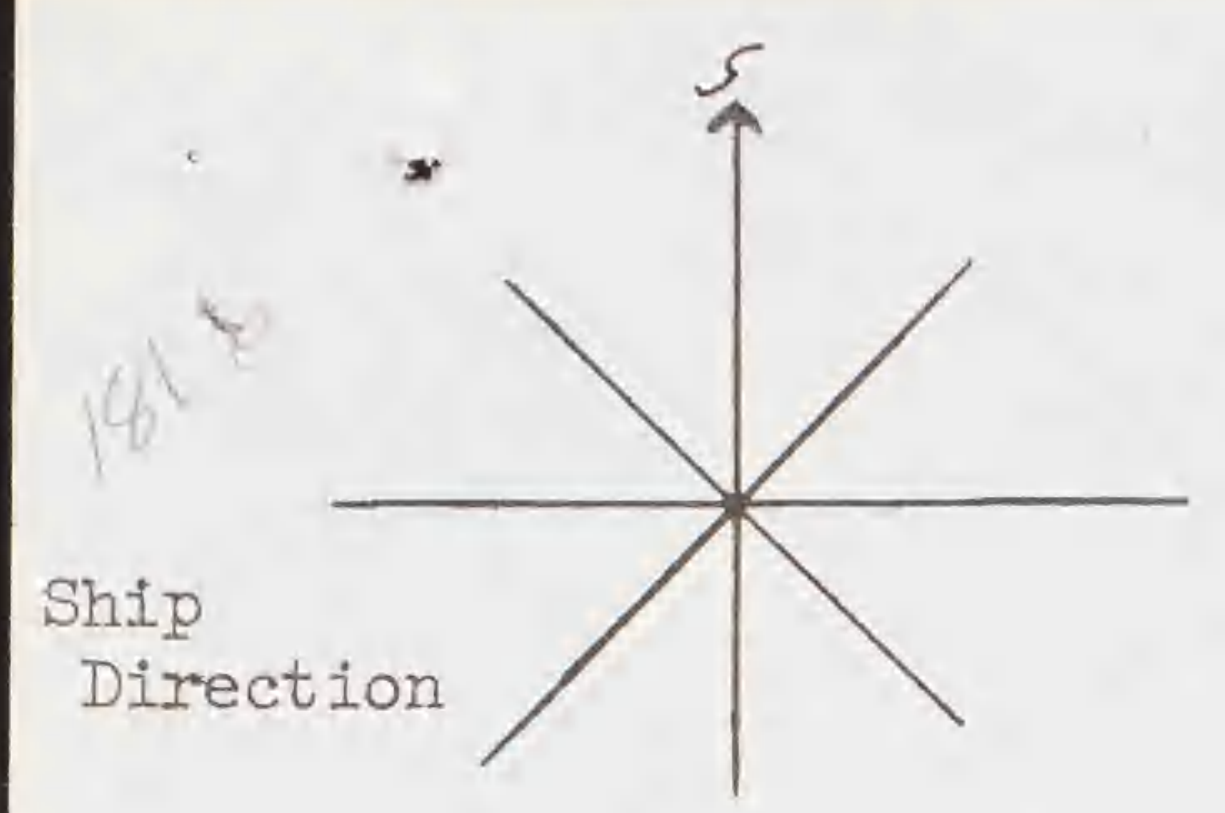

SMITHSONIAN INSTITUTION

DIVISION OF BIRDS

AT SEA DAILY LOG - E

SPEC IMEN

OBSERVERS :

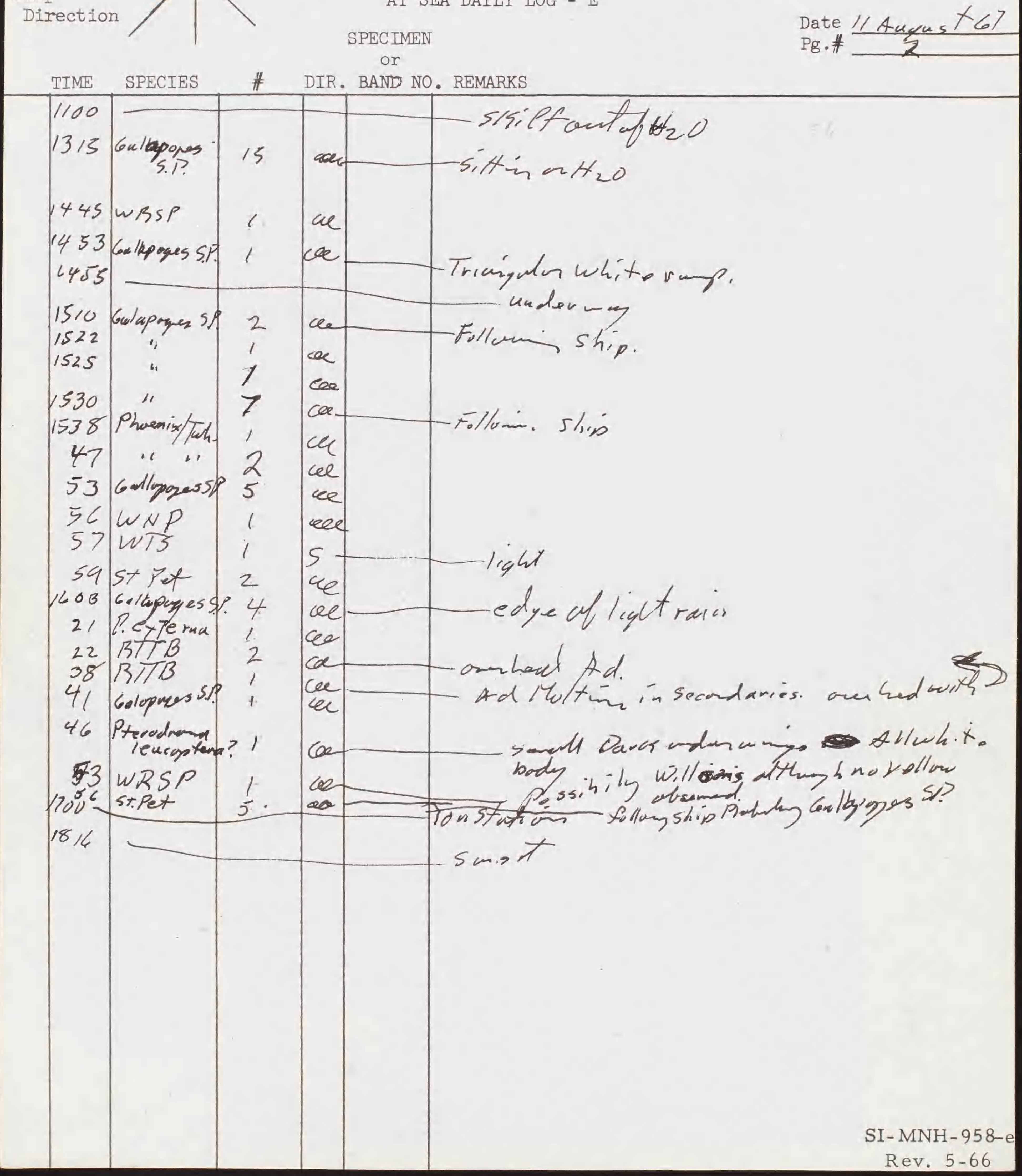




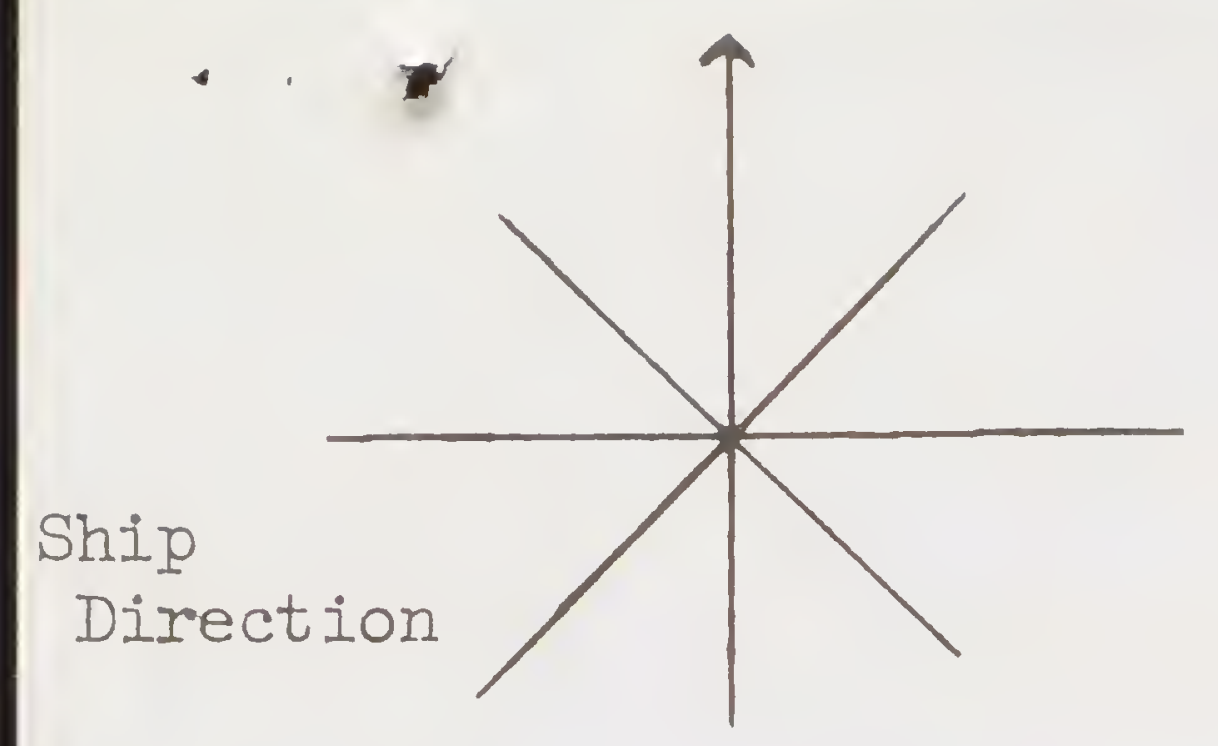

SMITHSONIAN INSTITUTION

DIVISION OF BIRDS

AT SEA DAILY LOG - E

SPECIMEN Nocturnal

OBSERVERS :

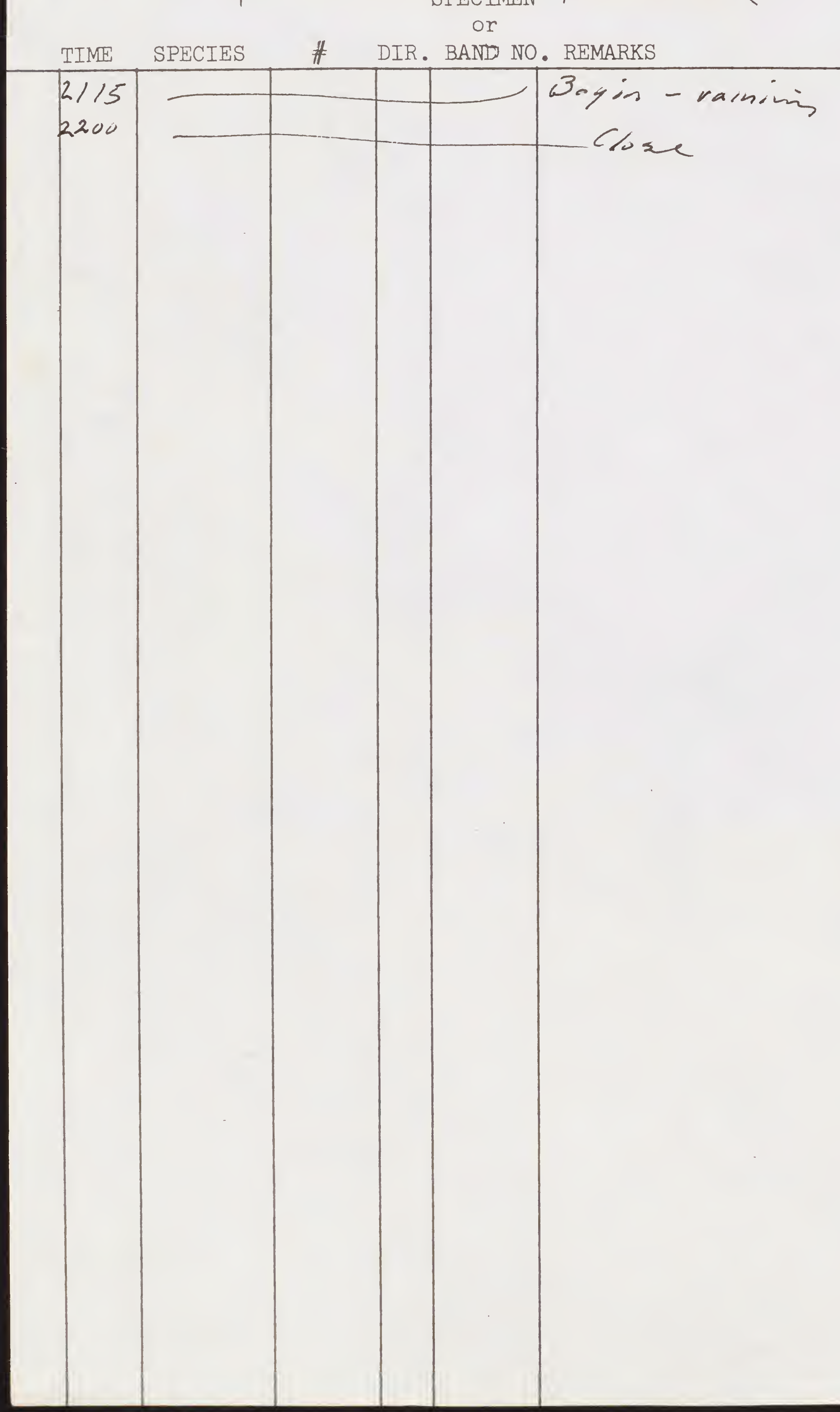

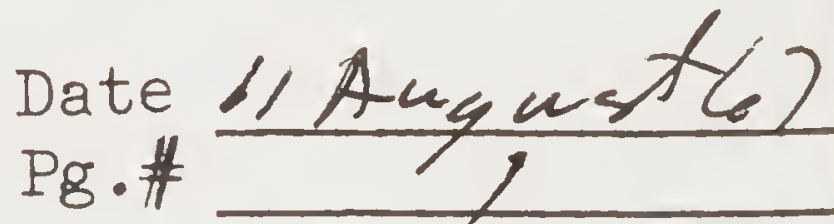

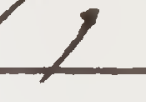




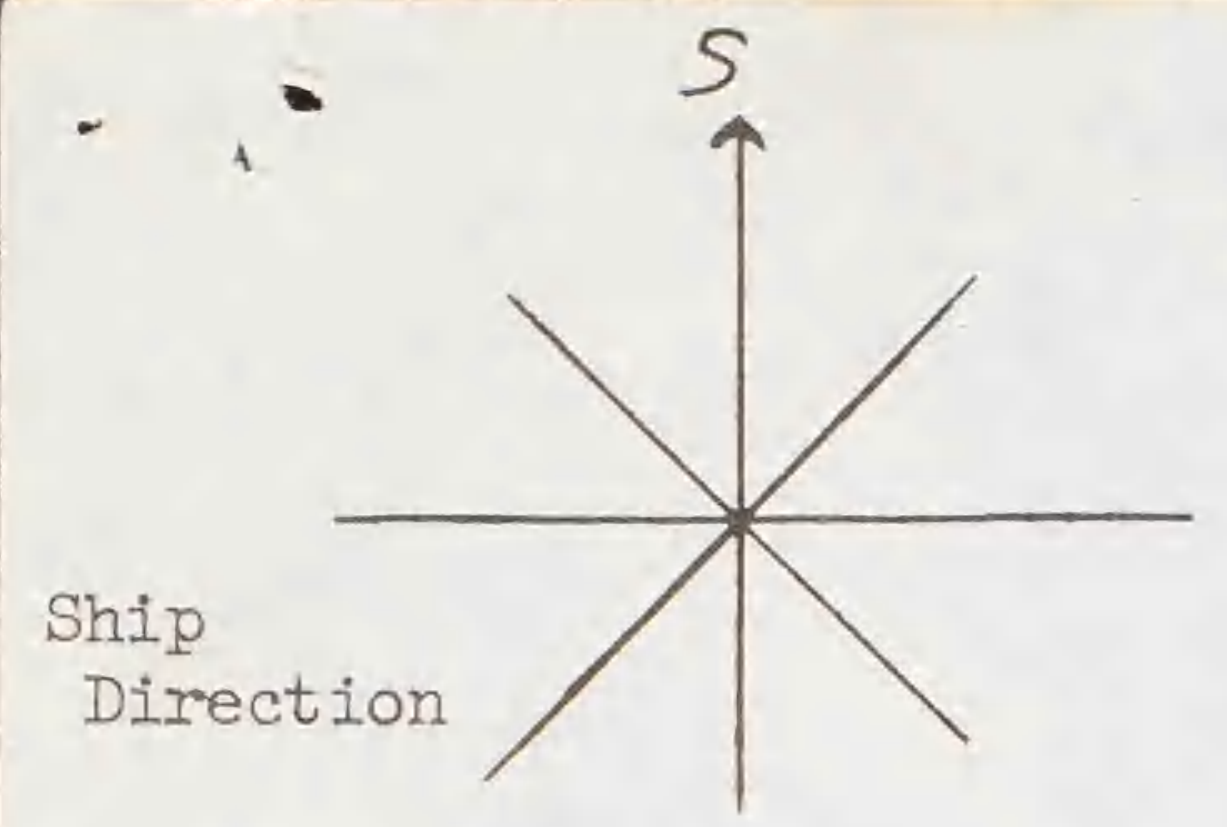

OBSERVERS :

Moleate suells How wans wid20-25

SMITHSONIAN INSTITUTION

DIVISION OF BIRDS

AT SEA DAILY LOG - E
SPEC IMEN

or
Higket seas twid ou fav antrip.

Date 22 Augus TCI

$\mathrm{Pg}$.\#

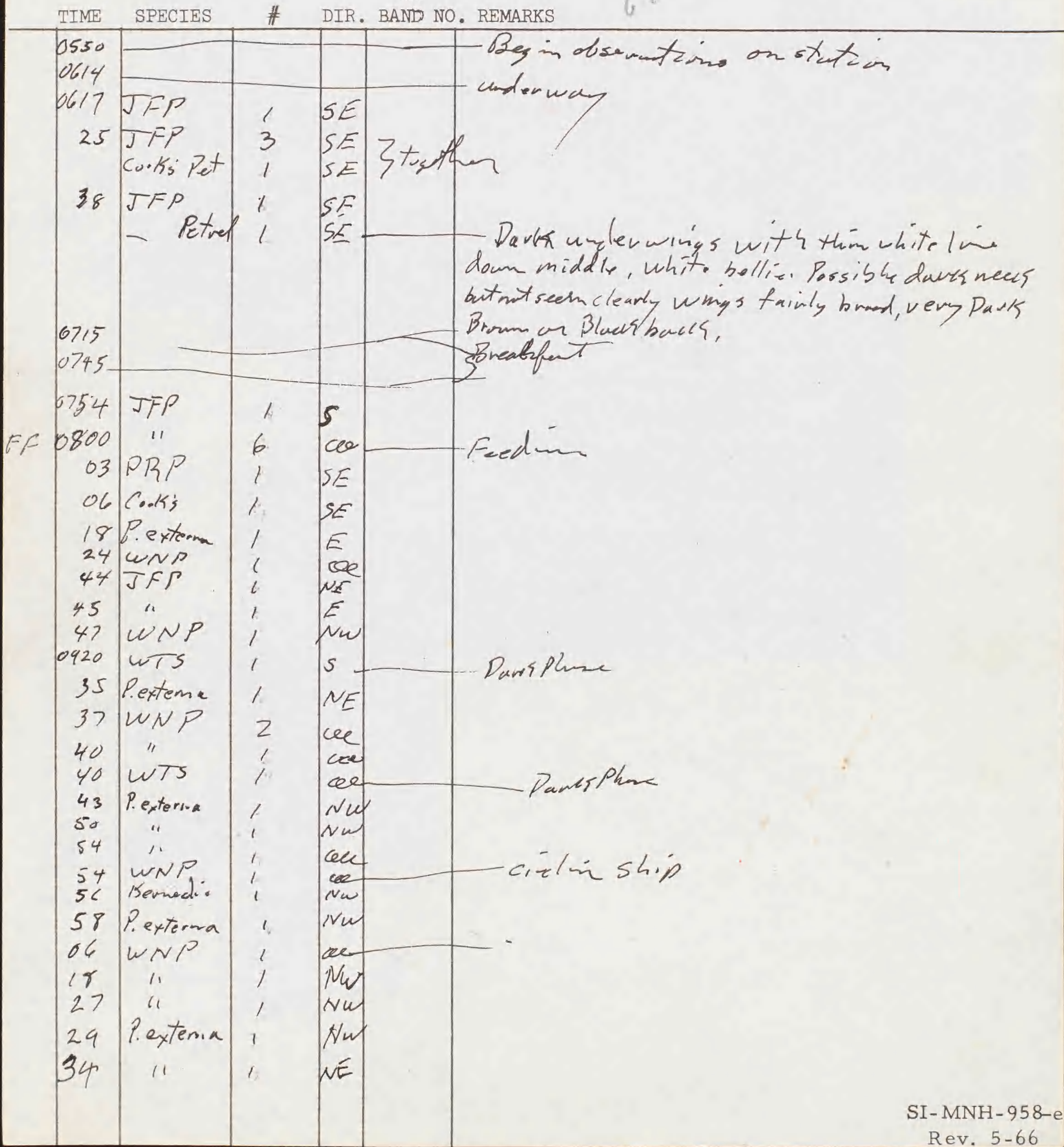




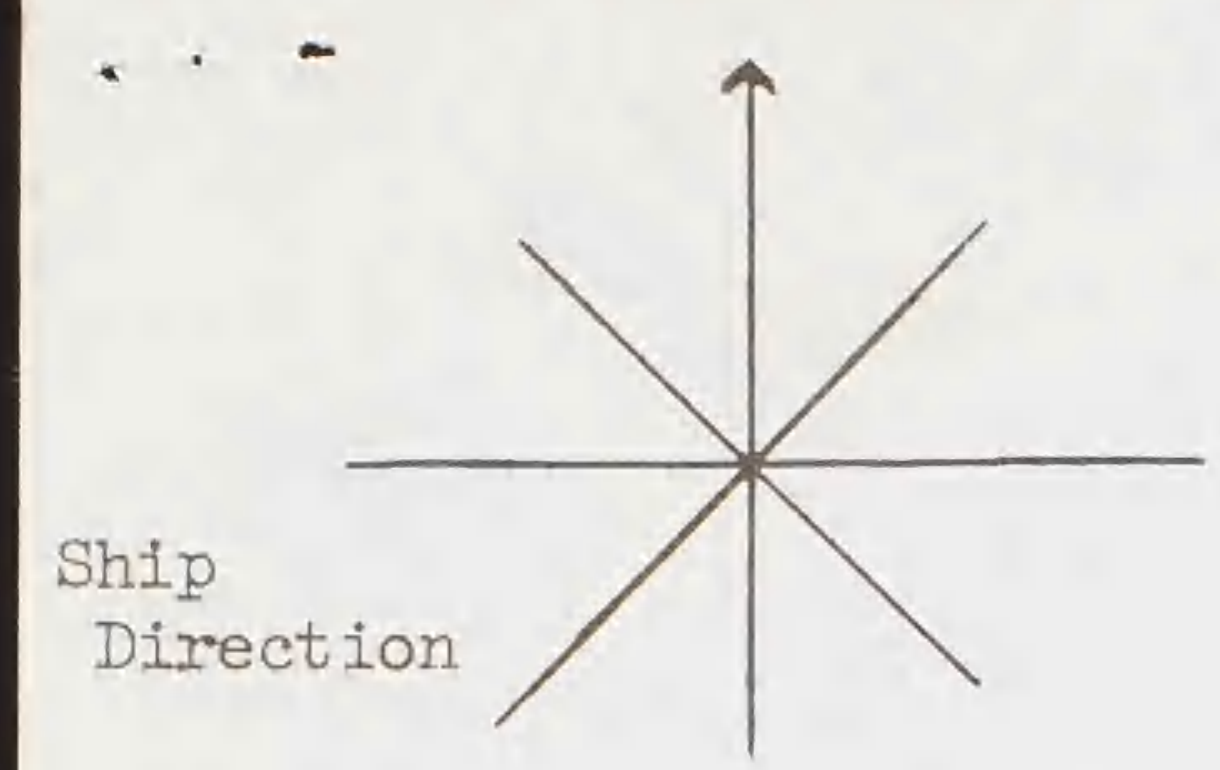

SMITHSONIAN INSTITUTION

DIVISION OF BIRDS

AT SEA DAILY LOG - E

SPECIMEN

Date 12 Angus $t$ $\mathrm{Pg} \cdot \# \frac{2}{2}$

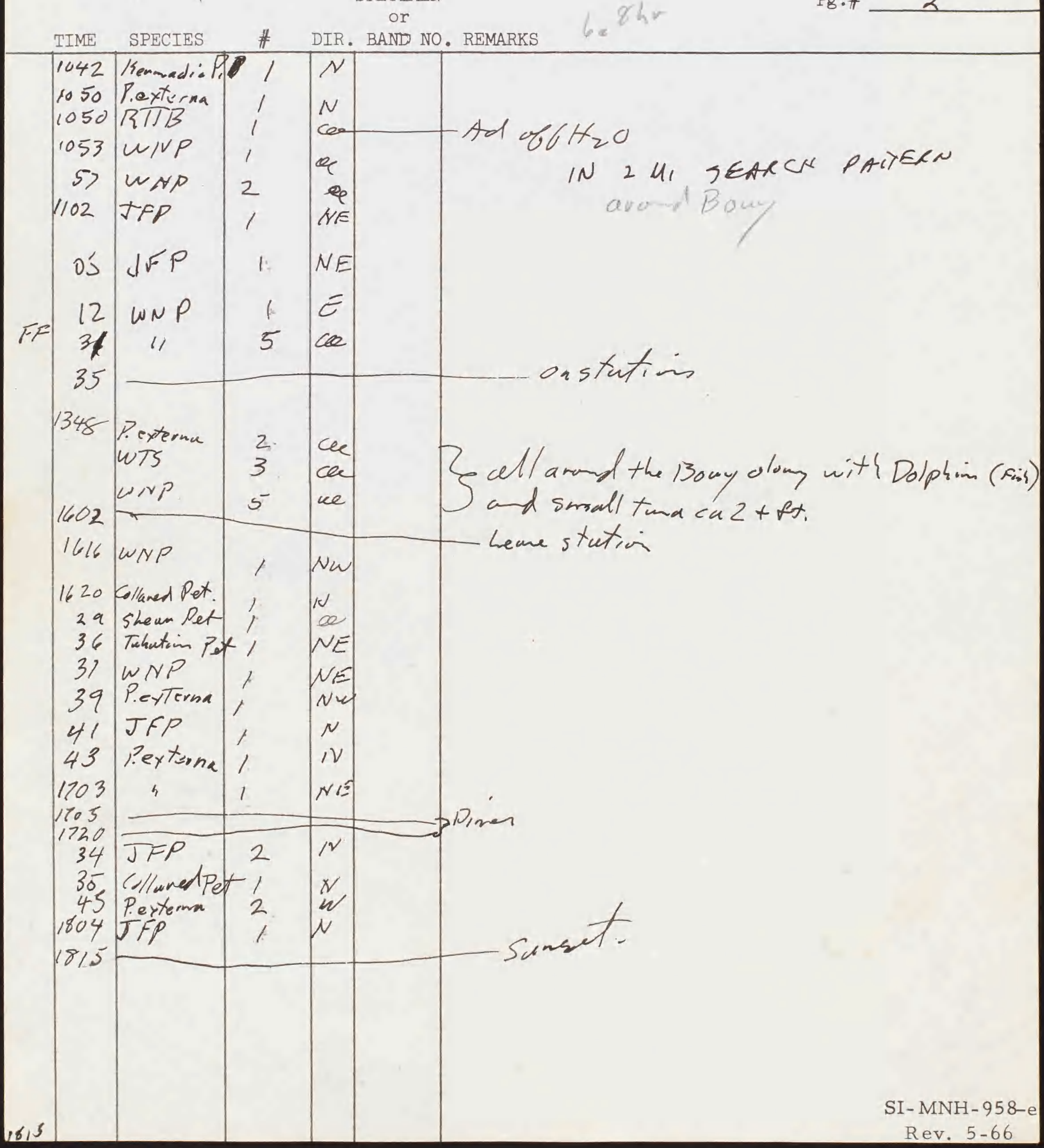




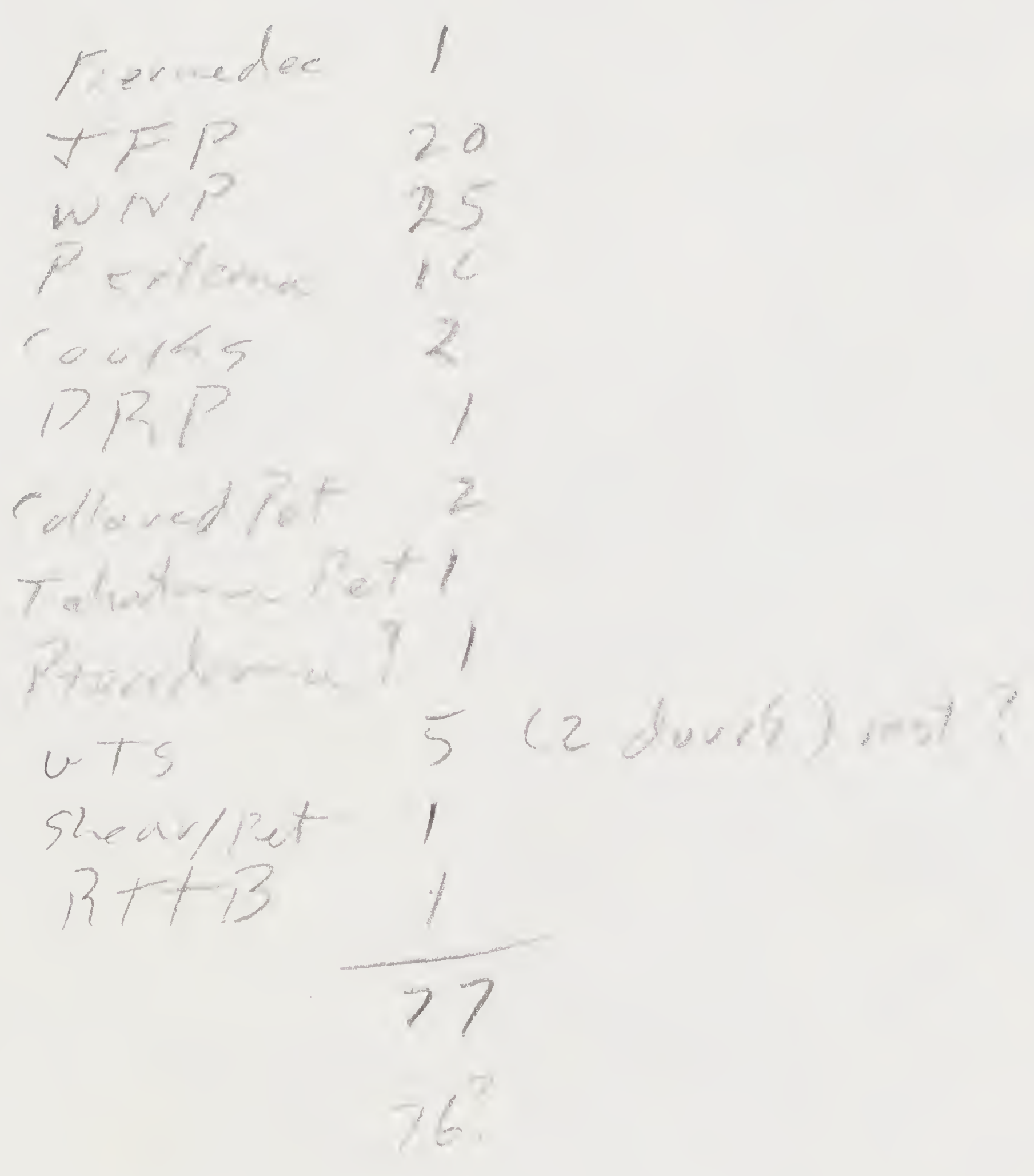




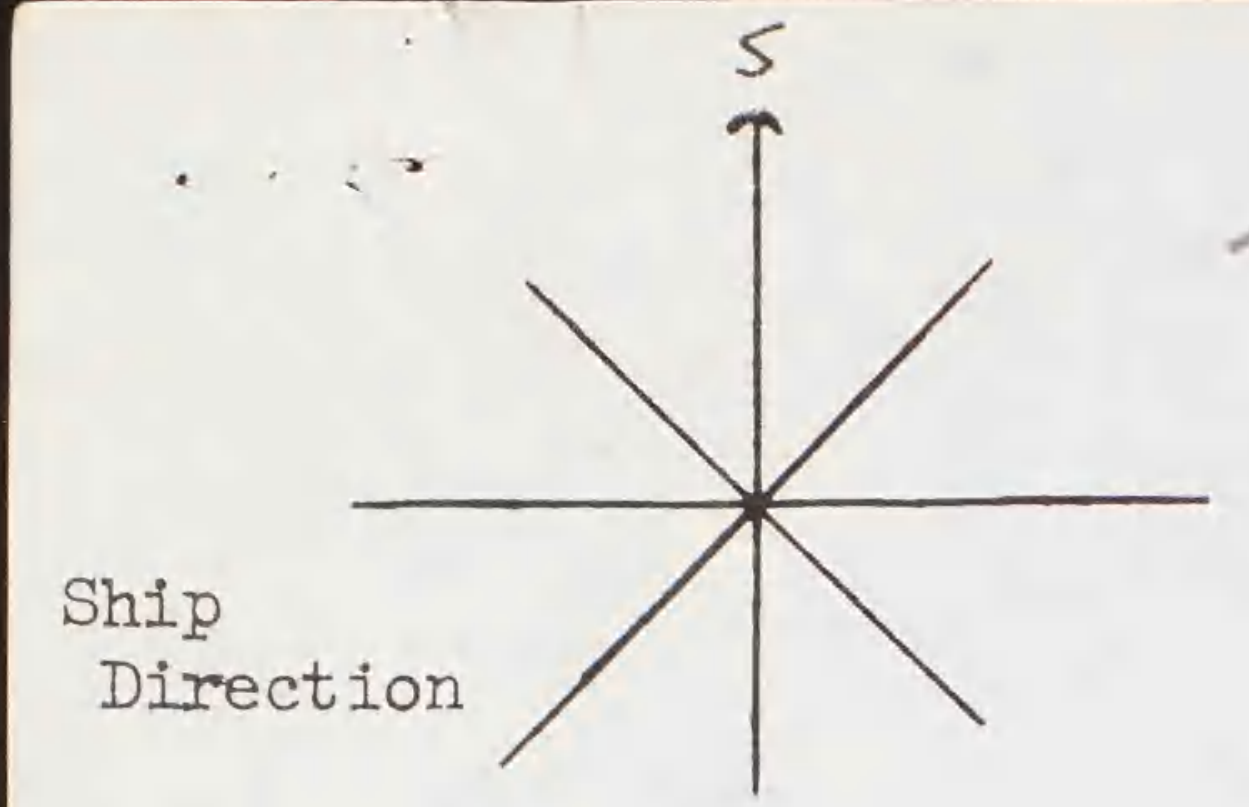

OBSERVERS :

SMITHSONIAN INSTITUTION DIVISION OF BIRDS AT SEA DAILY LOG - E
SPEC IMEN

or

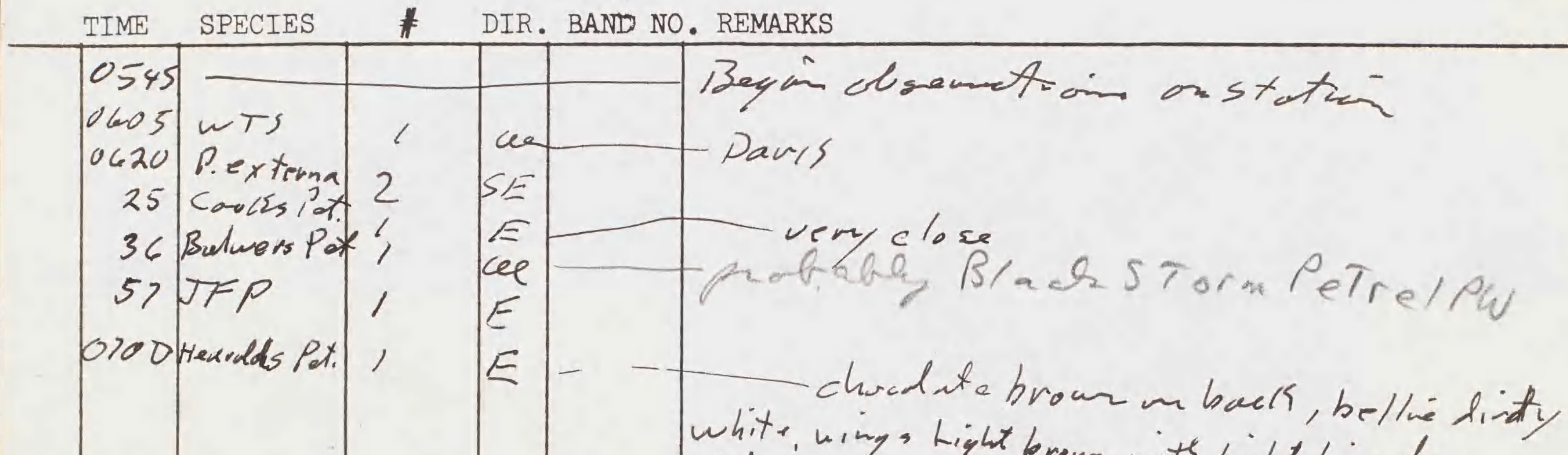
white, uings Light bromen with light line dun middh. Parkien on chest. thenkellis

6700
$\$>20$

$\$ 733$ Hearealstent

colloned Pext

54

WTS

58

Of 18 Sm.Pt.

35

\begin{tabular}{l|l|}
35 & JF, \\
39 & Phoenin/Tration \\
40 & WIVP
\end{tabular}

oq

43

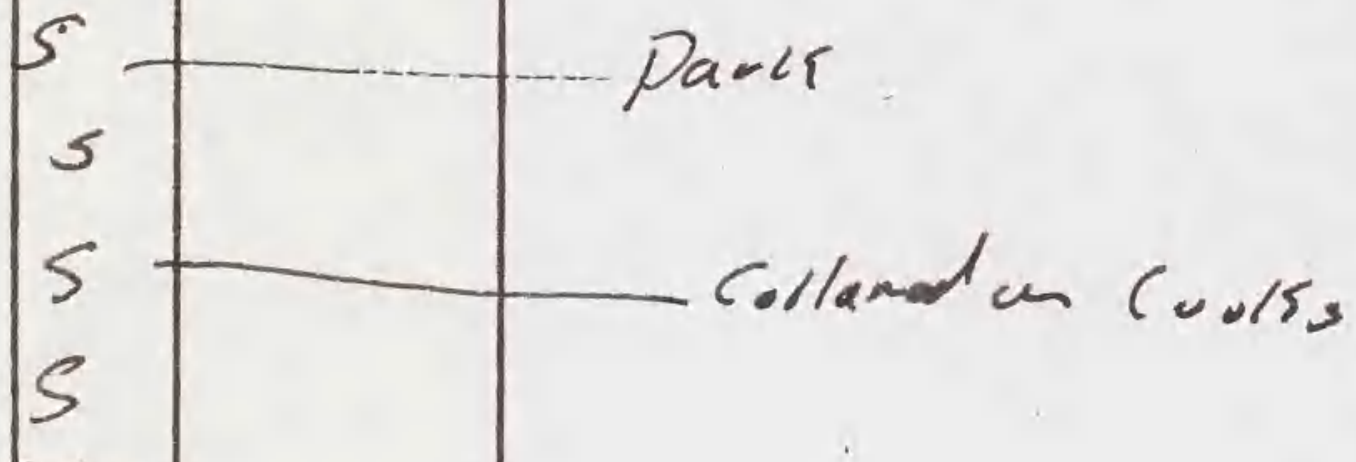

1 ac
20 Balwers

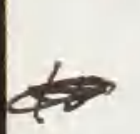

1000

or RTTB

11

Petrel

41 JFP

45 Galkapoges

1100

\begin{tabular}{l|l}
1 & 5 \\
5 & 5
\end{tabular}

1

1 SE

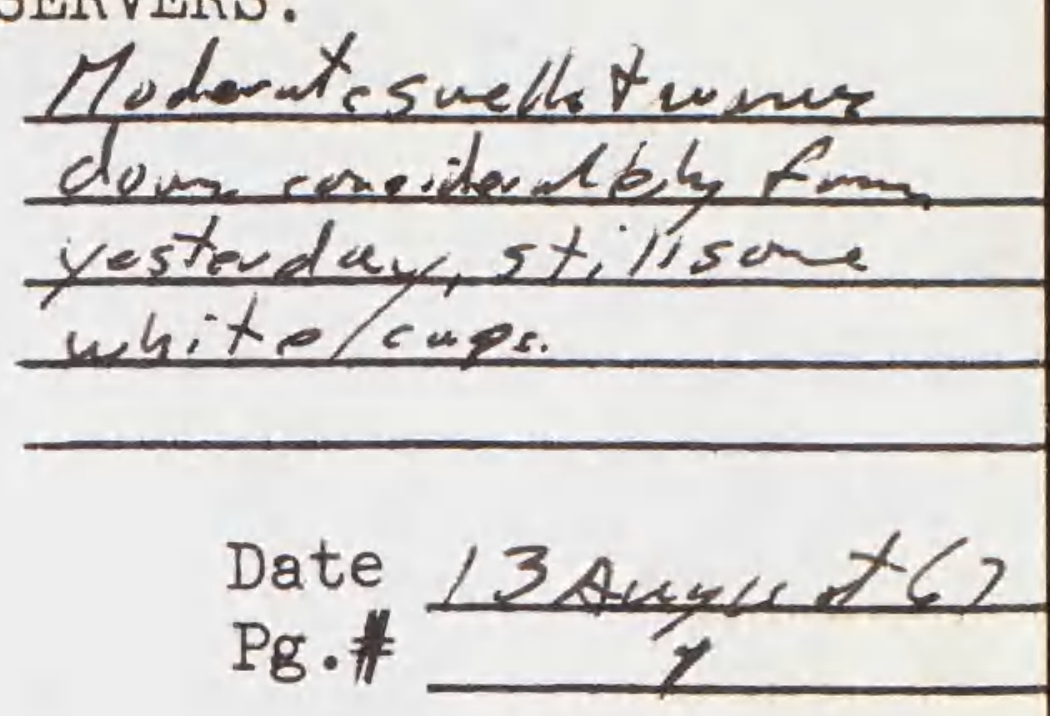
$\mathrm{Pg} \cdot \#$

dom corridededy fom

yestorday, stilison

whitelcups.

Date 13 suyud 62

r 


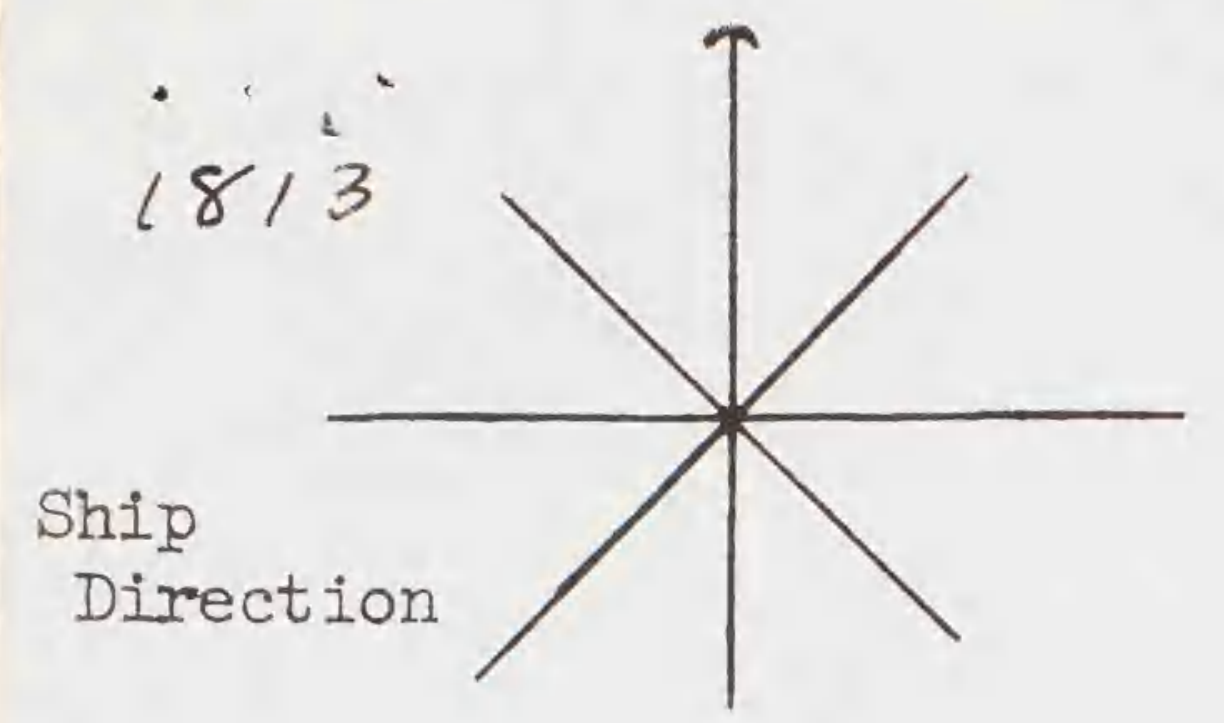

OBSERVERS:
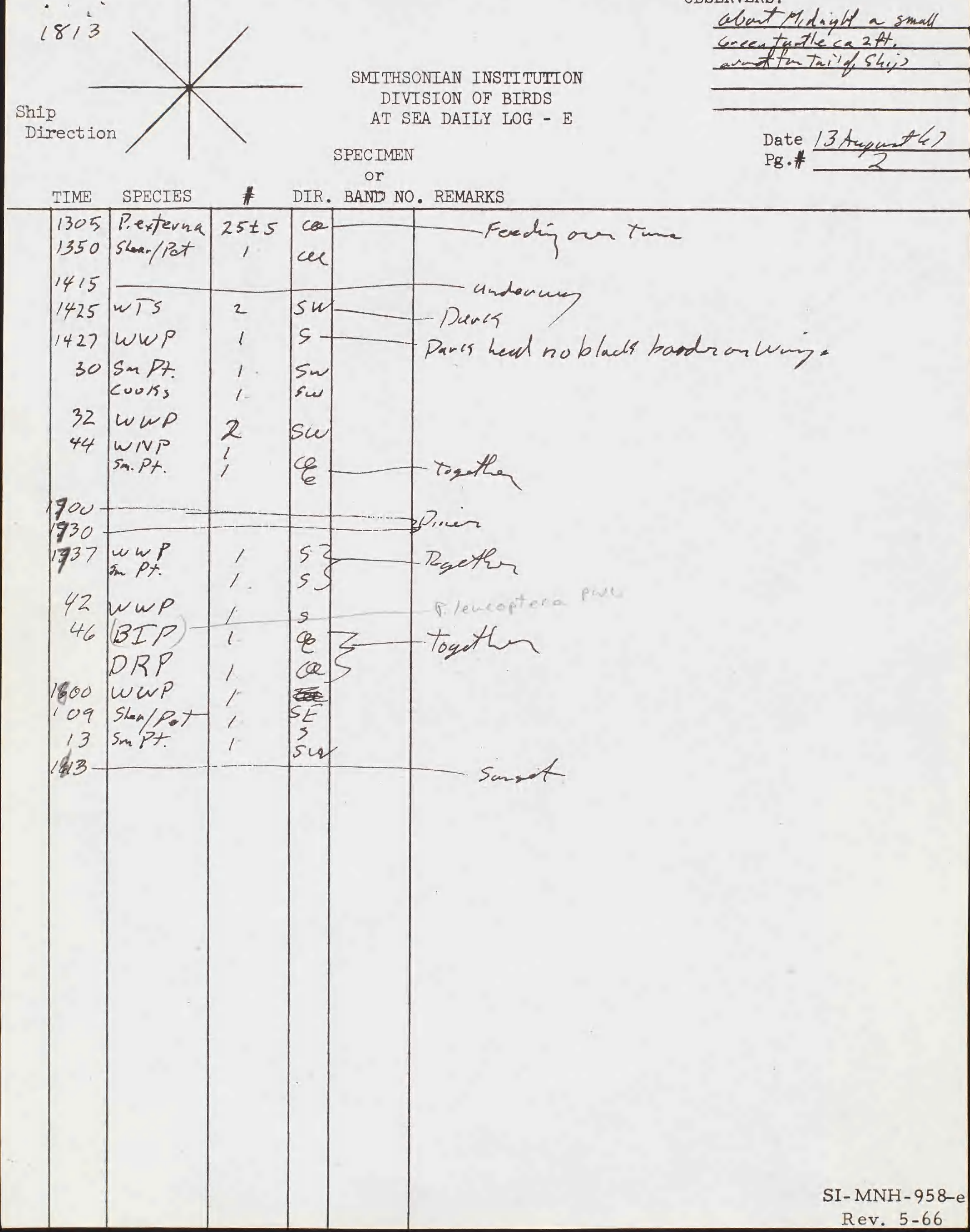


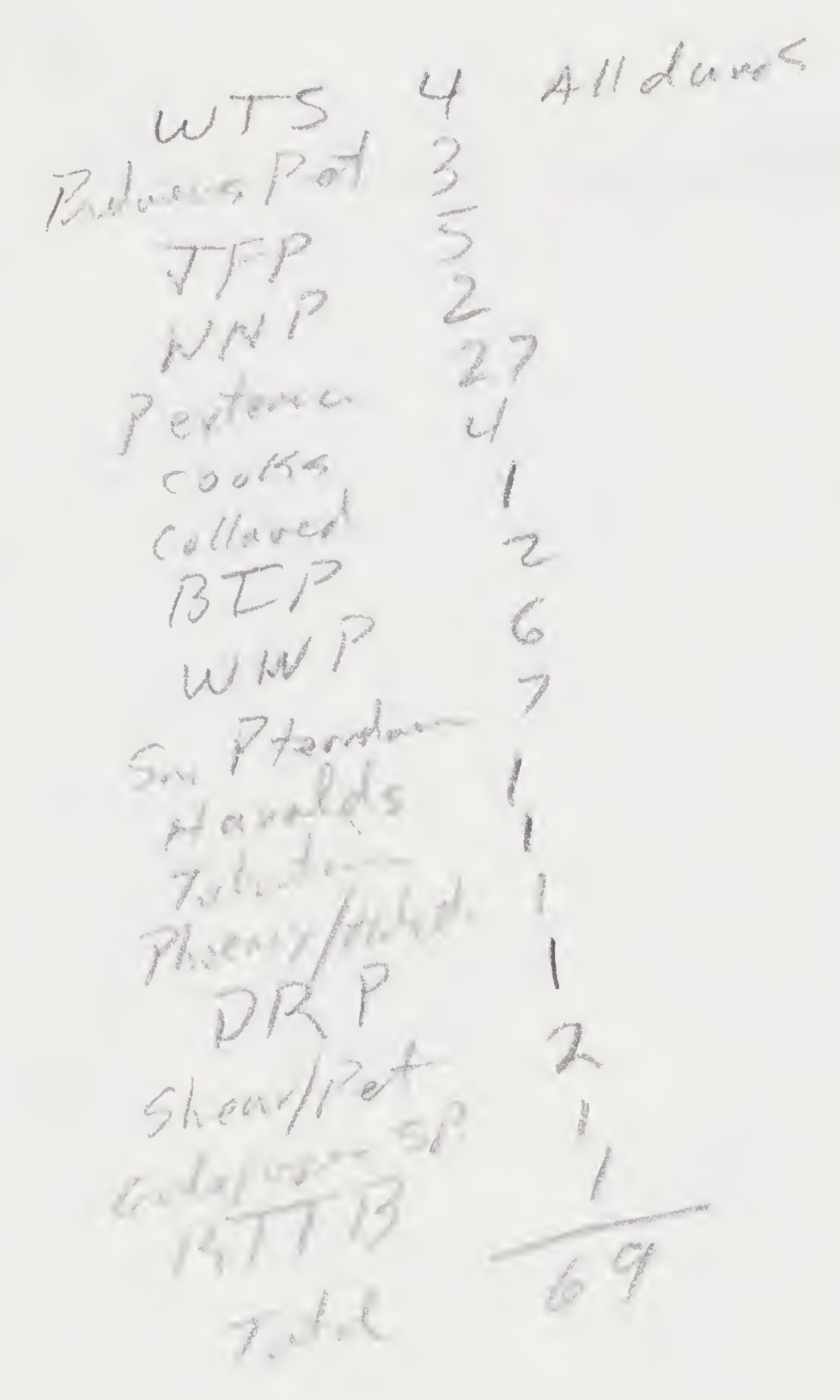




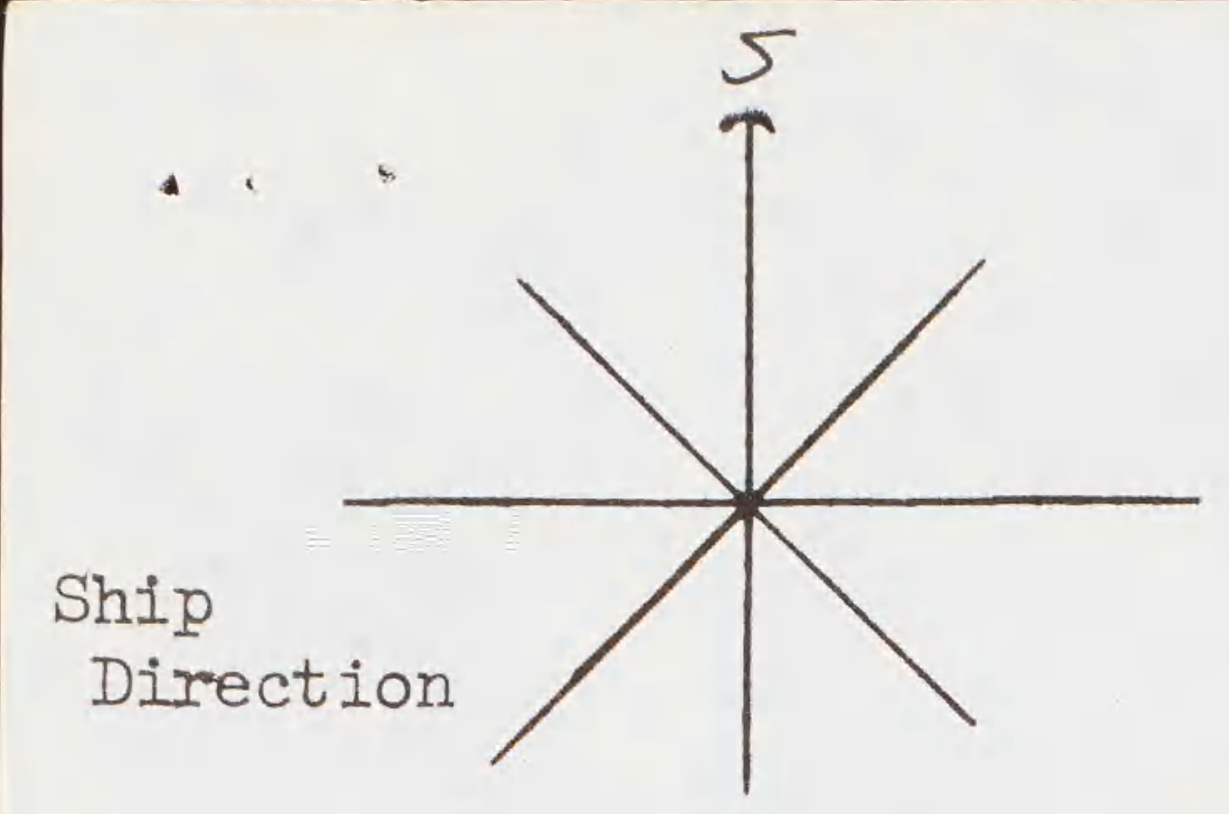

SMITHSONIAN INSTITUTION DIVISION OF BIRDS AT SEA DAILY LOG - E

SPECIMEN Nocturnal

or

OBSERVERS : 


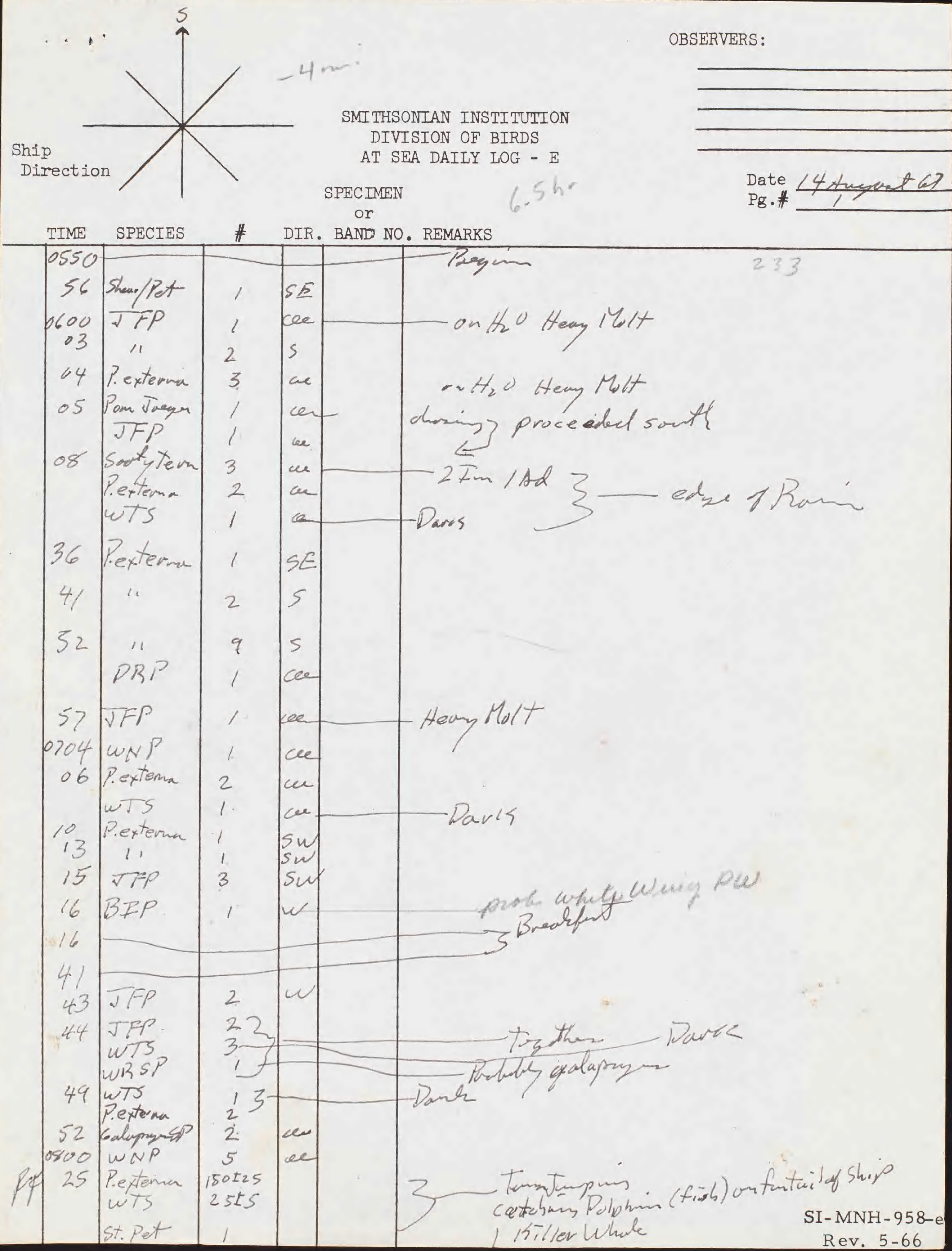




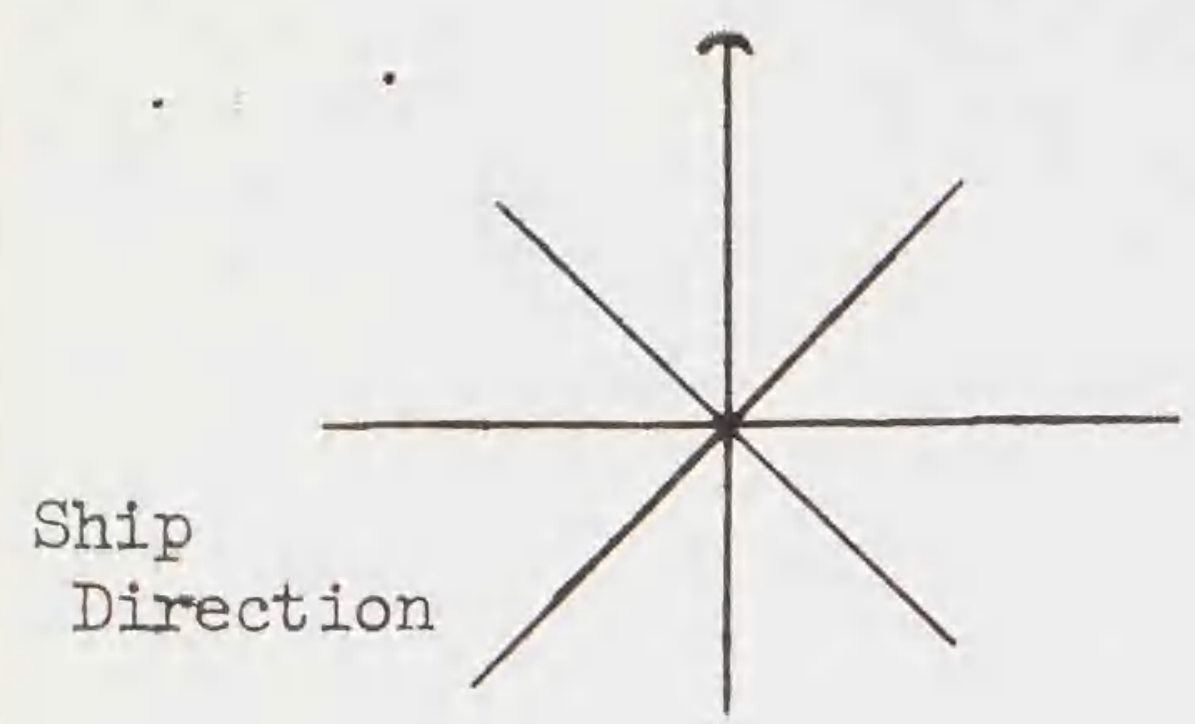

SMITHSONIAN INSTITUTION

DIVISION OF BIRDS

AT SEA DAILY LOG - E

OBSERVERS :

SPECIMEN

or
Date 14 Anger 67

$\mathrm{Pg} \cdot \#$

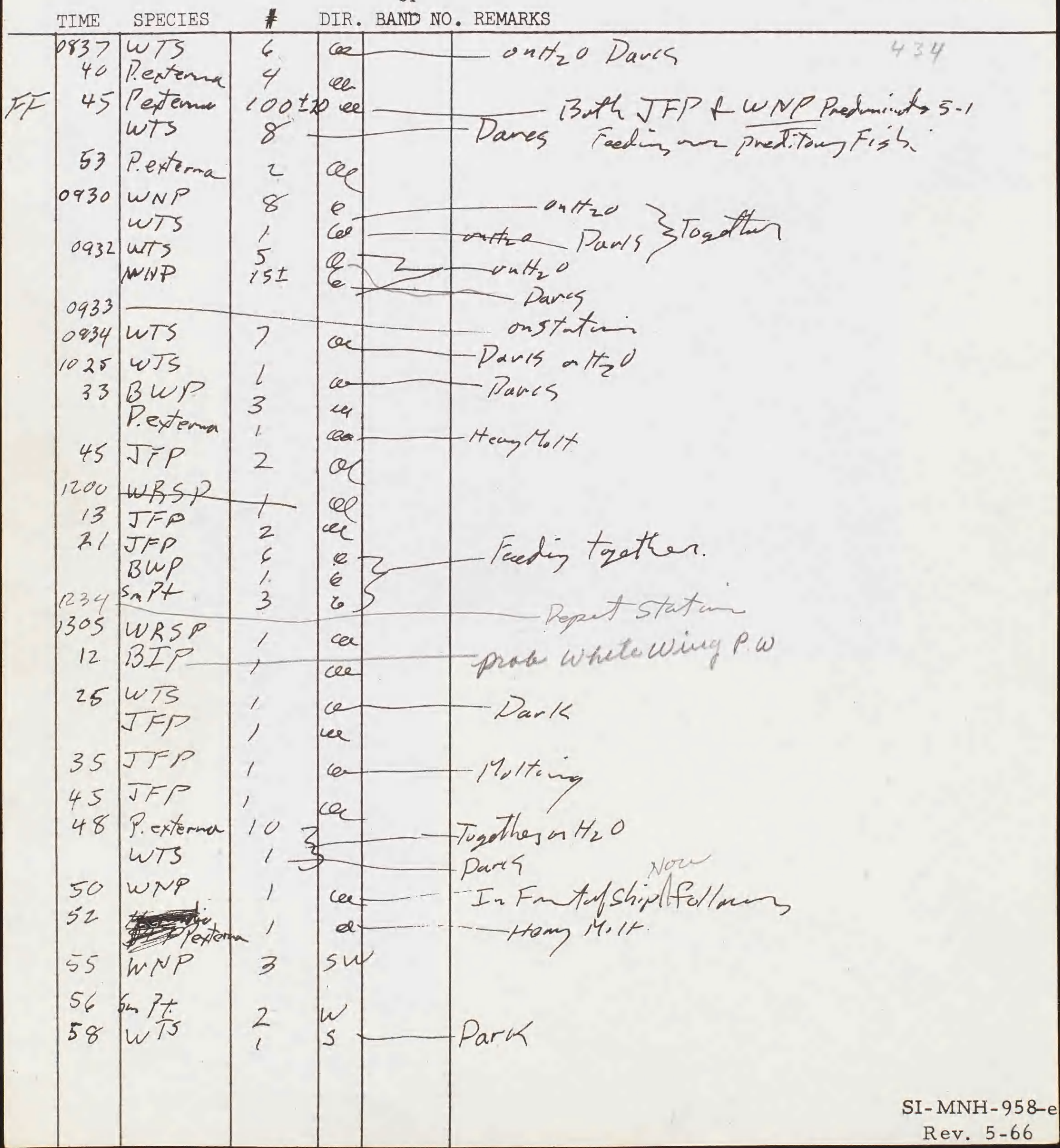




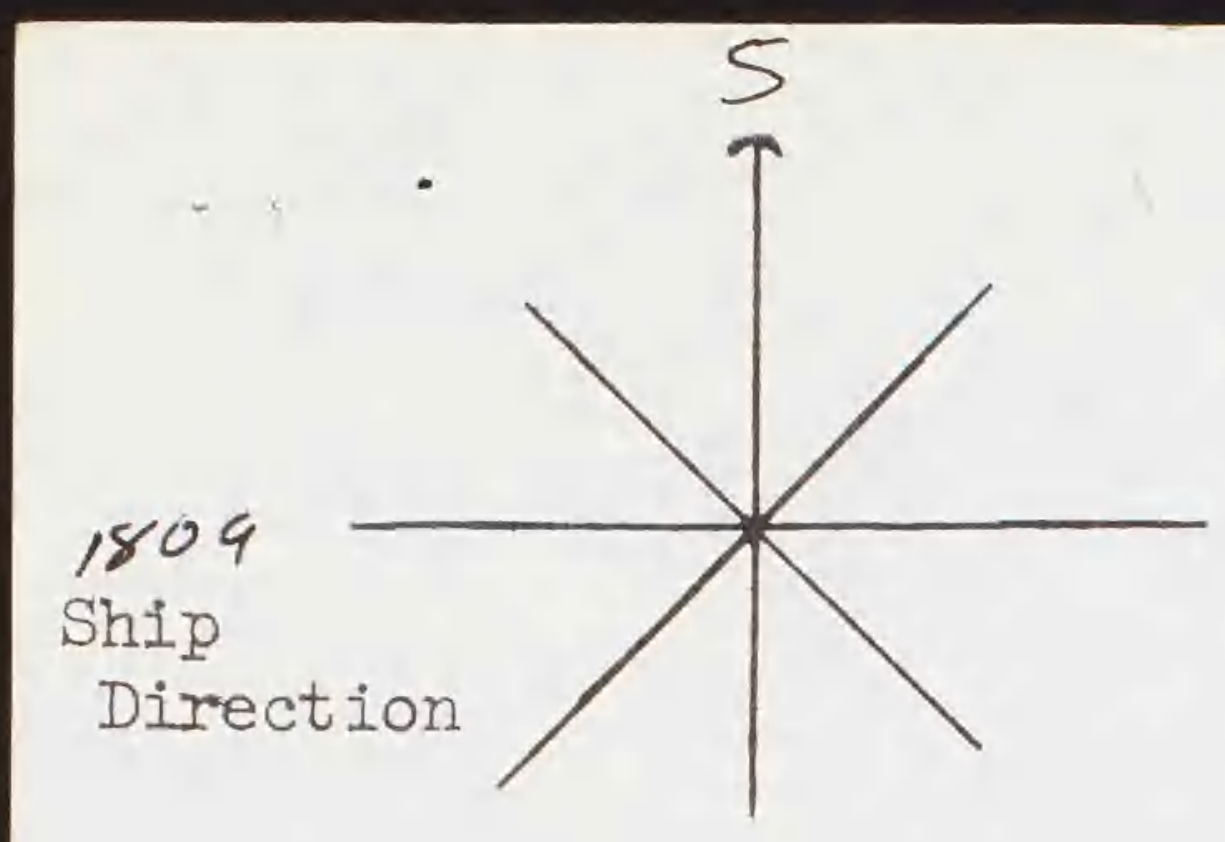

OBSERVERS :

\section{Muy Scheds of sinoll Flyini ind}

SMITHSONIAN INSTITUTION

DIVISION OF BIRDS

AT SEA DAILY LOG - E

SPEC IMEN

or

Date 14 Aupunt $<7$

$\mathrm{Pg} \cdot \#$

3

TIME SPECIES \# DIR. BAND NO. REMARKS

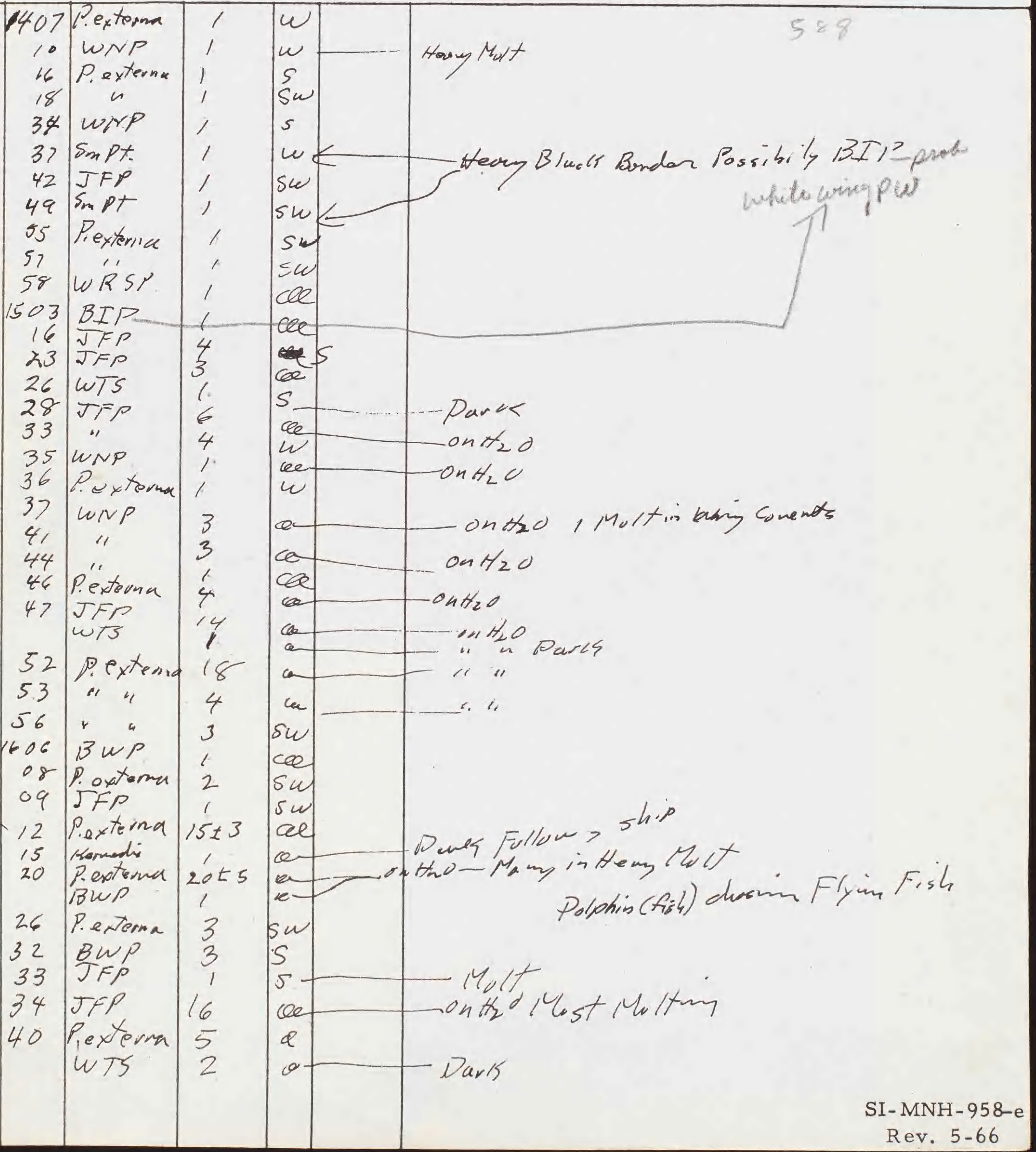




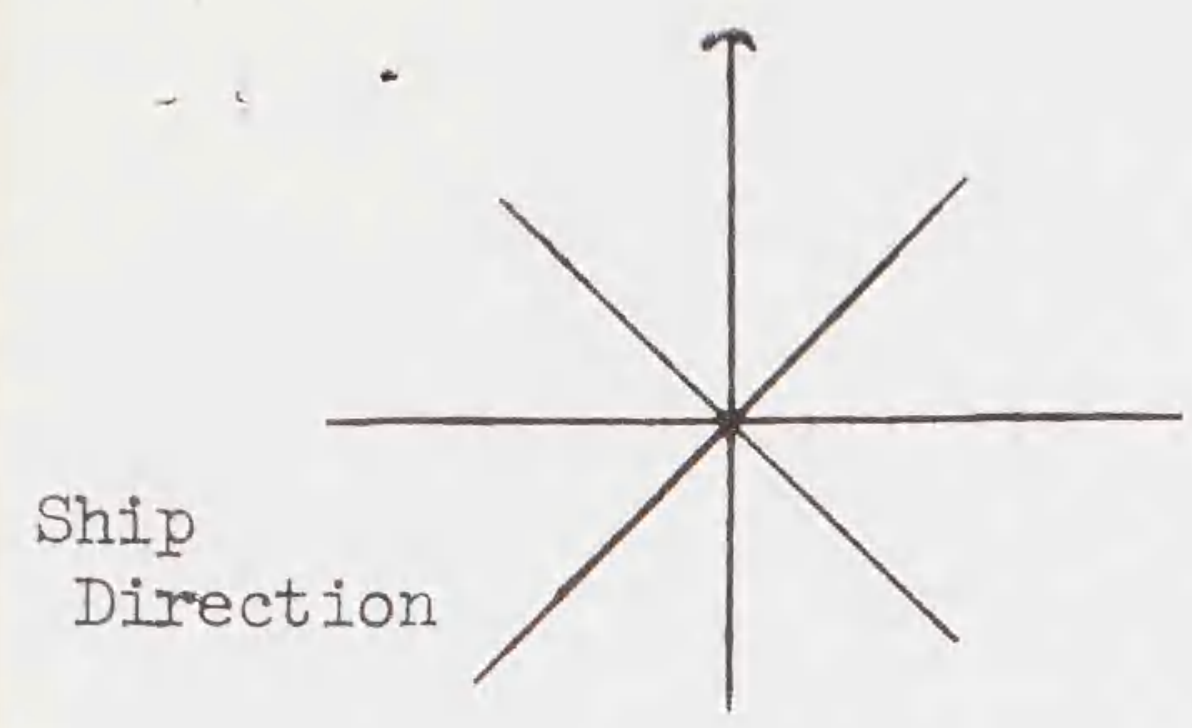

SMITHSONIAN INSTITUTION DIVISION OF BIRDS AT SEA DAILY LOG - E

SPEC IMEN

or

OBSERVERS: 


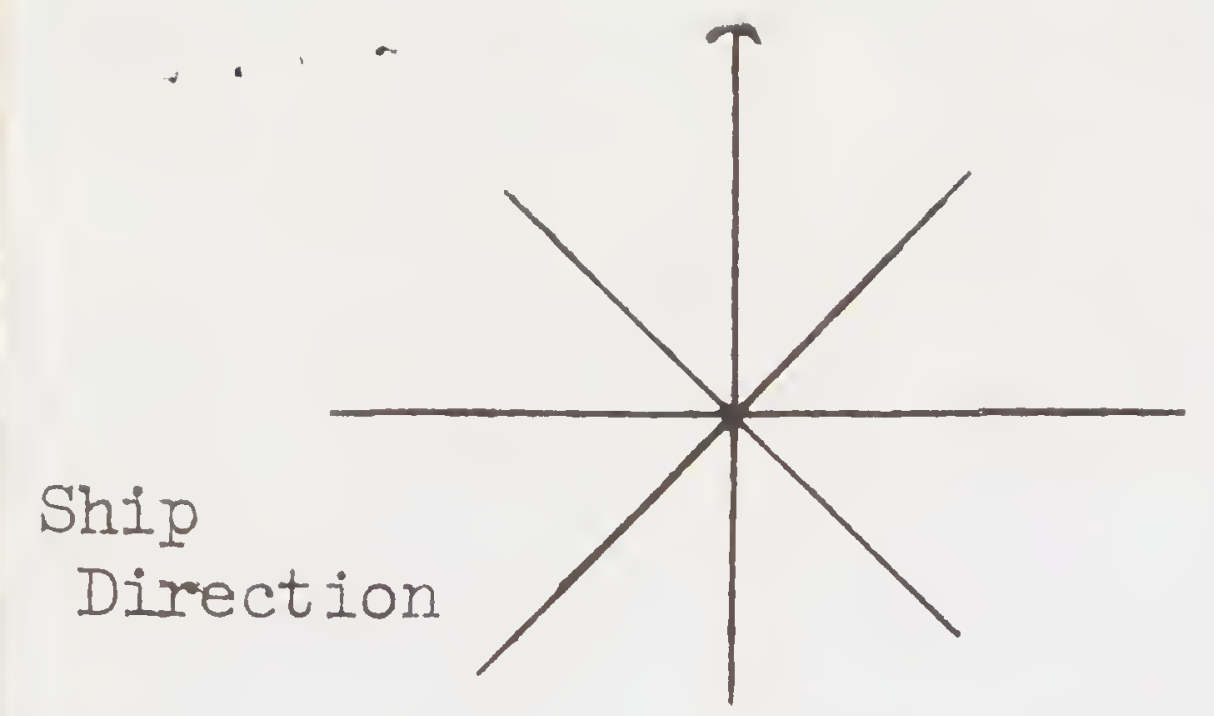

SMITHSONIAN INSTITUTION

DIVISION OF BIRDS

AT SEA DAILY LOG - E

SPECIMEN Nocturnal

or

OBSERVERS :

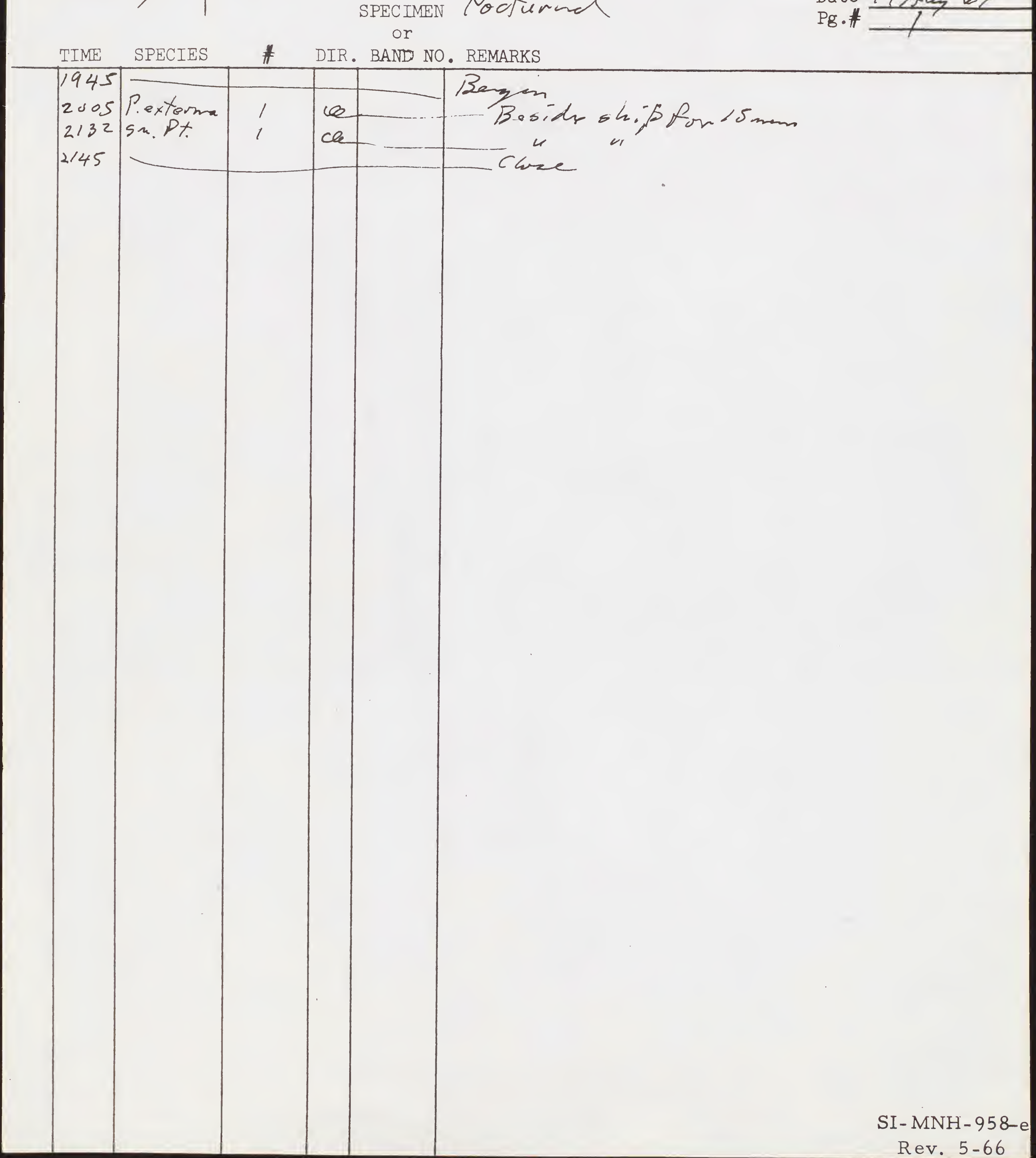




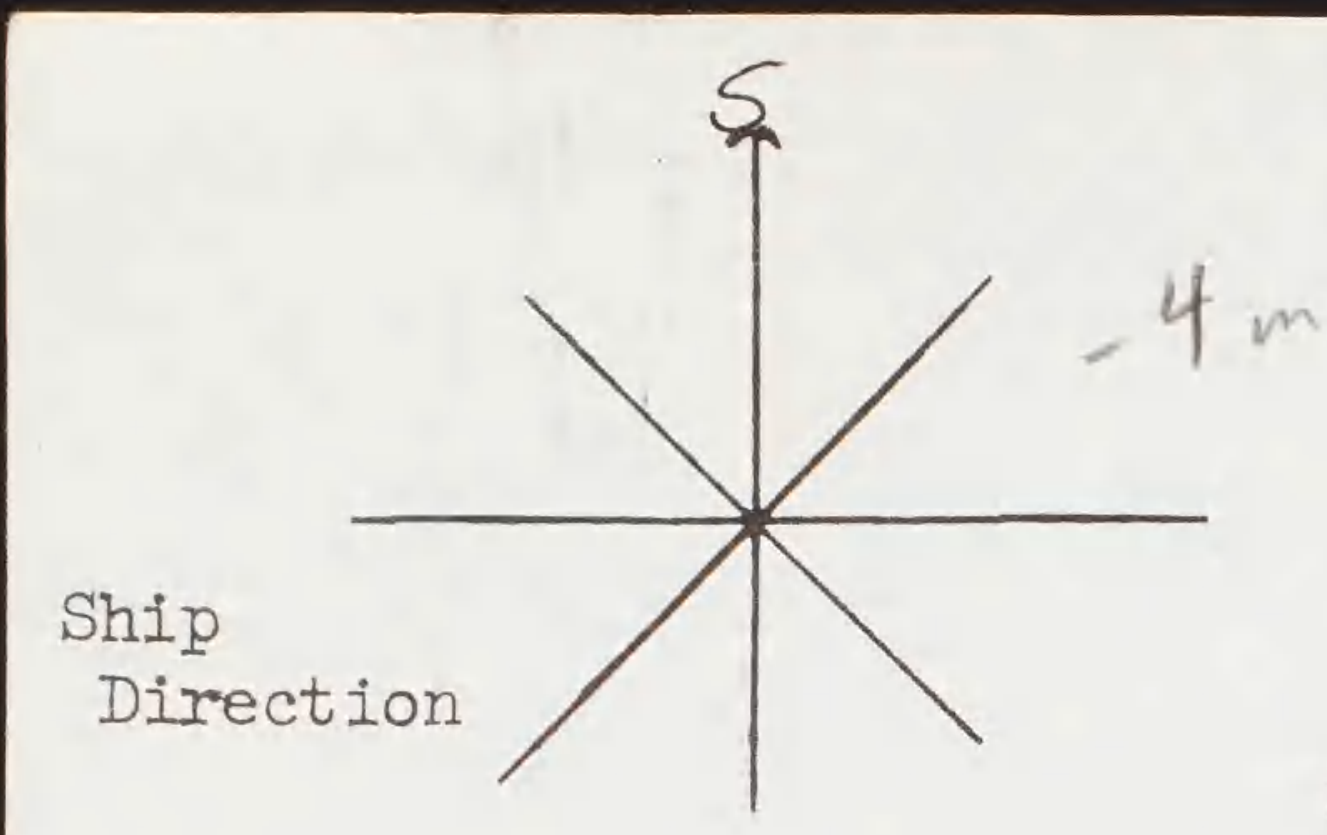

SMITHSONIAN INSTITUTION

DIVISION OF BIRDS

AT SEA DAILY LOG - E

\section{SPEC IMEN}

or

$3.1 h v$

Date 15 Anyust 67

$\mathrm{Pg} \cdot \#$

TIME SPECIES \# DIR. BAND NO. REMARKS

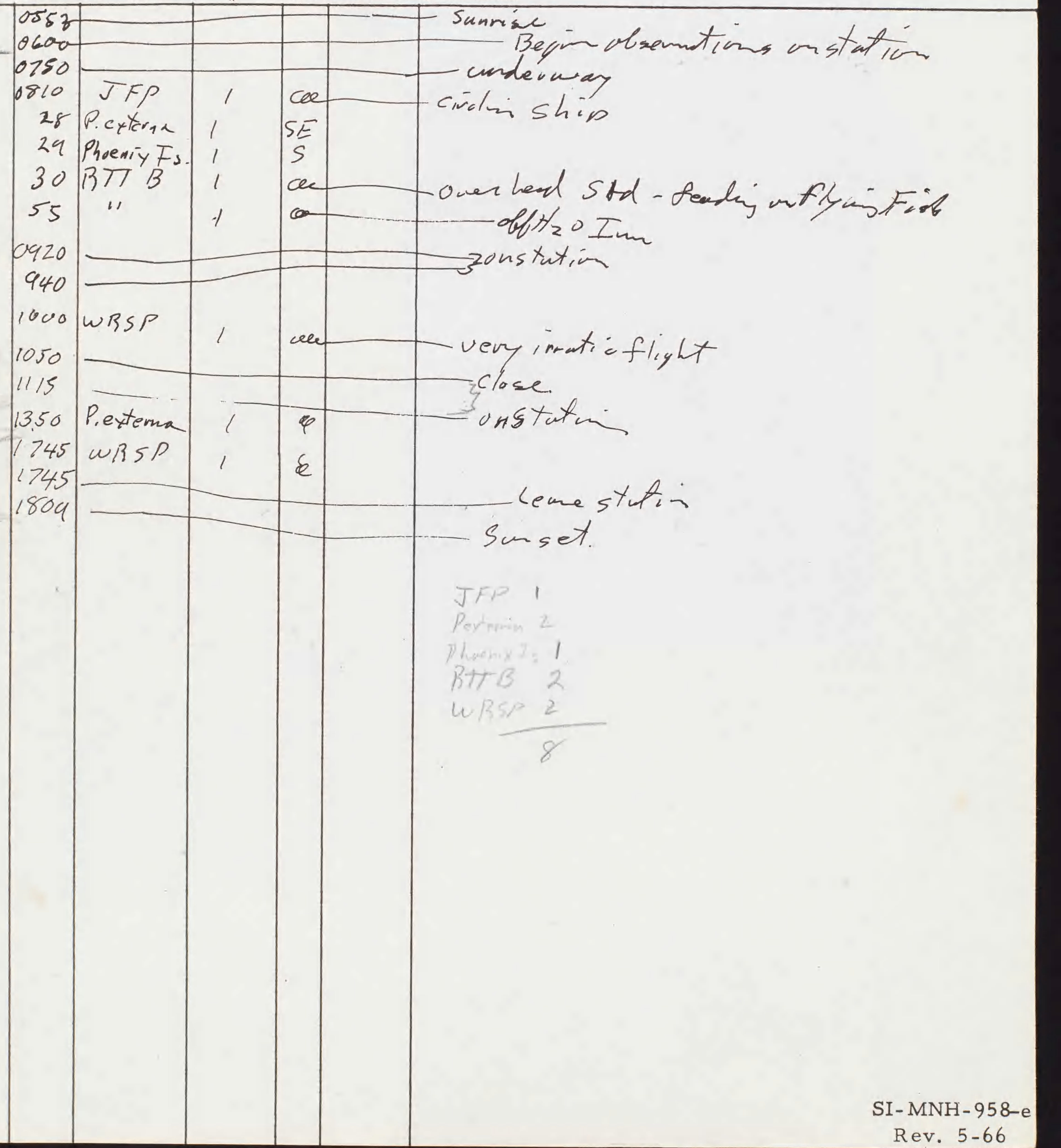

Rev. 5-66 


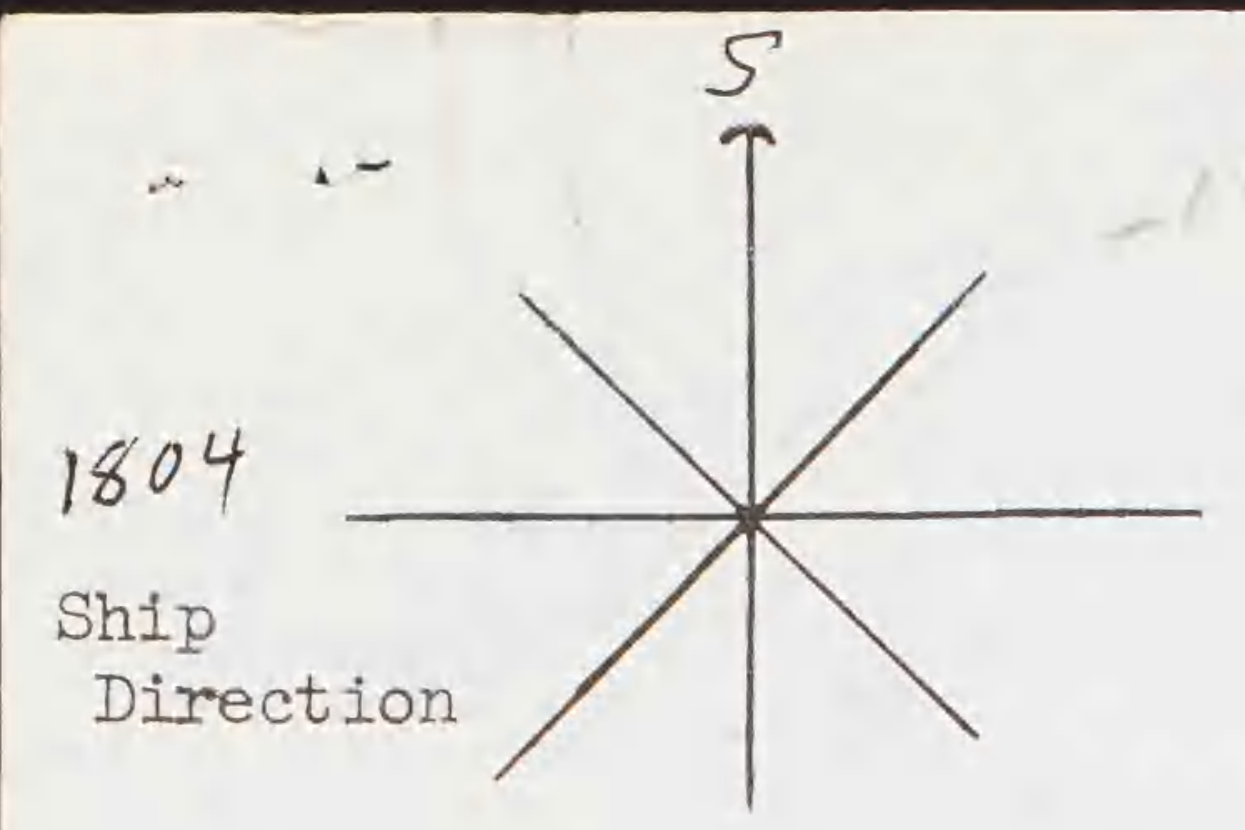

OBSERVERS :

SMITHSONIAN INSTITUIION DIVISION OF BIRDS

AT SEA DAILY LOG - E

SPEC IMEN

or

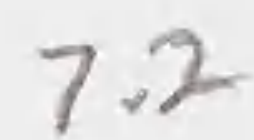

Date 16 Any, A 67 TIME SPECIES \# DIR. BAND NO. REMARKS

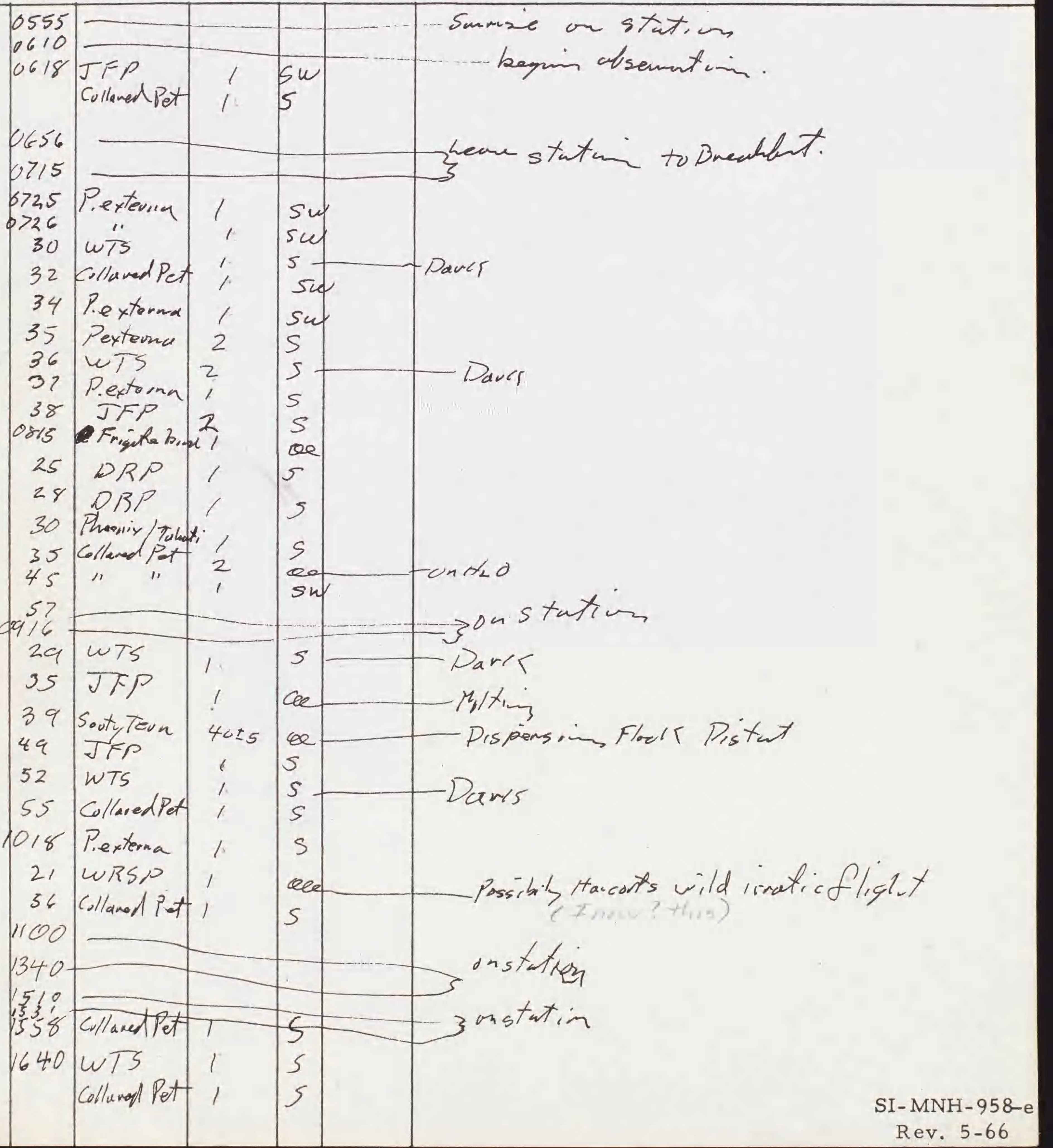




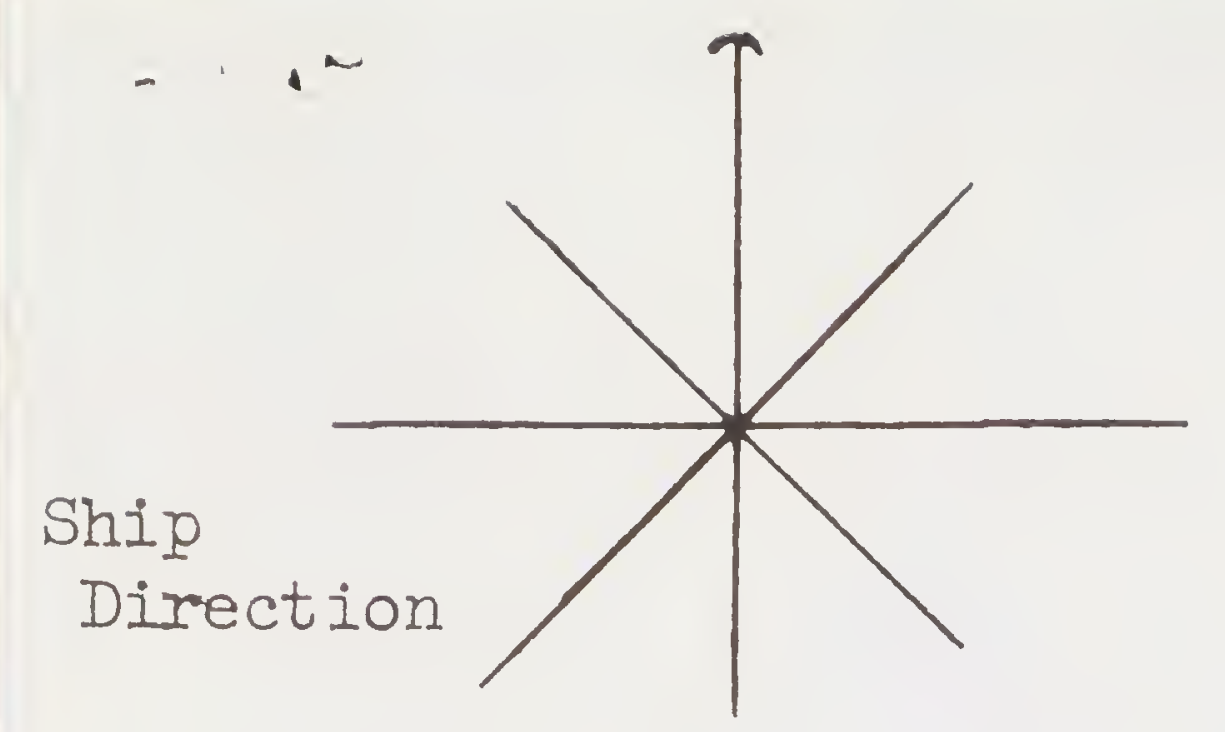

SMITHSONIAN INSTITUTION

DIVISION OF BIRDS

AT SEA DAILY LOG - E

SPEC AMEN

or

OBSERVERS:

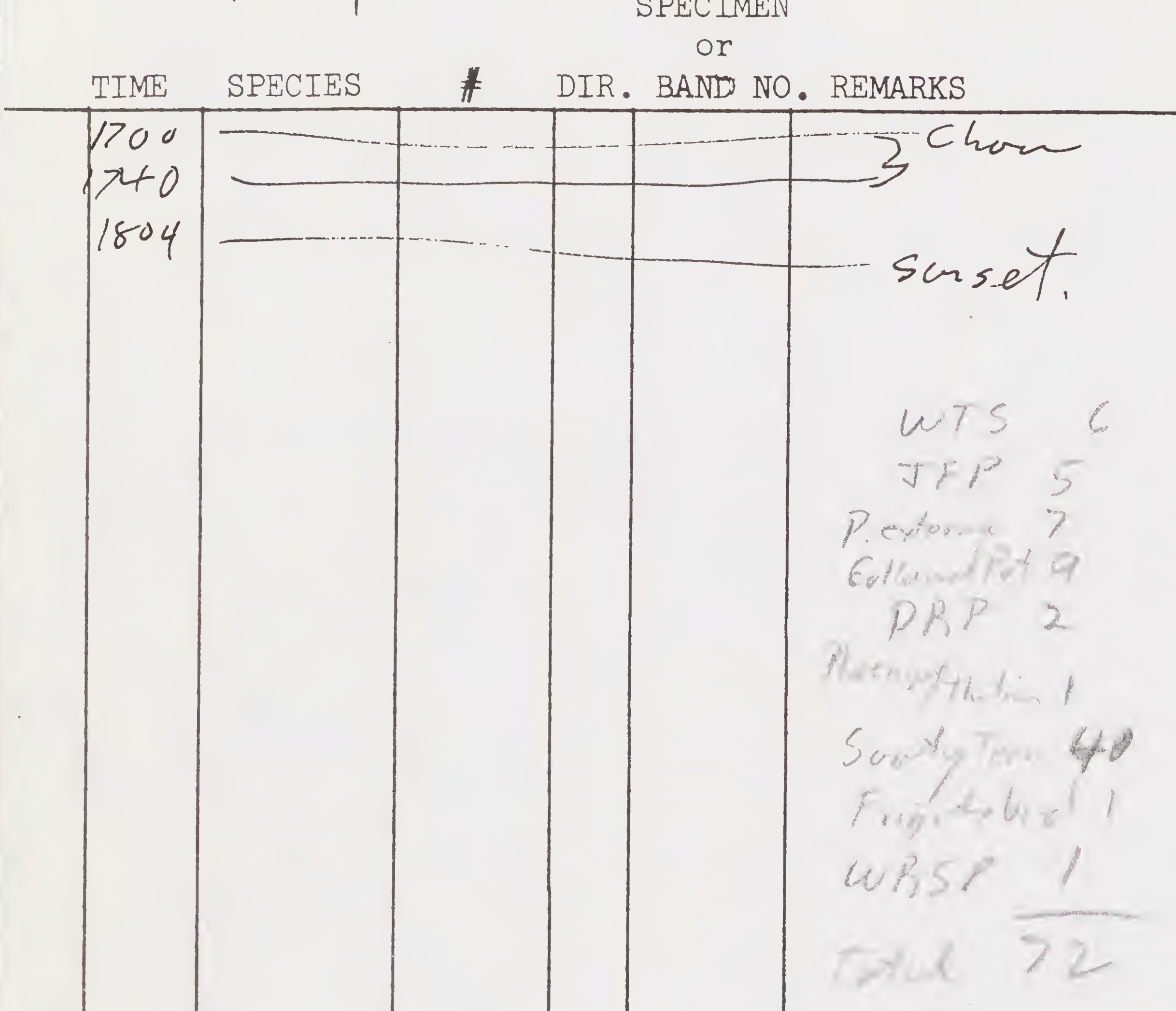

SI - MNH- 958-e

Rev. 5-66 


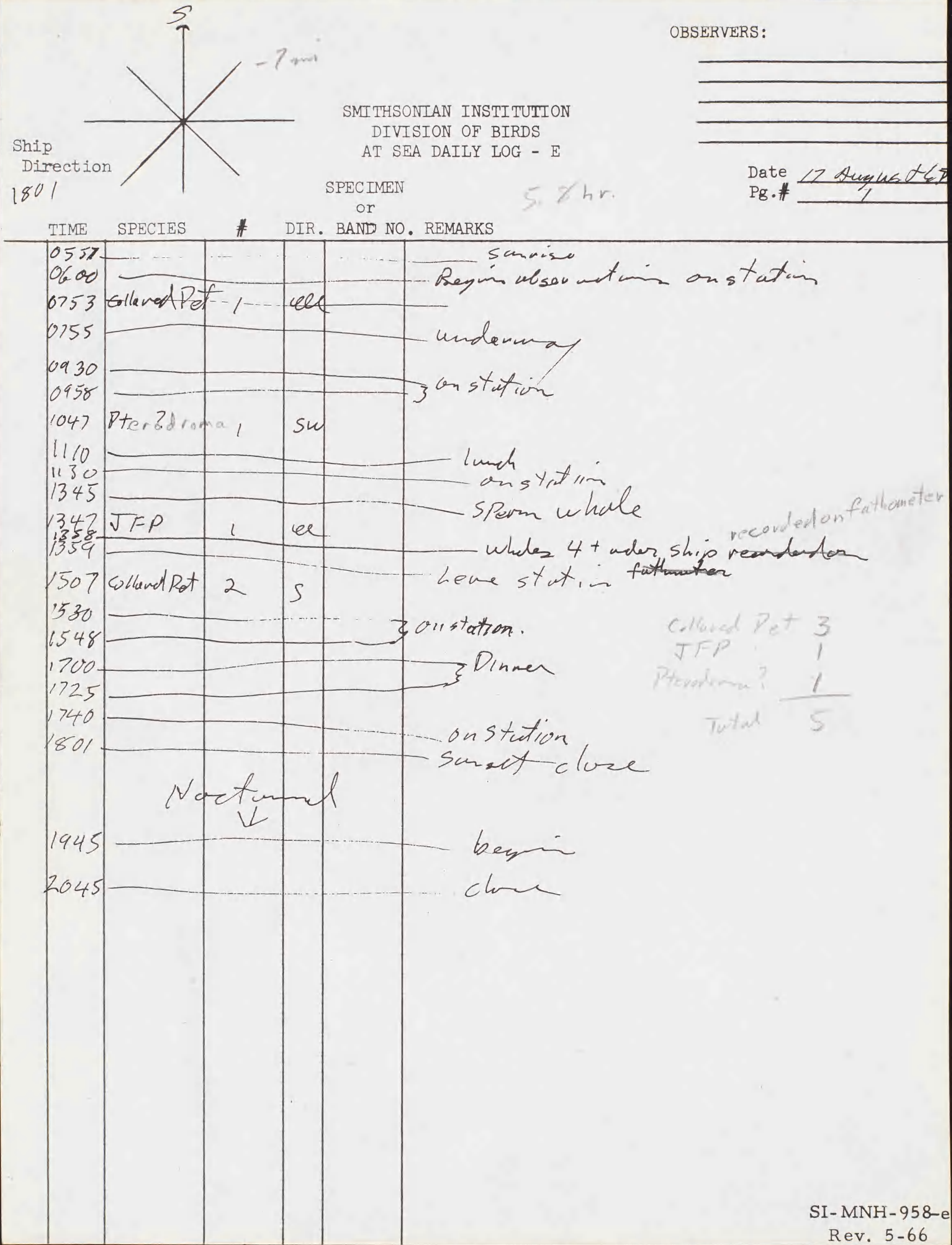




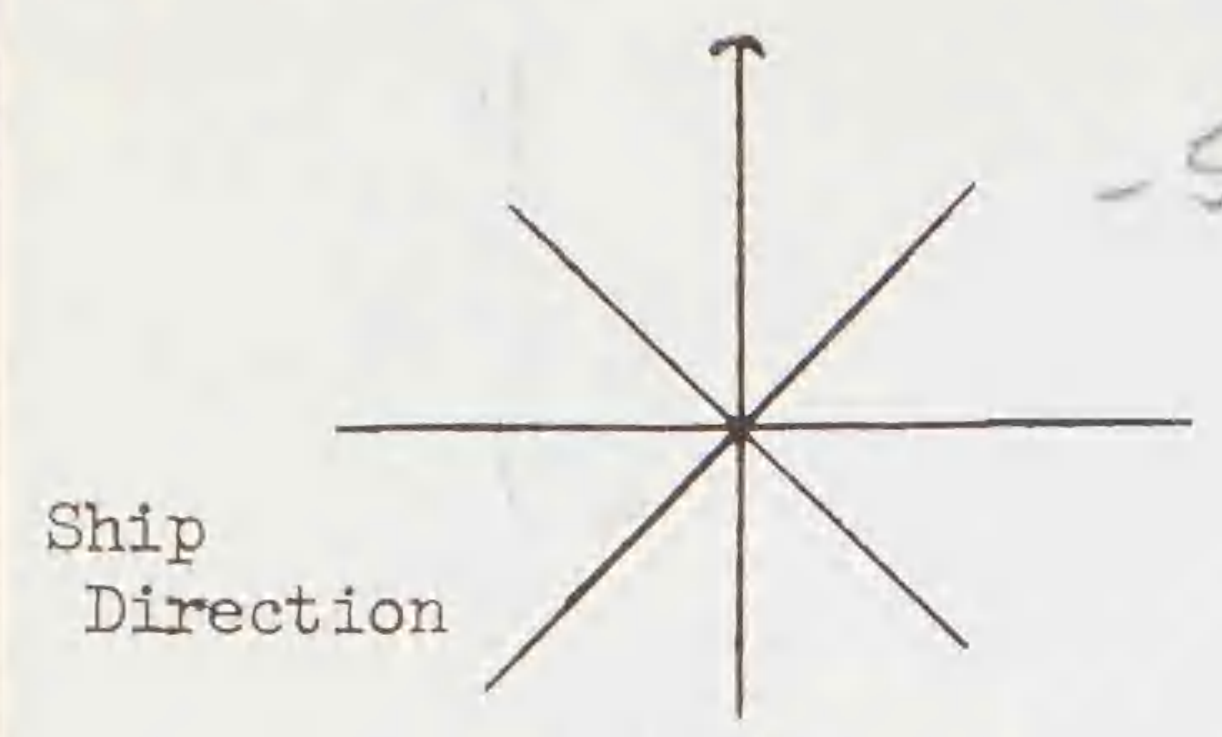

SMITHSONIAN INSTITUTION

DIVISION OF BIRDS

AT SEA DAILY LOG - E

SPECIMEN

OBSERVERS :

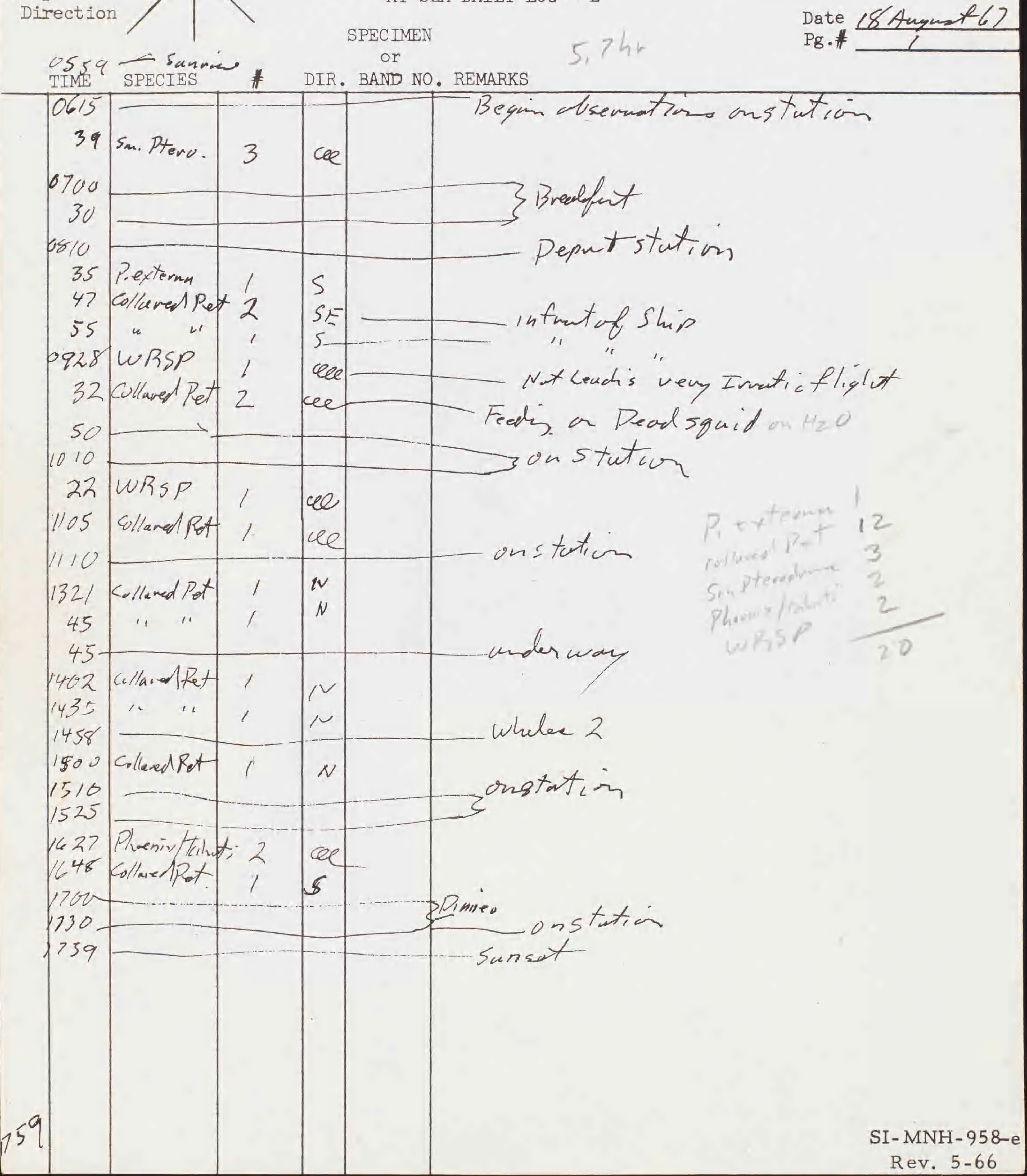




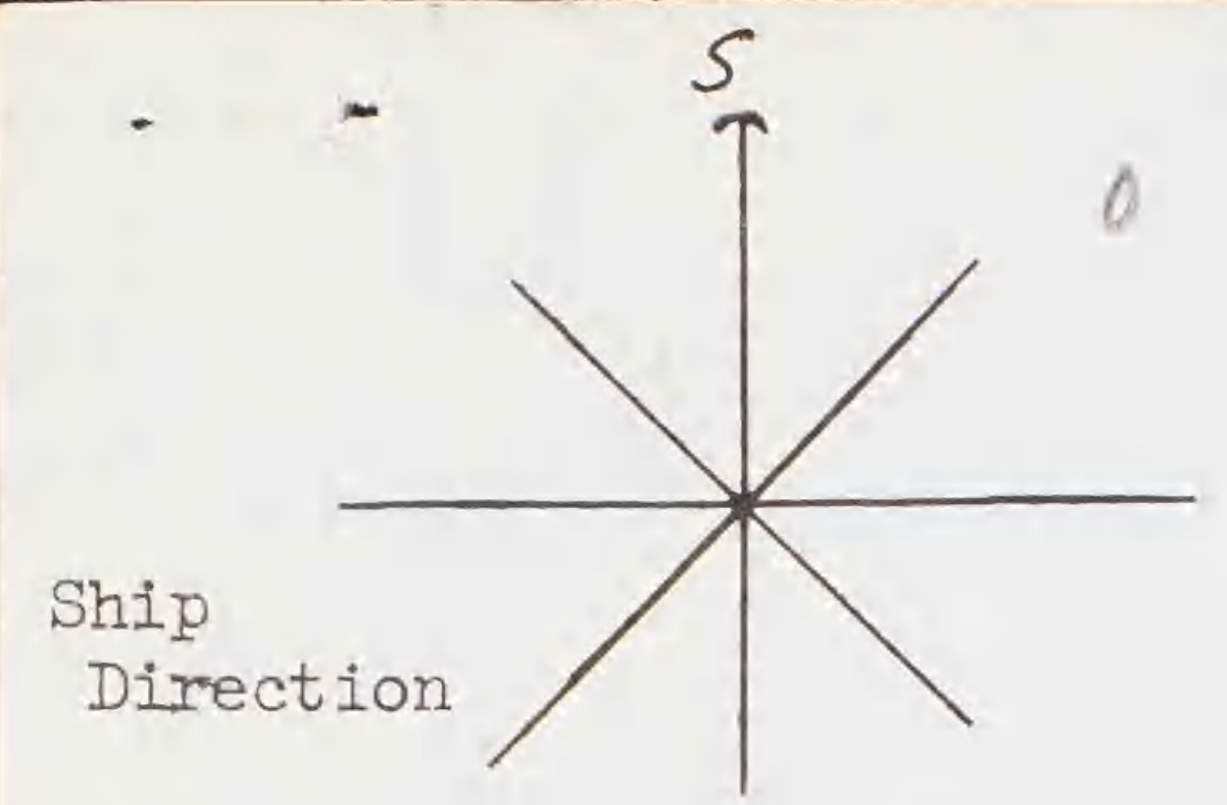

OBSERVERS:

SMITHSONIAN INSTITUTION DIVISION OF BIRDS AT SEA DAILY LOG - E

SPECIMEN $6.6 \mathrm{~h}$. or
Date 19 August 62 $\mathrm{Pg} \cdot \#$

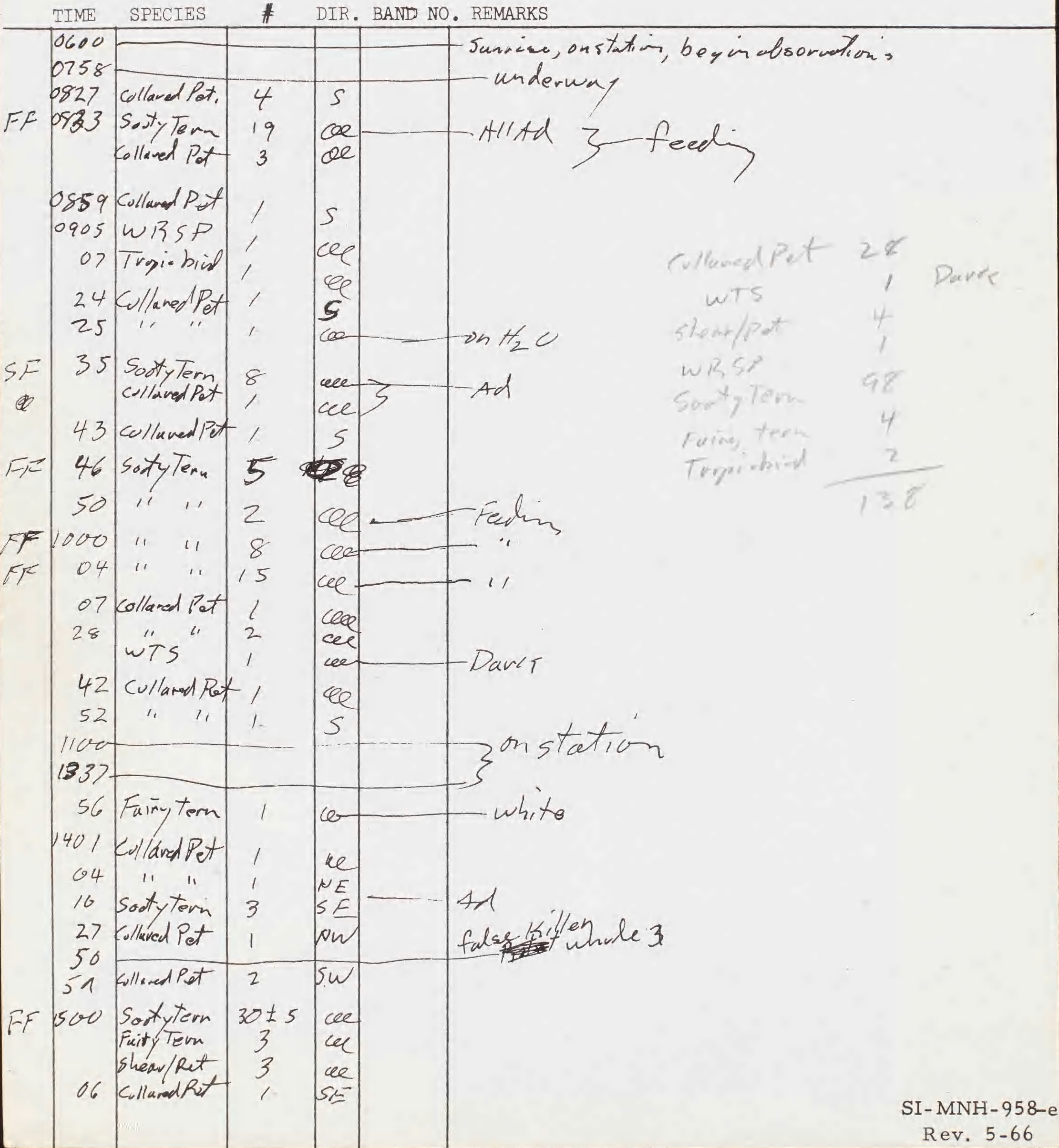




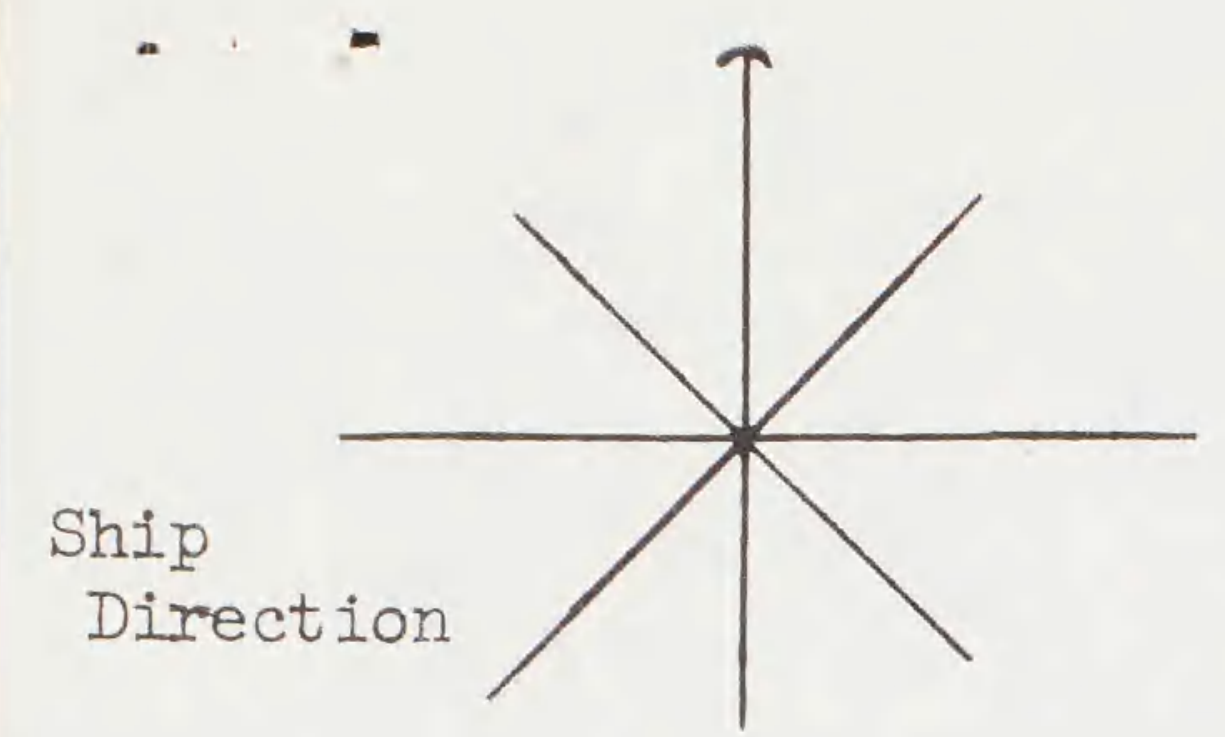

SMITHSONIAN INSTITUTION DIVISION OF BIRDS AT SEA DAILY LOG - E

SPEC IMEN

or

Date 19 August 6$\rangle$

$\mathrm{Pg} \cdot$.\#

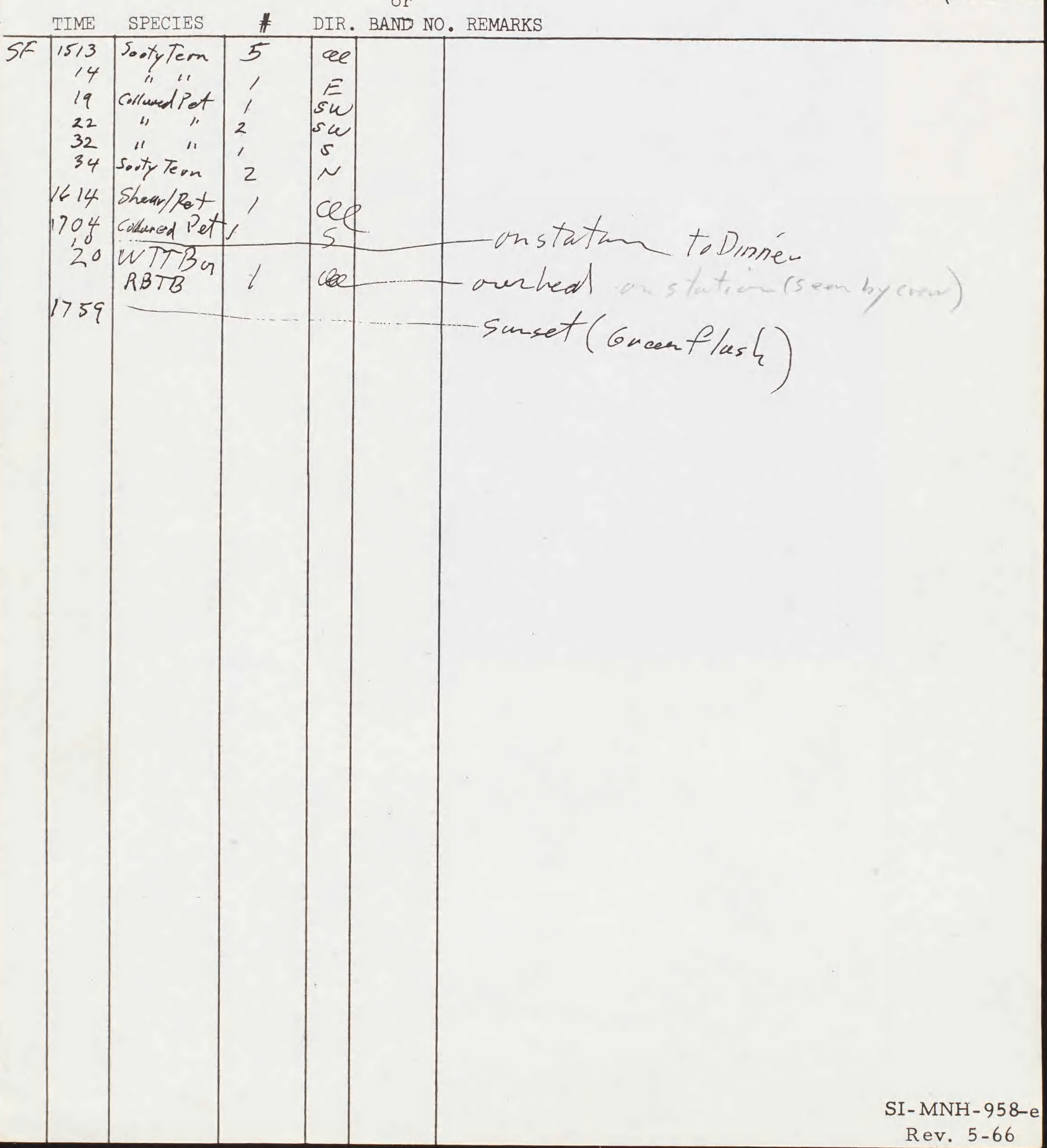




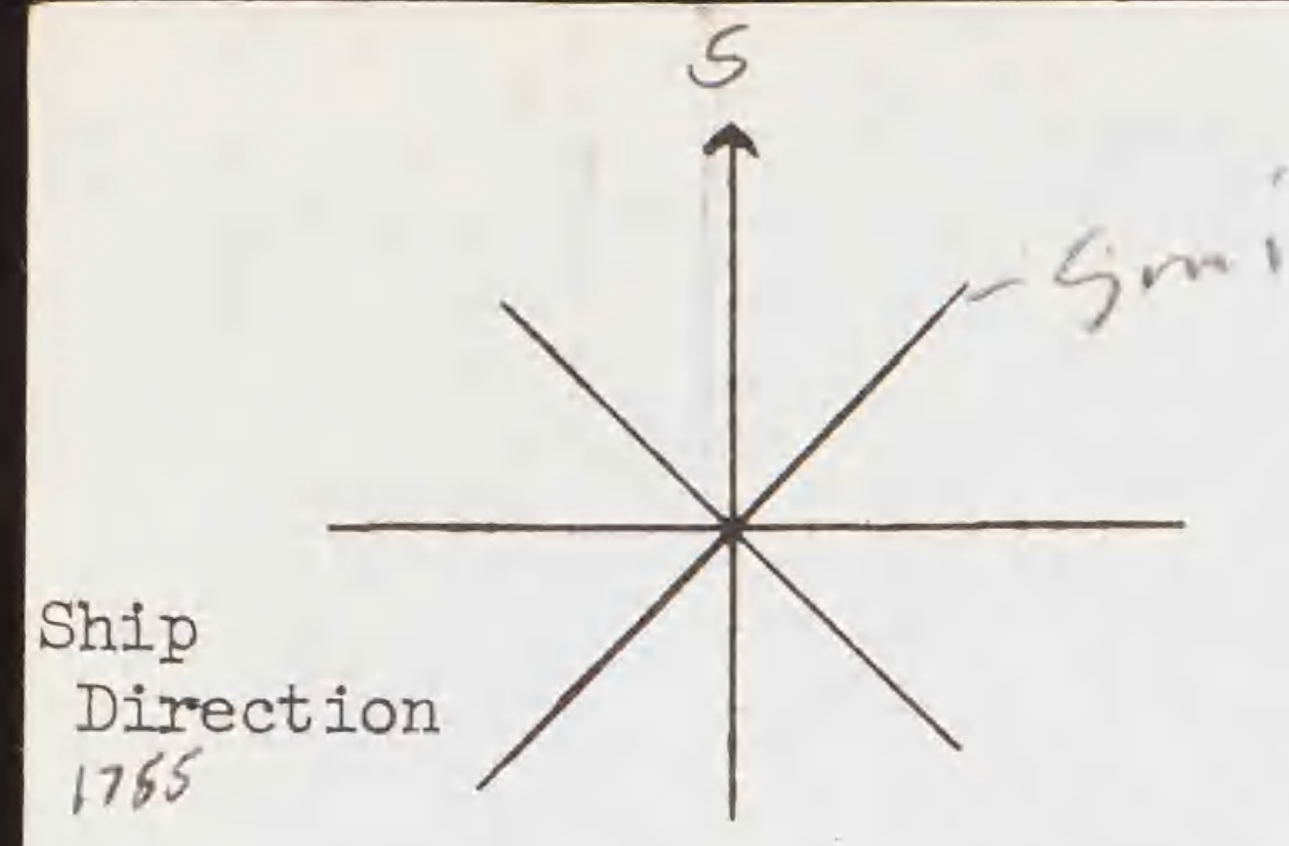

SMITHSONIAN INSTITUTION DIVISION OF BIRDS AT SEA DAILY LOG - E

OBSERVERS :

SPECIMEN $7.4 \mathrm{hr}$

Date 20 Aquas 47

or

$\mathrm{Pg}$.

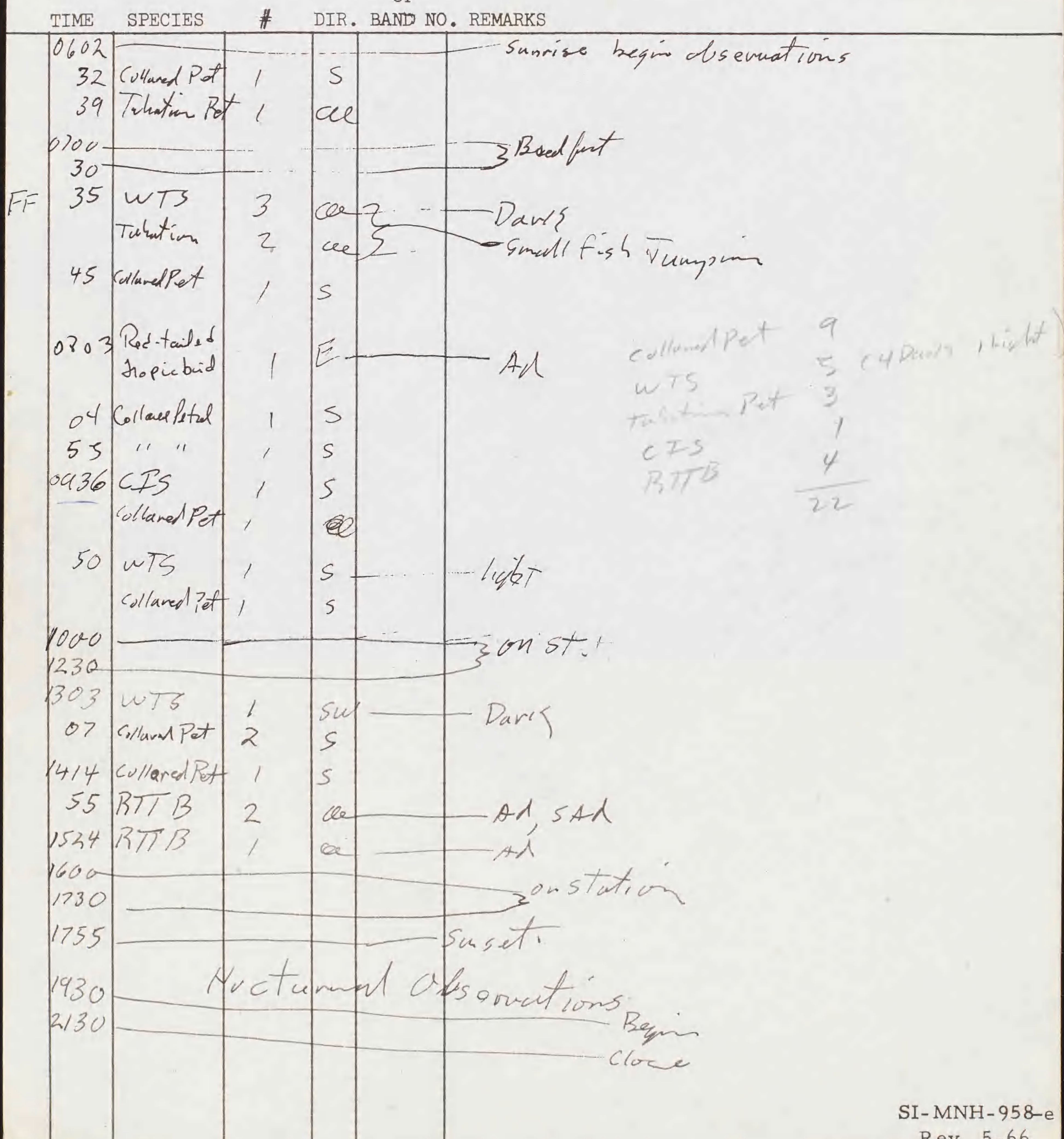

Rev. 5-66 


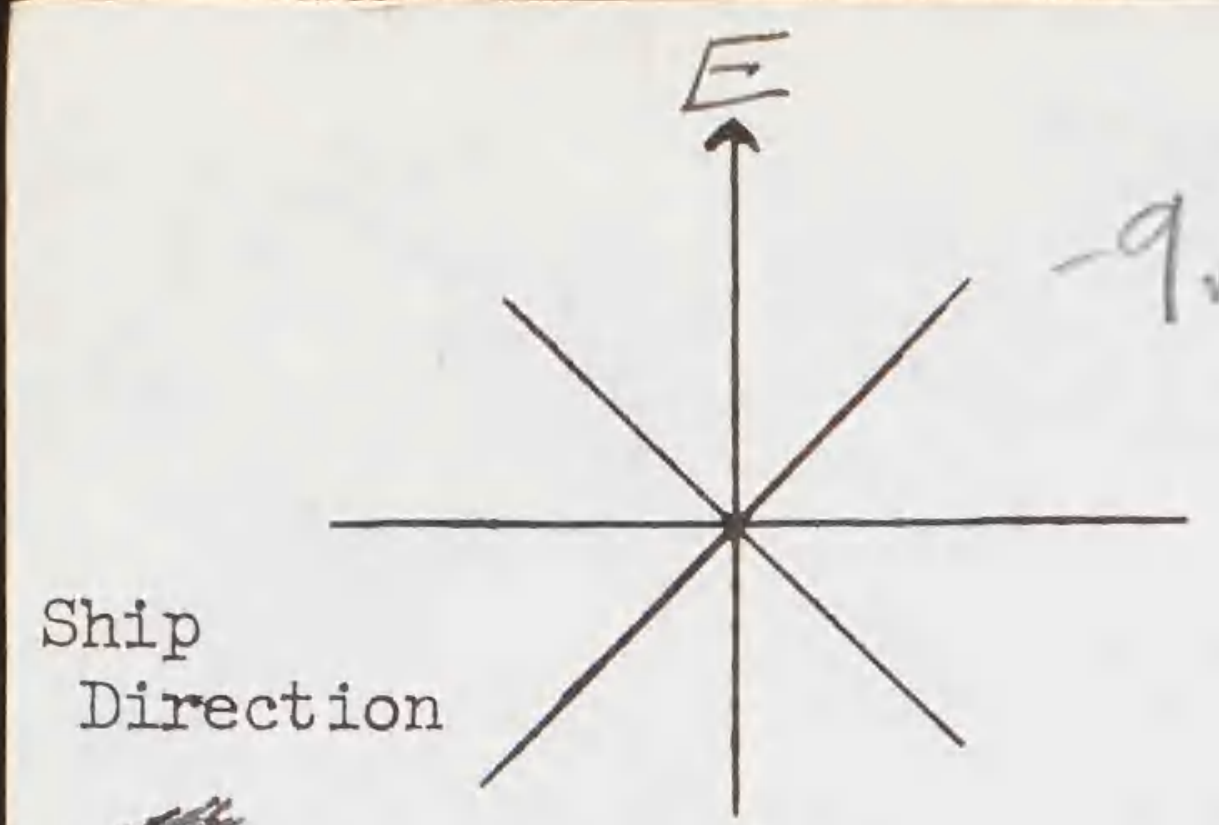

OBSERVERS :

SMITHSONIAN INSTITUTION

DIVISION OF BIRDS

AT SEA DAILY LOG - E

SPECIMEN

or

8. 6 hr.

Date 21 Angus 67

$\mathrm{Pg} \cdot \#$

DIR. BAND NO . REMARKS

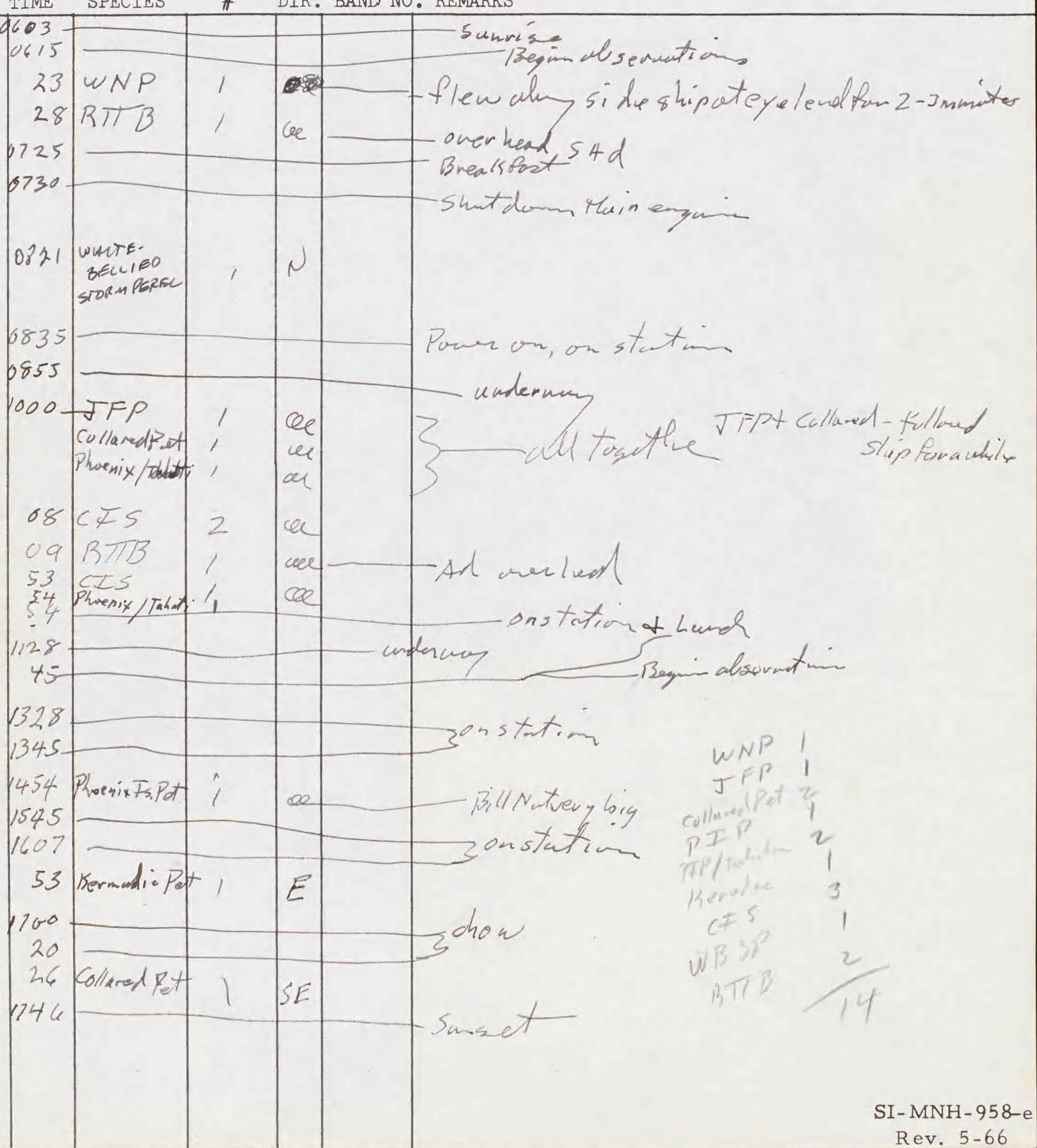

Rev. 5-66 


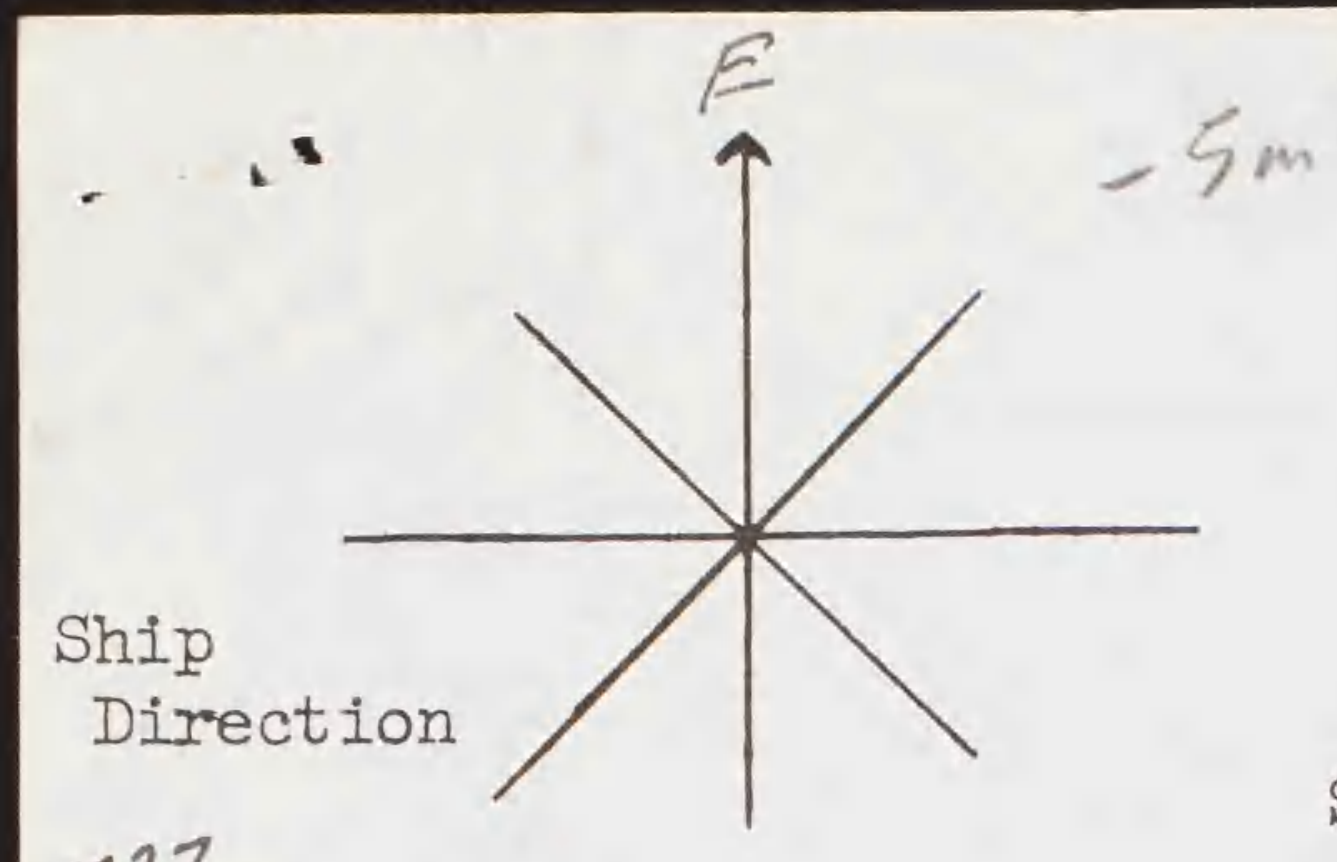

1727

SMITHSONIAN INSTITUTION DIVISION OF BIRDS

AT SEA DAILY LOG - E

SPEC IMEN

or
OBSERVERS :

TIME SPECIES \# DIR. BAND NO. REMARKS

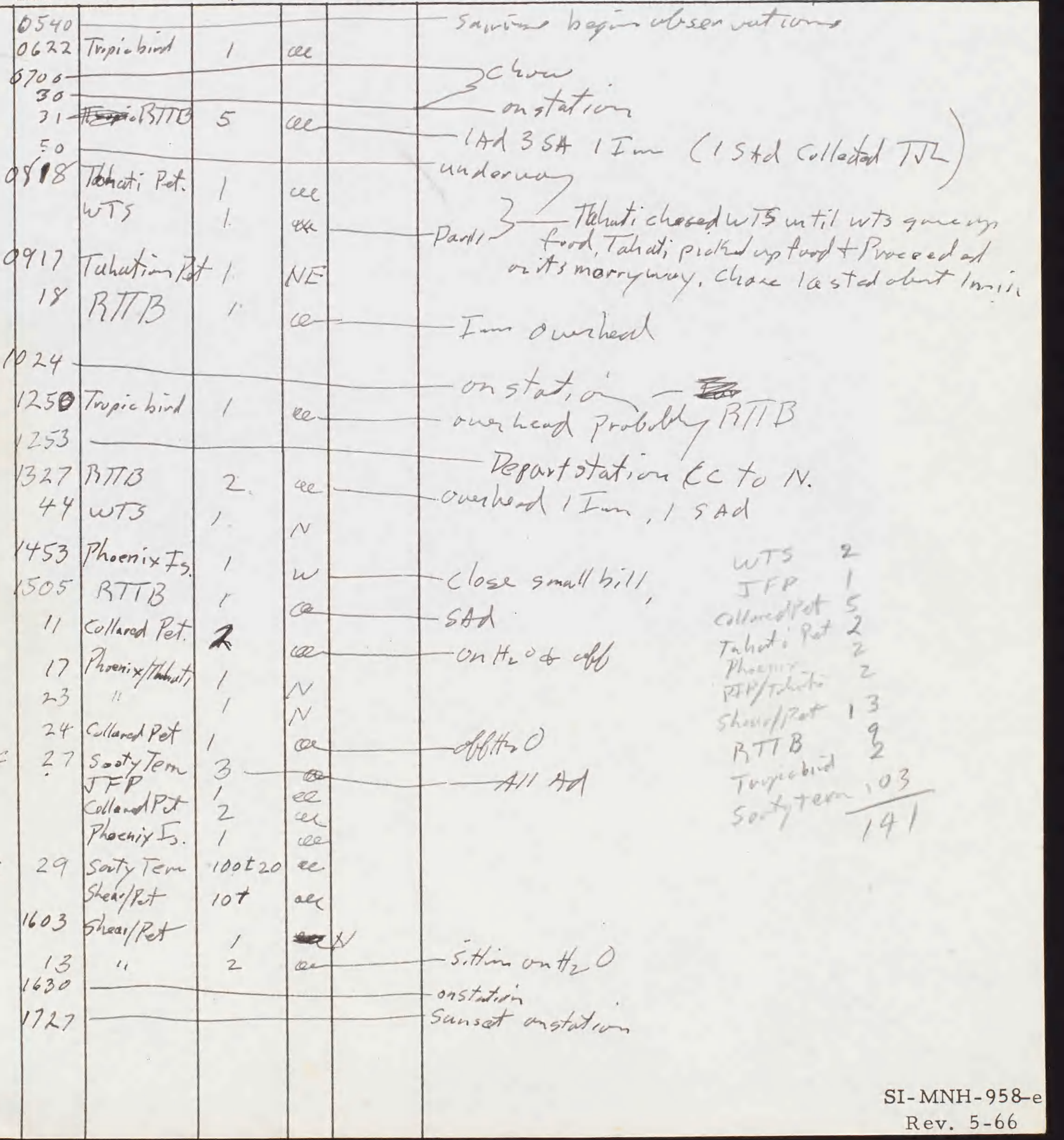




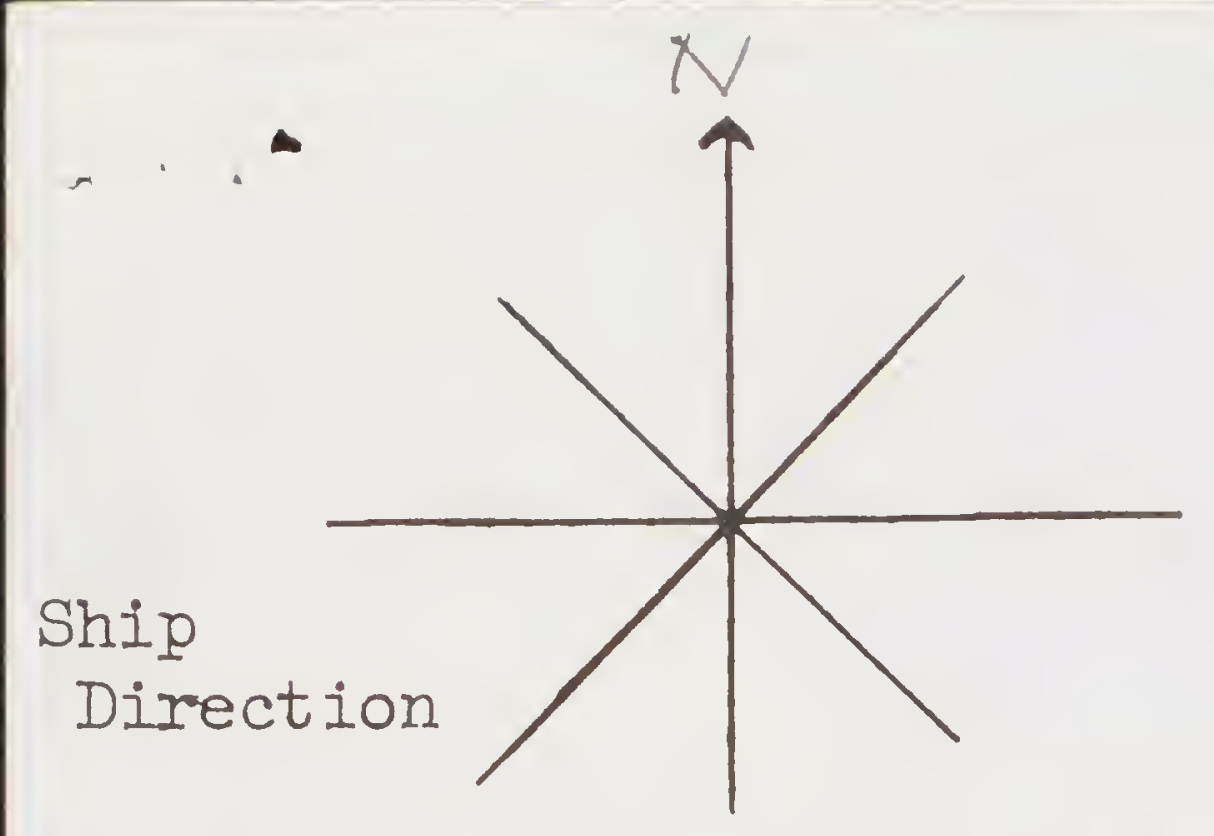

SMITHSONIAN INSTITUTION

DIVISION OF BIRDS

AT SEA DAILY LOG - E

SPECIMEN Nocturnal

Date 23 Amuse 62

or

$\mathrm{Pg} \cdot \#$

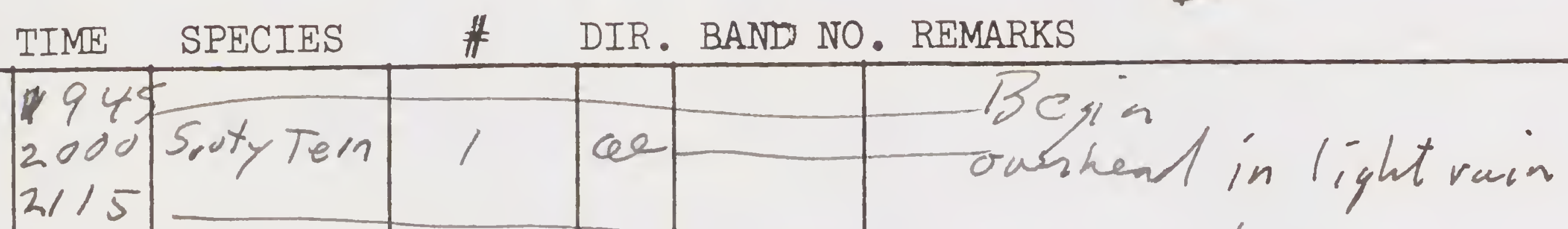

$$
\text { close }
$$

OBSERVERS: 
Ship

Direction 1828

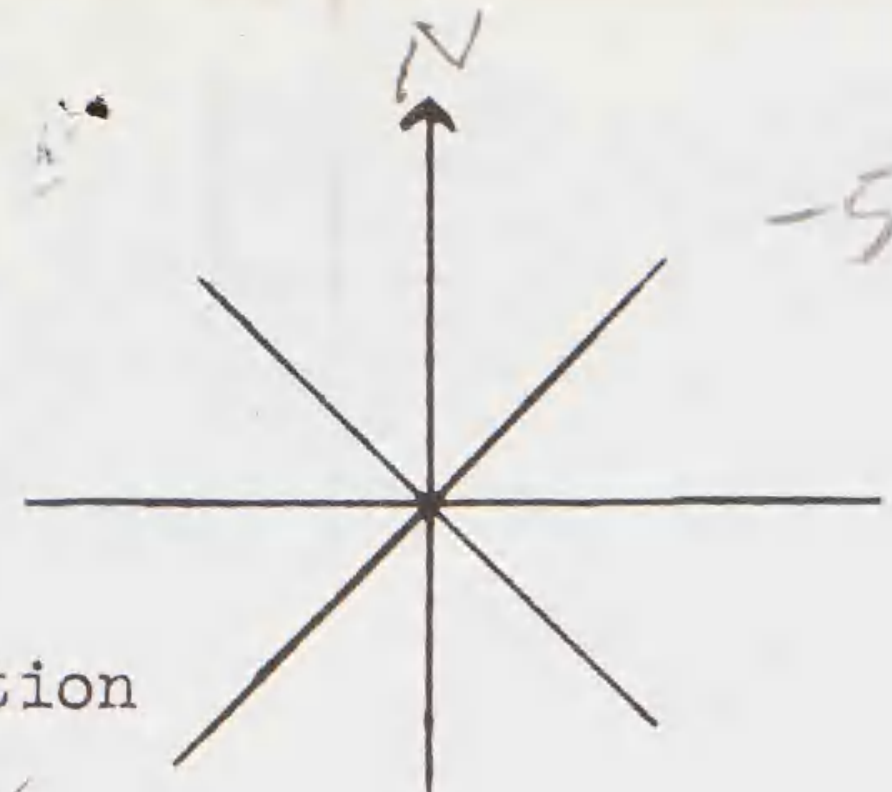

\section{OBSERVERS :}

SMITHSONIAN INSTITUTION DIVISION OF BIRDS

AT SEA DAILY LOG - E $6.8 \mathrm{hr}$

SPECIMEN +7 Tim $20 \mathrm{ce}$

Date 24 Anquat? $\mathrm{Pg} \cdot$.\#

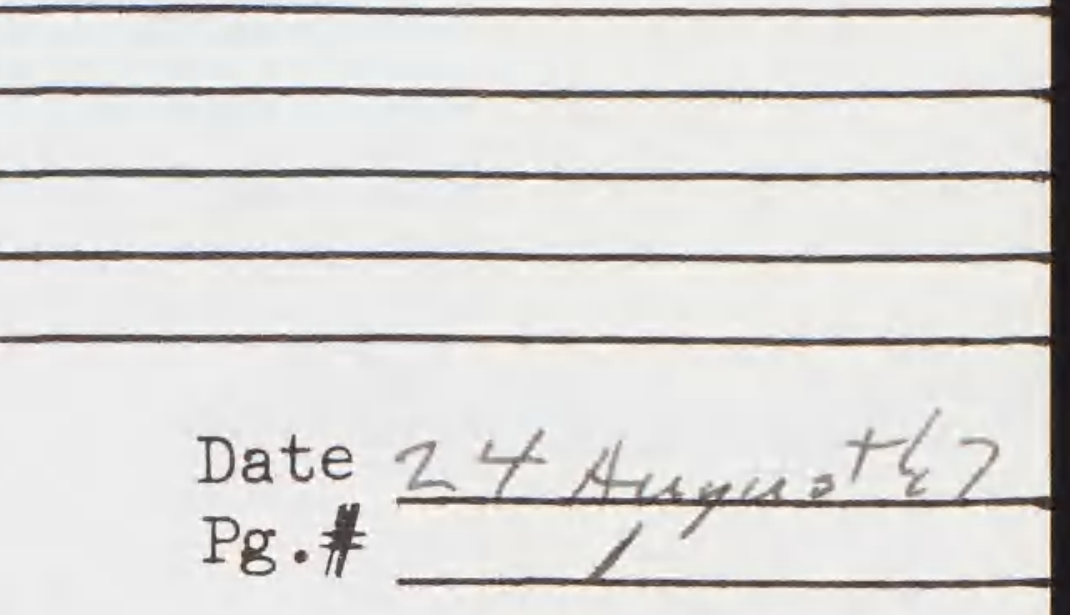

TIME SPECIES \# DIR. BAND NO. REMARKS

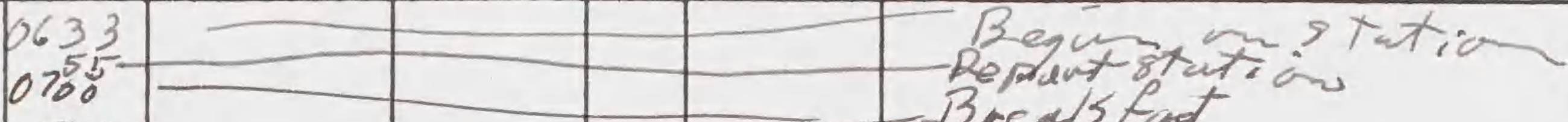

30

39 Colleralpet

54

6800

WBSP

04

1030

1368

09

1316

1342

Tuenix/Tahut:

cot

couared Pet

Sooty Tern

Fairytern

1417 st. Pet.

25 JFP

5) Cullared Pet

55

1509

1523

42

54

FF 1645

FF

1616

19

20

1630

1730

1800

1828 


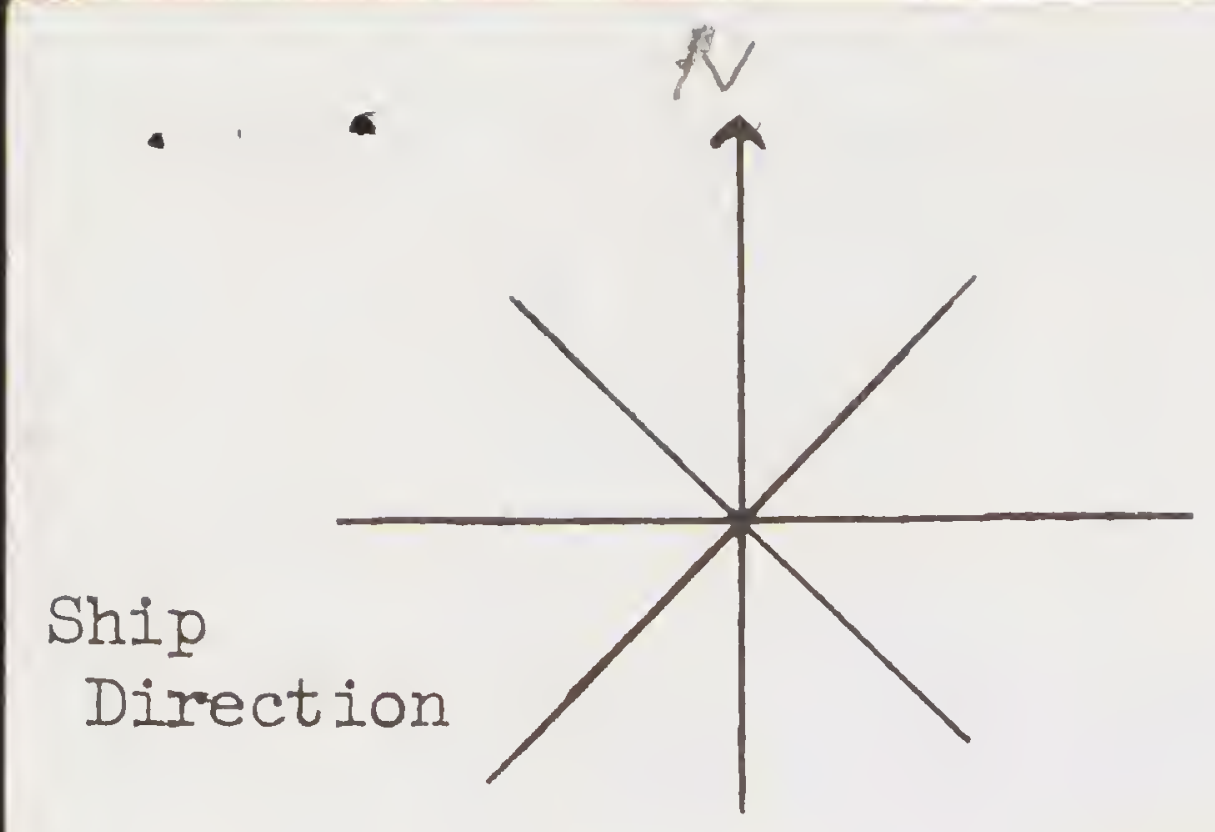

SMITHSONIAN INSTITUTION

DIVISION OF BIRDS

AT SEA DAILY LOG - E

SPECIMEN Foe turn d

or

OBSERVERS :

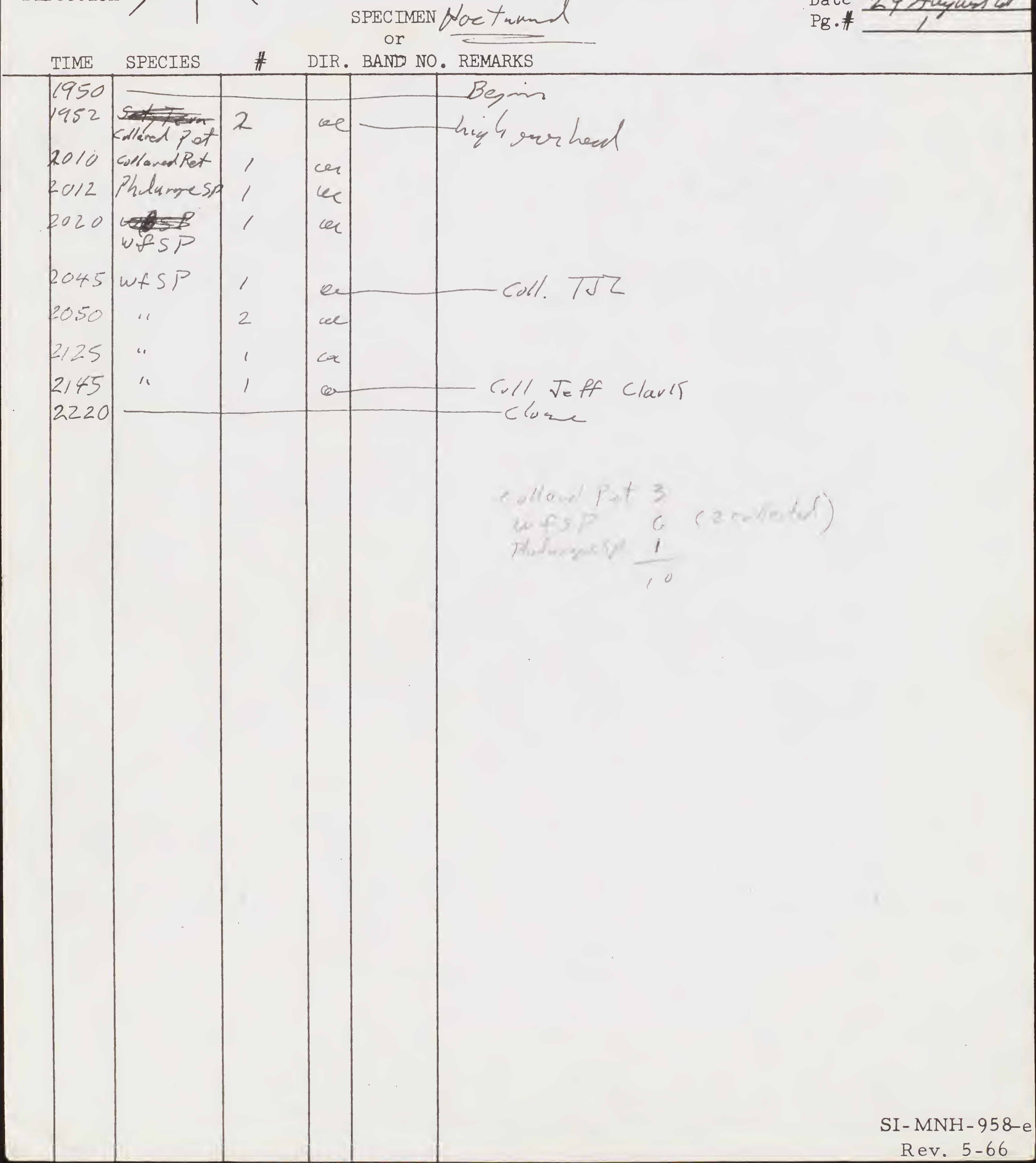




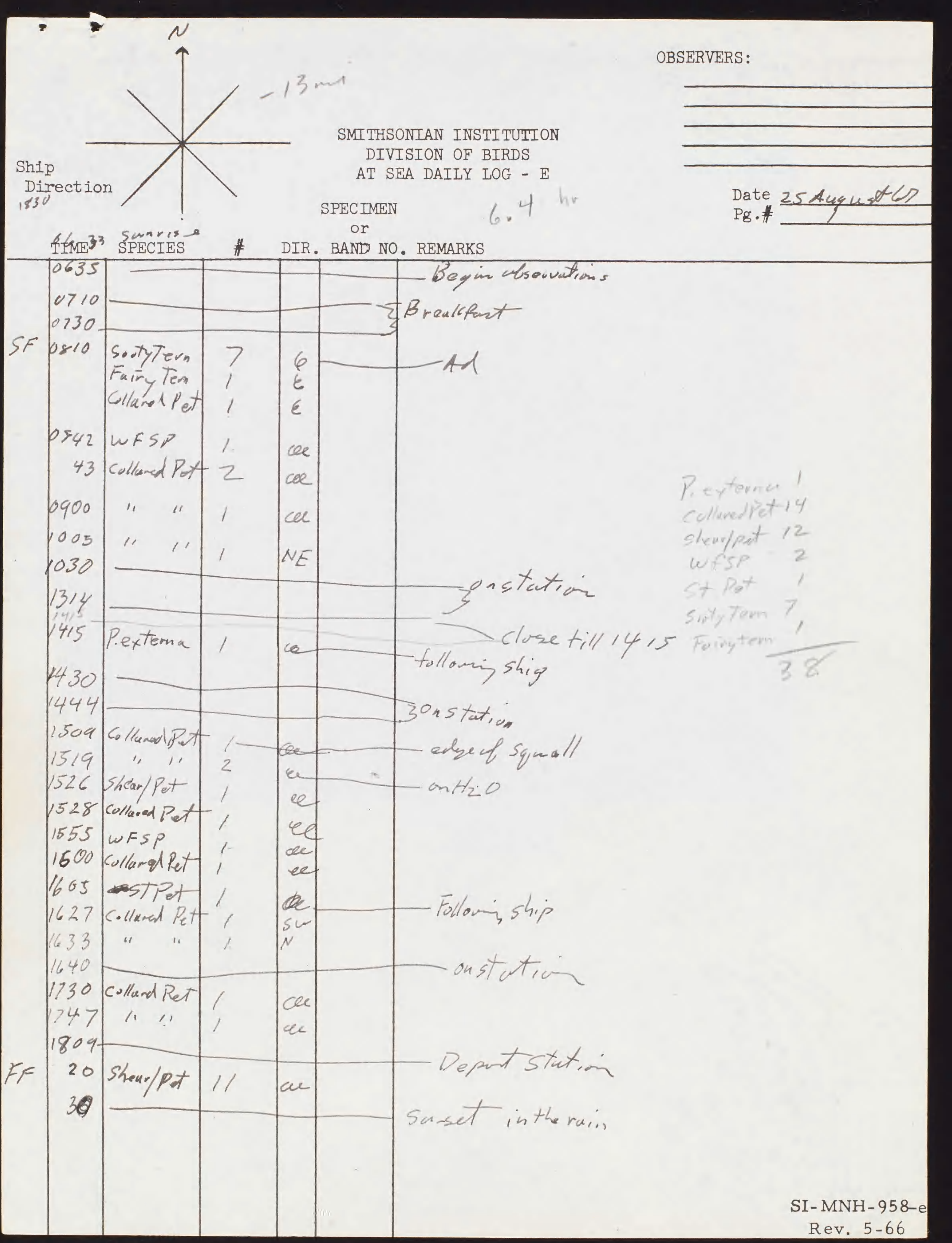




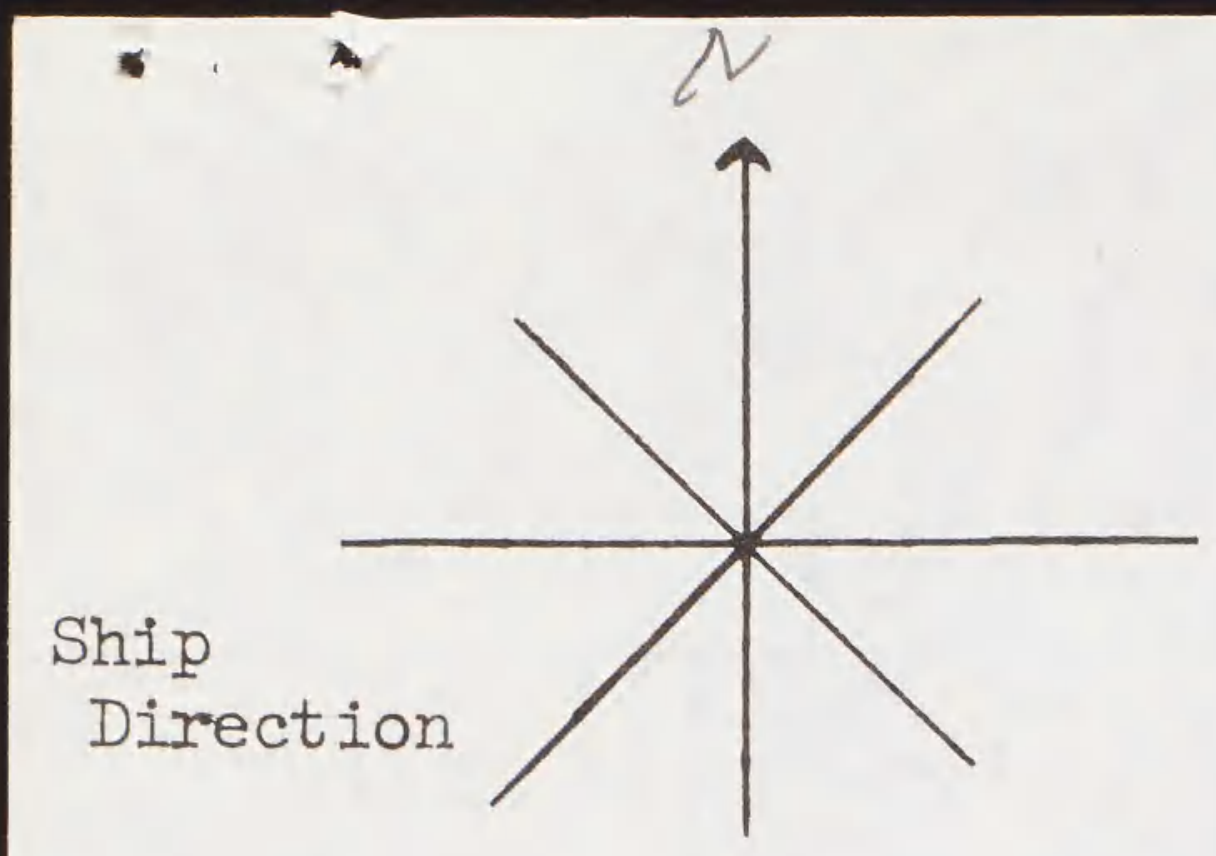

SMITHSONIAN INSTITUTION DIVISION OF BIRDS AT SEA DAILY LOG - E

SPECIMEN Nocturnal OBSERVERS :

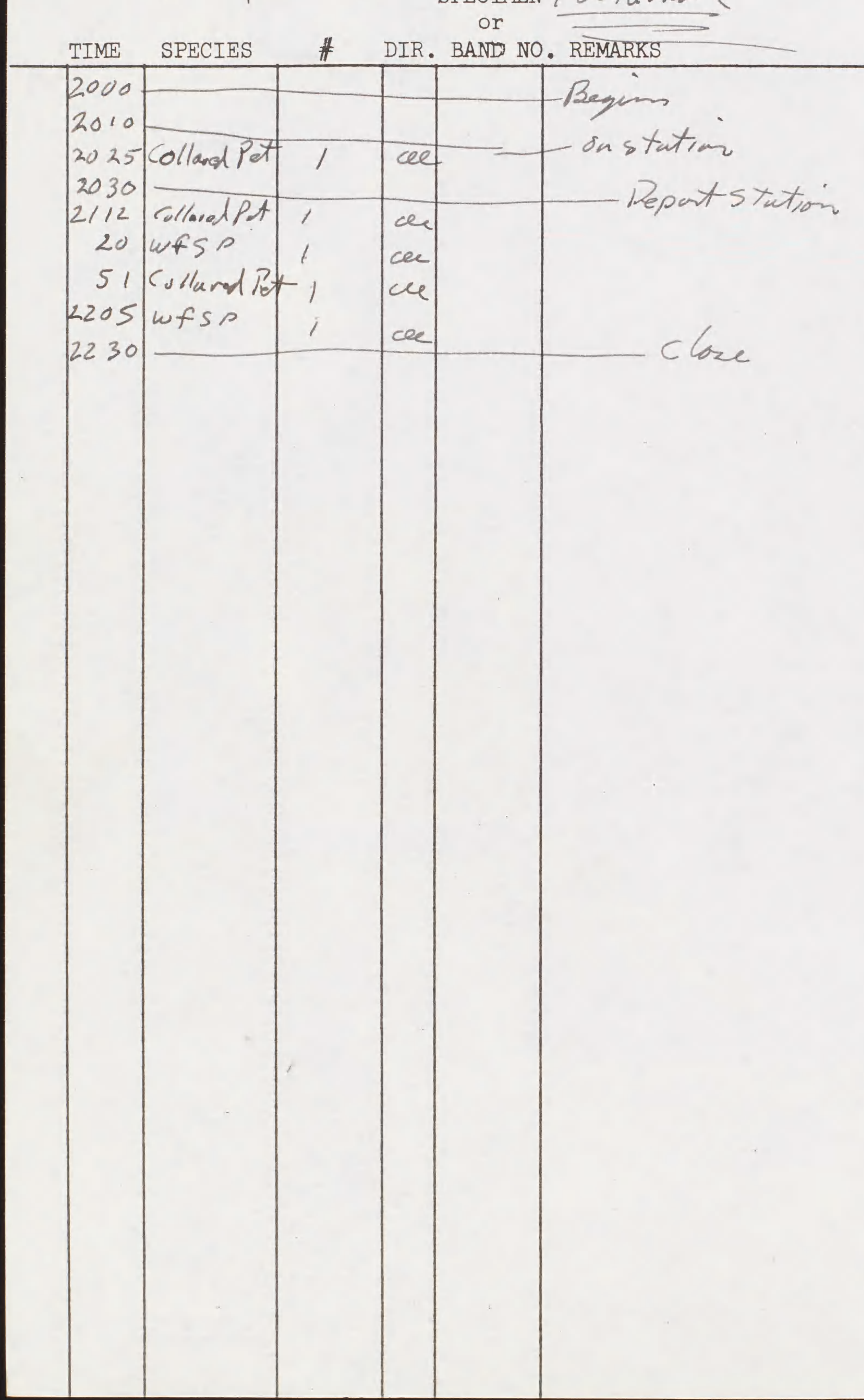
Date 25 Angus $f 6>$
$P g . \# \frac{1}{1}$ collandpot 3
we sp $\frac{2}{5}$ 


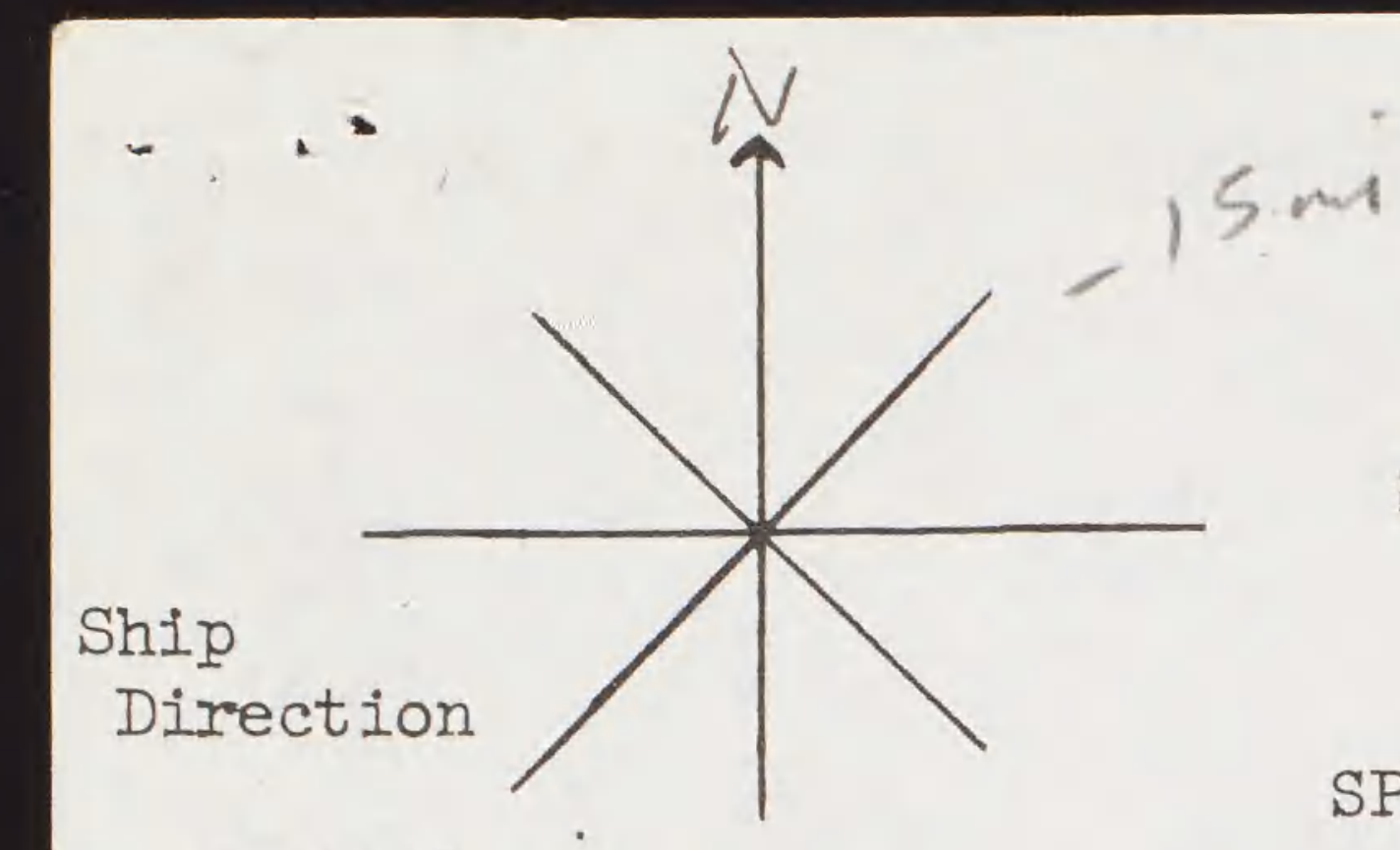

SMITHSONIAN INSTITUTION

DIVISION OF BIRDS

AT SEA DAILY LOG - E

SPECIMEN

or

OBSERVERS :

0633 Sunves * dir. band no. REMARKS

TIME SPECIES \#

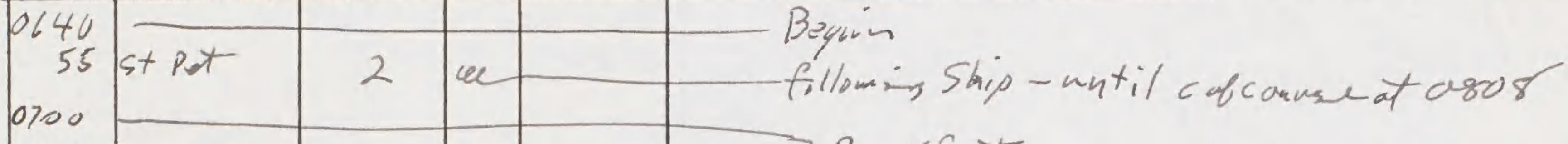

0720

0758 Culland Pet 6808

20 soutytern

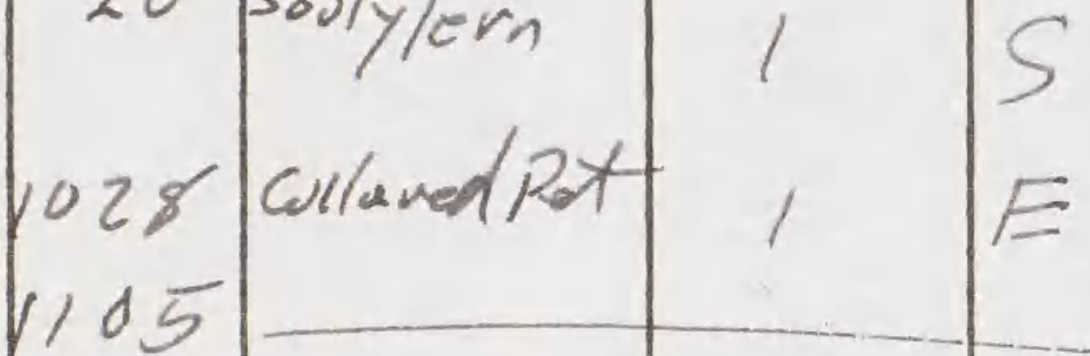

$1 / 50$

1153 shear/pet

1.58 st. pot.

1207 Gallupuess. 5

1242

1520

1715

730

1822

",

\begin{tabular}{|l|l|}
$\prime \prime \prime$ & 3 \\
\hline &
\end{tabular}

2

26 3 Brealsfeyt

ccto 103 to nreet undanted

Date

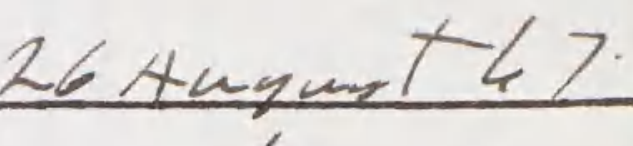

, 


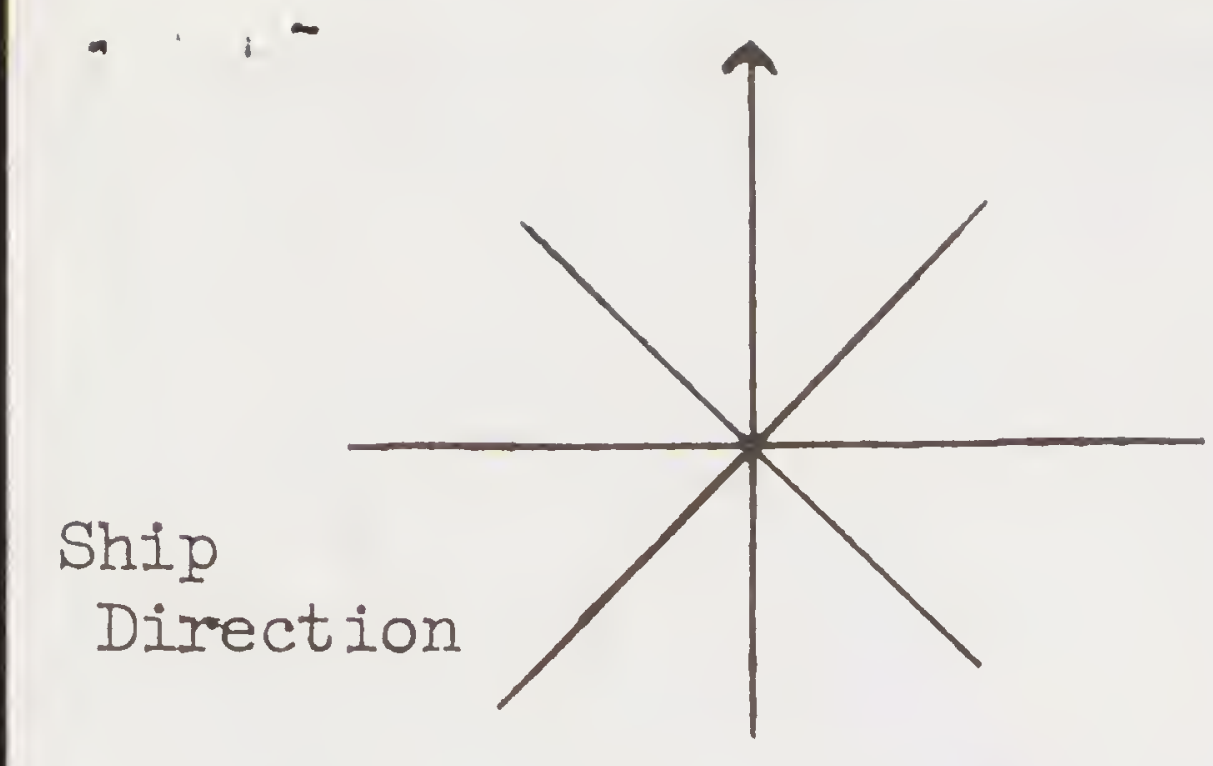

Ship

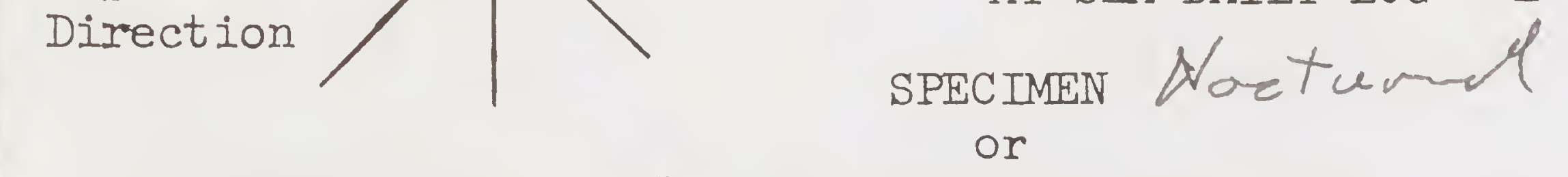

OBSERVERS :

SMITHSONIAN INSTITUTION

DIVISION OF BIRDS

AT SEA DAILY LOG - E

Date 264 w 62

$\mathrm{Pg} \cdot$ *

TIME SPECIES \# DIR. BAND NO. REMARKS

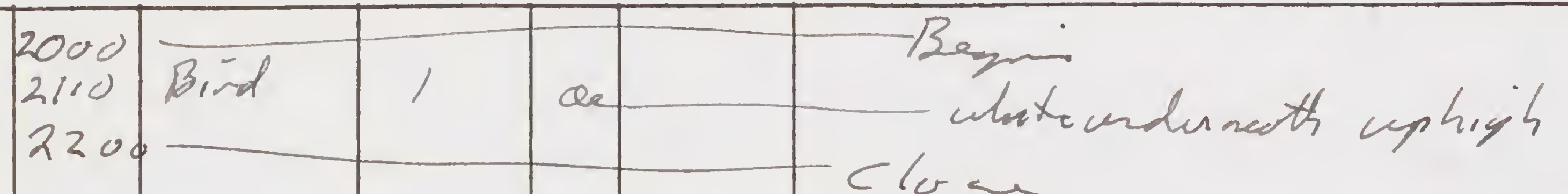

SI- MNH-958-e

Rev. 5-66 


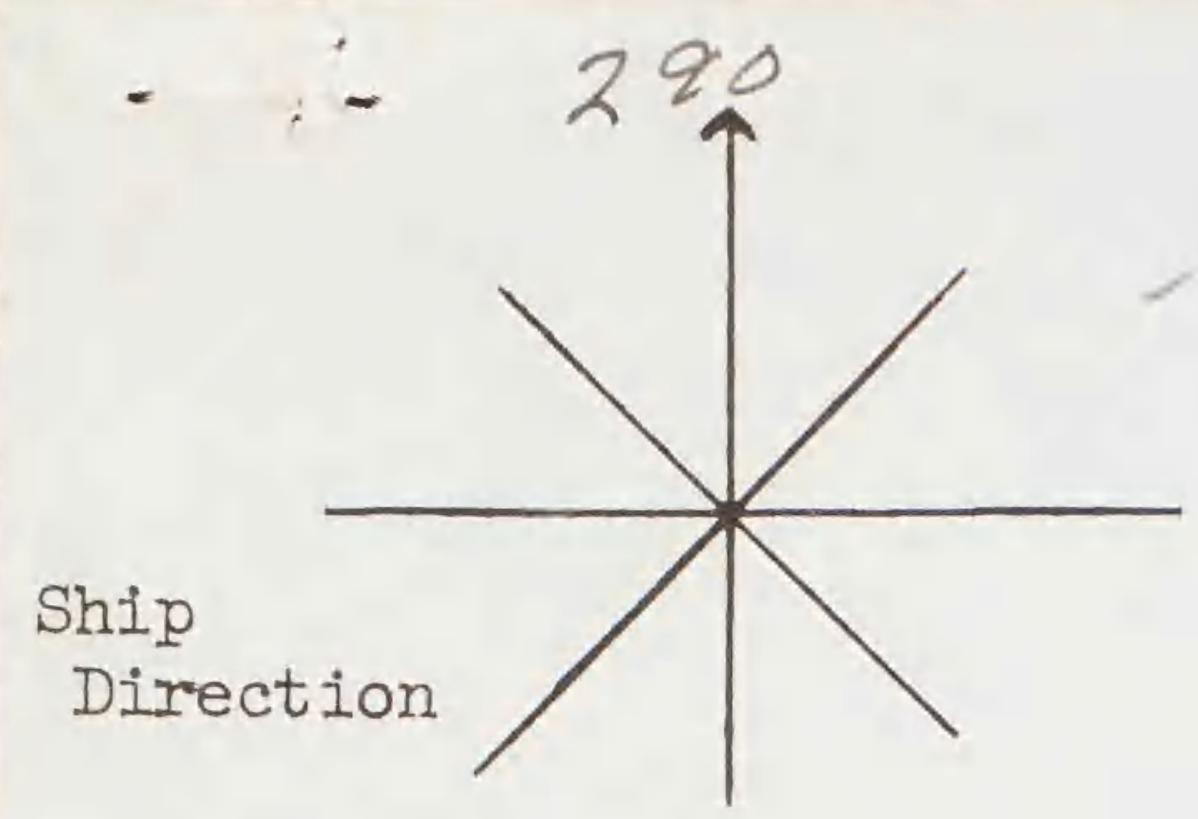

OBSERVERS:

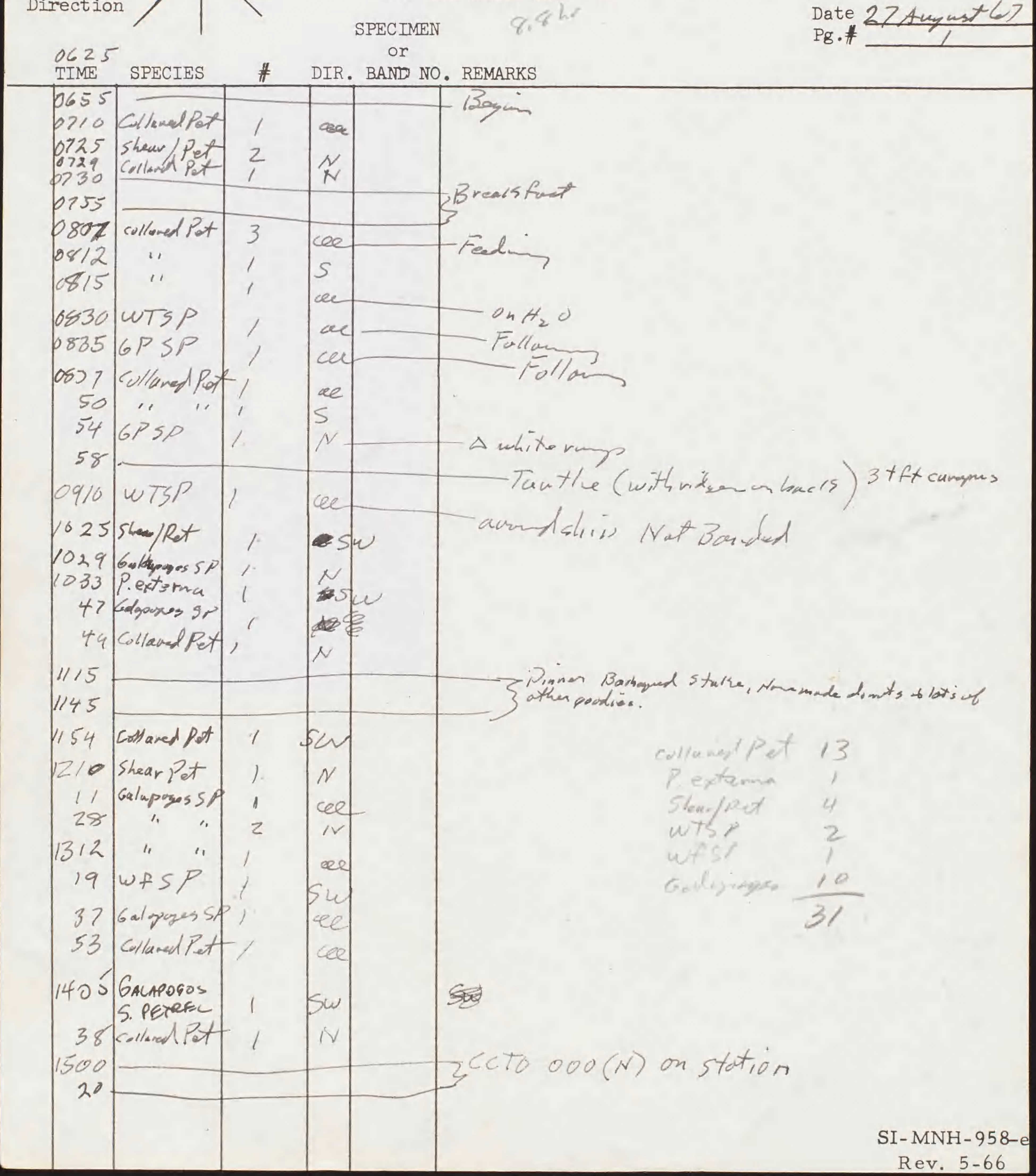




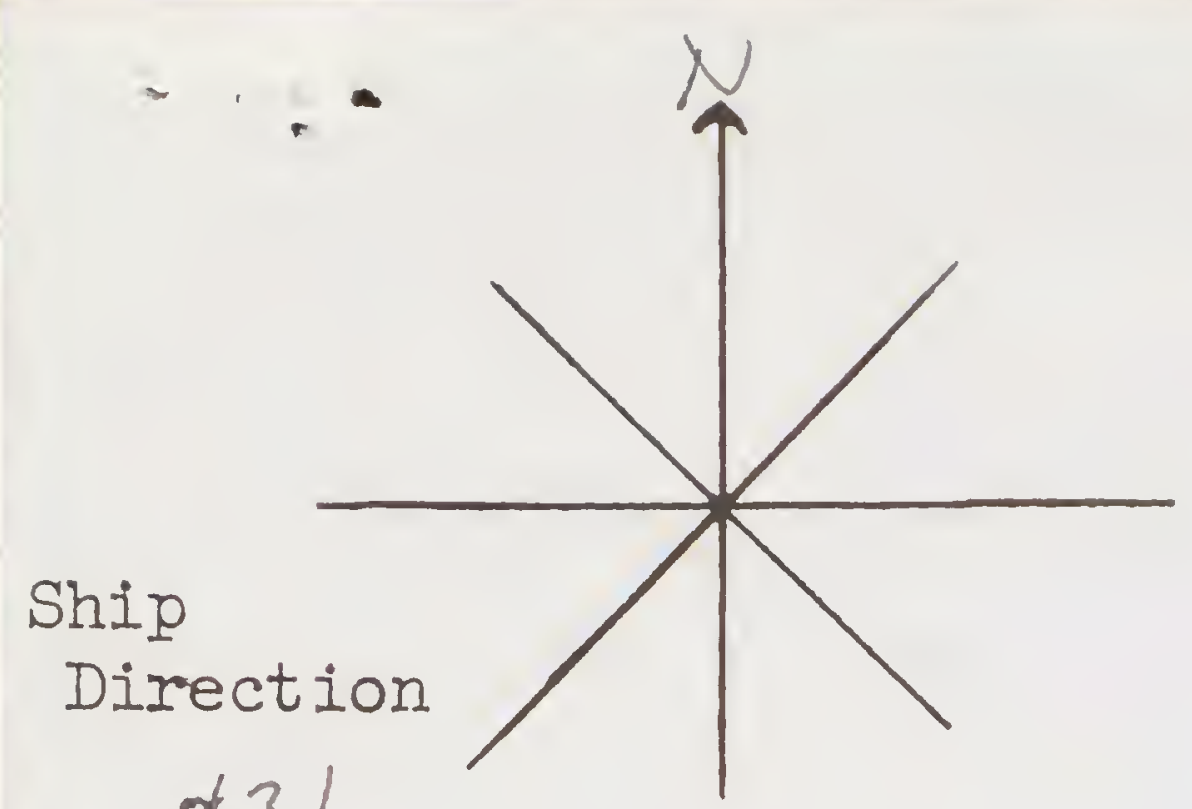

OBSERVERS:

SMITHSONIAN INSTITUTION

DIVISION OF BIRDS

AT SEA DAILY LOG - E

1831

SPEC IMEN

or
Date 27 August 67

$\mathrm{Pg} \cdot \#$

TIME SPECIES \# DIR. BAND NO. REMARKS

1630

1805
1831

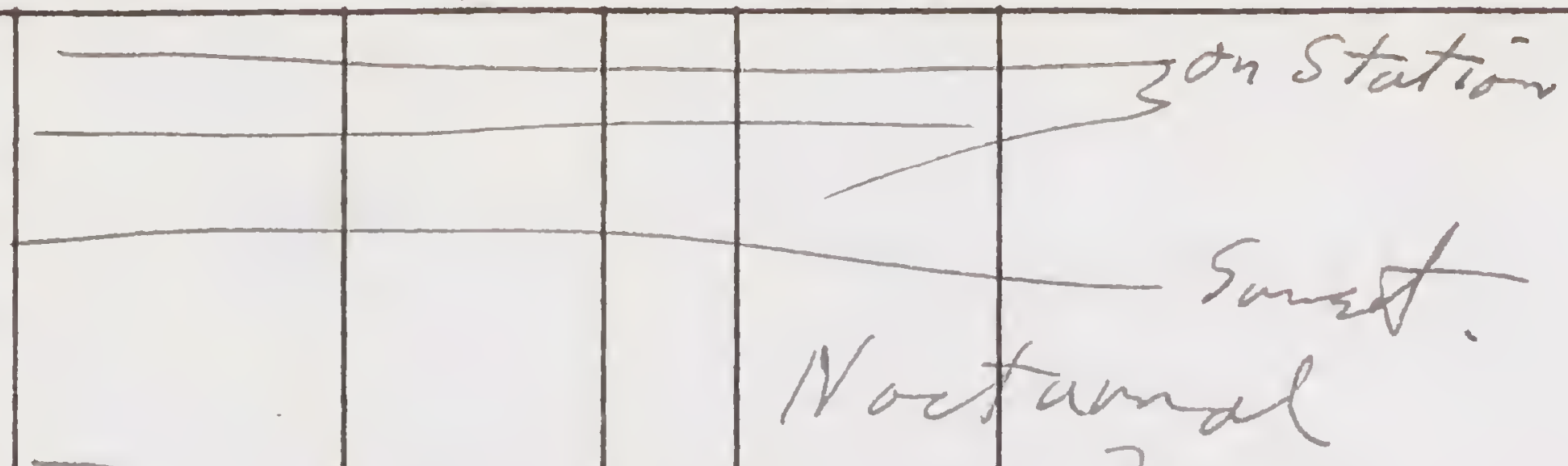

2000

- Beyer

2110

close

SI - MNH - 958-e

Rev. 5-66 


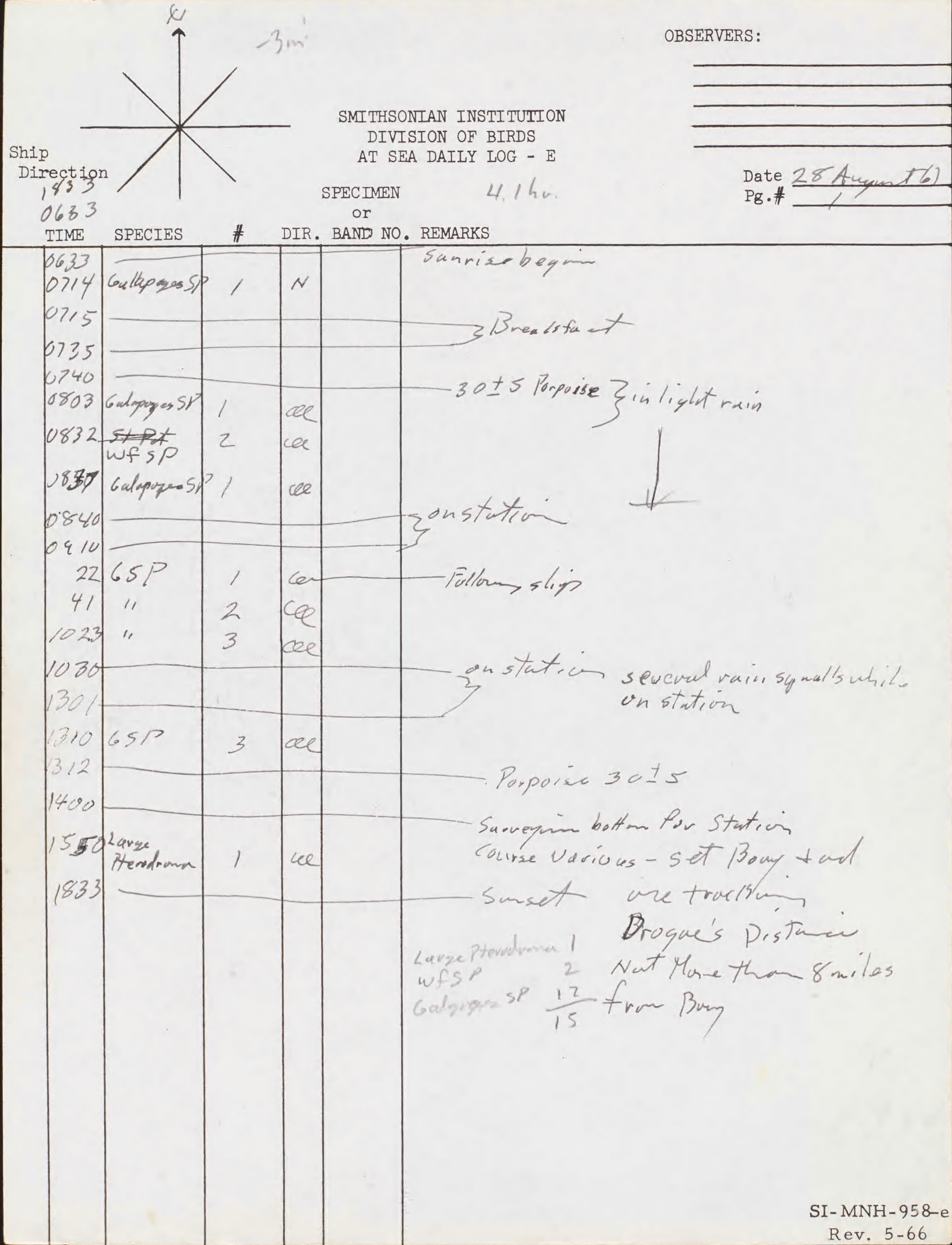




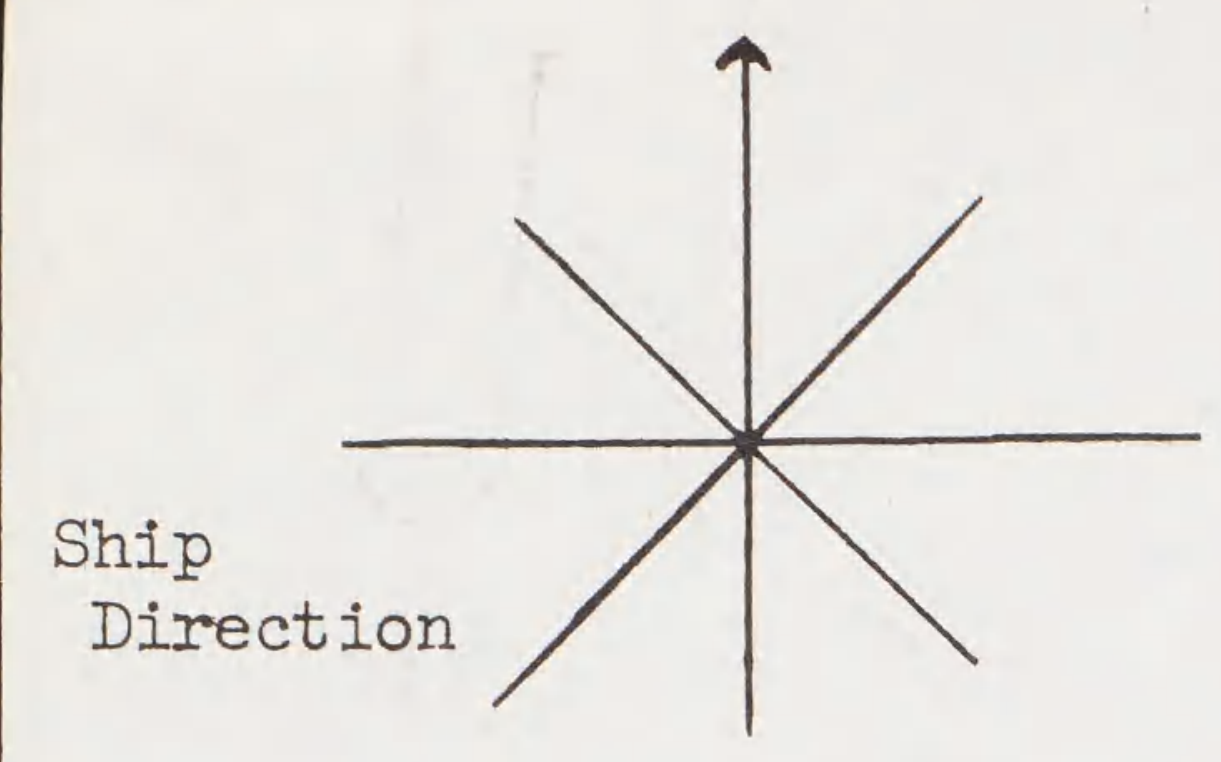

SMITHSONIAN INSTITUTION

DIVISION OF BIRDS

AT SEA DAILY LOG - E

SPEC MEN

or

OBSERVERS:

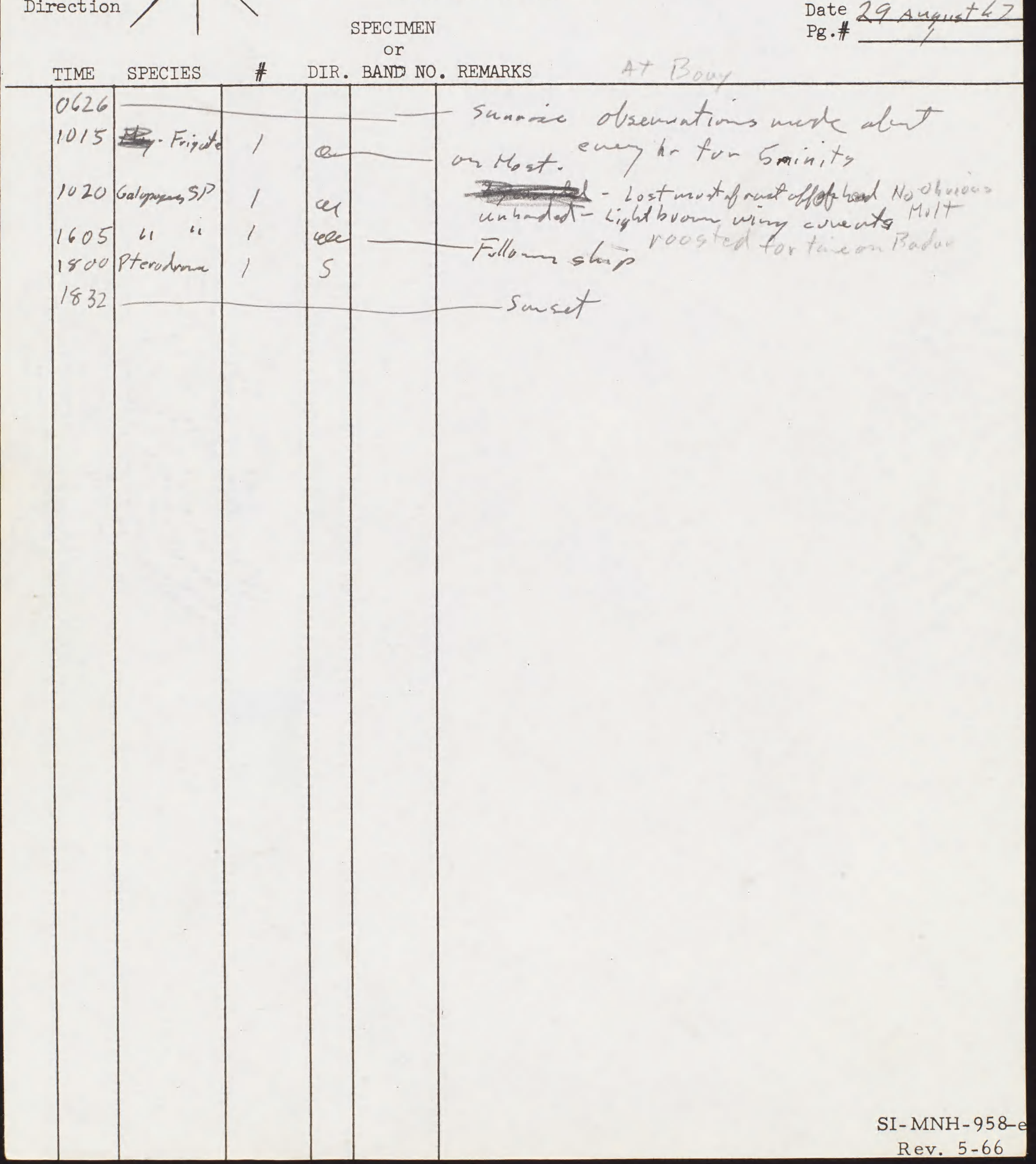




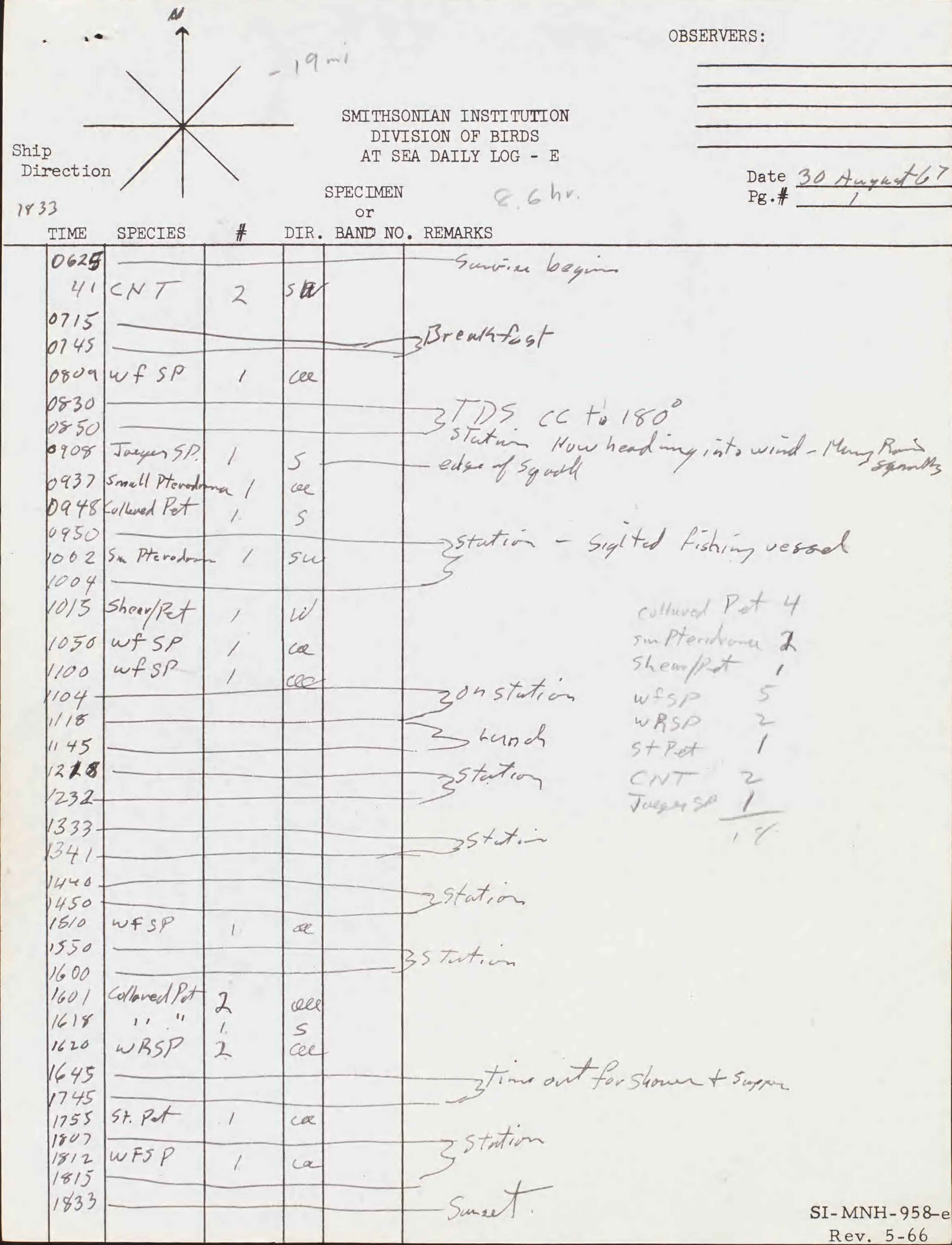




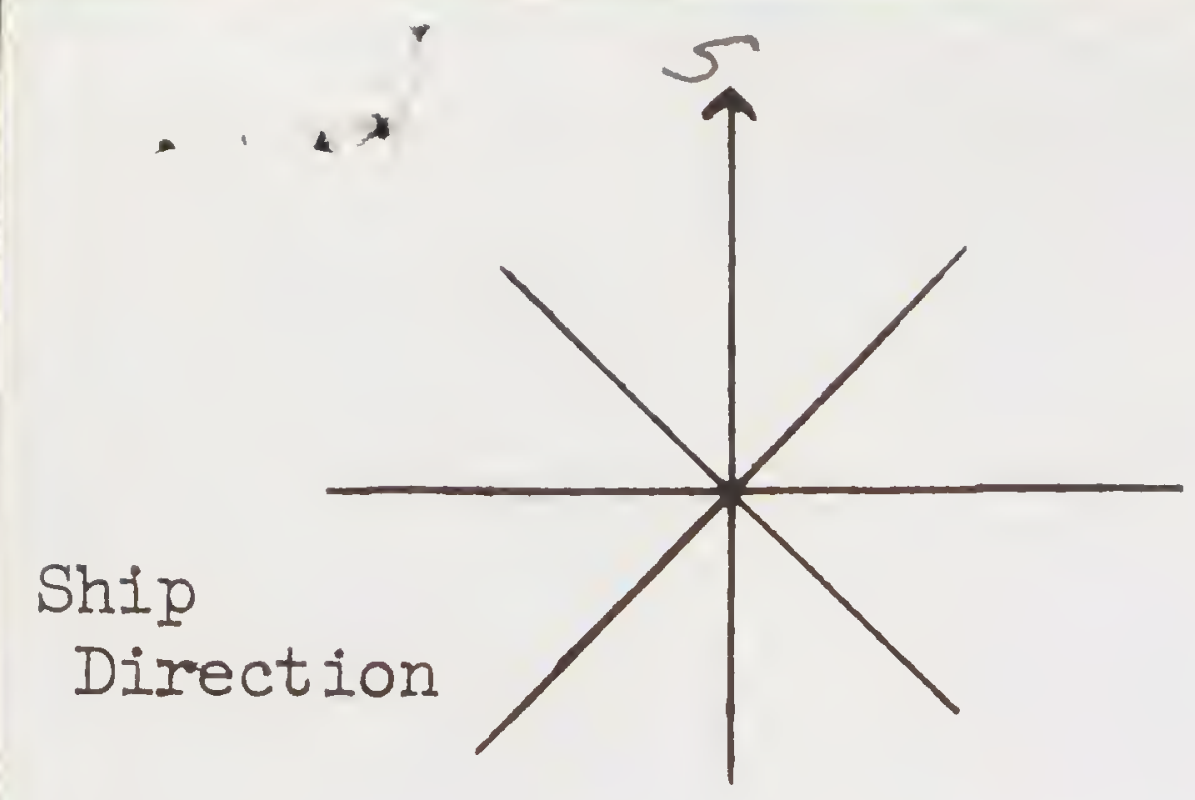

SMITHSONIAN INSTITUTION

DIVISION OF BIRDS

AT SEA DAILY LOG - E

SPECIMEN $N$ octurnal

OBSERVERS :

or

Date 30 August 167
$\mathrm{Pg} \cdot \# \frac{}{1}$

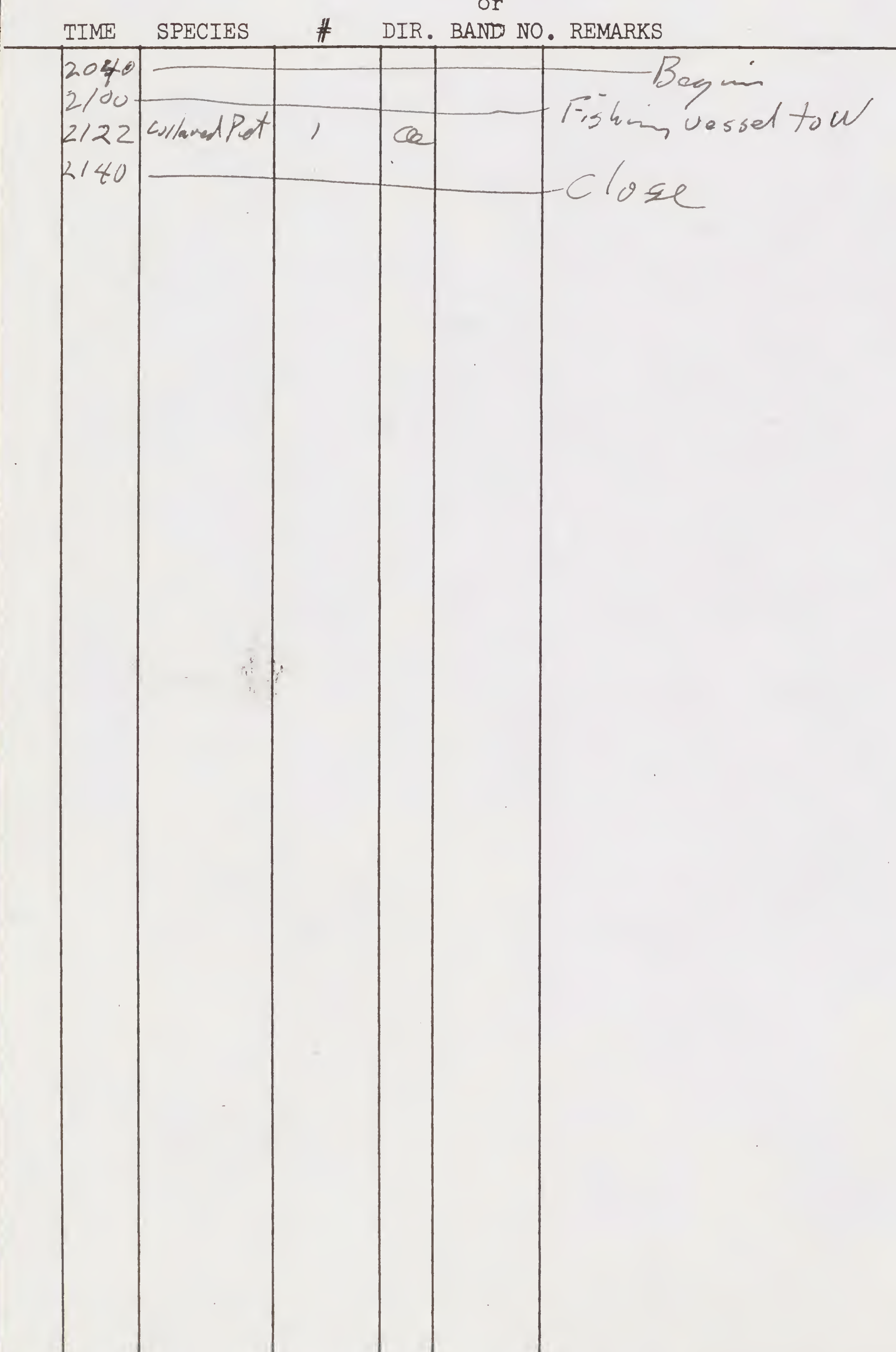

SI- MNH- 958-c

Rev. 5-66 


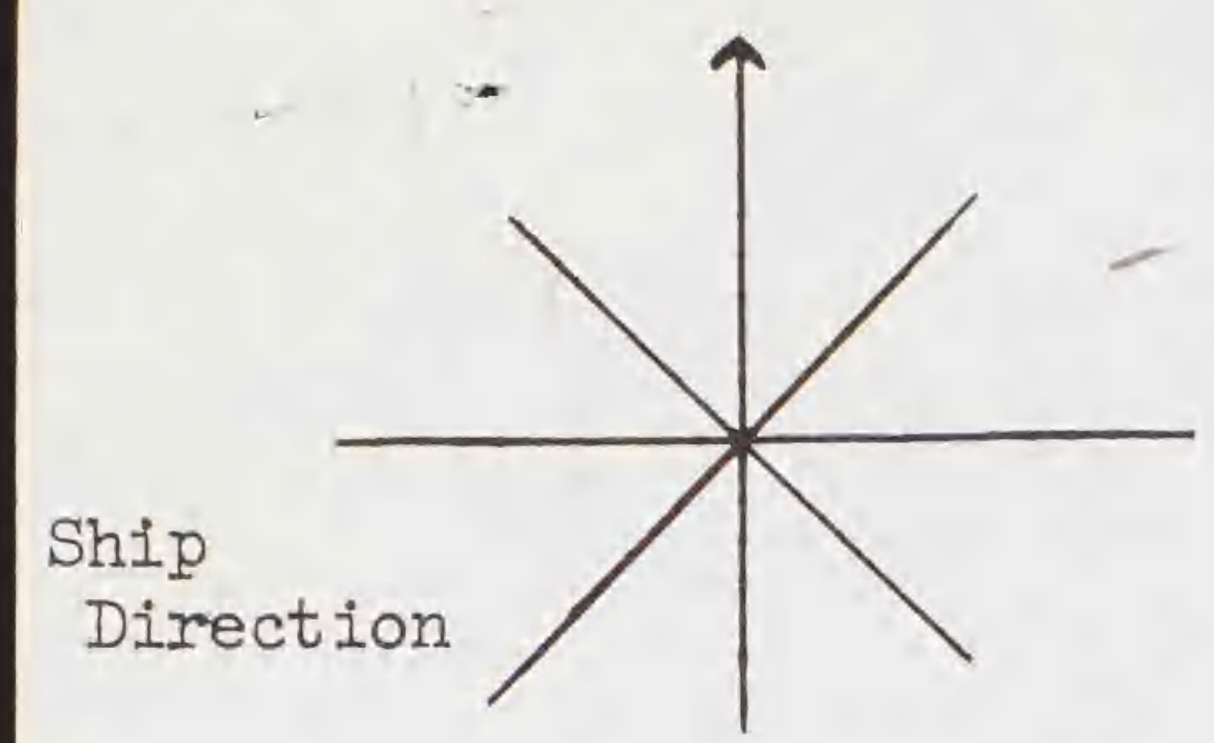

OBSERVERS :

SMITHSONIAN INSTITUTION

DIVISION OF BIRDS

AT SEA DAILY LOG - E

SPECIMEN

or

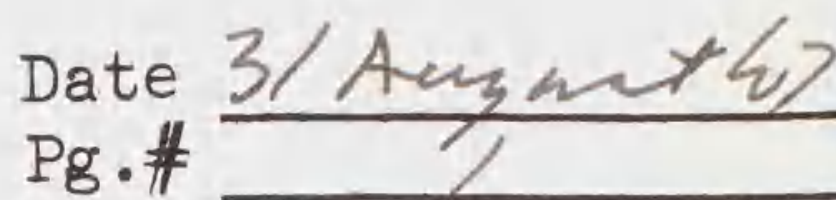

TIME SPECIES \# DIR. BAND NO. REMARKS

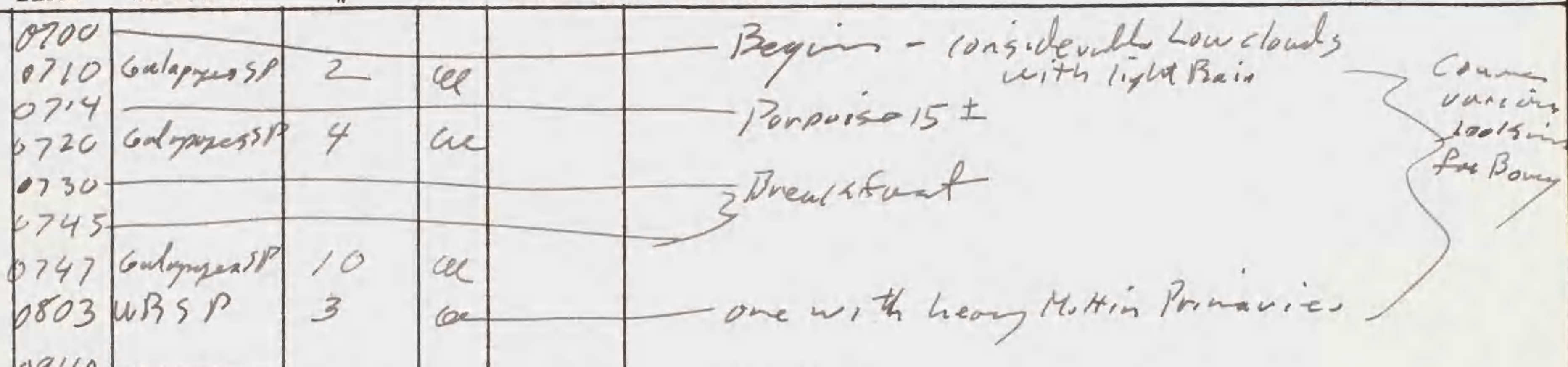

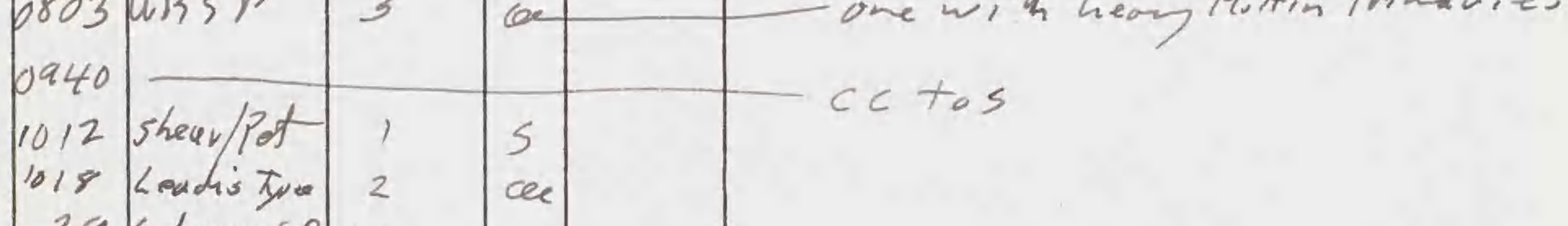

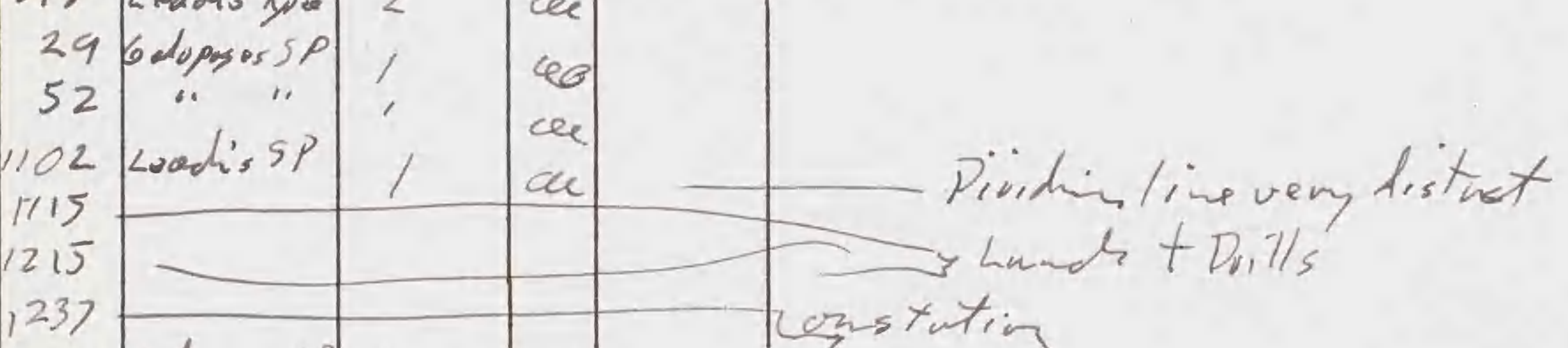

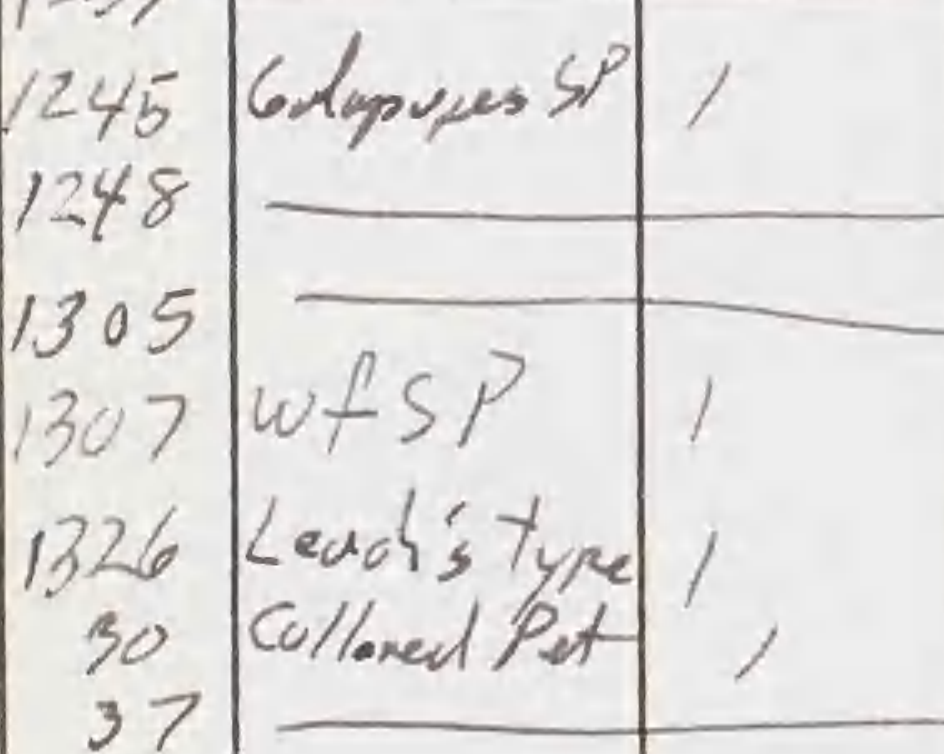

$1343-$

1442 Leach: SP

1457

1606

1616

1625

1730

174)

1924

st. Pot. cere

ie

constution

ce

Porpoise $150 \pm 25$

feeding - The mot erratic flight I. we ens seen Looked like aperient o octaves on

wholes (2) gustation

zonstatin

z station

Follow is ship

SPinner of Station

-arch type 3

6

\begin{tabular}{l|l|l} 
& \\
& &
\end{tabular}

27 Pat $\frac{1}{33}$

onstutur

SI-MNH-958-e

Rev. 5-66 


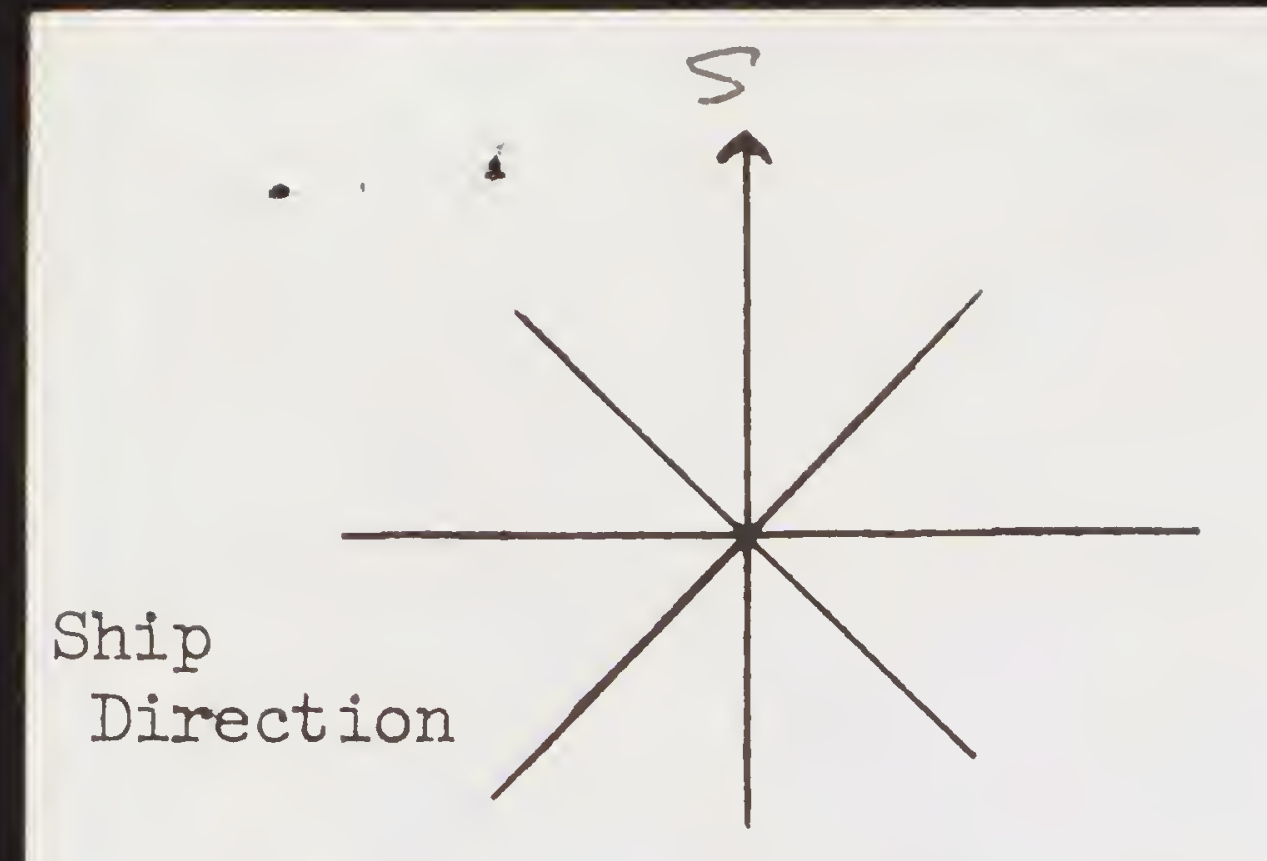

SMITHSONIAN INSTITUTION

DIVISION OF BIRDS

AT SEA DAILY LOG - E

SPECIMEN Nactum

or

Date 3 Anyeth?

$\mathrm{Pg}$ •\#

TIME SPECIES \# DIR. BAND NO. REMARKS

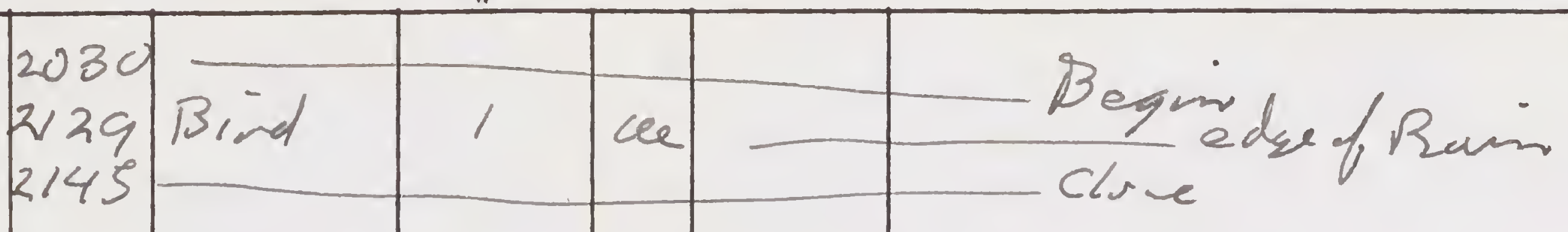

SI-MNH-958-e 


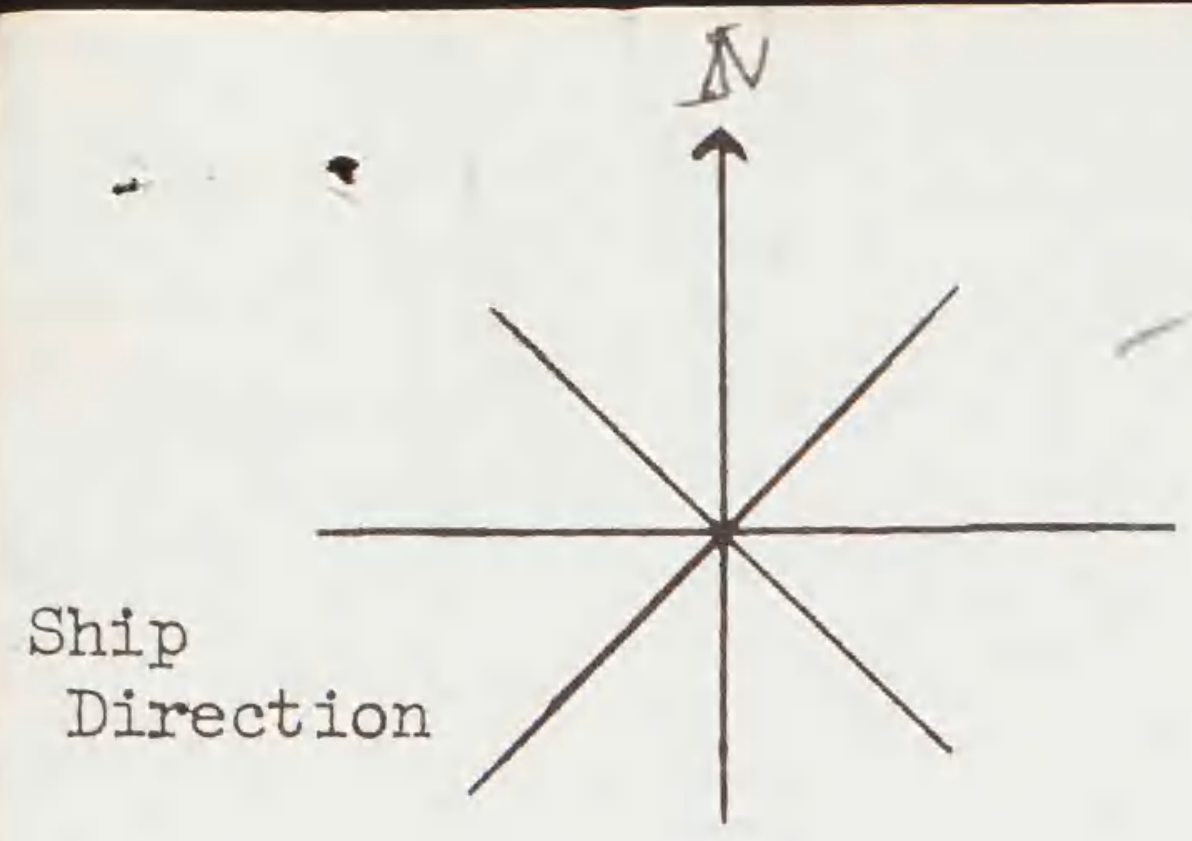

OBSERVERS :

SMITHSONIAN INSTITUTION

DIVISION OF BIRDS

AT SEA DAILY LOG - E

SPECIMEN

$9.2 \mathrm{hr}$

or
Date (Sent 6$)$

$\mathrm{Pg} \cdot \#$

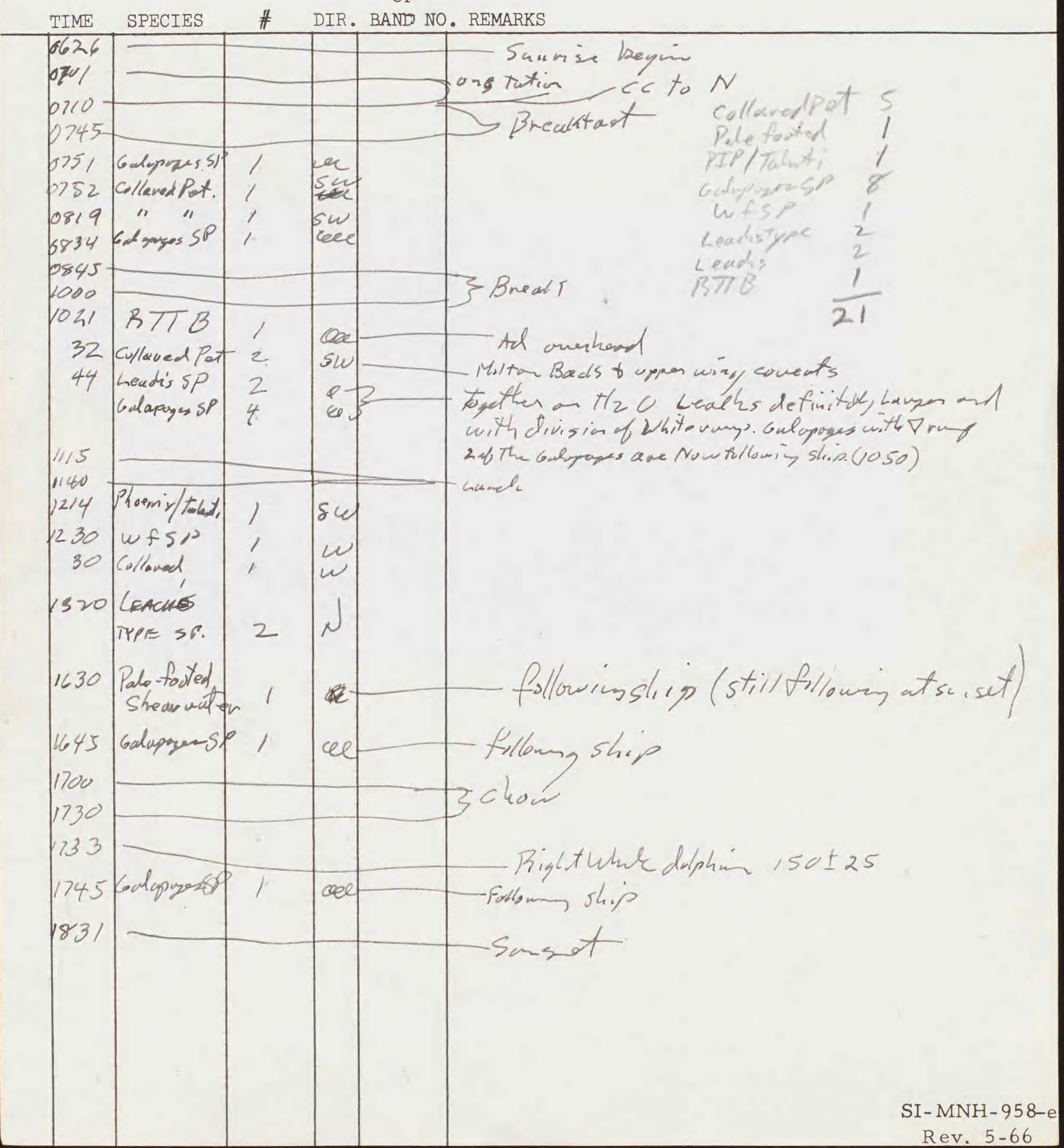




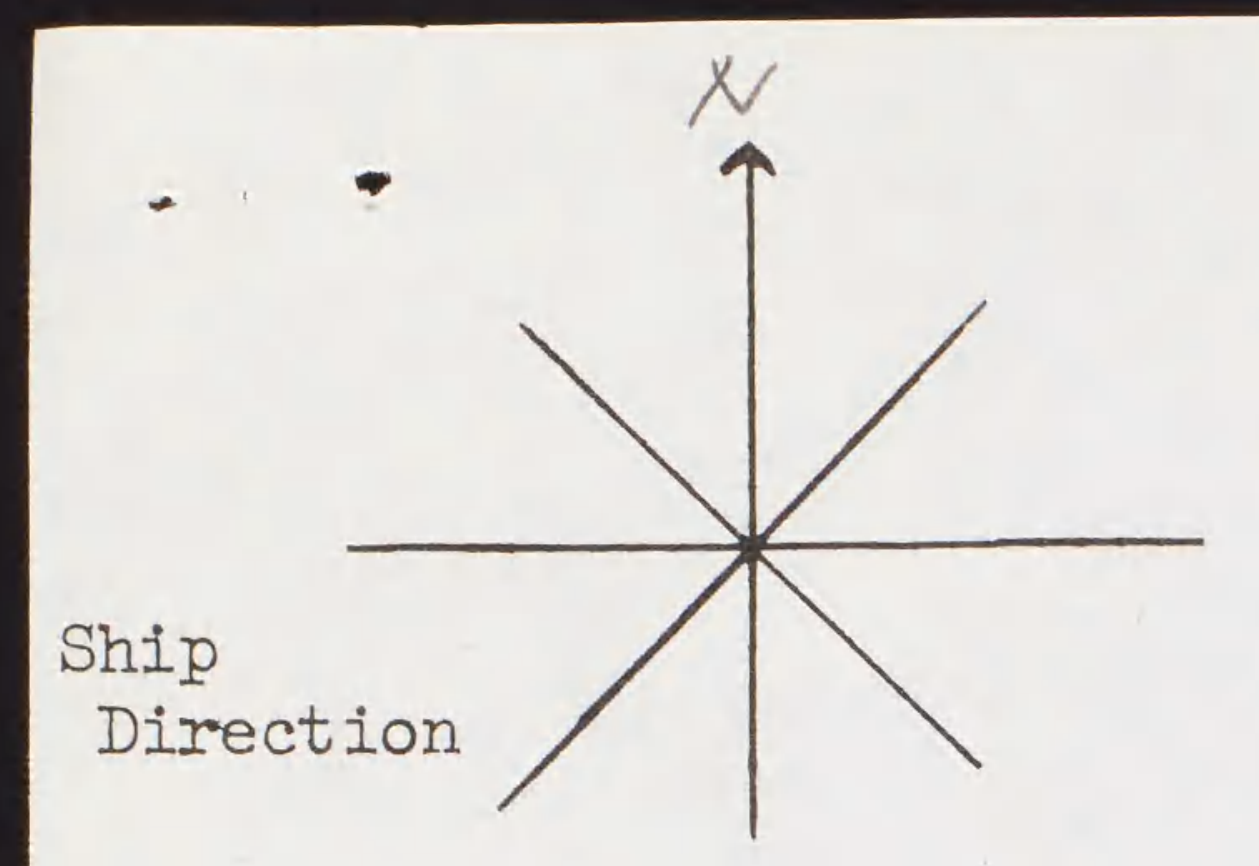

OBSERVERS:

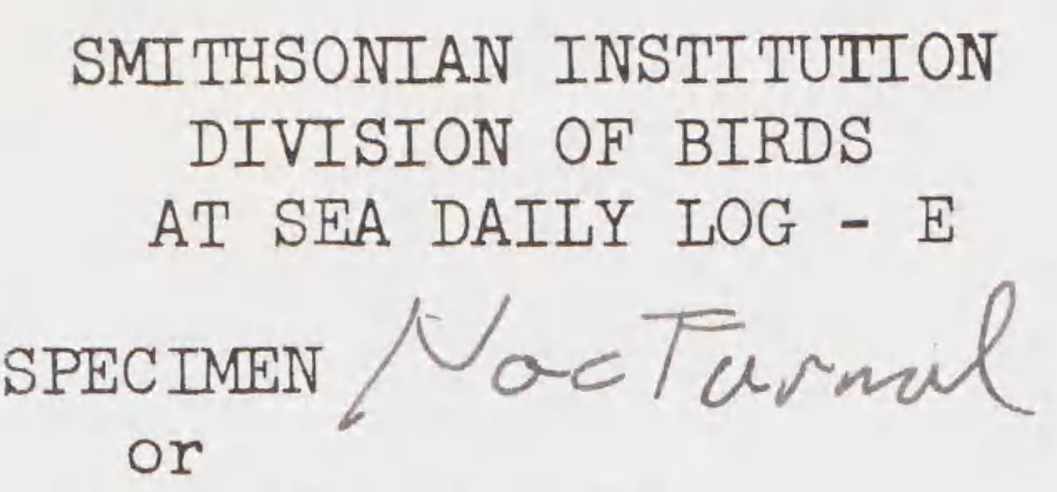

TIME SPECIES \# DIR. BAND NO. REMARKS

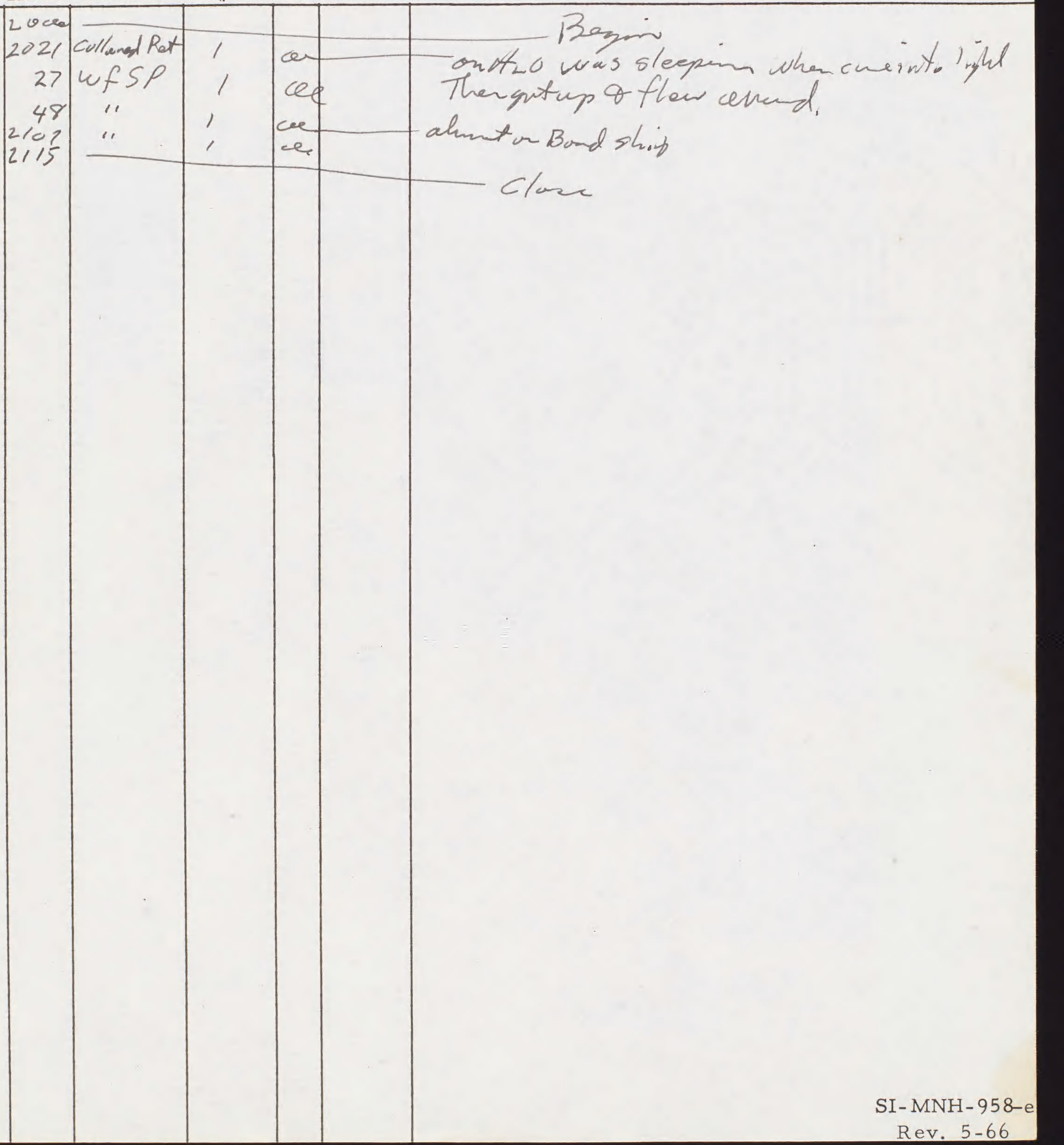




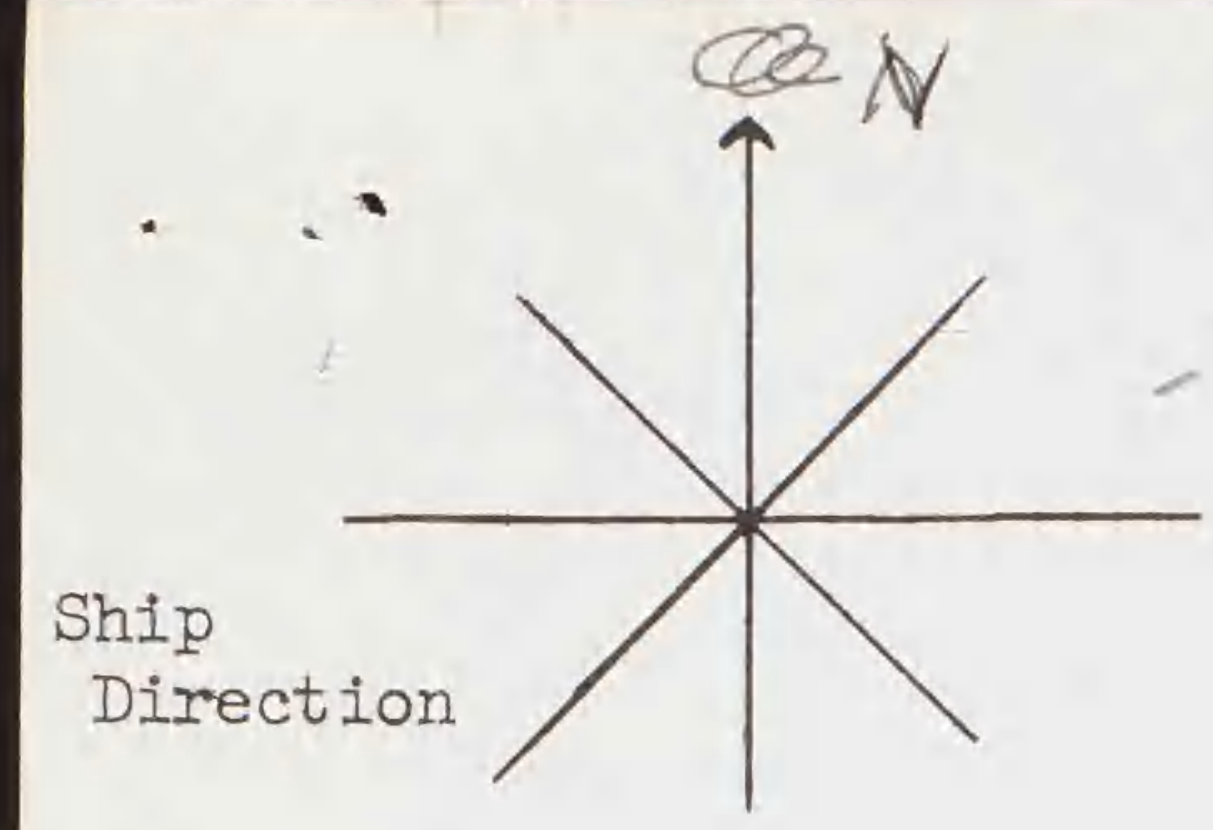

SMITHSONIAN INSTITUTION

DIVISION OF BIRDS

AT SEA DAILY LOG - E

SPECIMEN

OBSERVERS :

or

Date $25 e n t 67$
Pg.\#

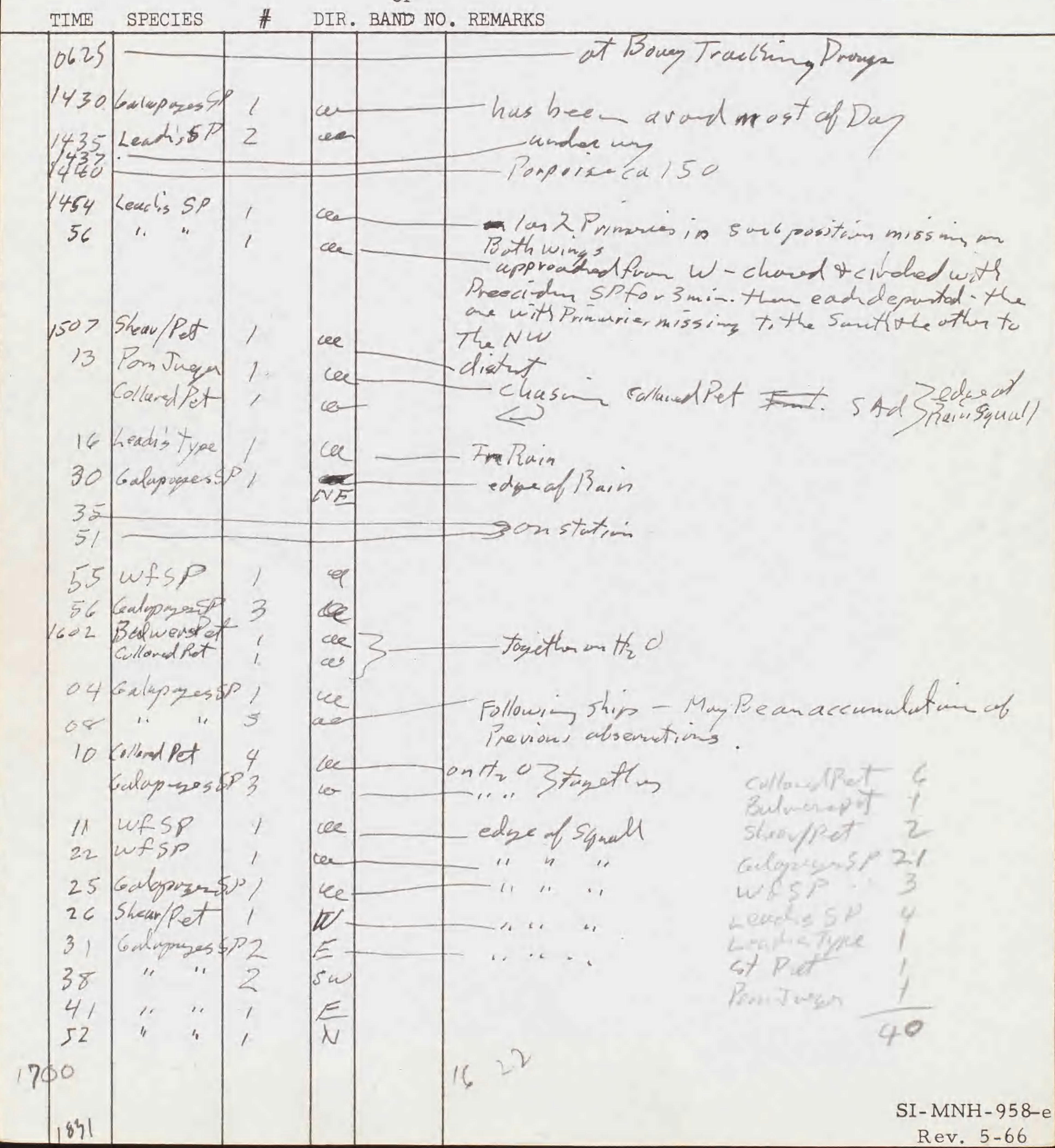




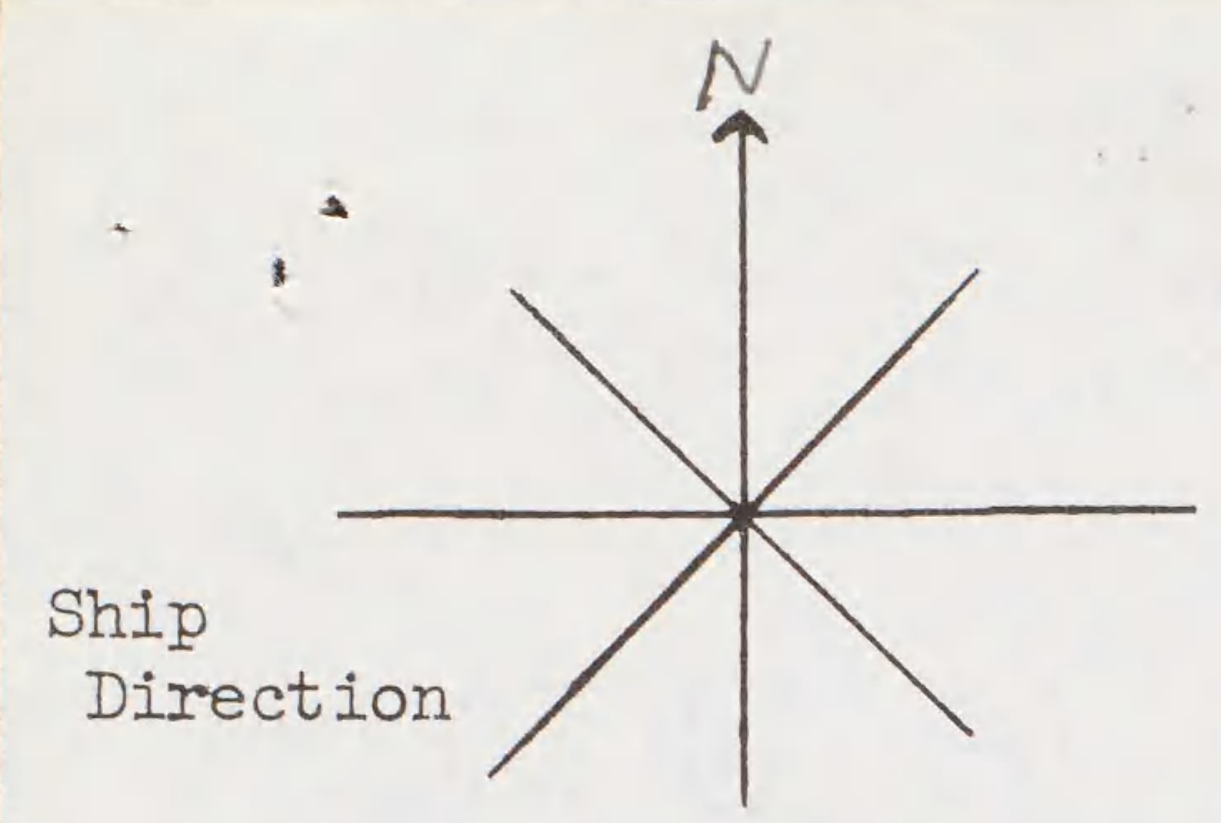

OBSERVERS :

SMITHSONIAN INSTITUTION DIVISION OF BIRDS AT SEA DAILY LOG - E

SPEC IMEN

or

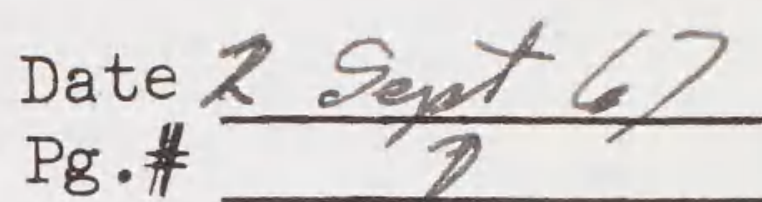

$\mathrm{Pg} \cdot \#$

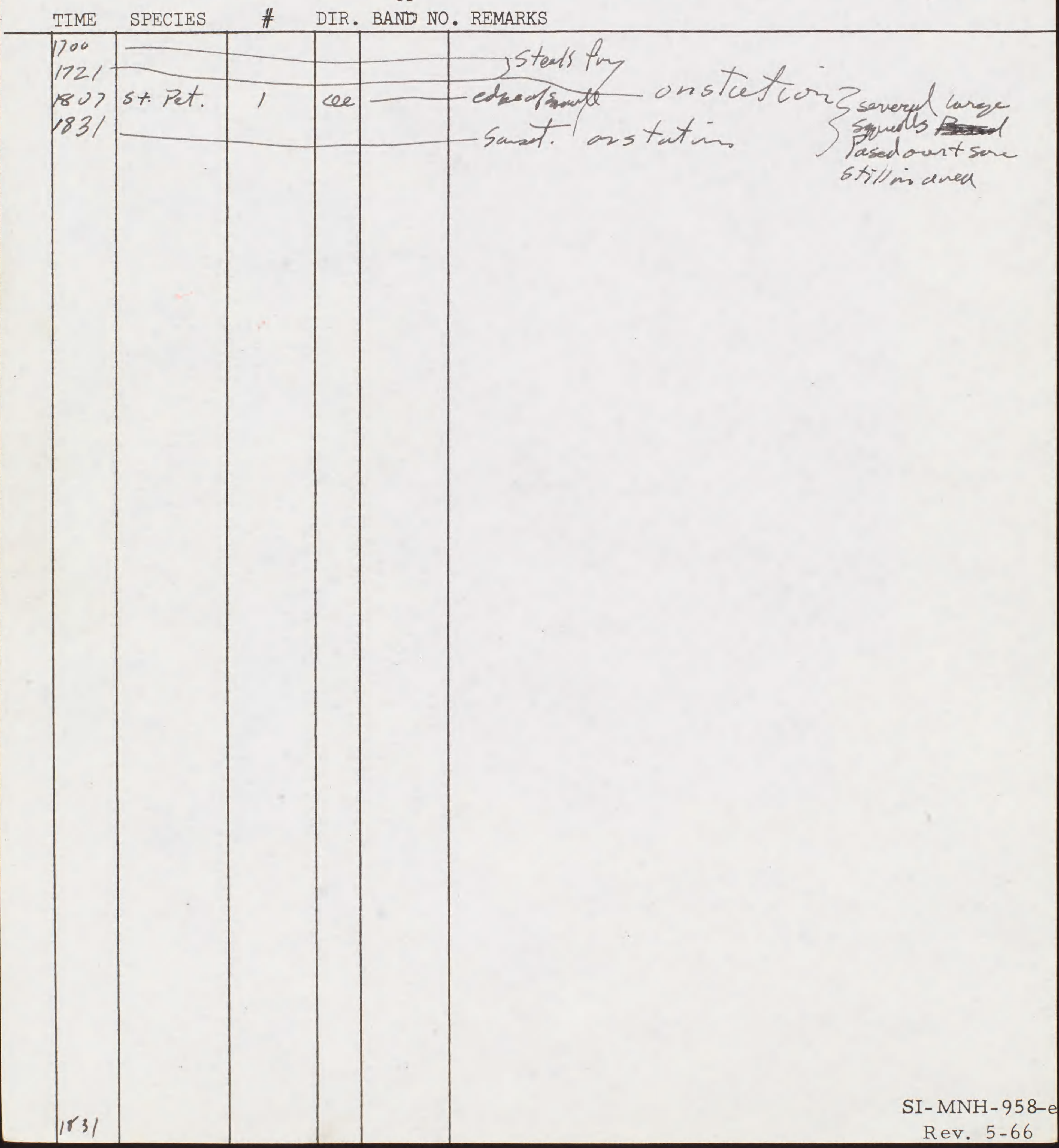




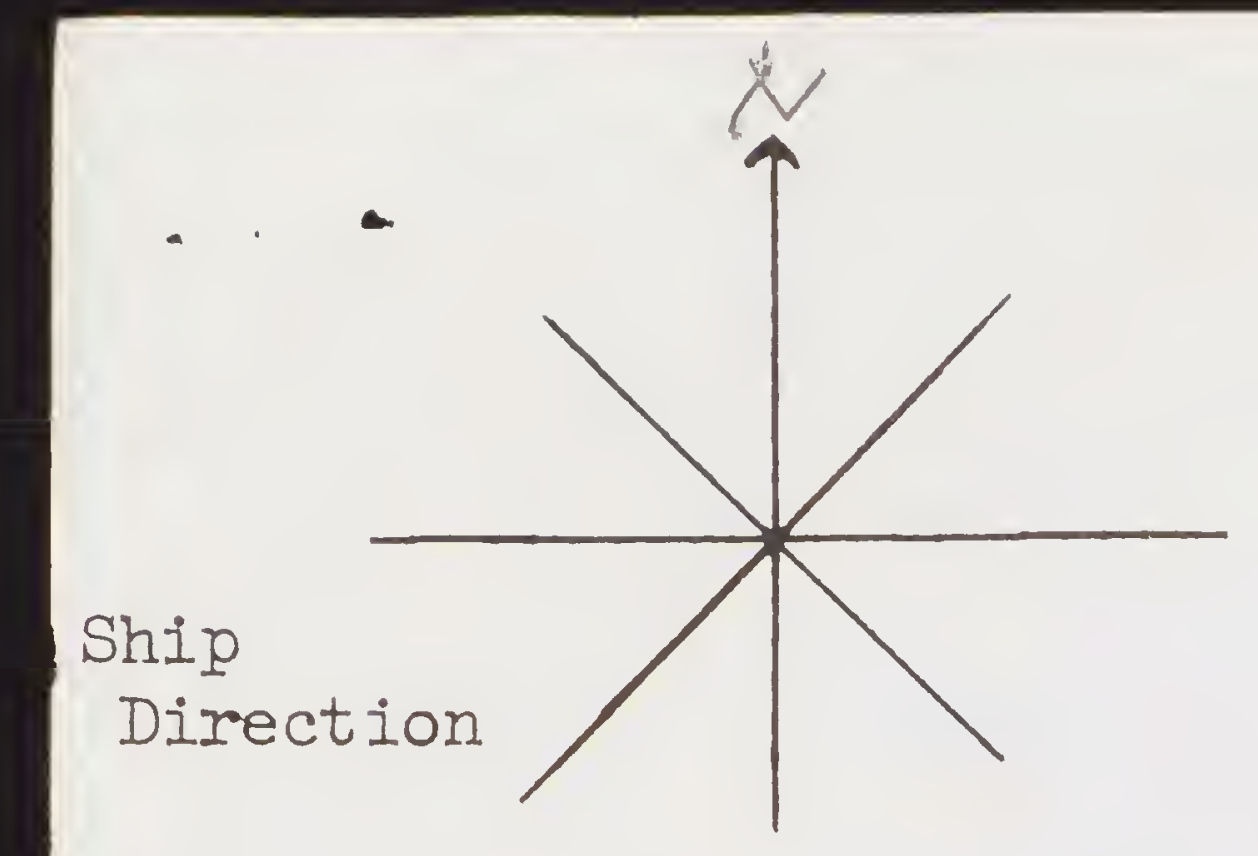

OBSERVERS :

SMITHSONIAN INSTITUTION

DIVISION OF BIRDS

AT SEA DAILY LOG - E

SPECIMEN Alocturach

or

Date 2 sept 67

$\mathrm{Pg}$ •\#

\section{TIME SPECIES \# DIR. BAND NO. REMARKS}

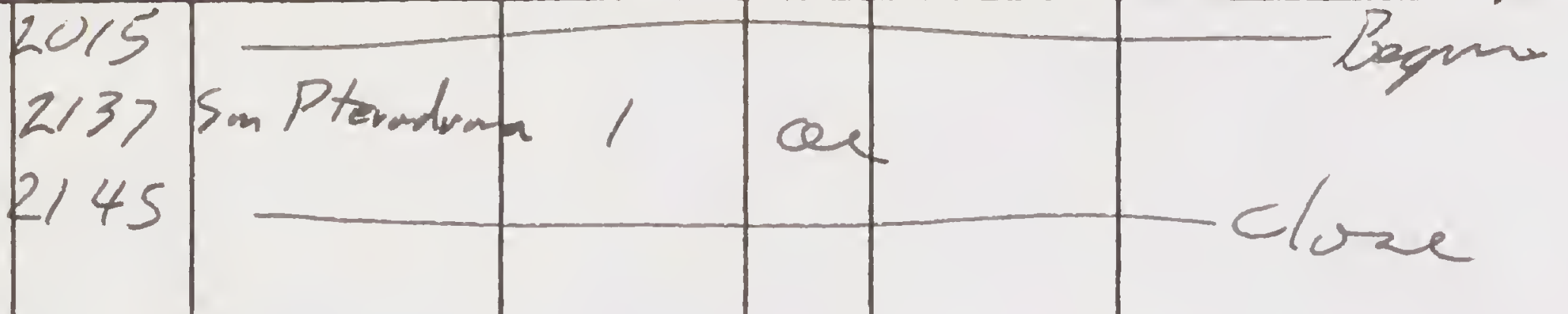

SI-MNH-958-e 


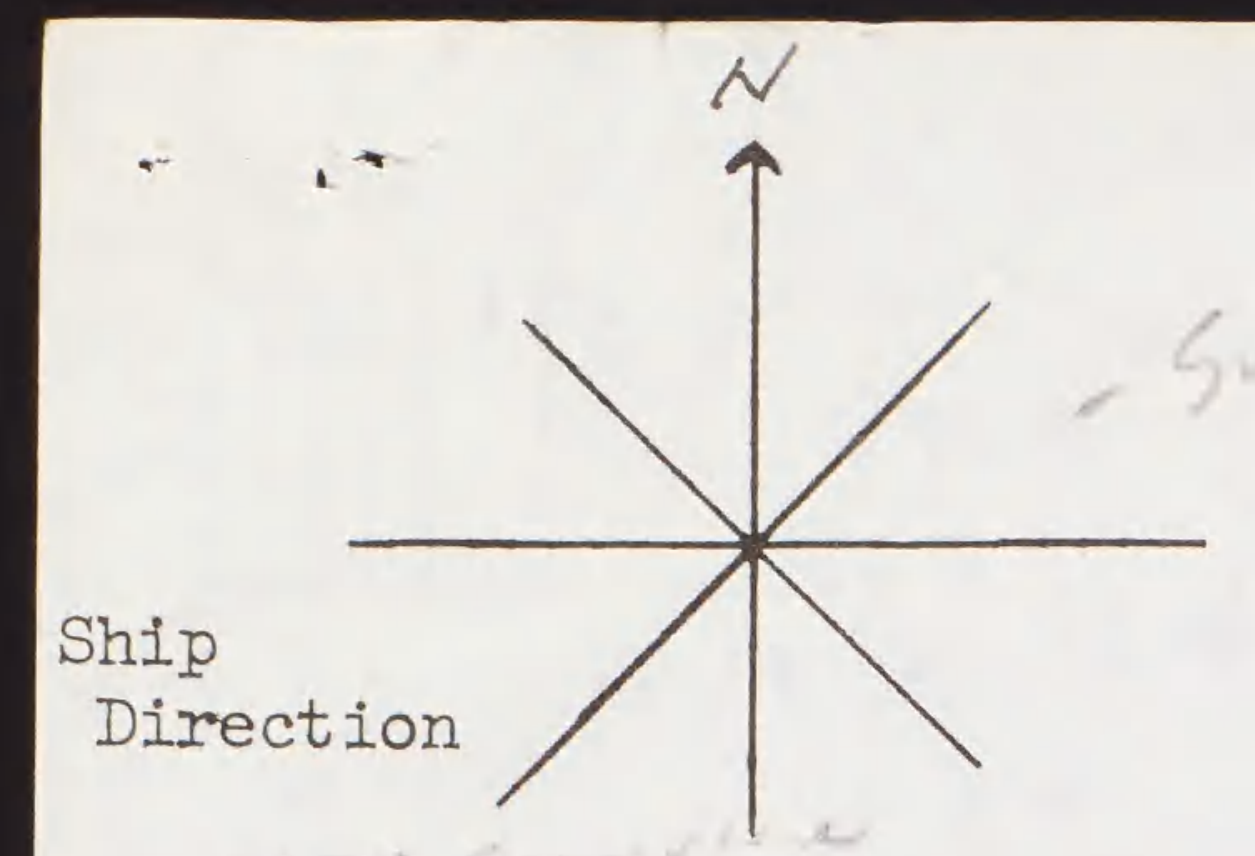

OBSERVERS :

SMITHSONIAN INSTITUTION DIVISION OF BIRDS AT SEA DAILY LOG - E

SPEC IMEN

or

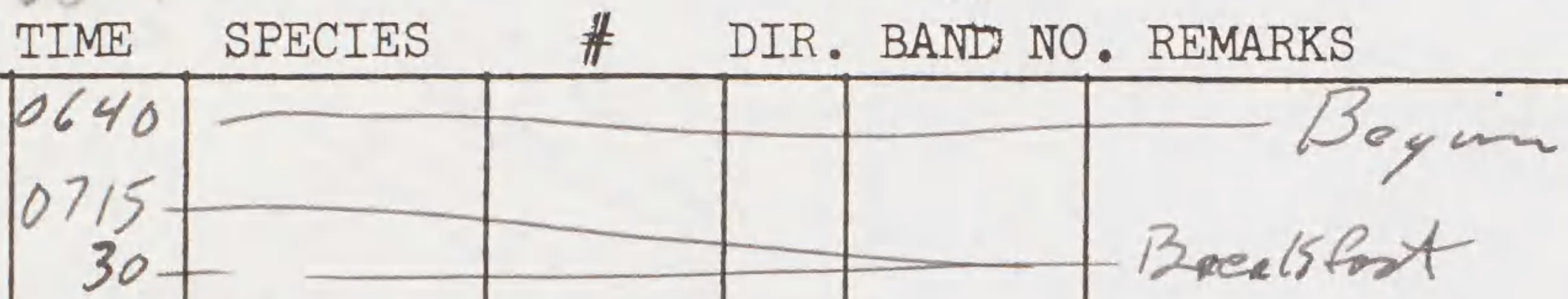
48 Colluredpot

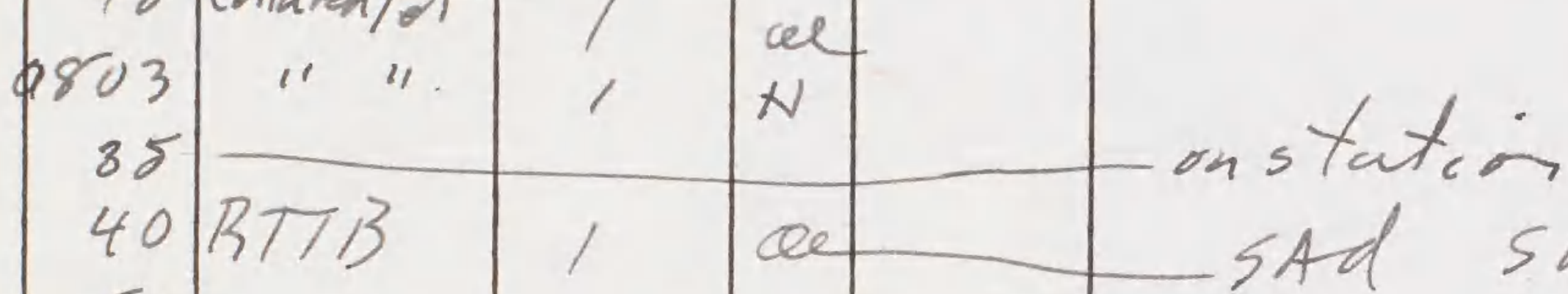
50
$\$ 204$ colland Pex
18 Sheurlet
1055
1310
Kromadic At!
1412 wf SP
1435
50
$15 / 3$

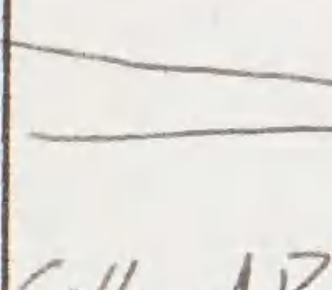
$1 \quad 5$
sAd shat at hent didist Depit stition
(1)

Date 3 Sent 67

Pg.\# 


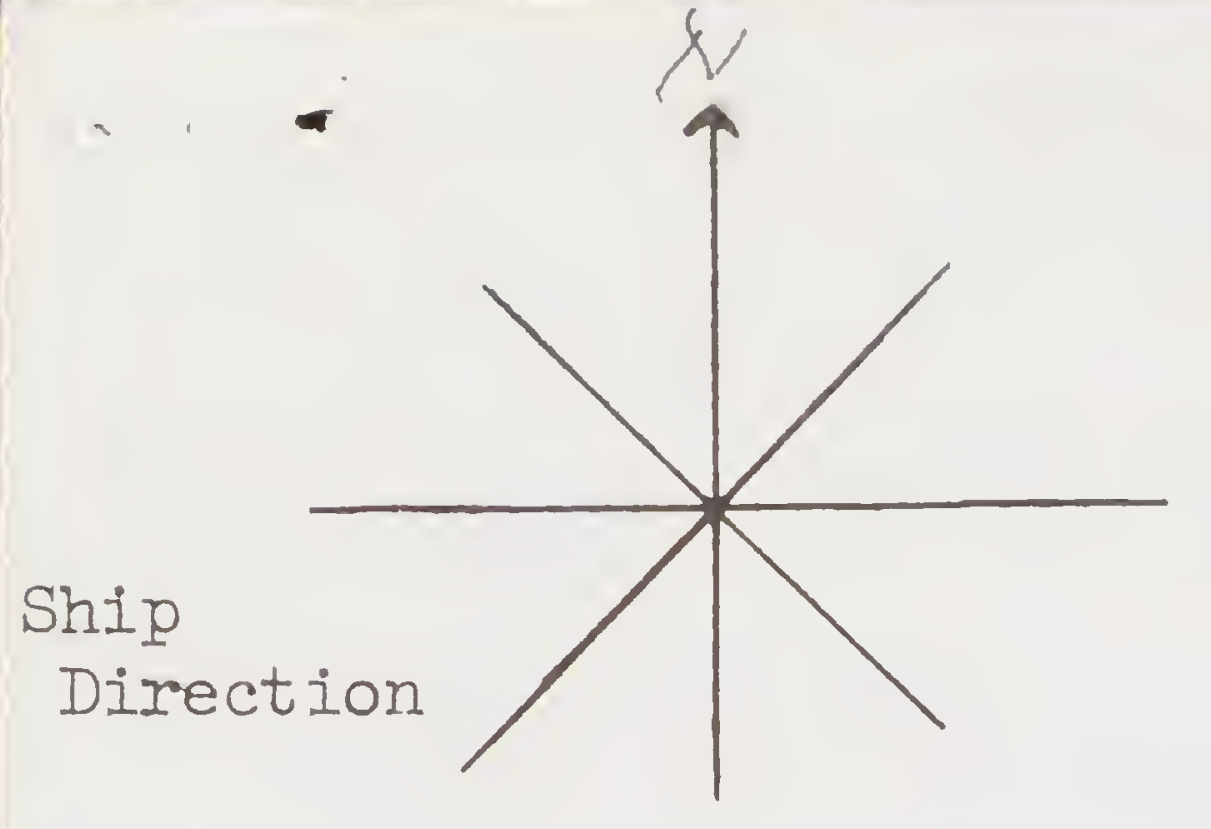

SMITHSONIAN INSTITUTION

DIVISION OF BIRDS

AT SEA DAILY LOG - E

SPECIMEN $/ V$ or tum at

OBSERVERS:

or

Date 3 Sente?

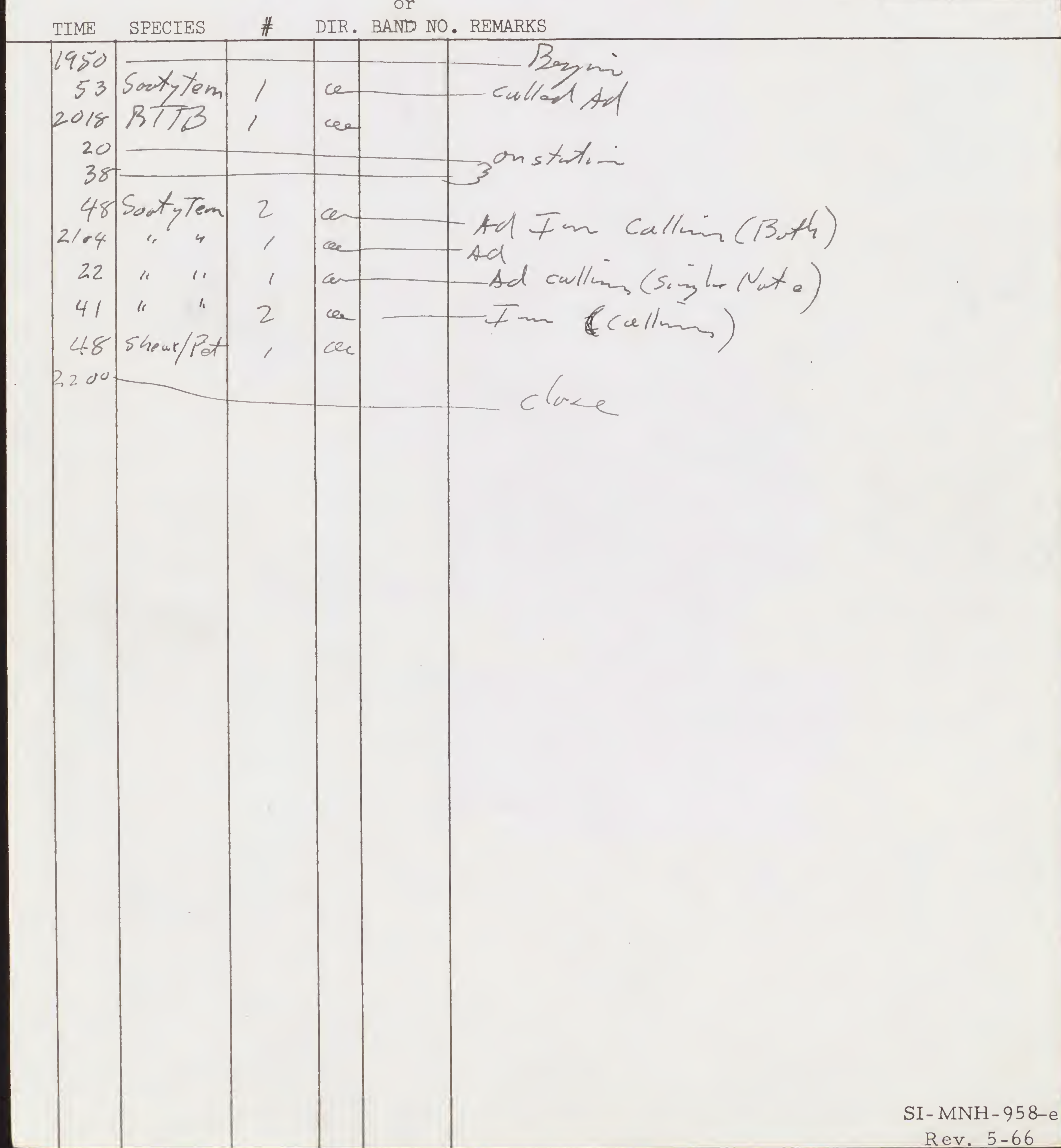




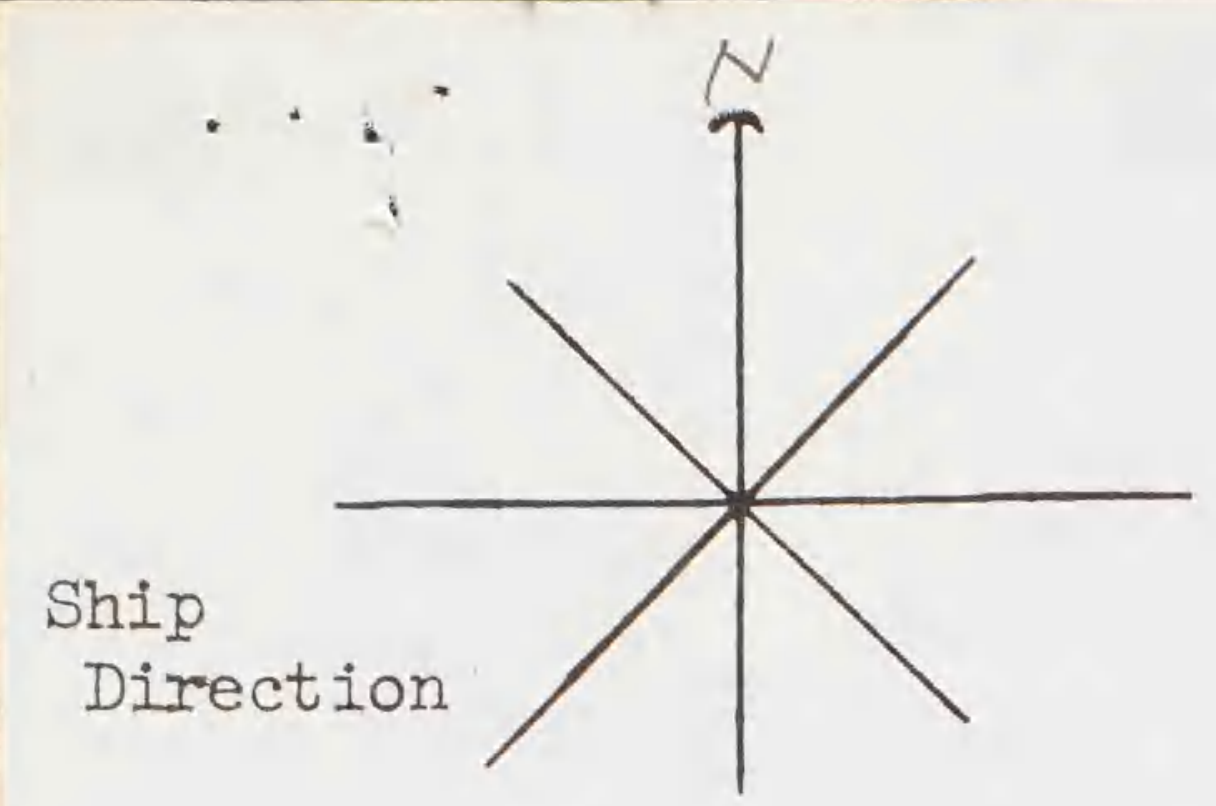

OBSERVERS :

SMITHSONIAN INSTITUTION DIVISION OF BIRDS AT SEA DAILY LOG - E

SPEC LIEN

or

$$
\text { Date } 4 \operatorname{sep} x<7
$$

$\mathrm{Pg} \cdot$ \#

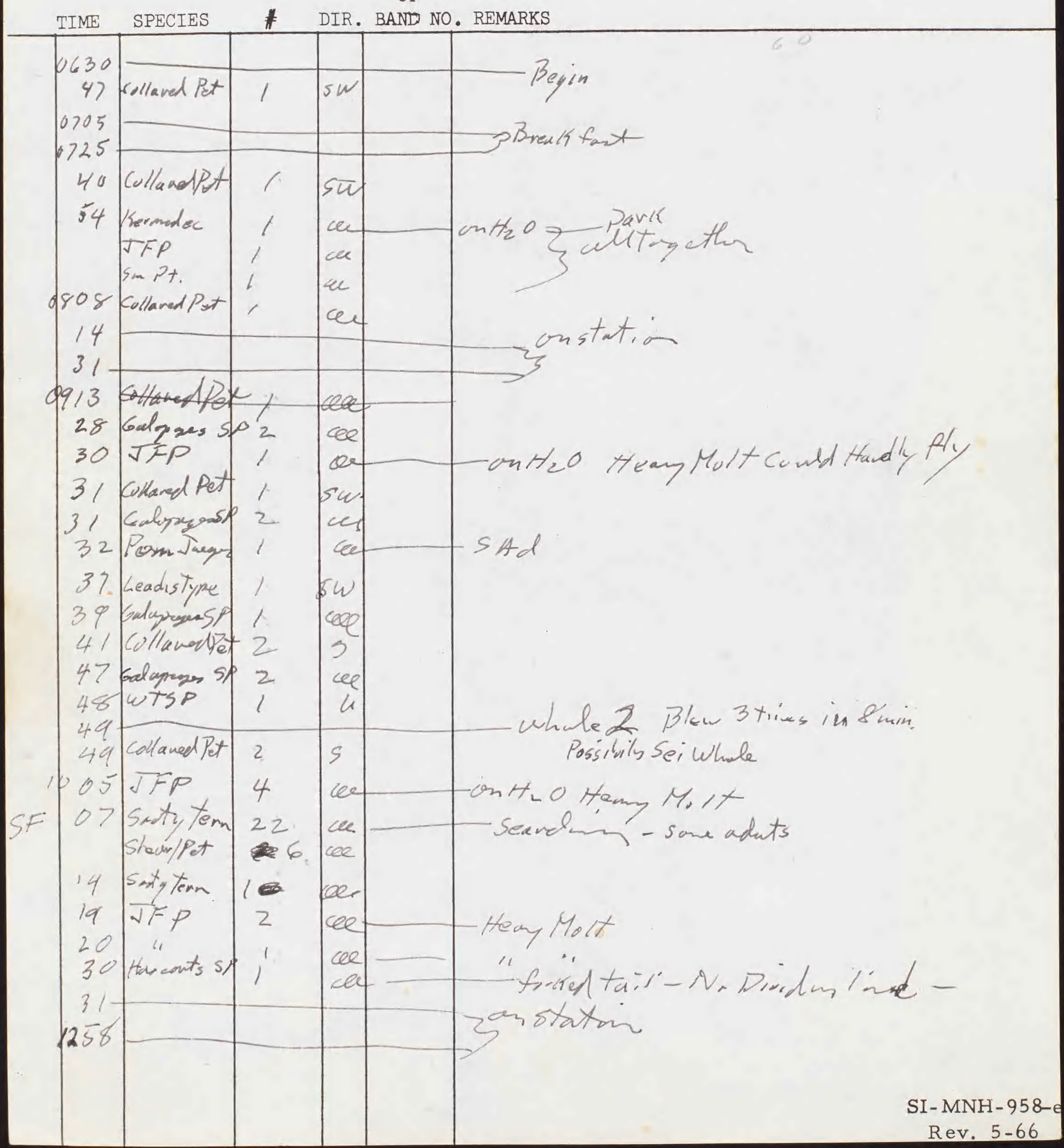




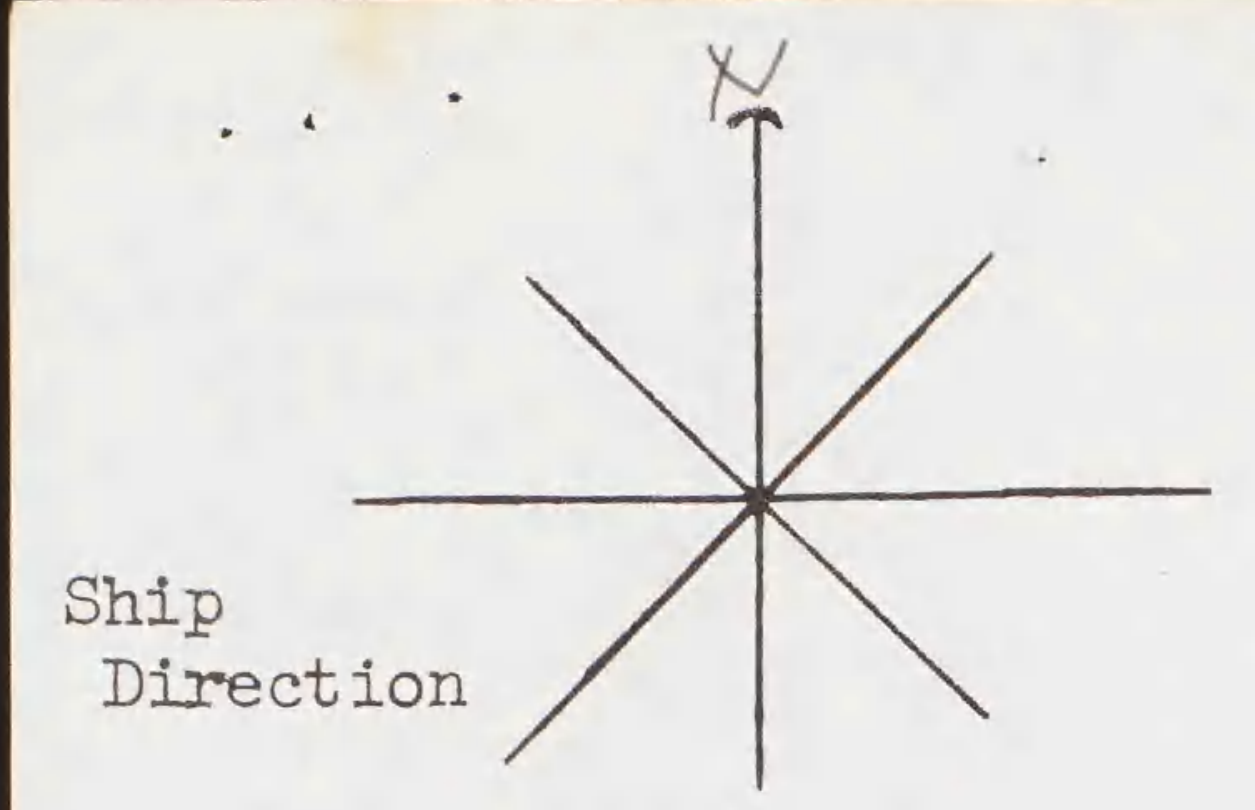

OBSERVERS :

SMITHSONIAN INSTITUTION

DIVISION OF BIRDS

AT SEA DAILY LOG - E

SPEC IMEN

or

Date $\frac{4 \sec 7 \cdot 67}{2}$

TIME SPECIES \# DIR. BAND NO. REMARKS

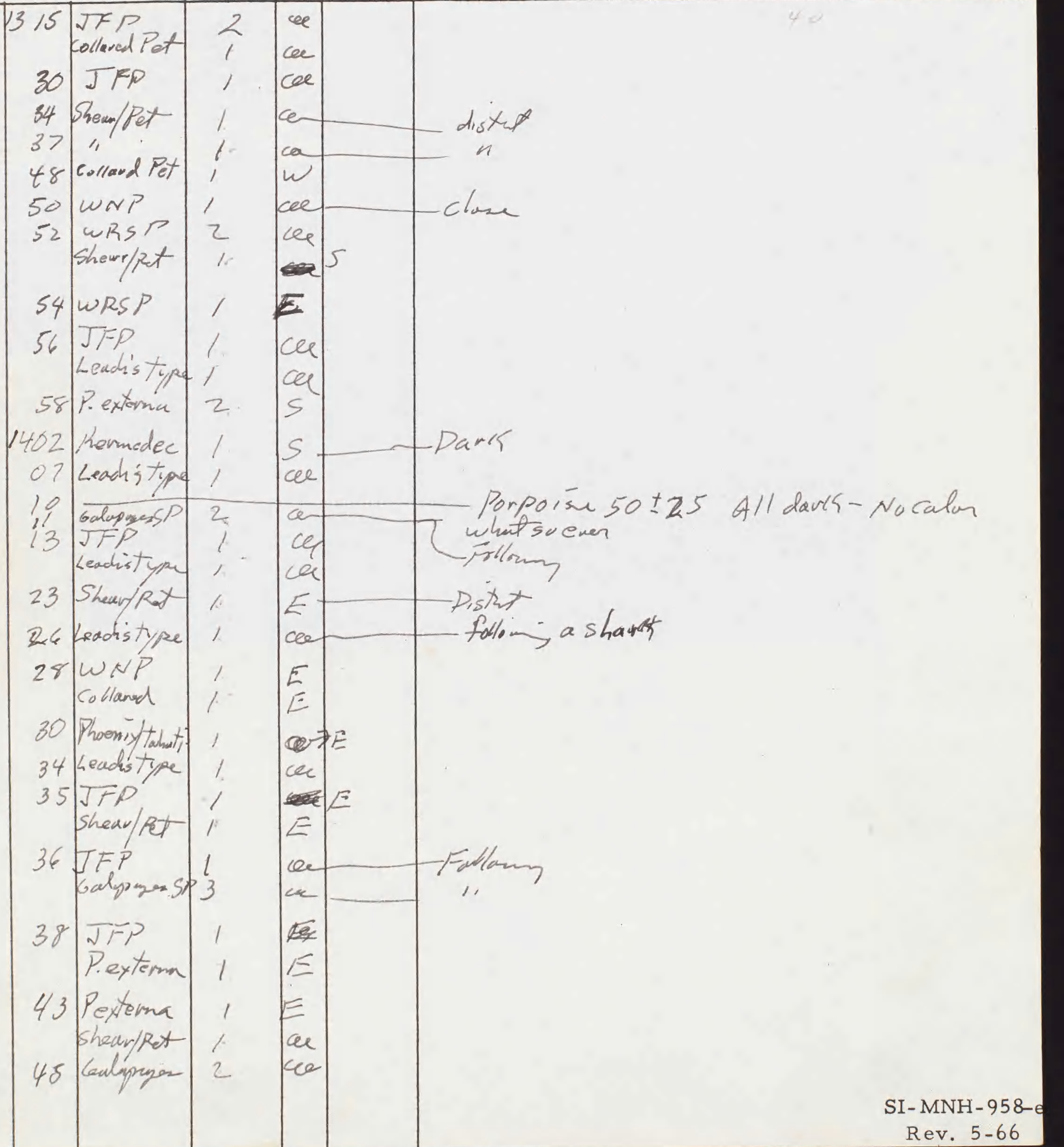

Rev. 5-66 


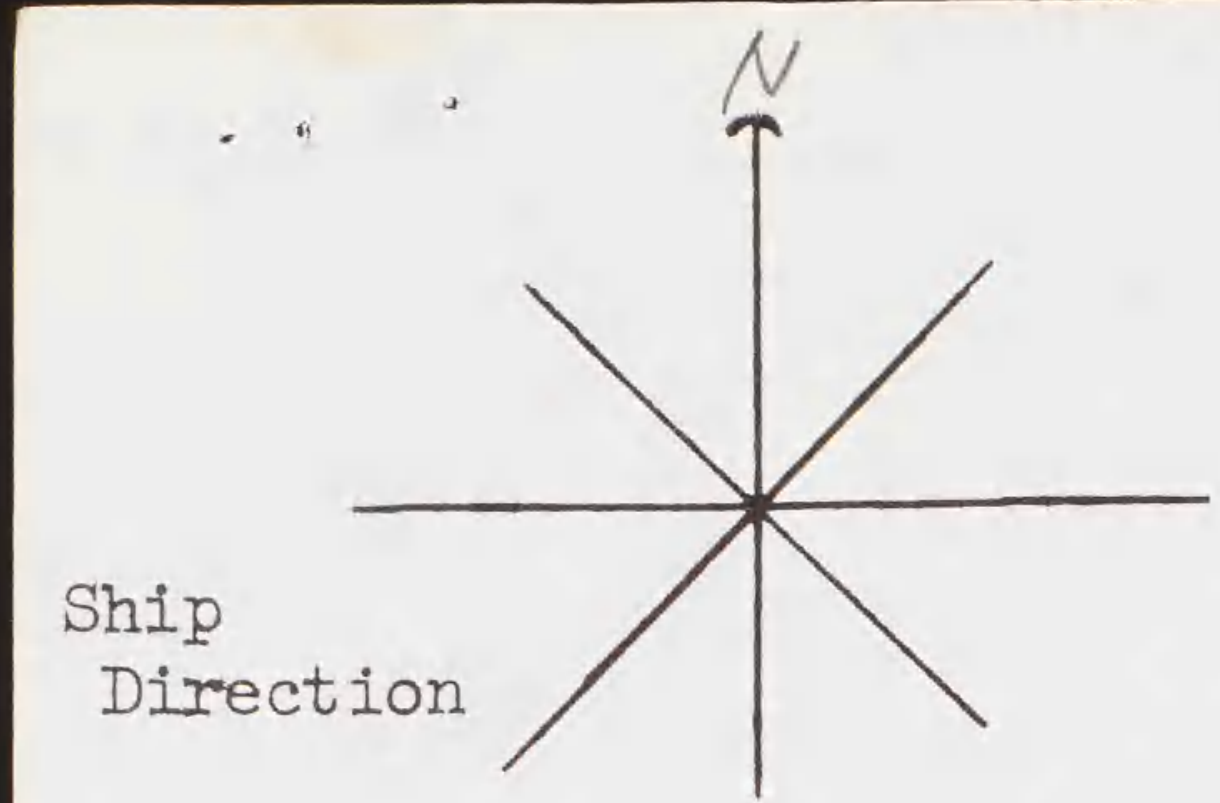

OBSERVERS :

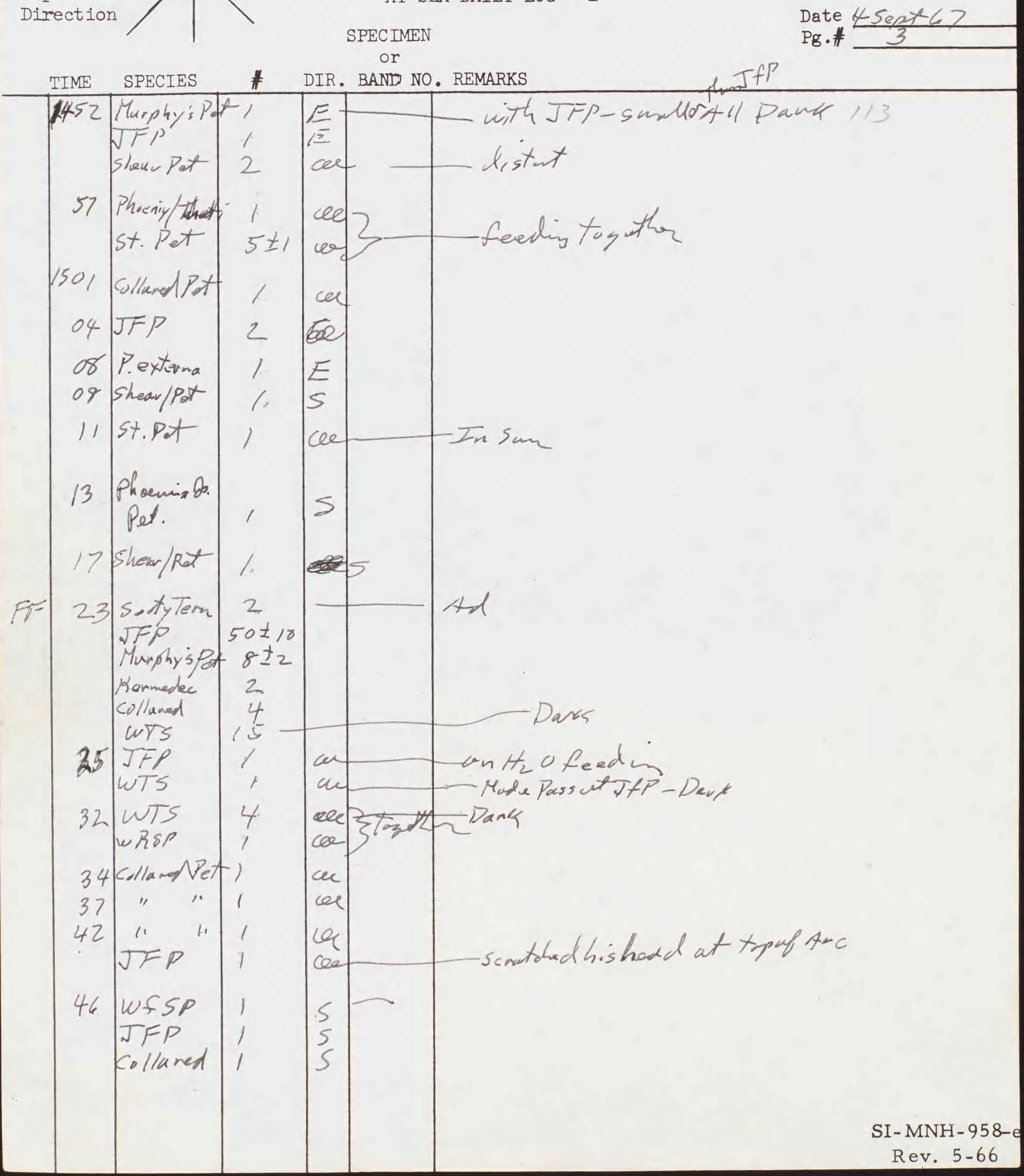




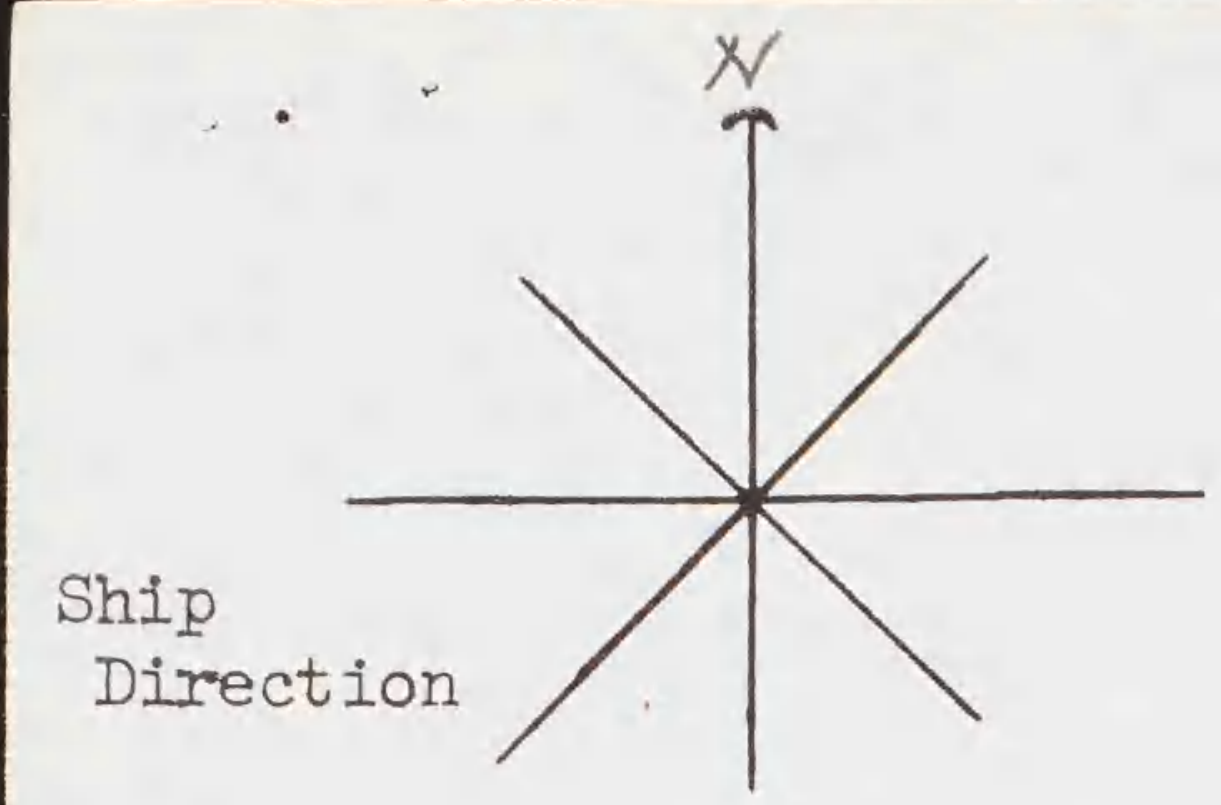

SMITHSONIAN INSTITUTION DIVISION OF BIRDS AT SEA DAILY LOG - E

SPEC IMEN or OBSERVERS :

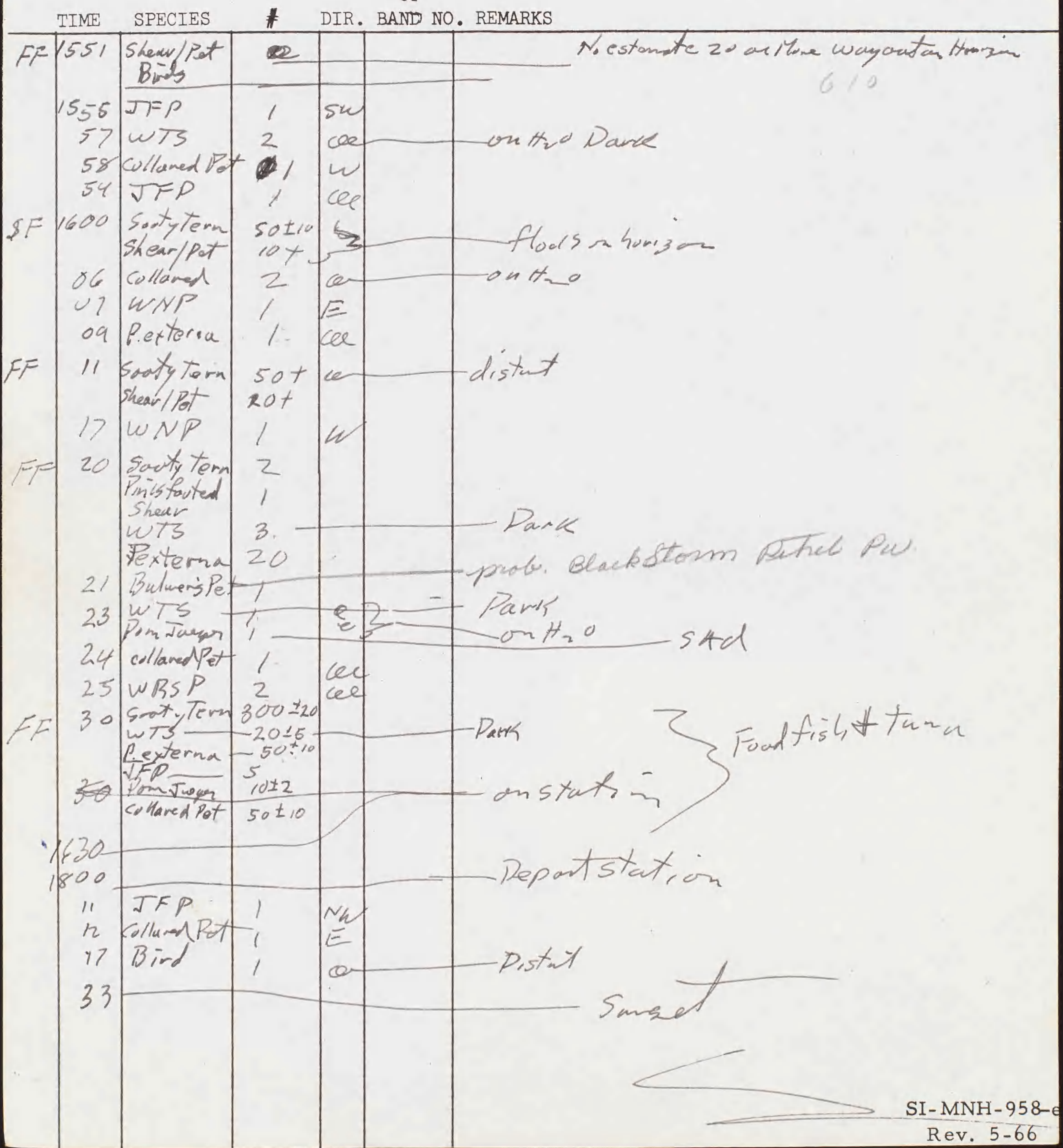




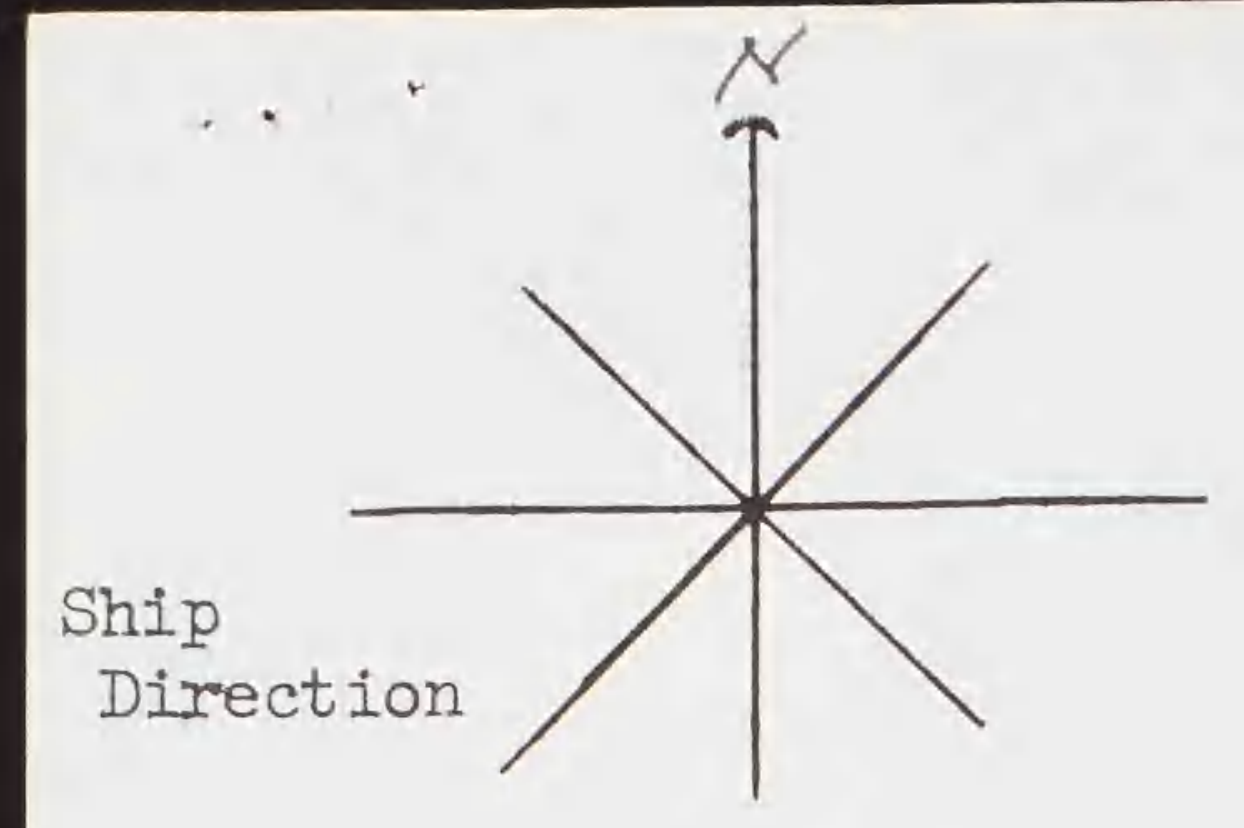

OBSERVERS :

SMITHSONIAN INSTITUTION DIVISION OF BIRDS AT SEA DAILY LOG - E SPECIMEN Nocturnal Date $4 \operatorname{sen} x 67$ or

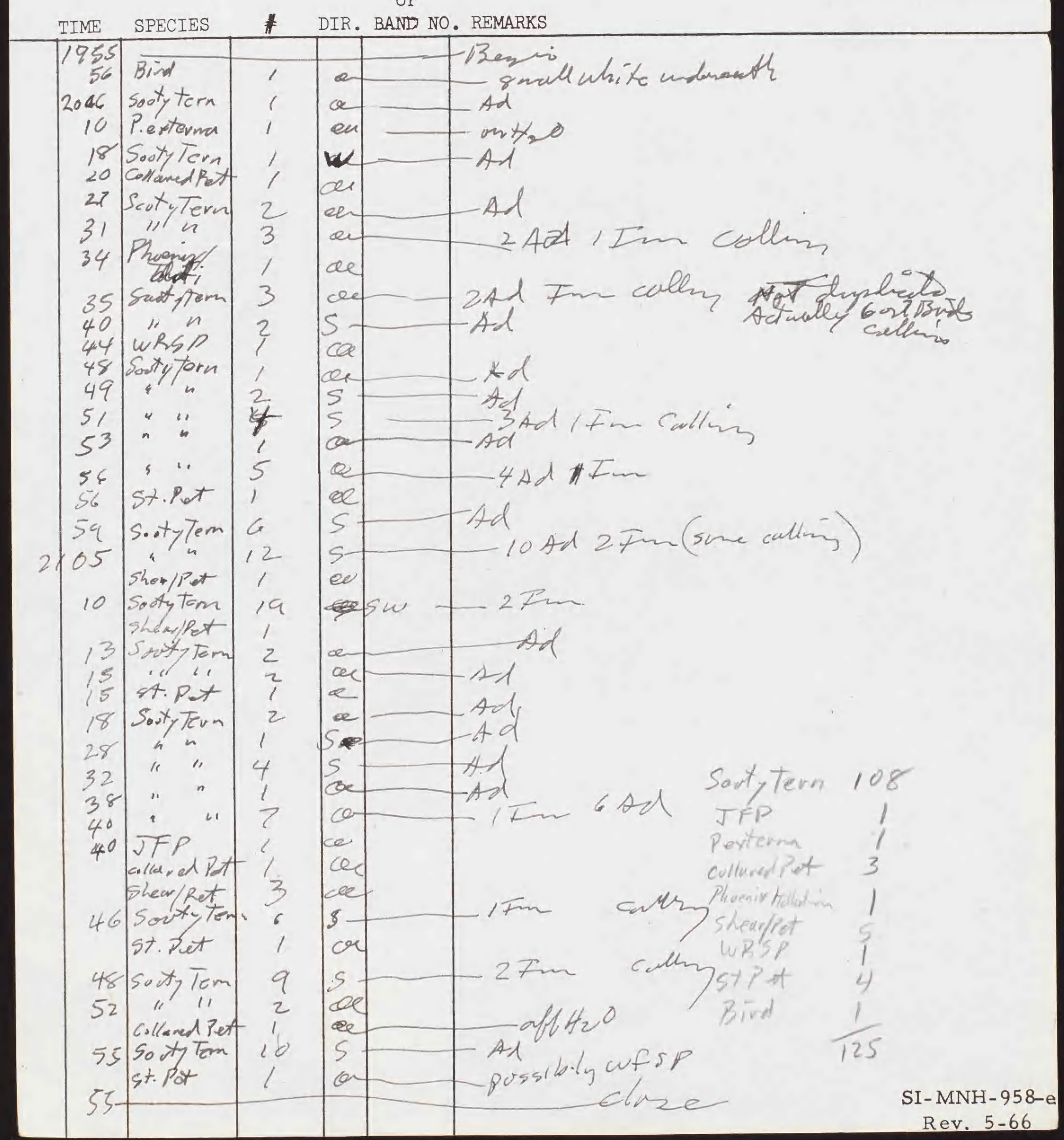




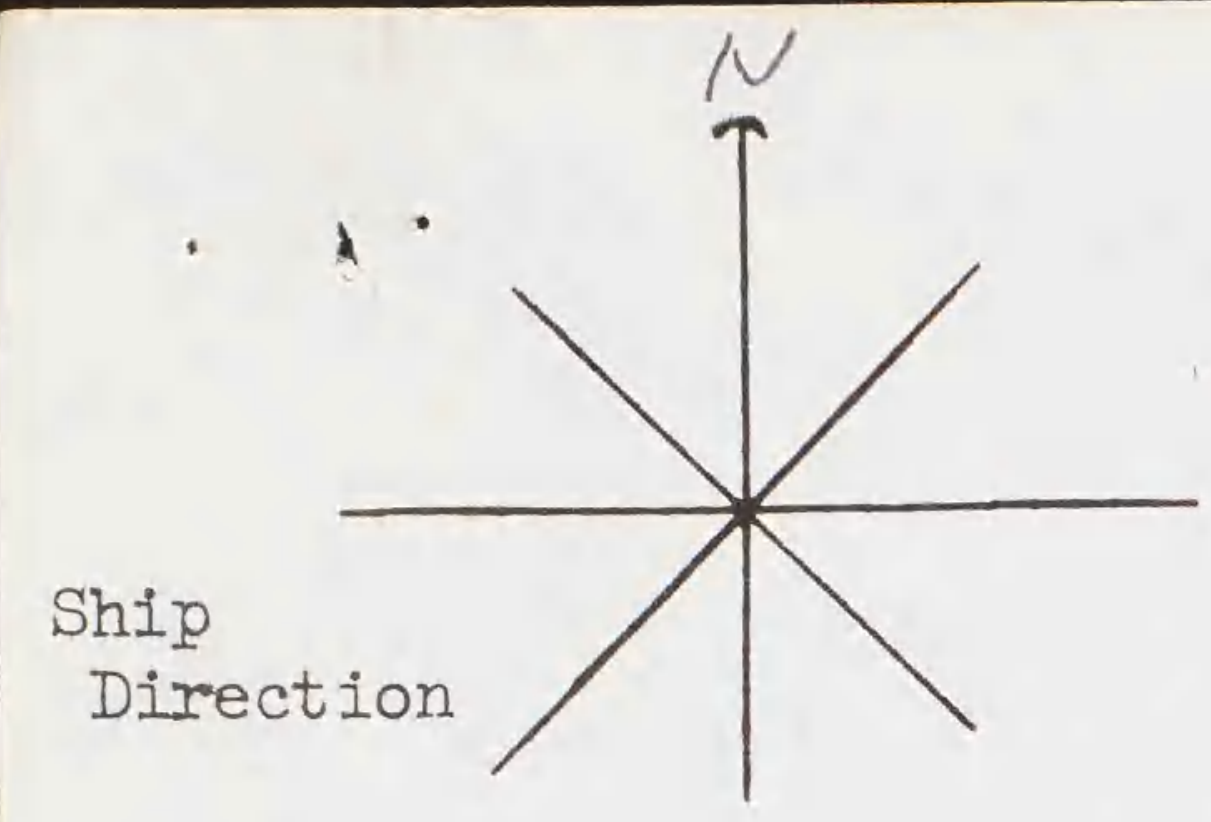

SMITHSONIAN INSTITUTION

DIVISION OF BIRDS

AT SEA DAILY LOG - E

OBSERVERS :

SPECIMEN

or
Date $\frac{5 \operatorname{sep} 467}{1}$

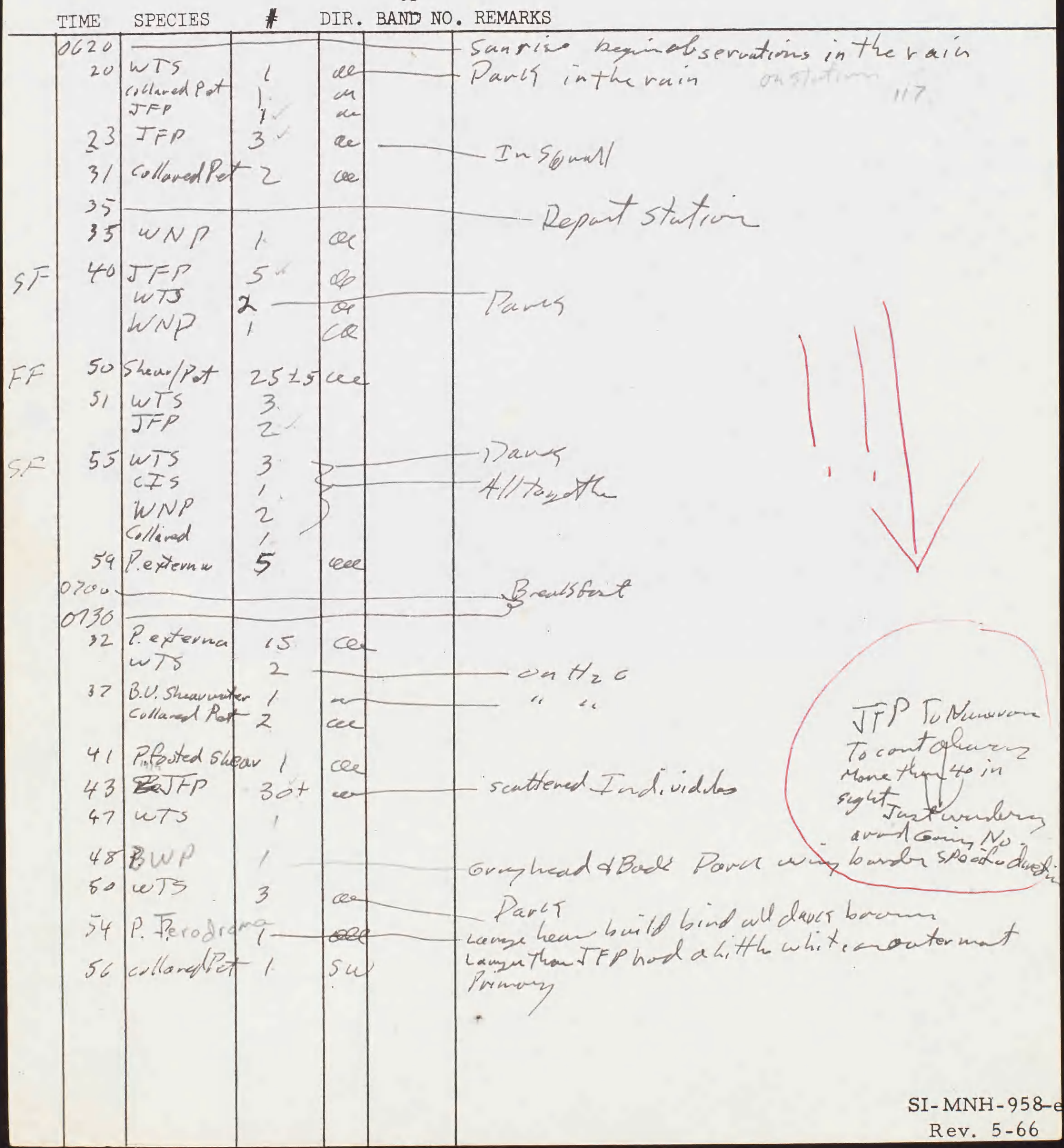




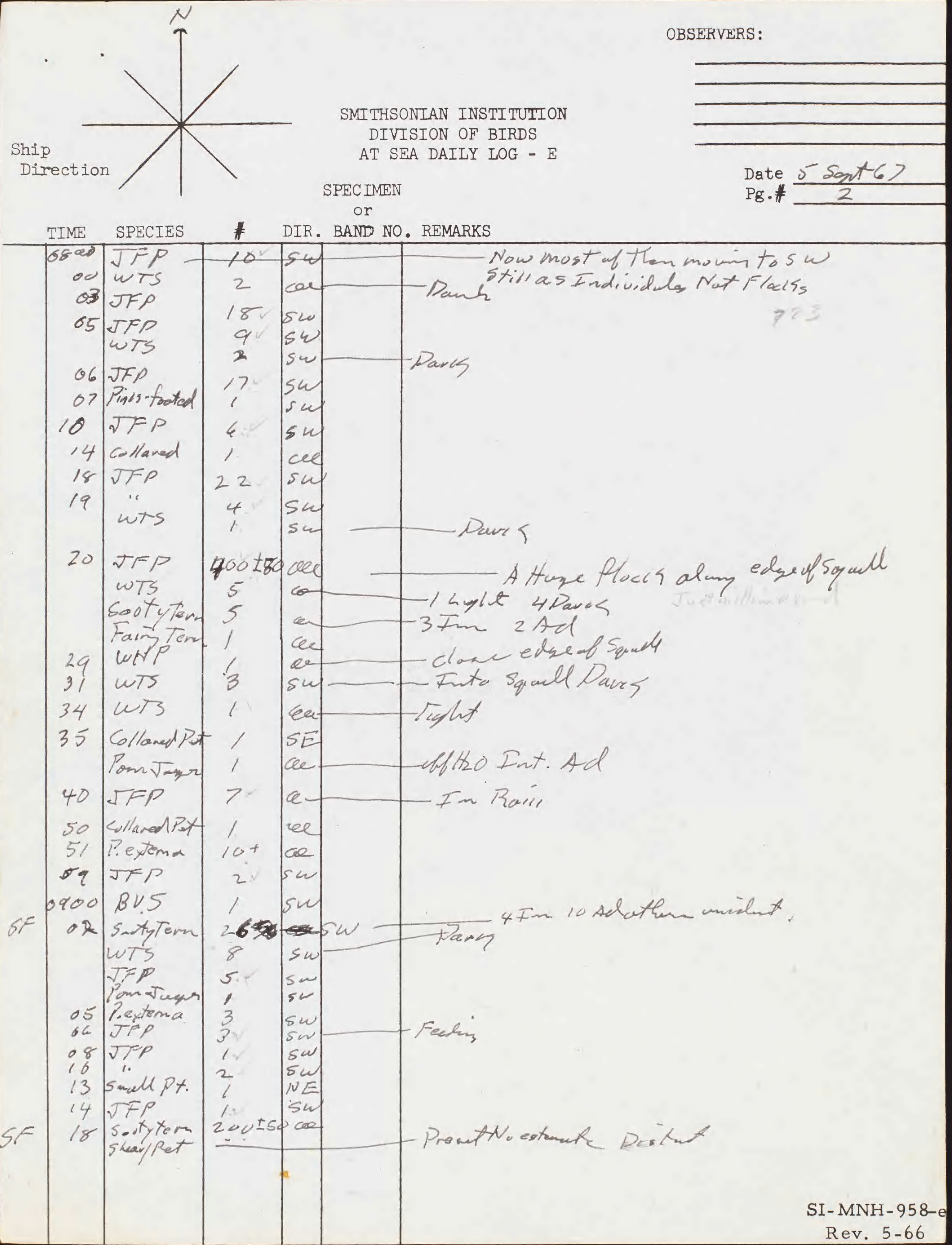




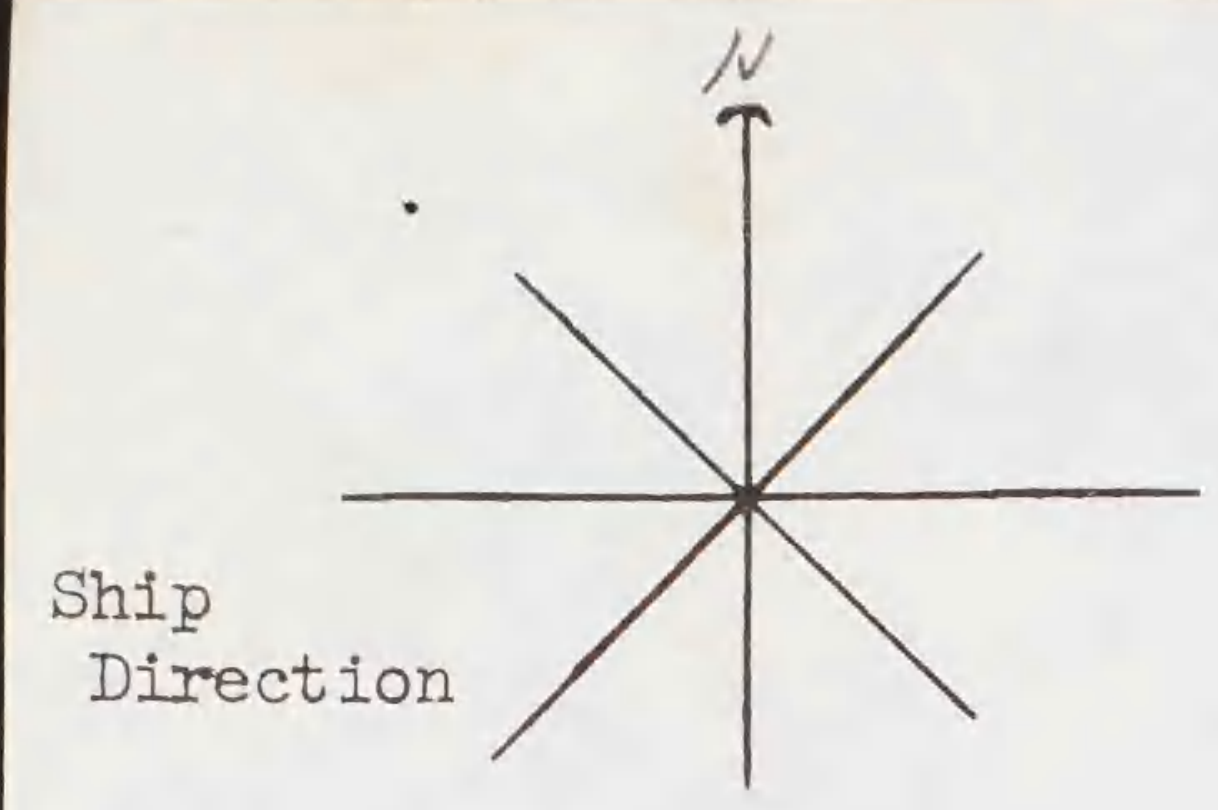

SMITHSONIAN INSTITUTION

DIVISION OF BIRDS

AT SEA DAILY LOG - E

OBSERVERS :

SPEC IMEN

or
Date
$\mathrm{Pg} . \# \frac{5 \operatorname{sen} t}{3}<7$

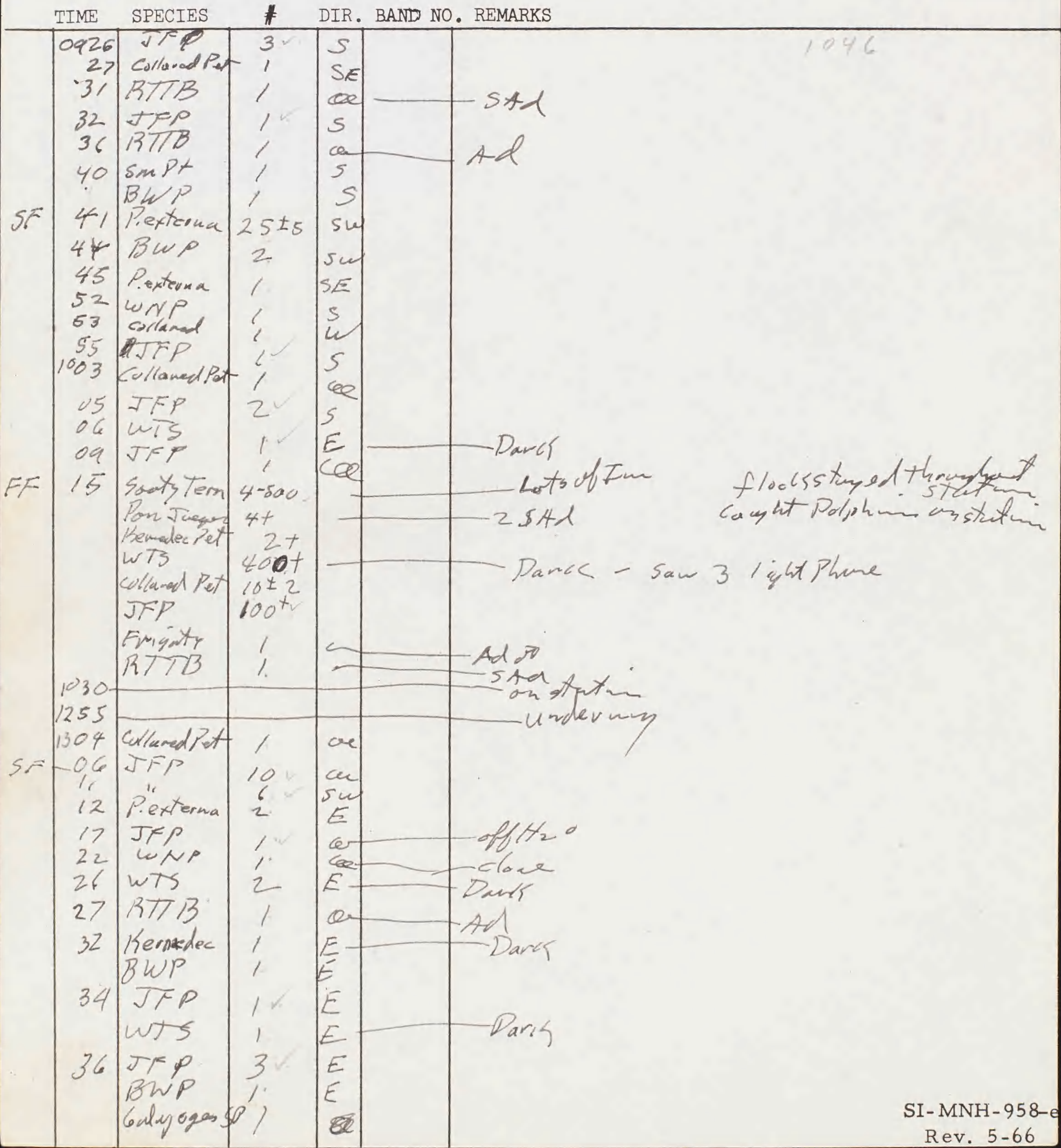




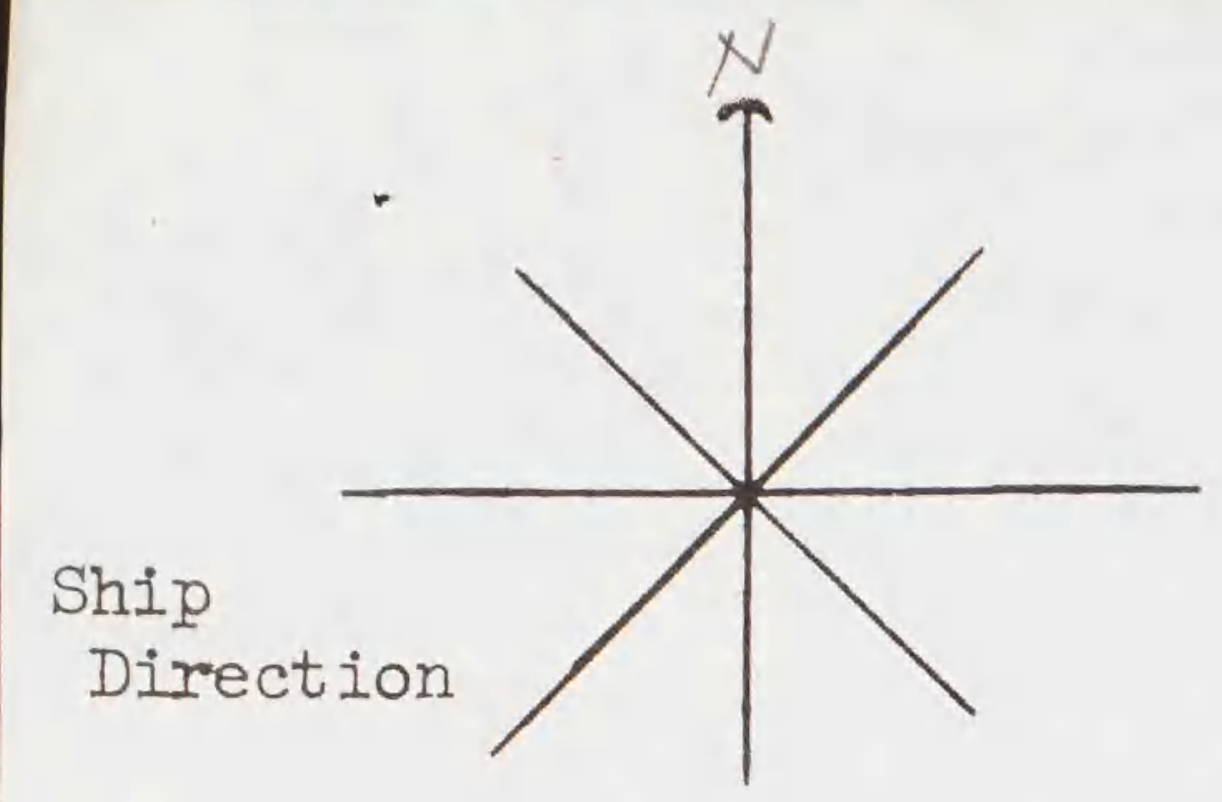

OBSERVERS :

SMITHSONIAN INSTITUTION

DIVISION OF BIRDS

AT SEA DAILY LOG - E

SPEC MEN

or

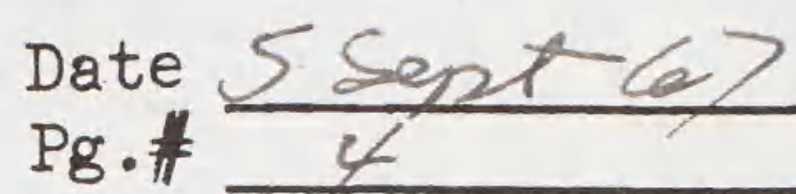

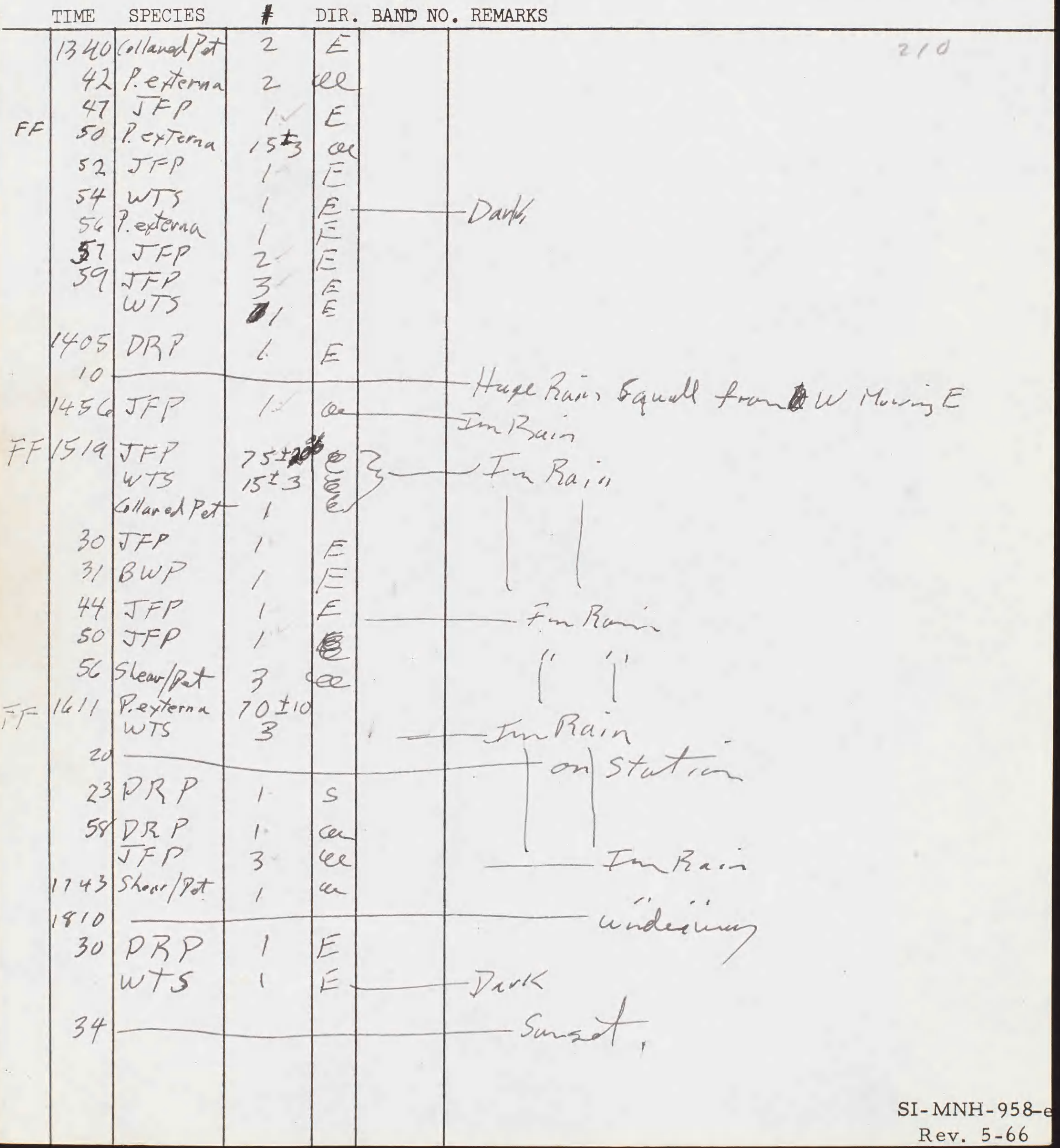




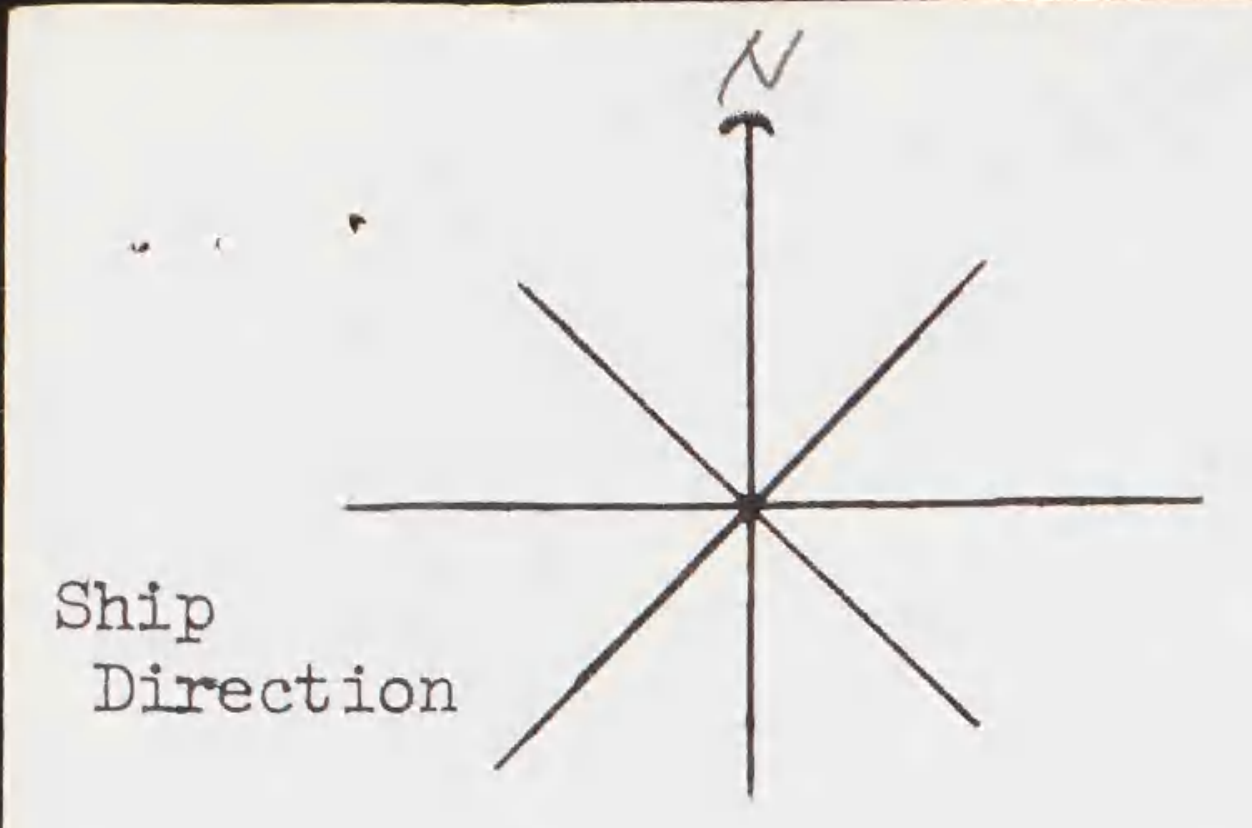

OBSERVERS :

SMITHSONIAN INSTITUTION DIVISION OF BIRDS

AT SEA DAILY LOG - ${ }^{\mathrm{E}}$

NoCIMEN No ta and

or

Date sent 67

DIR. BAND NO. REMARKS

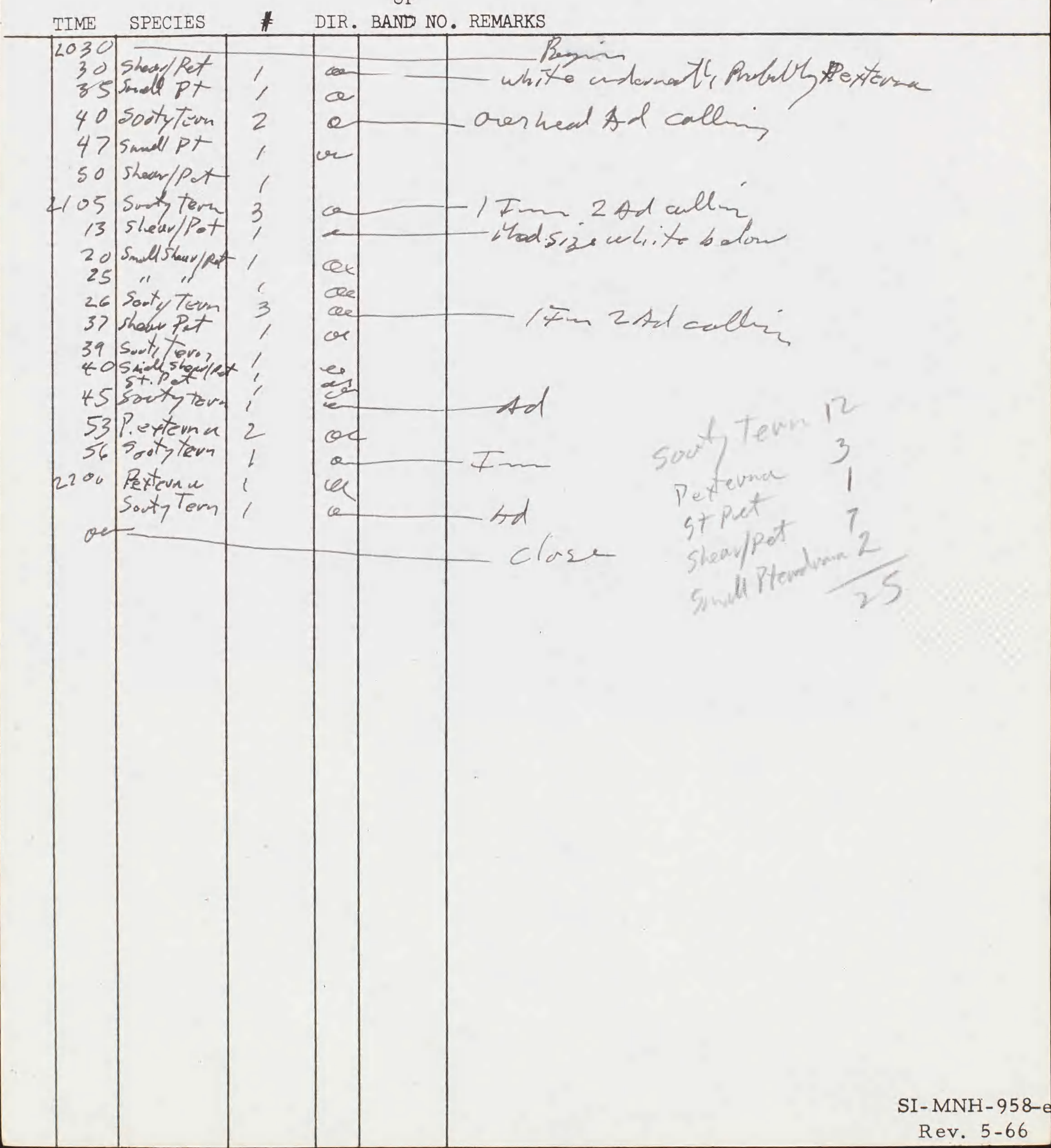




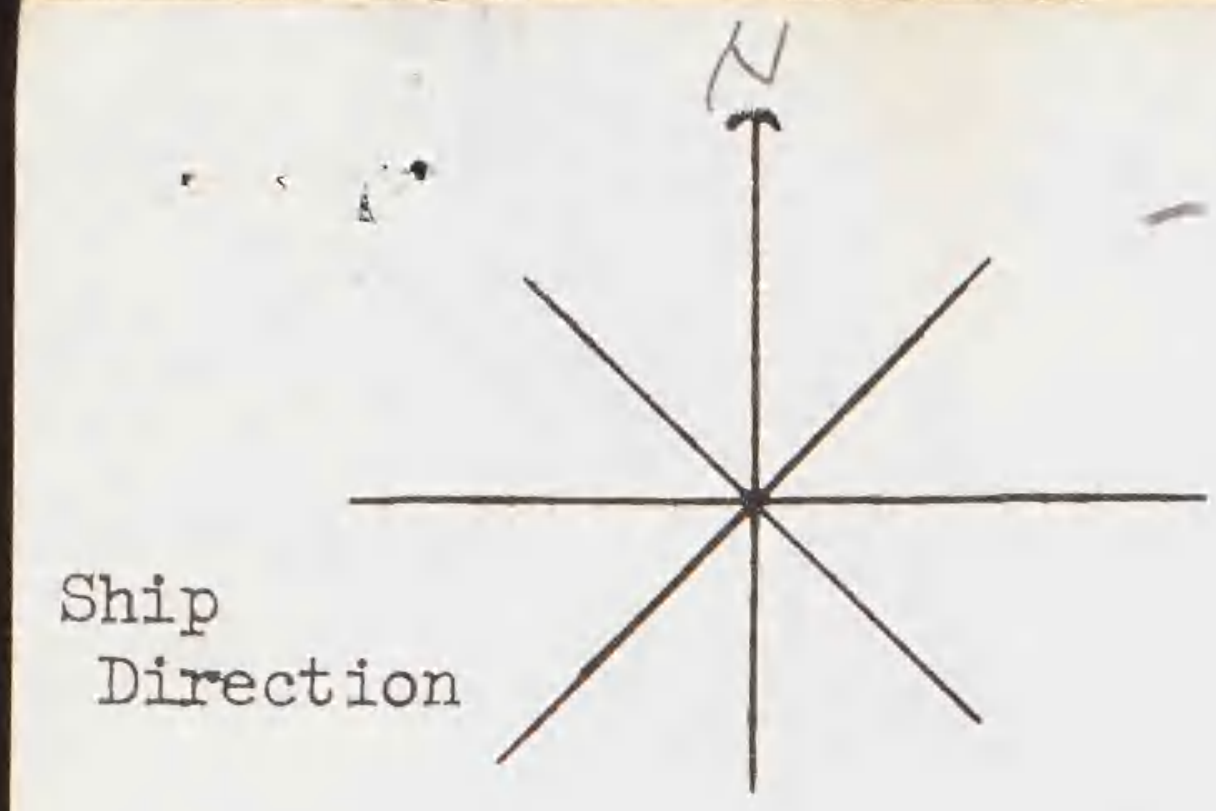

SMITHSONIAN INSTITUTION DIVISION OF BIRDS AT SEA DAILY LOG - E

OBSERVERS :

SPECIMEN or
5. 8 hr

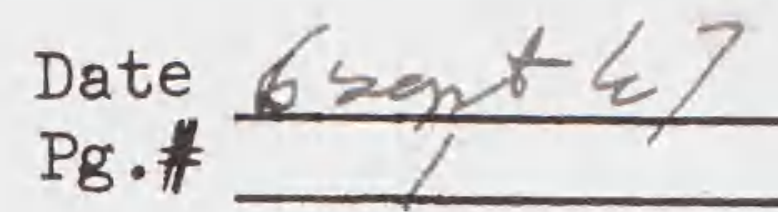

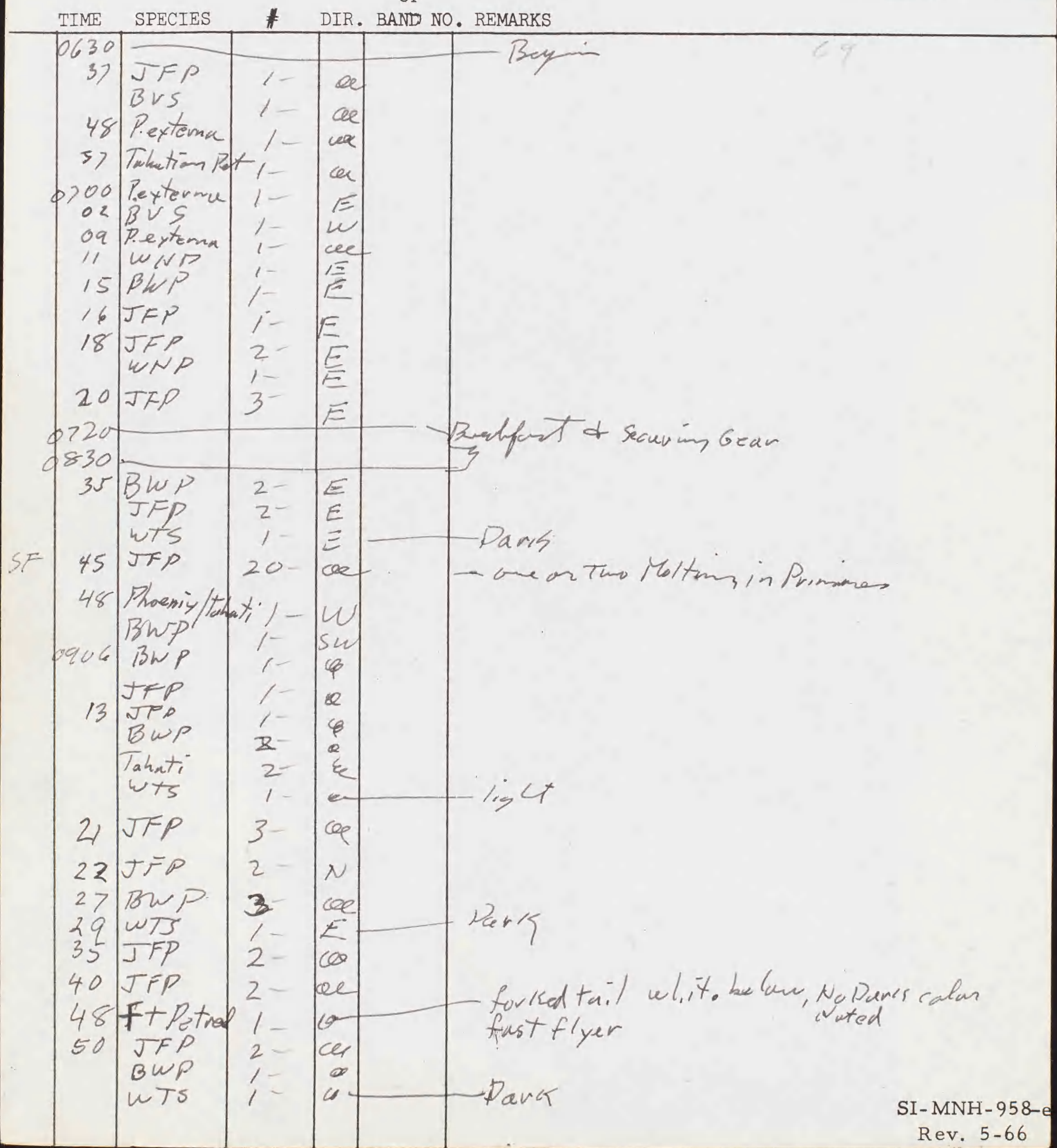




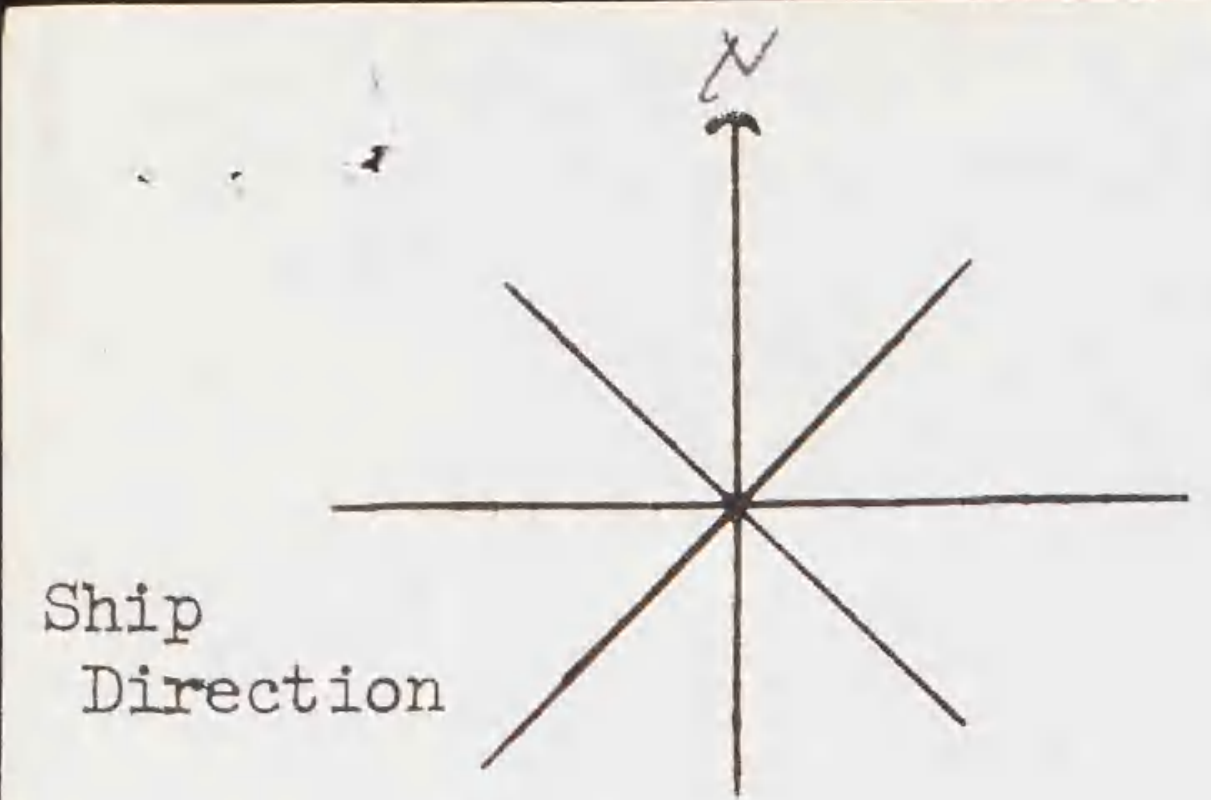

OBSERVERS :

SMITHSONIAN INSTITUTION DIVISION OF BIRDS AT SEA DAILY LOG - E

SPECIMEN

or
Date 65 ent 67

$\mathrm{Pg} \cdot \#$

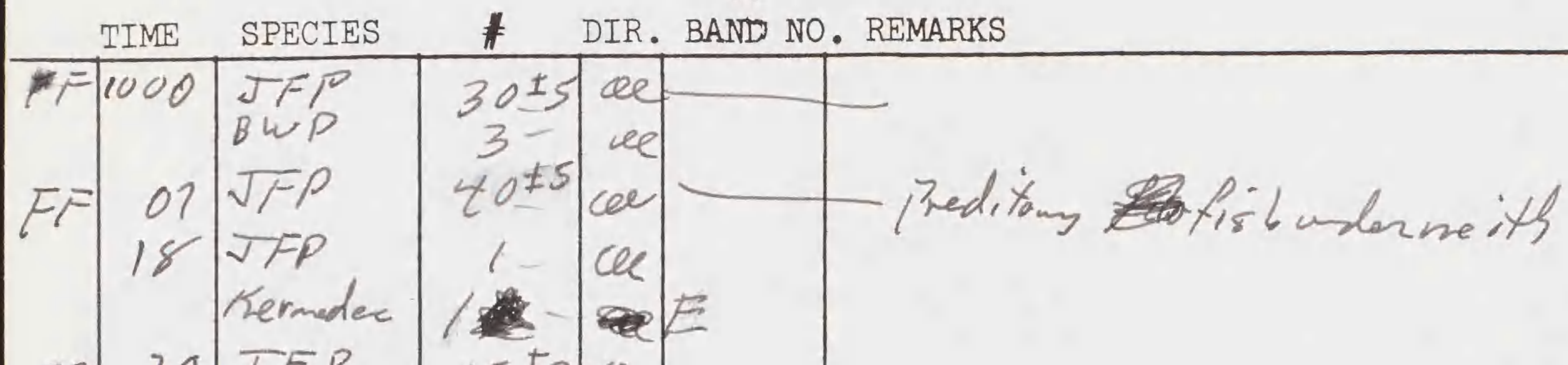

F.

\begin{tabular}{l|l}
20 & TFP \\
21 & BWP \\
WTS
\end{tabular}

\begin{tabular}{c|c}
$15 \pm 3$ & $\varphi$ \\
$2-$ & $\varphi$ \\
$2-$ & $N$
\end{tabular}$\quad \quad$ Dark

1300
430 Bepant Sf ition

430
38

JTFP

47

5) WTS

JFP

\begin{tabular}{l|l}
55 & BWP \\
58 & CIS \\
TFP
\end{tabular}

1503 BVVP

$06 \mathrm{~K}$

15
20
20 JFP
Harconts SA

30 JFA

34 BWP

37 CoLP

\begin{tabular}{r|l} 
प1 & JEP \\
1601 & TFP
\end{tabular}

WTS

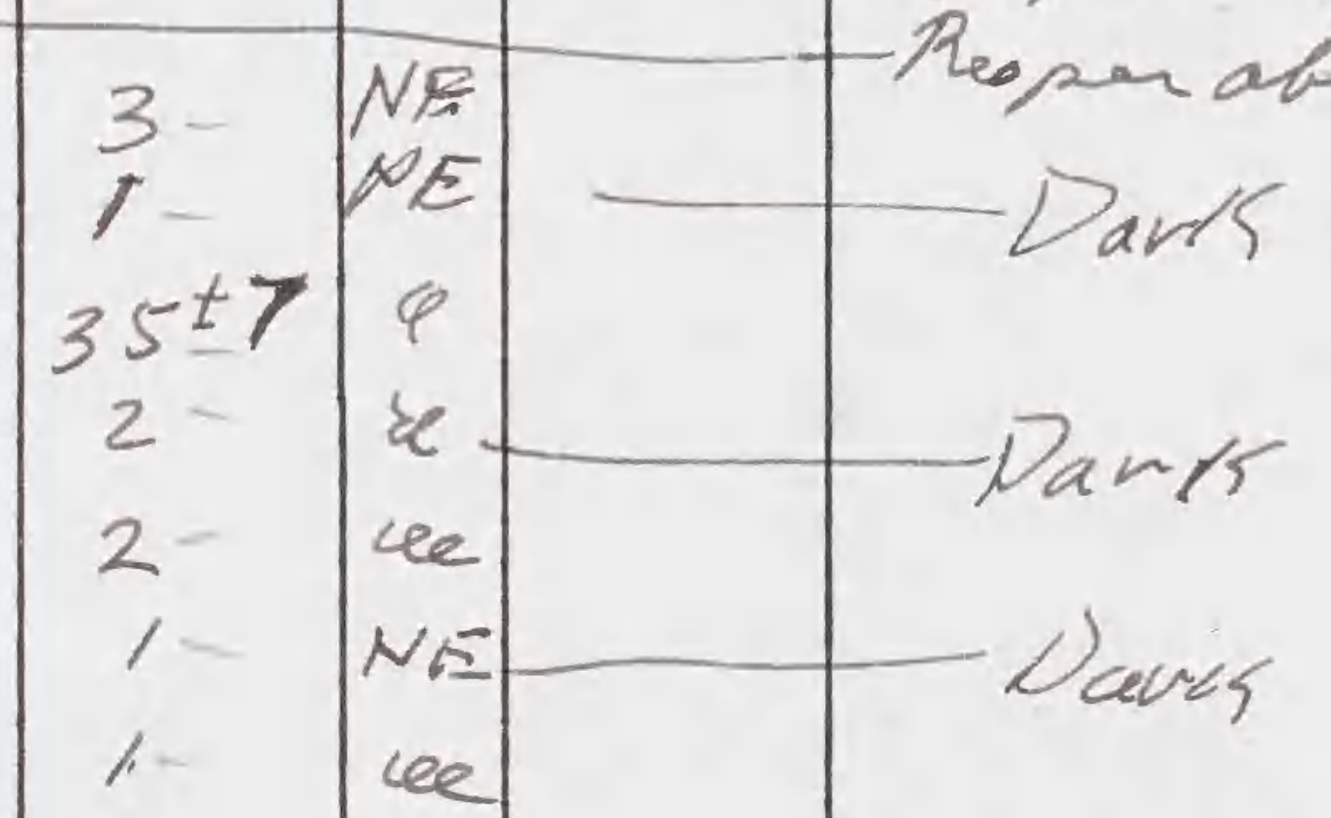

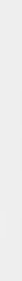

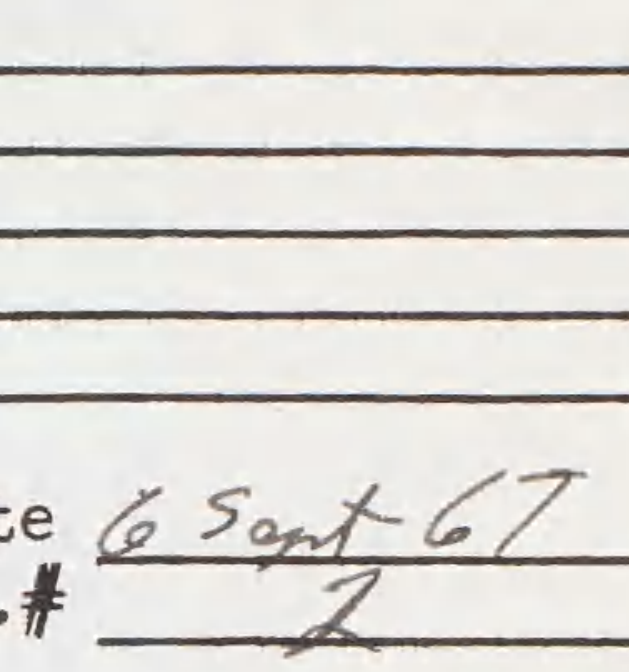




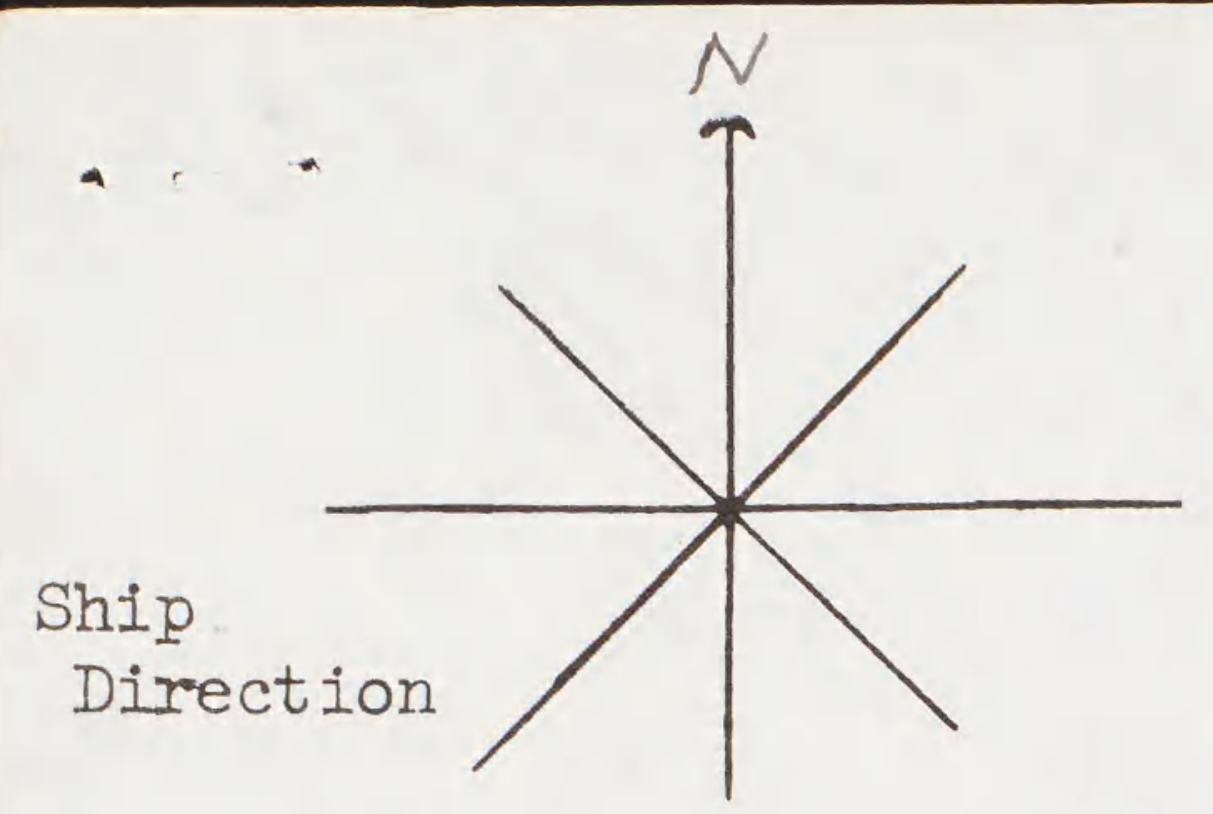

SMITHSONIAN INSTITUTION DIVISION OF BIRDS AT SEA DAILY LOG - E

SPEC MEN

or
OBSERVERS :

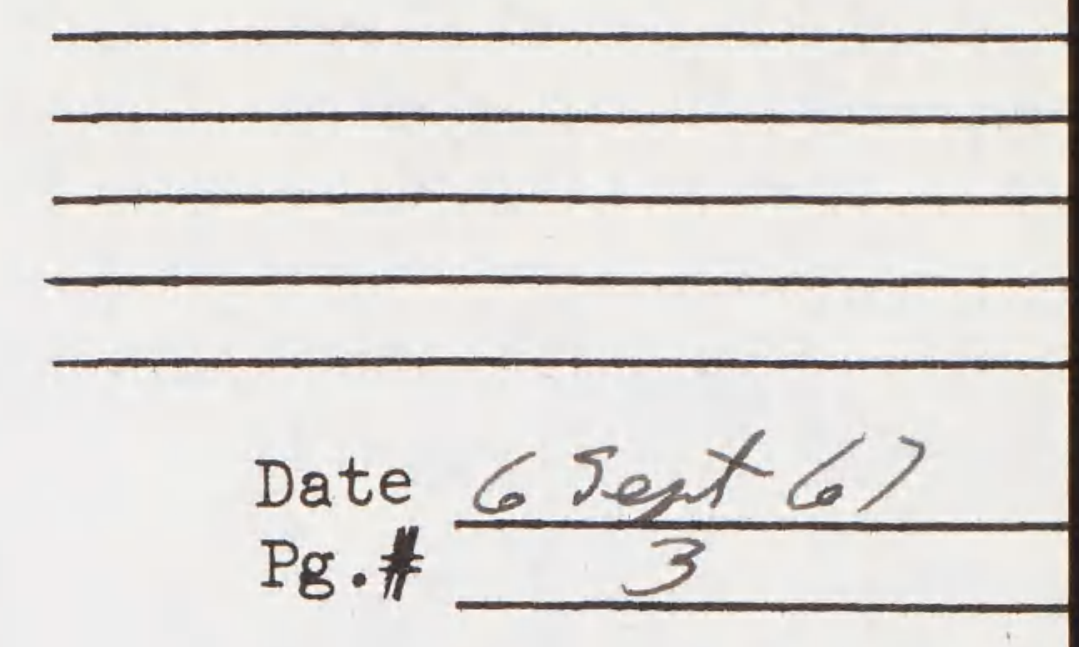

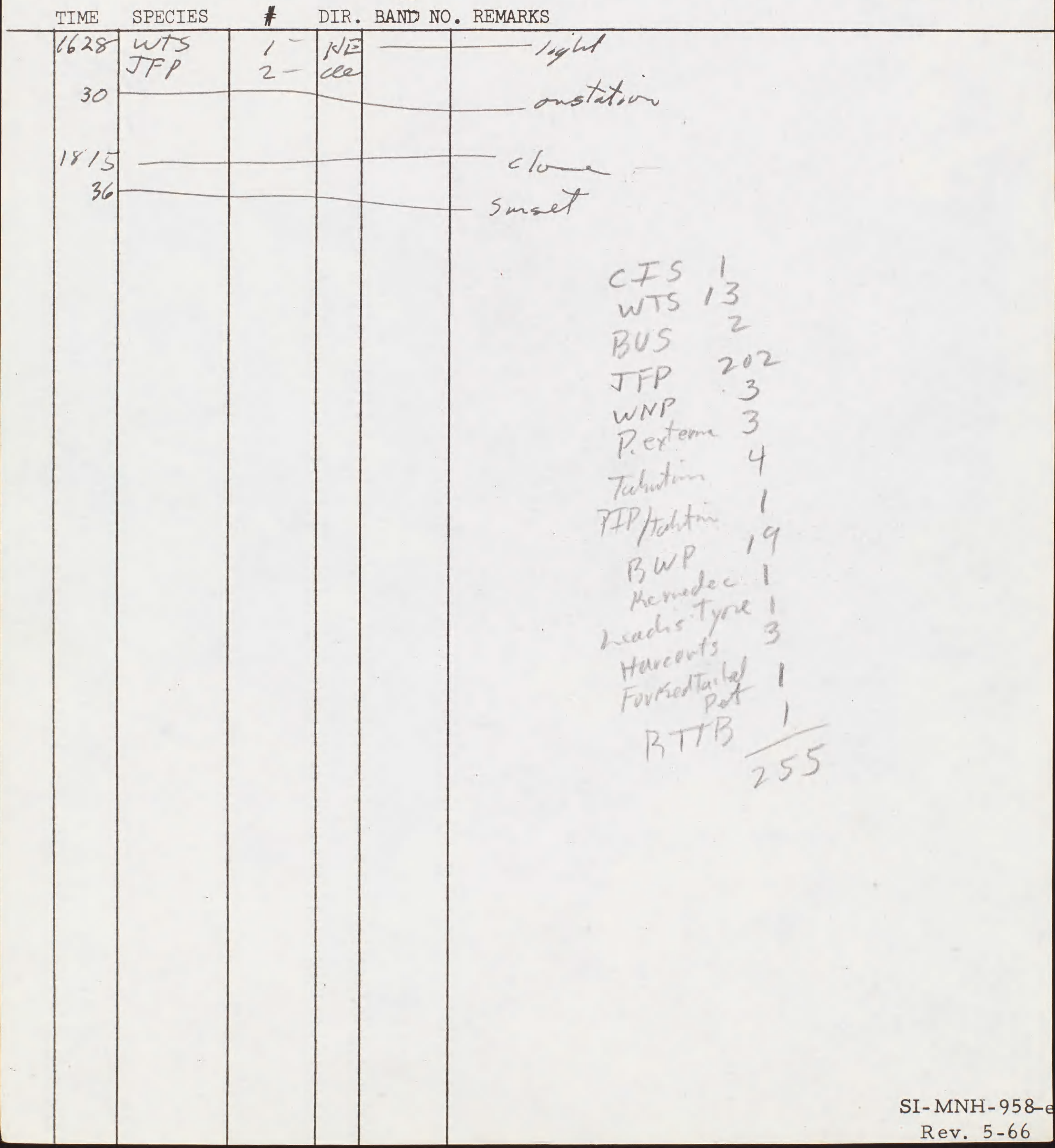




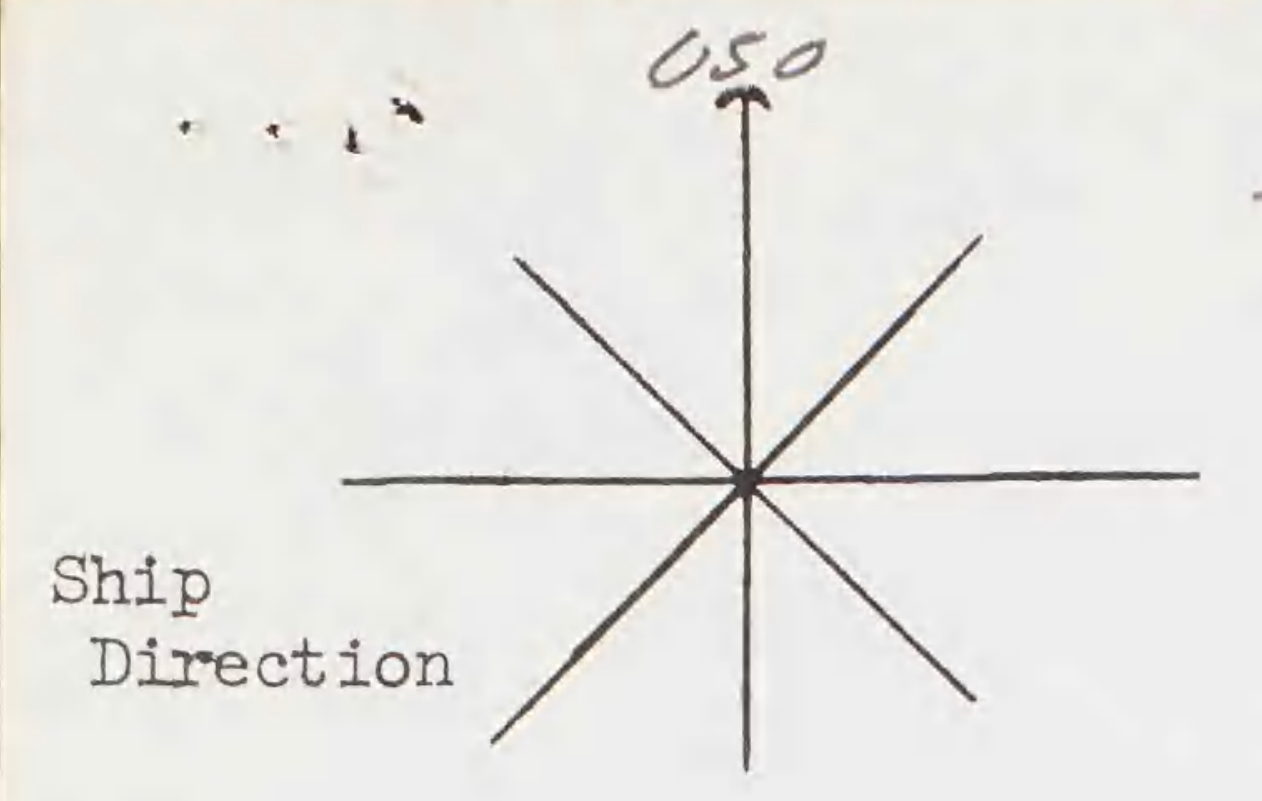

OBSERVERS:

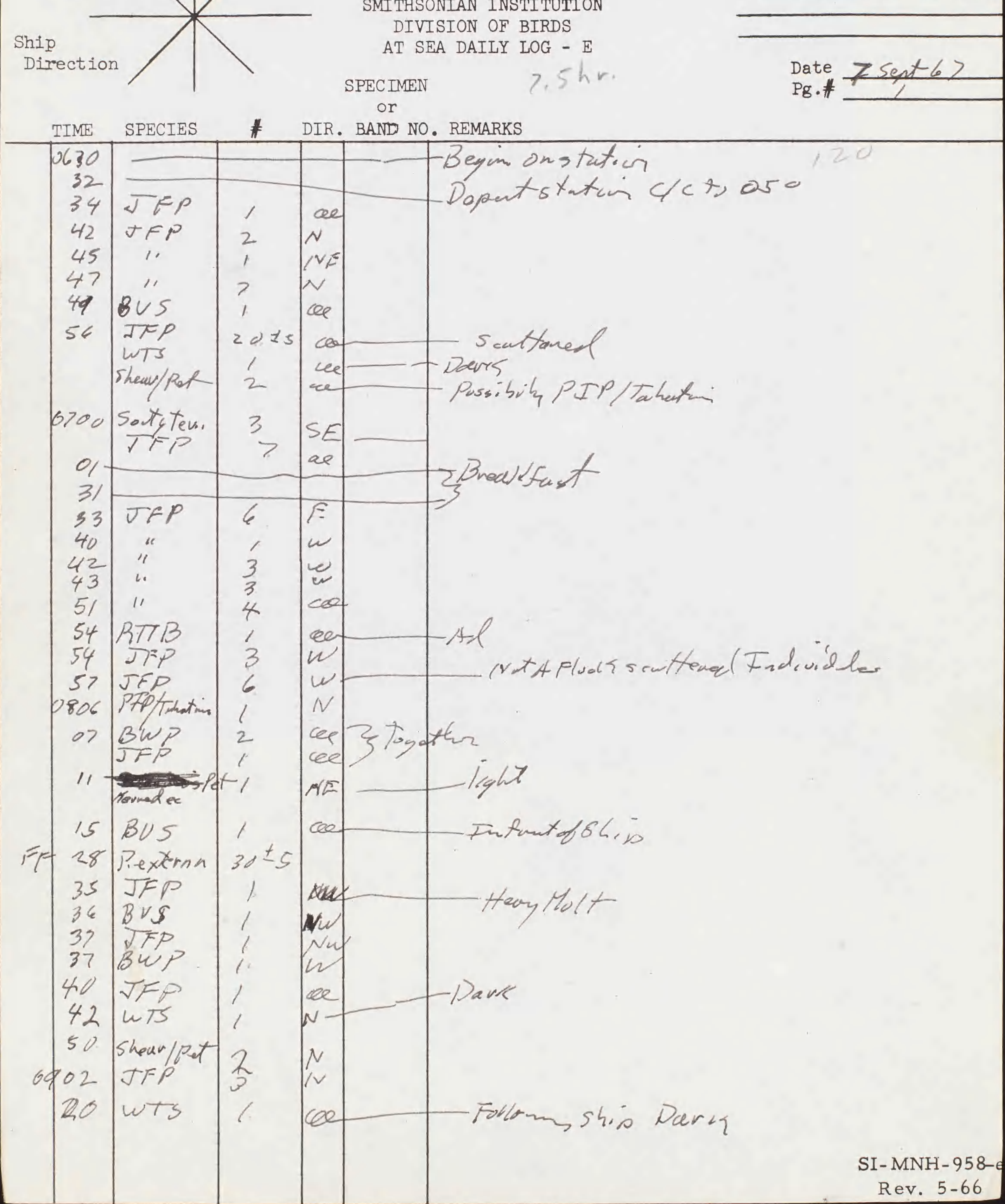




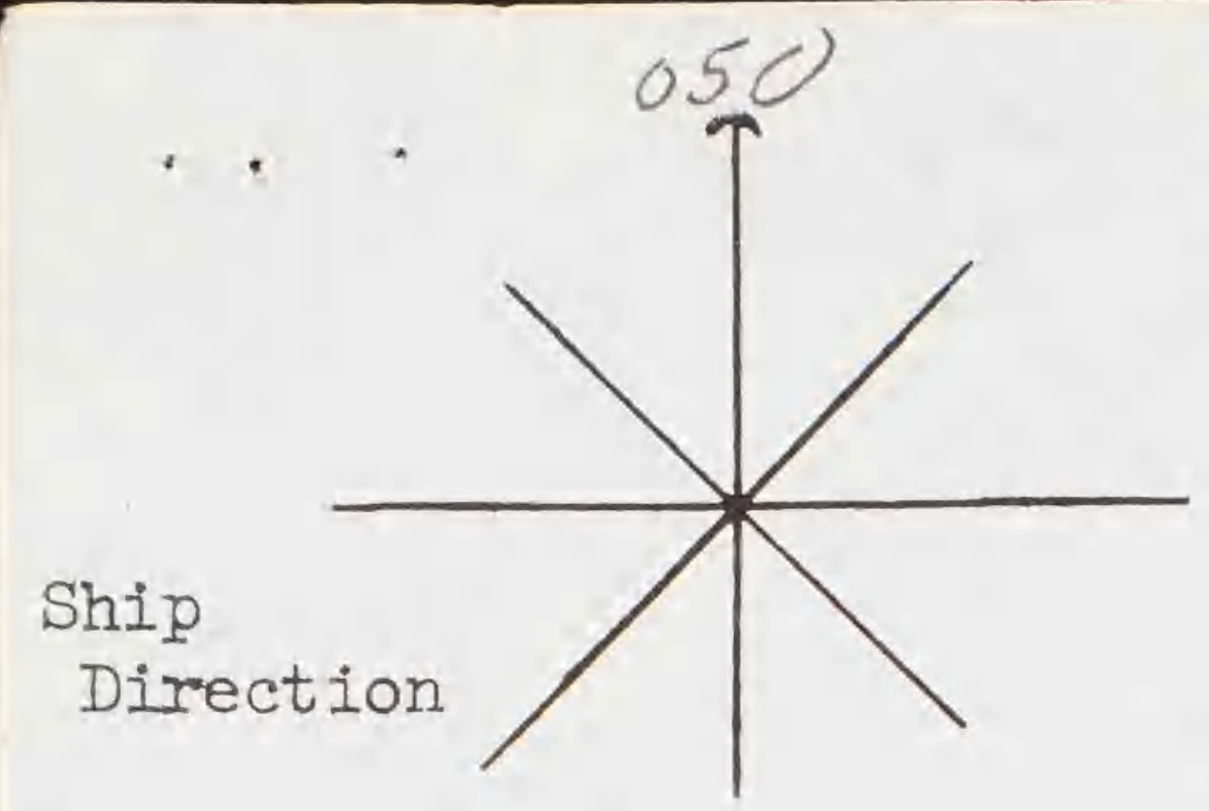

OBSERVERS :

\section{SMITHSONIAN INSTITUTION DIVISION OF BIRDS \\ AT SEA DAILY LOG - E \\ SPECIMEN}

or

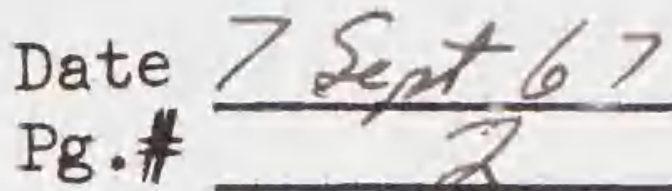

TIME SPECIES \# DIR. BAND NO. REMARKS

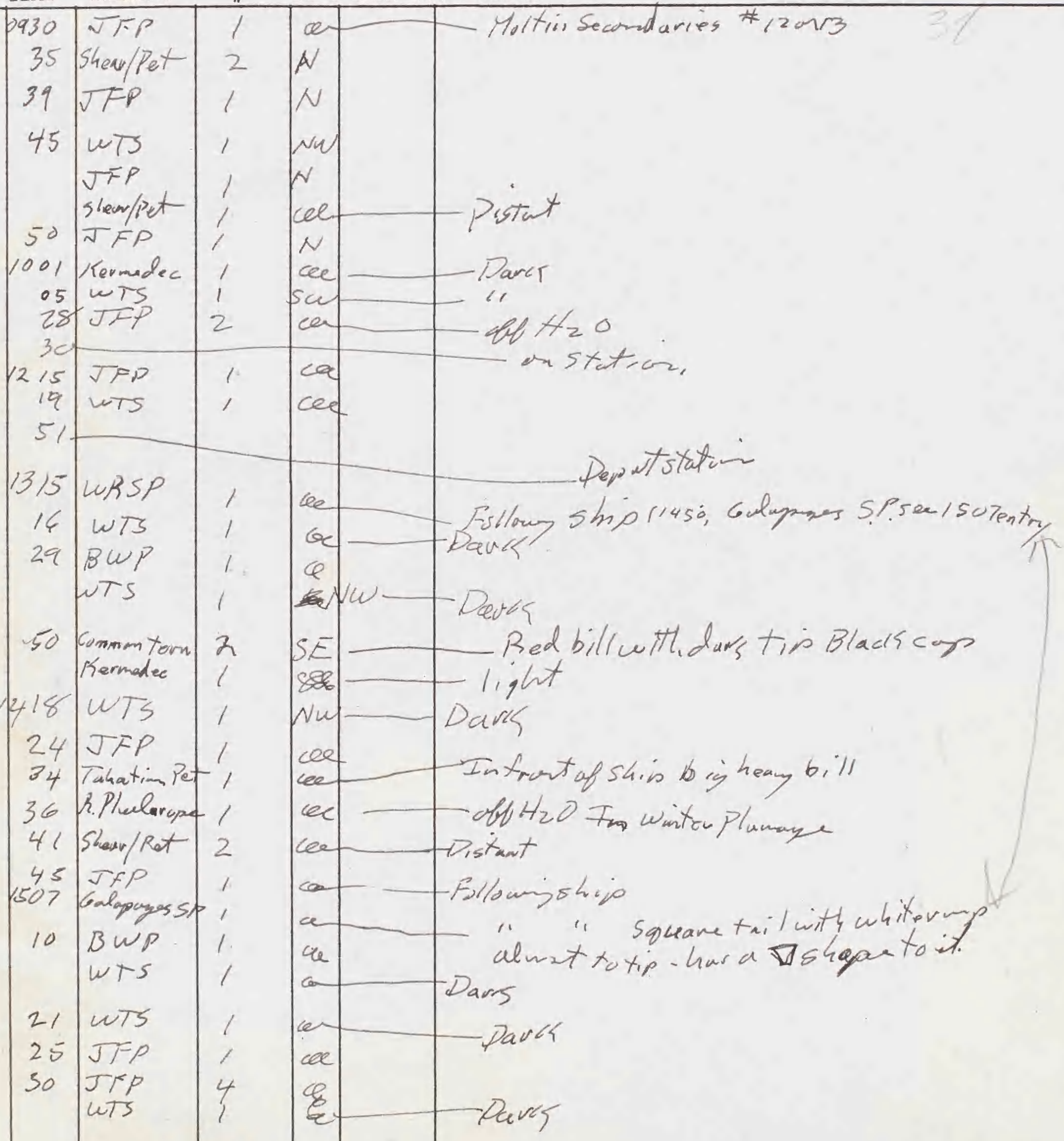




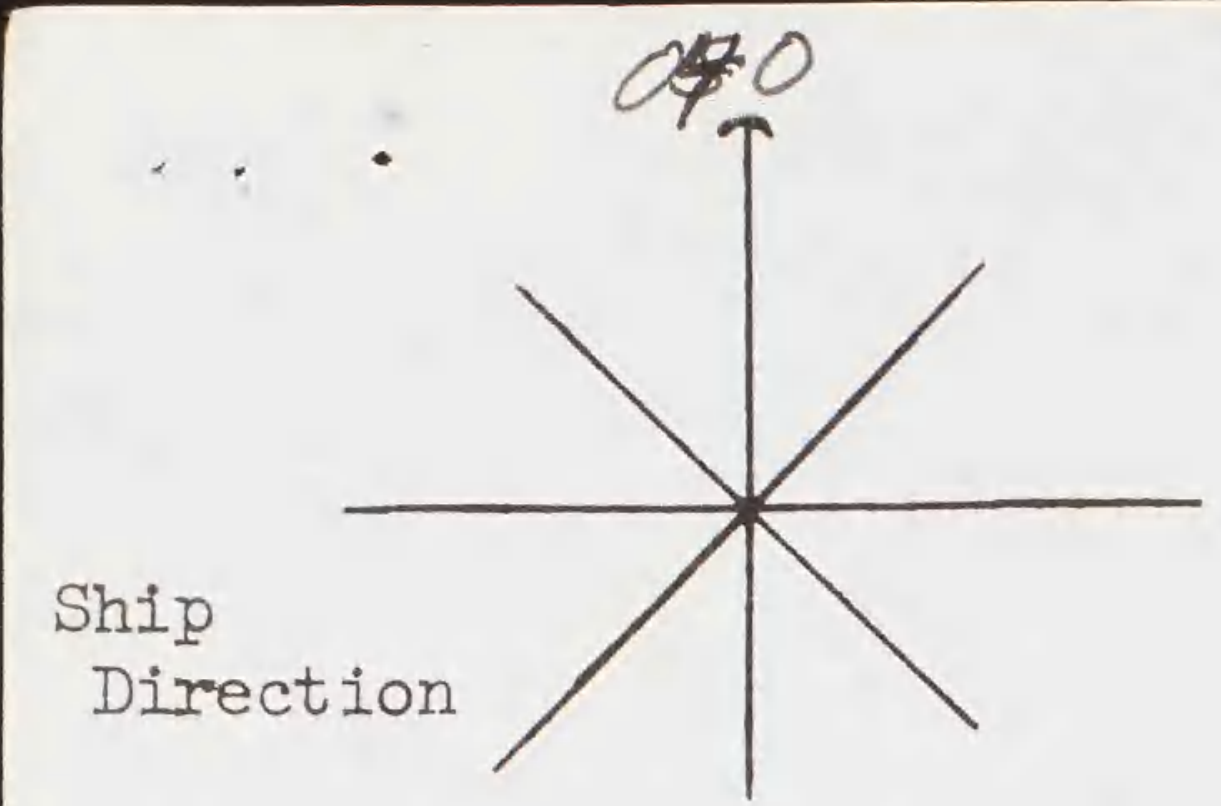

OBSERVERS :

SMITHSONIAN INSTITUTION DIVISION OF BIRDS AT SEA DAILY LOG - E

SPEC IMEN or

$$
\operatorname{Pate} \frac{7 \operatorname{sen} t 67}{3}
$$

DIR. BAND NO. REMARKS

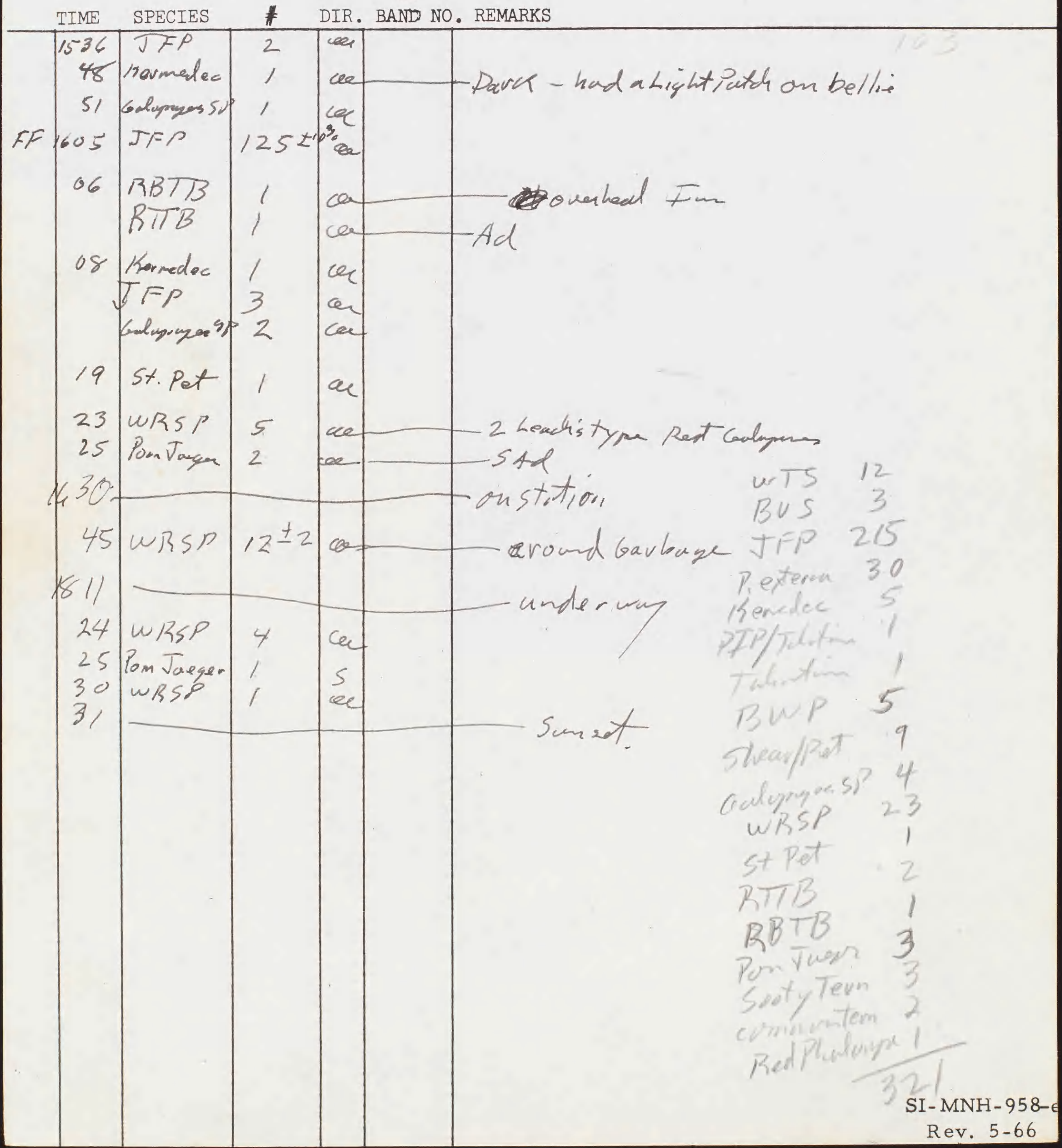




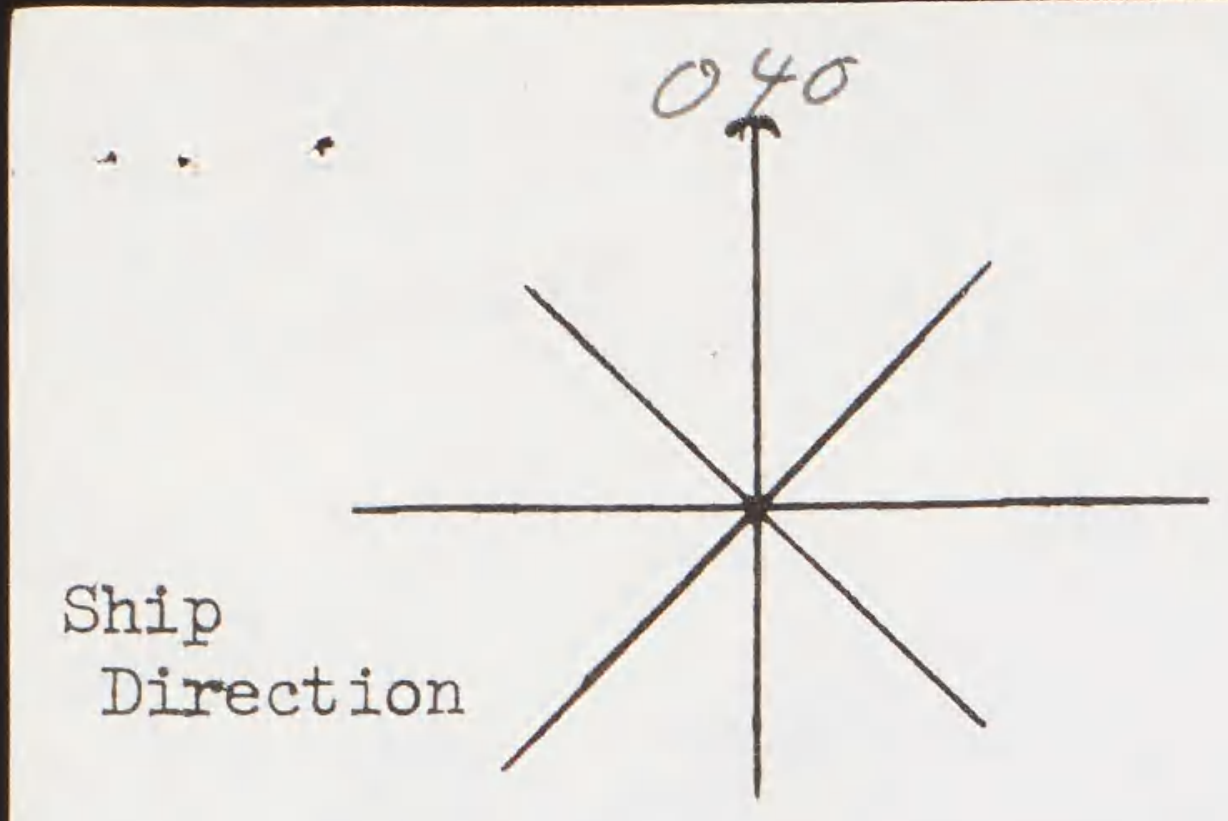

OBSERVERS: 
Ship

Direction

S 710 SPECIMEN

or

or
OBSERVERS :

SMITHSONLAN INSTITUIION

DIVISION OF BIRDS

AT SEA DAILY LOG - E

DIR. BAND NO. REMARKS
Date $8 \operatorname{sen} t / 6$ ?

$\mathrm{Pg} \cdot \#$

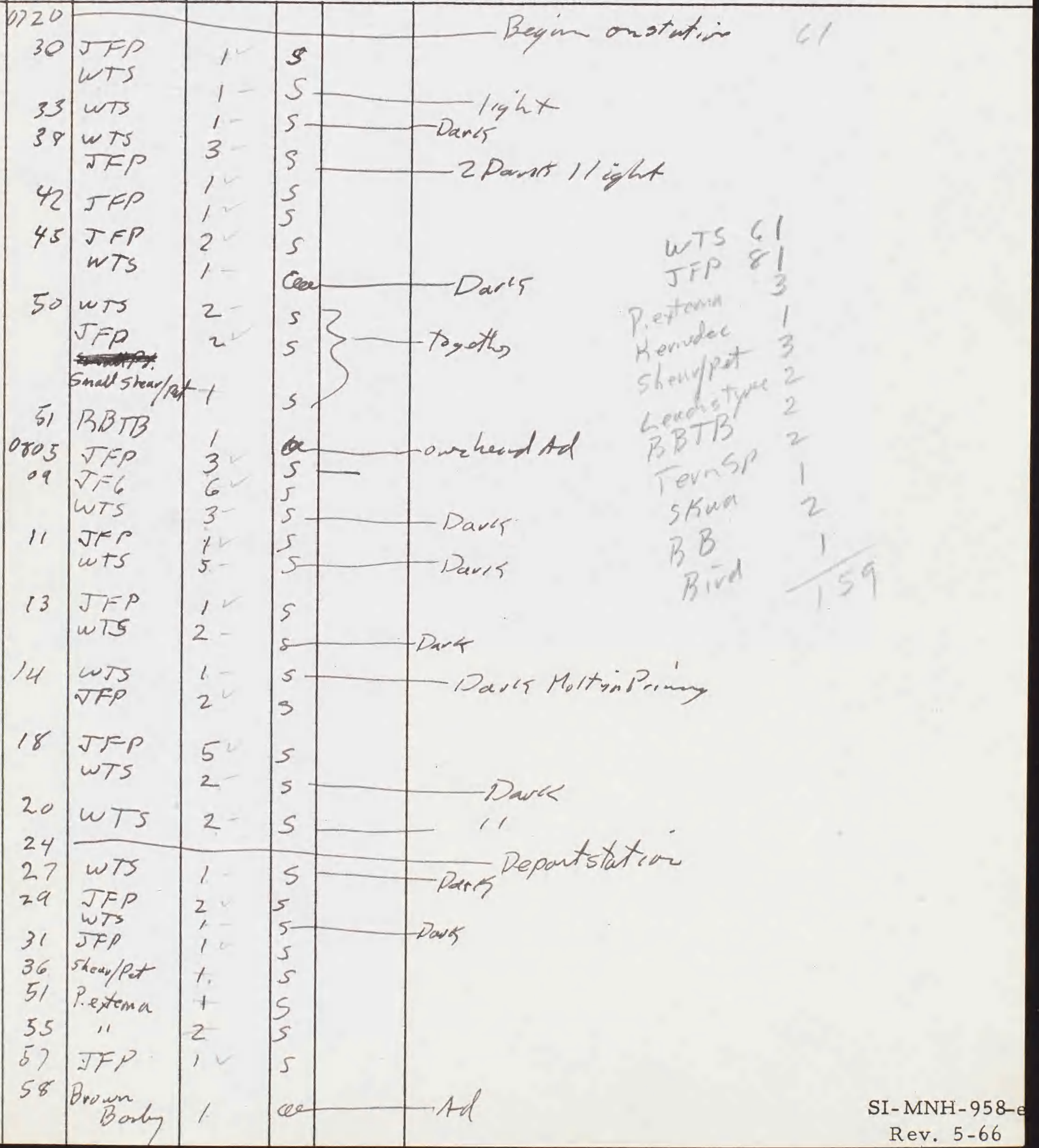




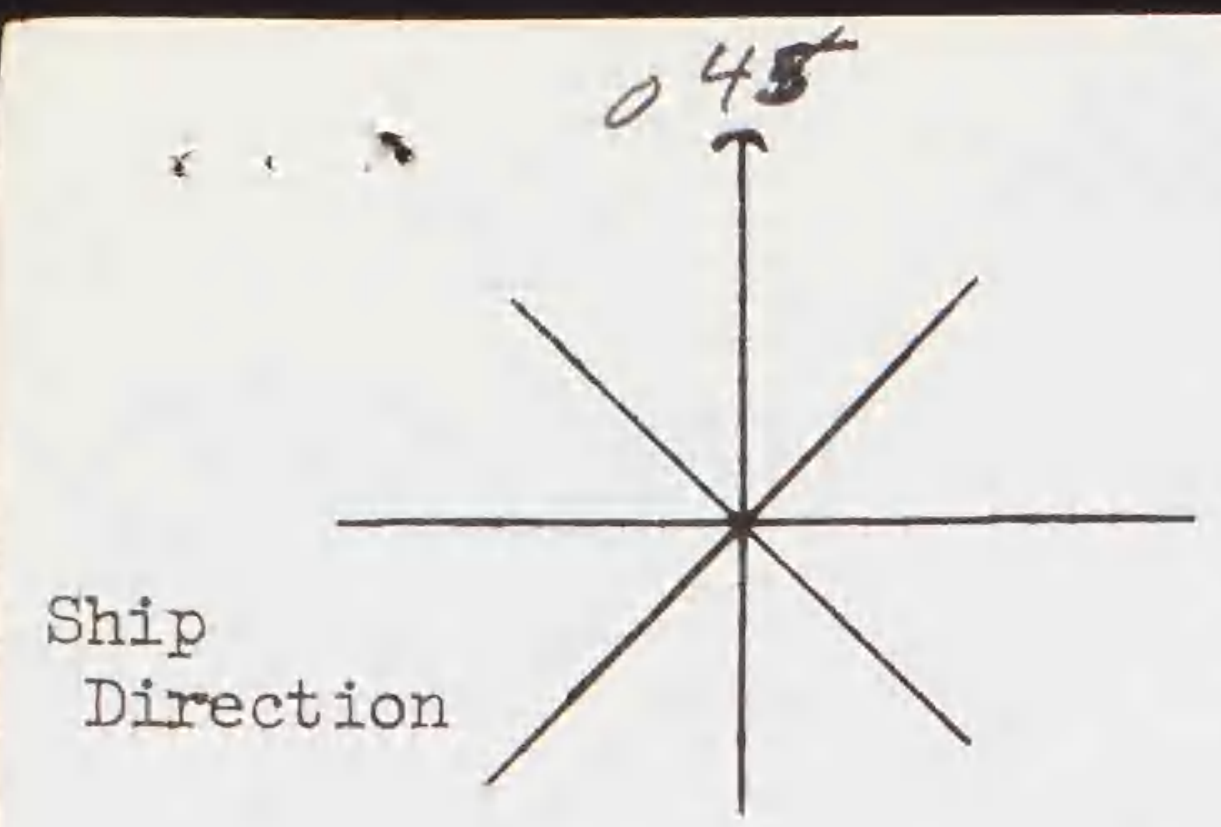

OBSERVERS :

SMITHSONIAN INSTITUTION DIVISION OF BIRDS AT SEA DAILY LOG - E

SPEC IMEN

or

$$
\begin{aligned}
& \text { Date } \\
& \mathrm{Pg} . \# \frac{8 \text { en }}{2}-67
\end{aligned}
$$

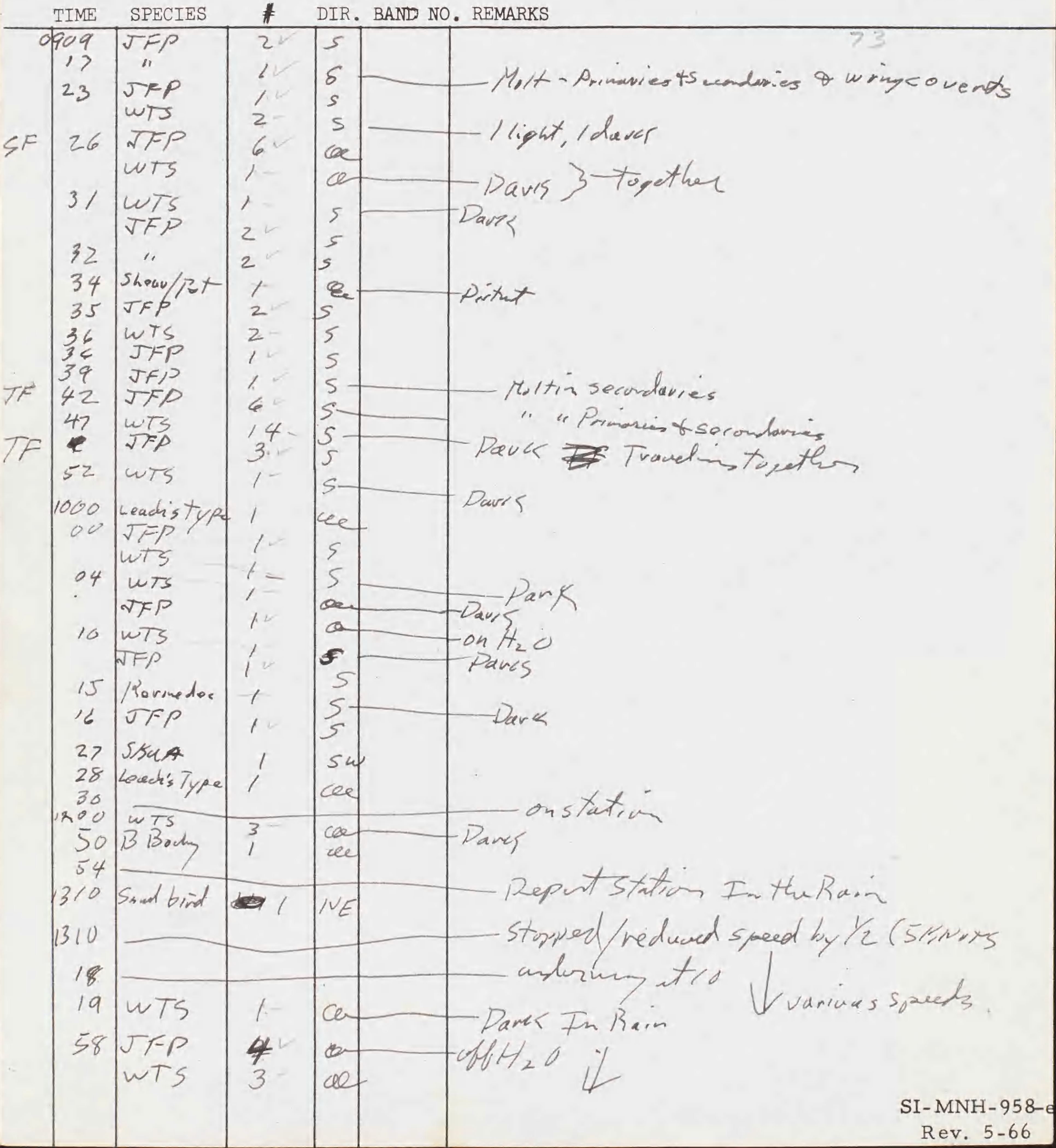




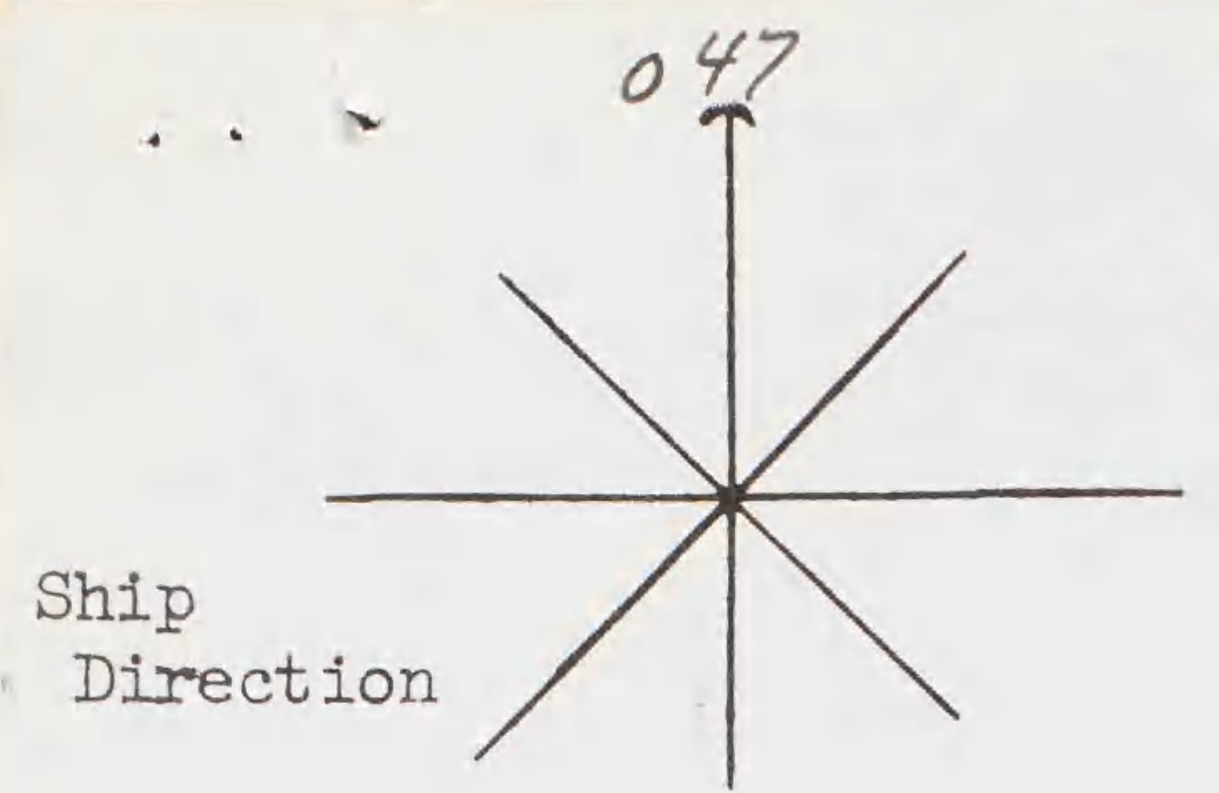

OBSERVERS :

SMITHSONIAN INSTITUTION DIVISION OF BIRDS AT SEA DAILY LOG - E

SPECIMEN

or
Date $\frac{8 \operatorname{sen} t 67}{3}$

$\mathrm{Pg} \cdot \#$

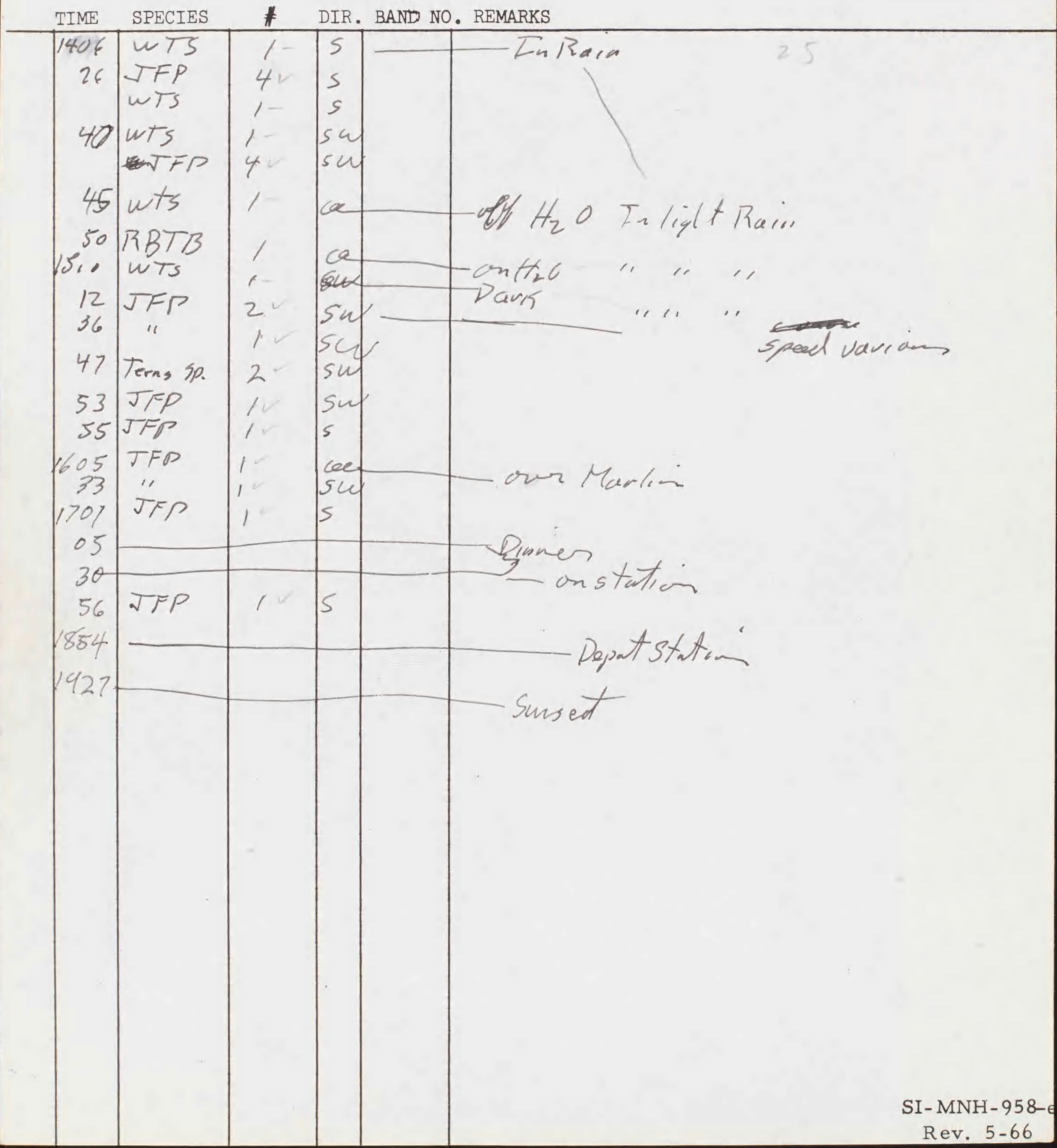




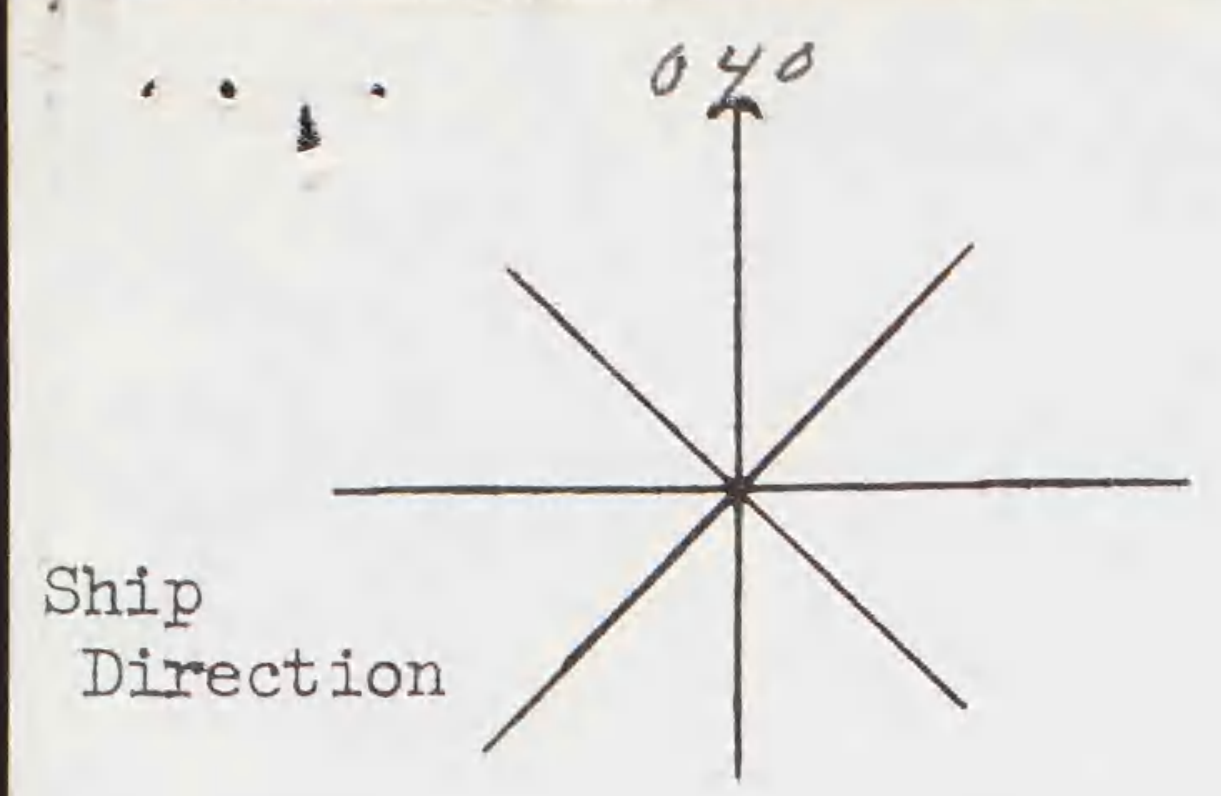

OBSERVERS :

SMITHSONIAN INSTITUTION DIVISION OF BIRDS AT SEA DAILY LOG - E
SPEC IMEN

or
Date $9 \operatorname{sen} x 67$

$\mathrm{Pg} \cdot \#$

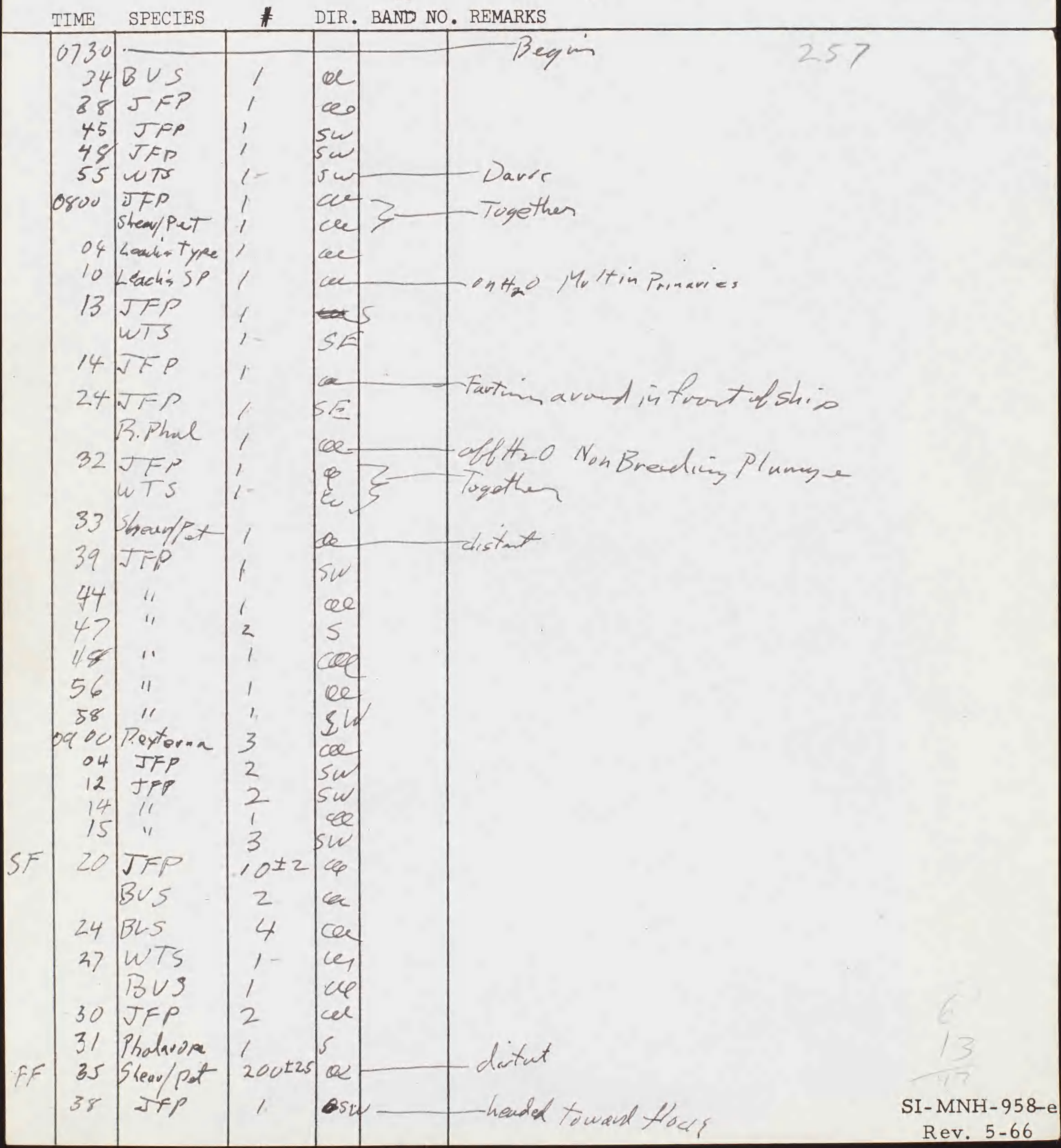




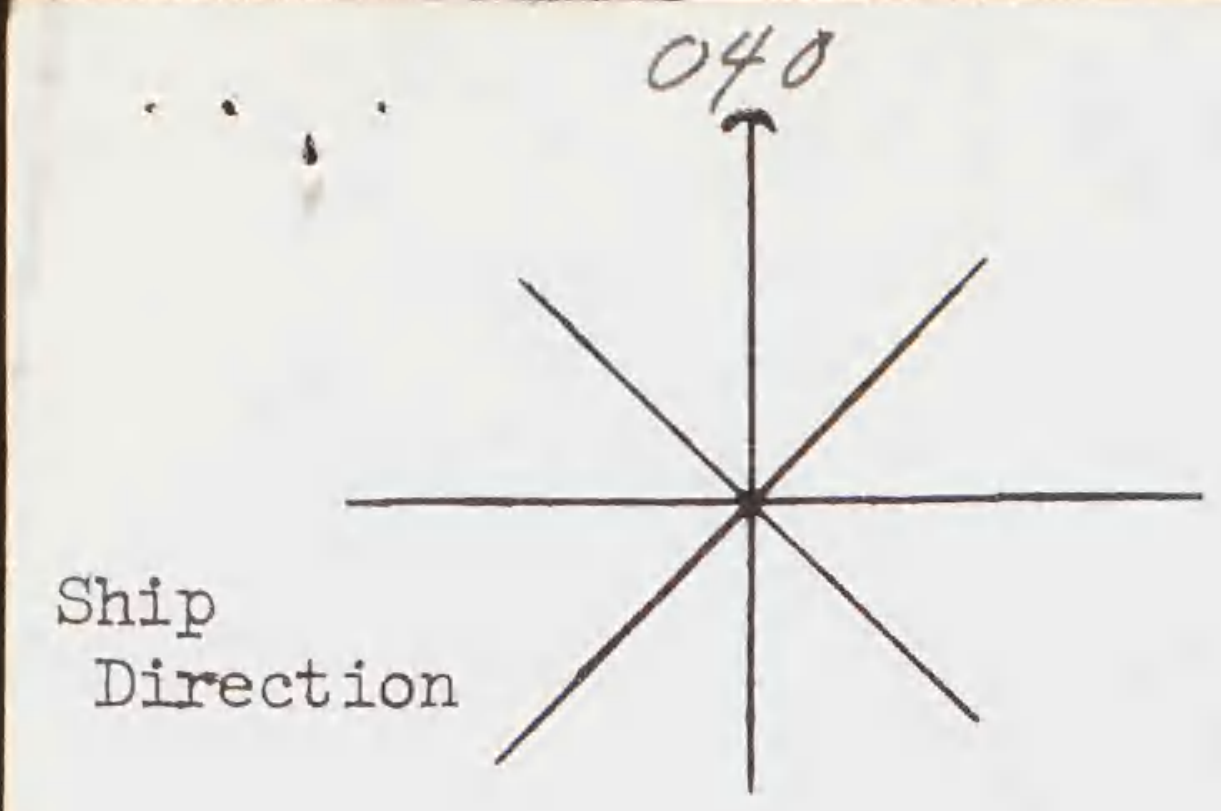

SMITHSONIAN INSTITUTION

DIVISION OF BIRDS

AT SEA DAILY LOG - E

SPEC IMEN

or

OBSERVERS :

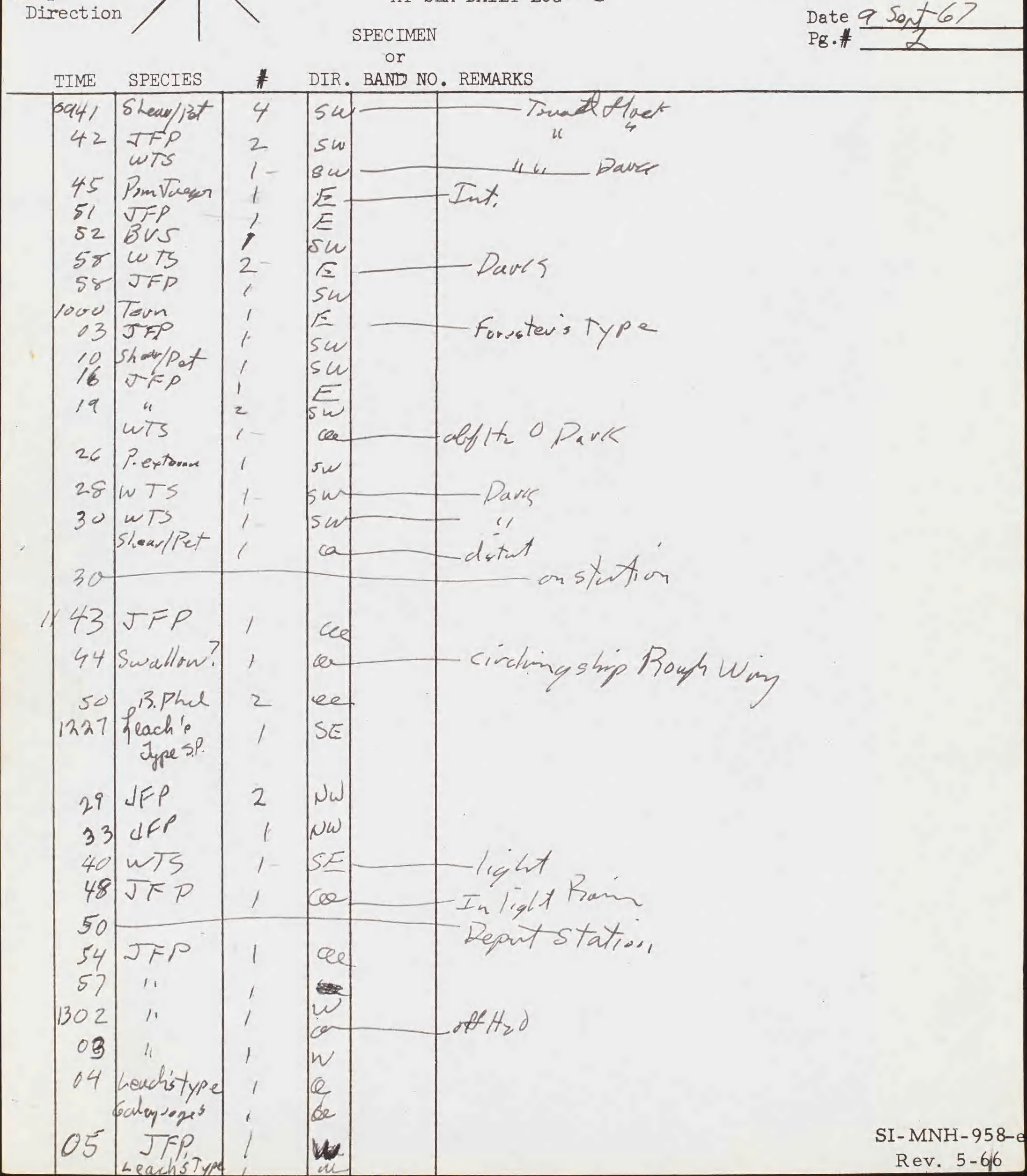




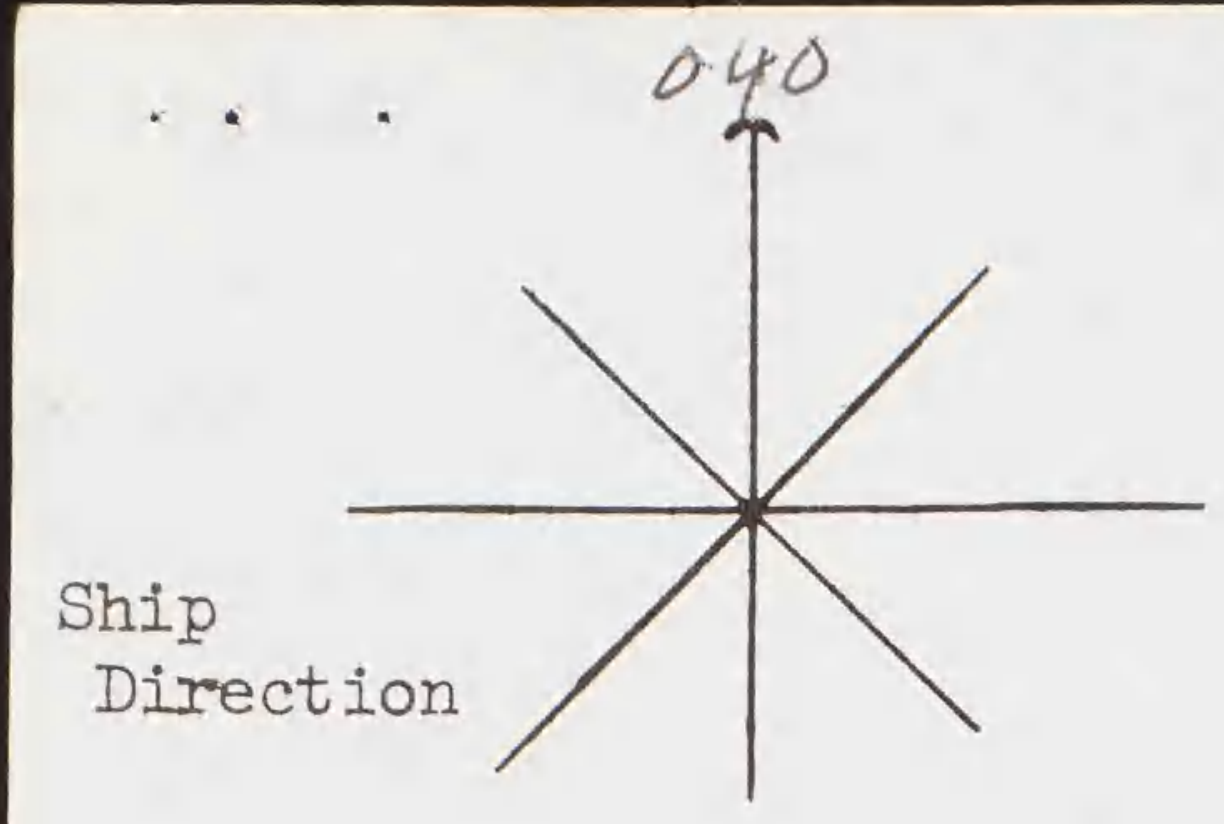

OBSERVERS :

SMITHSONIAN INSTITUTION

DIVISION OF BIRDS

AT SEA DAILY LOG - E

SPEC IMEN

Date 9 sept 67

or

$\mathrm{Pg} \cdot \#$

TIME SPECIES \# DIR. BAND NO. REMARKS

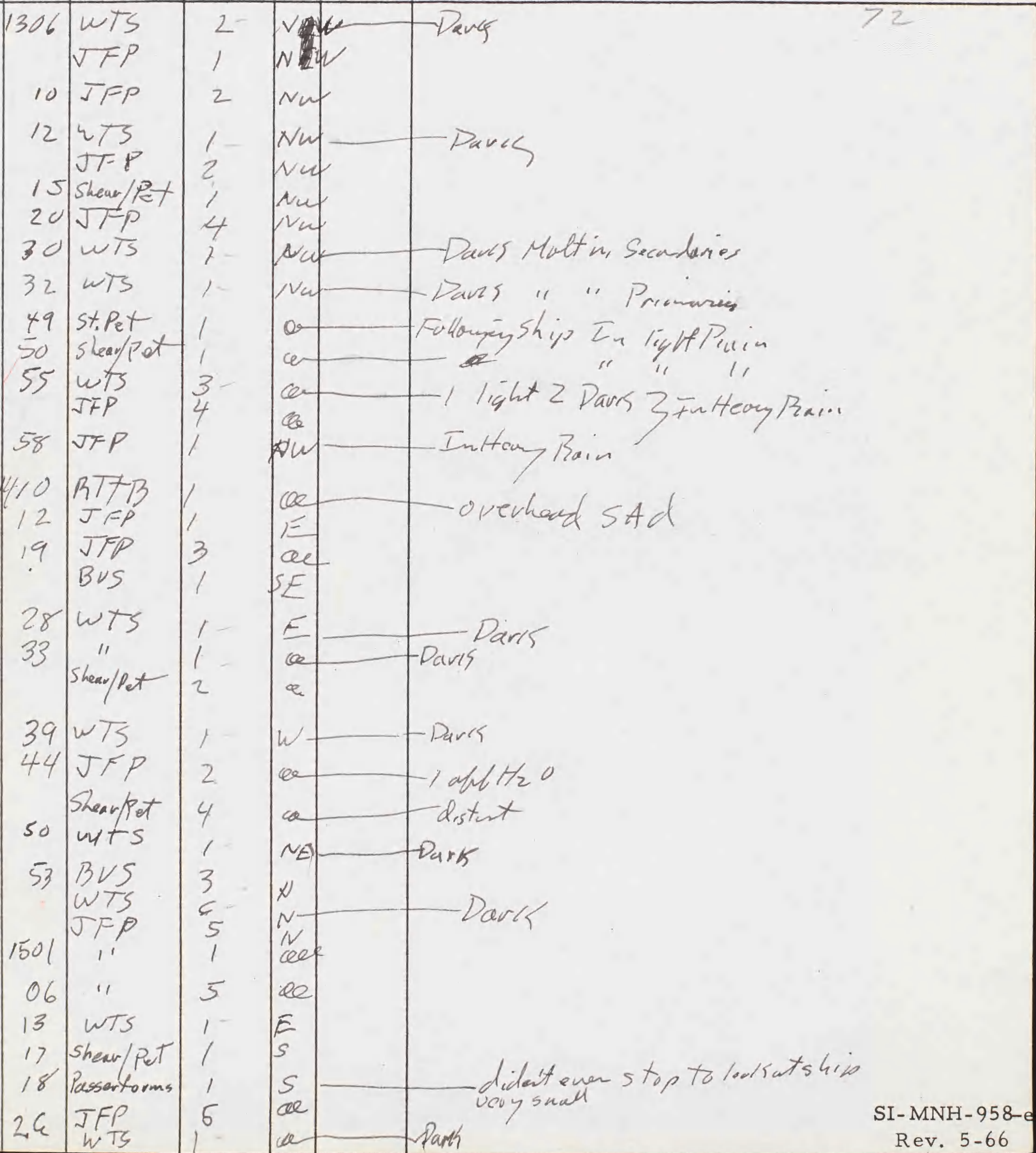



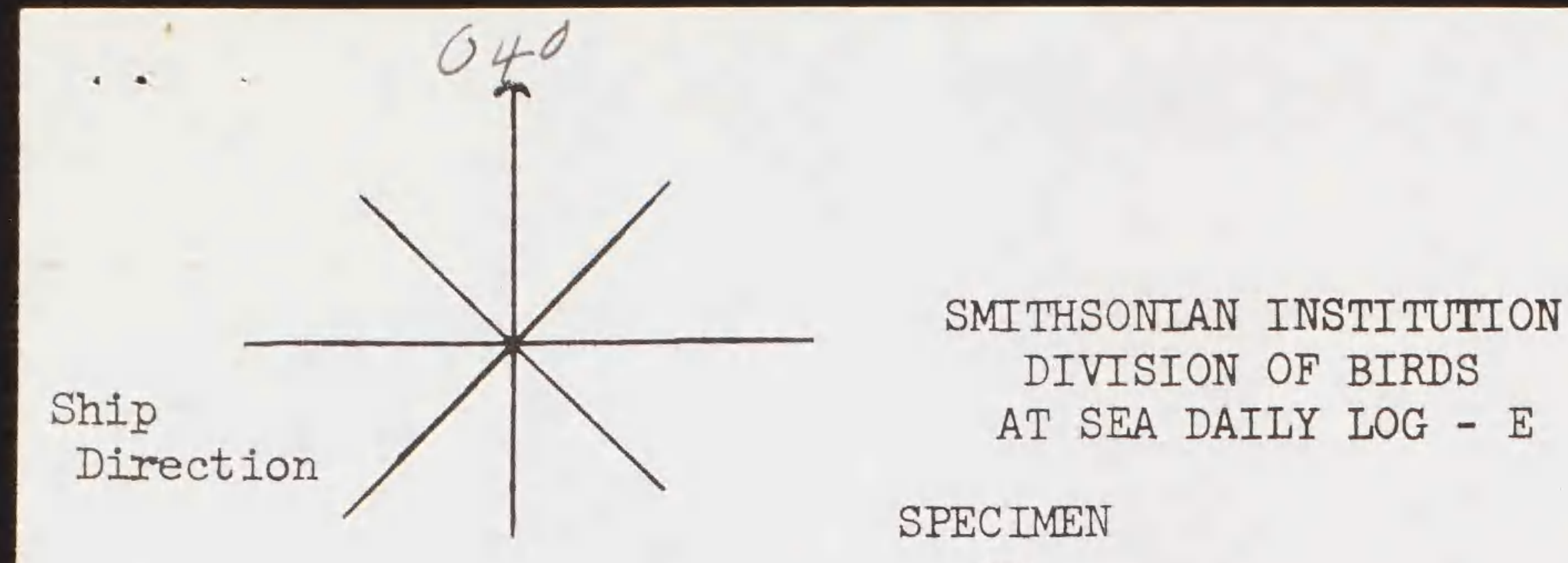

\section{OBSERVERS :}

or

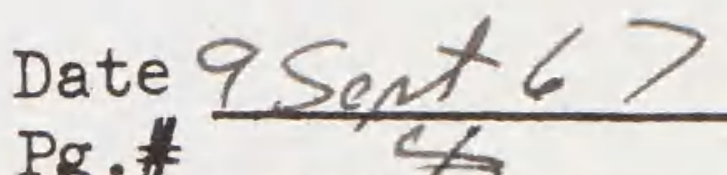

TIME SPECIES \# DIR. BAND NO. REMARKS

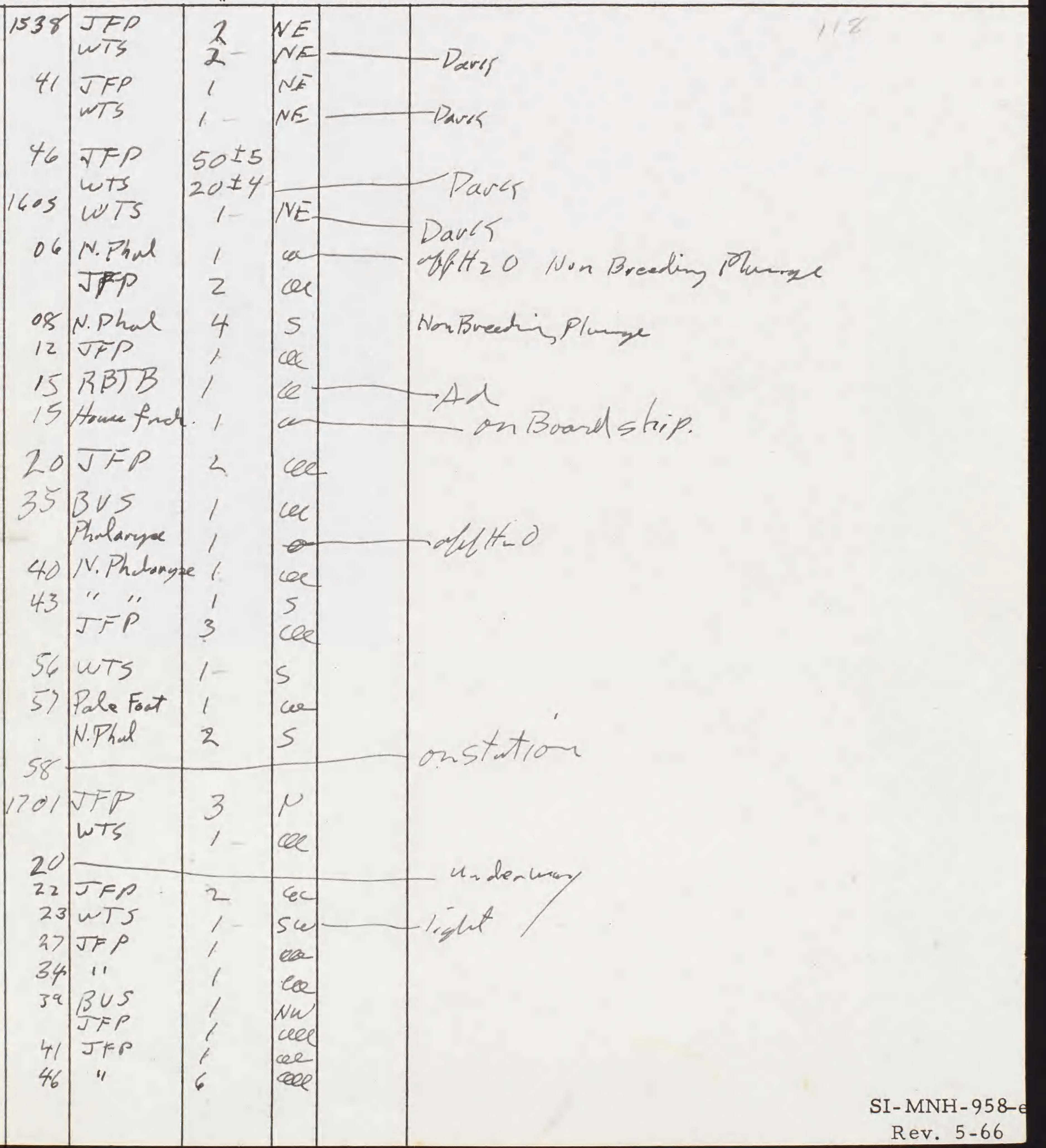




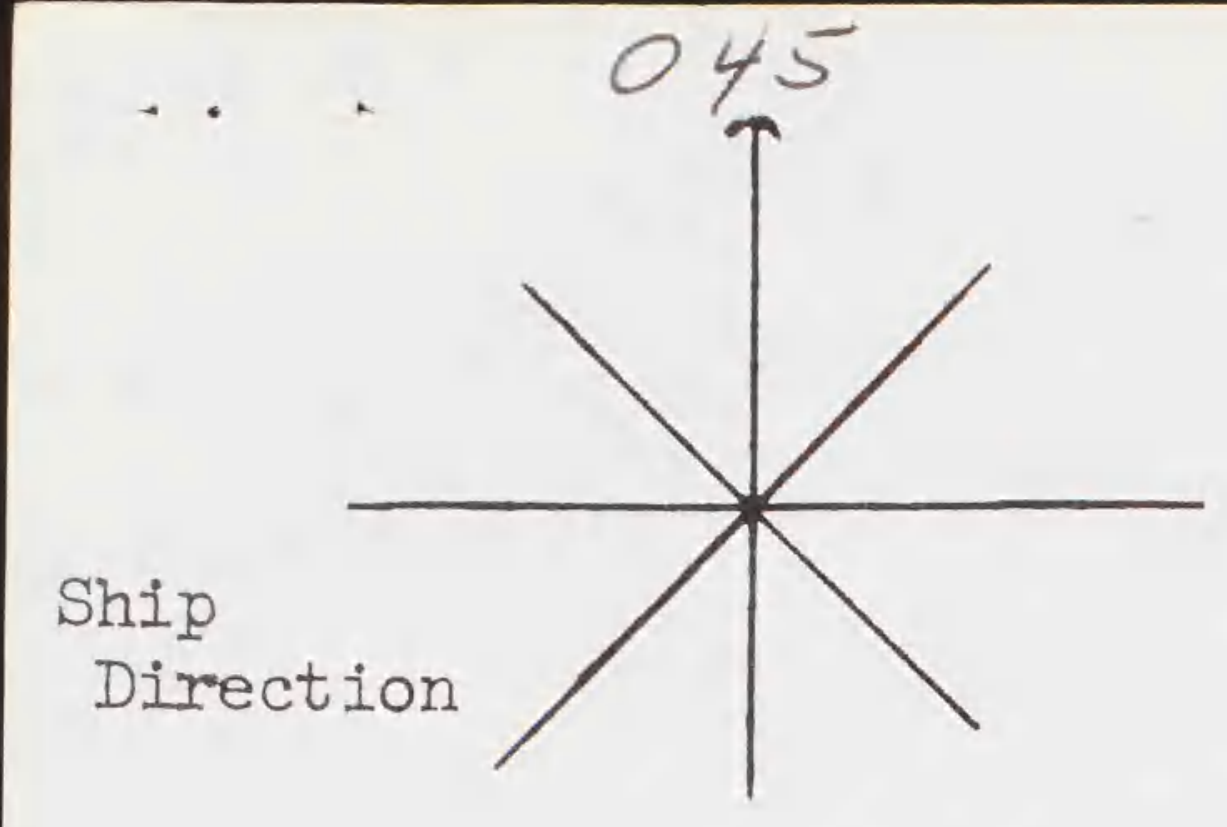

OBSERVERS:

SMITHSONIAN INSTITUTION DIVISION OF BIRDS AT SEA DAILY LOG - E

SPECIMEN

or
Date
$P g . \# \frac{95}{5} 67$

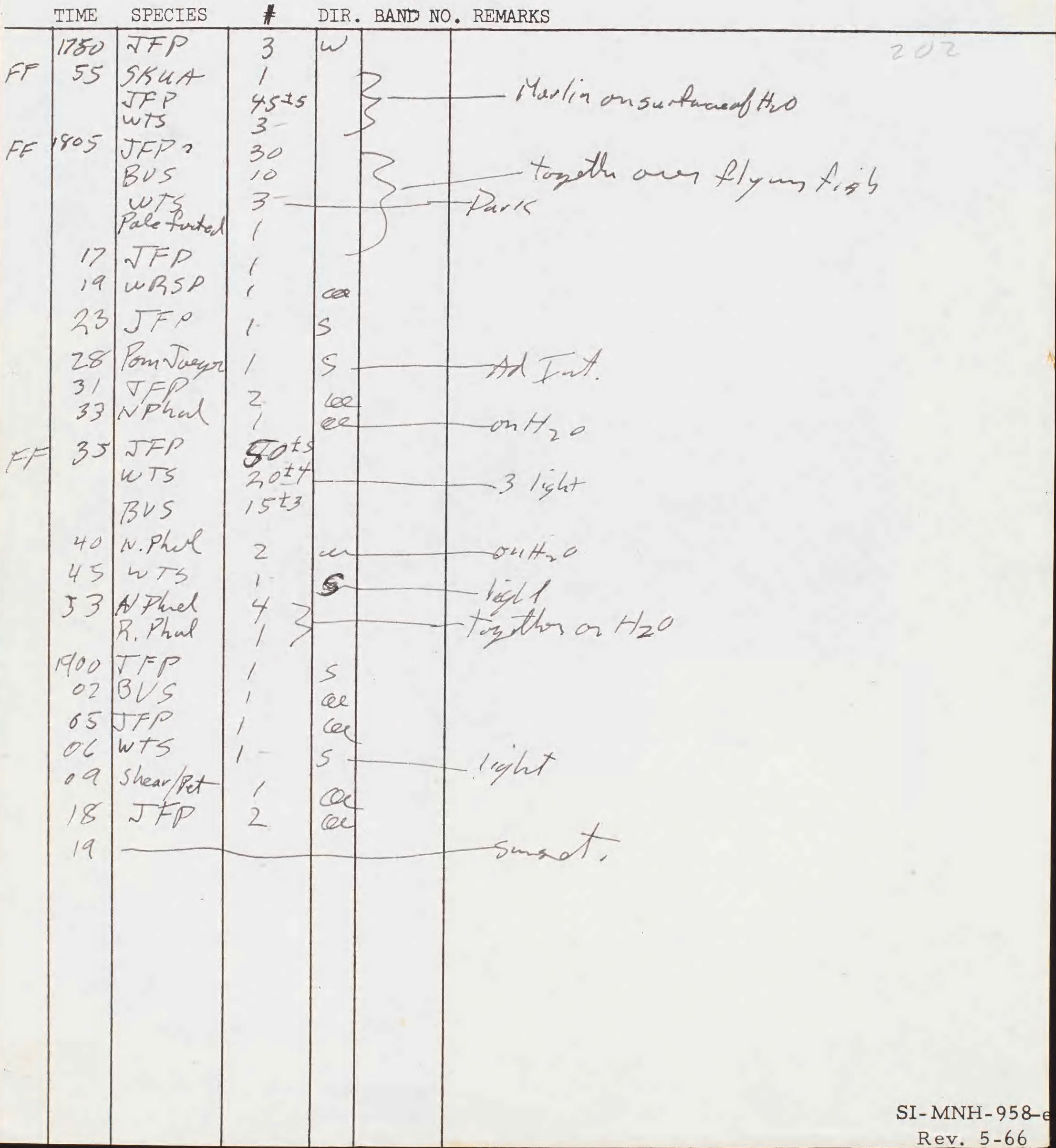




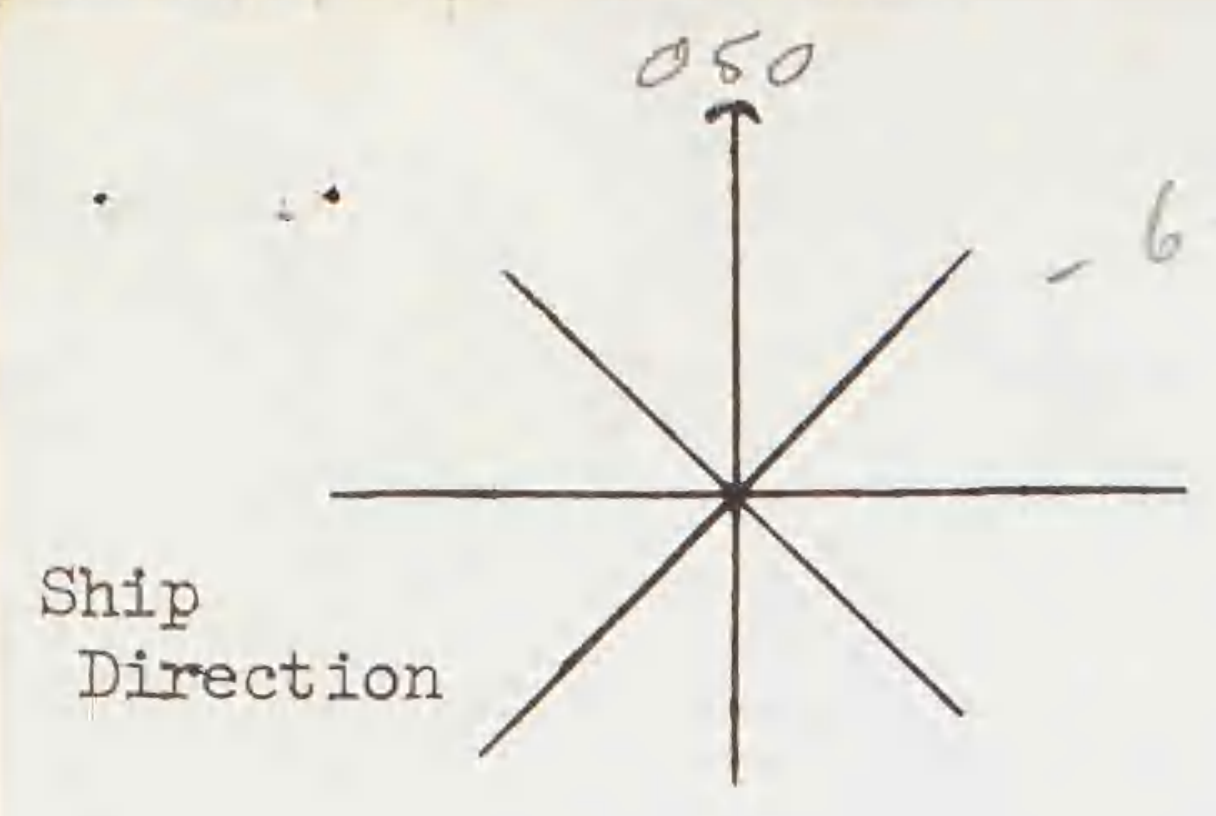

OBSERVERS :

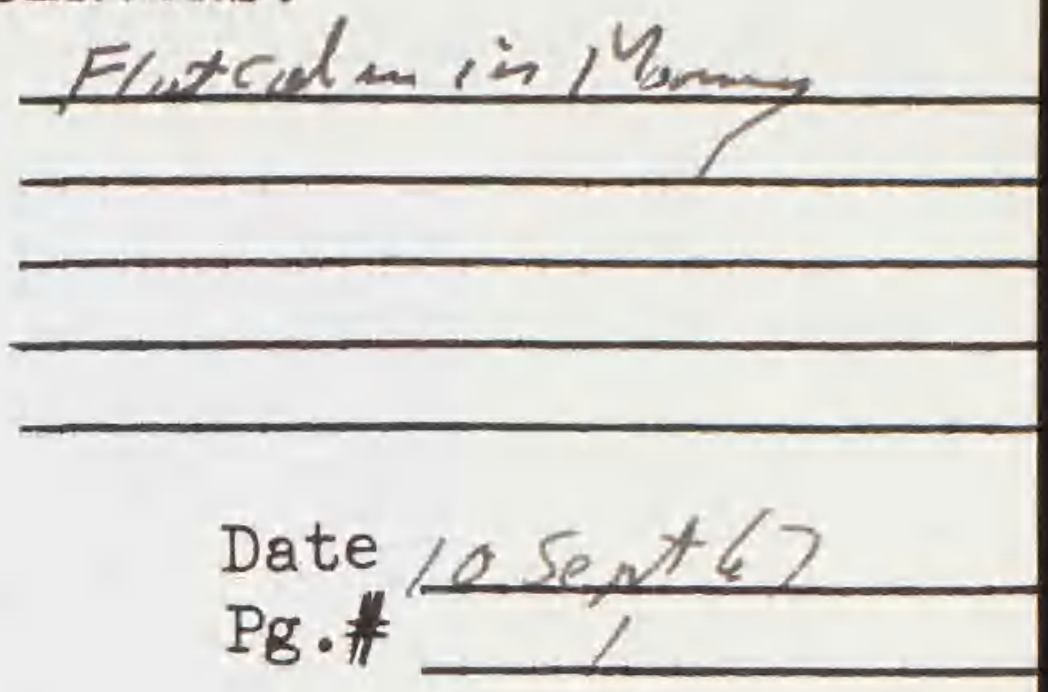

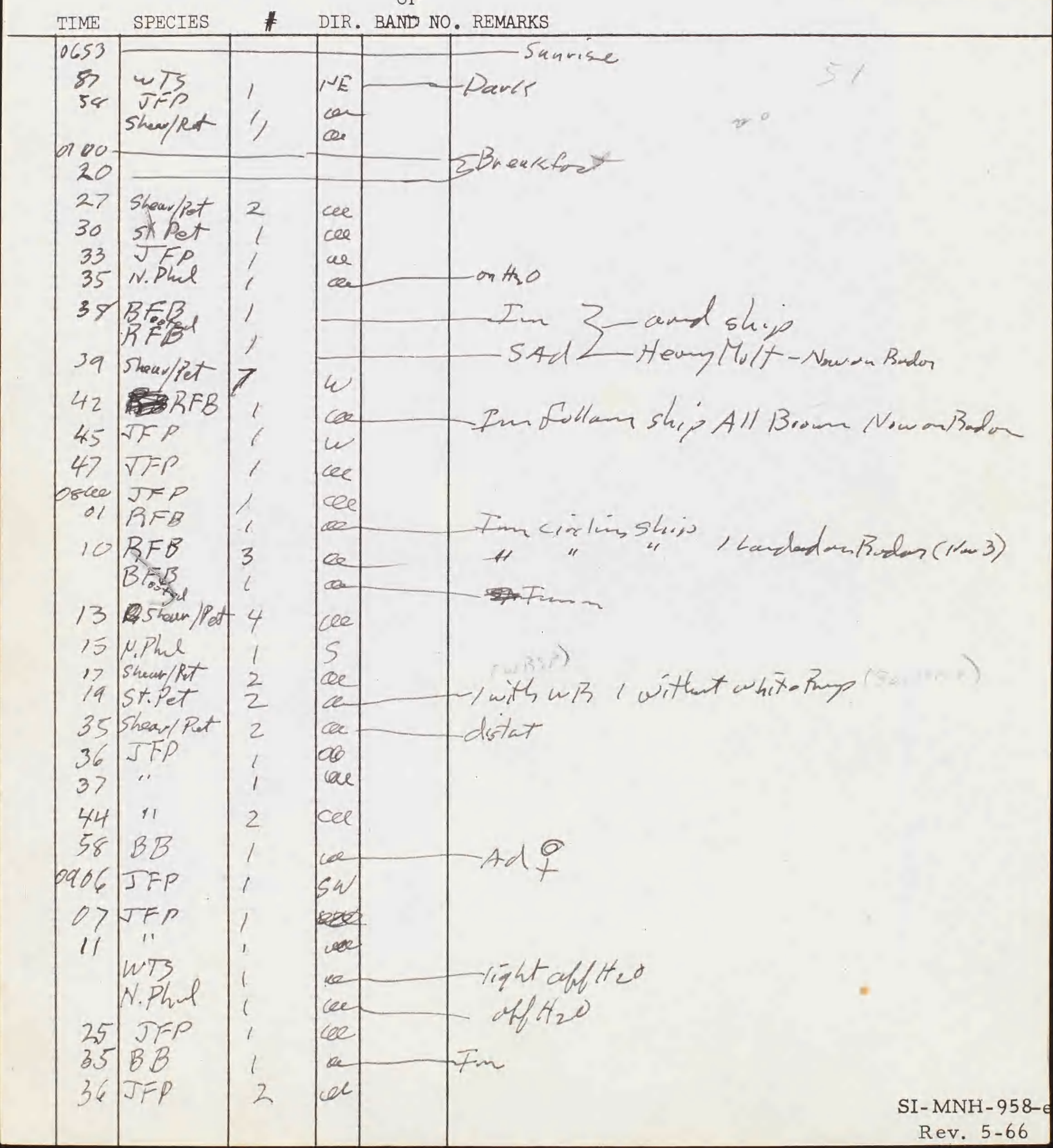




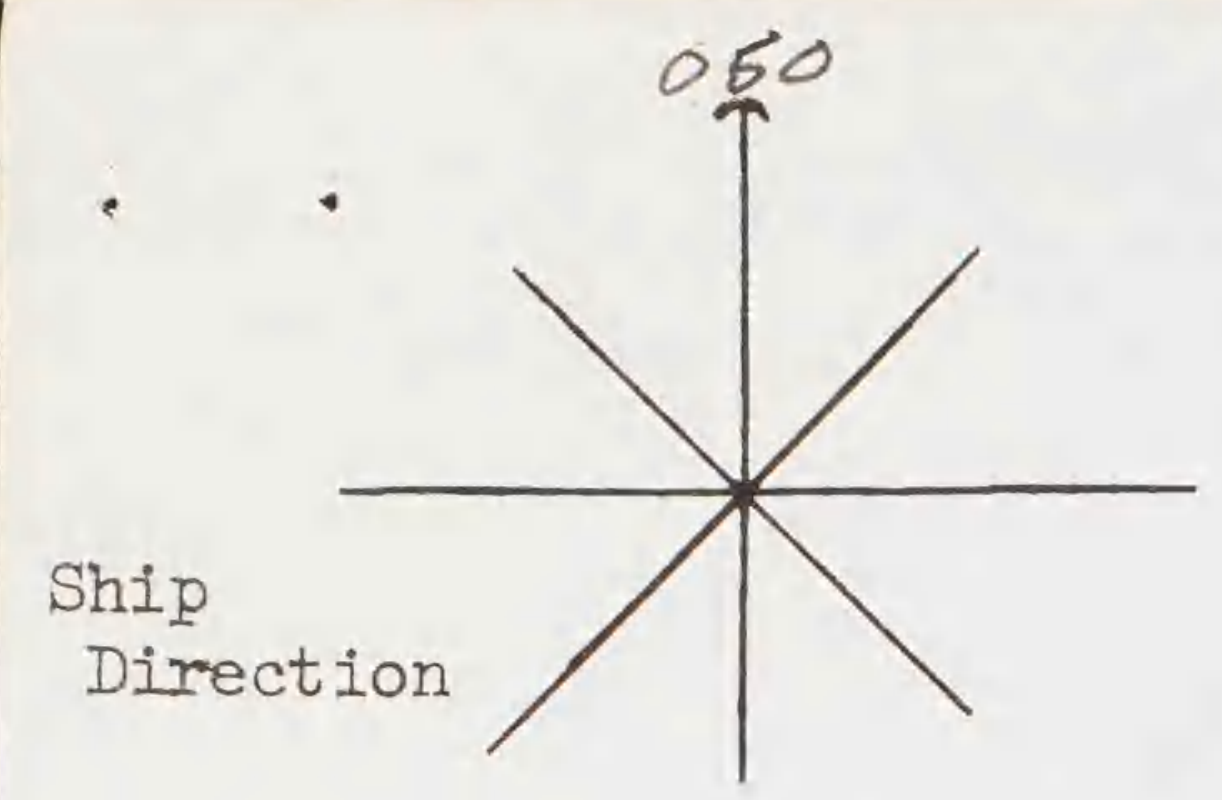

OBSERVERS: 


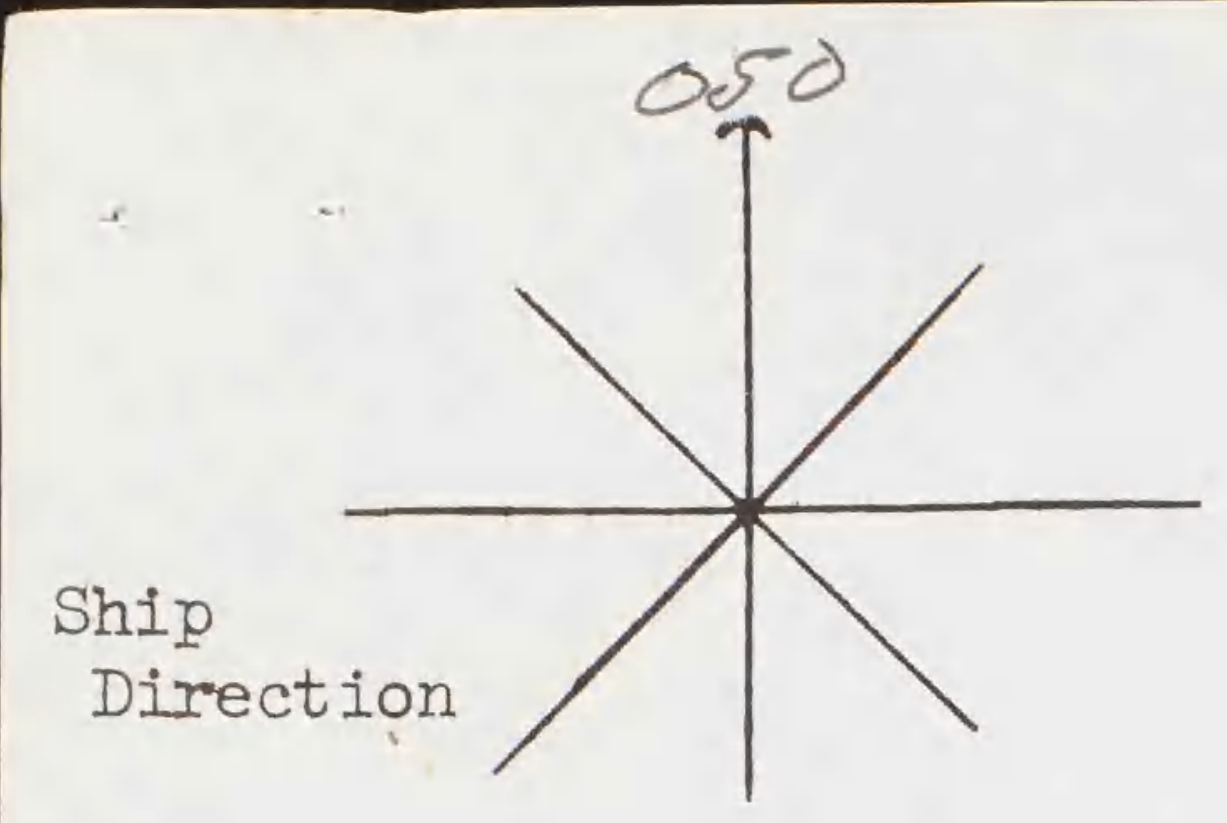

OBSERVERS :

SMITHSONIAN INSTITUTION

DIVISION OF BIRDS

AT SEA DAILY LOG - E

or

Date $10 \frac{\operatorname{sen} x-6>}{3}$

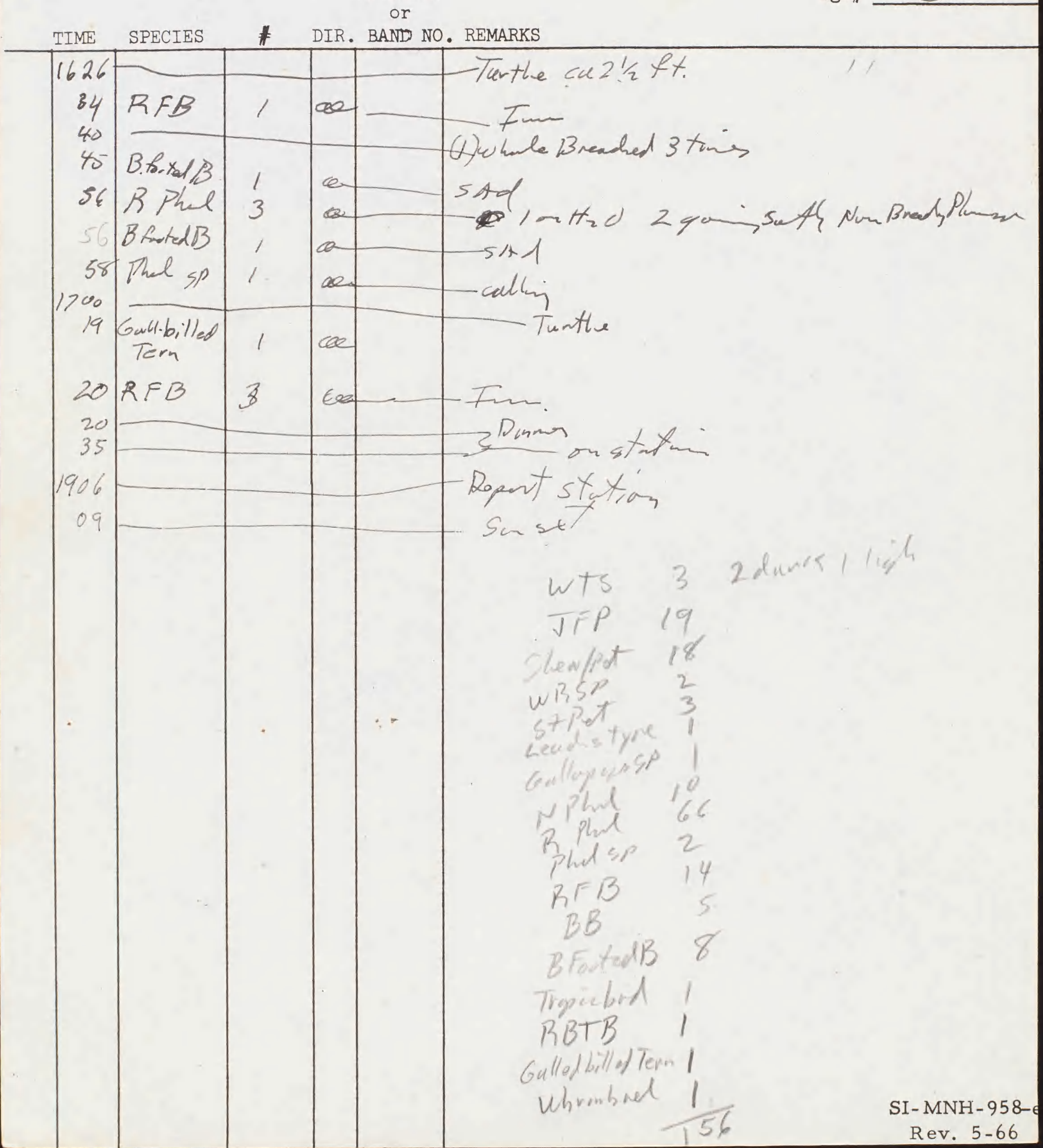




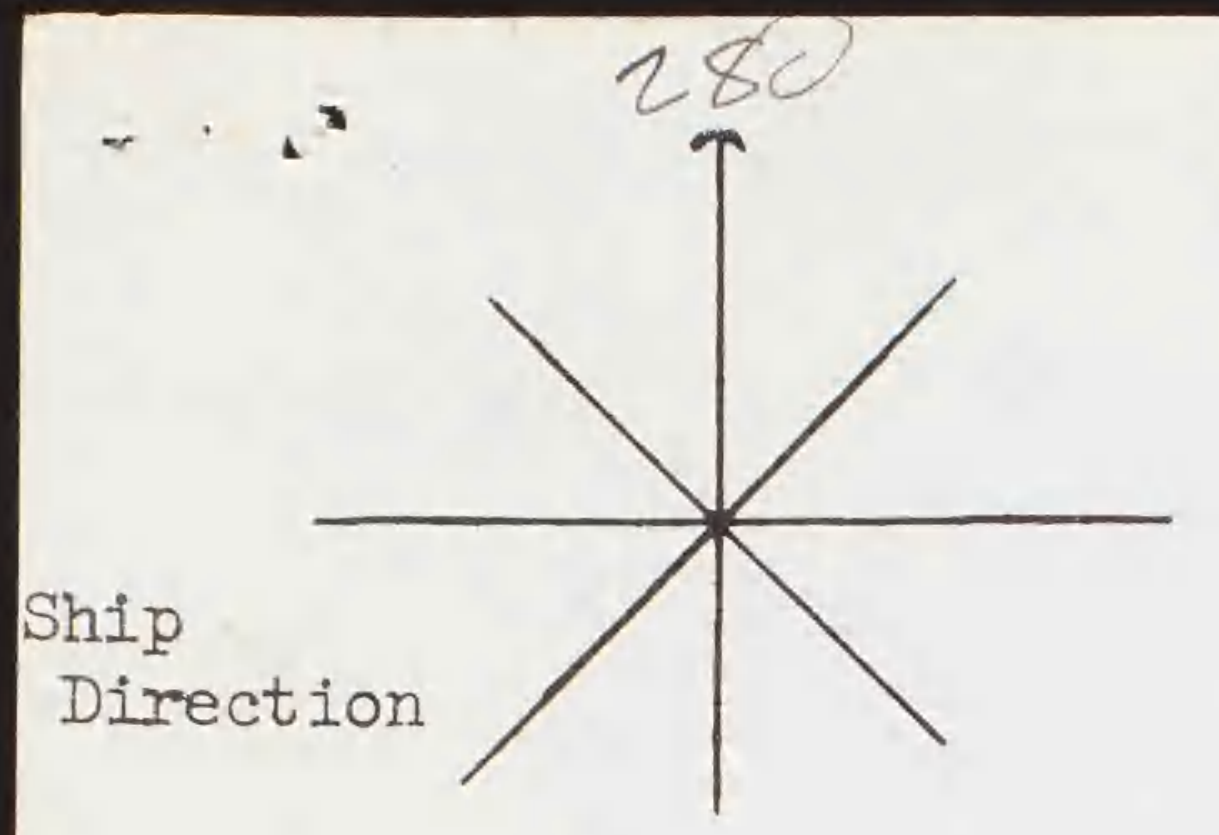

\section{SMITHSONIAN INSTITUTION DIVISION OF BIRDS AT SEA DAILY LOG - E}

SPEC IMEN

or
OBSERVERS :

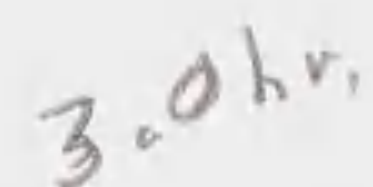

Date $135 \operatorname{sen} 67$

$\mathrm{Pg} \cdot \#$

TIME SPECIES \# DIR. BAND NO. REMARKS

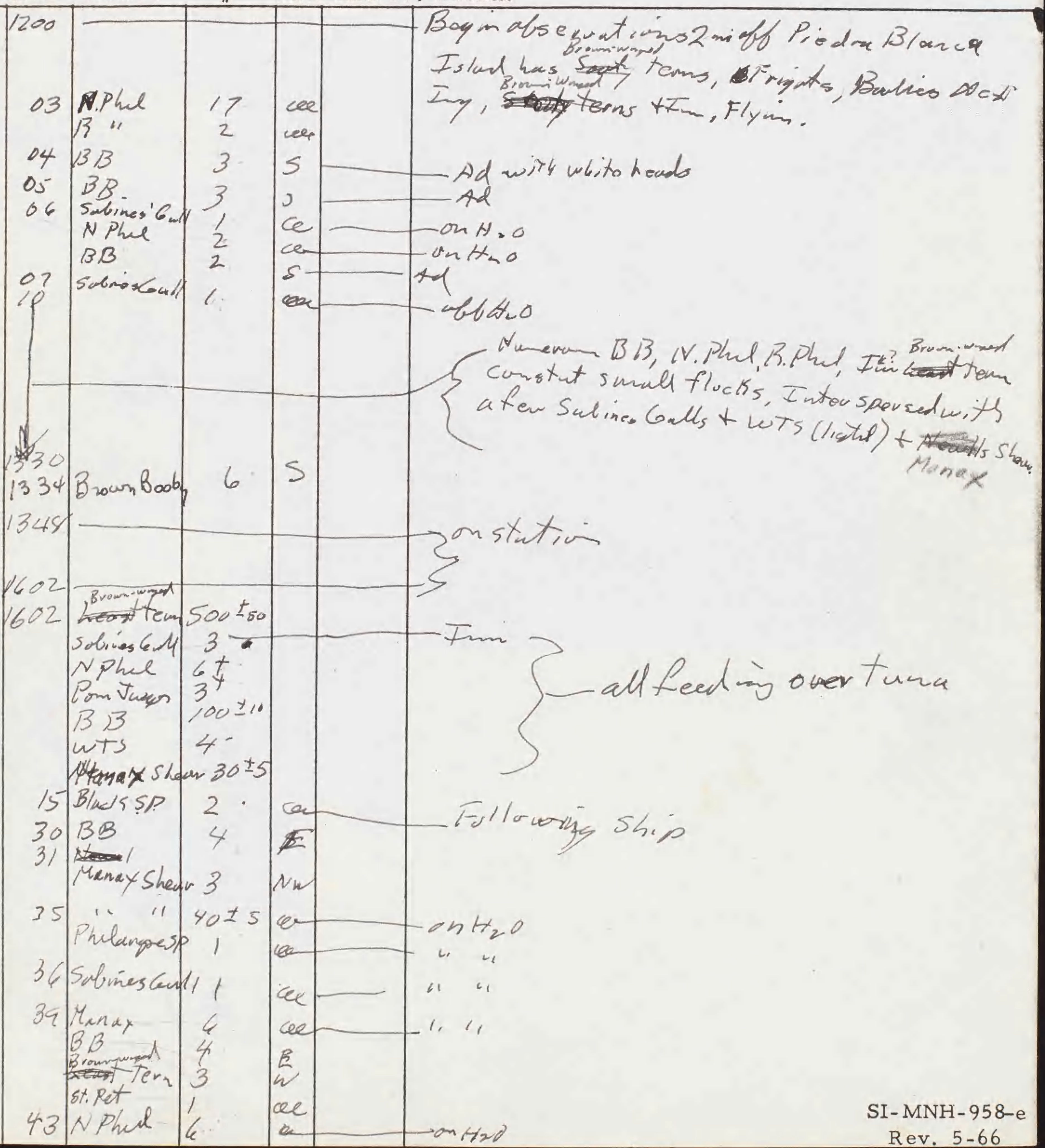




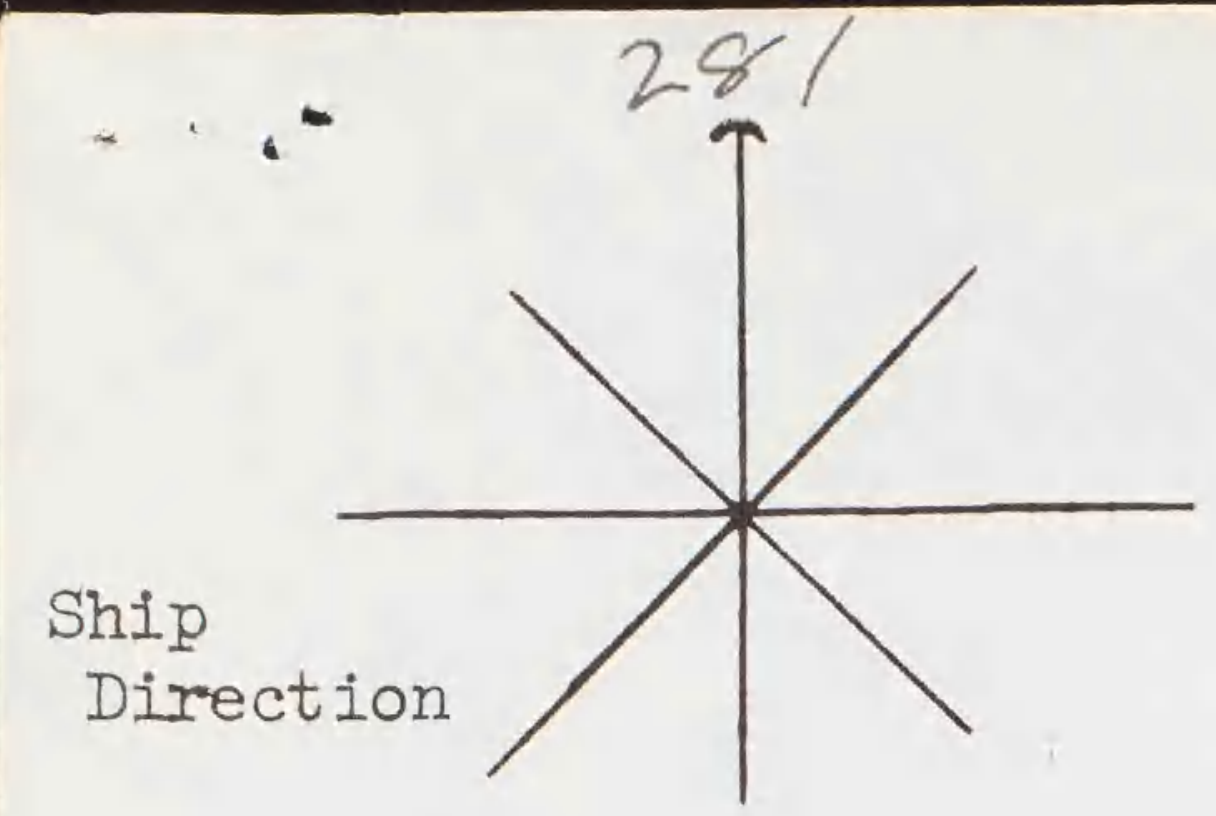

OBSERVERS :

SMITHSONIAN INSTITUTION DIVISION OF BIRDS AT SEA DAILY LOG - E

SPECIMEN

or
Date 13 sent 67

$\mathrm{Pg} \cdot \#$

DIR. BAND NO. REMARKS

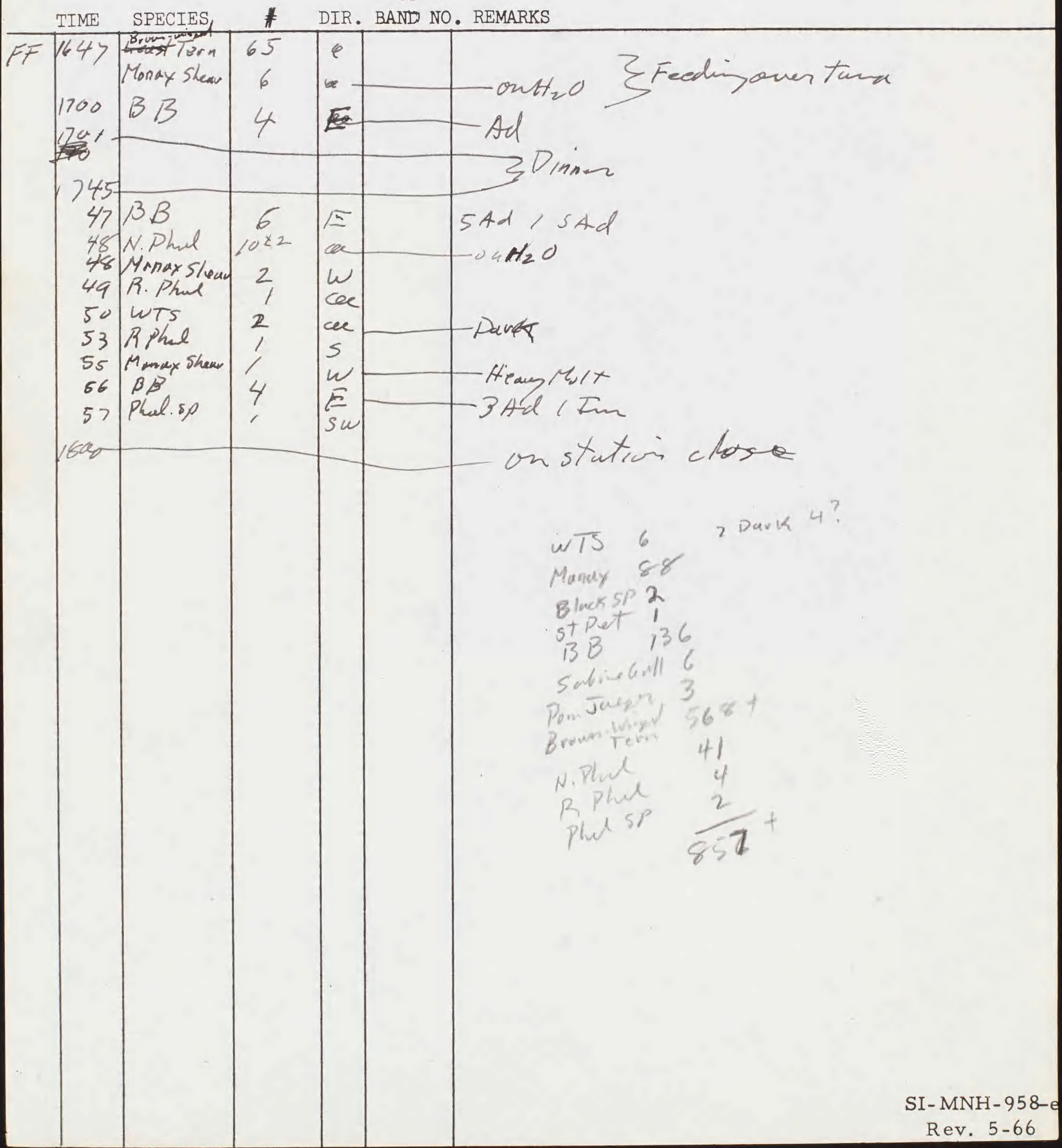




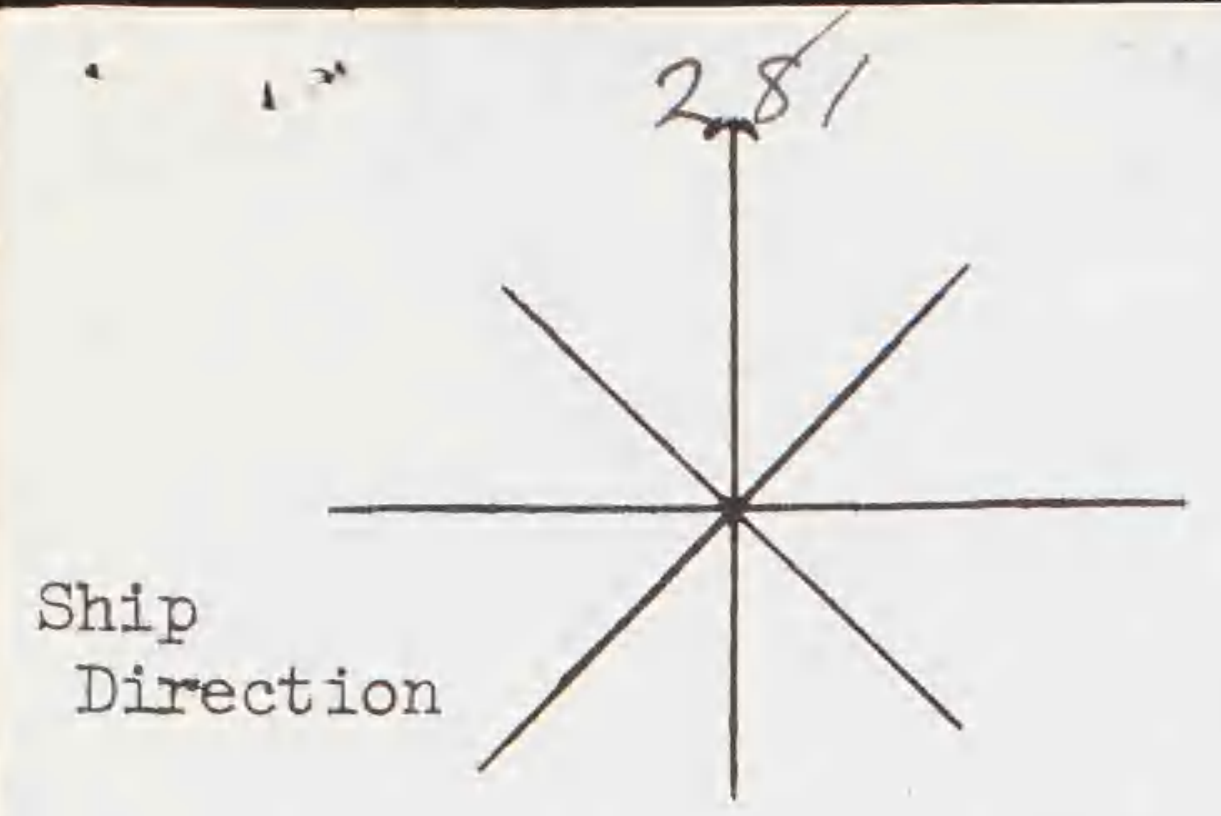

SMITHSONIAN INSTITUTION DIVISION OF BIRDS AT SEA DAILY LOG - E

SPECIMEN or
OBSERVERS:

Date $(4 \operatorname{sen} t 6)$ $\mathrm{Pg} \cdot \#$

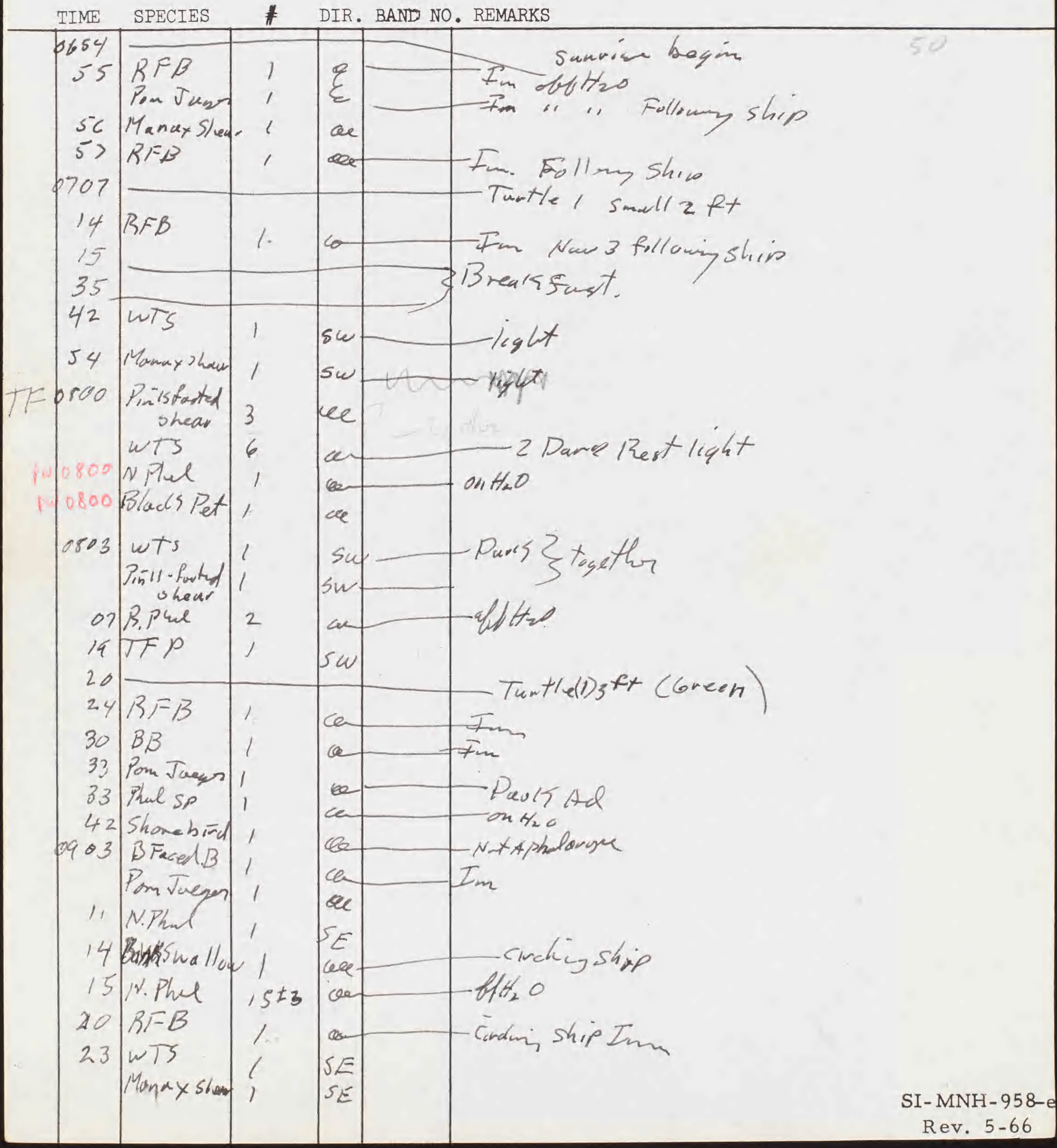




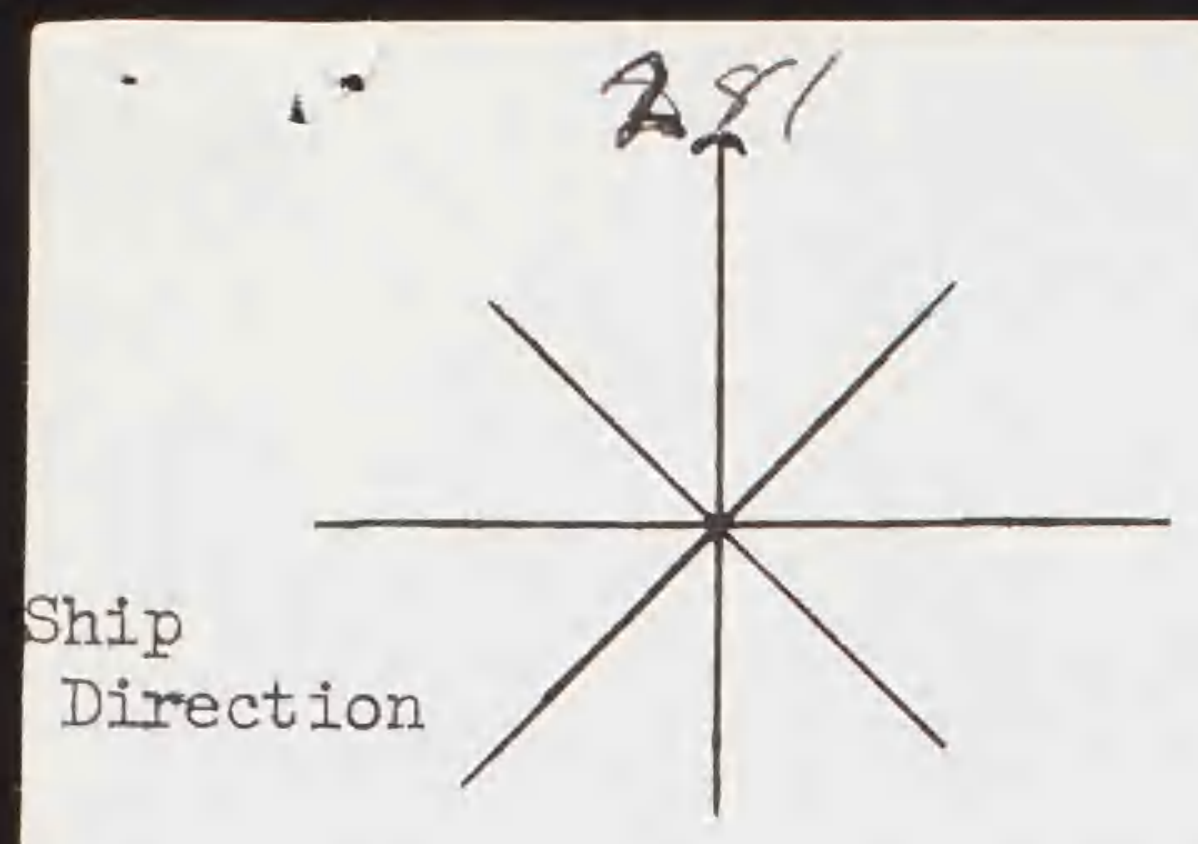

\section{OBSERVERS :}

SMITHSONIAN INSTITUTION

DIVISION OF BIRDS

AT SEA DAILY LOG - E

\section{SPEC IMEN}

or

Date

$\mathrm{Pg} \cdot \#$

TIME SPECIES \# DIR. BAND NO. REMARKS

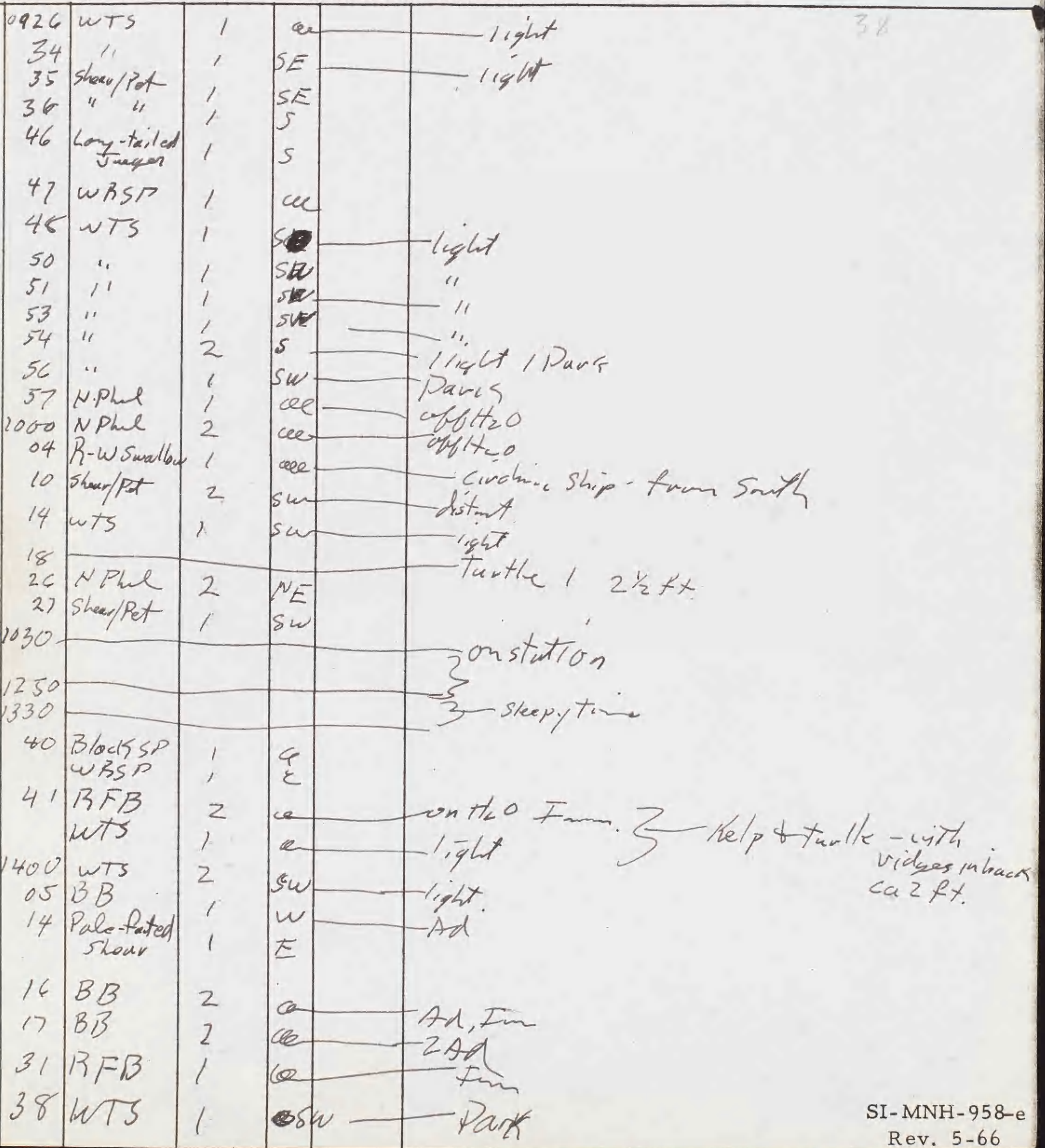




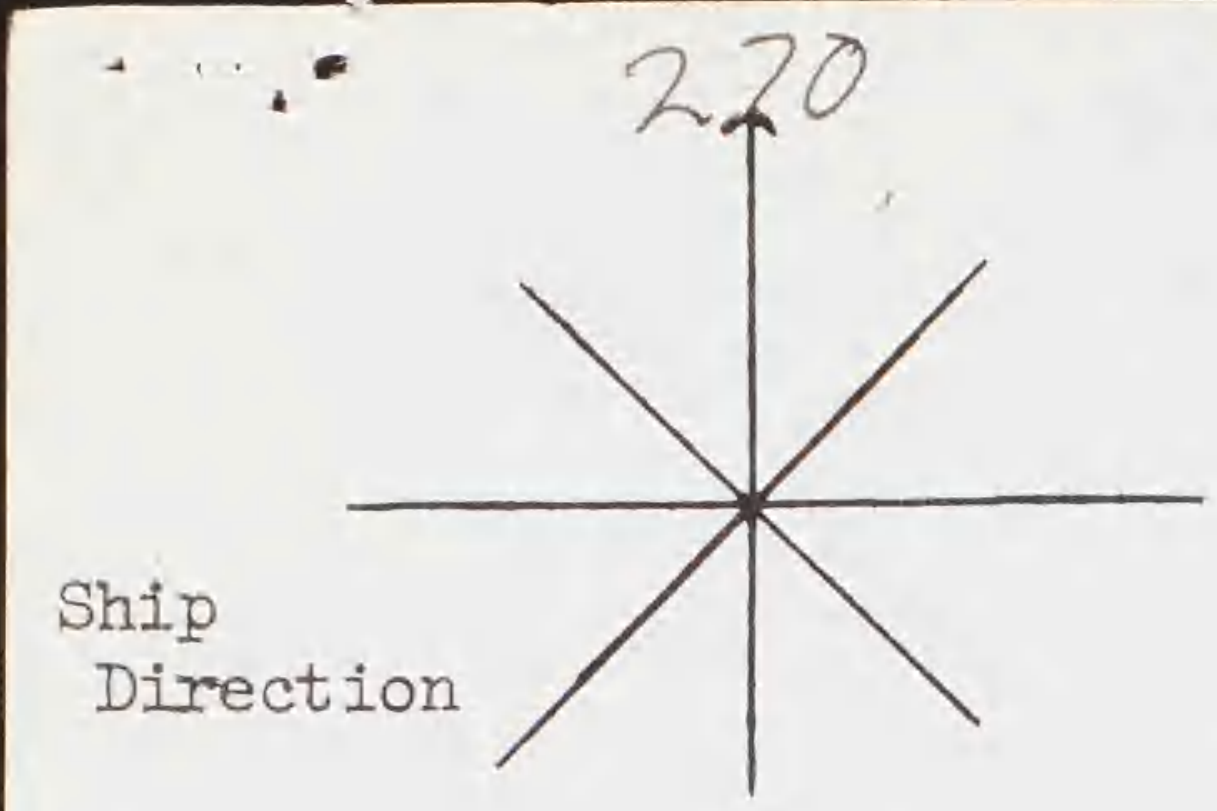

OBSERVERS :

SMITHSONIAN INSTITUTION DIVISION OF BIRDS AT SEA DAILY LOG - E

SPECIMEN

or
Date $\frac{14 \text { sent } 67}{3}$

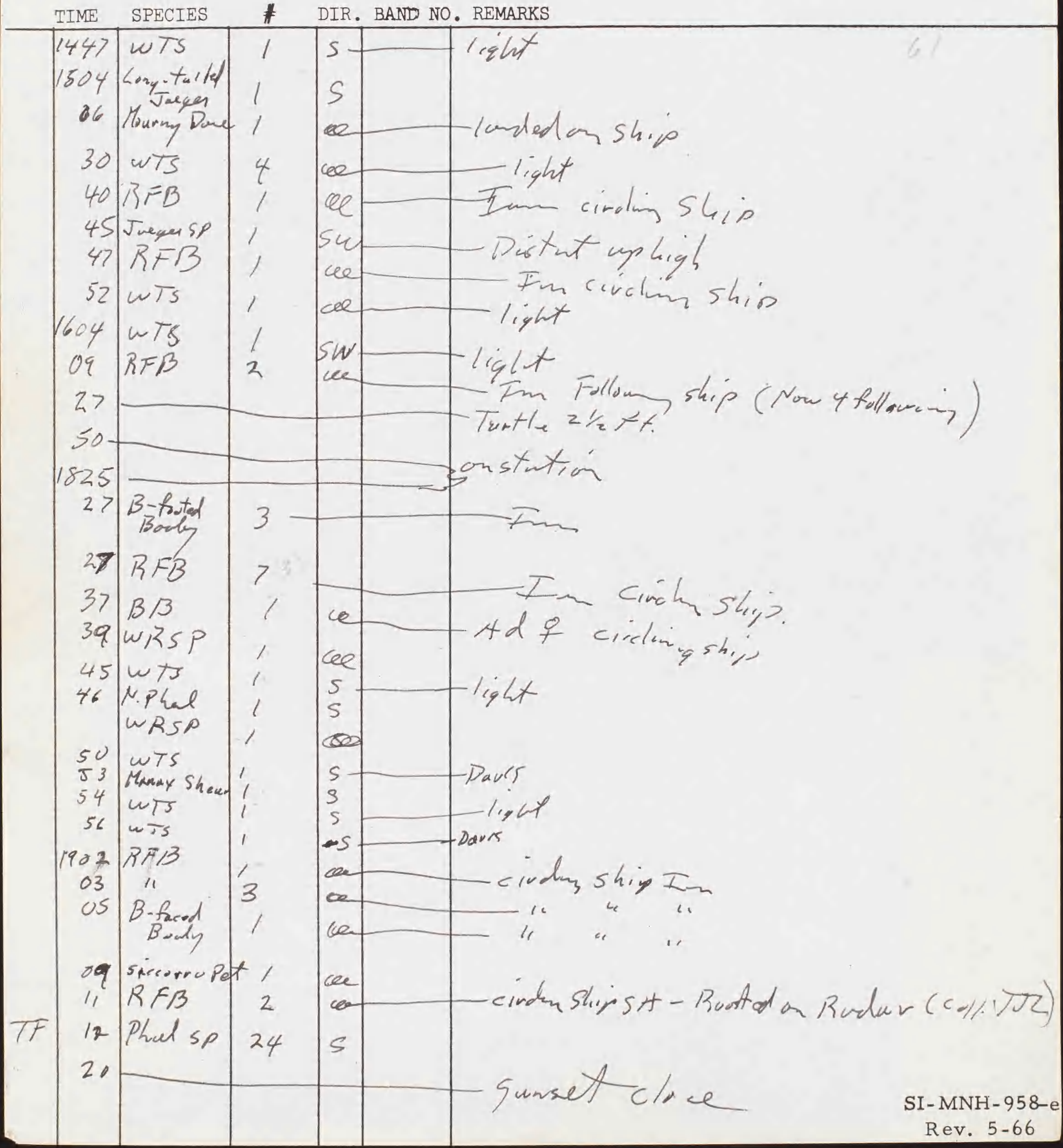




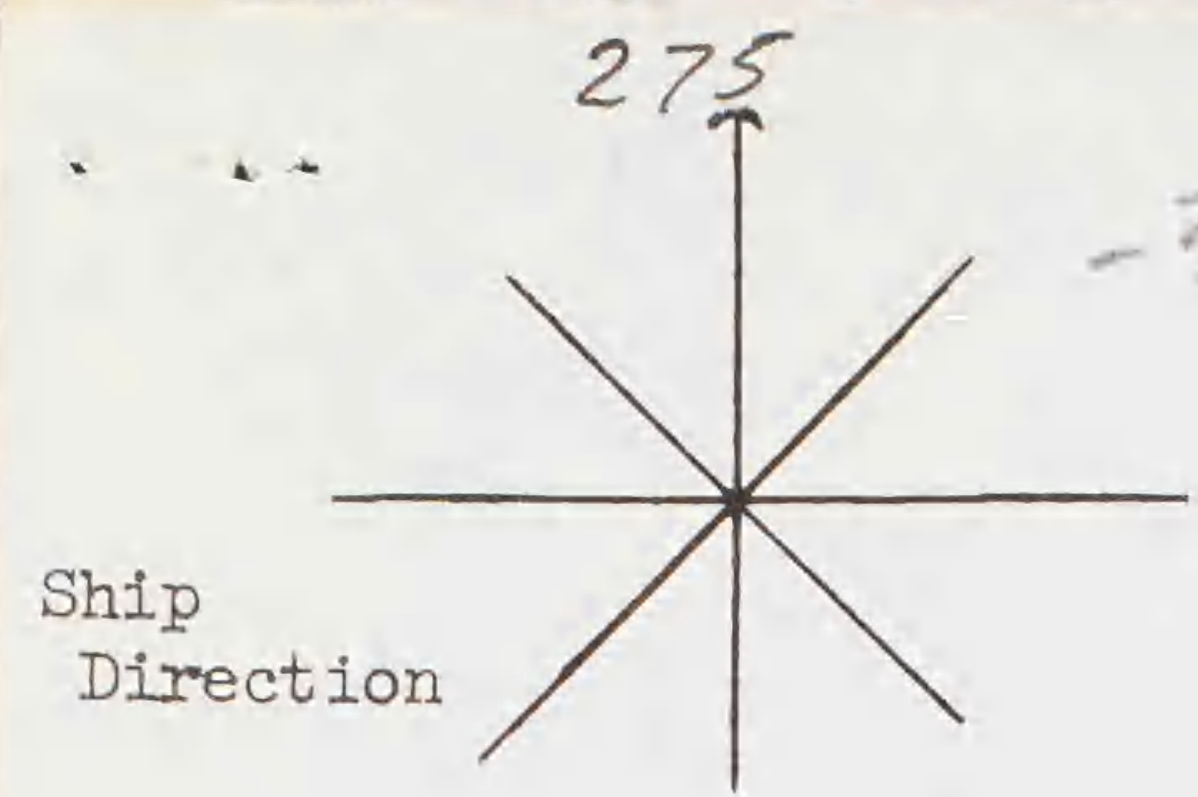

OBSERVERS:

Now ont> Tin

SMITHSONLAN INSTITUTION

DIVISION OF BIRDS

AT SEA DAILY LOG - E

SPECTMEN

or
Date $15 \operatorname{sen}+6\rangle$

$\mathrm{Pg} \cdot \#$

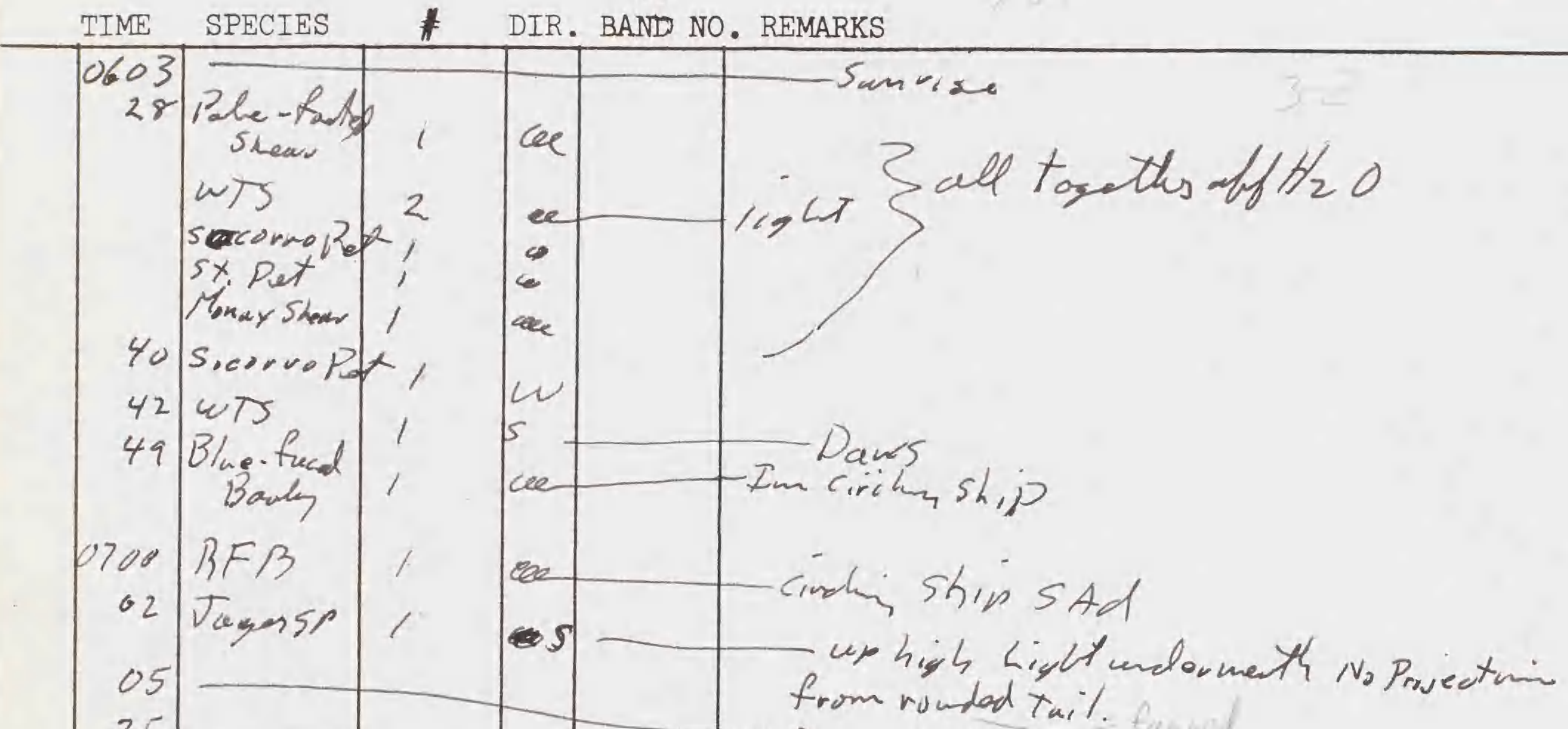

\begin{tabular}{r|l}
25 & \\
43 & WY5 \\
811 & BFB \\
17 & WTS \\
30 & Pale-tontd
\end{tabular}

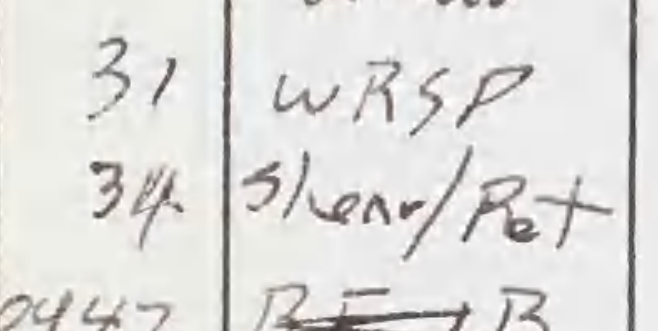

$0447 \frac{B D}{R F B}$

55 Phal SP

WR SP

\begin{tabular}{l|l|}
56 & $R F P$ \\
58 & Pale-fonted
\end{tabular}

shear.

1000

aa 3 Breakfast

5 Dav/5

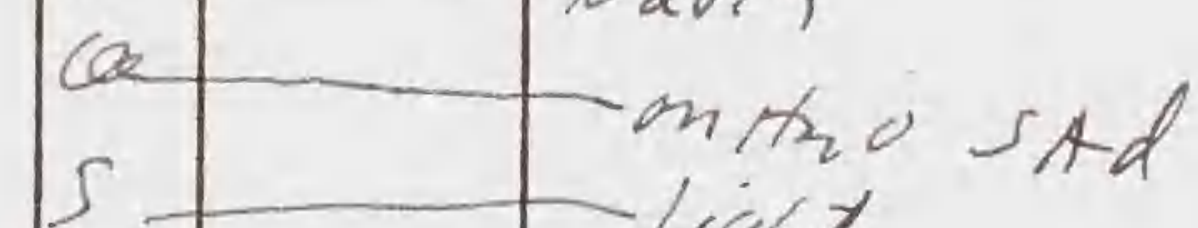

27 Pab-footed

Shearmata

1223

R254 WRSP

,

see

SI- MNH-958-e

Rev. 5-66 


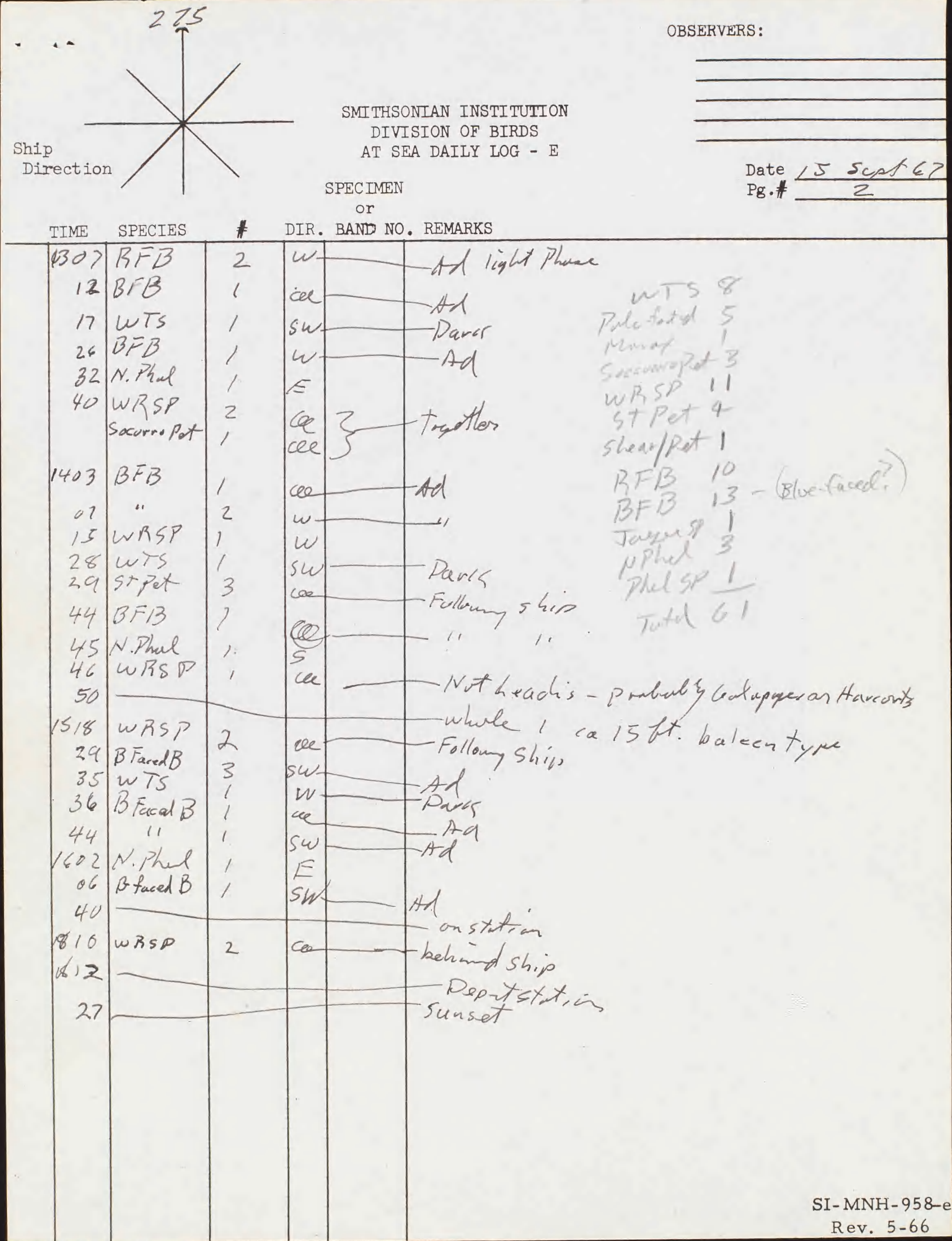



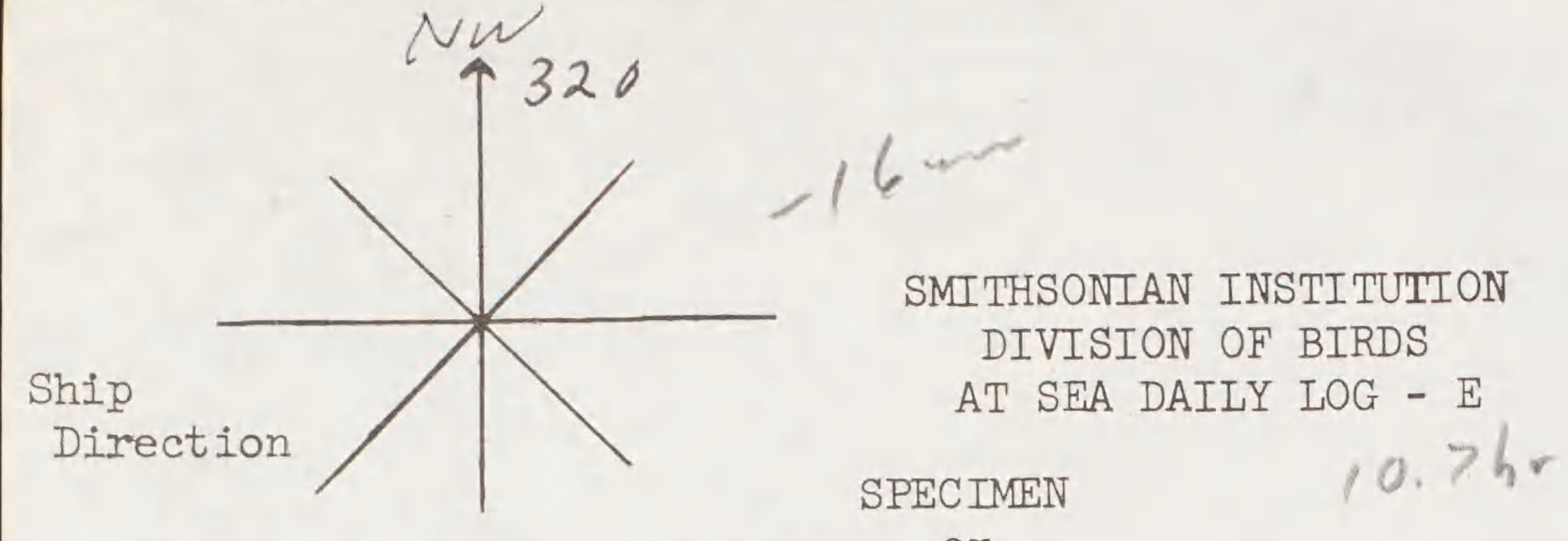

OBSERVERS :

Date $\frac{16 \operatorname{sen} t}{1} \leq 7$

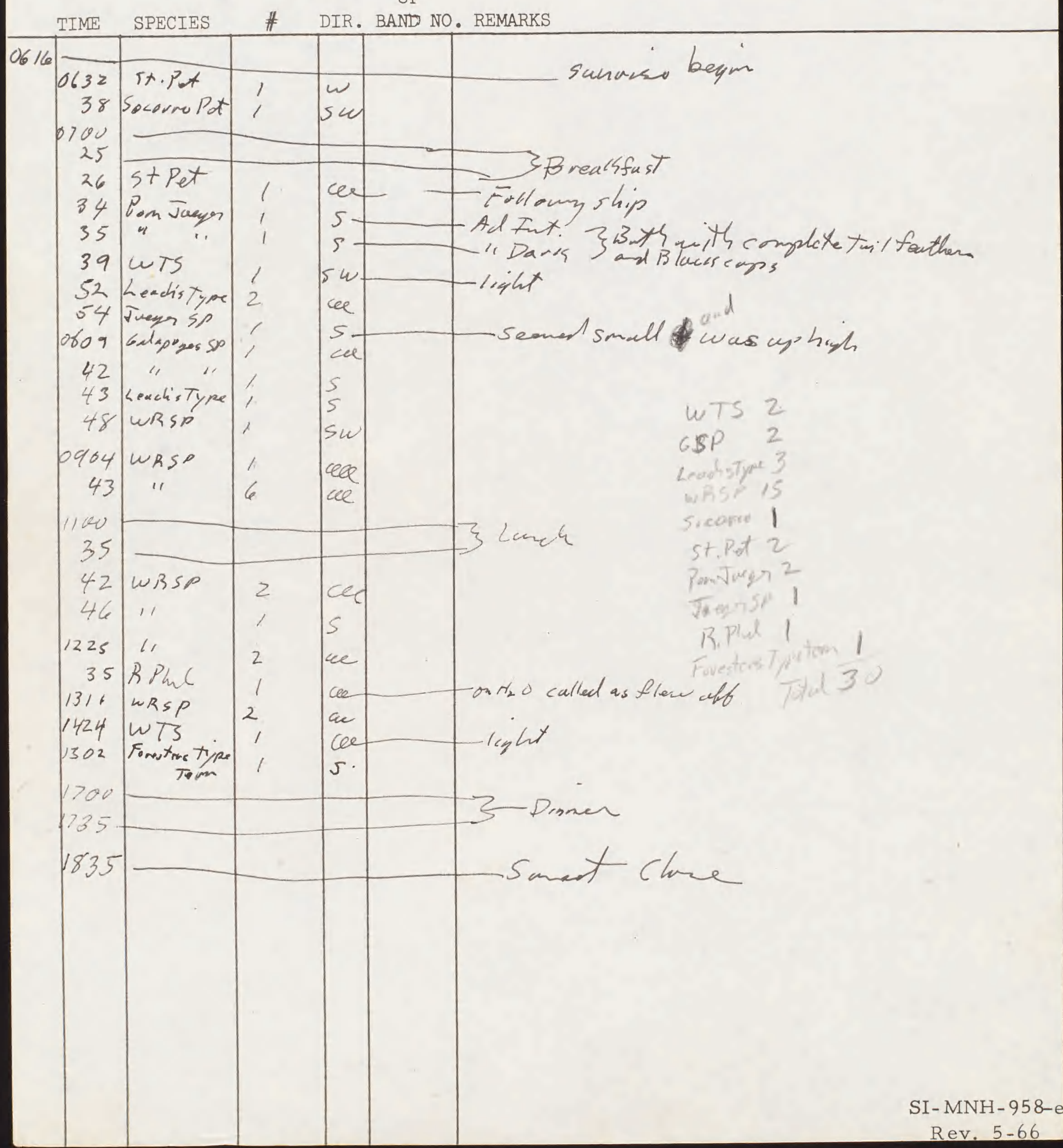


Ship

Direction

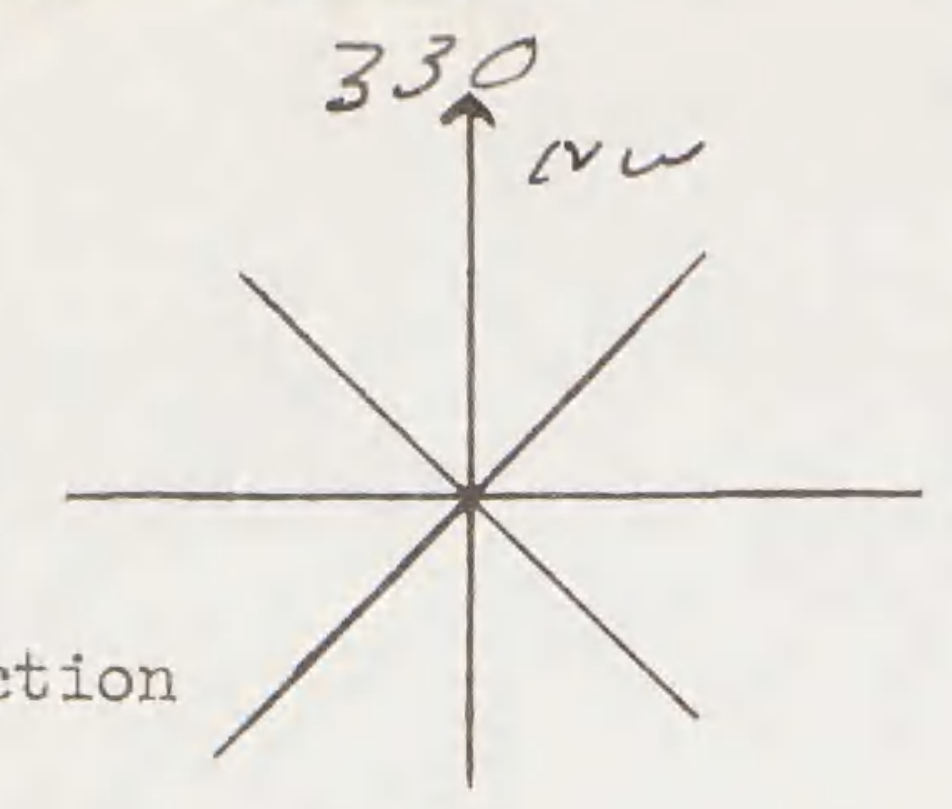

OBSERVERS :

SMITHSONIAN INSTITUTION

DIVISION OF BIRDS

AT SEA DAILY LOG - E

SPEC IMEN

or

$9.3 \mathrm{hr}$
Date 175en 67

$\mathrm{Pg} \cdot \#$

TIME SPECIES \# DIR. BAND NO. REMARKS

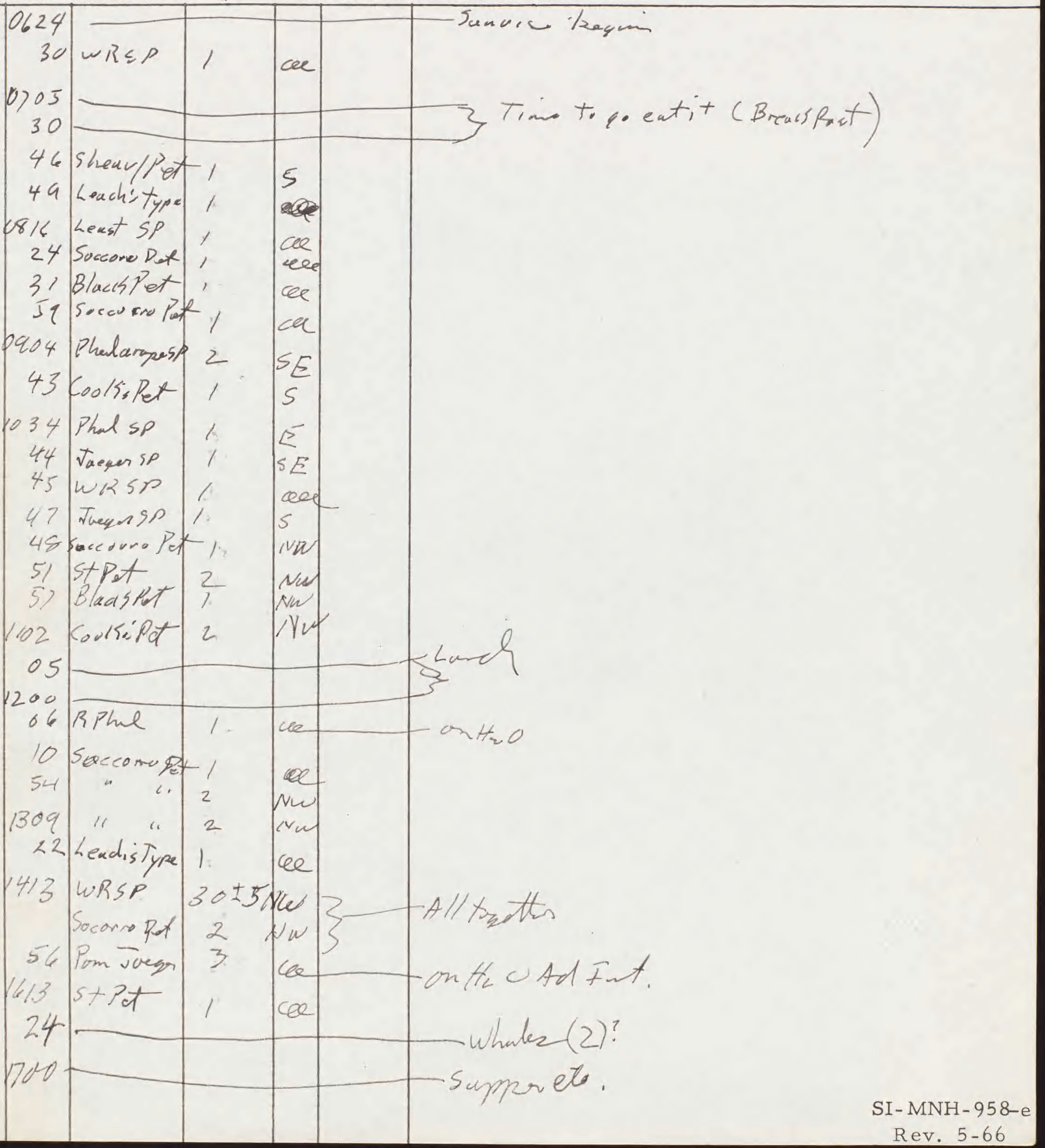




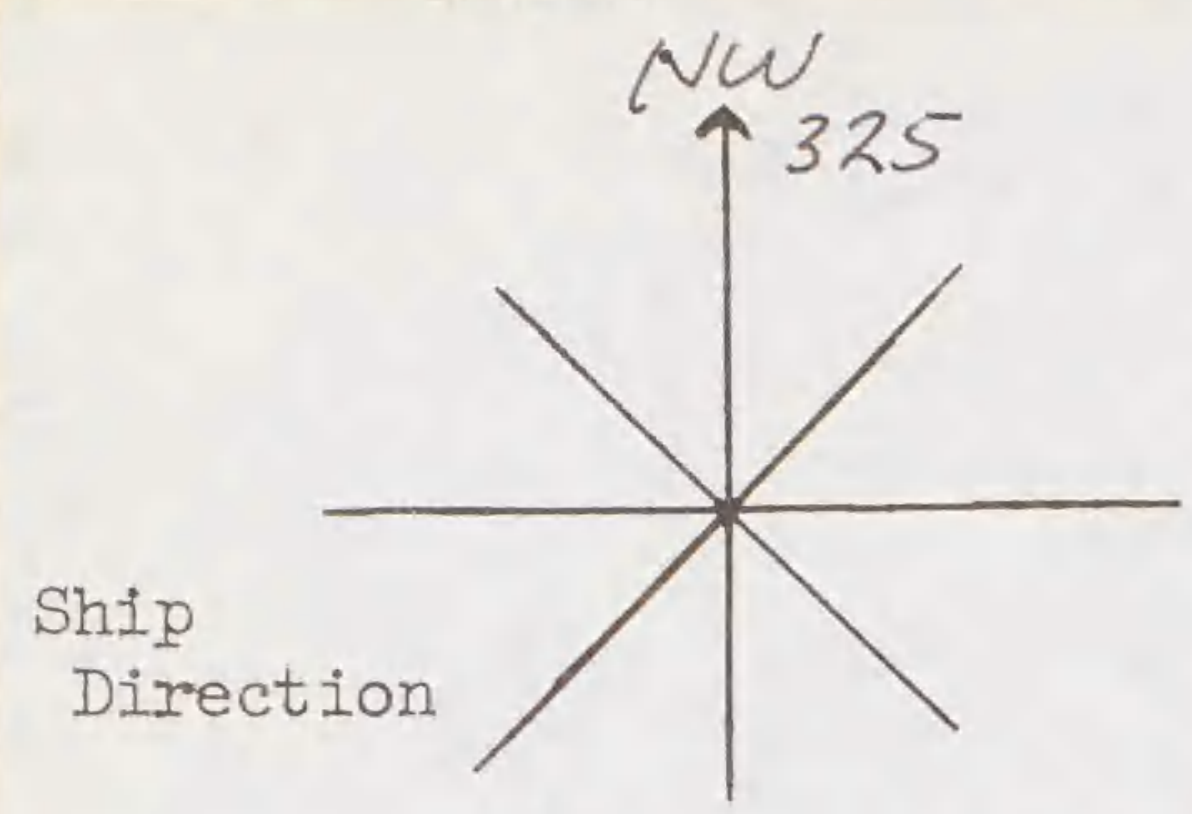

OBSERVERS :

SMITHSONIAN INSTITUTION DIVISION OF BIRDS AT SEA DAILY LOG - E

SPECIMEN 7. 6 her
Date $\langle 8$ Sept 6$\rangle$ $\mathrm{Pg} \cdot \#$

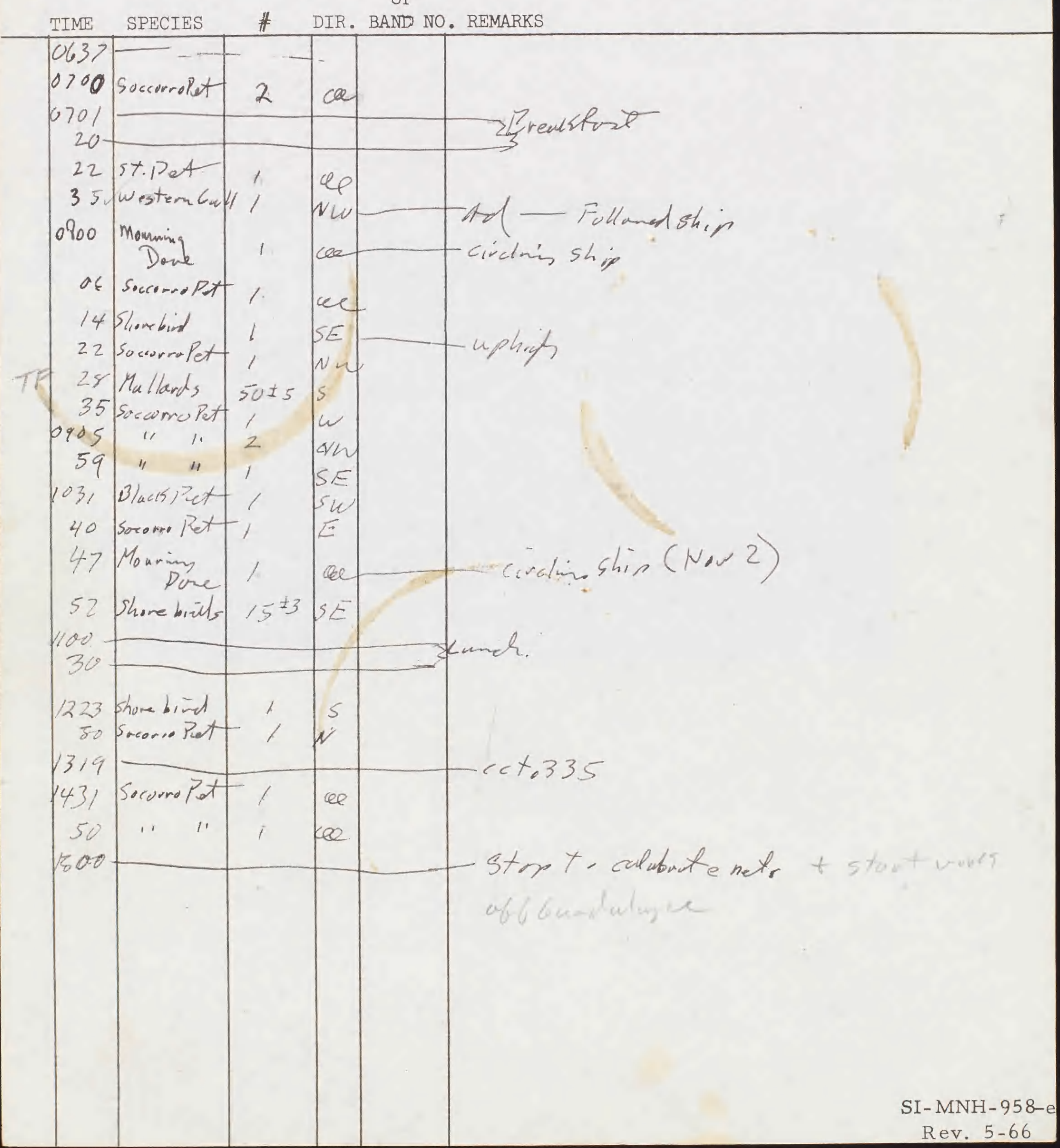




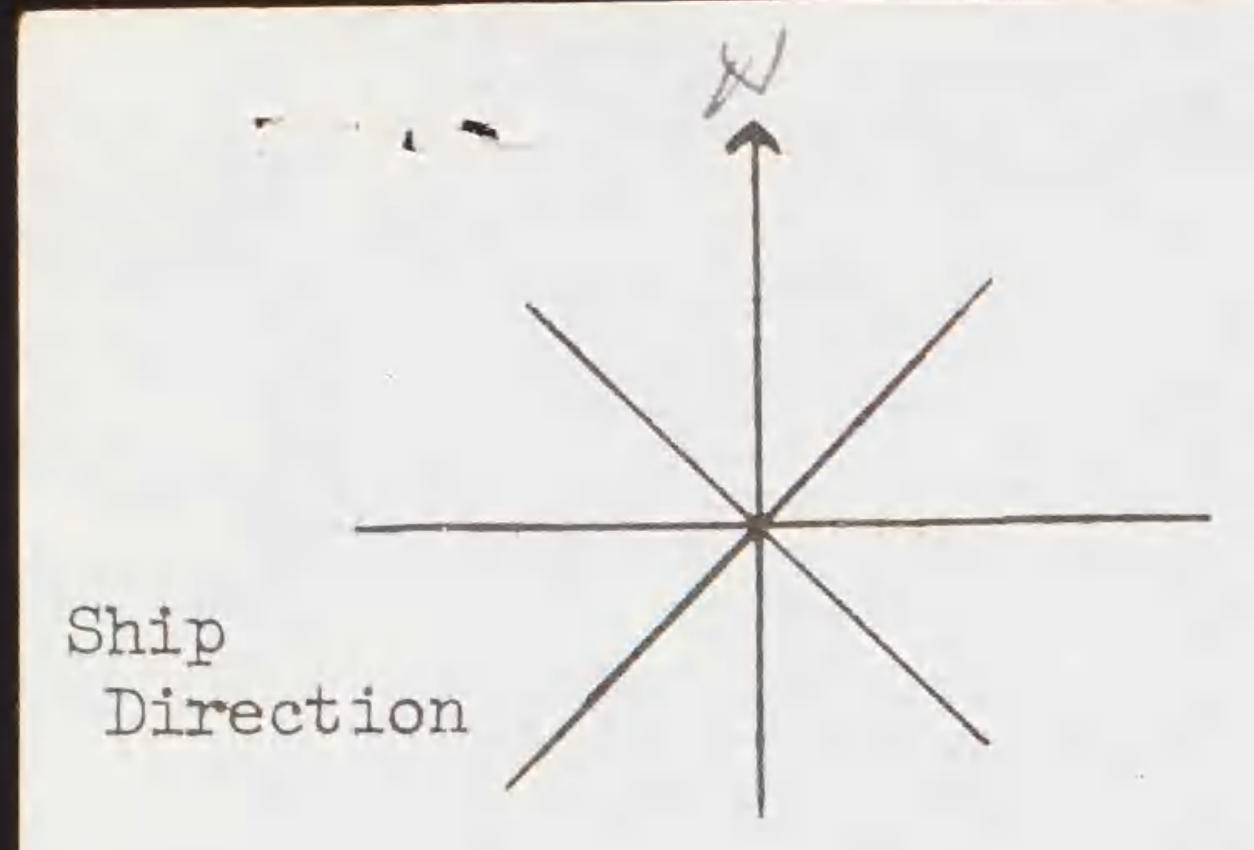

\section{OBSERVERS :}

SMITHSONLAN INSTITUTION DIVISION OF BIRDS AT SEA DAILY LOG - E

SPECIMEN

or

10.4 hr.

Date $24 \operatorname{sen} T 62$

$\mathrm{Pg} \cdot \#$

TIME SPECIES \# DIR. BAND NO. REMARKS

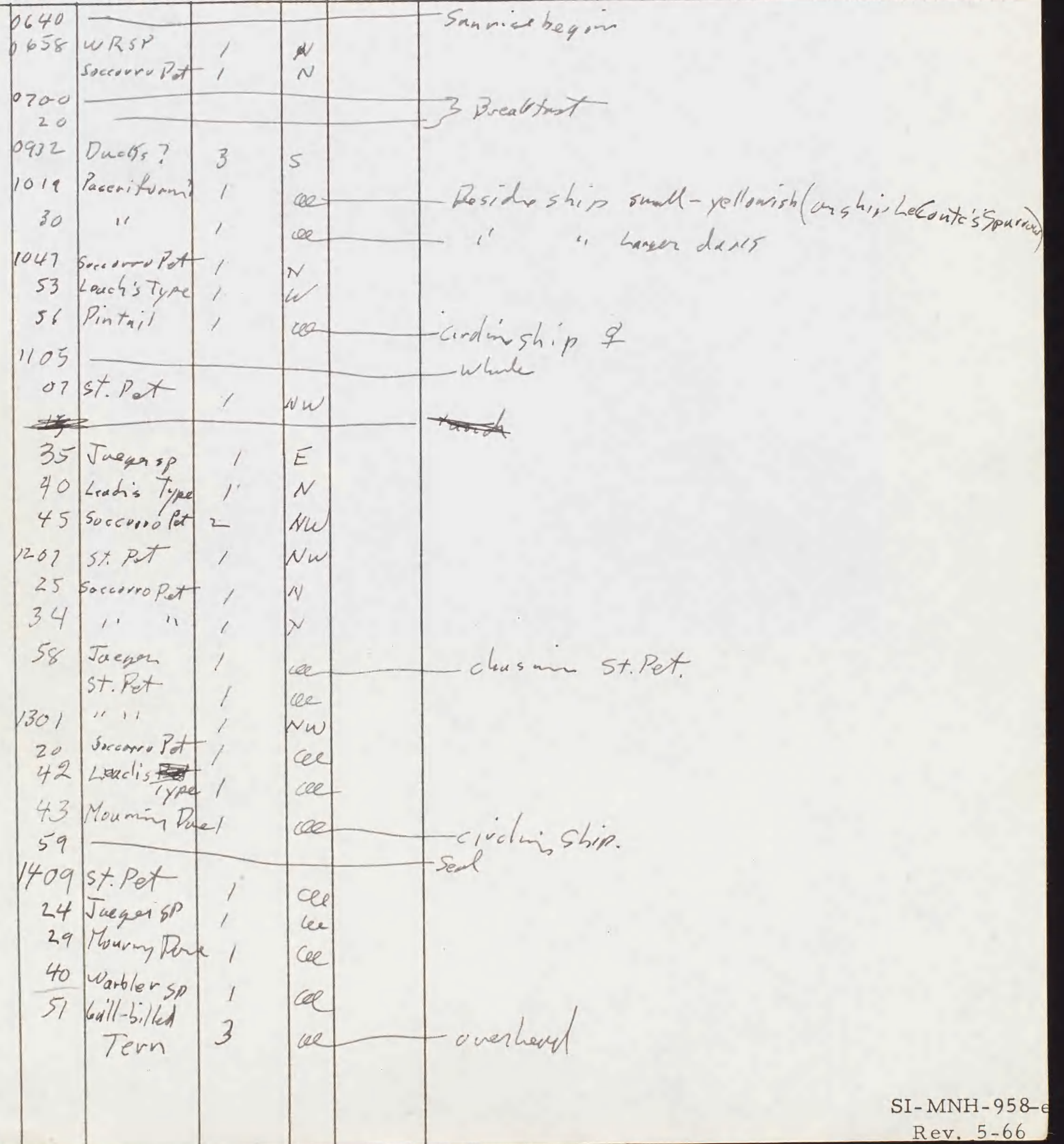

Rev. 5-66 

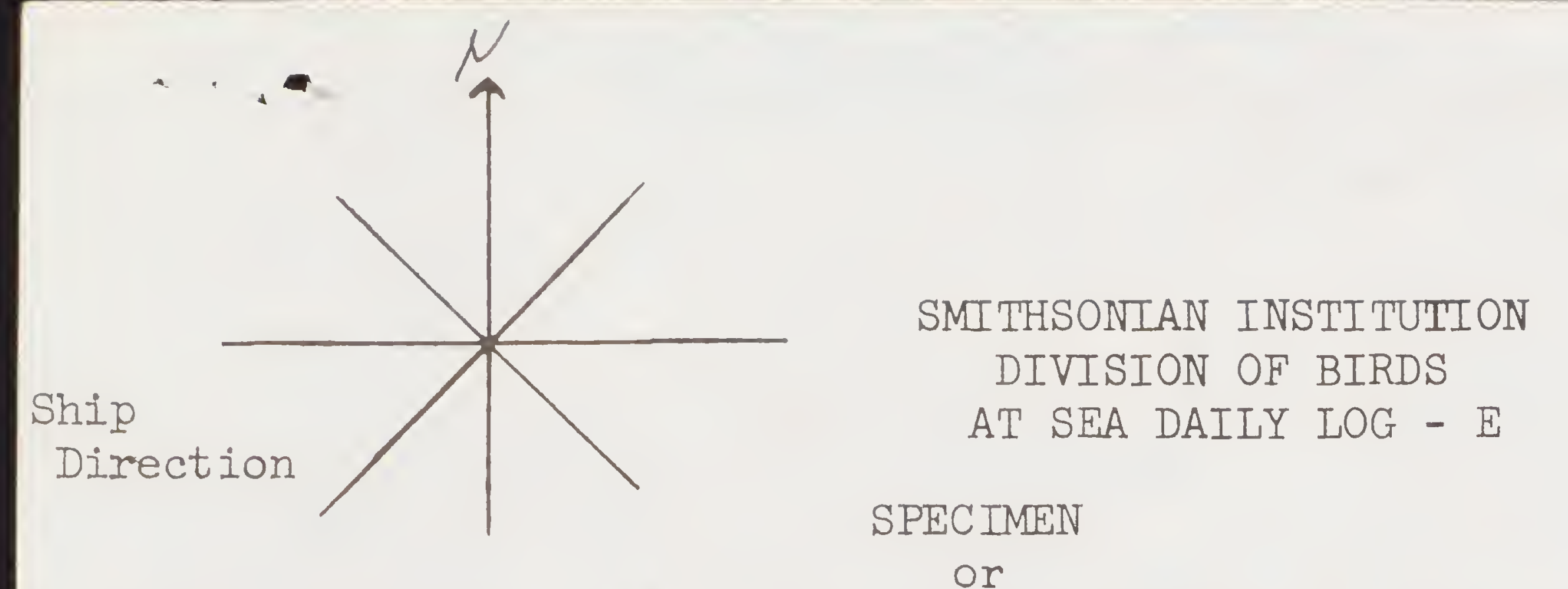

OBSERVERS :

Date 245 ant 62

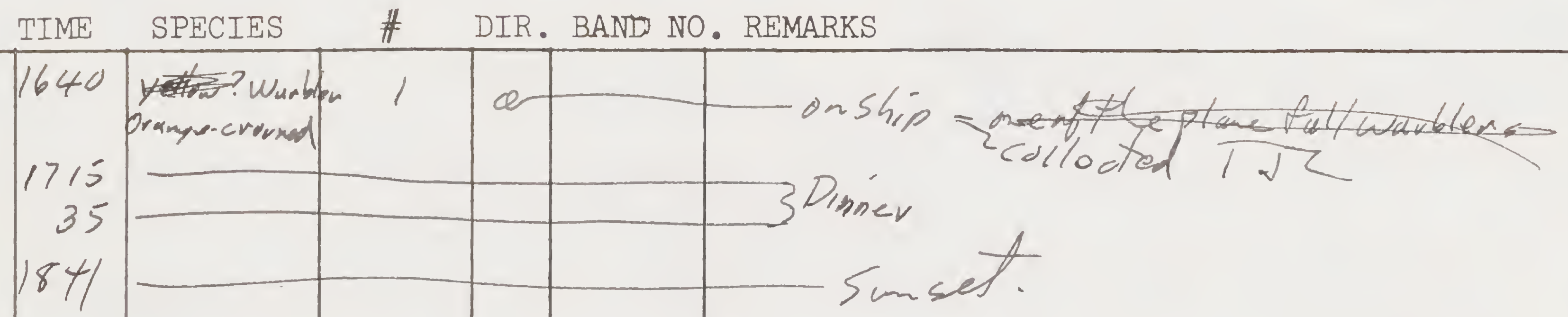


Date 3 Anqust 67

organization PUBSP
Ship T. Washington(

Recorder T.J. Lewes
Sunrise: Time

Sunset: Time 1944
Position: Lat.

Position: Lat.
Cruise No. $228-0002$

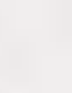

Miles travelled from 0000 hours to sunrise =

Miles travelled from sunrise to sunset $=-31$

Miles travelled from sunset to 2400 hours $=\frac{43}{2}$

TIME OF FIX TYPE OF FIX IATITUDE IONGITUDE

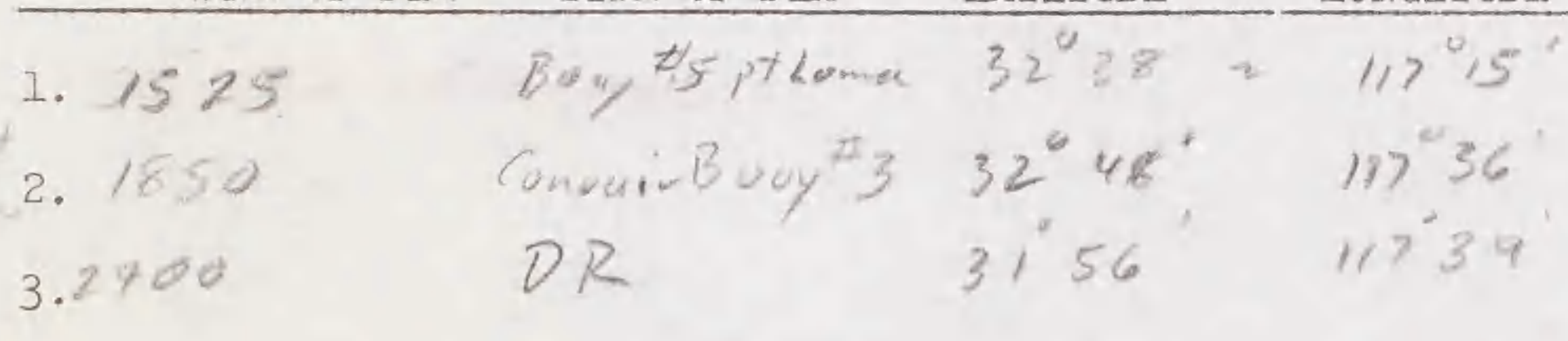

4.

5 .

Hourly Positions:

Time Latitude Longitude Wind Dir. Wind Sp. Wave Dir. Wave Hgt.

\begin{tabular}{|c|c|c|c|c|c|c|}
\hline 0100 & & & & & & \\
\hline 0200 & & & & & & \\
\hline 0300 & & & & & & \\
\hline 0400 & & & & & & \\
\hline 0500 & & & & & & \\
\hline 0600 & & & & & & \\
\hline 0700 & & & & & & \\
\hline 0800 & & & & & & \\
\hline 0900 & & & & & & \\
\hline$\frac{1000}{1100}$ & & & & & & \\
\hline$\frac{1100}{1200}$ & & & & & & \\
\hline 1300 & & & & & & \\
\hline 1400 & & & & & & \\
\hline 525 & 32038 & $117^{\circ} 15$ & & & & \\
\hline 1600 & 40 & 20 & & & & \\
\hline .700 & 44 & 29 & & & & \\
\hline 1800 & $32-48$ & $117^{\circ} 36$ & & & & \\
\hline 1900 & 3248 & 11736 & & & & {$[+$} \\
\hline 2000 & $32 \quad 31$ & & & & & \\
\hline 2100 & & C & & & & \\
\hline$\frac{2200}{2300}$ & 31104 & 11790 & & & & - \\
\hline$\frac{2300}{2400}$ & $3 / 34$ & 11722 & & & & \\
\hline
\end{tabular}


Date 4 Auqust 6 ?

Organization BOBSP
Ship T. Washington (

Recorder T.J. Lewis
Sunrise: Time $05 / 2$

Sunset: Time 1842
Position: Lat. $30^{\prime} 5$, Iong. $117^{\circ 4} 49$

Position: Lat. $28^{\prime \prime} 37$, Long. $11800^{\prime}$

Miles travelled from 0000 hours to sunrise $=6 /$

Miles travelled from sunrise to sunset $=$

Miles travelled from sunset to 2400 hours $=64$

TIME OF FIX TYPE OF FIX LATITUDE LONGITUDE

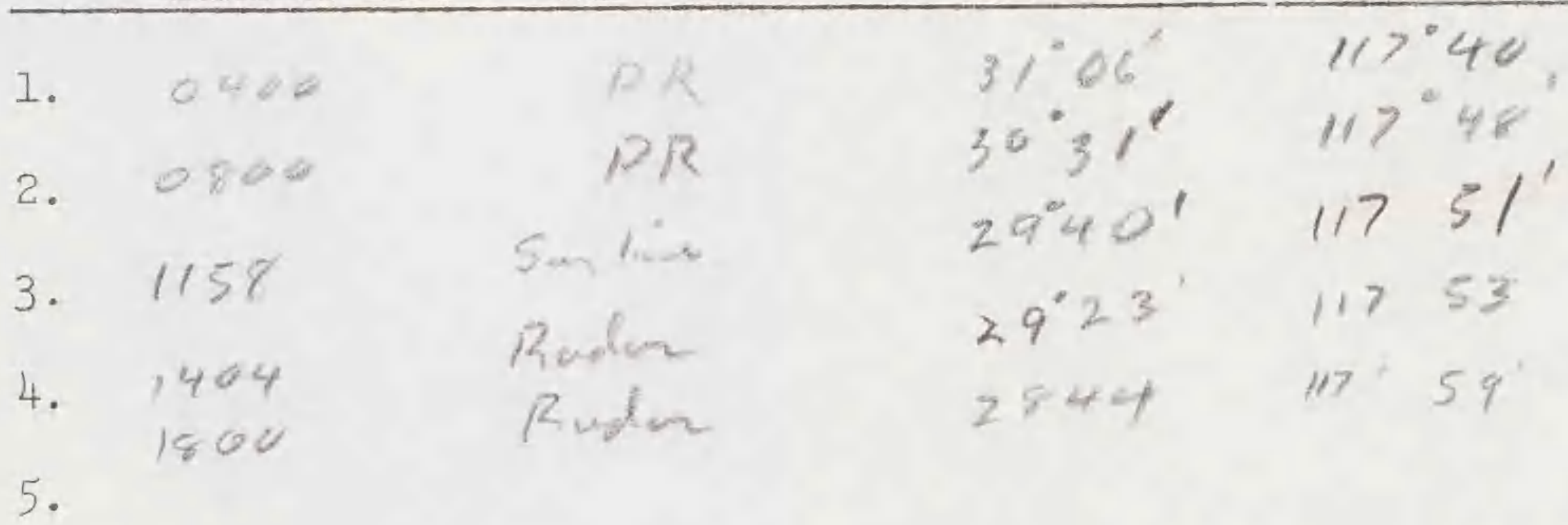

Hourly Positions:

theng to +8 The 2 we

Time Latitude Longitude Wind Dir. Wind Sp. Wave Dir. Wave Hgt.

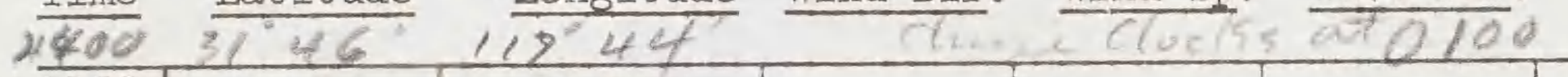

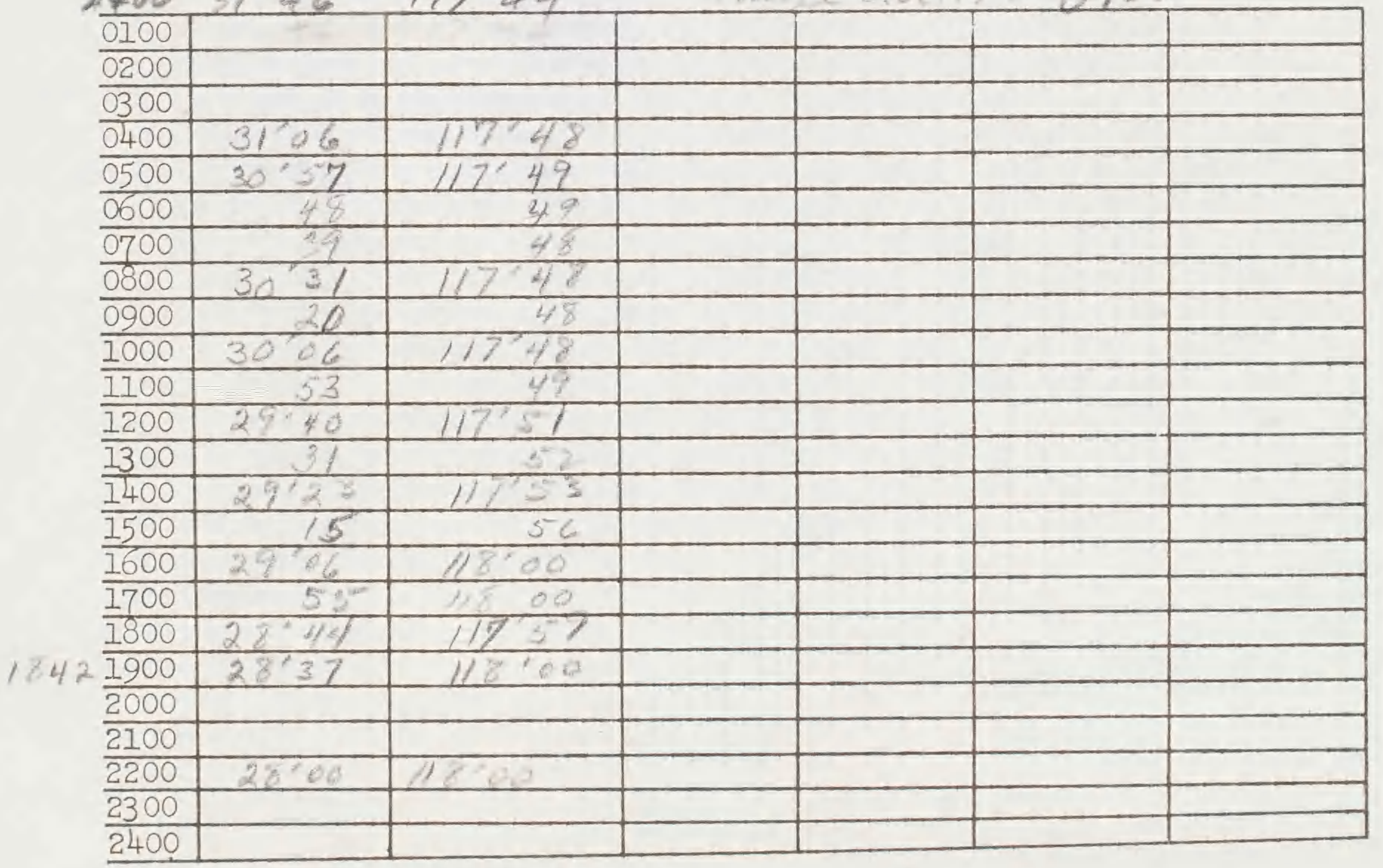


Date 5 August 67

organization BOBSP

Sunrise: Time

Sunset: Time 1835
Ship T. Washingtun

Recorder T.T.hewis

Position: Lat. $26^{\circ} 45^{\prime}$, Long. $118^{\prime} 09^{\prime}$

Position: Lat. $24^{\circ} 5 /{ }^{\prime}$, Iong. $1 / 8^{\prime} 15$

Miles travelled from 0000 hours to sunrise $=56$ m

Miles travelled from sunrise to sunset

$=114$

Miles travelled from sunset to 2400 hours $=46$

TIME OF FIX TYPE OF FIX LATITUDE

LONGITUDE

1 .

0808
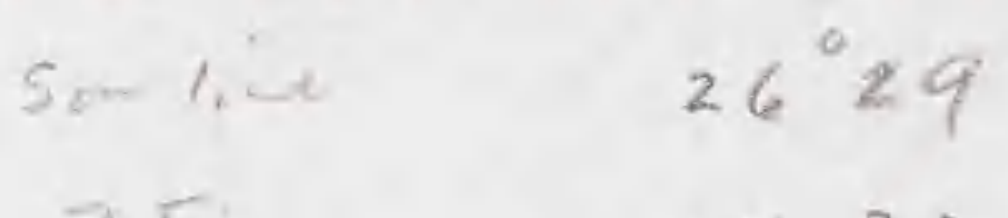

2. 1315

$3 F$ iy

2537

118,0

3.

4.

5. 1630

$D R$

$25^{\circ} 00^{\circ}$

$118^{\circ} 14$

Hourly Positions:

Time Latitude Longitude Wind Dir. Wind Sp. Wave Dir. Wave Hgt.

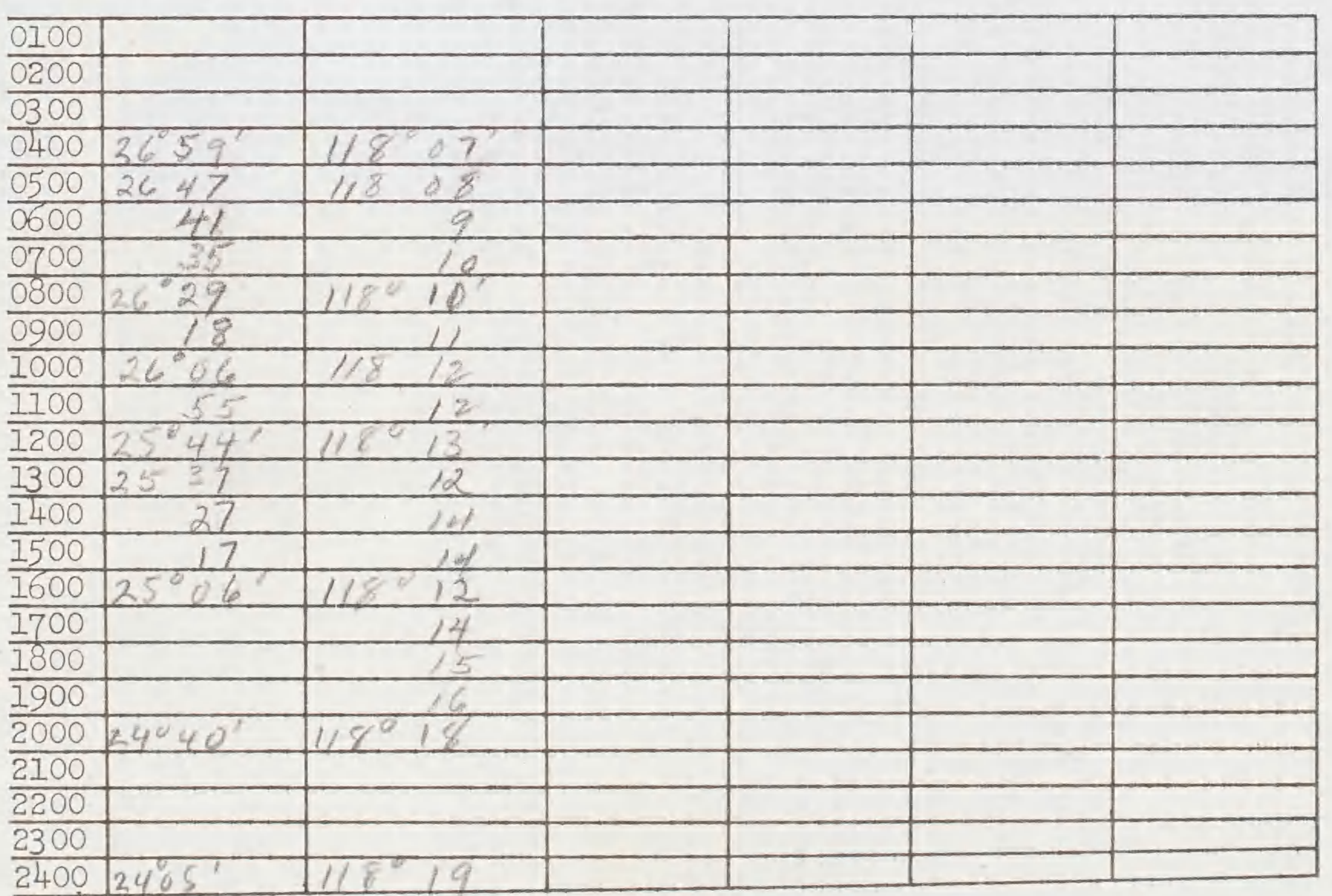


Date 6Auy 67 Ship T. Washington (_) Cruise No.028-0002 organization PUBSP RecorderI.J.Lewis

Sunrise: Time 0522 Position: Lat. $23^{\circ} 12^{\prime}$, Iong. $118^{\circ} 4$ Sunset: Time 1832 Position: Lat. $22^{\prime \prime} \mathrm{eq}^{\prime}$, Long. $118^{\circ} 31$

Miles travelled from 0000 hours to sunrise $=$

Miles travelled from sunrise to sunset $=-68$

Miles travelled from sunset to 2400 hours $=42$

TIME OF FIX TYPE OF FIX LATITUDE LONGITUDE

1.

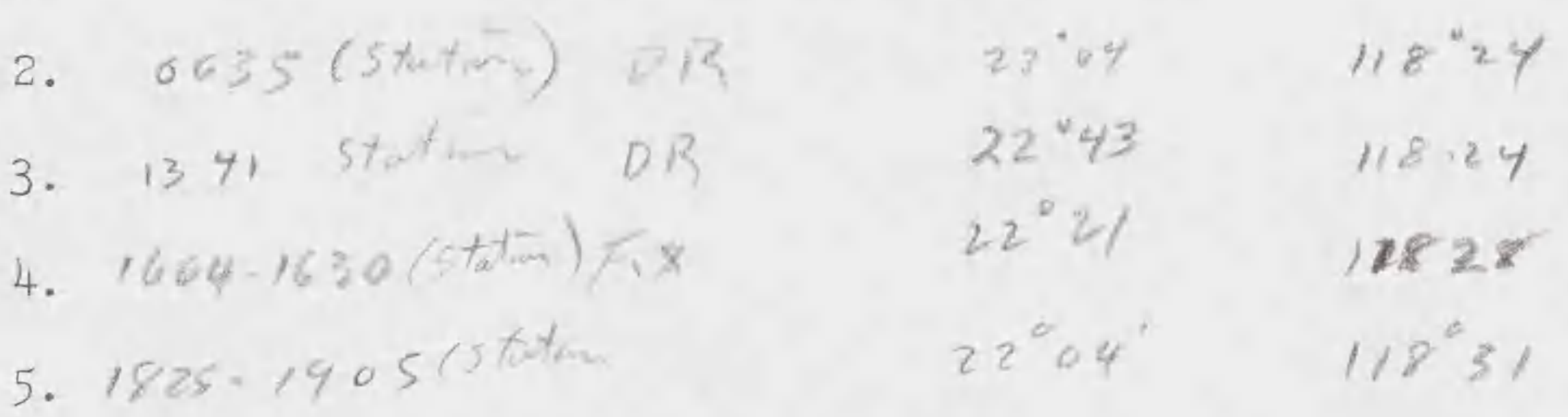

Hourly Positions:

Time Latitude Longitude Wind Dir. Wind Sp. Wave Dir. Wave Hgt.

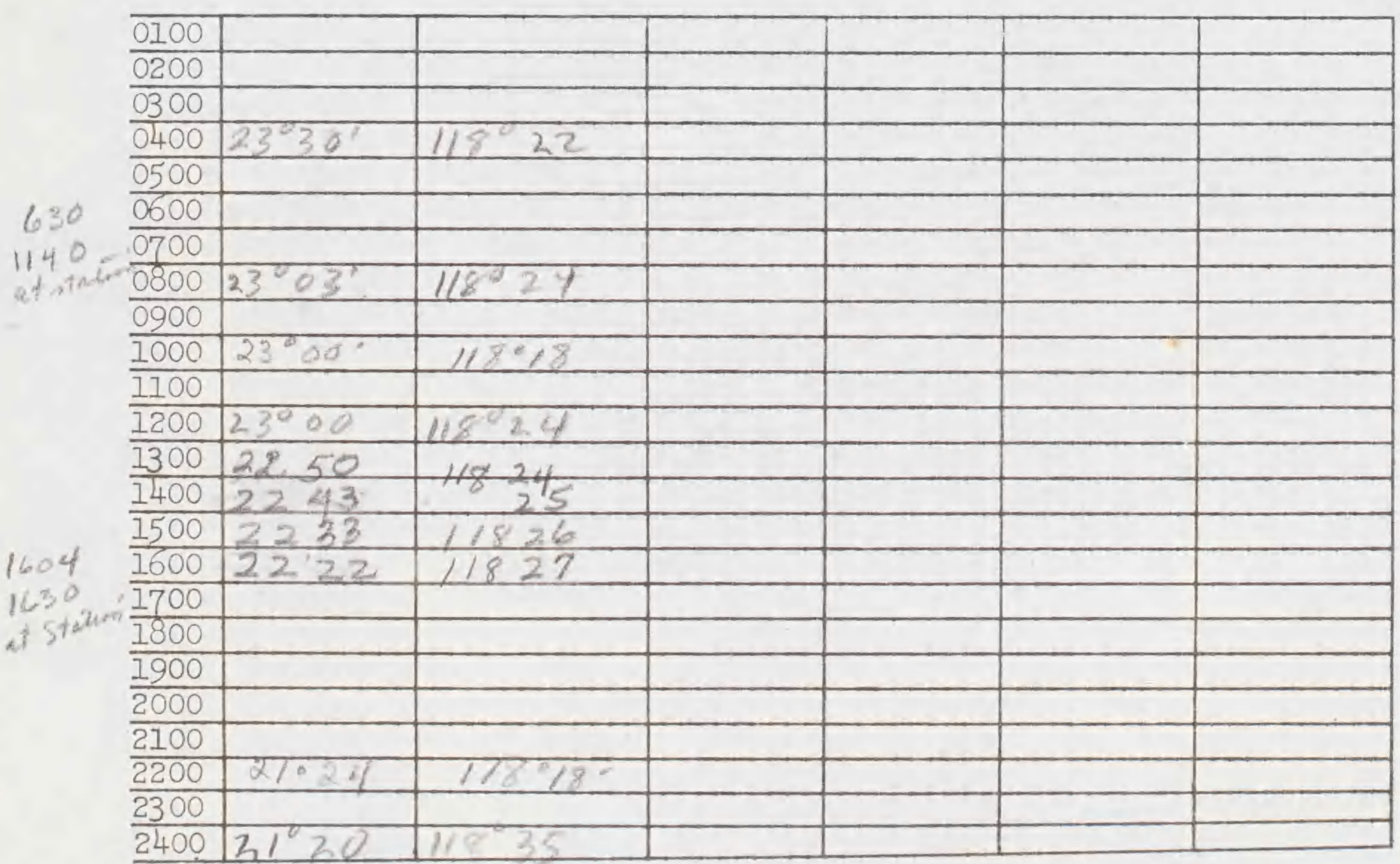


Date? Auqust 67

Organization POBSP
Ship T. Washinaturn

Recorder Lewis
Sunrise: Time 0528

Sunset: Time 1831
Position: Lat. $200^{\circ}$ it $8^{\circ}$

Position: Lat.
Cruise No.028-0002

Miles travelled from 0000 hours to sunrise $=40$

Miles travelled from sunrise to sunset $=49$

Miles travelled from sunset to 2400 hours $=39$

TIME OF FIX TYPE OF FIX LATITUDE IONGITUDE

1.

2 .

3.

4.

5 .

Hourly Positions:

Time Iatitude Longitude Wind Dir. Wind Sp. Wave Dir. Wave Hgt.

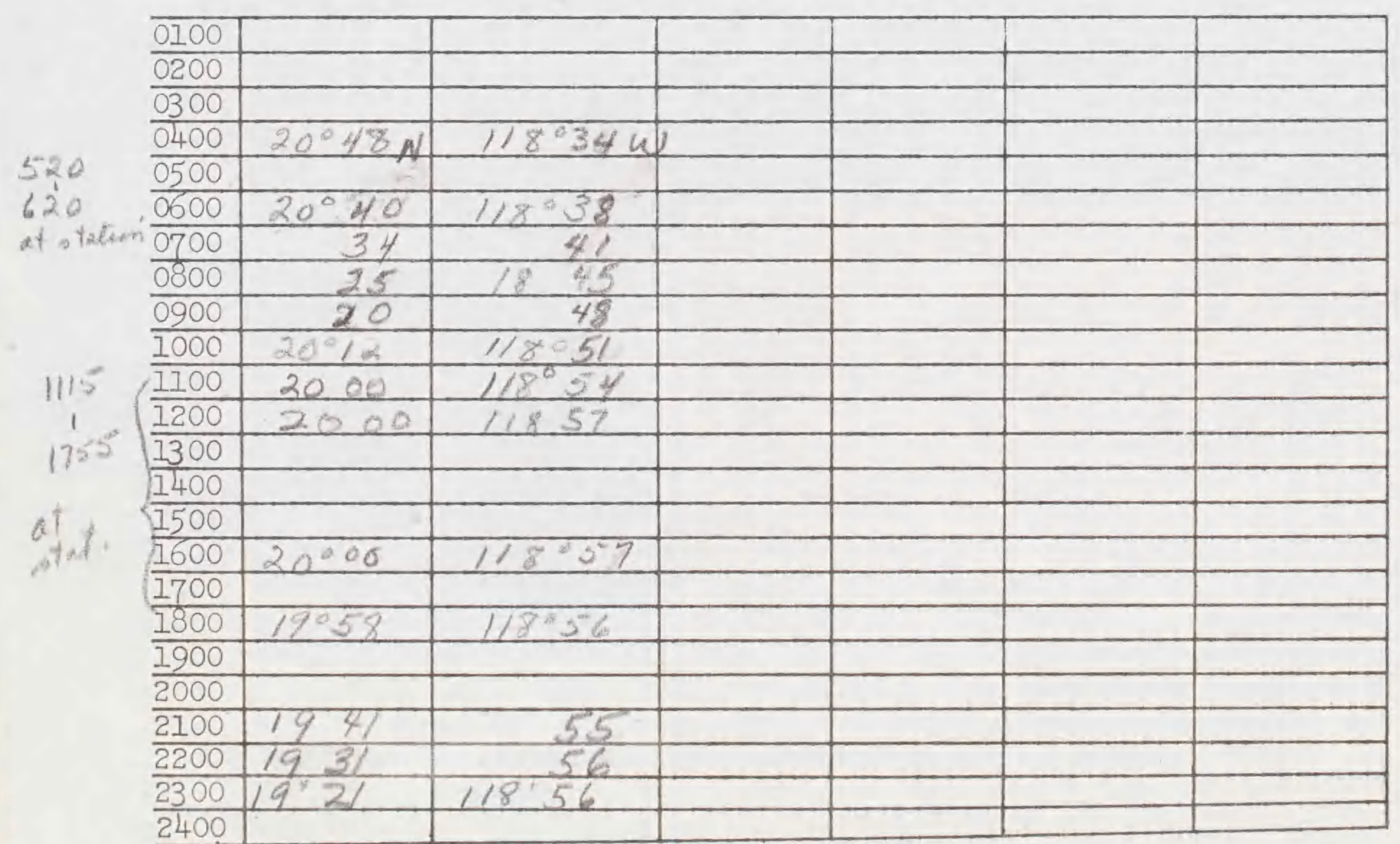


Date 8 August 6?

Organization POBCP
Ship I Washingtun (

Recorder
Sunrise: Time 0535

Sunset: Time 1827
Position: Lat. $18^{\prime} 42$, Long.

Position: Lat. $11^{\circ} \mathrm{y}^{2}$, Long.

Miles travelled from 0000 hours to sunrise $=$

Miles travelled from sunrise to sunset =

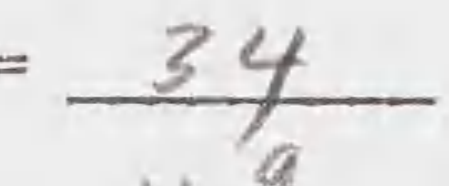

Miles travelled from sunset to 2400 hours $=32$

TIME OF FIX TYPE OF FIX LATITUDE LONGITUDE

1.

2.

3.

4.

5.

Hourly Positions:

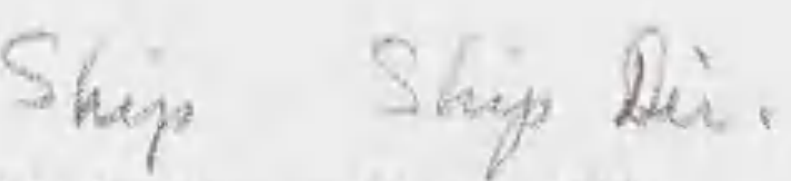

Time Latitude Longitude Wind Dir. Wind Sp. Wave Dir. Wave Hgt.

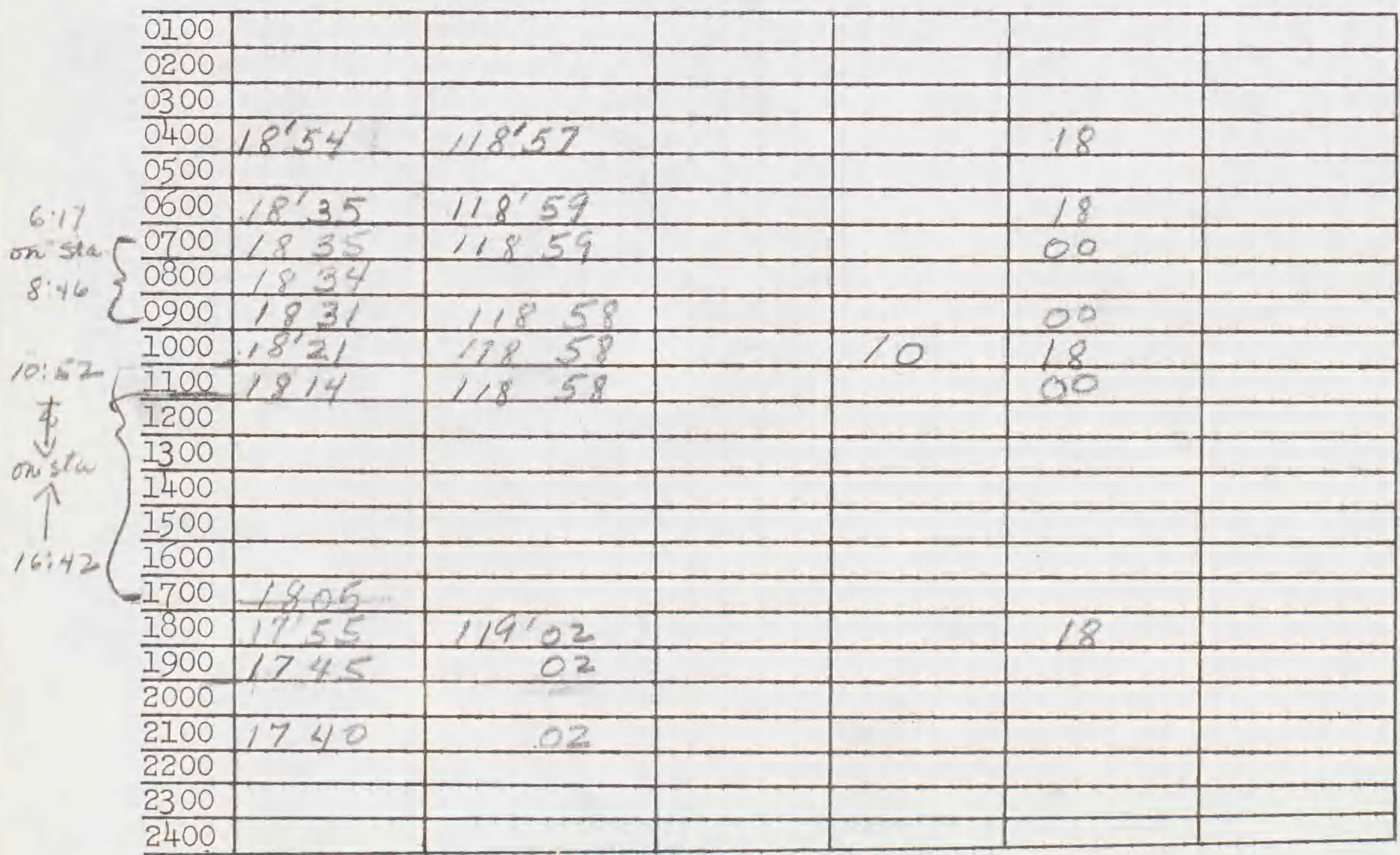


Date 9 Auqust 47

organization POBSP
Ship T. Washington

Recorder_Lewis
Sunrise: Time 0538

Sunset: Time 1823
Position: Lat. 16

Position: Lat.
Cruise No.028-0002

Miles travelled from 0000 hours to sunrise $=30$

Miles travelled from sunrise to sunset $=\frac{60}{36}$
Miles travelled from sunset to 2400 hours $=3$

TIME OF FIX TYPE OF FIX LATITUDE

LONGITUDE

1.

2.

3.

4.

5.

Hourly Positions:

Time Latitude Longitude Wind Dir. Wind Sp. Wave Dir. Wave Hgt.

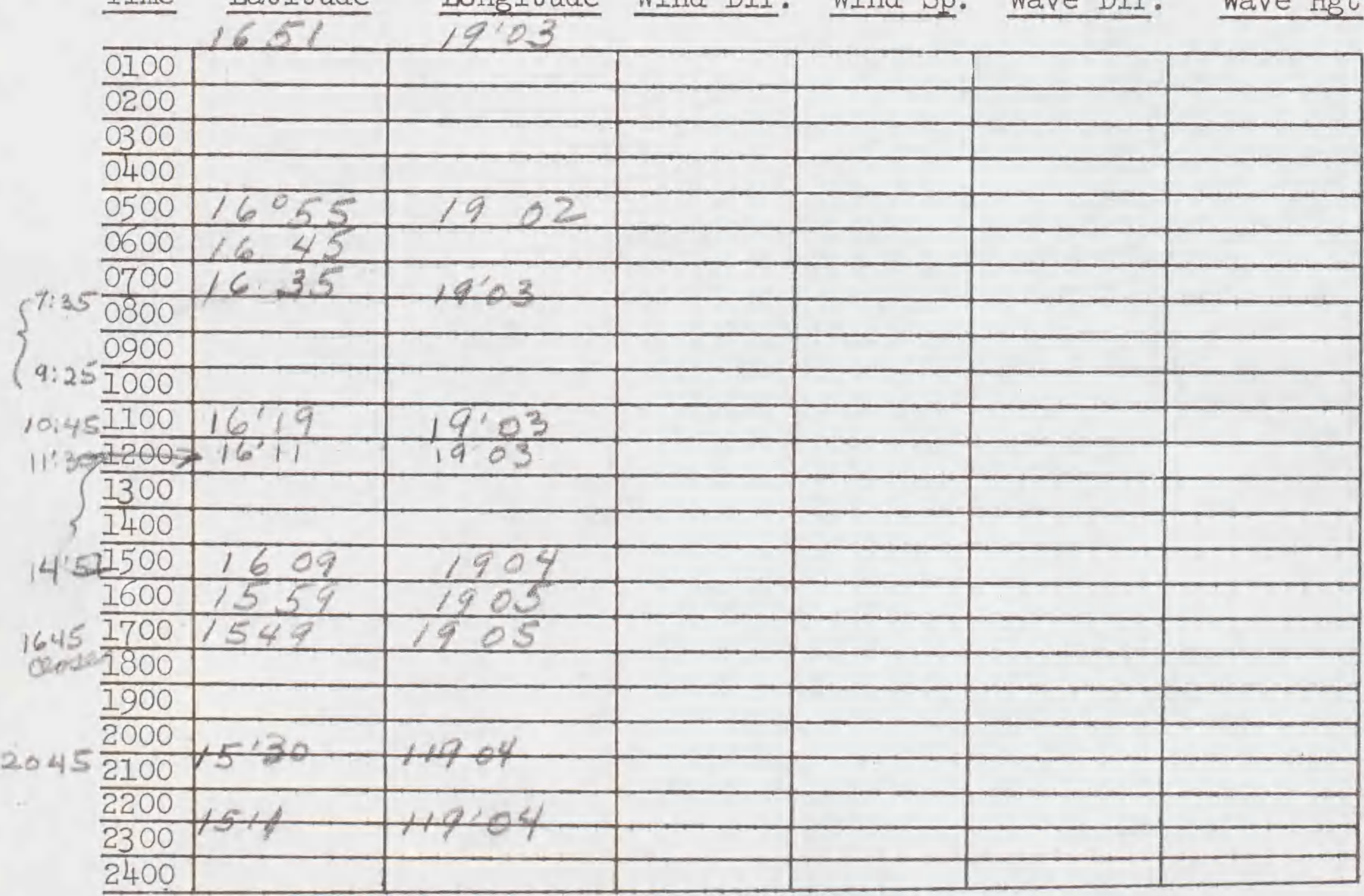


Date 10 Anyust 67

Organization POBSP

Sunrise: Time 0540

Sunset: Time 1821
Ship T. Waskingten (

Recorder Lewis

Position: Lat.

Position: Lat. 1344 , Long. $119^{\circ} 04$

Miles travelled from 0000 hours to sunrise $=40$

Miles travelled from sunrise to sunset $=50$

Miles travelled from sunset to 2400 hours $=43$

TIME OF FIX TYPE OF FIX LATITUDE LONGITUDE

1.

2.

3.

4.

5 .

Hourly Positions:

Time Latitude Longitude Wind Dir. Wind Sp. Wave Dir. Wave Hgt.

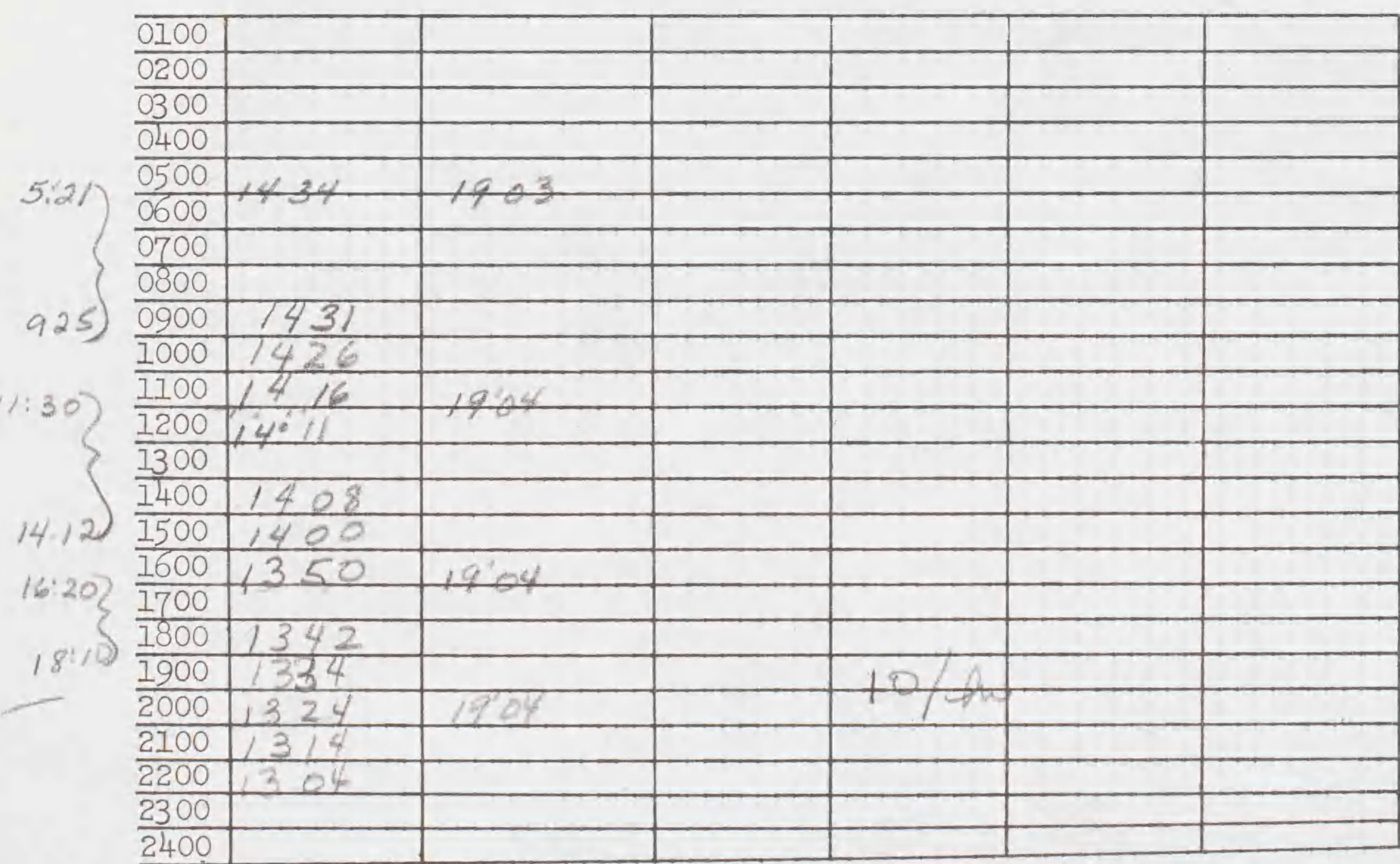


Date $1($ August 6 )

organization POBSP
ShipI Washington

Recorder hewis

Sunrise: Time 0544 Position: Lat. $12^{\circ} 31^{\prime}$, Long. $119^{\circ} 04$

Sunset: Time $18 / C$ Position: Lat. $11^{\circ} 29$, Long. $119^{\circ} 03$

Miles travelled from 0000 hours to sunrise $=$

Miles travelled from sunrise to sunset

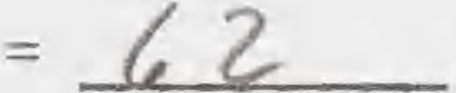

Miles travelled from sunset to 2400 hours

$=38$

TIME OF FIX TYPE OF FIX LATITUDE IONGITUDE

1.

2.

3.

4.

5 .

Hourly Positions:

Time Latitude Longitude Wind Dir. Wind Sp. Wave Dir. Wave Hgt.

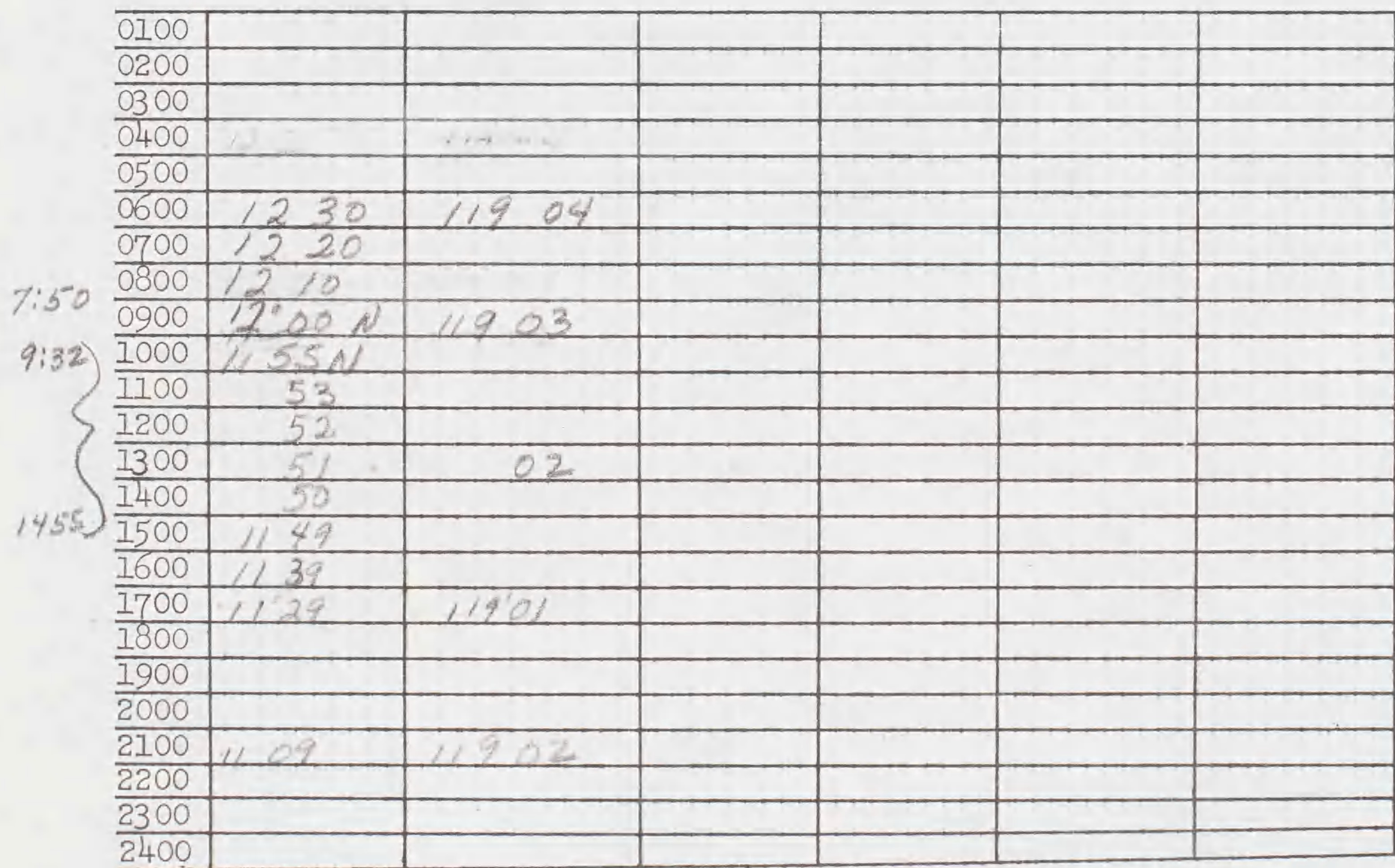


Date 12 August 6 ?

organization POBSP
Ship T. Washingten

Recorder
Cruise No. $028-002$

Sunrise: Time 0547 Position: Lat.1016, Long.119 03

Sunset: Time 18/5, Position: Lat. 913, Long. 11900

Miles travelled from 0000 hours to sunrise $=35$

Miles travelled from sunrise to sunset $=-4 \zeta$

Miles travelled from sunset to 2400 hours $=26$

TIME OF FIX TYPE OF FIX LATITUDE LONGITUDE

1.

2 .

3.

4.

5 .

Hourly Positions:

Time Latitude Longitude Wind Dir. Wind Sp. Wave Dir. Wave Hgt.

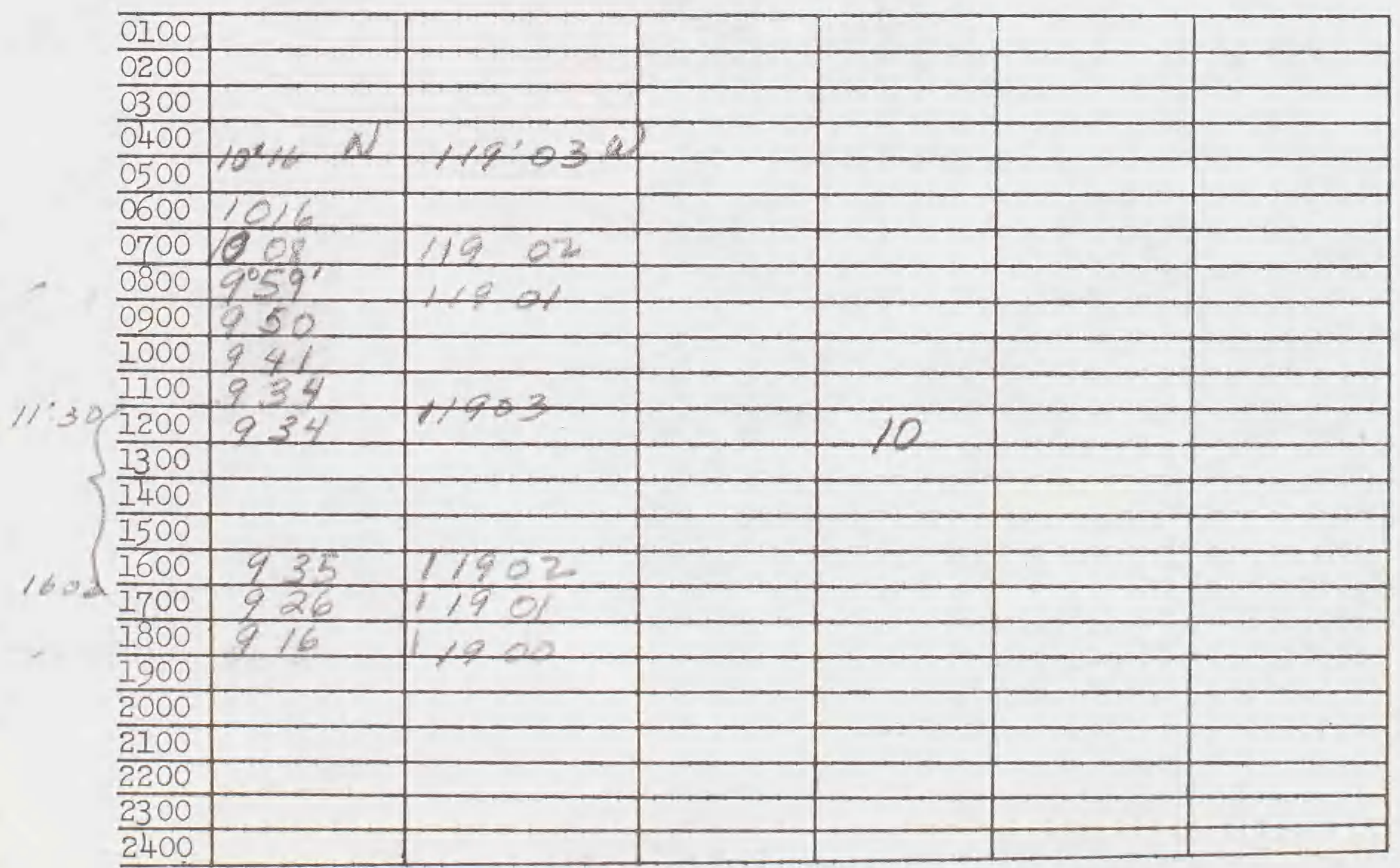


Date 13 Auy 67

Organization POBSP

Sunrise: Time 0549

Sunset: Time 1813
Ship T. Washimgten (

Recorder hewis

Miles travelled from 0000 hours to sunrise $=19$

Miles travelled from sunrise to sunset

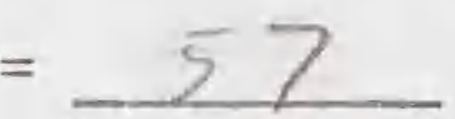

Miles travelled from sunset to 2400 hours $=38$

TIME OF FIX TYPE OF FIX LATITUDE LONGITUDE

1.

2 .

3 .

4.

5 .

Hourly Positions:

Time Latitude Longitude Wind Dir. Wind Sp. Wave Dir. Wave Hgt.

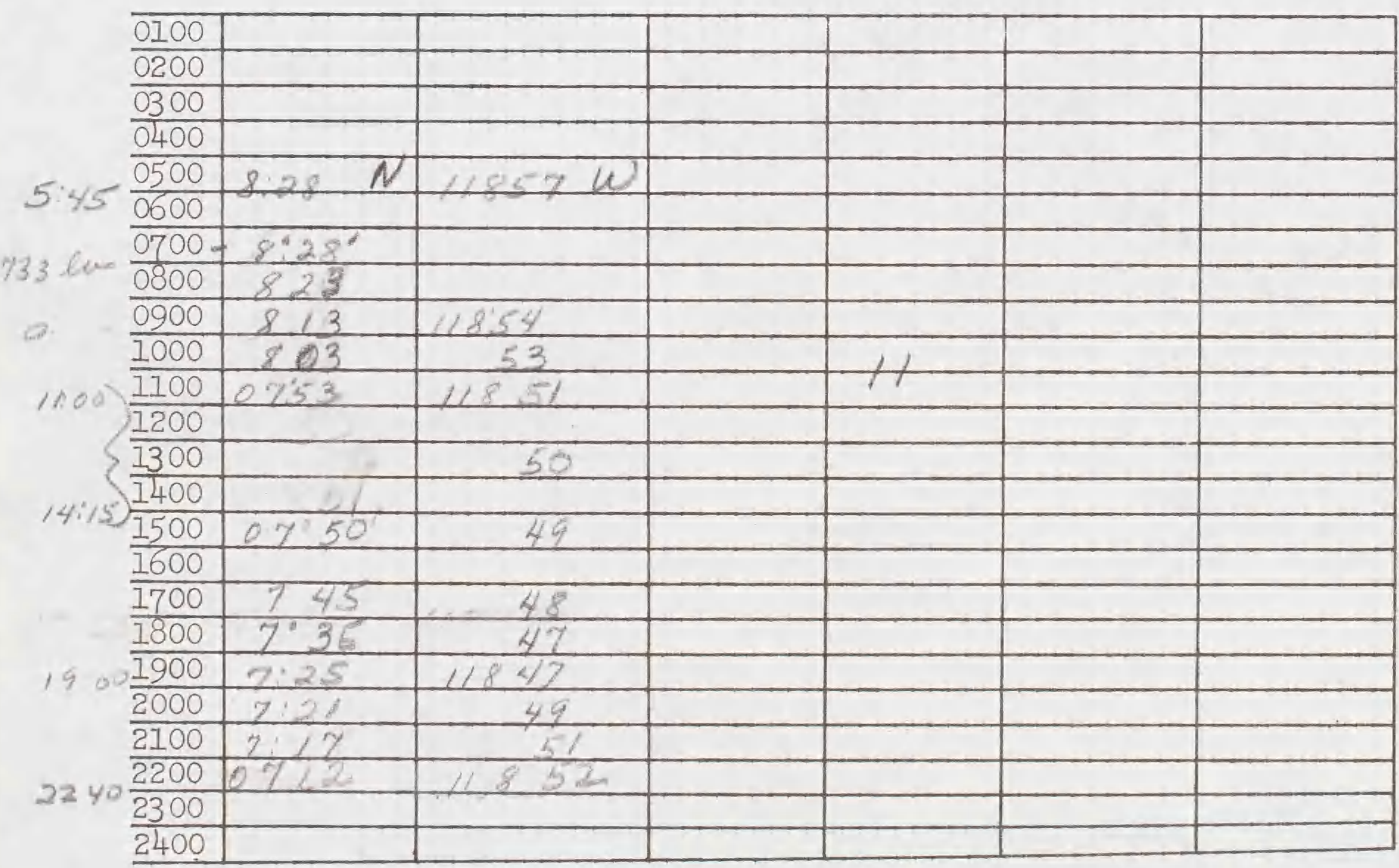


Date 14 Auqust $6 ?$

organization POBSP

Sunrise: Time 0553

Sunset: Time 1809
ShipT. Washington (

Recorder Lewis

Miles travelled from 0000 hours to sunrise $=$

Miles travelled from sunrise to sunset

$=\frac{71}{34}$

Miles travelled from sunset to 2400 hours

TIME OF FIX TYPE OF FIX IAATITUDE

LONGITUDE

I.

2 .

3.

4.

5 .

Hourly Positions:

Time Latitude Iongitude Wind Dir. Wind Sp. Wave Dir. Wave Hgt.

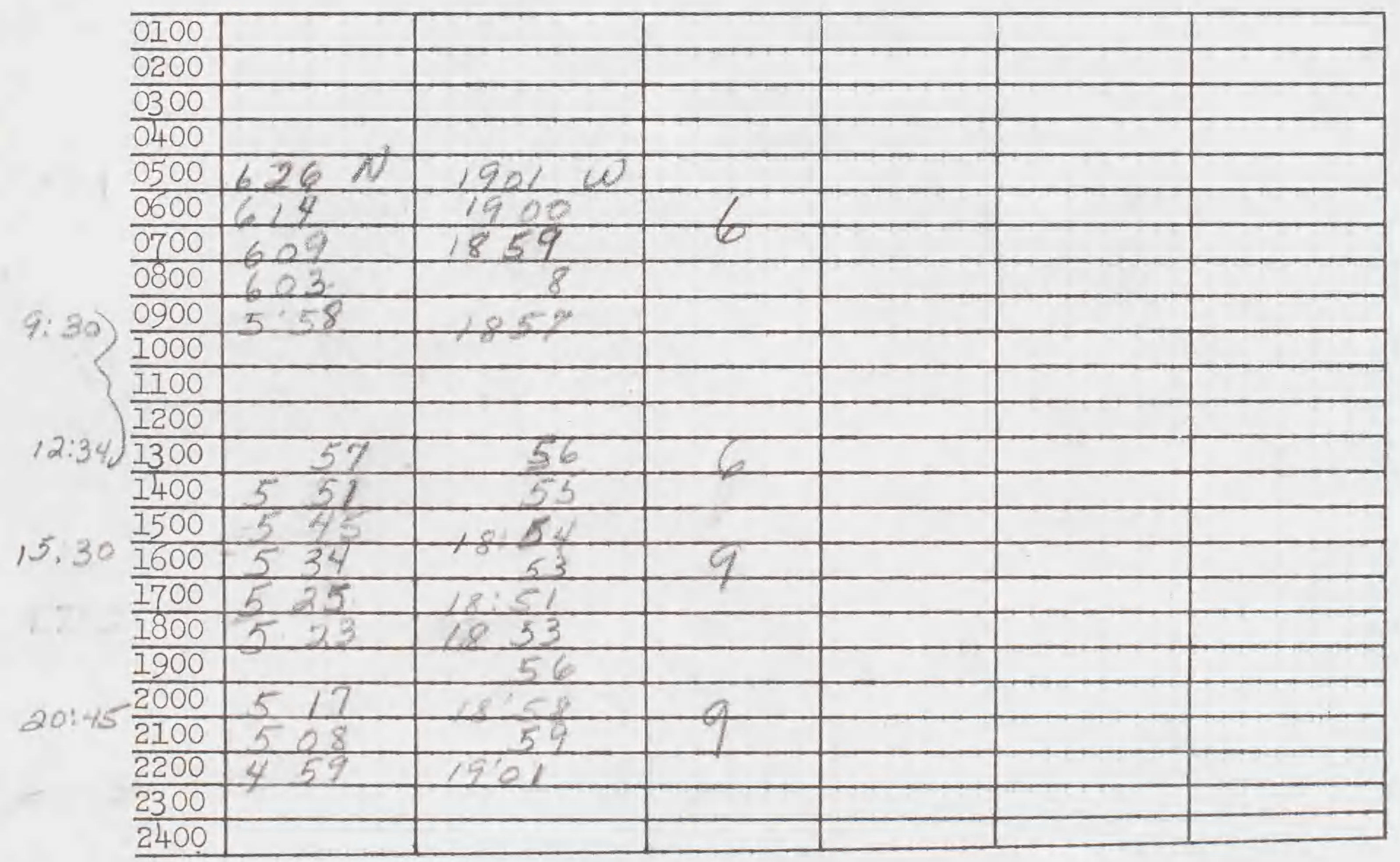


Date 15 Amurst 67

organization POBSP

ShipT Washingten

Cruise No. $228-000^{2}$

Sunrise: Time

Position: Lat.

Long. $119 \quad 15$

Sunset: Time 1809

Position: Lat.

, Long. $114 \quad 16$

Miles travelled from 0000 hours to sunrise $=47 \mathrm{~mm}$

Miles travelled from sunrise to sunset $=42$ -

Miles travelled from sunset to 2400 hours $=25$

TIME OF FIX TYPE OF FIX LATITUDE

LONGITUDE

1.

2 .

3.

4.

5 .

Hourly Positions:

Time Latitude Longitude Wind Dir. Wind Sp. Wave Dir. Wave Hgt.

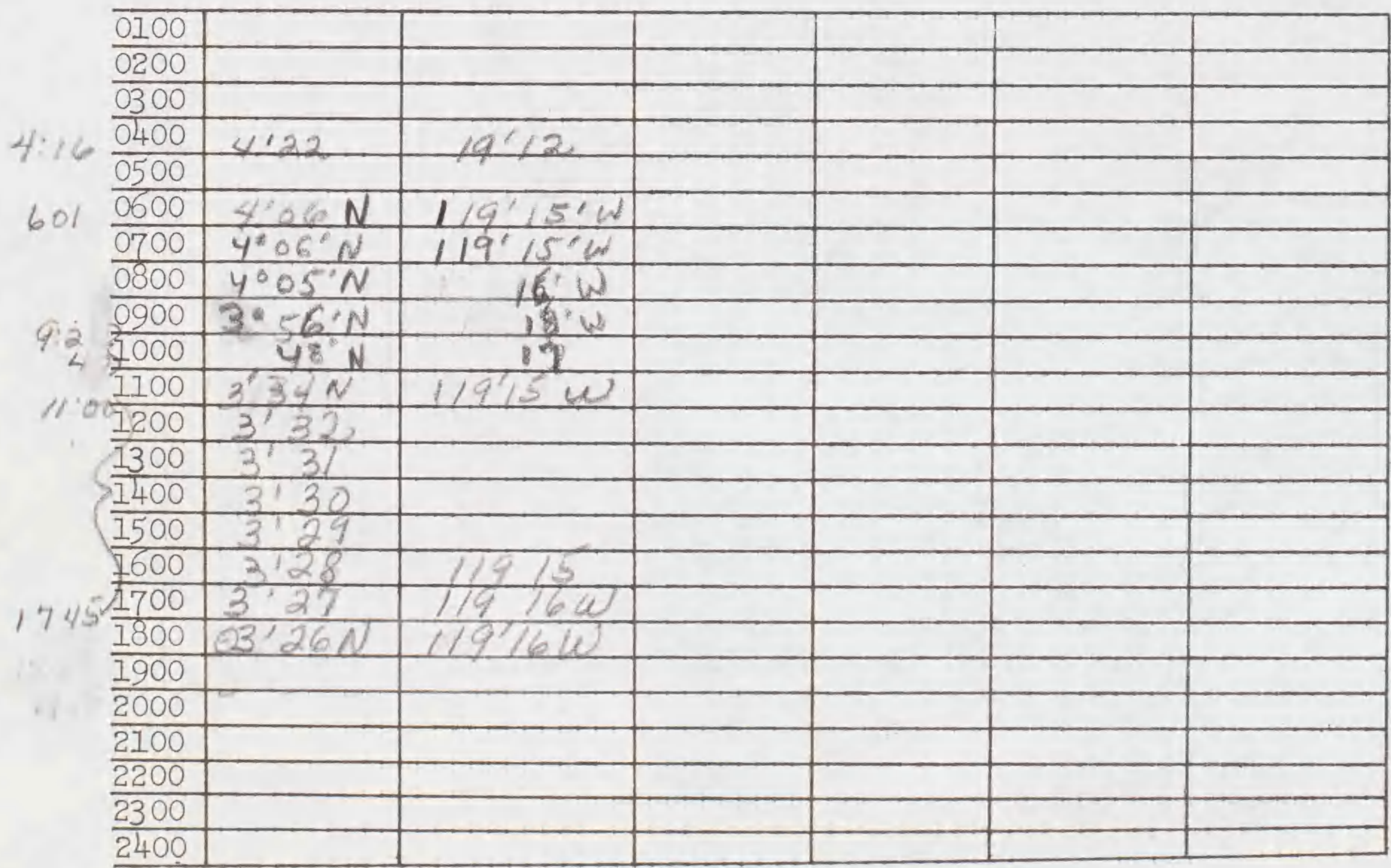


Date 16 August 6?

Organization POBSP
Ship T. Washingtun (

Recorder Lewis
Sunset: Time 1804
Position: Lat. $0211 \mathrm{~N}$, Long. 11905

Position: Lat. $0^{\circ 5} 5 \mathrm{~N}$, Long. 11904

Miles travelled from 0000 hours to sunrise $=37$

Miles travelled from sunrise to sunset $=? 4$

Miles travelled from sunset to 2400 hours $=33$

TIME OF FIX TYPE OF FIX LATITUDE IONGITUDE

1.

2.

3.

4.

5 .

Hourly Positions:

Time Latitude Iongitude Wind Dir. Wind Sp. Wave Dir. Wave Hgt.

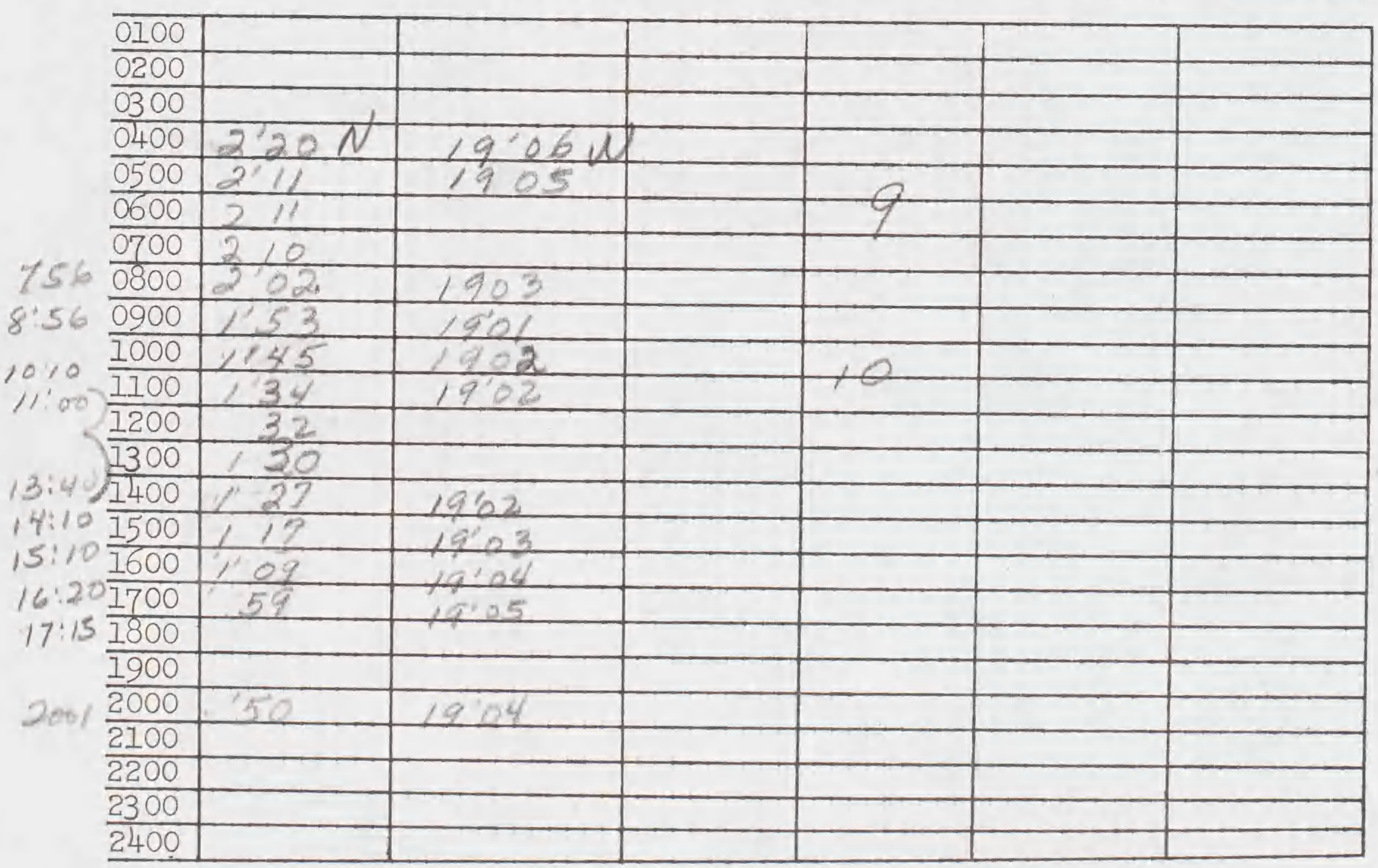


Date 1) August 6?

Organization POBSP
ShipT. Washington

Recorder Lewis
Sunrise: Time

Sunset: Time 1801
Position: Lat. 00135 , Long.

Position: Lat.

Cruise No. $028-0002$

Miles travelled from 0000 hours to sunrise $=37$

Miles travelled from sunrise to sunset $=\& 9$

Miles travelled from sunset to 2400 hours $=39$

TIME OF FIX TYPE OF FIX IATITUDE LONGITUDE

1.

2 .

3.

4.

5 .

Hourly Positions:

Time Latitude Longitude Wind Dir. Wind Sp. Wave Dir. Wave Hgt.

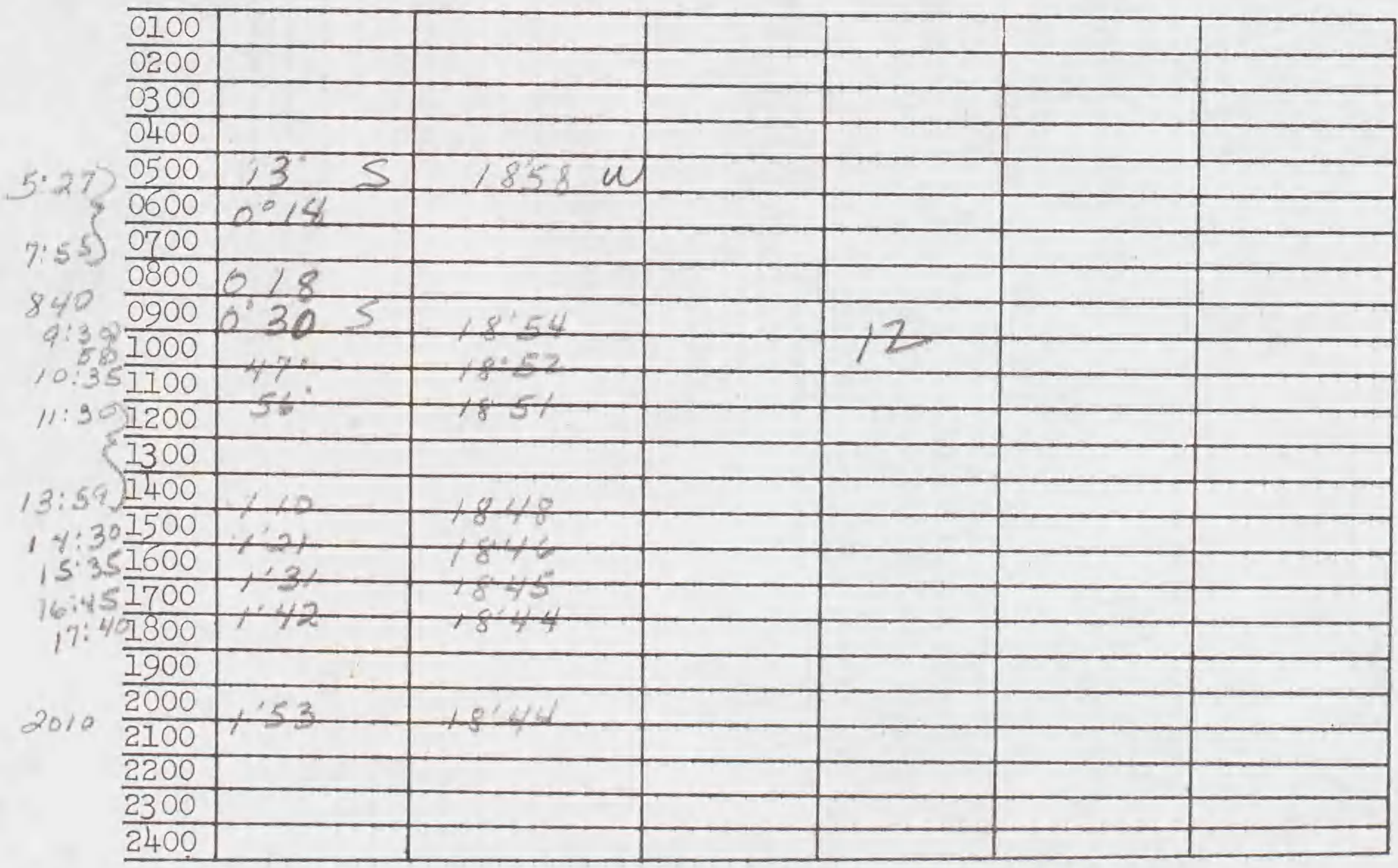


Date 14 Angus 6 ?

Organization POBSP

Sunrise: Time

Sunset: Time $\angle 259$
Ship IWushington (

Recorder hewis
Cruise No. $028=0002$

Miles travelled from 0000 hours to sunrise =

Miles travelled from sunrise to sunset

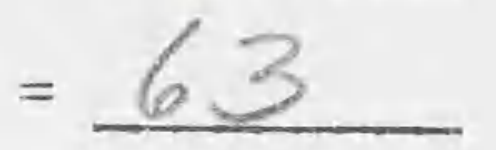

Miles travelled from sunset to 2400 hours $=32$

TIME OF FIX TYPE OF FIX IATITUDE

IONGITUDE

1 .

2 .

3 .

4.

5 .

Hourly Positions:

Time Latitude Longitude Wind Dir. Wind Sp. Wave Dir. Wave Hgt.

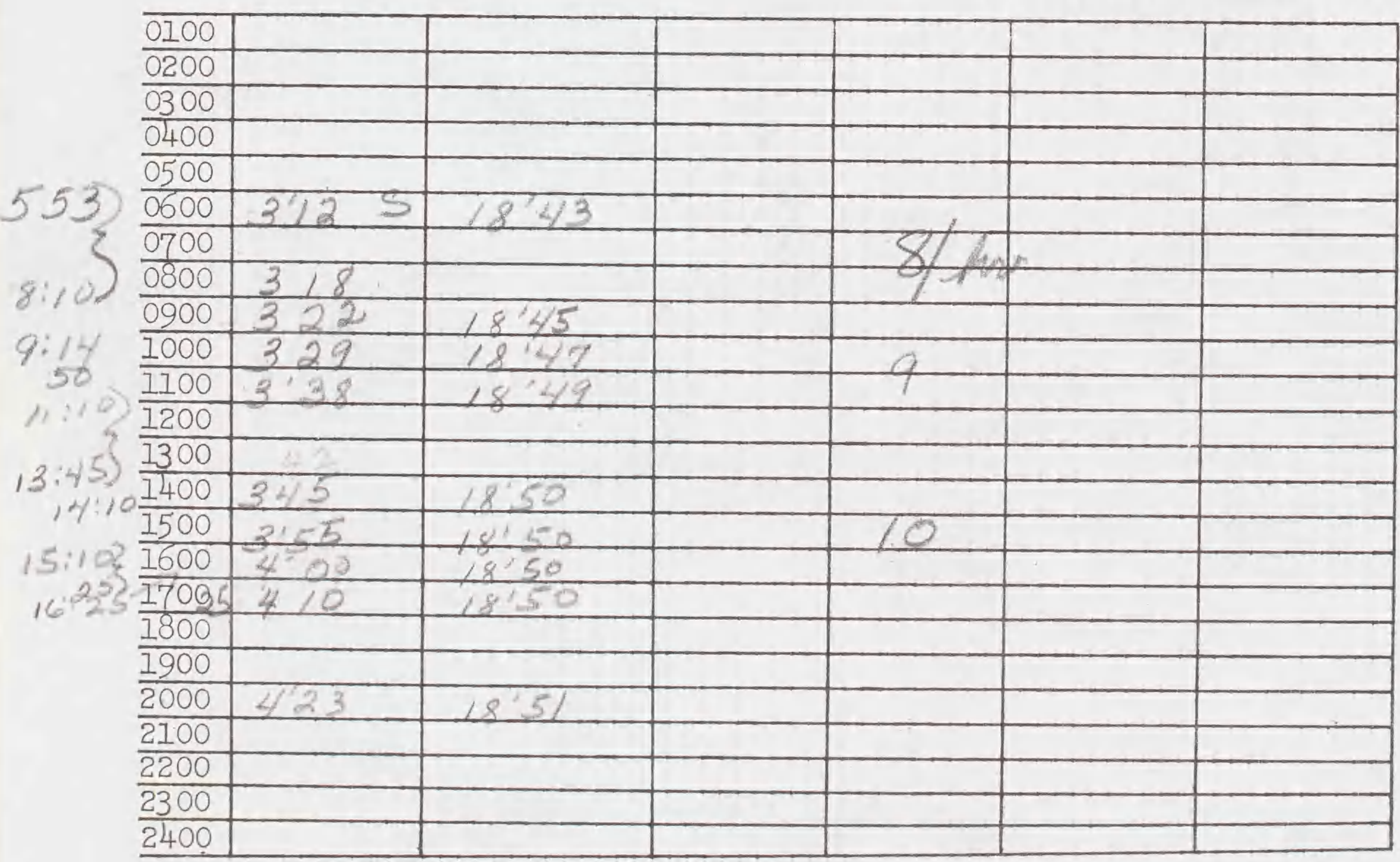


Date 19 4uyus 62

organization POBSP

Sunrise: Time 0600

Sunset: Time 1759
ShipT. Washington (

Recorder Lewis
Cruise No. $028-0002$

Miles travelled from 0000 hours to sunrise $=\underline{25}$

Miles travelled from sunrise to sunset $=70$

Miles travelled from sunset to 2400 hours $=39$

TIME OF FIX TYPE OF FIX LATITUDE LONGITUDE

1 .

2 .

3.

4.

5 .

Hourly Positions:

Time Latitude Longitude Wind Dir. Wind Sp. Wave Dir. Wave Hgt.

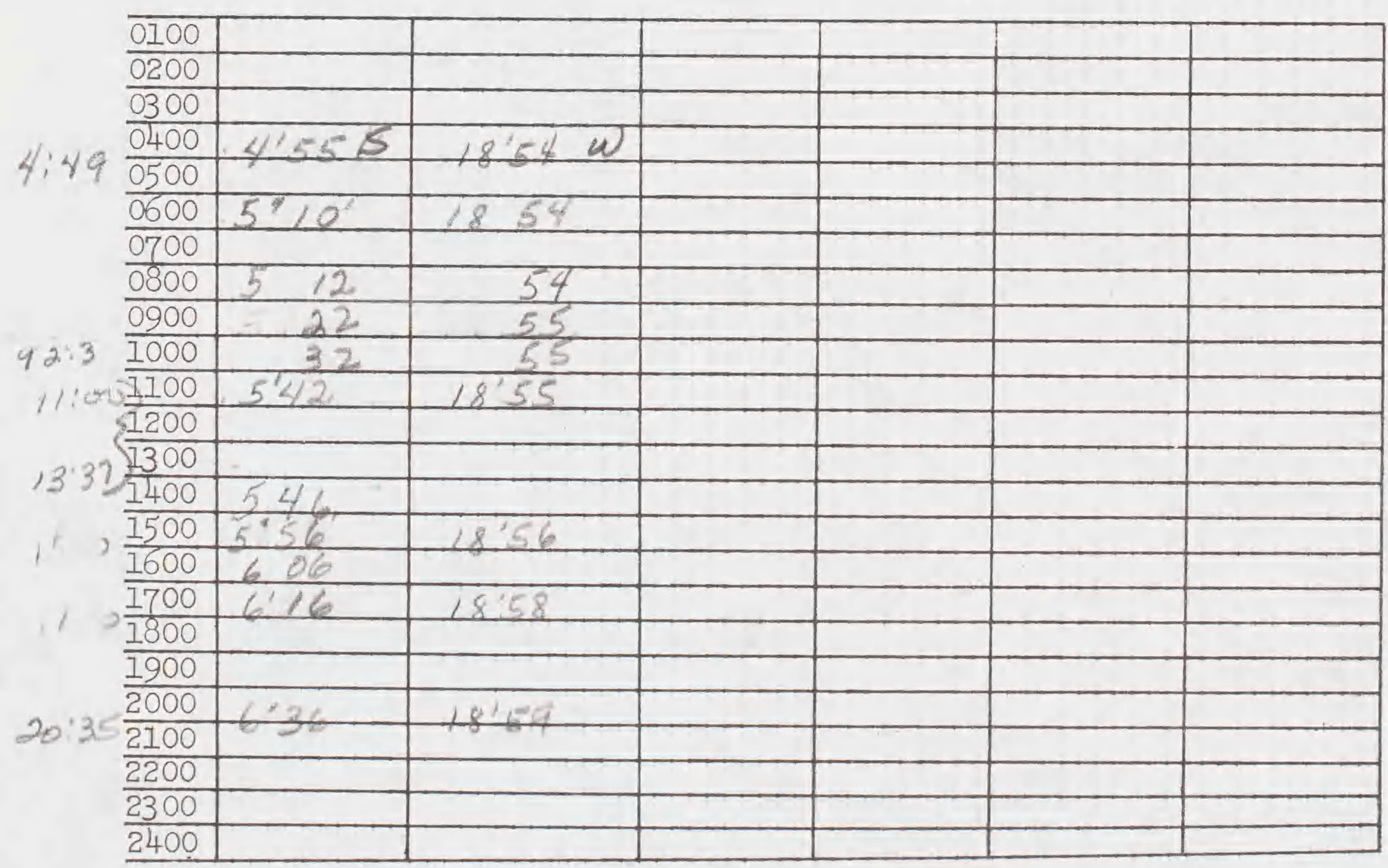


Date 20 Augus +67

Organization POBSP

Sunrise: Time 0602

Sunset: Time 1755
Ship T. Washingtou (

Recorder hewis
Cruise No. $028-0002$

Miles travelled from 0000 hours to sunrise $=33$

Miles travelled from sunrise to sunset $=82$

Miles travelled from sunset to 2400 hours $=52$

TIME OF FIX TYPE OF FIX LATITUDE LONGITUDE

1 .

2.

3.

4.

5 .

Hourly Positions:

Time Latitude Longitude Wind Dir. Wind Sp. Wave Dir. Wave Hgt.

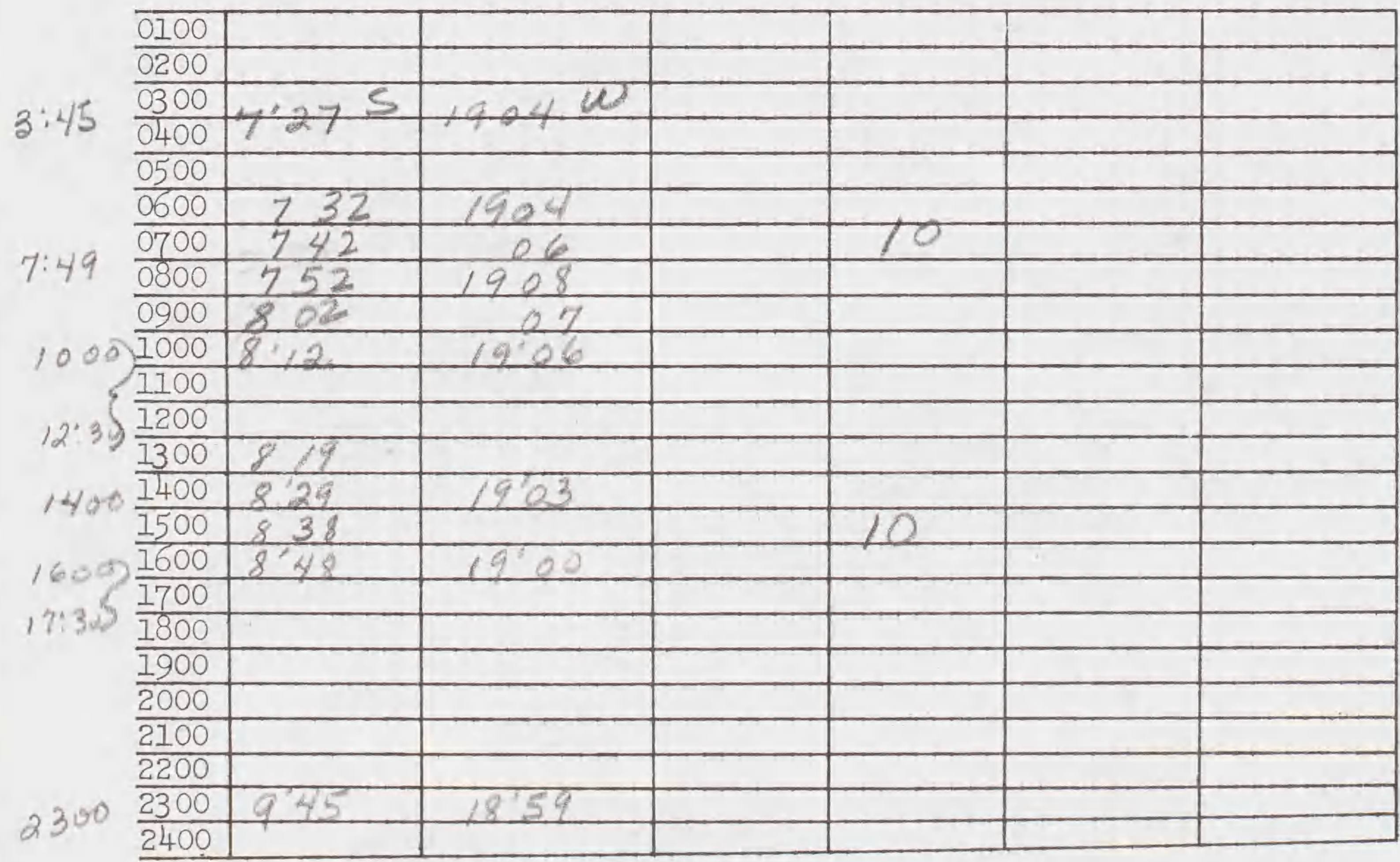


Date L1 Hupul 6?

Organization POBSP
Ship I. Washington (

Recorder Lewis
Sunrise: Time 0603

Sunset: Time 1746
Position: Lat.

Position: Lat. 9465 , Long. $117 \%$

Miles travelled from 0000 hours to sunrise $=19$

Miles travelled from sunrise to sunset

Miles travelled from sunset to 2400 hours $=43$

TIME OF FIX TYPE OF FIX

LATITUDE

LONGITUDE

1.

2.

3.

4.

5 .

Hourly Positions:

Time Latitude Iongitude Wind Dir. Wind Sp. Wave Dir. Wave Hgt.

\begin{tabular}{|c|c|c|c|c|c|c|}
\hline 0100 & & & & & & \\
\hline$\frac{0200}{0300}$ & & & & & & \\
\hline$\frac{0300}{0400}$ & & & & & & \\
\hline$\frac{0400}{0500}$ & & & & & & \\
\hline 0600 & $0^{\prime} 45$ & $1840 w$ & & 12 & & \\
\hline$\frac{0700}{0800}$ & 42 & 28 & 07 & & & \\
\hline 0800 & & & & & & \\
\hline$\frac{0900}{1000}$ & 943 & 1821 & & & & \\
\hline$\frac{1000}{1100}$ & 244 & 1811 & & & & \\
\hline $28 \frac{1100}{1200}$ & 4.45 & 1800 & & & & \\
\hline $8 \frac{7300}{9400}$ & $9: 45$ & $17: 43$ & & & & \\
\hline $5 \frac{1400}{1500}$ & & $1734^{\circ}$ & 09 & 0. & & \\
\hline 1600 & & 1727 & & & & \\
\hline 1700 & & 1718 & & & & \\
\hline 1800 & 945 & 1768 & & & & \\
\hline$\frac{1900}{2000}$ & & & & & & \\
\hline$\frac{2000}{2100}$ & & & & & & \\
\hline 200 & & & & & & \\
\hline 300 & & & & & & \\
\hline & & & & & & \\
\hline
\end{tabular}


Date 23 Angas 167

Organization POBSP

Sunrise: Time 0540

Sunset: Time $1>27$
Ship T. Washingter (

Recorder Lowi,
Cruise No. $028=0002$

Miles travelled from 0000 hours to sunrise $=5 /$

Miles travelled from sunrise to sunset $=29$

Miles travelled from sunset to 2400 hours $=39$ TIME OF FIX TYPE OF FIX LATITUDE LONGITUDE

1 .

2.

3.

4.

5 .

Hourly Positions:

Time Latitude Longitude Wind Dir. Wind Sp. Wave Dir. Wave Hgt.

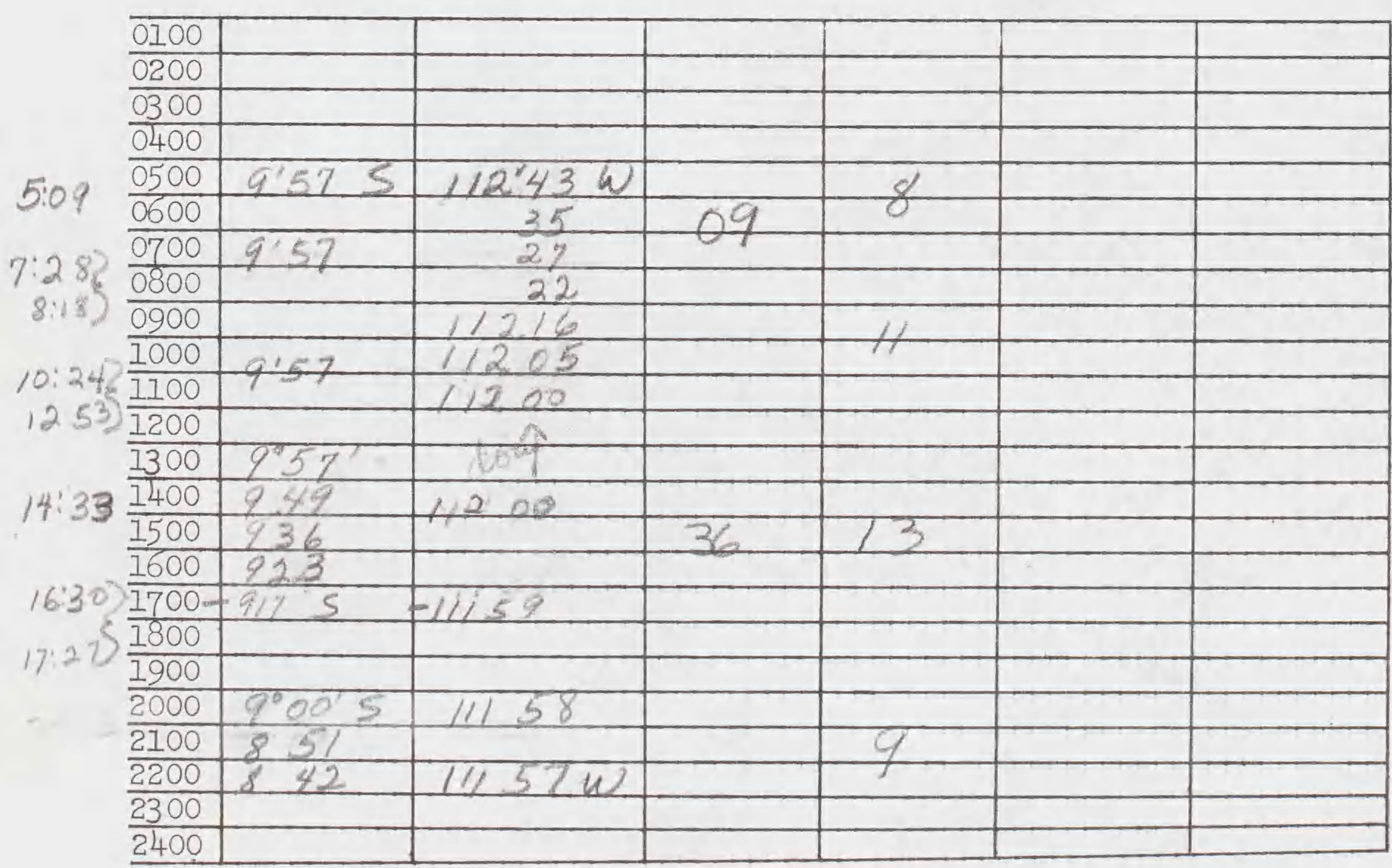


Date 24 Anqust 67

organization POBSP
ShipI. Washington

Recorder_Lew/s
Sunrise: Time 0629

Sunset: Time $182 \ell$
Position: Lat. 08015

Position: Lat. 06435 , Iong. $111^{\circ} 53 \omega$

Miles travelled from 0000 hours to sunrise $=$

Miles travelled from sunrise to sunset

Miles travelled from sunset to 2400 hours $=41$

TTME OF FIX TYPE OF FIX IATITUDE LONGITUDE

1.

2.

3.

4.

5 .

Hourly Positions:

Time Latitude Longitude Wind Dir. Wind Sp. Wave Dir. Wave Hgt.

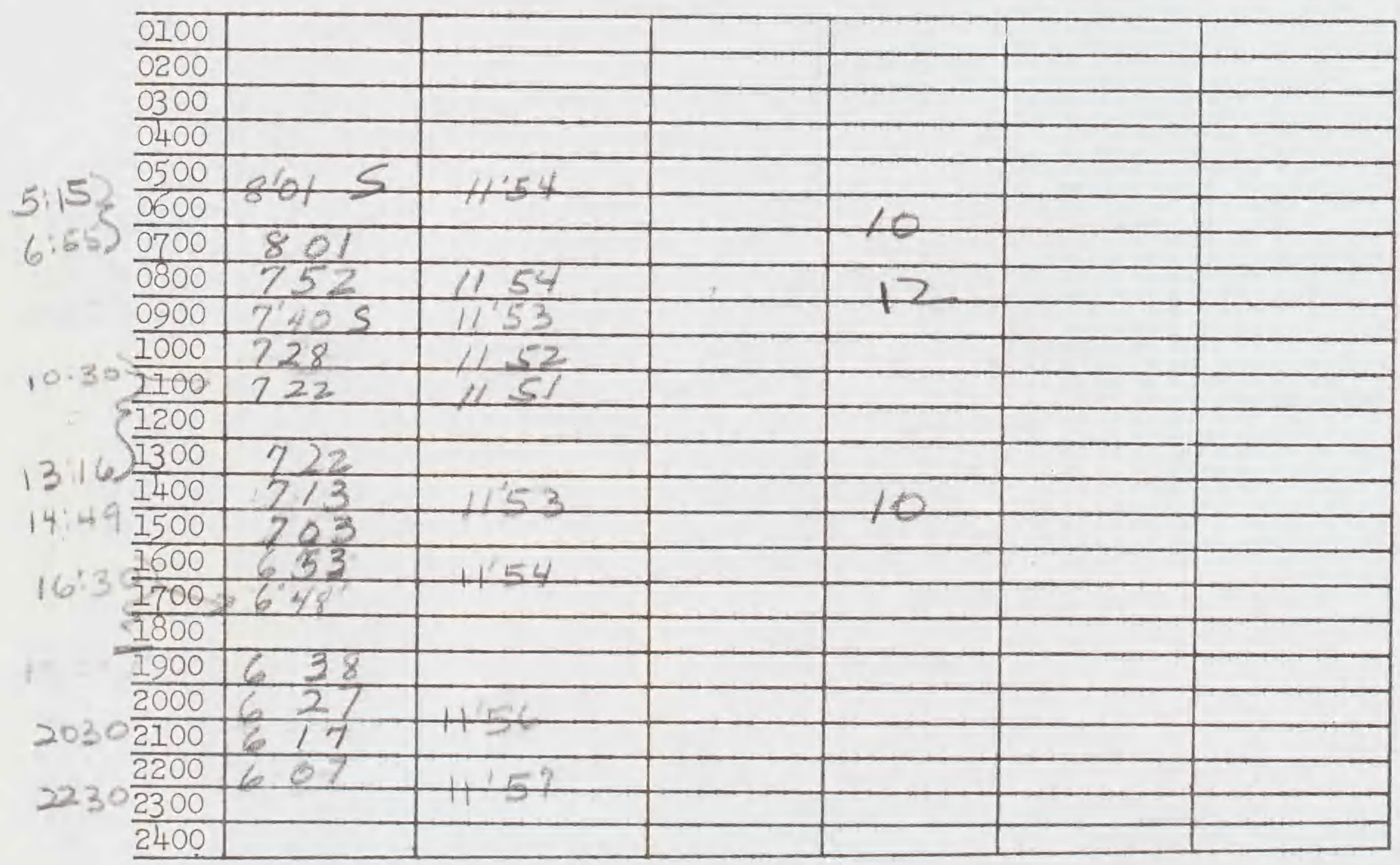


Date 25 Auqus 6 ?

Organization POBSP
Ship T. Waskington -

Recorder Lewis

Sunrise: Time 0633 Position: Lat. $05^{\circ} 19^{\prime}$, Iong. $112^{\circ} 00^{\circ}$

Sunset: Time 1830 Position: Lat. $04^{\circ} 0$ 's Iong. $112^{\circ}$ of w

Miles travelled from 0000 hours to sunrise $=43$

Miles travelled from sunrise to sunset

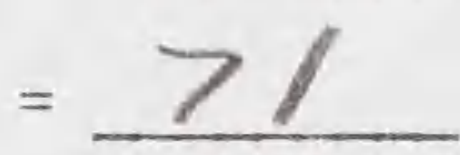

Miles travelled from sunset to 2400 hours $=39$

TIME OF FIX TYPE OF FIX IATITUDE IONGITUDE

1.

2.

3 .

4.

5 .

Hourly Positions:

Time Latitude Longitude Wind Dir. Wind Sp. Wave Dir. Wave Hgt.

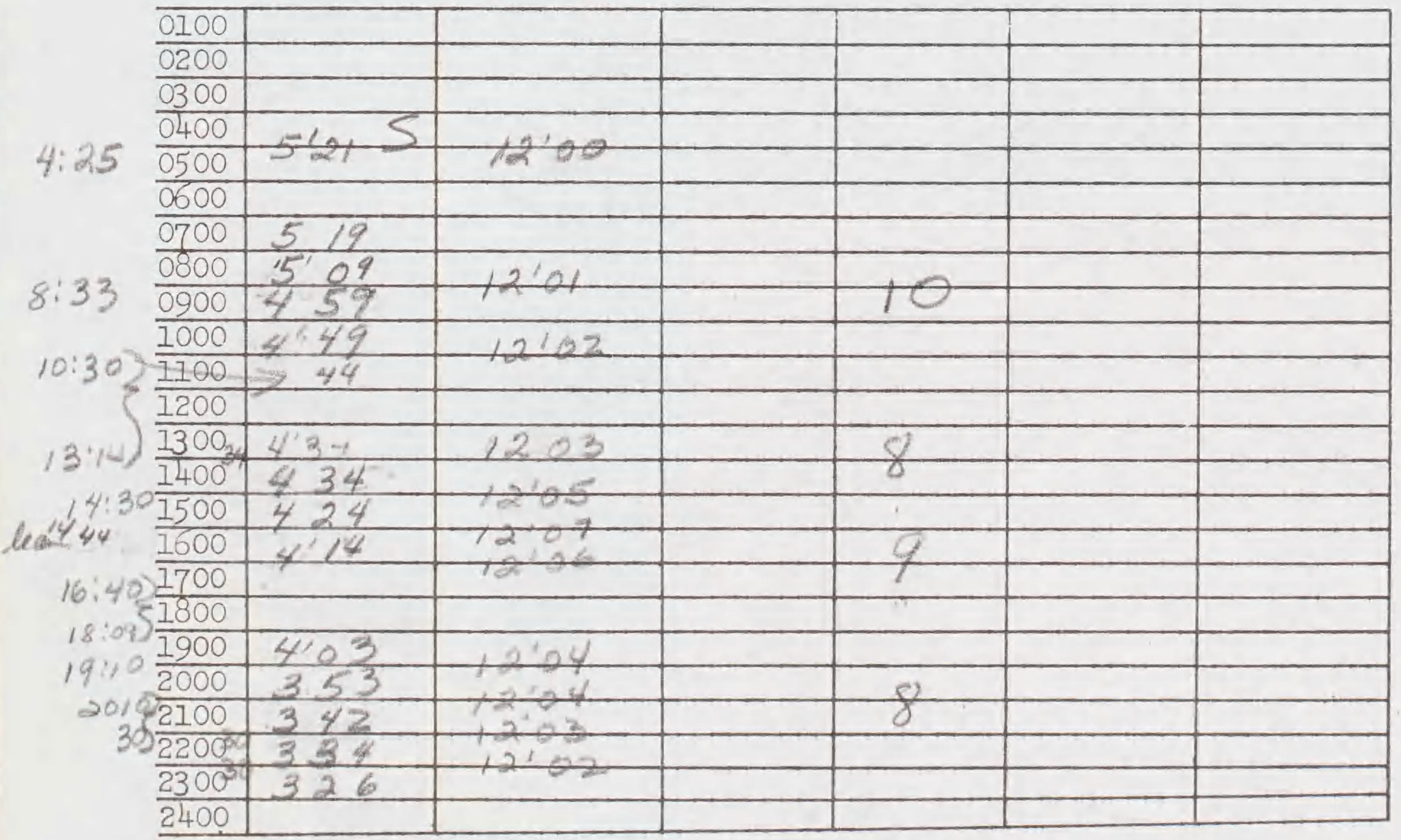


Date 2 August 67 organization POBSP
Ship I. Washington

Recorder Lewis's

Sunrise: Time 0633 Position: Lat. $0255^{\prime} S$ Long. $111^{\prime \prime} 58$

Sunset: Time 1822 Position: Lat. 03005 , Iong.11030

$028-$

Cruise No.000z

Miles travelled from 0000 hours to sunrise $=34$

Miles travelled from sunrise to sunset $=10\rangle$

Miles travelled from sunset to 2400 hours $=\$ 54$

TIME OF FIX TYPE OF FIX LATITUDE - LONGITUDE

1.

2.

3.

4.

5.

Hourly Positions: 23 to Rendezvous with Undaunted

Time Latitude Longitude Wind Dir. Wind Sp. Wave Dir. Wave Hgt.

$4 \cdot 45$

7.27

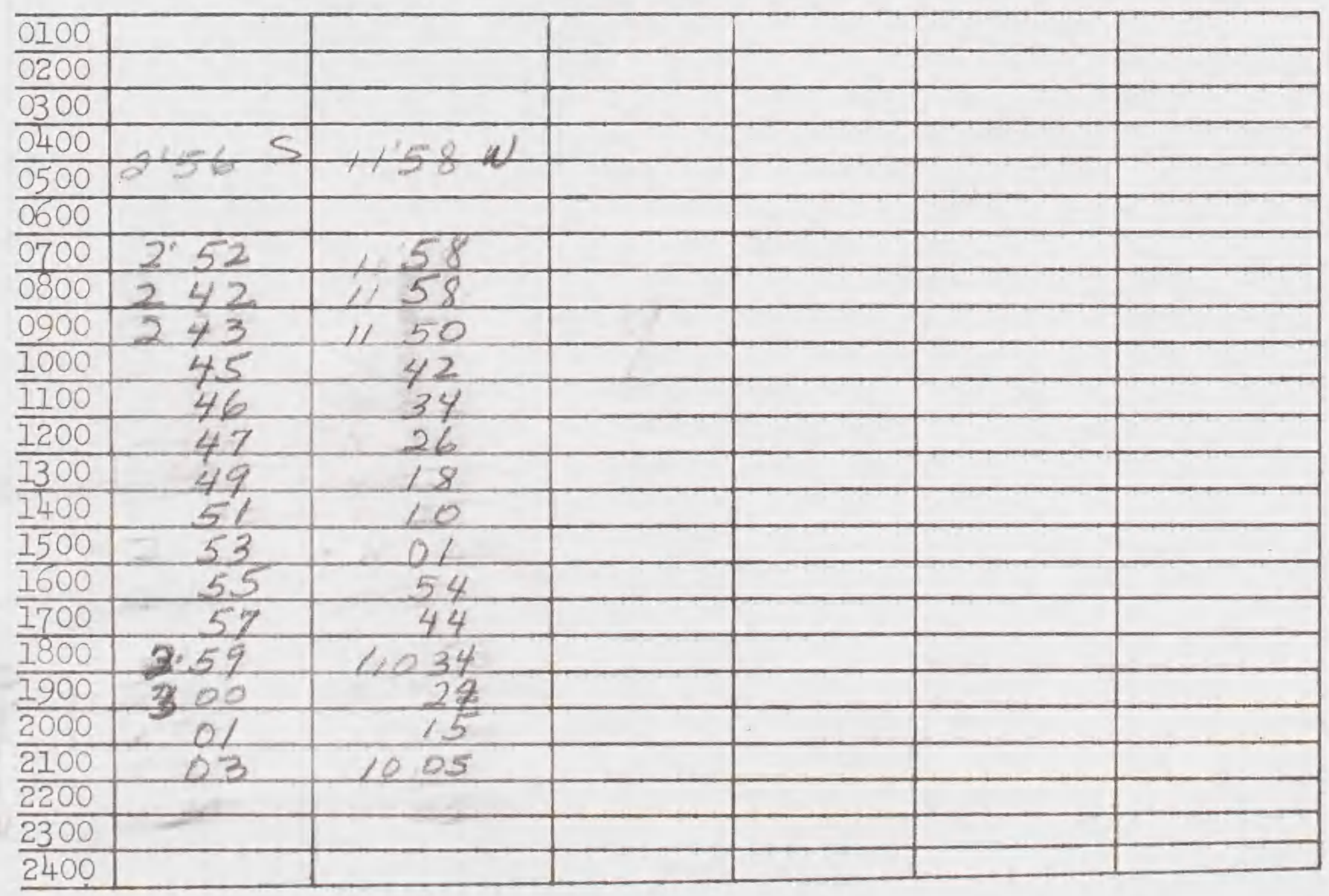

958b-SI-MNH

Rev. 9/28/66 
Date 27 August C7 ShipT. Wushington (_) Cruise No. 228-0002

Organization POBSP Recorder Lewis

Sunrise: Timel625 Position: Lat. 2575 , Long. $11033 \mathrm{~W}$

Sunset: Time183/ Position: Lat. 2105 , Long. $11158 \mathrm{w}$

Miles travelled from 0000 hours to sunrise $=58$

Miles travelled from sunrise to sunset $=111$

Miles travelled from sunset to 2400 hours $=41$

TIME OF FIX TYPE OF FIX IATITUDE LONGITUDE

I.

2 .

3.

4.

5 .

Hourly Positions:

1822 Time Latitude Iongitude Wind Dir. Wind Sp. Wave Dir. Wave Hgt.

\begin{tabular}{|c|c|c|c|c|c|c|}
\hline 0100 & & & & & & \\
\hline 0200 & & & & & & \\
\hline 0300 & & & & & & \\
\hline 0400 & & & & & & \\
\hline 0500 & & & & & & \\
\hline 0600 & 2585 & 32 & & & & \\
\hline 0700 & 56 & 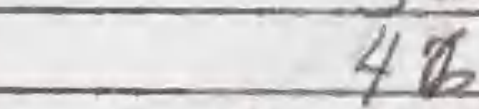 & & & & \\
\hline 0800 & 53 & 11057 & & & & \\
\hline 0900 & 50 & 06 & 29 & & & \\
\hline 1000 & 47 & 111.15 & & & & \\
\hline 1100 & 44 & 24 & & 10 & & \\
\hline 1200 & 40 & + & & 19114 & & \\
\hline 1300 & 37 & $\quad 42$ & & & & \\
\hline 1400 & 33 & 11151 & & & & \\
\hline 7500 & 229 & $1 \pm 200^{\prime}$ & & & & \\
\hline$\frac{1600}{1700}$ & & & 5 & & & \\
\hline 1800 & & & & & & \\
\hline 1900 & 204 & 1158 & & & & \\
\hline 2000 & +53 & +16 & & & & \\
\hline$\frac{2100}{200}$ & 14 & 11.55 & 36 & & & \\
\hline$\frac{6200}{2300}$ & & & & & & \\
\hline 2400 & & & & 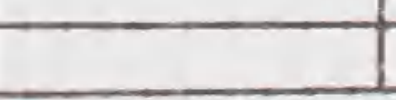 & & \\
\hline
\end{tabular}


Date 28 August 6?

Organization POBSP

Sunrise: Time 0633

Sunset: Time 1833
ShipI. Washingtun (

Recorder Lewis

Position: Lat. $00^{\circ} 4$ 's, Long. $111^{\circ} 47 \mathrm{~W}$

Position: Lat. $0026 \mathrm{~N}$, Iong. $11203,5 \mathrm{~W}$

Miles travelled from 0000 hours to sunrise $=42$

Miles travelled from sunrise to sunset $=-54$

Miles travelled from sunset to 2400 hours $=0$

TIME OF FIX TYPE OF FIX LATITUDE LONGITUDE

1.

2.

3.

4.

5 .

Hourly Positions:

Time Latitude Longitude Wind Dir. Wind Sp. Wave Dir. Wave Hgt.

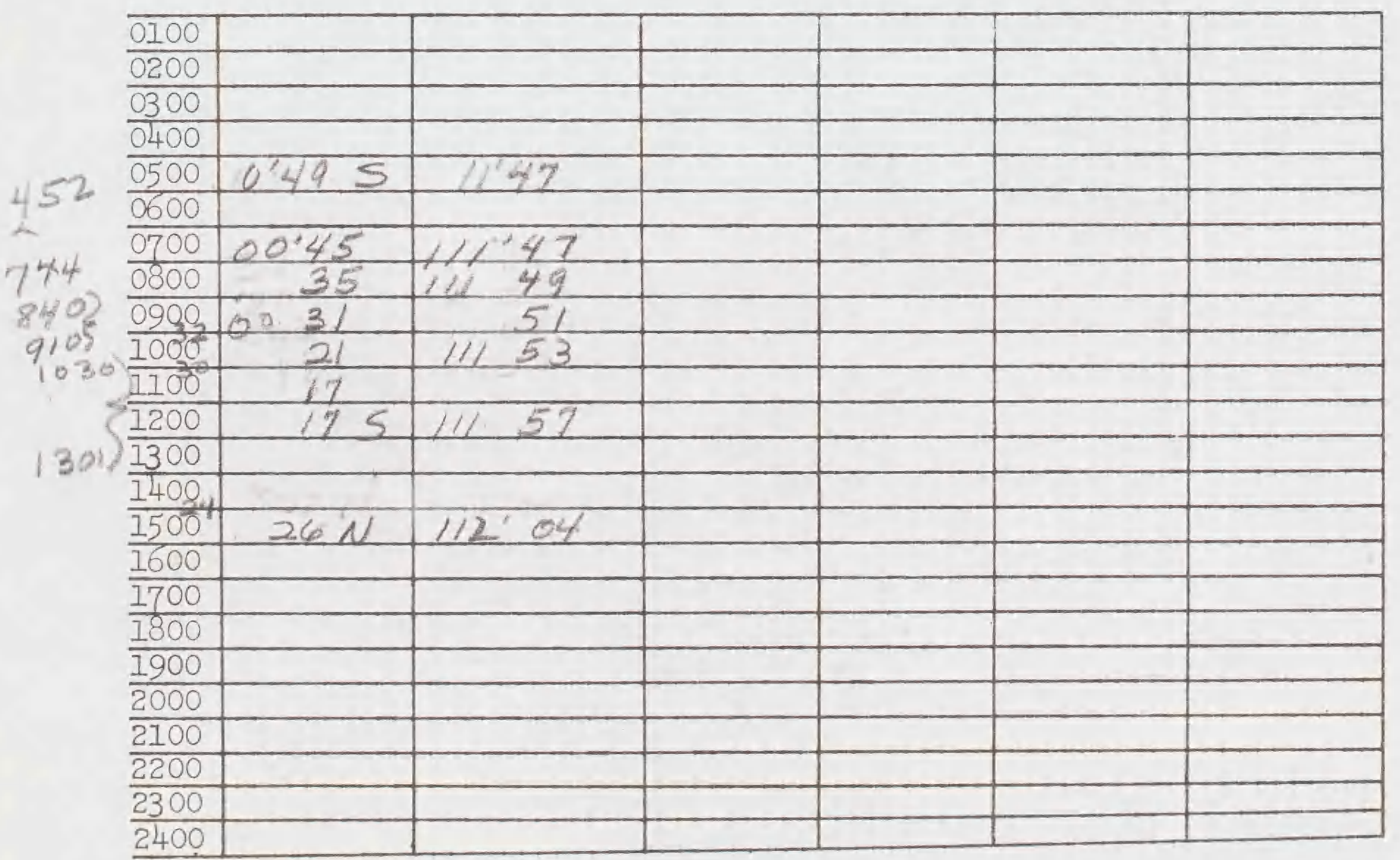


Date 29 Anyust 6?

organization POBSP
ShipT. Washirgton

Recorder
Cruise No.028.0002
Sunrise: Time 0626

Sunset: Time 1832
Position: Iat. $00^{\circ} 26^{\circ} \mathrm{N}$,

Long. $112 " 03.5 \mathrm{~m}$ Position: Lat. $00^{\circ} 26^{\circ} \mathrm{iv}$, Iong. $112^{\circ} 03.5 \mathrm{w}$

Miles travelled from 0000 hours to sunrise $=$ 0

Miles travelled from sunrise to sunset

$=$

Miles travelled from sunset to 2400 hours $=59$

TIME OF FIX TYPE OF FIX LATITUDE

IONGITUDE

1.

2.

3.

4.

5 .

Hourly Positions:

Time Latitude Longitude Wind Dir. Wind Sp. Wave Dir. Wave Hgt.

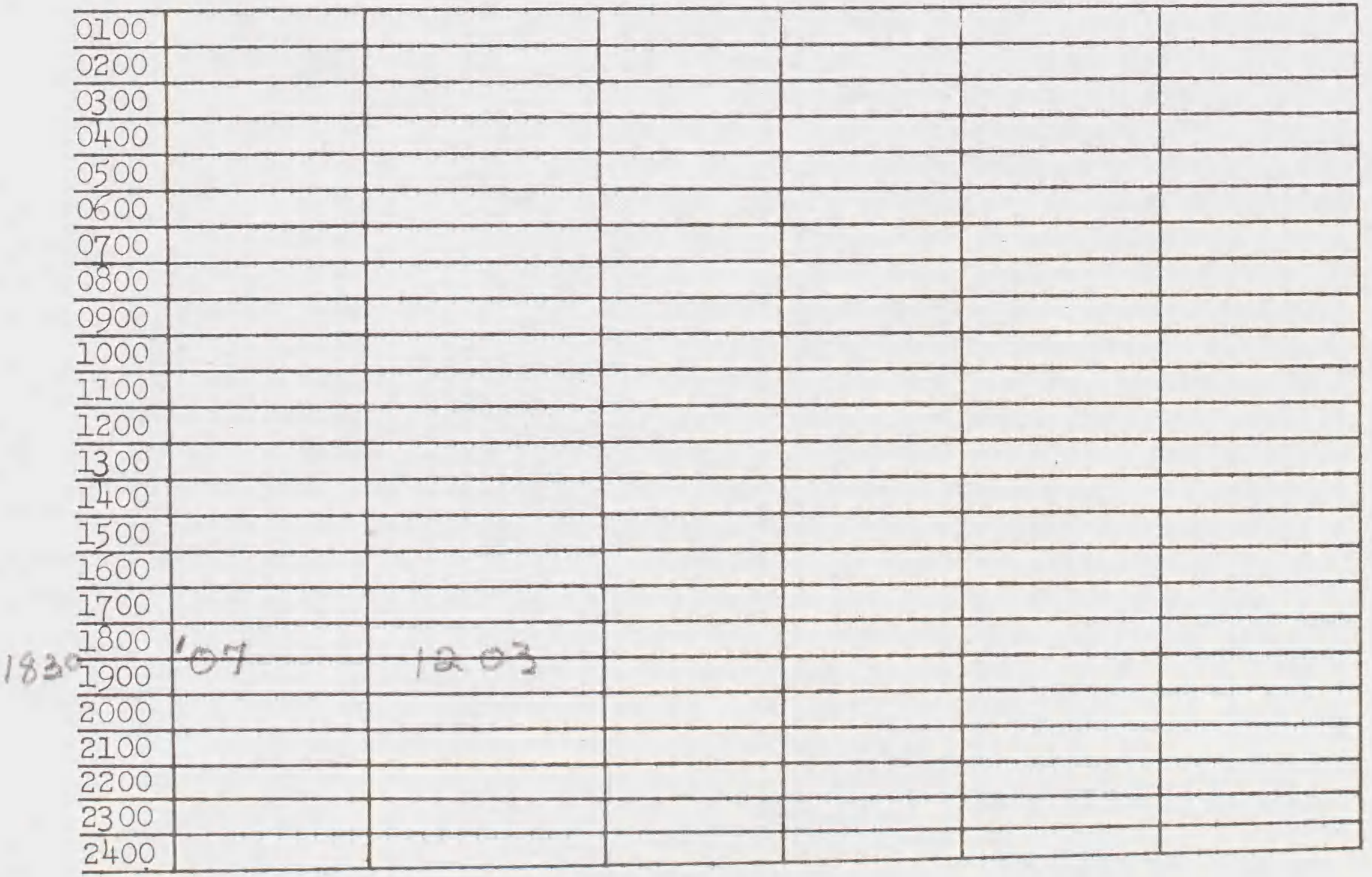


Date 30 Anqust 6 ?

Organization POBSP
Ship I. Washington (

Recorder Lewis

Sunrise: Time 0625 Position: Lat. $02^{\circ} 06 \mathrm{~N}$, Long. 11220 w

Sunset: Time 1833 Position: Lat. $18 \mathrm{i}^{\circ} \mathrm{iv}$, Iong.11207w

Miles travelled from 0000 hours to sunrise $=64$

Miles travelled from sunrise to sunset $=93$

Miles travelled from sunset to 2400 hours $=40$

TIME OF FIX TYPE OF FIX LATITUDE LONGITUDE

1.

2 .

3.

4.

5 .

Hourly Positions:

Time Latitude Longitude Wind Dir. Wind Sp. Wave Dir. Wave Hgt.

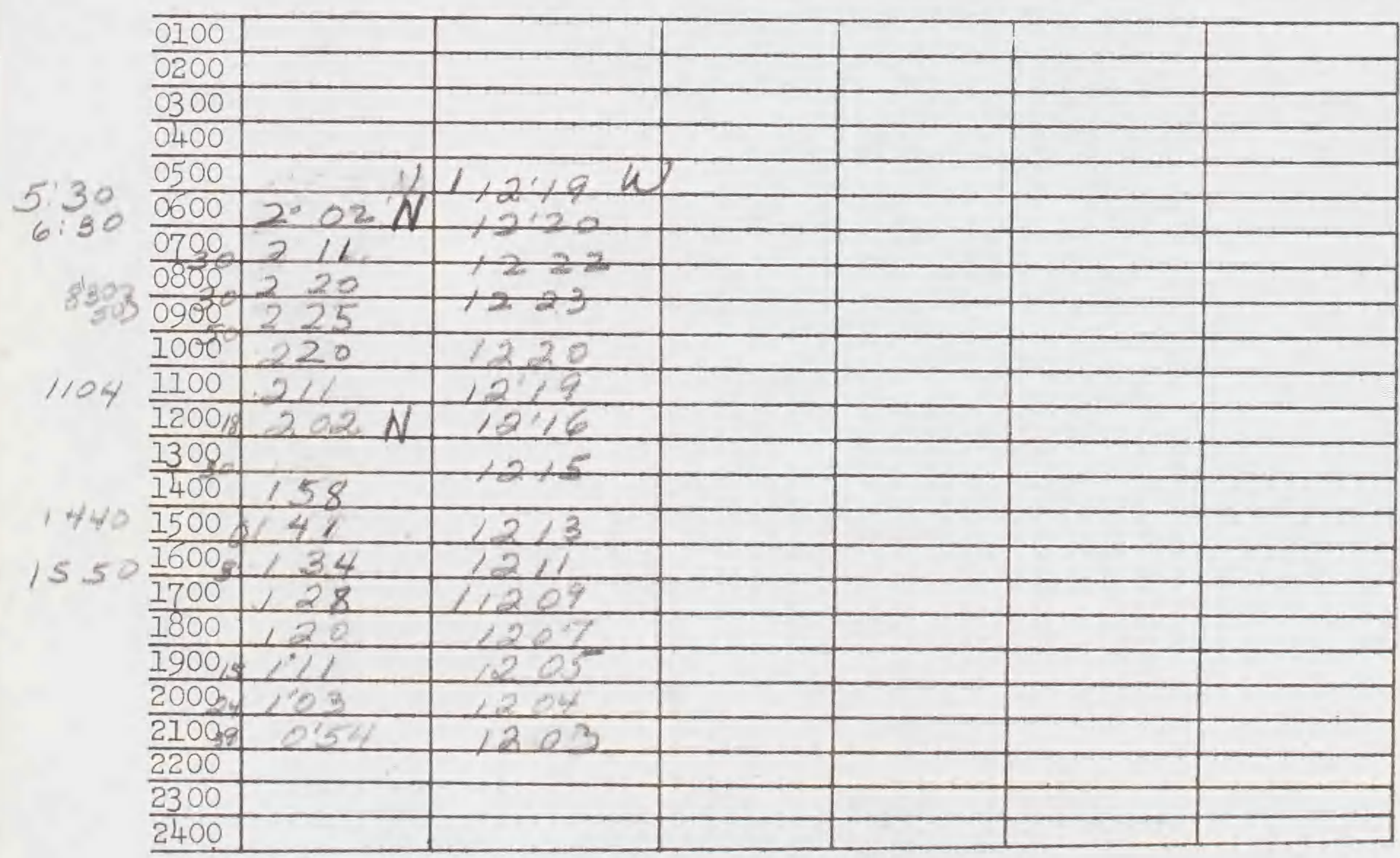


Date 31 Anqust 6 ?

organization Po bSP

ShipI. Washingtun

Cruise No.028-0002

Sunrise: Time

Sunset:
Recorder Lewis

Position: Lat. 0

Position: Iat. 00545 , Iong.11/ 5/w

Miles travelled from 0000 hours to sunrise $=42$

Miles travelled from sunrise to sunset

Miles travelled from sunset to 2400 hours $=38$

TIME OF FIX TYPE OF FIX LATITUDE - LONGITUDE

1 .

2.

3.

4.

5.

Hourly Positions:

Time Iatitude Iongitude Wind Dir. Wind Sp. Wave Dir. Wave Hgt.

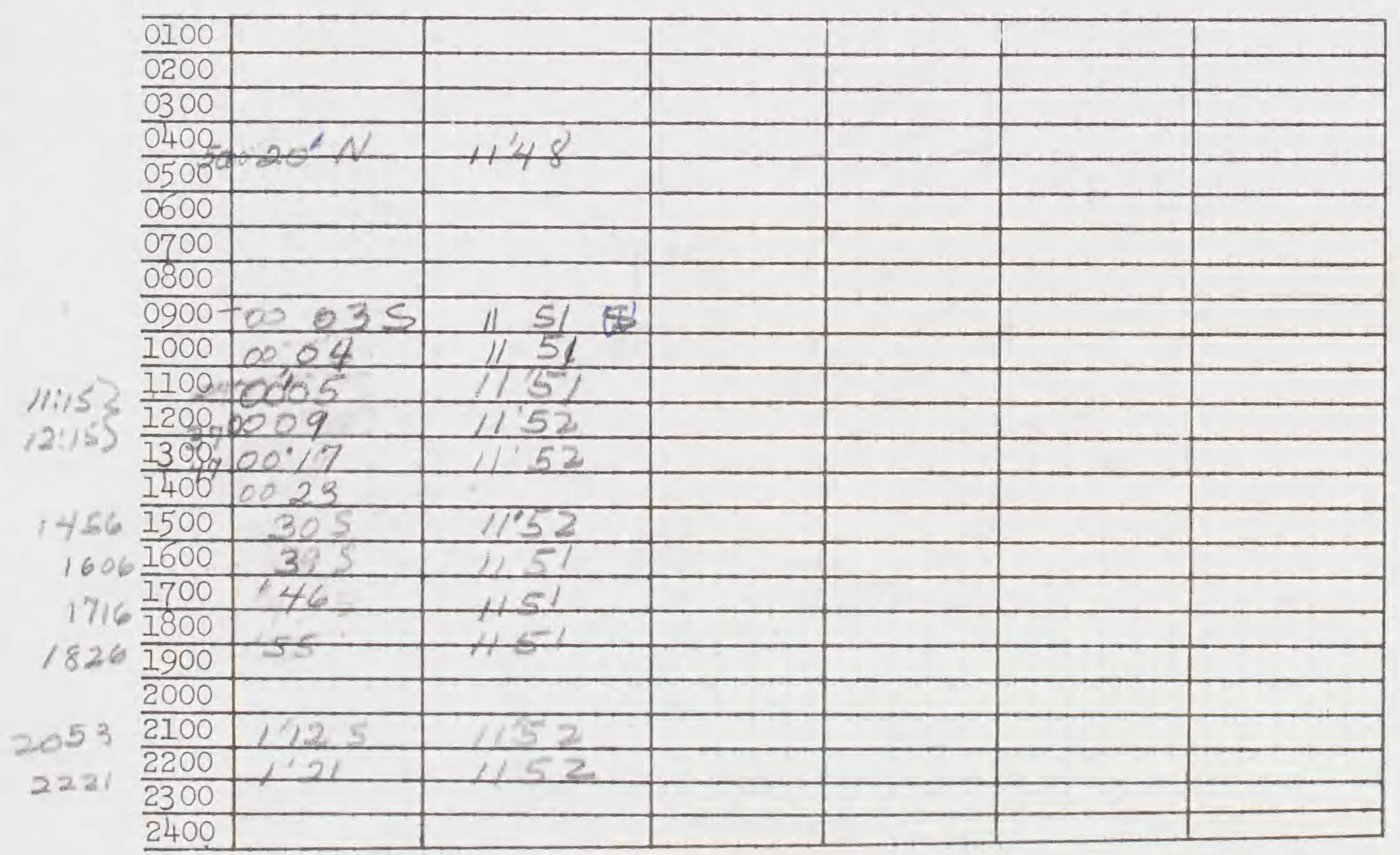


Date, Sept 67 ShipI. Wushingtan (_) Cruise No.028-0002 organization POBSP Recorder Lewis

Sunrise: Timel6 26 Position: Lat.2 24'5, Long. $1 / 154$ Sunset: Time $183 /$ Position: Lat. $0^{\prime} 335$, Iong.11/ $59 \mathrm{w}$

Miles travelled from 0000 hours to sunrise $=5 /$

Miles travelled from sunrise to sunset $=115$

Miles travelled from sunset to 2400 hours $=38$

TIME OF FTX TYPE OF FIX LATITUDE LONGITUDE

1.

2.

3.

4.

5 .

Hourly Positions:

Time Latitude Longitude Wind Dir. Wind Sp. Wave Dir. Wave Hgt.

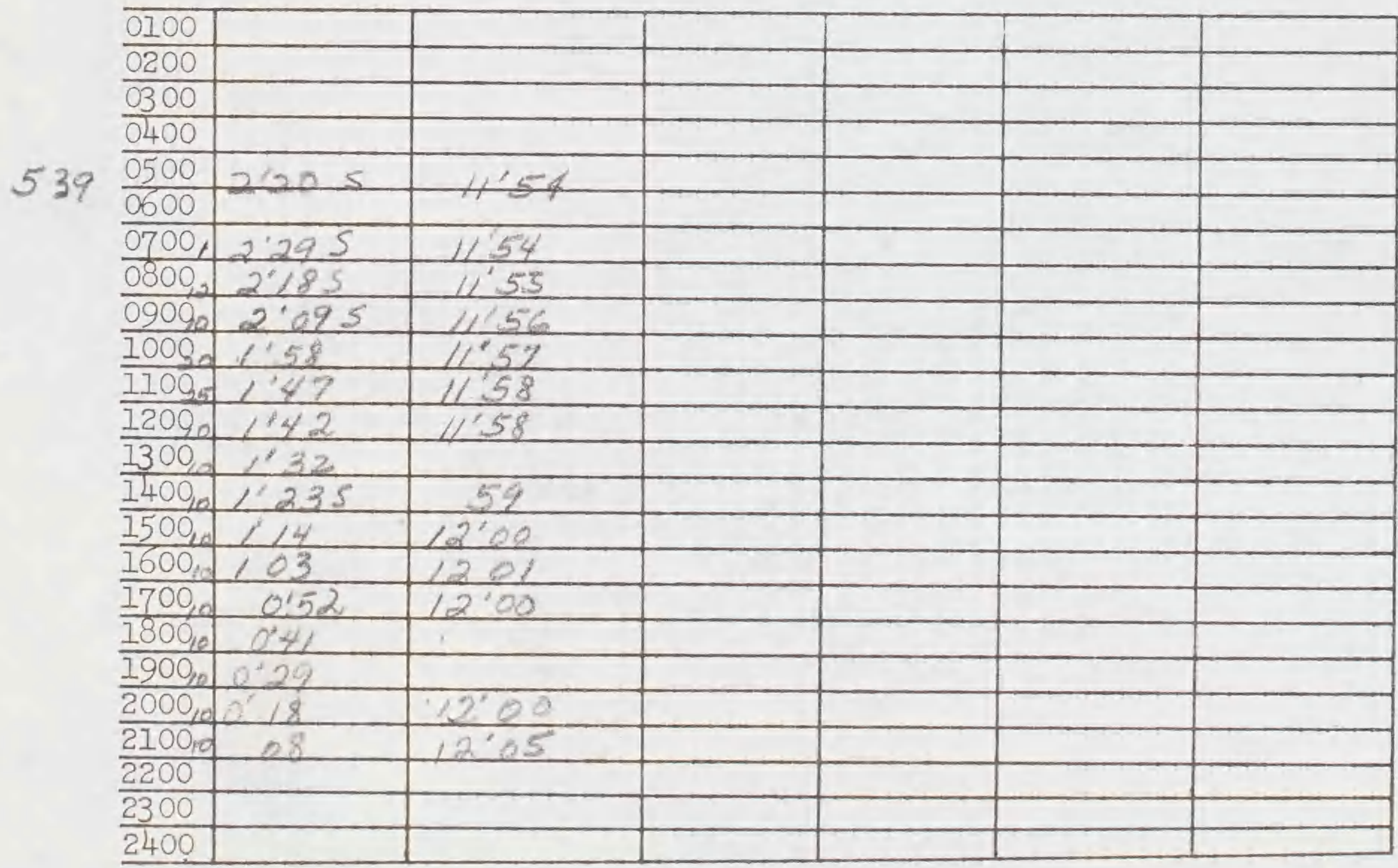


Date 2 Sept 67 organization POBSP

Sunrise: Time 0625 ?

Sunset: Time 1831
ShipT Washingtun

Recorder Lewis
Cruise No.028-0002

Miles travelled from 0000 hours to sunrise $=$

Position: Lat. 00'04 IN Iong. $111 \mathrm{Sg}^{\prime} \mathrm{w}$

Position: Lat. $00^{\circ} 32^{\circ} \mathrm{N}$, Iong. $112 \mathrm{c4} \mathrm{W}$

Miles travelled from sunrise to sunset $=\frac{29}{21}$
Miles travelled from sunset to 2400 hours $=2$

TIME OF FIX TYPE OF FIX LATITUDE LONGITUDE

1.

2 .

3.

4.

5 .

Hourly Positions:

Time Latitude Longitude Wind Dir. Wind Sp. Wave Dir. Wave Hgt.

\begin{tabular}{|c|c|c|c|c|c|}
\hline \multirow{2}{*}{\multicolumn{6}{|c|}{$\frac{100}{0200}=0008 \mathrm{~N}$}} \\
\hline & & & & & \\
\hline \multicolumn{6}{|l|}{0300} \\
\hline \\
\hline \multirow{2}{*}{\multicolumn{6}{|c|}{$\frac{0500}{0600}$}} \\
\hline & & & & & \\
\hline \multirow{2}{*}{\multicolumn{6}{|c|}{$\frac{0700}{0800}$}} \\
\hline & & & & & \\
\hline \multicolumn{6}{|c|}{0900} \\
\hline$\frac{1000}{1100}$ & & & & & \\
\hline \multicolumn{6}{|c|}{$12004000^{\circ} 04 \mathrm{~N}$} \\
\hline \\
\hline & & & & & \\
\hline \multicolumn{6}{|c|}{$112^{\prime} 02 \omega$} \\
\hline \multirow{2}{*}{\multicolumn{6}{|c|}{$\frac{112.034}{112.08}$}} \\
\hline & & & & & \\
\hline \multirow{2}{*}{\multicolumn{6}{|c|}{$112^{\prime} 04$}} \\
\hline & & & & & \\
\hline \multicolumn{6}{|c|}{2000} \\
\hline 2100 & $.043 N$ & $11204 \omega$ & & & \\
\hline 2200 & $0,50 \mathrm{~N}$ & $112^{\prime} 03$ & & & \\
\hline$\frac{2300}{2400}$ & & & & & 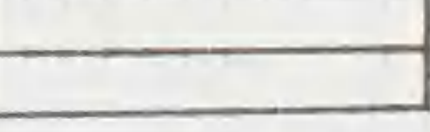 \\
\hline
\end{tabular}


Datesept 3, 62

organization POBSP
ShipT. Woshingten (

Recorder Lewis
Cruise No.028-0002

Sunrise: Time 0624 Position: Lat. $0126 \mathrm{~N}$ Iong. $11201 \mathrm{w}$

Sunset: Time 1833 Position: Lat. $02^{\circ} 477^{\circ}$ Iong.11202 W

Miles travelled from 0000 hours to sunrise $=32$

Miles travelled from sunrise to sunset $=81$

Miles travelled from sunset to 2400 hours $=39$

TIME OF FIX TYPE OF FIX LATITUDE LONGITUDE

1.

2 .

3.

4.

5 .

Hourly Positions:

Time Latitude Longitude Wind Dir. Wind Sp. Wave Dir. Wave Hgt.

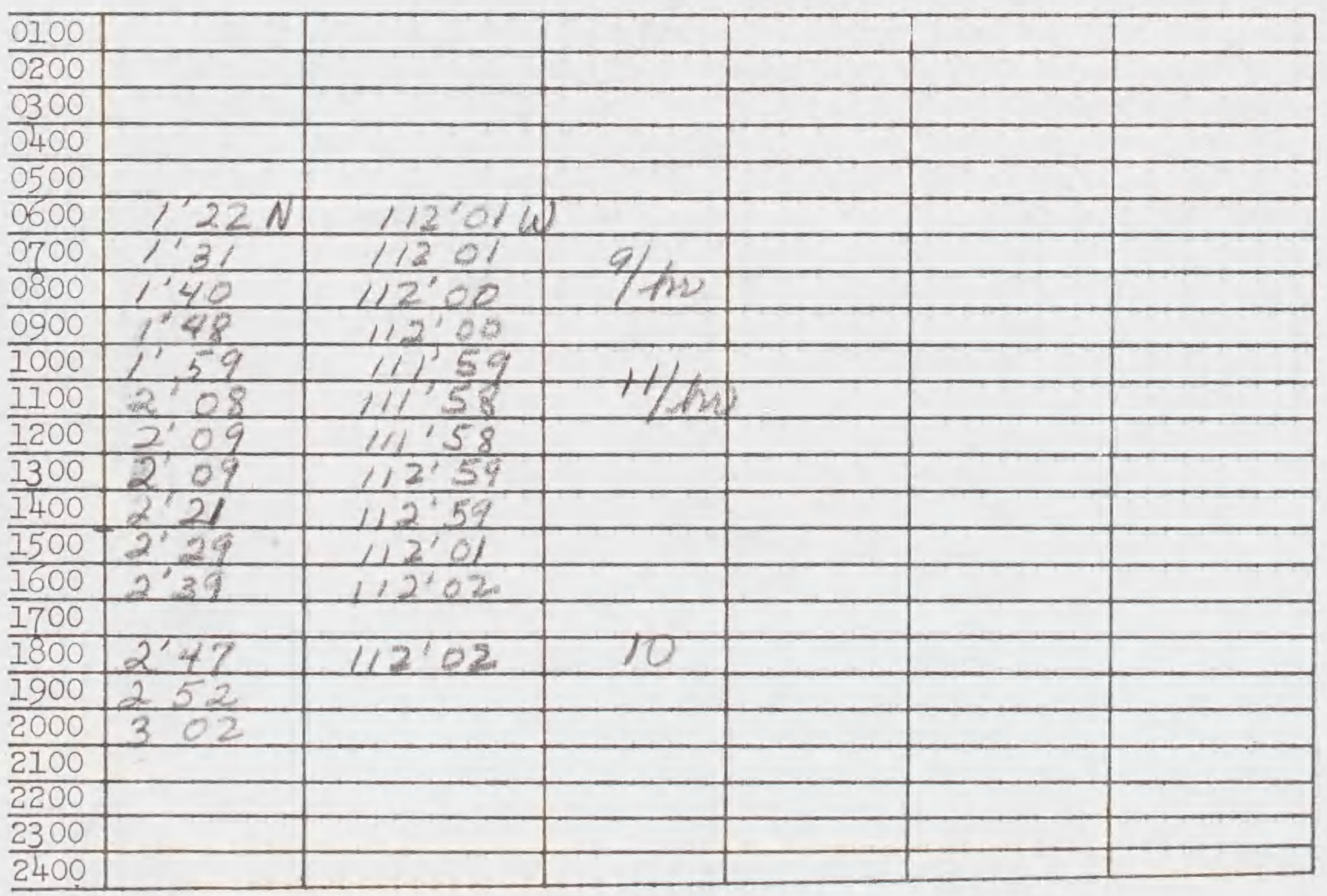


Date 4 Sept 62

organization POBSP
Ship I. Woskingturs.

Recorder Lewis

Sunrise: Time

Sunset: Time 1833
Position: Lat.

Position: Lat.
Cruise No. $028-0002$

Miles travelled from 0000 hours to sunrise $=42$

Miles travelled from sunrise to sunset $=93$

Miles travelled from sunset to 2400 hours $=42$

TIME OF FIX TYPE OF FIX IATITUDE IONGITUDE

1.

2.

3.

4.

5 .

Hourly Positions:

Time Latitude Longitude Wind Dir. Wind Sp. Wave Dir. Wave Hgt.

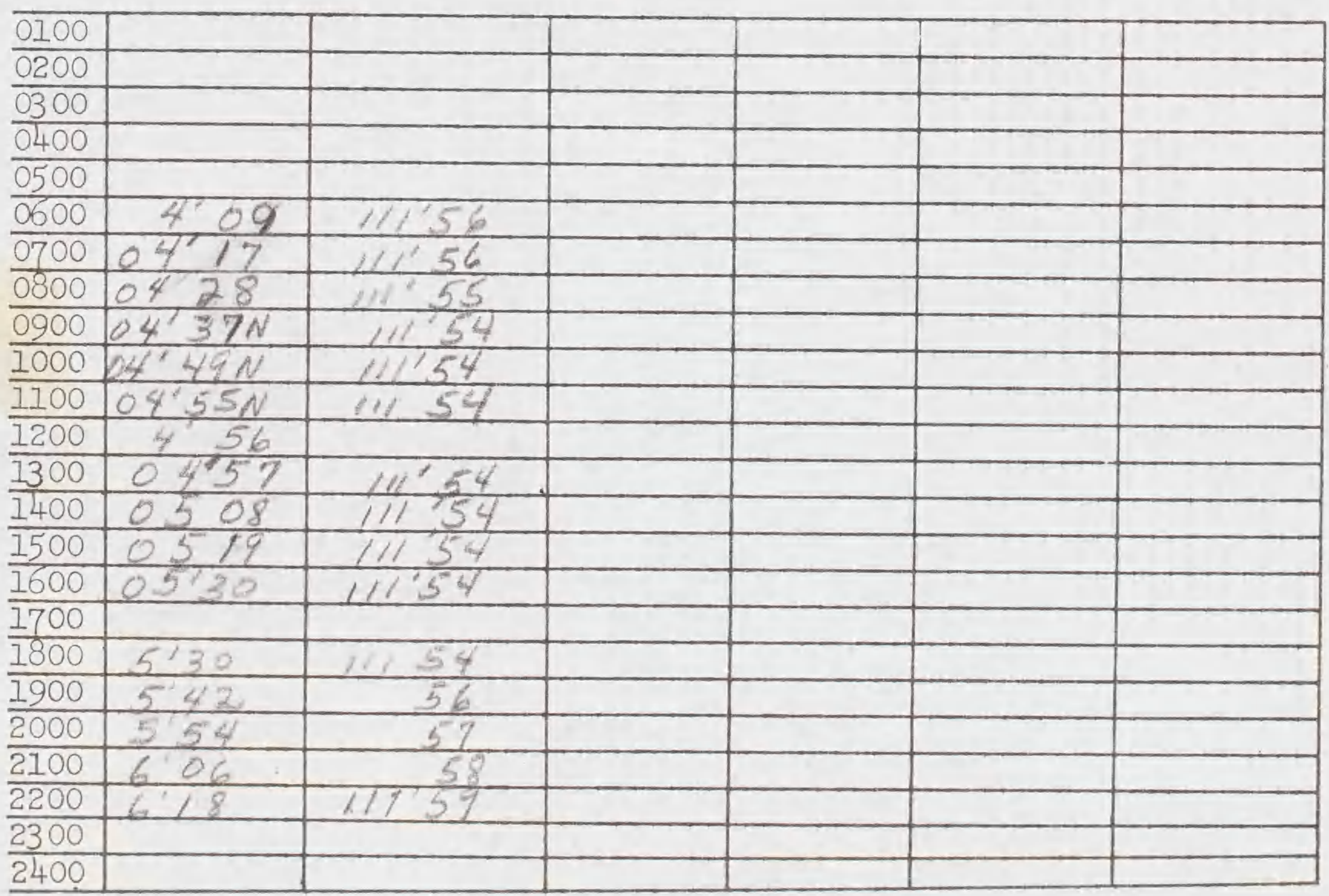


Date $\frac{5 \text { Sept } 67}{\text { organization } 70,36 p}$

Ship. Washington

Cruise No. $028=0002$

Sunrise: Time 9620 Position: Lat. $07^{\circ} 02^{\circ} \mathrm{V}$, Long. $112^{\circ} 05^{\prime} \mathrm{W}$

Sunset: Time 1834 Position: Lat. $08^{\circ} 22 \mathrm{~N}$, Long. $11200 \mathrm{~W}$

Miles travelled from 0000 hours to sunrise $=40$

Miles travelled from sunrise to sunset $=8 /$

Miles travelled from sunset to 2400 hours $=40$

TIME OF FIX TYPE OF FIX LATITUDE LONGITUDE

1.

2.

3.

4.

5 .

Hourly Positions:

Time Latitude Longitude Wind Dir. Wind Sp. Wave Dir. Wave Hgt.

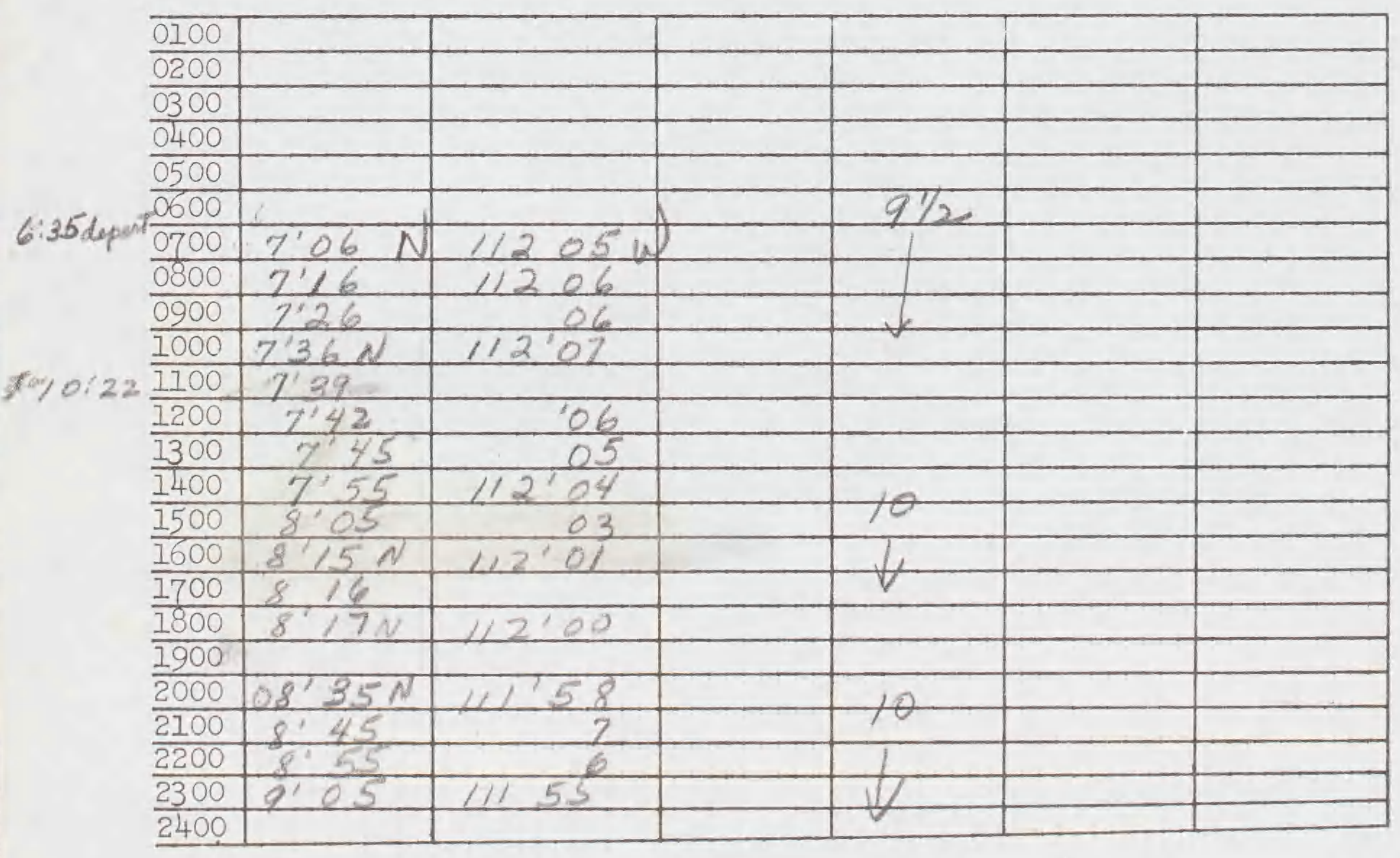

$958 b-\mathrm{SI}-\mathrm{MNH}$

Rev. $9 / 28 / 66$ 
Date 6 Sent 67

organization POBSP
Ship T. Washingtons

Recorder Lewis
Sunrise: Time $6 / 9$

Sunset: Time 1836
Position: Lat. $0938 \mathrm{~N}$ Long. 11150 Position: Lat. 1051 , Long. $1 / 139$

Miles travelled from 0000 hours to sunrise $=37$

Miles travelled from sunrise to sunset

Miles travelled from sunset to 2400 hours $=38$

TIME OF FIX TYPE OF FIX IATITUDE IONGITUDE

1 .

2 .

3.

4.

5 .

Hourly Positions:

Time Latitude Longitude Wind Dir. W/nd-Gp. Wave Dir. Wave Hgt.

\begin{tabular}{|c|c|c|c|c|c|}
\hline 0100 & & & & & \\
\hline$\overline{0200}$ & & & & & \\
\hline 0300 & & & & & \\
\hline 0400 & & & & & \\
\hline 0500 & & & & & \\
\hline 0600 & $935 N$ & $111.50 \mathrm{~W}$ & & & \\
\hline 0700 & $945 \mathrm{~N}$ & 48 & 10 & & \\
\hline 0800 & $955 \mathrm{~N}$ & 42 & & & \\
\hline 0900 & $1005 \mathrm{~N}$ & 45 & & & \\
\hline 1000 & $1015 N$ & 1114461 & & & \\
\hline 1100 & & & & & \\
\hline 1200 & & & & & \\
\hline 1300 & & & & & \\
\hline 1400 & $10^{\prime} 28$ & 111192 & q & & \\
\hline 1500 & 10.374 & 111.41 & 7 & & \\
\hline 1600 & 10 4h & 111.40 & & & \\
\hline 1700 & & & & & \\
\hline$\frac{1800}{1900}$ & & & & & \\
\hline$\frac{1900}{2000}$ & & & & & \\
\hline 2100 & & & & & \\
\hline 200 & & & & & \\
\hline 30 & 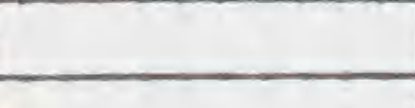 & & & & \\
\hline 2400 & & & & & \\
\hline
\end{tabular}


Date 7 Sent 6 ?

Ship T. Washin tun

Cruise No.

organization POBSP

Recorder Lewis

Sunrise: Time 0617 Position: Lat.1206N, Long. 11209 W

Sunset: Time $183 /$ Position: Lat. $1300 \mathrm{~N}$, Long. 11112 'W

Miles travelled from 0000 hours to sunrise $=42$

Miles travelled from sunrise to sunset $=-79$

Miles travelled from sunset to 2400 hours $=40$

TIME OF FIX TYPE OF FIX LATITUDE LONGITUDE

1.

2.

3.

4.

5.

Hourly Positions:

Time Latitude Longitude Wind Dir. Wind Sp. Wave Dir. Wave Hgt.

$6: 30$
$8: 30$
1217

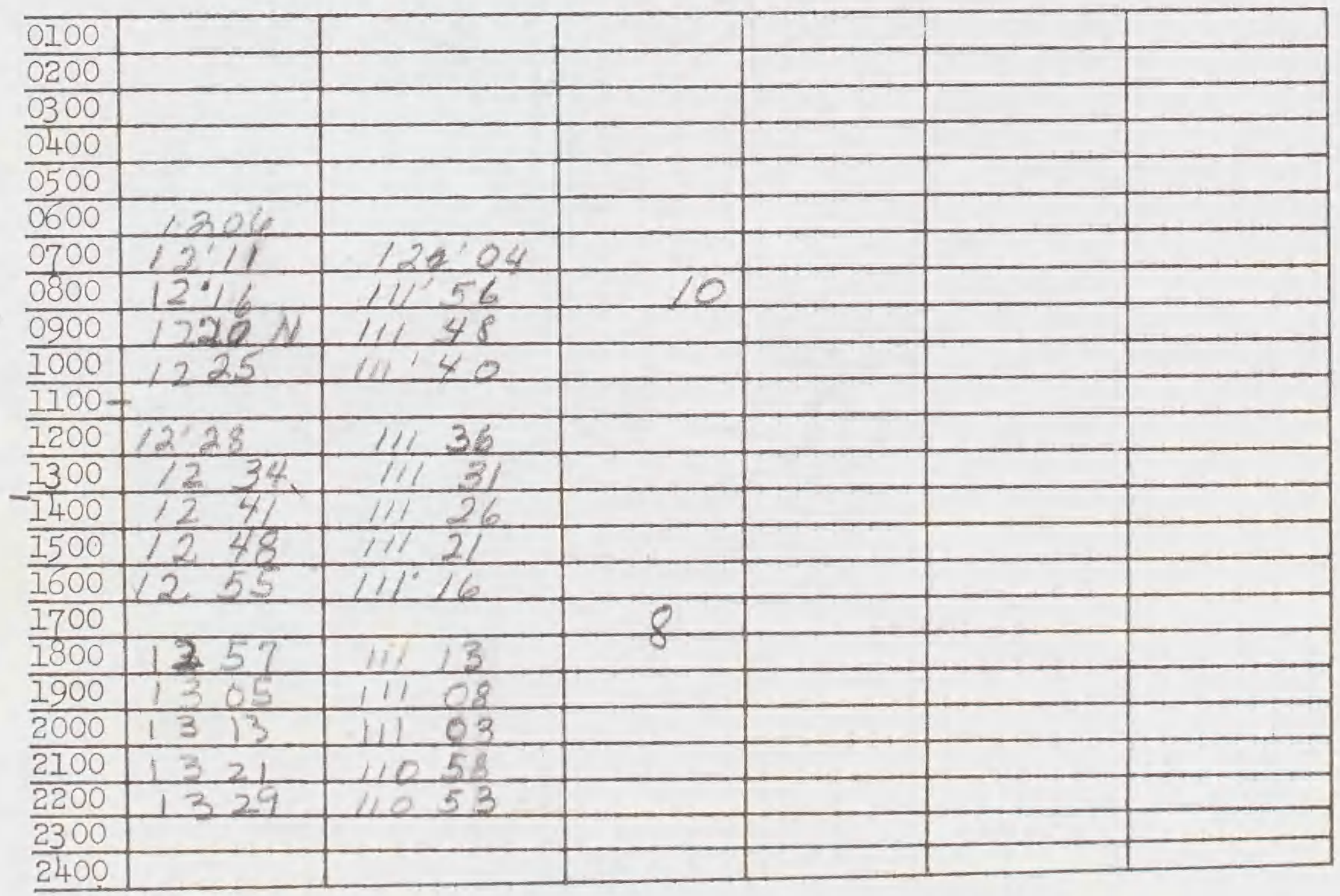

$958 b-\mathrm{SI}-\mathrm{MNH}$

Rev. $9 / 28 / 66$ 
Date 8 Sept 67

Organization POBSP

Sunrise: Time $\geq 10$

Sunset: Time1927
Ship T. Washingtun (

Recorder Lewi's
Cruise No.

Miles travelled from 0000 hours to sunrise $=28$

Miles travelled from sunrise to sunset $=79$

Miles travelled from sunset to 2400 hours $=29$

TIME OF FIX TYPE OF FIX IATITUDE IONGITUDE

1.

2.

3.

4.

5.

Hourly Positions:

Time Latitude Longitude Wind Dir. Wind Sp. Wave Dir. Wave Hgt.

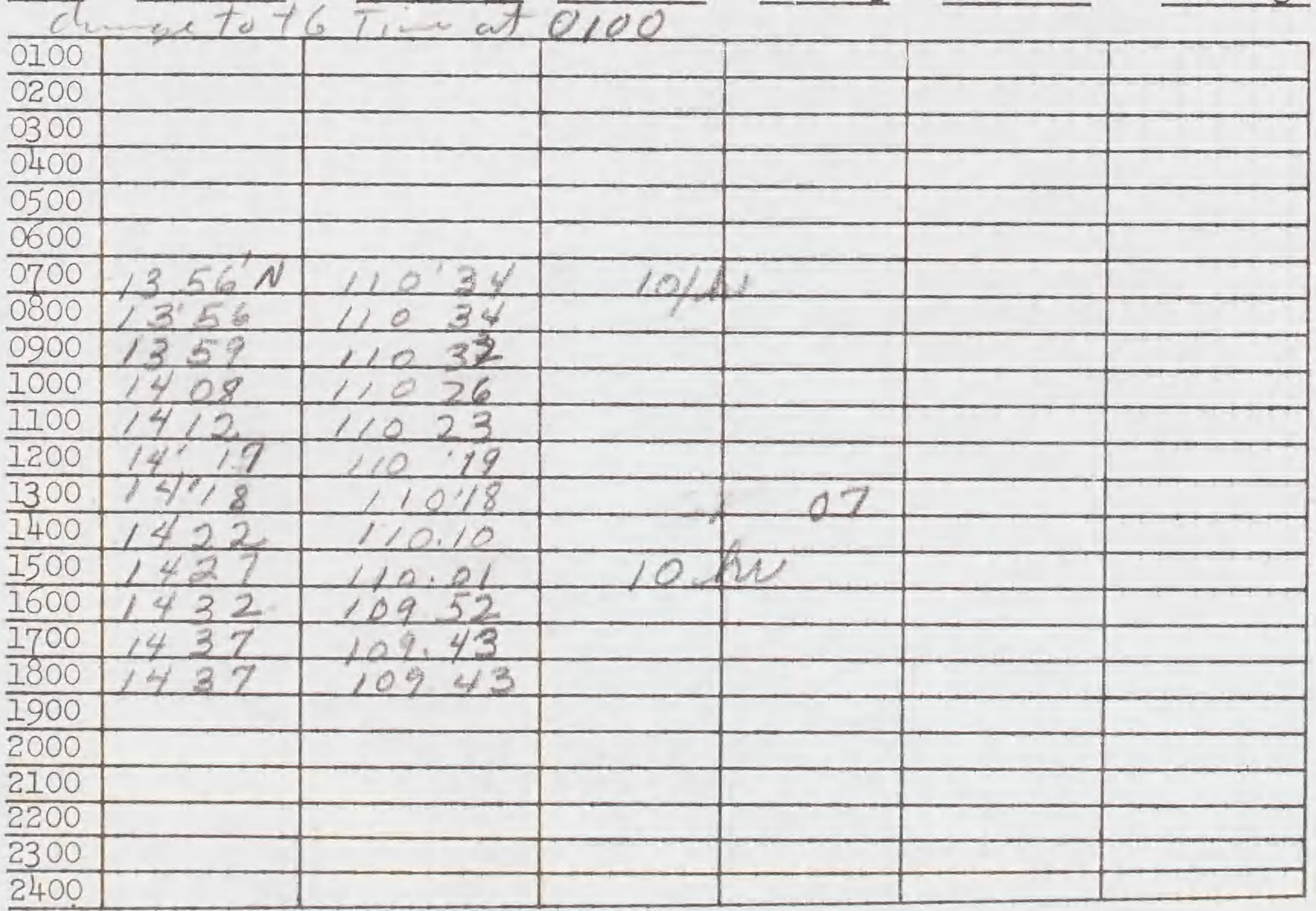


Date 9 Sent 67

Organization POPSP
Ship T. Washington

Recorder hewis
Sunrise: Time) 202

Sunset: Time $19 / 9$
Position: Lat. $15^{\circ} 22 \%$ Iong.10829'w

Position: Lat.163/N, Long.10727

Miles travelled from 0000 hours to sunrise $=4 /$

Miles travelled from sunrise to sunset $=92$

Miles travelled from sunset to 2400 hours $=34$

TIME OF FIX TYPE OF FIX IATITUDE LONGITUDE

1.

2.

3.

4.

5.

Hourly Positions:

Time Latitude Longitude shep Dir. Sp. Wave Dir. Wave Hgt.

\begin{tabular}{|c|c|c|c|c|c|c|}
\hline \multirow{2}{*}{\multicolumn{7}{|c|}{$\frac{0100}{0200}$}} \\
\hline & & & & & & \\
\hline \multirow{2}{*}{\multicolumn{7}{|c|}{$\frac{0300}{0400}$}} \\
\hline \multirow{2}{*}{\multicolumn{7}{|c|}{$\frac{0400}{0500}$}} \\
\hline & & & & & & \\
\hline 0600 & $1521 \mathrm{~N}$ & 108304 & 04 & $10 / \mathrm{hat}$ & & \\
\hline 0800 & 1528 & 10824 & & & & \\
\hline 0900 & 1535 & 10818 & & & & \\
\hline 1000 & $1542 N$ & 10812 & & & & \\
\hline 1100 & 1545 & 10809 & & & & \\
\hline 1200 & 1547 & 10808 & & & & \\
\hline \multicolumn{7}{|c|}{10806} \\
\hline 1400 & 1557 & 10259 & & & & \\
\hline \multicolumn{7}{|c|}{150011604} \\
\hline 1600 & 1611 & 10745 & & & & \\
\hline 1700 & 1618 & 10738 & 04 & $10 \mathrm{Ca}$ & & \\
\hline 1800 & 1622 & 10734 & & & & \\
\hline 1900 & 1629 & 10729 & & & & \\
\hline$\frac{2000}{2100}$ & 1635 & 10723 & 04 & 09 & & \\
\hline$\frac{2100}{2200}$ & & & & & & \\
\hline 00 & & & & & & \\
\hline 2400 & & & & & & \\
\hline
\end{tabular}


Datelo Sent 47

organization POBSP
ShipI. Washingto, (

Recorder Lewis
Sunrise: Time 0653

Sunset: Time1909
Position: Lat. 12 28' 28

Position: Lat. $1825 \mathrm{~N}$
Cruise No.

Miles travelled from 0000 hours to sunrise $=5 /$

Miles travelled from sunrise to sunset $=89$

Miles travelled from sunset to 2400 hours $=33$

TIME OF FIX TYPE OF FIX LATITUDE LONGITUDE

1.
2.
3.
4.
5.

Hourly Positions:

Time Latitude Longitude ShP Dir. Wind Sp. Wave Dir. Wave Hgt.

11: 30001

\begin{tabular}{|c|c|c|c|c|c|c|}
\hline \multirow{2}{*}{\multicolumn{7}{|c|}{$\frac{0100}{0200}$}} \\
\hline & & & & & & \\
\hline \multirow{2}{*}{\multicolumn{7}{|c|}{$\frac{0300}{0400}$}} \\
\hline \\
\hline & & & & & & \\
\hline 0700 & 1728 & 10621 & & & & \\
\hline 0800 & 17.35 & 10613 & 05 & 12 & & \\
\hline 0900 & 1742 & 10604 & & & & \\
\hline 1000 & 1749 & 10556 & & & & \\
\hline 1100 & 1736 & 10548 & & & & \\
\hline 1200 & 1801 & 10541 & & & & \\
\hline \multicolumn{7}{|l|}{1300} \\
\hline 1400 & 1804 & 10537 & 0.5 & It & & \\
\hline 1500 & 1810 & 10528 & & & & \\
\hline 1600 & 812 & 10526 & & & & \\
\hline 1700 & 1815 & 10523 & & & & \\
\hline \multirow{2}{*}{\multicolumn{7}{|c|}{$\frac{7800}{1900 / 1817}$}} \\
\hline & 1827 & 10509 & & & & \\
\hline \multicolumn{7}{|c|}{$\frac{2000}{2100}$} \\
\hline \multicolumn{7}{|l|}{$\frac{2100}{2200}$} \\
\hline 2300 & & 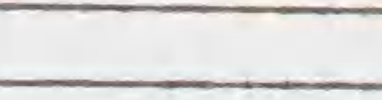 & & & & \\
\hline 2400 & & & & & & \\
\hline
\end{tabular}


Date 13 Sent 67

Ship T. Washingten

Cruise No.

Organization POBSP

Recorder Lewss

Sunrise: Time

Position: Lat.

Long.

Sunset: Time 1904 Position: Lat. $19^{2} 05^{\circ} \mathrm{N}$, Iong. 10509

Miles travelled from 0000 hours to sunrise $=$

Miles travelled from 1100

Miles travelled from sunset to 2400 hours

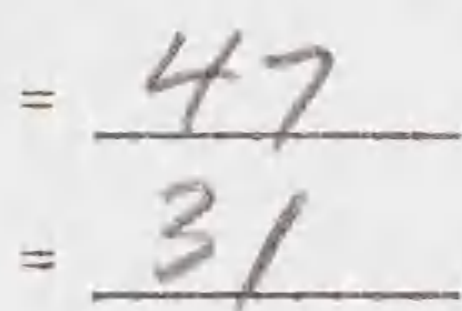

TIME OF FIX TYYPE OF FIX IATITUDE

IONGITUDE

1 .

2.

3.

4.

5 .

Hourly Positions:

Time Latitude Longitude Wind Dir. Wind Sp. Wave Dir. Wave Hgt.

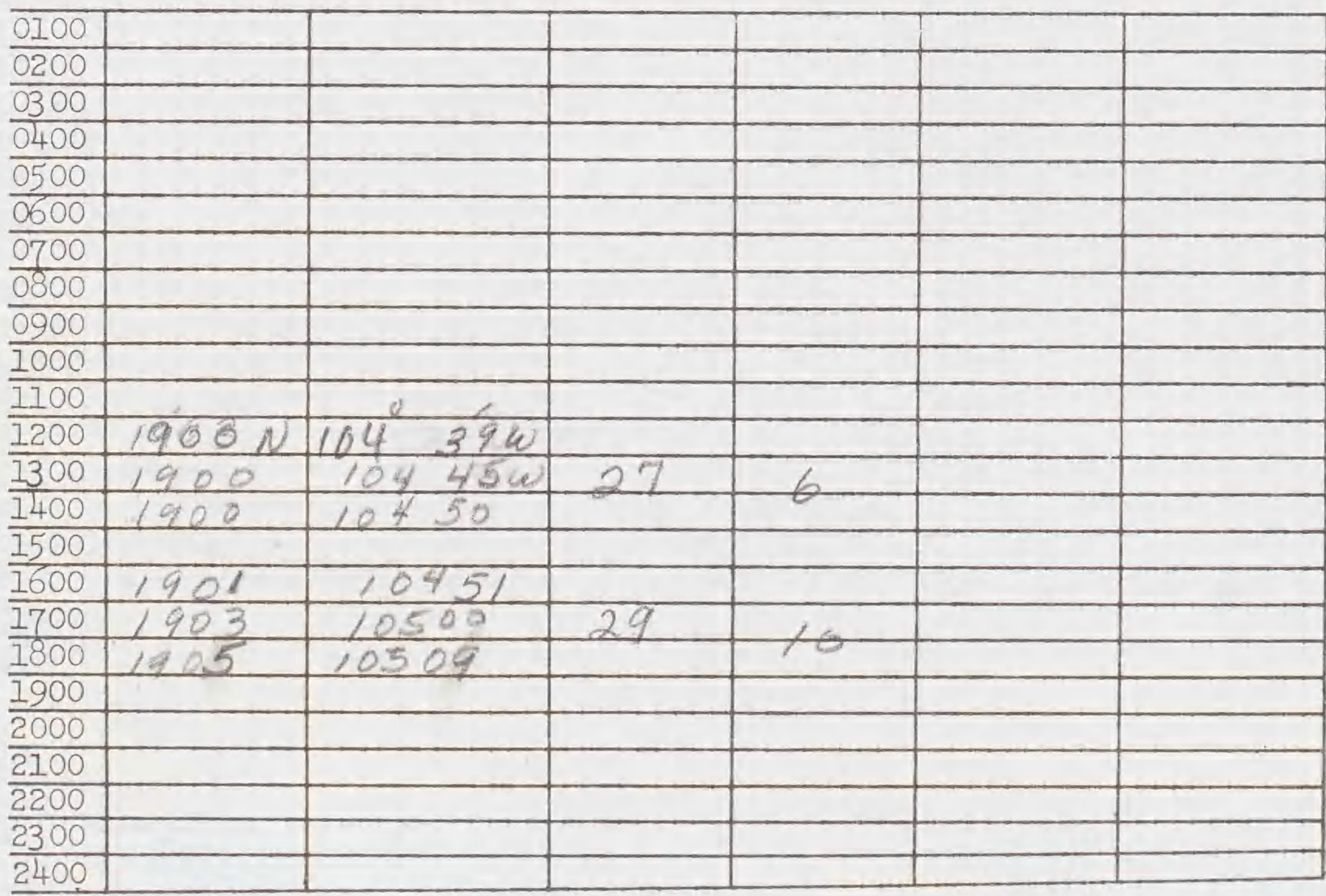


Date 14 Sent 6 ?

organization POBSP

Sunrise: Time 06 54

Sunset: Time 1920
Ship T. Washington(

Recorder hew's

Cruise No.

Miles travelled from 0000 hours to sunrise $=$

Miles travelled from sunrise to sunset

Miles travelled from sunset to 2400 hours

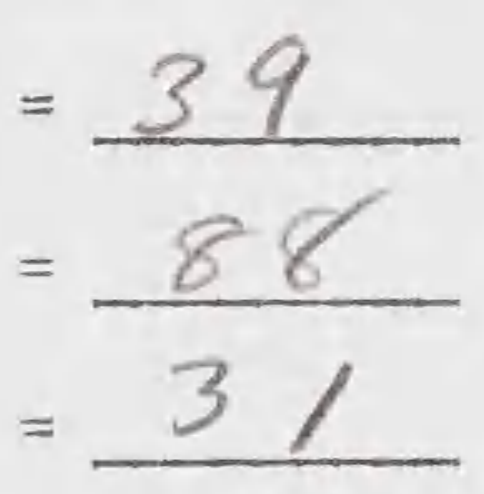

TIME OF FIX TYPE OF FIX IATITUDE

IONGITUDE

I.

2.

3.

4.

5.

Hourly Positions:

Time Latitude Longitude Ship Dir. Ship Sp. Wave Dir. Wave Hgt.

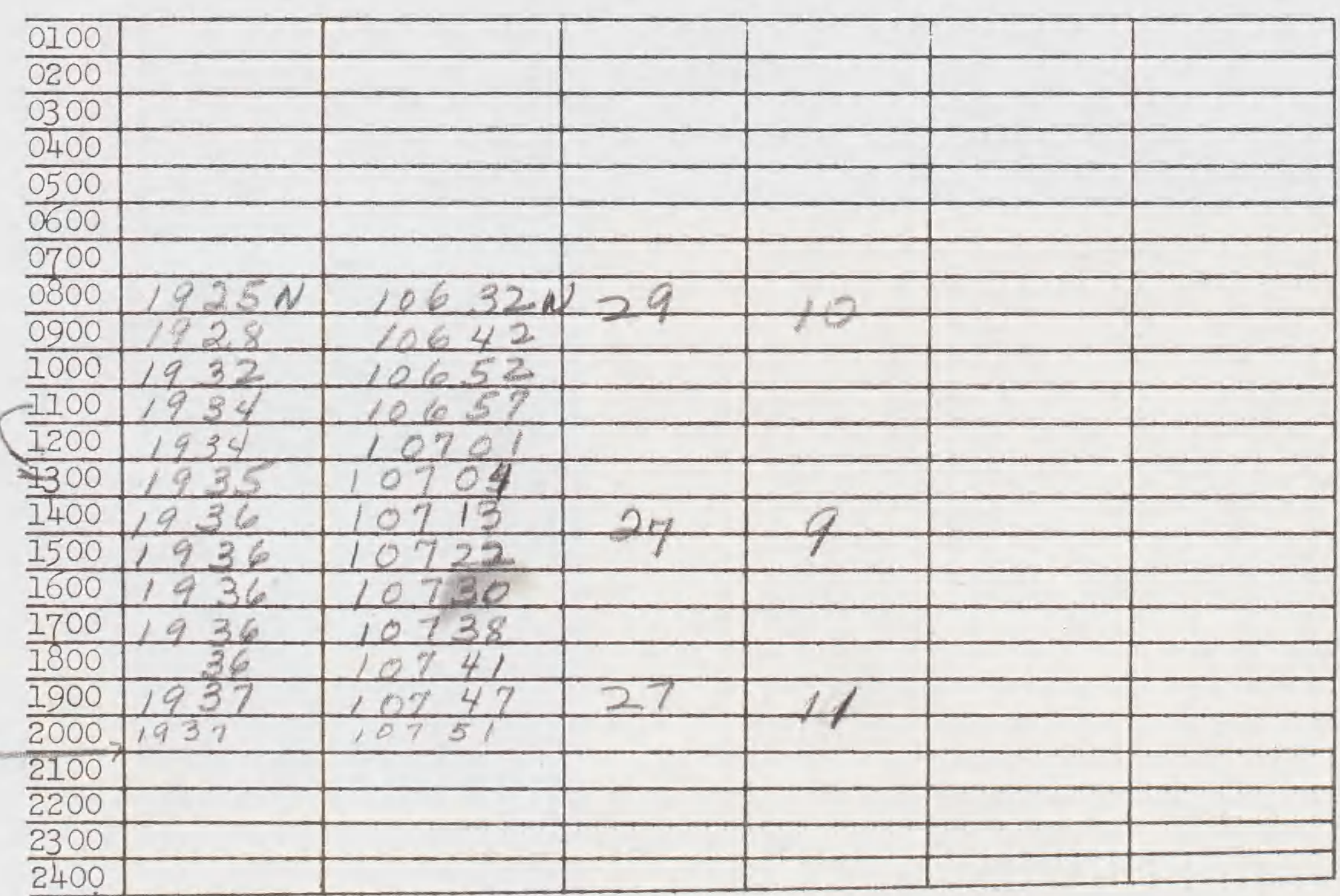


Date 15 Se,2t 67

organization POBSP
Ship T. Washingten (

Recorder lewis
Sunrise: Time 0603

Sunset: Time 1822
Position: Lat. 1943 , Long. 10914 Position: Lat. 1953 , Iong. $110^{\circ} 46$

Miles travelled from 0000 hours to sunrise $=47$

Miles travelled from sunrise to sunset $=-90$

Miles travelled from sunset to 2400 hours $=37$

TIME OF FIX TYPE OF FIX LATITUDE LONGITUDE

1.

2 .

3.

4.

5.

Hourly Positions:

Time Latitude Iongitude Wind Dir. Wind Sp. Wave Dir. Wave Hgt.

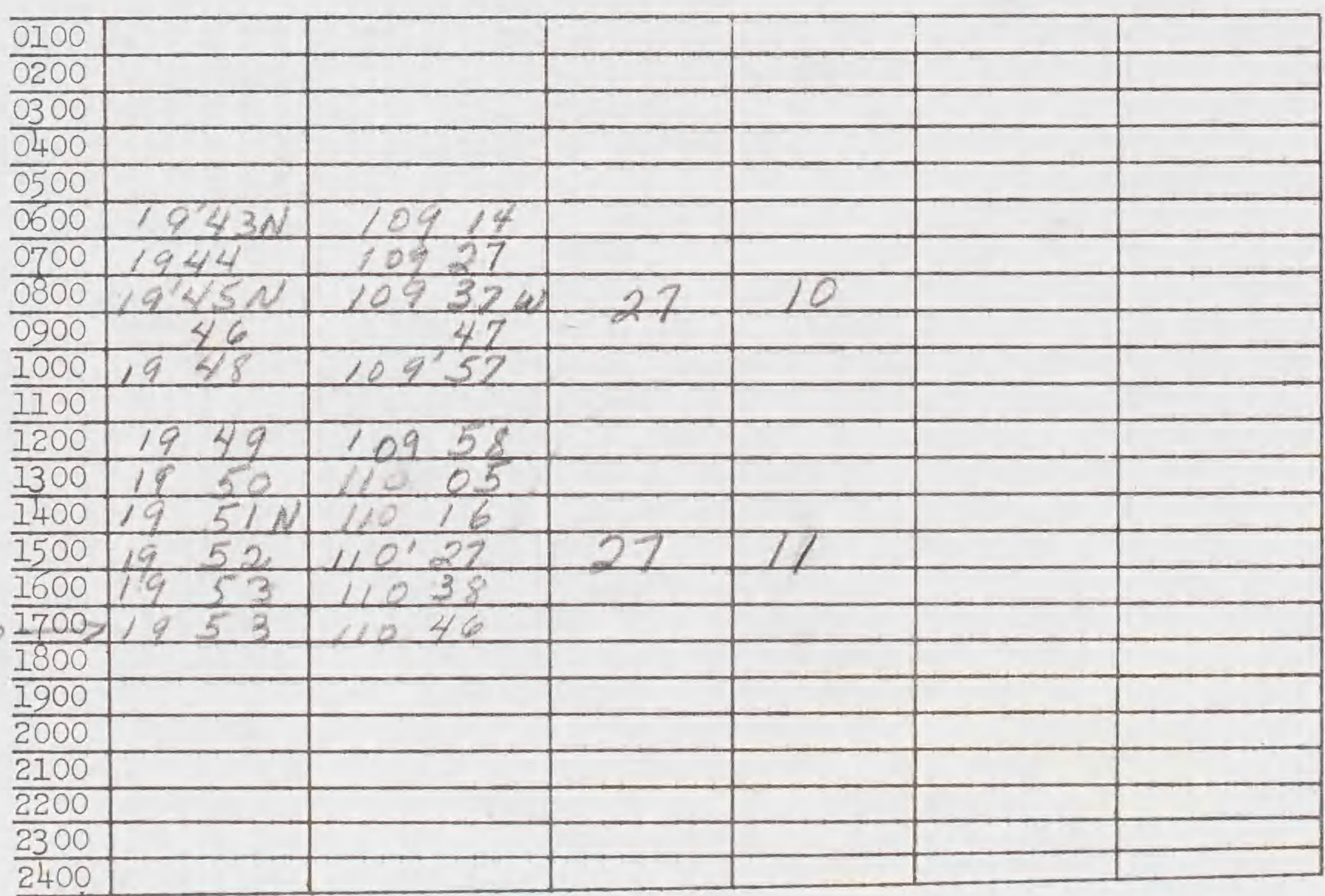


Date 16 Sent

organization POBSP
Ship Washington

Recorder hewis
Sunrise: Time $16 / 6$

Sunset: Time
Position: Lat. $20^{\circ} 42$, Iong. 11139 Position: Lat. $22^{2} \geq 5$, Iong. 1313

Miles travelled from 0000 hours to sunrise $=56$

Miles travelled from sunrise to sunset $=12 /$

Miles travelled from sunset to 2400 hours $=52$

TIME OF FIX TYPE OF FIX LATITUDE LONGITUDE

$\begin{array}{llll}\text { 1. } 0546 & * & 20^{\circ} 4 b^{\prime} & 111^{\circ} 56^{\prime} \\ \text { 2. } 1225 & \text { LAN } & 21^{\circ} 36^{\prime} & 11238 \\ \text { 3. } 1900 & * & 2230 & 1136^{\circ}, \\ \text { 4. } 2400 & D R & 2310 & 11344\end{array}$

5.

Hourly Positions:

Time Latitude Longitude Ship Dir. Wind Sp. Wave Dir. Wave Hgt.

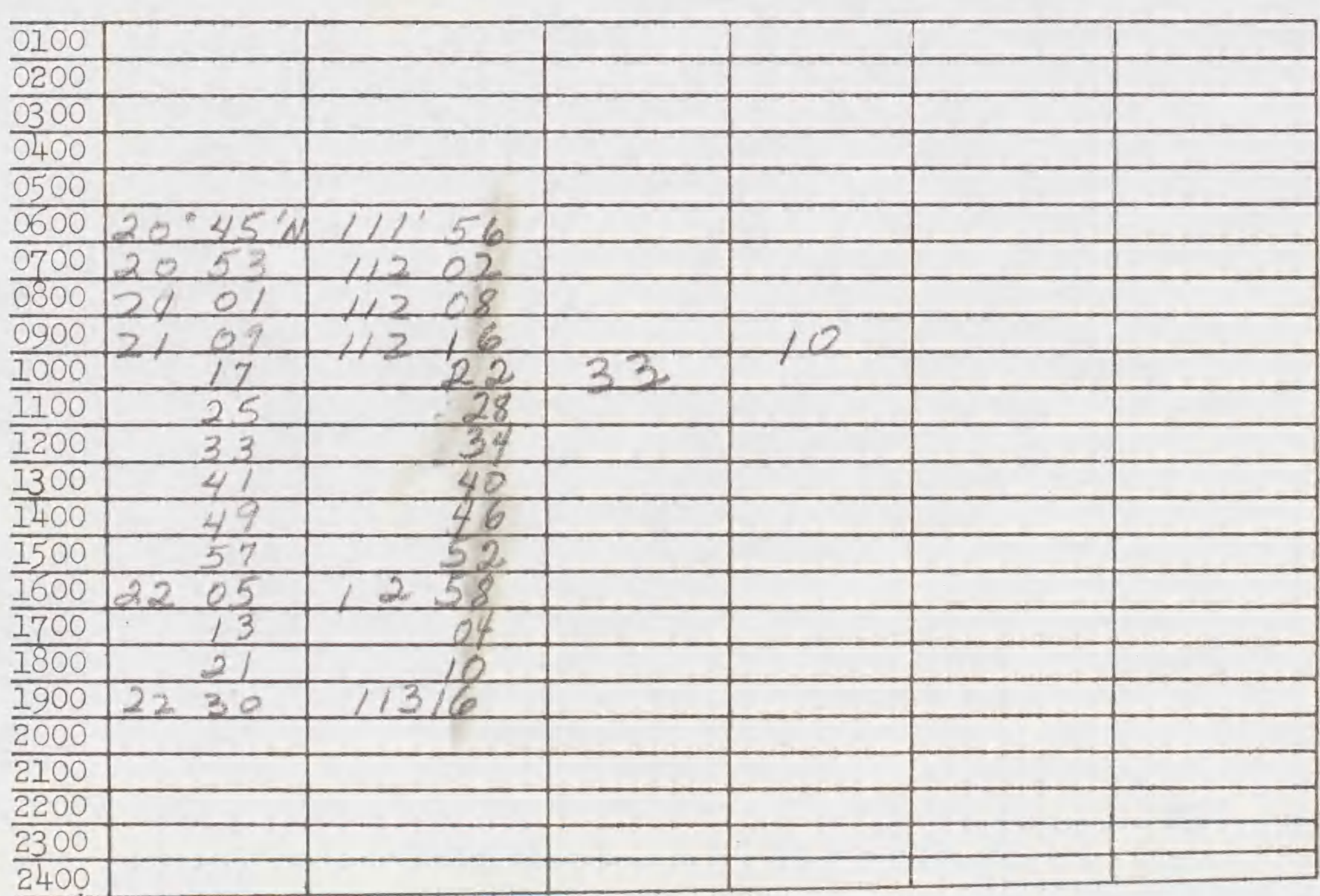


Date 17 Sept 67

Organization POBSP
Shipt Washington (__ Cruise No.

Recorder Lewis

Sunrise: Time 0624 Position: Lat. $24 \mathrm{~d}$, Iong. $114 \mathrm{22}$

Sunset: Time 1845 Position: Lat. 2535 , Long. 11543

Miles travelled from 0000 hours to sunrise $=\underline{C L}$

Miles travelled from sunrise to sunset $=118$

Miles travelled from sunset to 2400 hours $=49$

TIME OF FIX TYPE OF FIX LATITUDE LONGITUDE

$\begin{array}{llllll}1.0356 & * & 2341 N 11407 \\ 3.0554 & * & 2357 & 11419 \\ 3.1235 & \angle A N & 2448 & 11503 \\ 4.1921 & * & 2539 & 11547\end{array}$

5 .

Hourly Positions:

Time Latitude Iongitude Wind Dir. Wind Sp. Wave Dir. Wave Hgt.

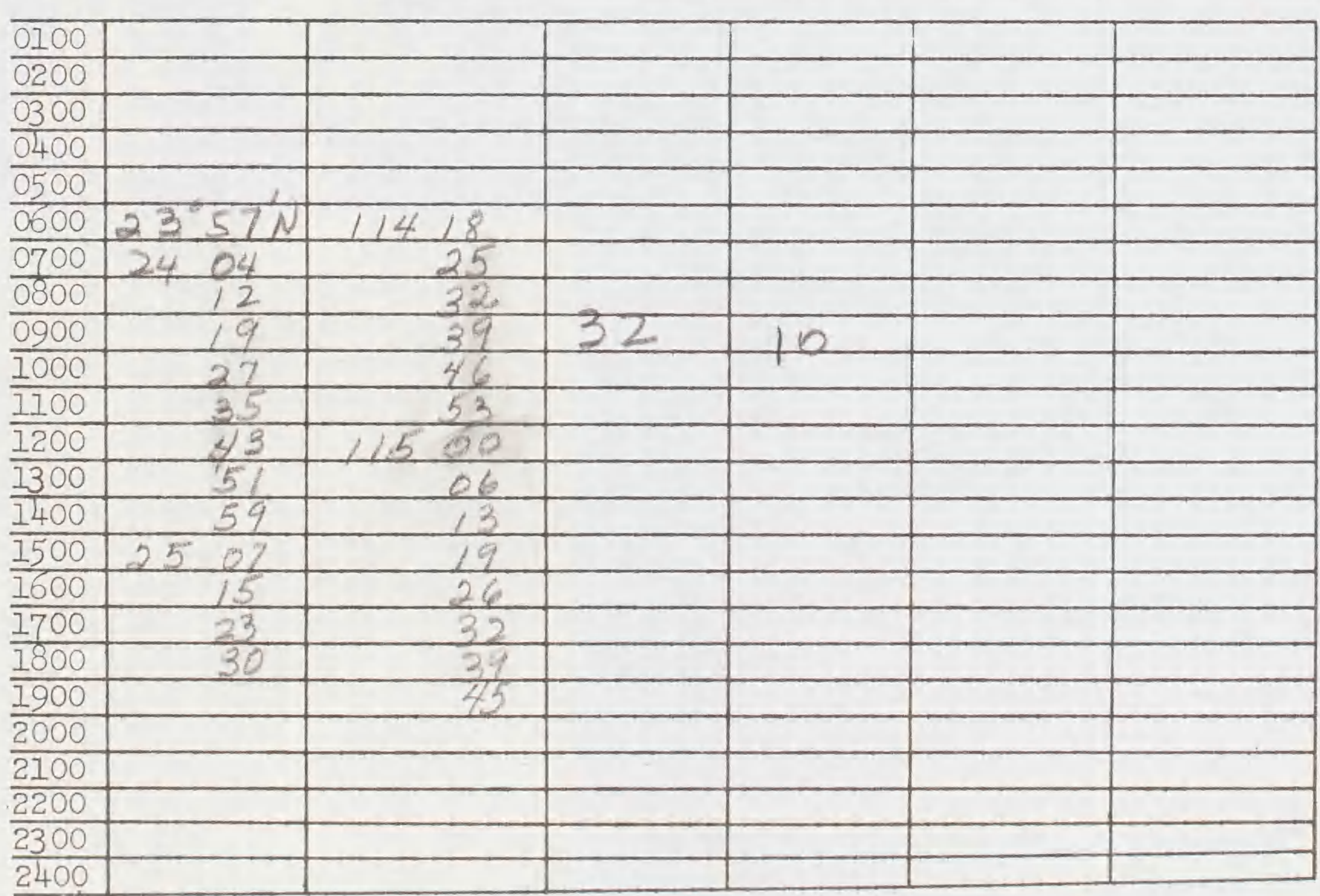


Date 18 sent 67

organization POBSP
Ship T. Washingtun

Recorder

Cruise No.

Sunrise: Time 0637 Position: Lat. 2706 , Iong. $117 / 1$

Sunset: Time Position: Lat. , Iong.

Miles travelled from 0000 hours to sunrise $=$

Miles travelled from sunrise to sunset

Miles travelled from sunset to 2400 hours $=$ TIME OF FIX TTYPE OF FIX IATITUDE LONGITUDE

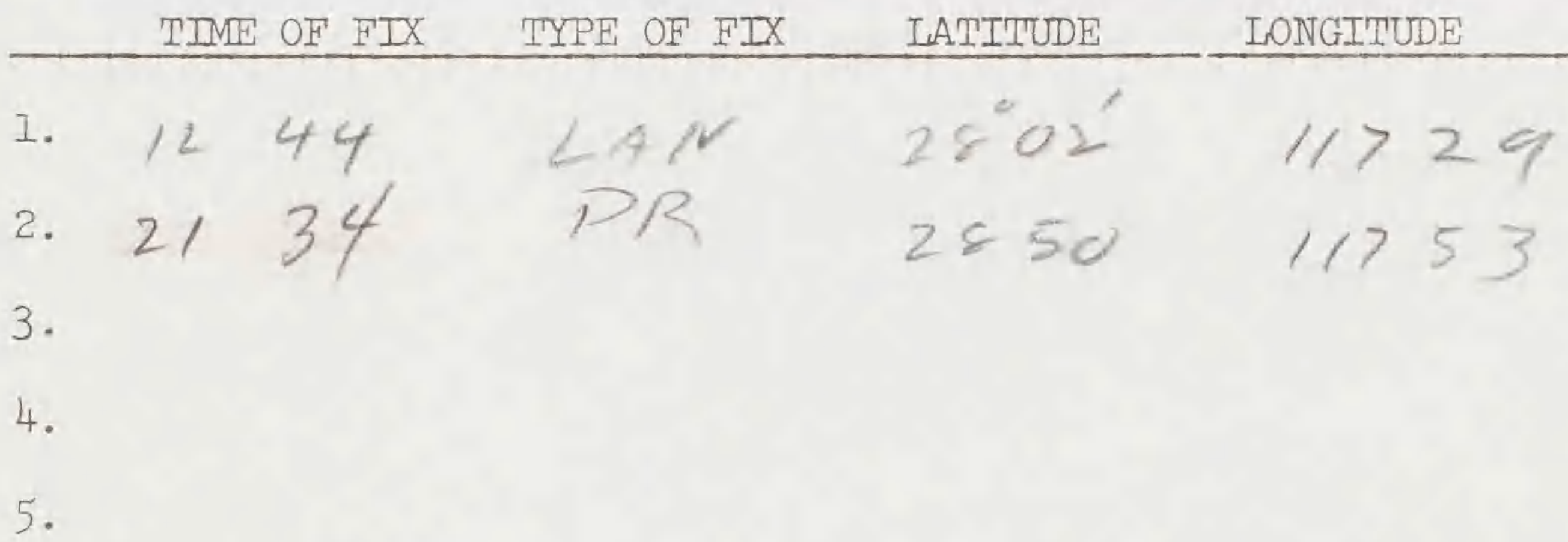

Hourly Positions:

Time Latitude Longitude Wind Dir. Wind Sp. Wave Dir. Wave Hgt.

\begin{tabular}{|c|c|c|c|c|c|c|}
\hline 0100 & & & & & & \\
\hline 0200 & & & & & & \\
\hline 0300 & & & & & & \\
\hline 0400 & & & & & & \\
\hline 0500 & & & & & & \\
\hline 0600 & $27^{\circ} 00$ & $117^{\circ} 08^{\prime}$ & 34 & $01 / 6$ & & \\
\hline 0700 & 2209 & $<2$ & & & & \\
\hline 0800 & 2718 & $\angle 5$ & & & & \\
\hline 0900 & 2727 & 18 & & & & \\
\hline 1000 & 2736 & 21 & & & & \\
\hline 1100 & 2745 & 24 & & & & \\
\hline 1200 & 2754 & 27 & & & & \\
\hline 1300 & 2803 & 30 & & & & \\
\hline 1400 & 2812 & 33 & & & & \\
\hline 1500 & 2821 & 36 & & & & \\
\hline 1600 & & & & & & \\
\hline 1700 & & & & & & \\
\hline 1800 & & & & & & \\
\hline 1900 & & & & & & 4 \\
\hline 00 & & & & & & \\
\hline 00 & & & & & & \\
\hline 0 & & & & & & \\
\hline 00 & & & & & & \\
\hline 00 & & & & & & \\
\hline
\end{tabular}




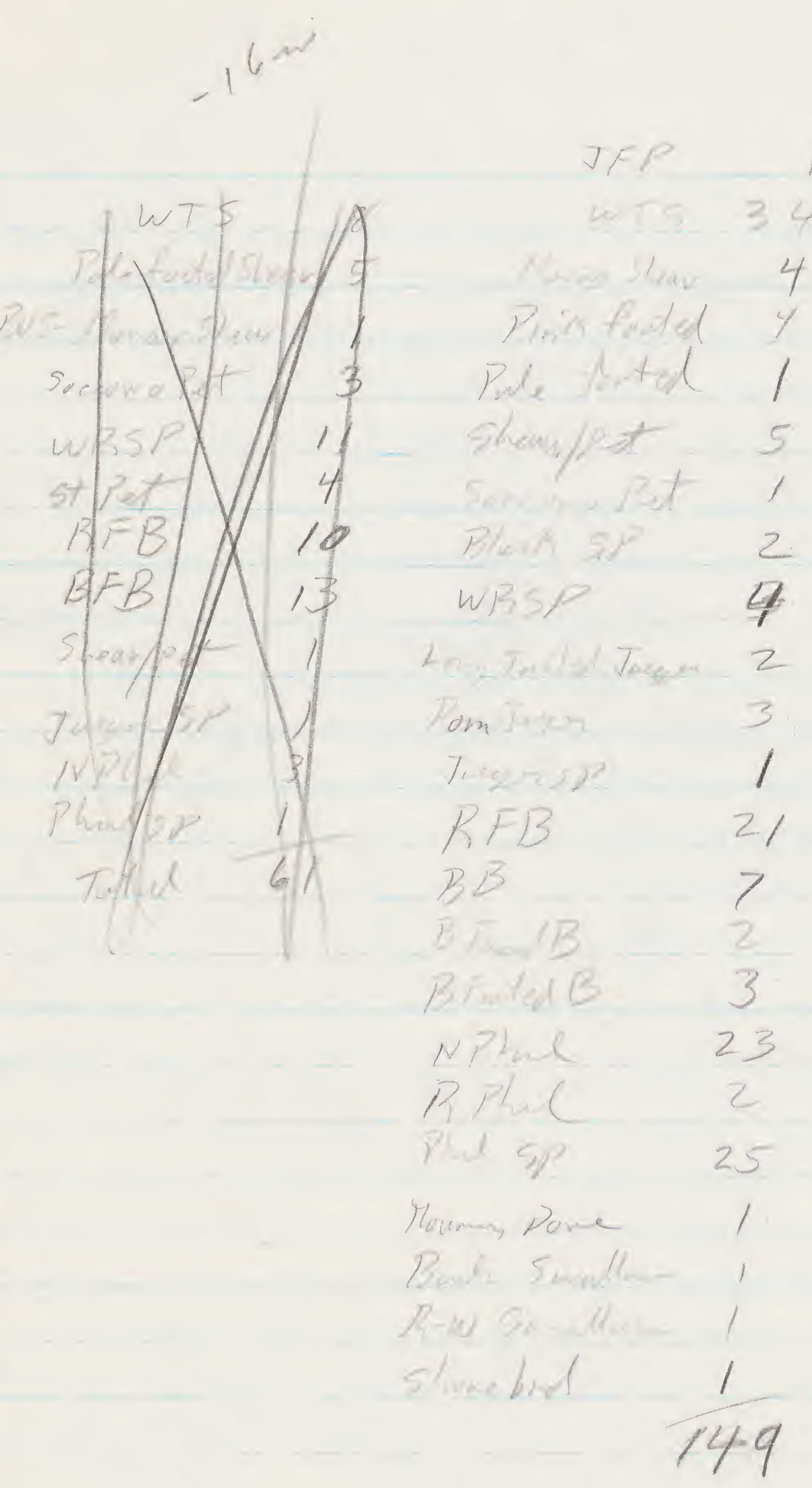


Date $24 \operatorname{sen} 67$

Organization POBSP
Ship T. Washingtour (

Recorder hewis

Sunrise: Time 0640 Position: Lat. $29^{\circ} 15.5 \mathrm{~N}$ Long. $117^{\circ} 48^{\circ} \mathrm{J}^{\mathrm{W}}$

Sunset: Time $184 /$ Position: Lat. 31020's, Iong.

Miles travelled from 0000 hours to sunrise $=29^{\prime}$

Miles travelled from sunrise to sunset $=126^{\prime}$

Miles travelled from sunset to 2400 hours $=$

TIME OF FIX TYPE OF FIX LATITUDE IONGITUDE

1.

2.

3.

4.

5 .

Hourly Positions:

Time Latitude Longitude Wind Dir. Wime Sp. Wave Dir. Wave Hgt.

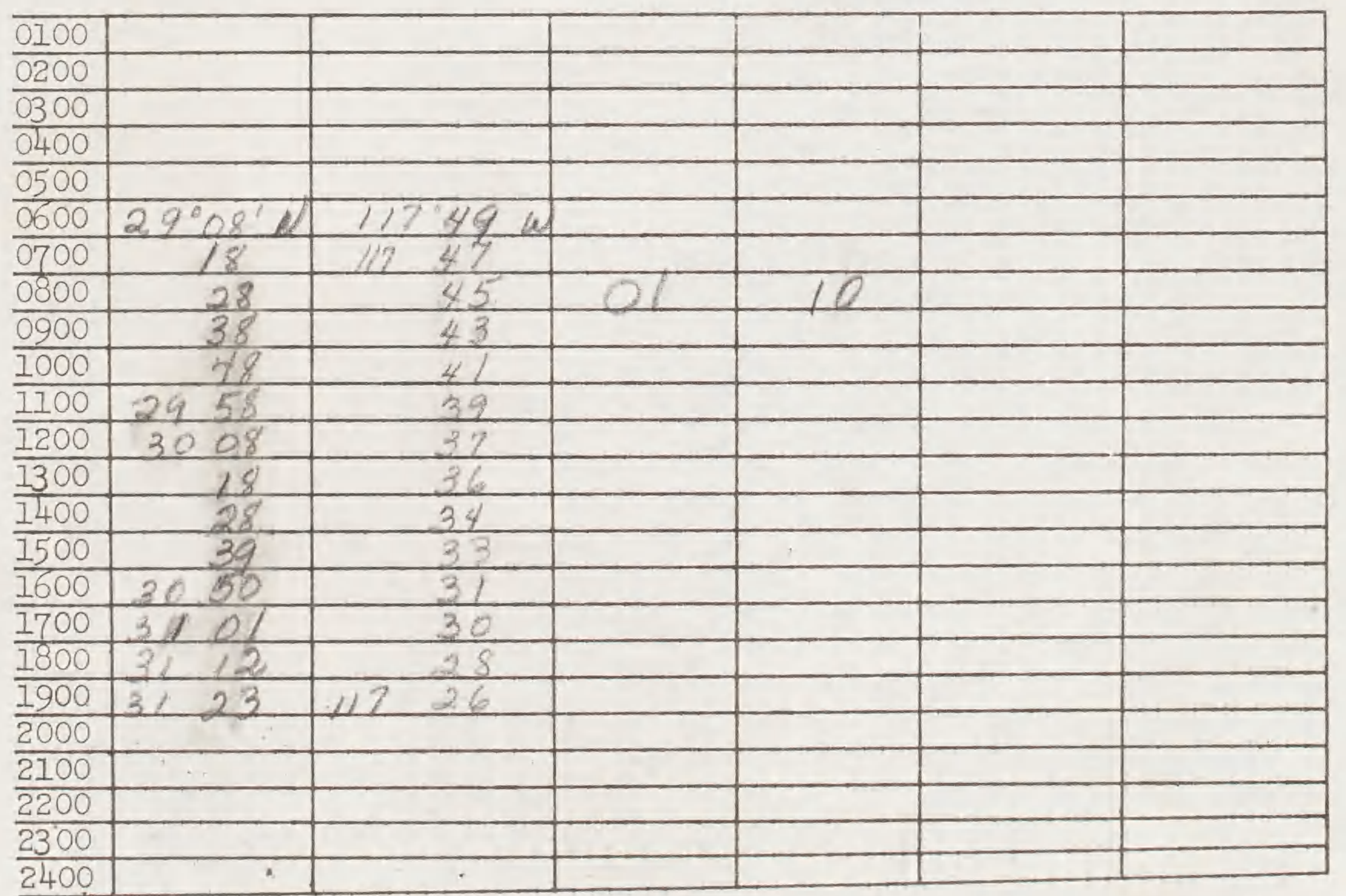




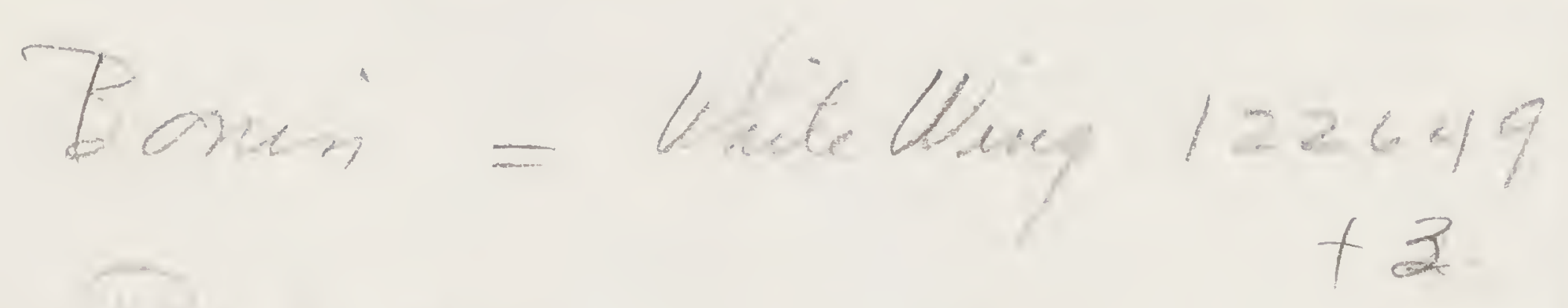

Close of $=123418+3$ Caclawe Petue $12=451+2$

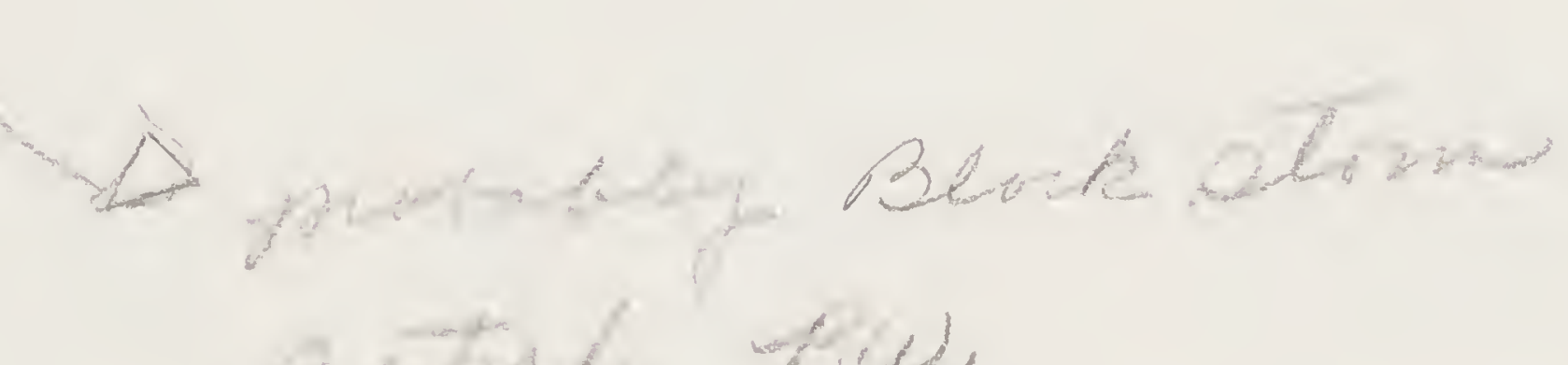
potal tw.

Am Pentemin 122600 
Sooty chencenta

$$
122519
$$

onty slendwbil 2

$$
\begin{aligned}
& \text { WPSP-123400-0 } \\
& \text { DRSP - } 123422+3 \\
& \text { DRPRA } 122607
\end{aligned}
$$

DRPhA $122607^{+3}$

manx - 12zszz

T White phency Relz

White necked Pithela 122601 if omp whet nowe $\rightarrow 609$ 
$-7 m i$

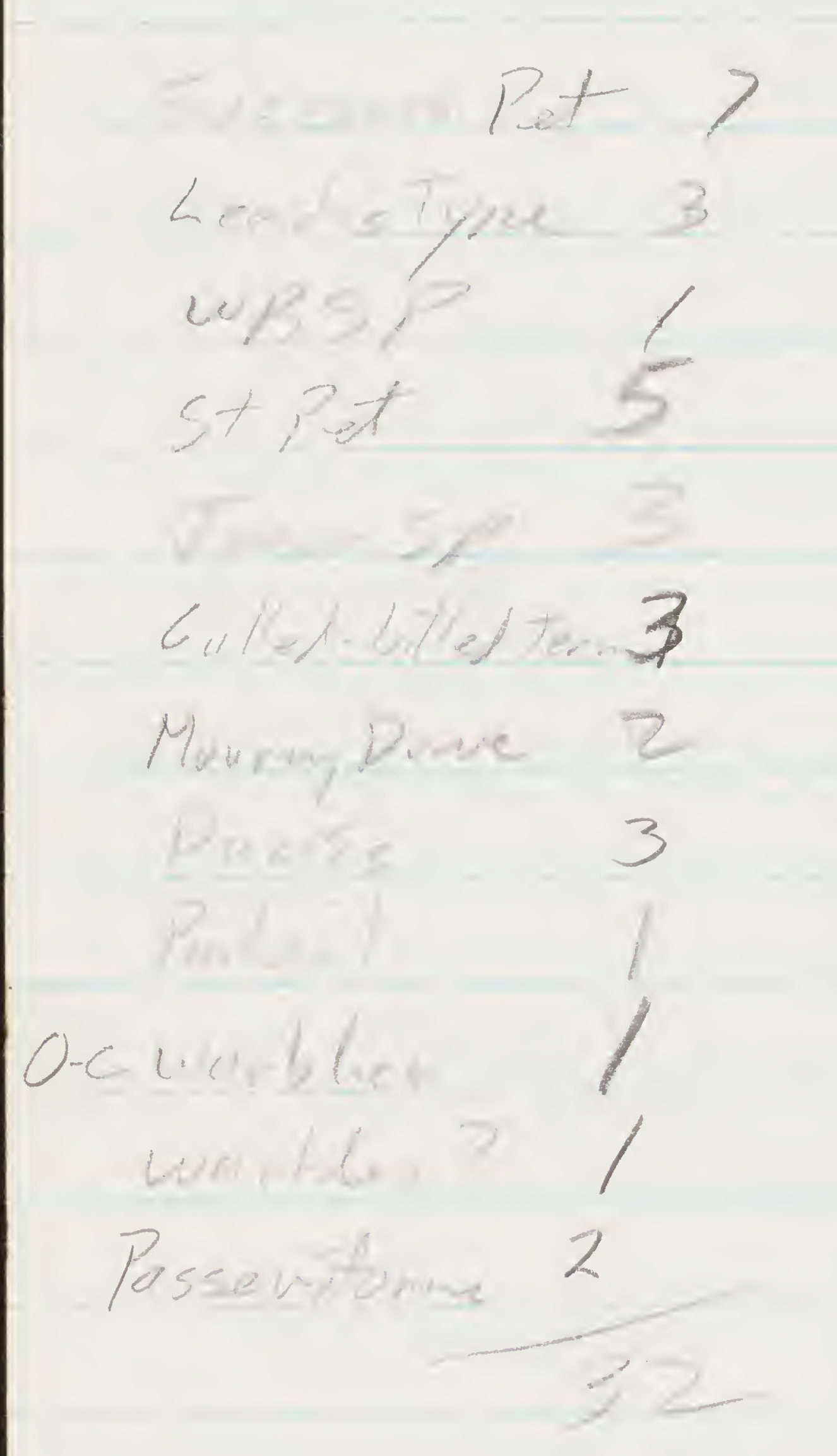




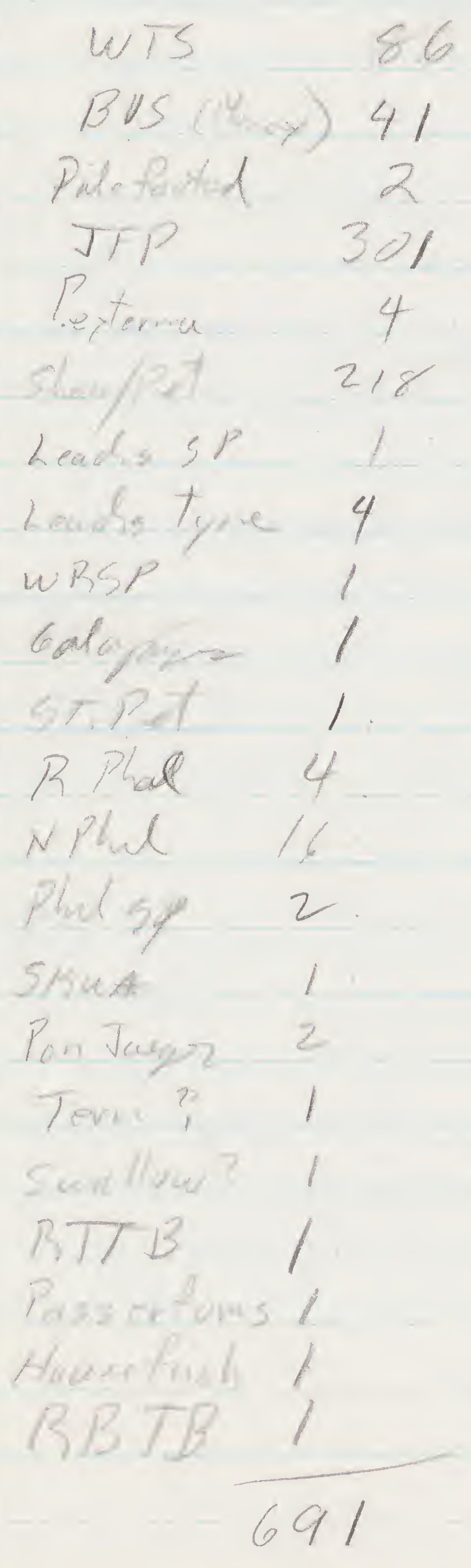



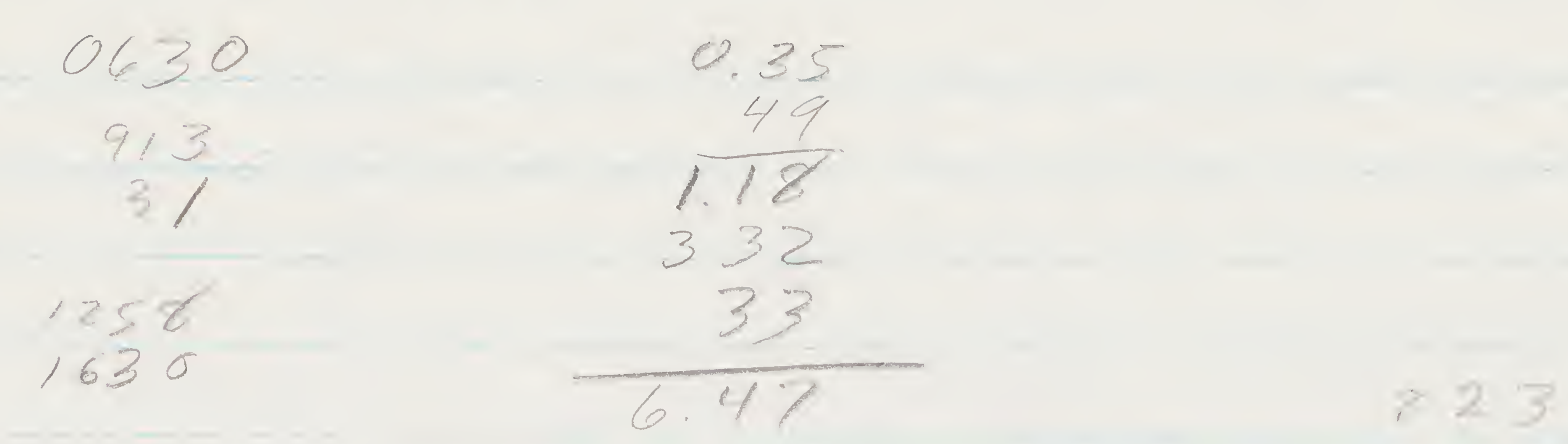

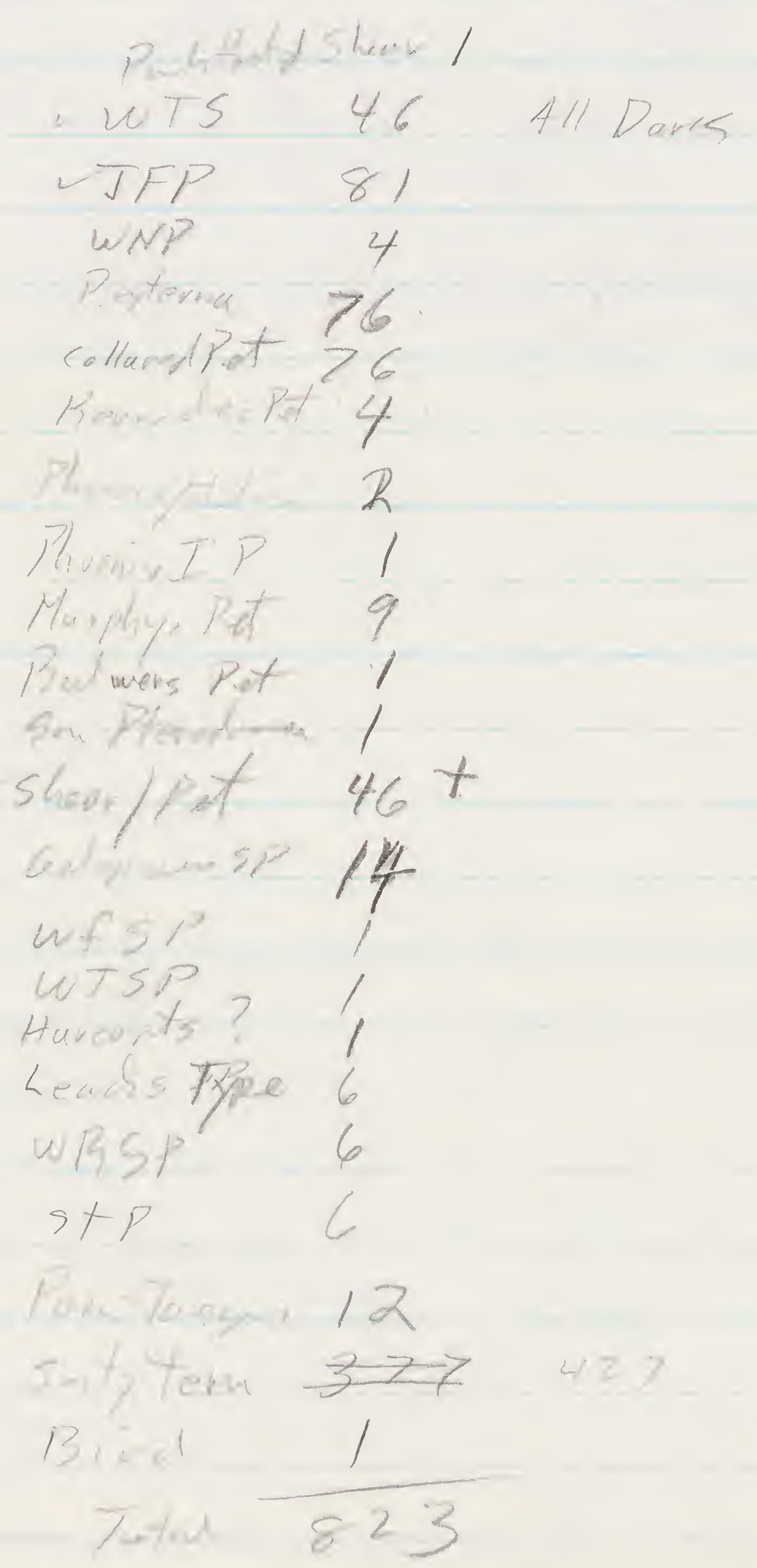




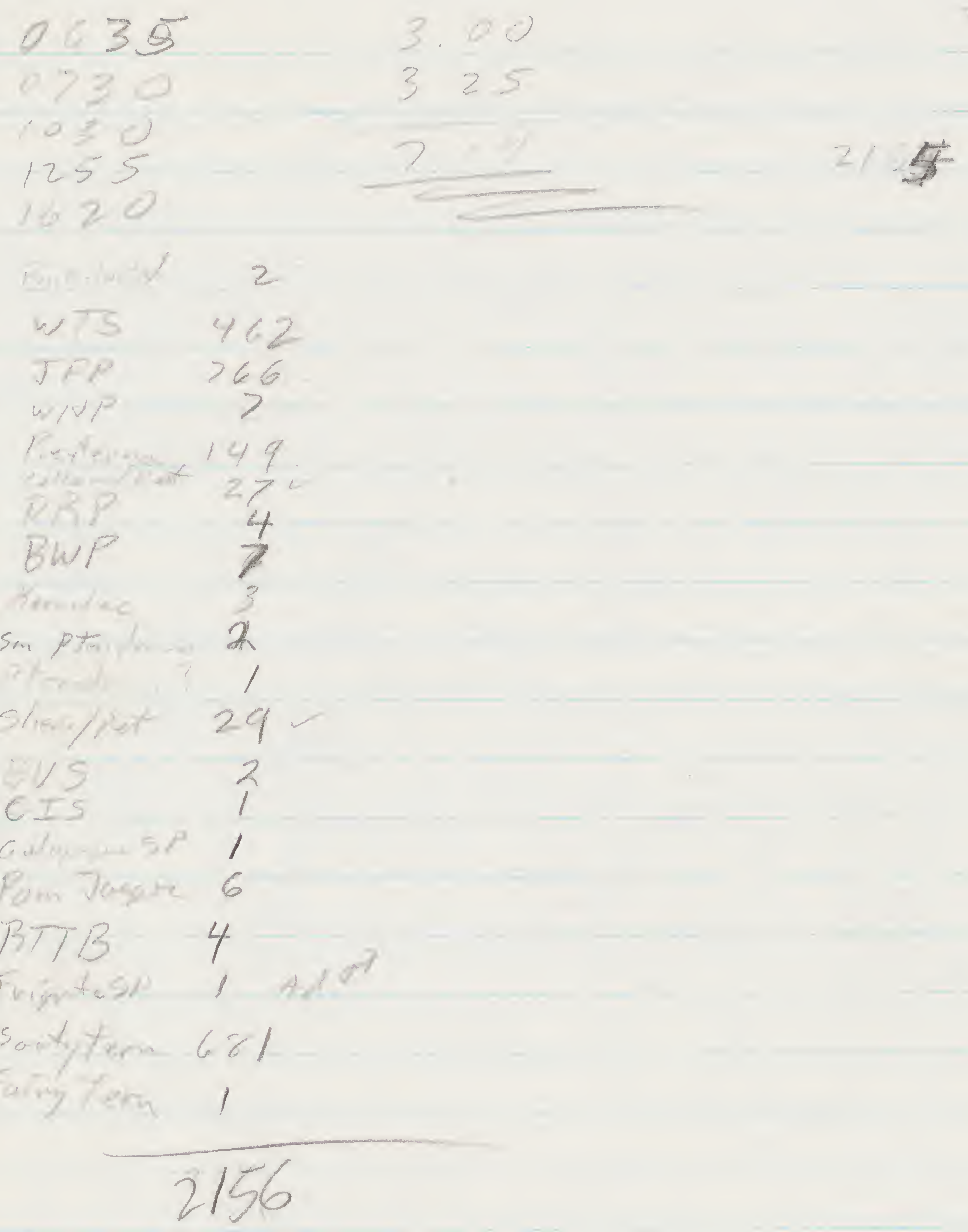




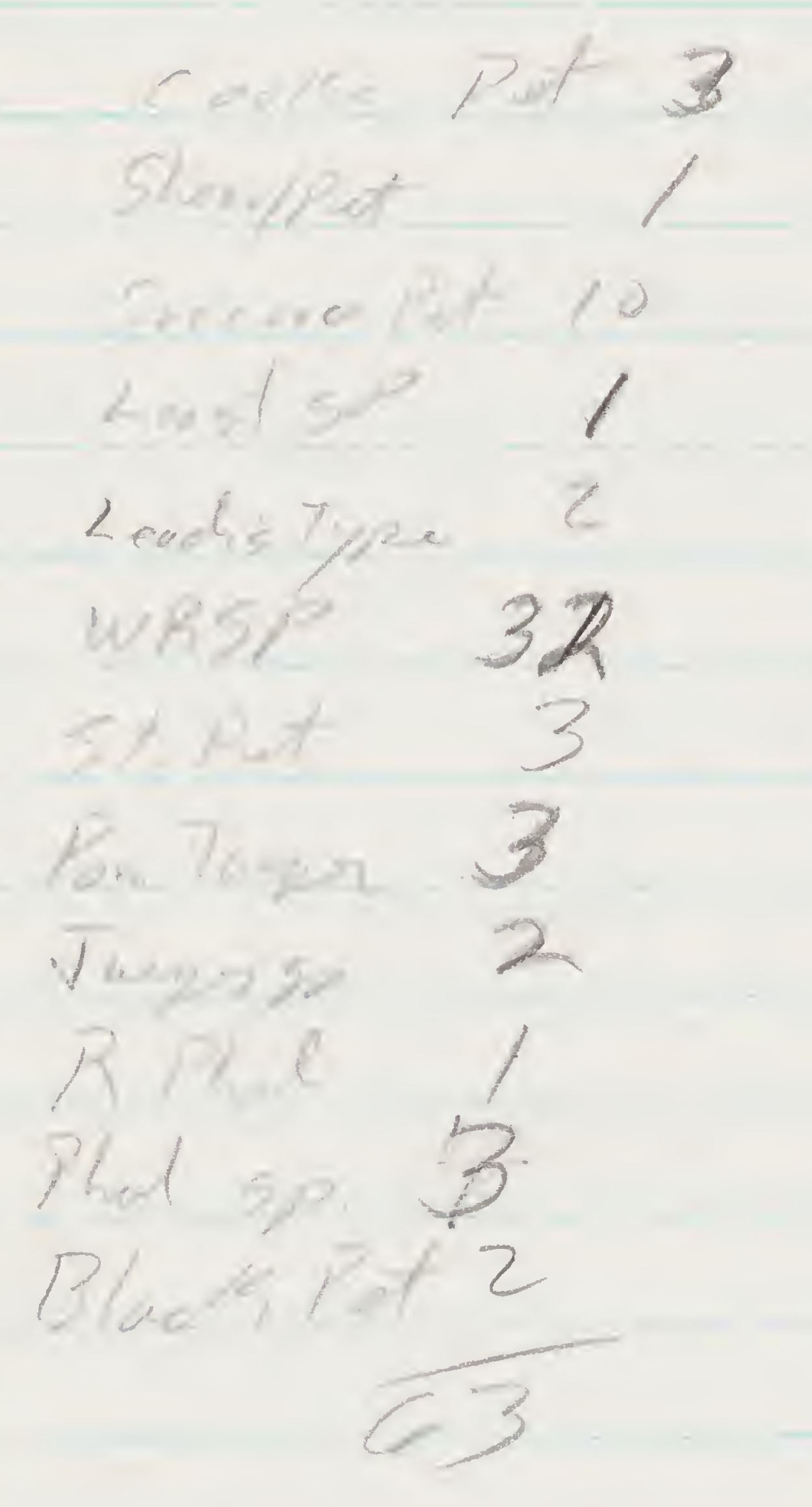


<smiles>C=CC</smiles>

$$
\begin{aligned}
& \text { Sowe Pet } 12 \\
& \text { Blow pt ! } \\
& \text { St. Ret ! }
\end{aligned}
$$

Mollands. 50t

Hewarg 2

Shuseletis 17

wosterali $\frac{1}{24}$ 
Thumas Washng ton

Eastropar. Fel -Apuit 68

\begin{tabular}{|c|c|c|c|c|c|}
\hline 16 Fol 68 & $29^{\circ} 00^{\prime} \mathrm{N}=117^{\circ} 26 \mathrm{~W}$ & 10.3 & 112 & 108 & 0.96 \\
\hline 7 & $24^{\circ} 28^{\prime} v=11^{\circ} 46^{\prime} \omega$ & 9.9 & 112 & 65 & .58 \\
\hline 18 & $21^{\circ} 34^{\prime} \mathrm{V}=118^{\circ} 08^{\circ} \mathrm{W}$ & 5.8 & 61 & 12 & .19 \\
\hline & $19^{\circ} 16^{\prime} N=118^{\circ} 52^{\prime} \omega$ & 7.1 & 75 & 19 & 125 \\
\hline 0 & $14^{\circ} 29^{\prime} \mathrm{N}-118^{\circ} 59^{\prime} \mathrm{W}$ & 7.1 & 80 & 18 & .22 \\
\hline I & $13^{\circ} 43^{\prime} N=119^{\circ} 00^{\circ}$ & 6.7 & 76 & 33 & .43 \\
\hline 2 & $11^{\circ} \mathrm{OIN}^{\prime}=118^{\circ} 53^{\prime}$ & 2. 3 & 75 & 96 & 1.28 \\
\hline 3 & $8^{\circ} 09^{\prime} V-1185^{\circ}$ & 6.4 & 71 & 64 & .90 \\
\hline 4 & $5^{\circ} 32^{\prime} \mathrm{N}-118^{\circ} 36^{\prime}$ & 5.6 & 56 & 61 & 1.09 \\
\hline 23 & $2^{\circ} 44^{\prime} \mathrm{V}=118^{\circ} 56^{\prime}$ & 8.0 & 83 & 16 & .19 \\
\hline 26 & $0^{\circ} 015=118^{\circ} 54$ & 6.3 & 21 & 7 & .10 \\
\hline 2.7 & $0^{\circ} 31^{\prime} 1^{\prime}=118^{\circ} 50^{\circ}$ & 6.2 & 70 & 13 & .18 \\
\hline 28 & $5^{\circ} 10^{\prime}=119^{\circ}$ of & 7.7 & 84 & 158 & 1.95 \\
\hline & $8^{\circ} 04^{\prime} 5 \sim 118^{\circ} 55^{\circ}$ & 6.3 & 67 & 3 & .04 \\
\hline Moud 68 & $10^{\circ} 48^{\prime} 5-118^{\circ} 56$ & 7.3 & 29 & 20 & .25 \\
\hline$\chi$ & $13^{\circ} \circ 7^{\prime} 5^{\prime} \sim 119^{\circ} \circ 2^{\prime}$ & 7.2 & 85 & 29 & .34 \\
\hline 7 & $16^{\circ} 025^{\circ}=118^{\circ} 59^{\prime}$ & 8.0 & 86 & 23 & .29 \\
\hline & $18^{\circ} 315^{\prime} \sim 118^{\circ} 56$ & 7.4 & 84 & 10 & .11 \\
\hline & $20^{\circ} 00^{\prime} S^{\prime} \sim 17^{\circ} 26^{\prime} \omega$ & 9.0 & 86 & 7 & .08 \\
\hline & $19^{\circ} 58^{\prime} 5 \sim 114^{\circ} 06^{\prime} \dot{w}$ & 9.6 & 89 & 3 & .033 \\
\hline & $19^{\circ} 2 / s^{\prime}=111^{\prime} 55^{\prime}$ & 5.7 & 57 & 6 & .10 \\
\hline & $1734^{\prime} 5 \sim 11154^{\prime}$ & 5.9 & 63 & 50 & .79 \\
\hline & $14^{\circ} 60^{\prime} 5=11^{\circ} 59$ & 4.2 & 72 & 9 & .125 \\
\hline & $1117{ }^{\circ}=111,55$ & 7.5 & 74 & 33 & .446 \\
\hline & $8^{\circ} 295 \sim 11^{\circ} 56$ & $7 \cdot 1$ & $7 ?$ & 12 & .155 \\
\hline & $6^{\circ} 3$ is $2109^{\circ} 12^{\prime}$ & 11.0 & $\begin{array}{l}107 \\
99\end{array}$ & 9 & $\begin{array}{l}1084 \\
474\end{array}$ \\
\hline & $5225=10557$ & 10.6 & 9.9 & 4 & .474 \\
\hline & $\begin{array}{l}4^{\circ} 33^{\circ} 5=10311 \\
3^{\circ} 35^{\prime} 5=100^{\circ} 05\end{array}$ & $\begin{array}{l}11.3 \\
10.2\end{array}$ & 99 & $\begin{array}{l}18 \\
199\end{array}$ & $\begin{array}{l}1.098 \\
2.01\end{array}$ \\
\hline & $2^{\circ} 30^{\prime} 5=96^{\prime} 12^{\prime}$ & 11,2 & 109 & $1 / 3$ & 1.036 \\
\hline 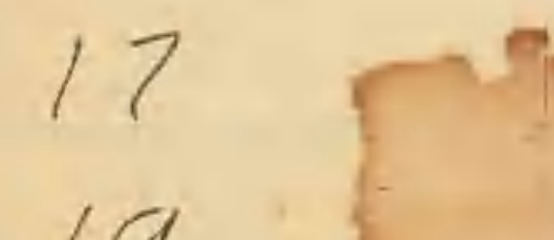 & $1355 \sim 92,12$ & 11.7 & 118 & 100 & .842 \\
\hline & $6^{\circ} 46^{\prime} 5=95^{\circ} 08$ & $\begin{array}{l}10.2 \\
10.6\end{array}$ & $\begin{array}{l}121 \\
112\end{array}$ & 216 & $\begin{array}{r}693 \\
192\end{array}$ \\
\hline 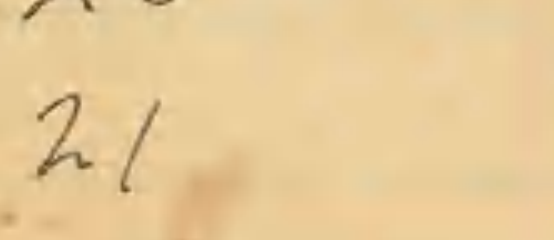 & $9^{\circ} 54^{\prime} 5 \sim 97^{\circ} 56$ & 6.4 & 73 & 229 & 3.137 \\
\hline
\end{tabular}




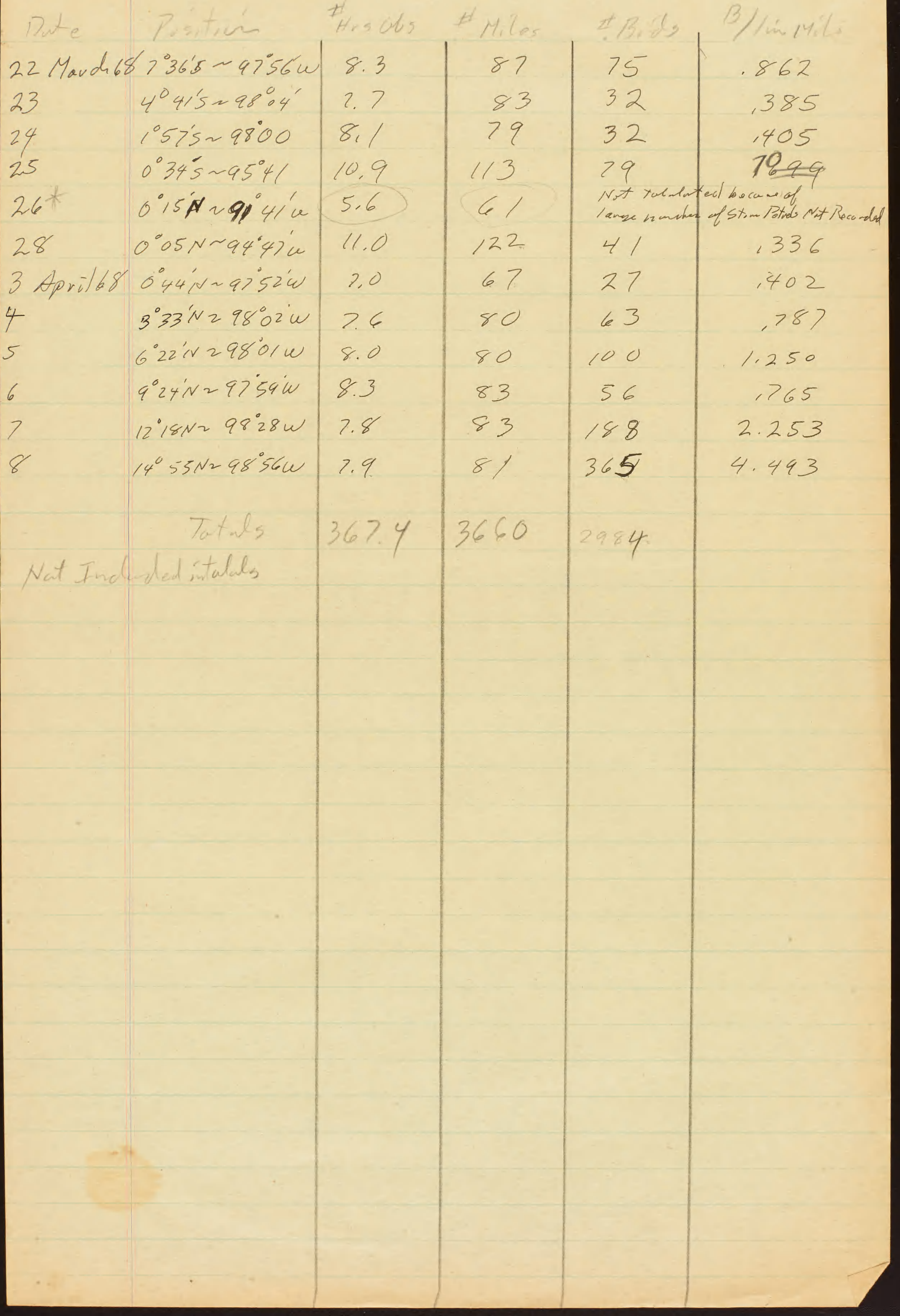




$$
\begin{aligned}
& \text { Wooster, Warren S. Dr. Screistist Incharge SFO } \\
& \text { Bremnan, Robent E. Marion Techinicion SFO } \\
& \text { Forsbergh, Eviap. Associate Scientist, IATTC } \\
& \text { De Alvere 3, Saul Student, Institute of } \\
& \text { Marimé Sciences, Ensenada, Mexico } \\
& \text { Graham, Jerry Blectronics tech, SFO } \\
& \text { Frani, Rustam H. Maviwe techuicion } 570 \\
& \text { Tones, James H. Researd Assistat SFO }
\end{aligned}
$$

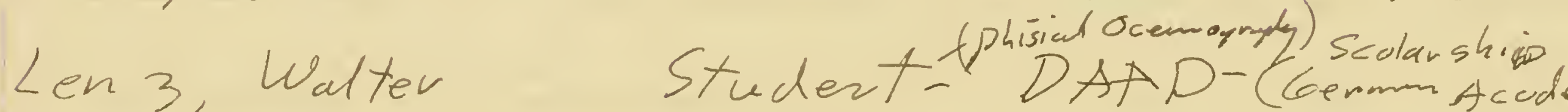

$$
\begin{aligned}
& \text { Lewis, Thomas Vunes Biolorist } \\
& \text { Lynn, Bonald J. Oceanographer BCF } \\
& \text { Maucls, Willion W. Mavine Techricion } 570 \\
& \text { Nishimoto, Bubeut, Biologist BCF } \\
& \text { Whitert, Wayne Technician BCF } \\
& \text { Wong, chi Shing student SEO }
\end{aligned}
$$


Weather - zerof on do if timin

Plot cruace traclat Paily Positions

Rayh in Ocenogmphic featues troverad

Make vayk Salmity. Nutrialt, t Tersp groxles

Plat B/limmi, Overcell, t some Fudicidah speries.

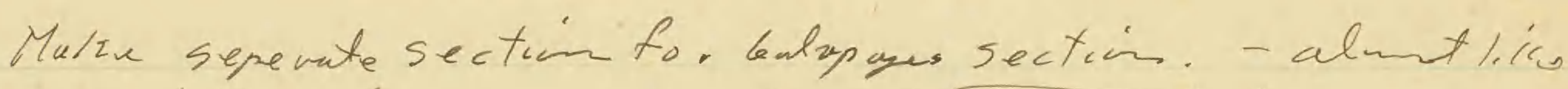
Pasund Repunt.

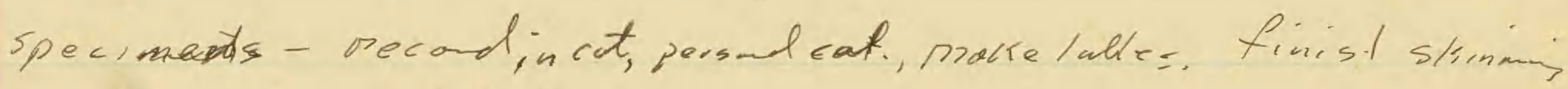
conphete-Mokeen.

hetters To.

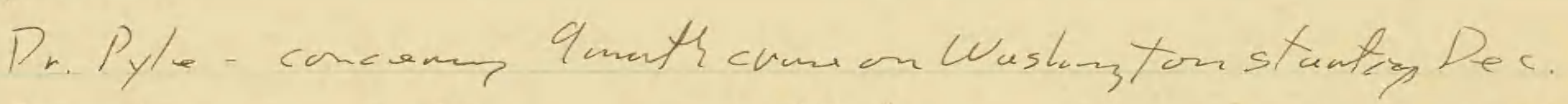

Pater Harper - Smittsmin Furstitution - Has woured on bieds in santwend of Pruvean Curvent. Also whet Dota-sperimins et reednd form off shore arean of S. Aneviciu

P. Bown possibiluty of loned salud.

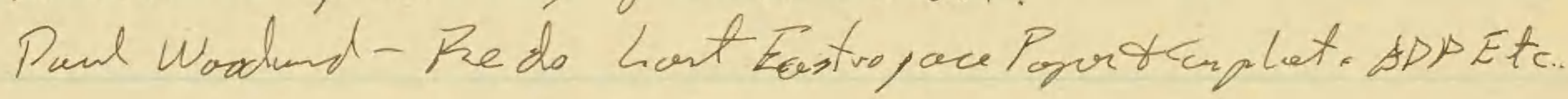

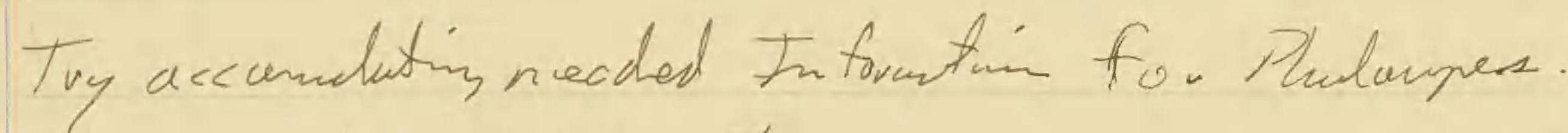

Leam molt natutain ets

Letter of thath to Dr. Wouter. + Copt. Fevris

tist of Rersmal. complated. 


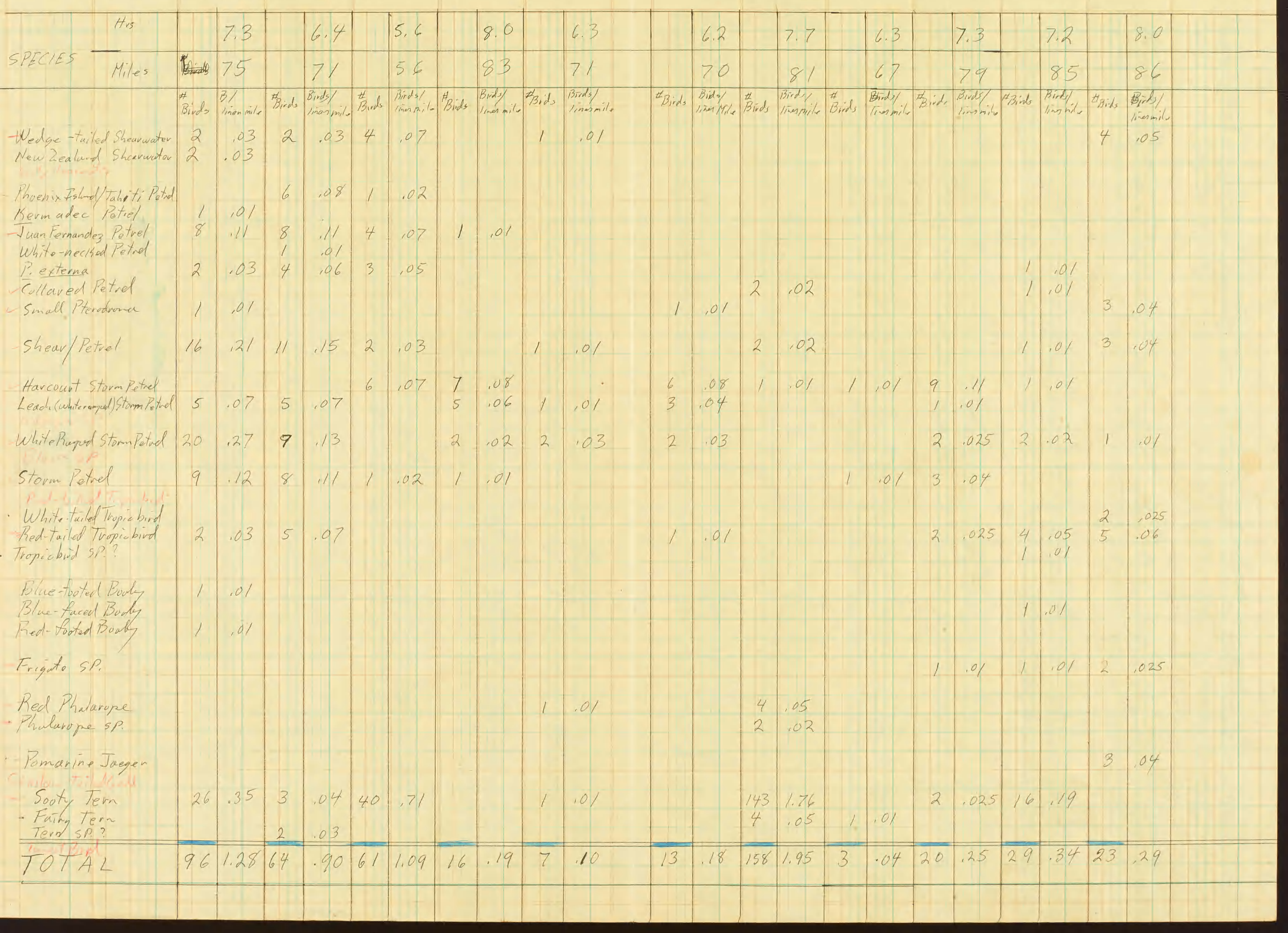




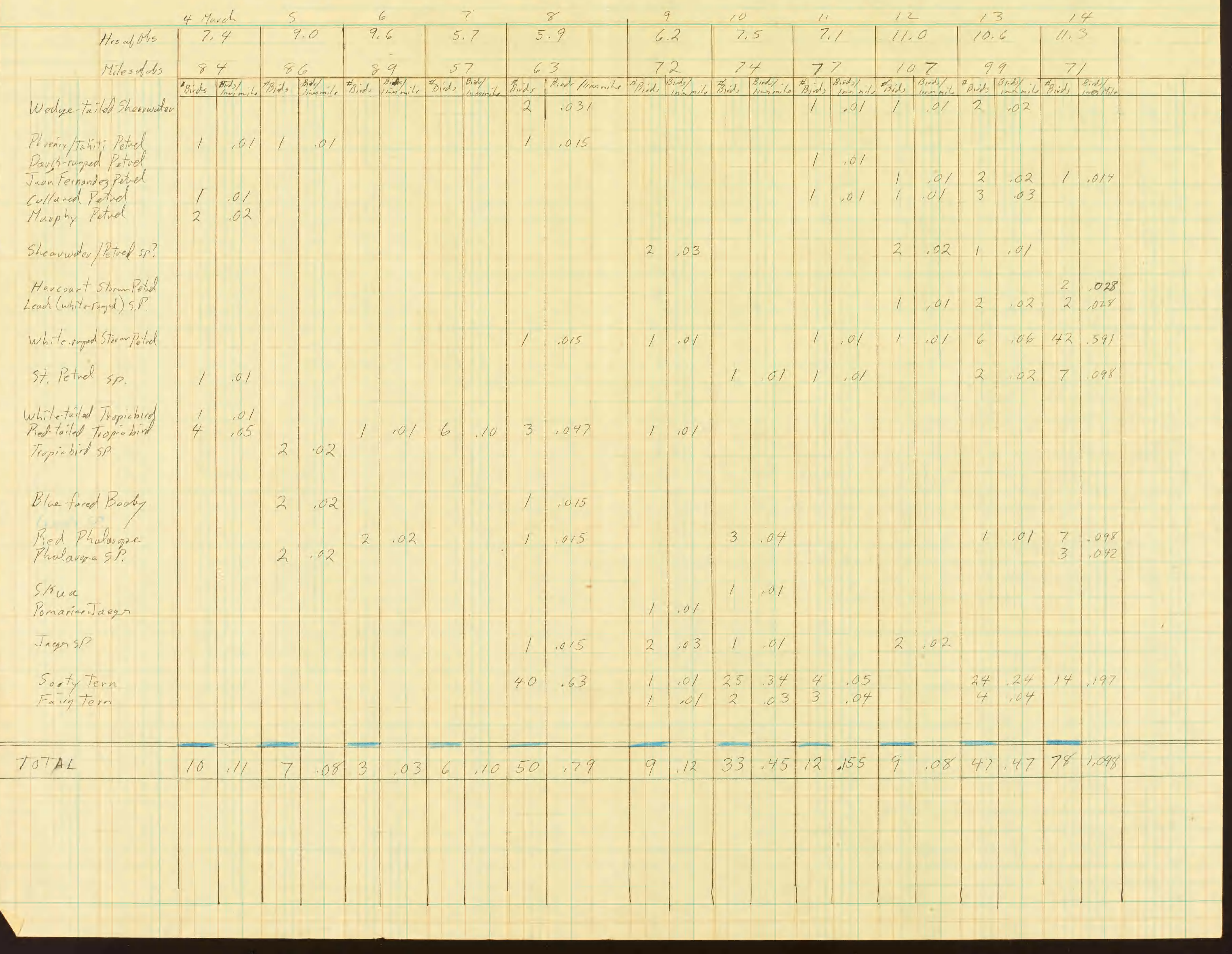





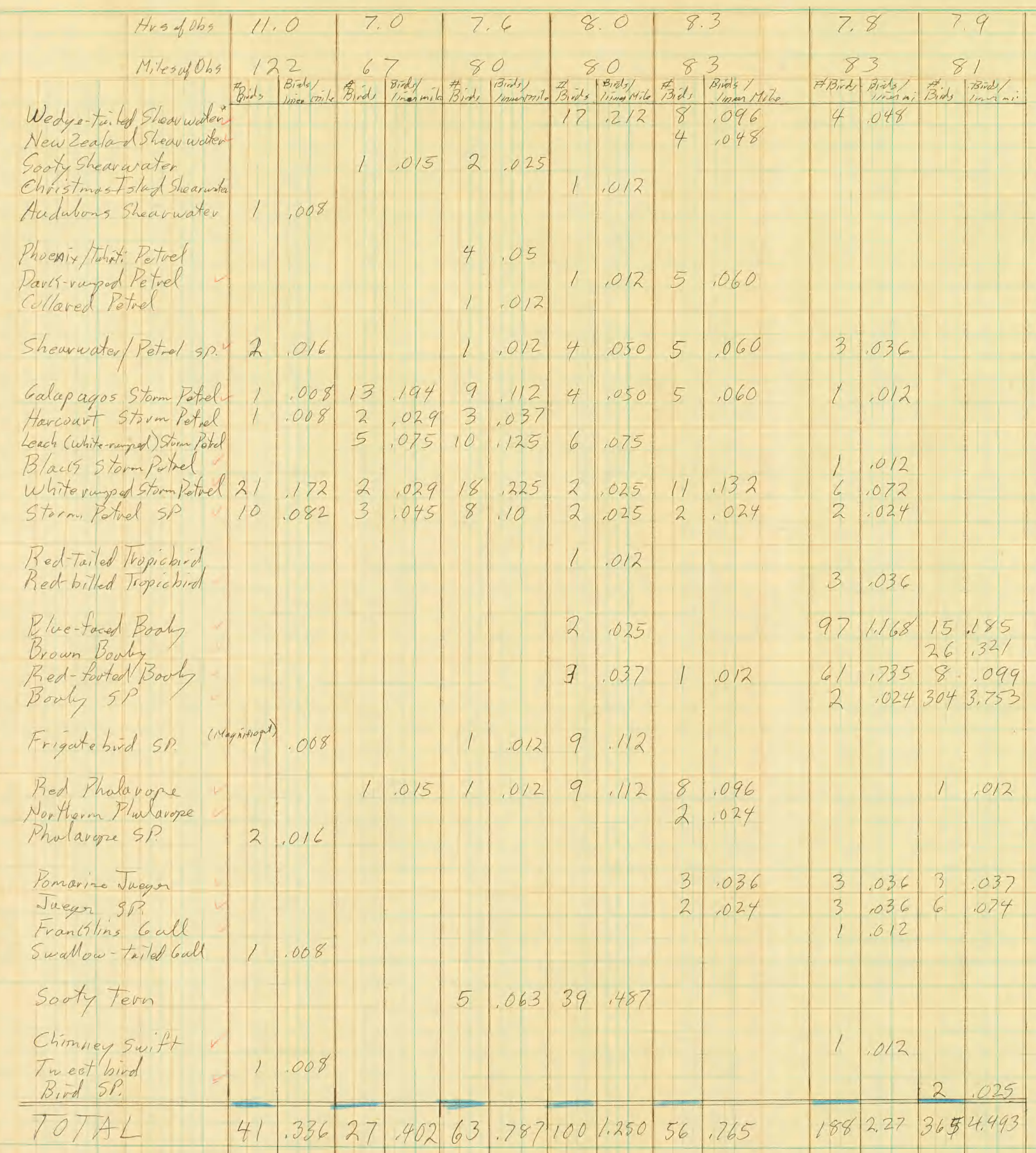




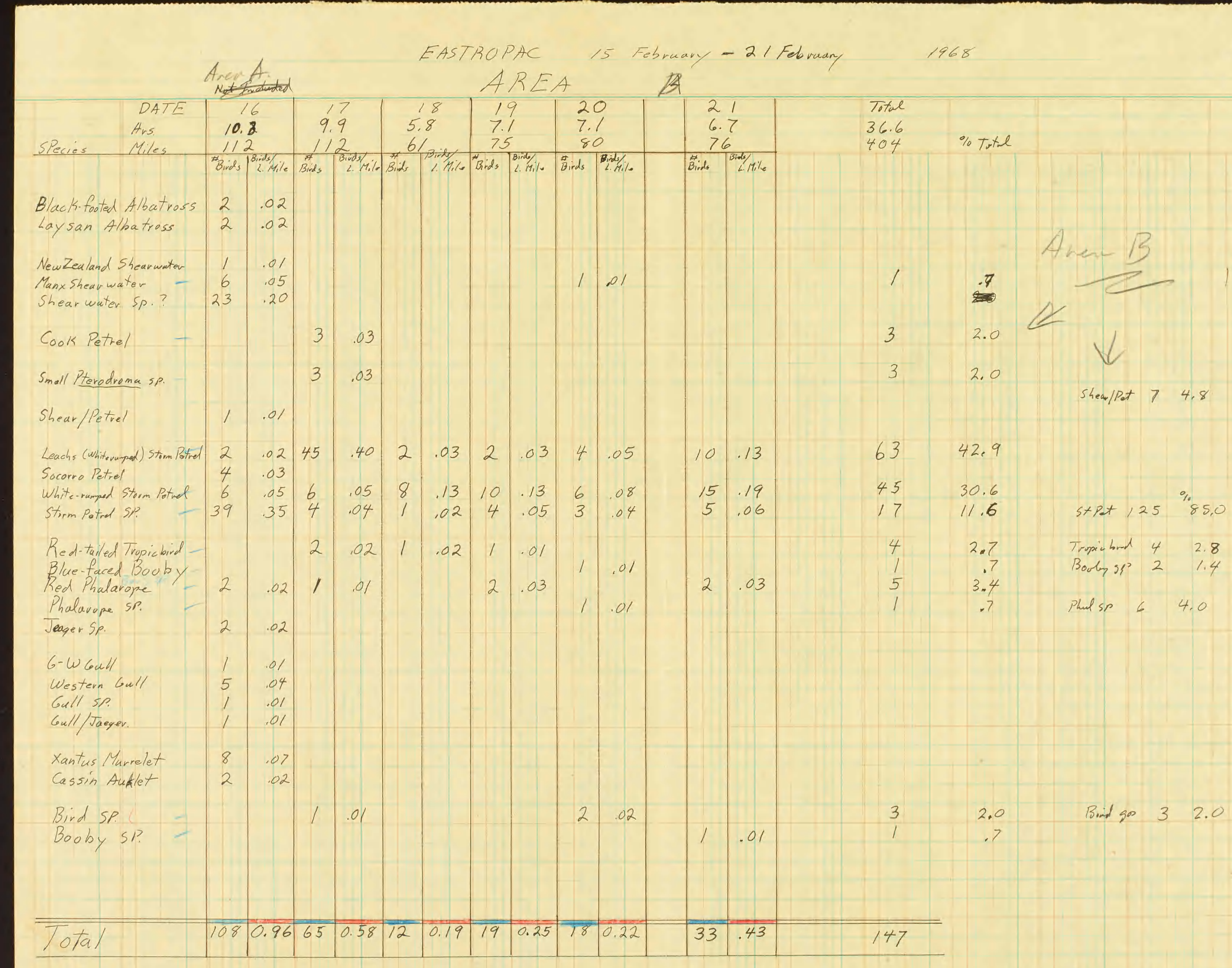




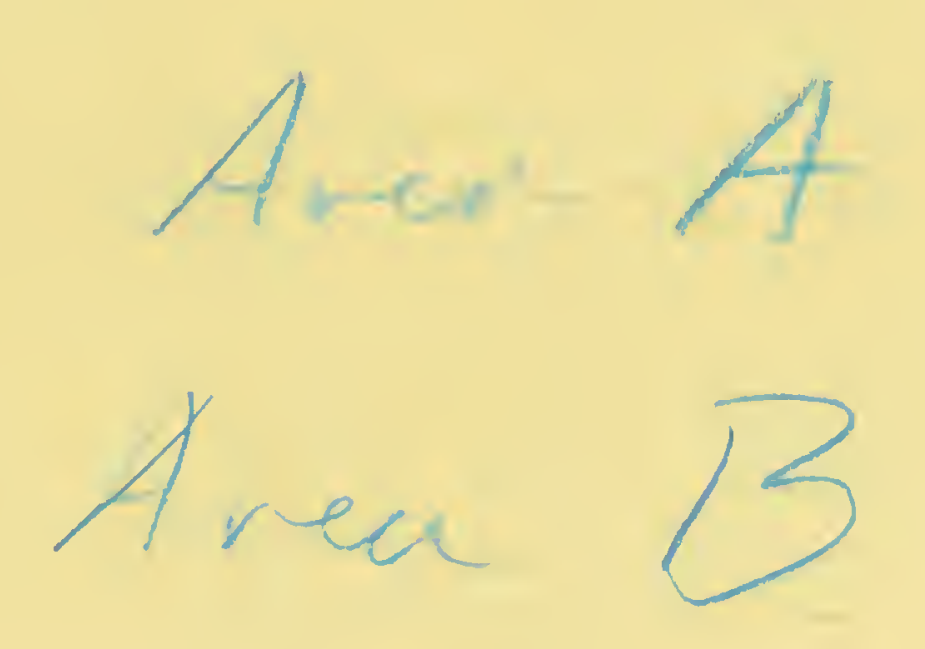


Area C

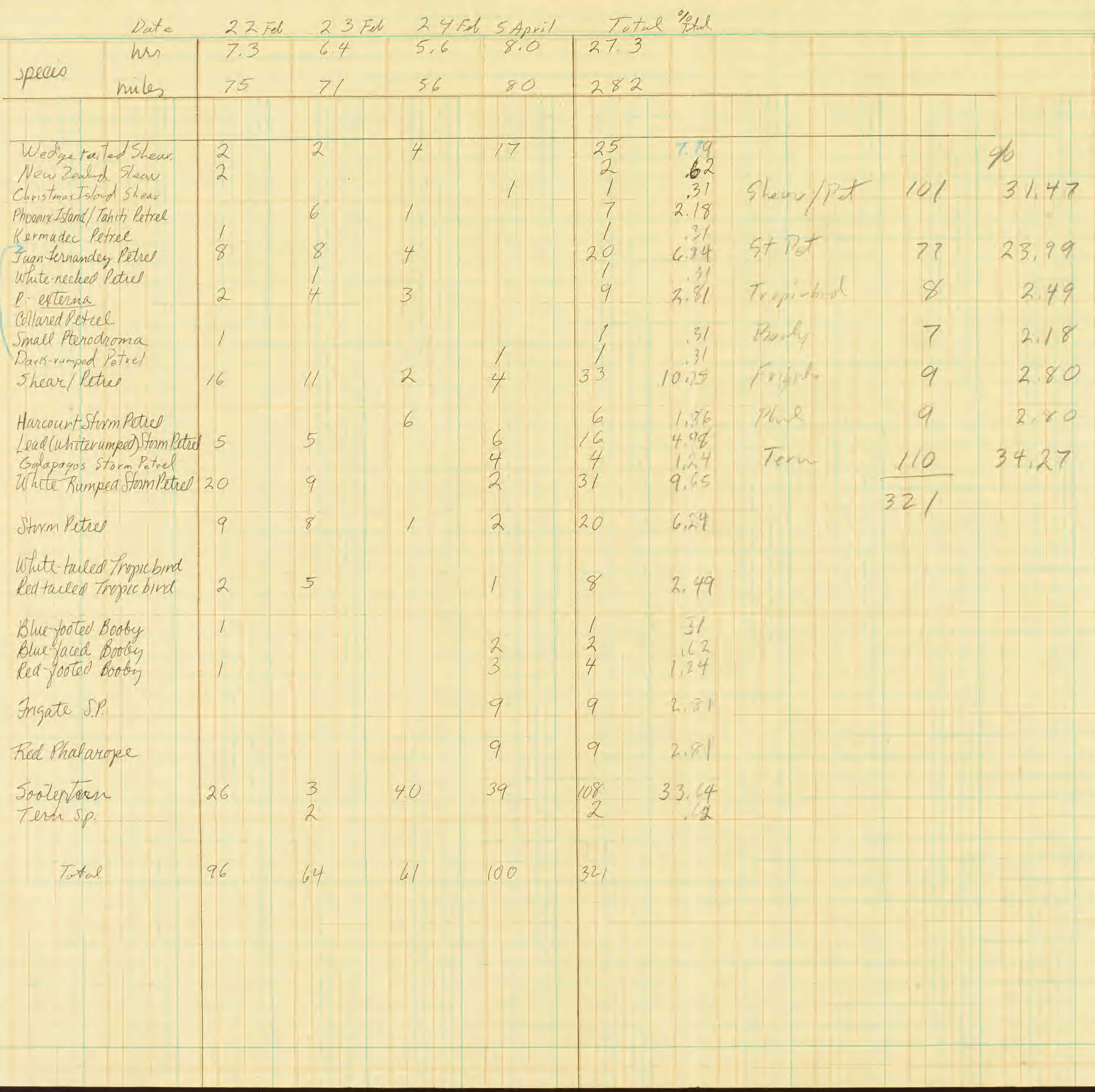




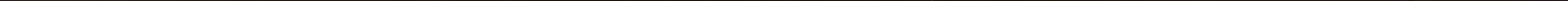




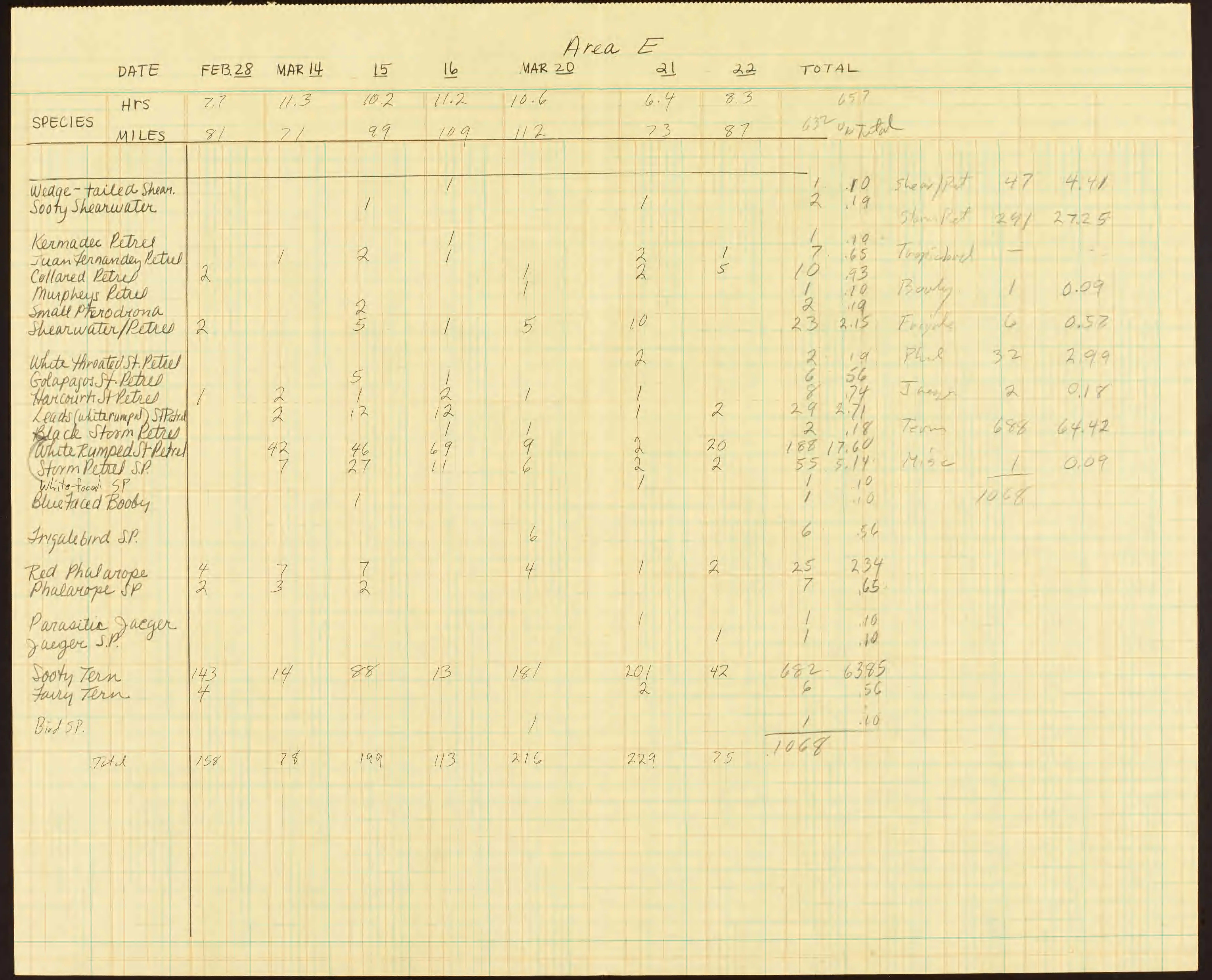




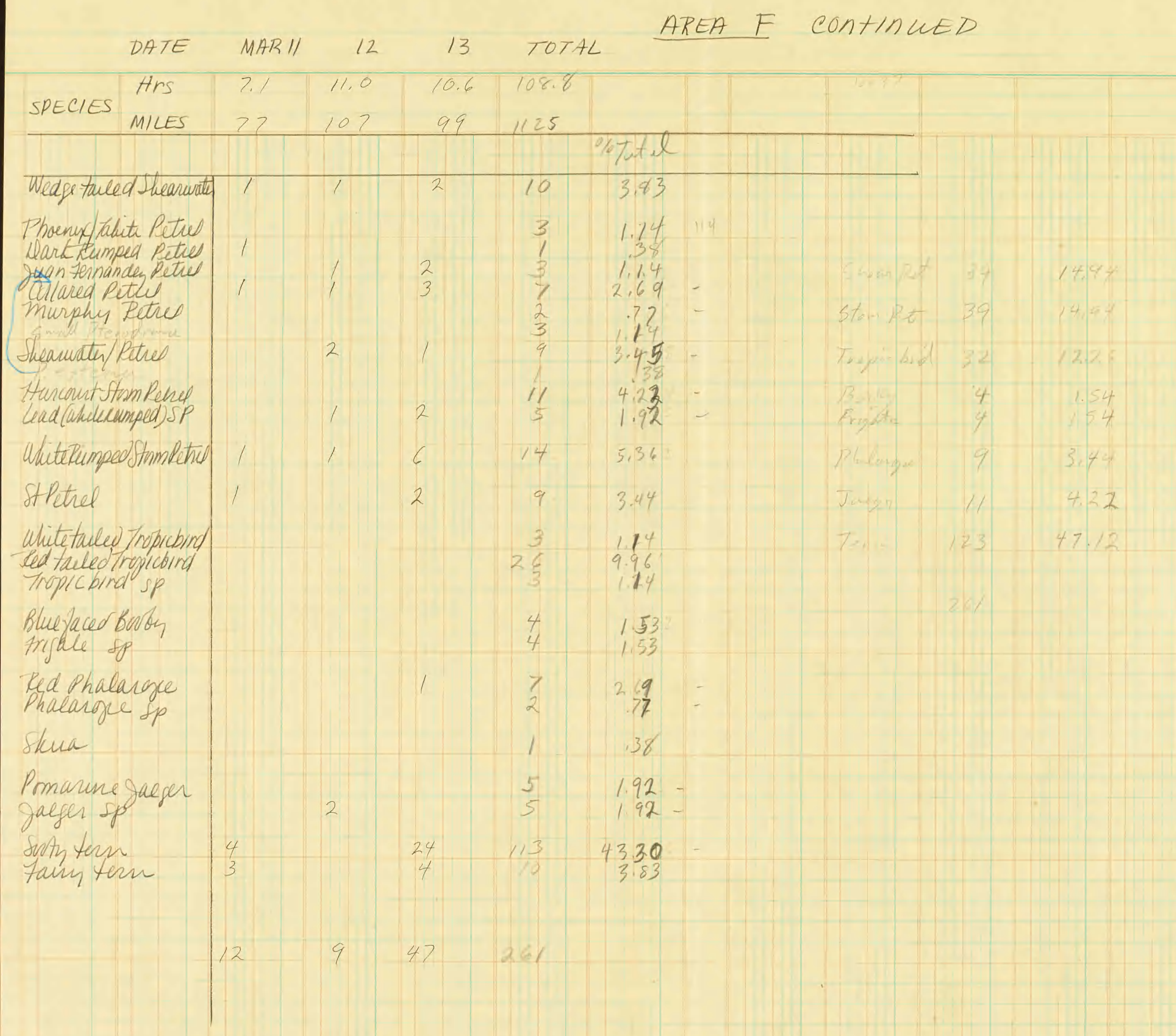




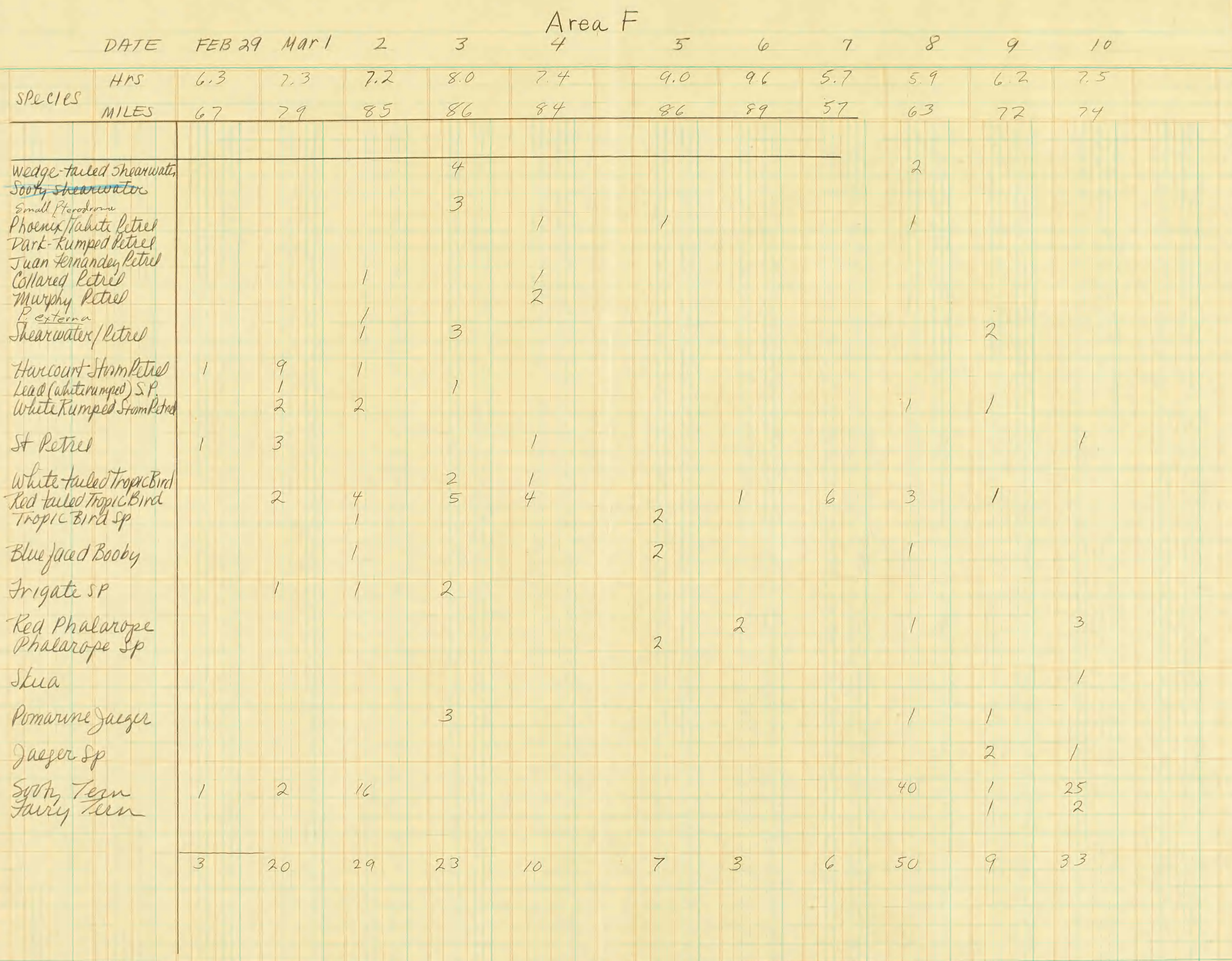





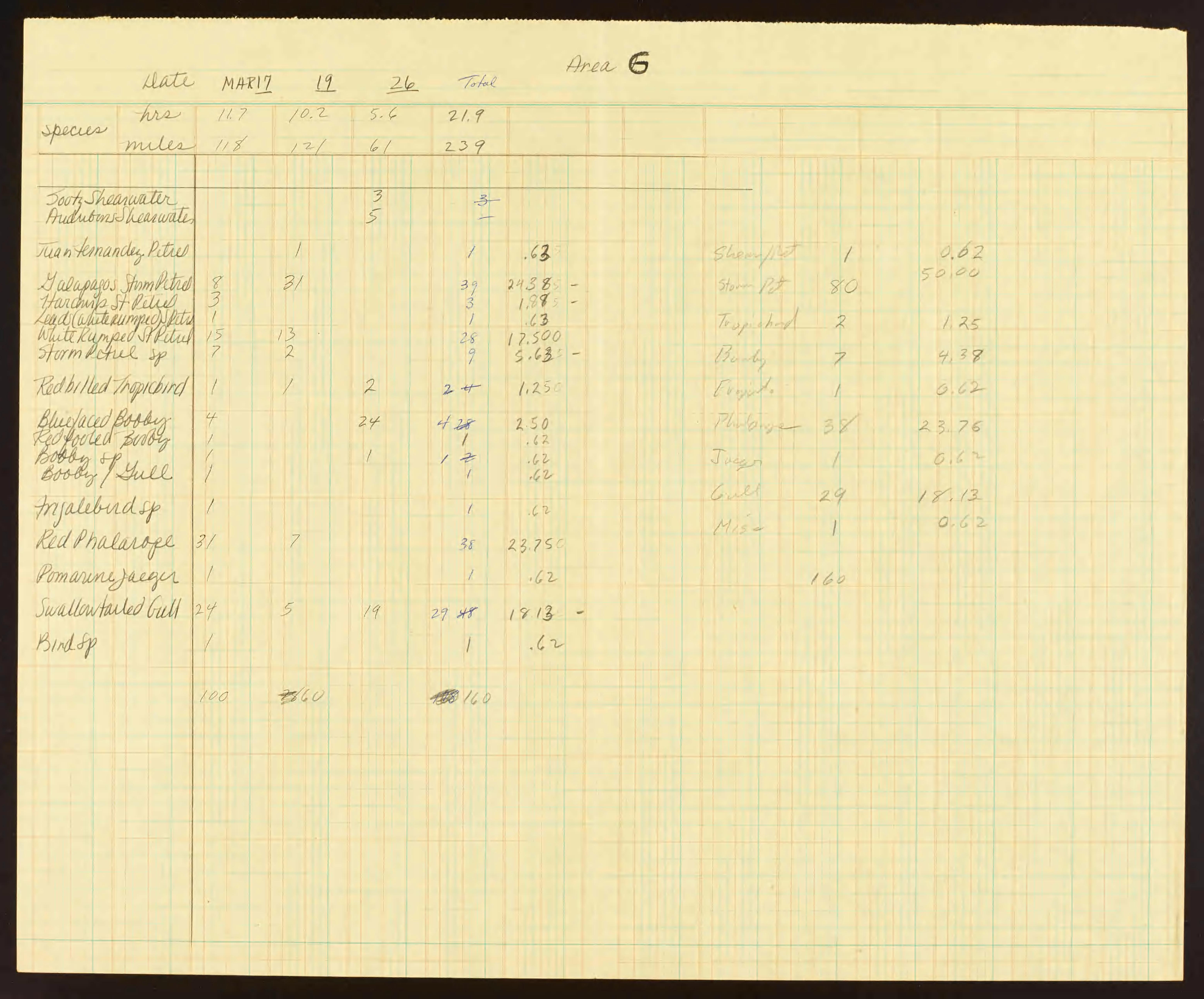




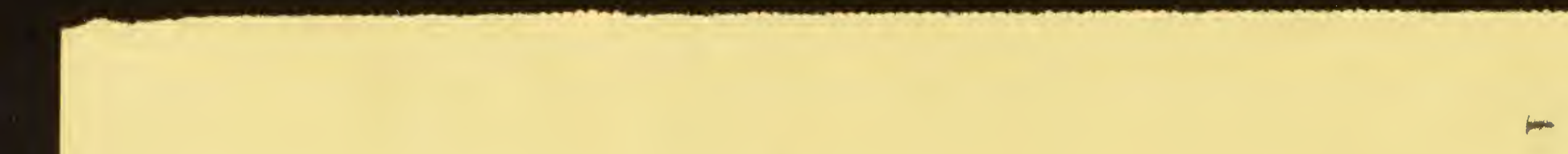




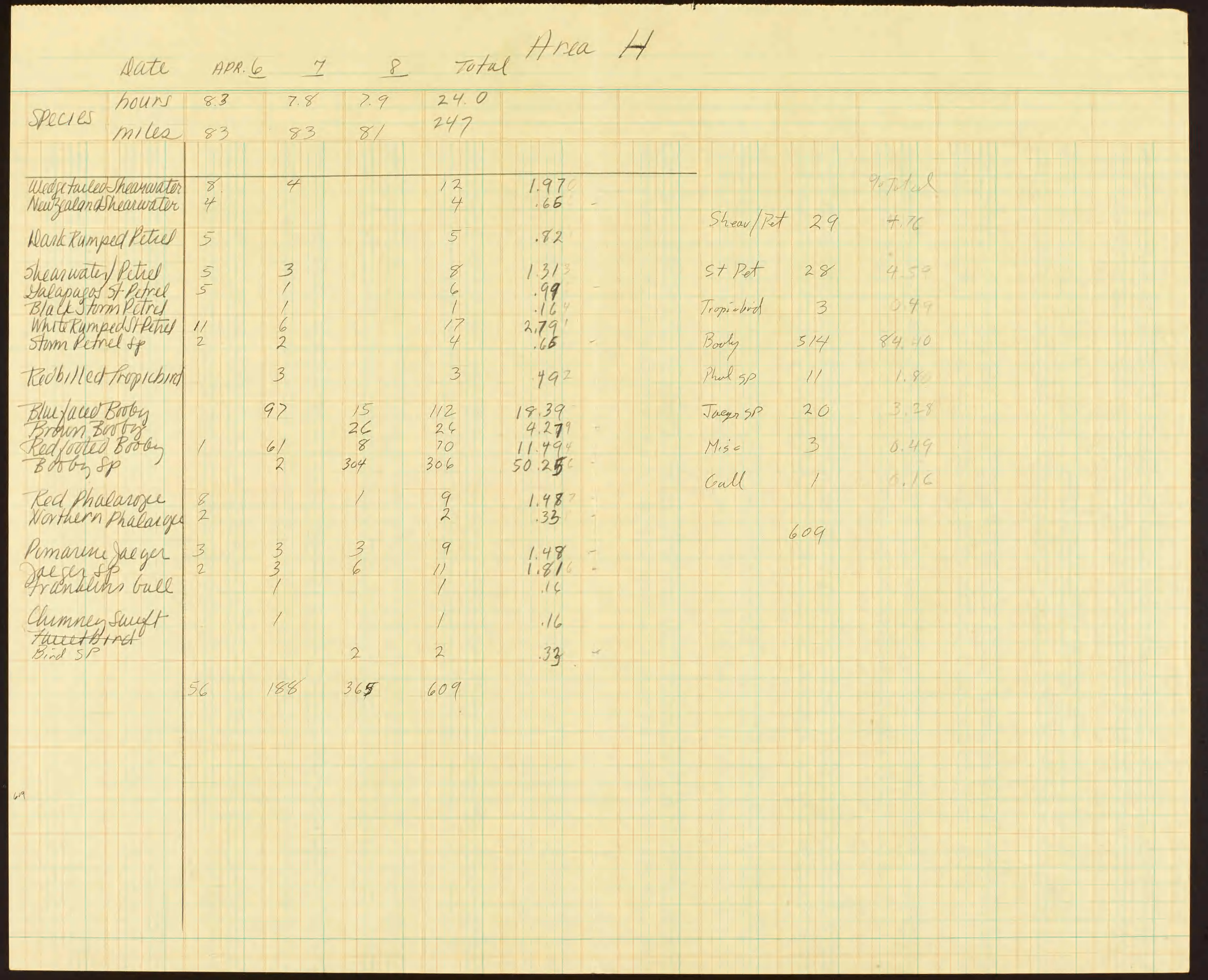





$$
\text { Abundonce of Specie= Gromss by Areas }
$$

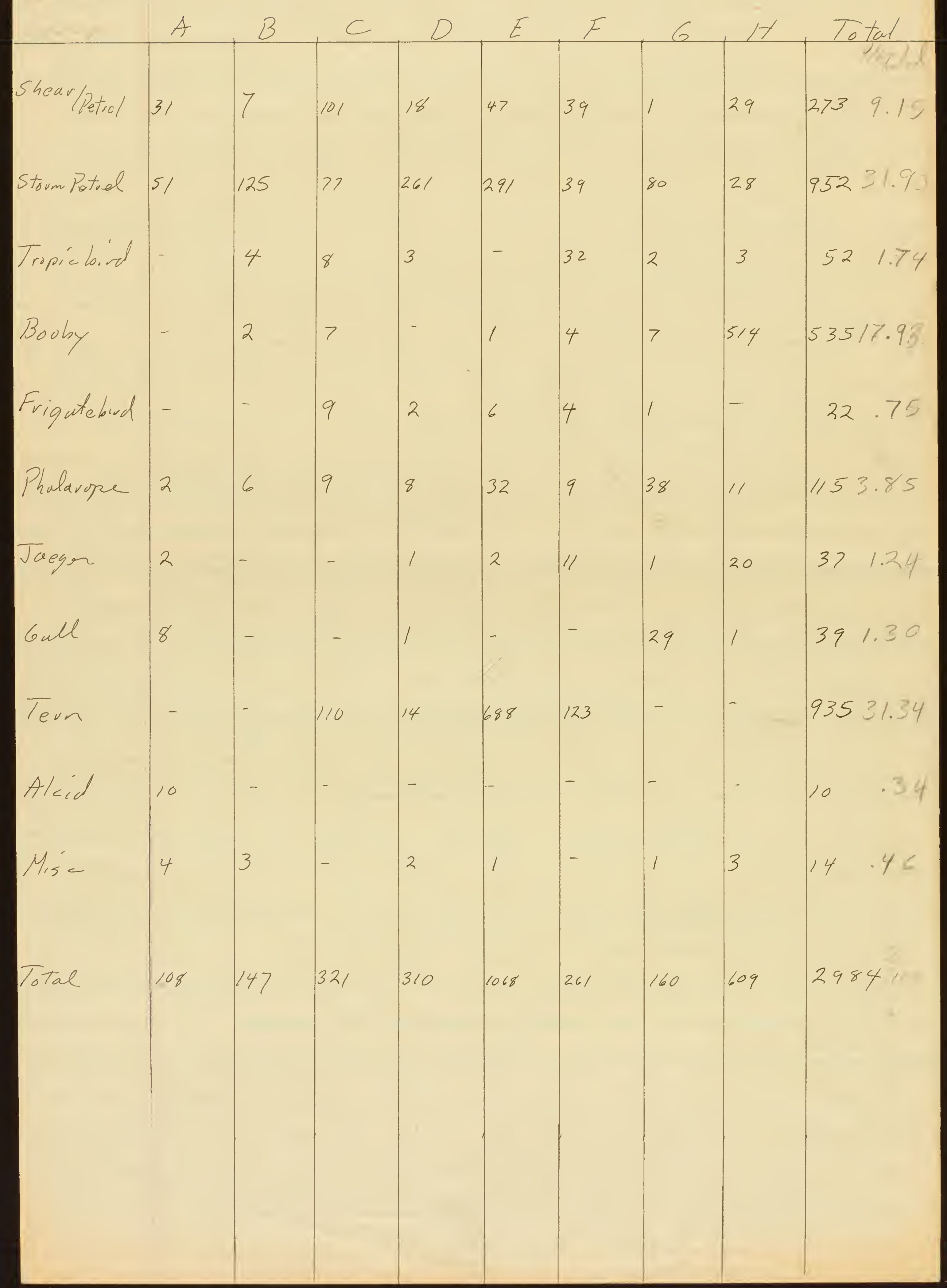

